\title{
APET Methodology for Defense Waste Processing Facility: Mode C Operation
}

by

R. P. Taylor, Jr

Westinghouse Savannah River Company

Savannah River Site

Aiken, South Carolina 29808

This paper was prepared in connection with work done under the above contract number with the U. S.

Department of Energy. By acceptance of this paper, the publisher and/or recipient acknowledges the U. S. Government's right to retain a nonexclusive, royalty-free license in and to any copyright covering this paper, along with the right to reproduce and to authorize others to reproduce all or part of the copyrighted paper. 


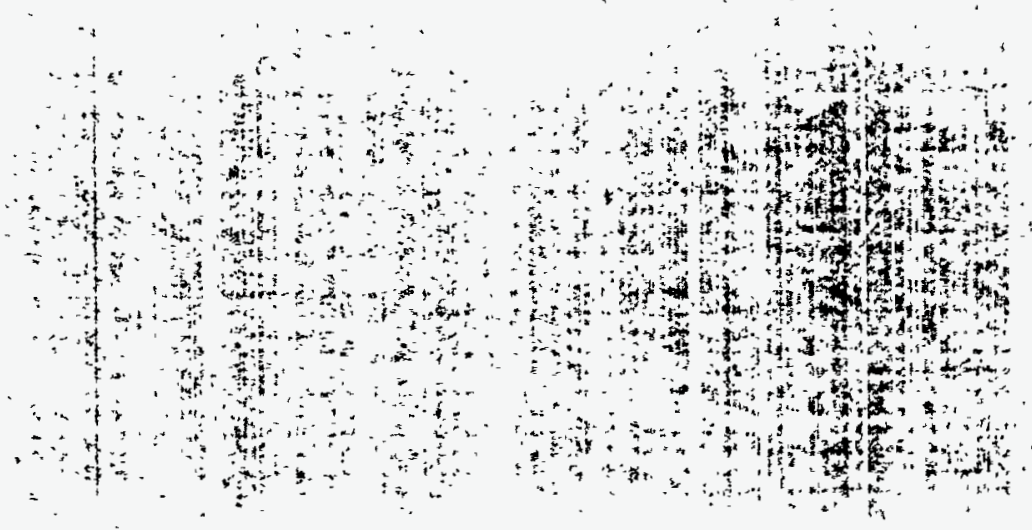

\section{DISCLATMER}

This report was preparsd as an account of work sponsored by an agency of the United States Government. Neither the United States Government nor any agency thereof, nor any of their employees, makes any warranty, express or implied, or assumes any legal liability or responsibility for the accuracy, completeness, or usefulness of any information, apparatus, product, or prscess disclosed, or represents that its use would not infringe privately owned rights. Reference herein to any specific commercial product, process, or service by trade name, trademark, manufacturer, or otherwise does not necessarily constitute or imply its endorsement, recomrnendation, or favoring by the United States Government or any agency thereof. The views and opinions of authors expressed herein do not necessarily state or reflect those of the United States Government or any agency thereof.

This report has been reproduced directly from the best available copy.

Available to DOE and DOE contractors from the Office of Scientific and Technical Information, P.O. Box 62, Oak Ridge, TN 37831; prices available from (615) 576-8401.

Available to the public from the National Technical Information Service, U.S. Department of Commerce; 5285 Port Royal Road, Springfield, VA 22161. 


\section{DISCLAIMER}

Portions of this document may be illegible in electronic image products. Images are produced from the best available original document. 
WSRC-TR-95-01.13

SAFETY ENGINEERING DEPARTMENT

KEYWORDS:

DWPF

Progression

Accident Analyses

Probabilistic

\section{APET METHODOLOGY FOR DEFENSE WASTE PROCESSING FACILITY: MODE C OPERATION}

R. P. Taylor, Jr

William M. Massey

Issued: April 1995

Approvals:

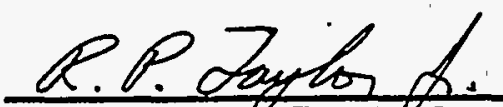

R. P. Taylor, Jr. Alathor

Risk Analysis Group

M Mpasen

W. M. Massey Author

DBA/Phenomena Analysis Group

Dawt.R. Rradel

D. R. Bradley, Technical Refewer

Integrated Safety Assessment Division, SAIC

Deflazs

D. A. Sharp, Manager

Risk Analysis Group

La Nooten

L. A. Wooten, Manager

DBA/Phenomena Analysis Group

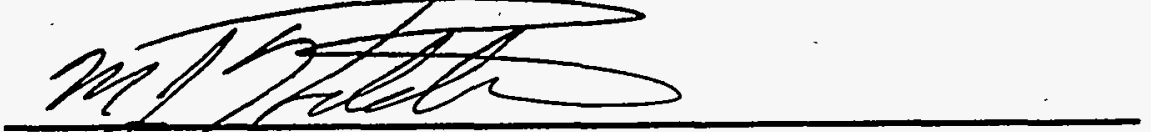

M. 1. Hitchler, Manager

SALS

Westinghouse Savannah River Company

Engineering and Construction Services Division

Aiken, SC 29808

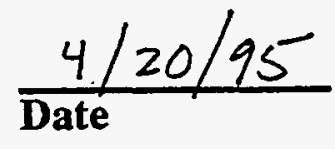

$\frac{4 / 20 / 95}{\text { Date }}$

$\frac{3-28-95}{\text { Date }}$

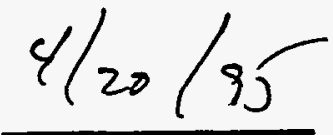

Date
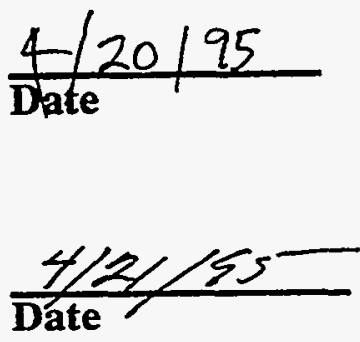


\begin{abstract}
The Savannah River Site [SRS] Defense Waste Processing Facility [DWPF] is a facility designed to convert the waste currently stored in tanks at the 200-Area tank farm into a form that is suitable for long term storage in engineered surface facilities and ultimately geologic isolation. The DWPF is currently undergoing cold chemical runs and some limited construction preparatory to hot startup in 1995. A Safety Analysis Report [SAR] has been prepared for the facility which describes postulated accidents and consequences of operation. [1] The usual practice in preparation of Safety Analysis Reports is to include a conservative analysis of certain 'design basis accidents'.

The methodology used to determine the risk of operation of the DWPF borrows heavily from methods applied to the Probabilistic Safety Assessment of SRS reactors and to some commercial reactors.[2] Risk of operation is associated with the breach of confinement barriers. The primary barrier to the release of fission products is the vessel or piping containing the waste that is being processed. The remaining barrier to the release of fission products is the confinement system which includes the buildings housing the process and the filter/ventilation systems. The potential consequences of an accident in the DWPF is determined by the systematic tracking of the progression of the accident in terms of the extent of damage to the confinement barriers. Accident progression analysis involves the application of logic models, supplemented by mechanistic evaluations, to track possible accident sequences from the initiating event, through damage to the primary confinement barrier and to the final state of the confinement system. The principal goal of the analysis is to evaluate the success of the confinement system in mitigating the release of fission products to the environment.
\end{abstract}

The Accident Progression Event Tree or APET, provides a probabilistic representation of potential sequences along which an accident may progress. The fundamental objective of the APET is to evaluate the success of the confinement system in mitigating releases. The APET consists of questions that are phenomenologically oriented and address important aspects of. accident progression as well as questions that address the integrity and performance of the confinement system. Each question in the APET is analogous to a top event in a classical event tree. The possible answers to the questions are specified in terms of two or more discrete outcomes represented as branches. The probability that a particular branch is taken is evaluated based on the answers to previous questions [i.e., accident progression is sequence dependent] and on mechanistic analysis of accident progression and confinement response.

Use of the EVNTRE/APET for modeling of accidents in the DWPF involves a significant departure from the methodology developed for reactor analysis as described in Reference 2. The APET does describe the sequence of events that may occur following an accident initiating failure as in conventional EVNTRE/APET methodology. However, it also determines the frequency of certain internally initiated energetic events. This determination is made by analysis of the cut sets for fault trees of systems in which failure can cause energetic events, using the capability of EVNTRE to interchange information with an external FORTRAN program. 


\section{CONTENTS}

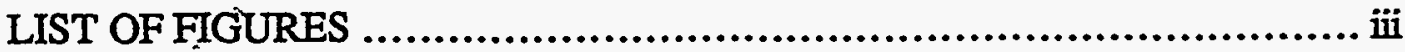

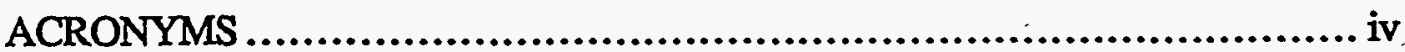

1. INTRODUCTION..................................................... 1

2. SUMMARY ............................................................ 1

2.1 The Accident Progression Event Tree..................................2

2.2 Quantification of the APET ...............................................2

3.0 SUMMARY DESCRIPTION OF THE DEFENSE WASTE PROCESSING FACILITIES ............................................

3.1 The Confinement System ............................................. 3

3.2 The Salt Process Cell............................................... 3

3.3 The Chemical Process Cell ............................................... 4

3.4 The Melter Cell......................................................5

3.5 Organic Waste Storage ...............................................6

3.6 The Low Point Pump Pit.......................................................6

4. THE ACCIDENT PROGRESSION EVENT TREE ........................6

4.1 Outcome Probabilities............................................6

4.2 Summary Outline of DWPF APET ..................................16

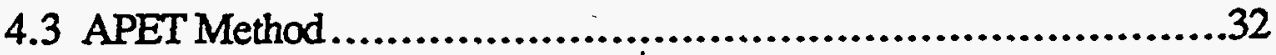

4.4 Explanation of the APET Questions .....................................33

5. THE SOURCE TERM BINNER ......................................207

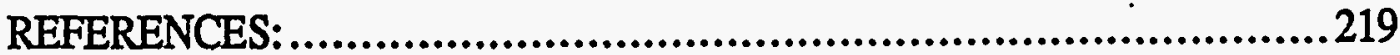

APPENDIX A: EXCEL Spreadsheet of the Accident Progression Event Tree

for Defense Waste Processing Facility: MODE C. 220 


\section{LIST OF FIGURES}

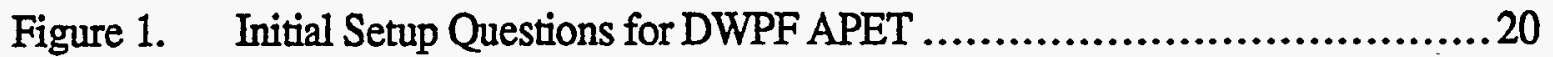

Figure 2. Internal Energetic Events...............................................21

Figure 3. Progressions in SPC and CPC ..........................................22

Figure 4. Progression in Melter, LPPP, OWST ...................................23.

Figure 5. Zone 1 Ventilation Failure Progression.................................................224

Figure 6. Seismic Progressions...................................................25

Figure 7. Seismic Progression in SPC and CPC ...................................26

Figure 8. Seismic Progression in Melter, LPPP and OWST .......................27,

Figure 9. ' Zone 1 Ventilation Failure Progression Seismic................................... 28

Figure. 10. C Crane Drop Accident Progressions ......................................29

Figure 11." Leak Progressions .......................................................... 30

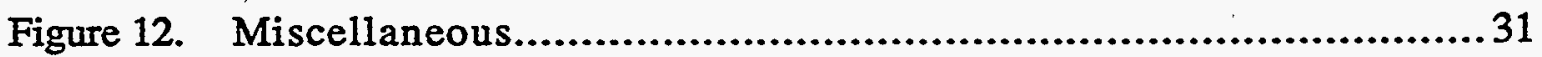




\section{ACRONYḾS .}

\begin{tabular}{|c|c|}
\hline APET & Accident Progression Event Tree \\
\hline $\mathrm{CPC}$ & Chemical Processing Cell \\
\hline $\mathrm{CPCV}$ & Chemical Process Cell Vent \\
\hline LFL & Lower Flammability Limit \\
\hline LPPP & Low Point Pump Pit \\
\hline MFT & Melter Feed Tank \\
\hline MOG & Melter Offgas System \\
\hline OEV & Organic Evaporator Vessel \\
\hline OECT & Organic Evaporator Condensate Tank \\
\hline OWST & Organic Waste Storage Tank \\
\hline PR & Precipitate Reactor \\
\hline PRFT & Precipitate Reactor Feed Tank \\
\hline PRBT & Precipitate Reactor Bottoms Tank \\
\hline PVVH & Process Vessel Vent Header \\
\hline PPPT & Pump Pit Precipitate Tank \\
\hline PPPC & Pump Pit Precipitate Cell \\
\hline PPST & Pump Pit Sludge Tank \\
\hline PPSC & Pump Pit Sludge Cell \\
\hline PPRT & Pump Pit Recycle Tank \\
\hline PHA & Precipitate Hydrolysis Aqueous \\
\hline $\mathrm{RCT}$ & Recycle Collection Tank \\
\hline $\mathrm{RPC}$ & Remote Process Cell \\
\hline SPCV & Salt Process Cell Vent \\
\hline SRS & Savannah River Site \\
\hline SAR & Safety Analysis Report \\
\hline
\end{tabular}



ACRONYMS (continued)
SPC . Salt Processing Cell
SME Slurry Mix Evaporator
SRAT Sludge Receipt and Adjustment Tank 


\section{INTRQODUCTION}

Safe operation of SRS facilities continues to be the highest priority of the Savannah River Site [SRS]: One of these facilities, the Defense Waste Processing Facility or DWPF, is currently undergoing cold chemical runs to verify the design and construction preparatory to hot startup in 1995. The DWPF is a facility designed to convert the waste currently stored in tanks at the 200-Area tank farm into a form that is suitable for long term storage in engineered surface facilities and, ultimately, geologic isolation. As a part of the program to ensure safe operation of the DWPF the Safety Analysis Engineering Section of E\&CSD has completed a Probabilistic Safety Assessment of the DWPF. The results of this analysis are incorporated into the Safety Analysis Report [SAR] for DWPF.[1] The usual practice in preparation of Safety Analysis Reports is to include only a conservative analysis of certain 'design basis accidents'.

A major part of a Probabilistic Safety Assessment is the development and quantification of an Accident Progression Event Tree or APET. The APET provides a probabilistic representation of potential sequences along which an accident may progress. The methodology used to determine the risk of operation of the DWPF borrows heavily from methods applied to the Probabilistic Safety Assessment of SRS reactors and to some commercial reactors.[2] This report describes the Accident Progression Event Tree developed for the Probabilistic Safety Assessment of the DWPF.

\section{SUMMARY}

In the Defense Waste Processing Facility [DWPF] there are three barriers to the release of fission products to the environment: the process vessels and piping [the primary barrier], the DWPF buildings, and the ventilation/filter system. These last two barriers are equivalent to the Confinement System of an SRS reactor and are so designated in this report. In the unlikely event of an accident that breaches the primary barrier, radionuclides will be released into the process cells and building. The main accidents of concern at DWPF are energetic events due to deflagration or detonation of the hydrogen and benzene vapors produced in the process vessels at DWPF during waste processing operations. In order to determine the consequences of an accident, systematic tracking is required of the progression of the accident from the time of the event or events leading to the breach of the primary barrier until the plant is in a stable state. The principal goals of the accident progression analysis are:

[1] to evaluate the performance of the Confinement System in mitigating the release of fission products to the environment and.

[2] to provide sufficient information such that the magnitude of any release to the environment can be estimated as well as the timing and energy associated with that release.

The logic model used to describe the progression of the accident and determine the success [or failure] of the confinement system in mitigating the consequences of an accident is called an Accident Progression Event Tree or APET. The APET described in this document is based on Salt Processing Cell (SPC) Mode C. This is the full radioactive mode processing. 


\subsection{The Accident Progression Event Tree}

The APET that was developed for DWPF contains 193 events [or questions] that detail the possible progression of postulated accidents. In general, the APET incorporates sufficient detail so that the answers to questions leading up to any point in the accident progression supply information adequate to [1] provide a reasonable estimate of the outcome of the next event considered or [2] establish bounding conditions for the use of mechanistic calculations to provide the answer.

In the development of the APET a series of events [questions] was postulated that could occur during progression of the accident. In general these events pertain to

[1] the cause of the accident [i.e., initiating event],

[2] the status of the plant at the time of the accident,

[3] the energetics of the accident,

[4] the impact of the accident on adjacent equipment/processes, and

[5] the response of the Confinement System to the challenge posed by the energetics accompanying the accident and/or the radionuclides that breach the primary barrier.

A probability of occurrence [branch point probability] is associated with each outcome of each event or question in the APET. The sequence of outcomes then determines the course of the accident with an associated probability of occurrence and is referred to as an accident progression sequence. An accident progression sequence is a specific set of events associated with the accident and the subsequent performance of the Confinement System in mitigating the consequences of the accident.

\subsection{Quantification of the APET}

Determination of the probability of each outcome [branch point probability] is the major effort in the quantification of the APET. The probability that a particular branch is taken is evaluated based on

[1] known information related to the condition of the facility or the accident under consideration,

[2] fault tree analysis of active systems within the facility,

[3]. the answers to previous questions [i.e., accident progression is sequence dependent], and/or

[4] mechanistic analysis of accident phenomena and confinement response.

Once the branch probabilities for all questions are determined, solution of the APET is performed using the EVNTRE program [2].

The result of quantifying the APET is an estimate of the relative frequency of the various accident progression sequences. A potential radionuclide release to the environment, or source term, can be associated with each accident progression sequence defined by the APET. The magnitude and content of the source term will depend on the course of the accident and performance of the confinement system. However, the tens of thousands of source terms that can result from a single quantification of the APET are still too many for further analysis of consequence. Therefore, the accident sequences that have similar characteristics, in terms of the radionuclide source to the environment are grouped or "binned". Nineteen characteristics 
[analogous to top events in the APET] are used in the source term binner. These source term bins are used in quantifying the risk of operation of DWPF[3].

\subsection{SUMMARY DESCRIPTION OF THE DEFENSE WASTE PROCESSING FACILITIES}

This section provides a summary description of the DWPF to aid in understanding the logic model for the progression of severe accidents. The following paragraphs present a brief description of the DWPF at SRS. The descriptive information for the DWPF, which is extracted from the DWPF SAR [1], is brief and is intended only to orient the reader with the process.

\subsection{The Confinement System}

The primary barrier to the release of radionuclides from the DWPF is the process vessels and associated piping that contain the radioactive waste. The secondary barrier to release of radionuclides is designated "the Confinement System". The Confinement System for the DWPF [equivalent to the Zone 1 ventilation system as described in the SAR] operates on a principle similar to that used for the SRS reactors: an enclosed confinement volume, a once through ventilation system that maintains the pressure in the confinement volume lower than atmospheric, and a filter system to remove radionuclides that may be in the exhausted air. The confinement volume is mainly at and above grade and enclosed by a steel reinforced concrete structure. The exhaust fans are located downstream of the sand filters, both at grade level. The exhaust air is discharged through an $\sim 150$-ft. stack.

\subsection{The Salt Process Cell}

Radioactive waste from the production of nuclear materials at the Savannah River Site is currently stored in large underground tanks. This waste consists of two parts, an insoluble sludge and a supernatant liquid containing soluble salts. The most abundant radioactive element in the supernate is cesium-137, although some strontium-90 and plutonium are also present. The cesium-137 is removed from the supernate by adding sodium tetraphenylborate to precipitate the Cs-137. Sodium titanate is added to the supernate to adsorb the ST-90 and Pu. The resulting solution is filtered, producing a decontaminated liquid which is converted to saltstone and interred in large engineered disposal buildings located in Z-Area. The remaining precipitate slurry, which has been concentrated, is the feed material to the precipitate hydrolysis process. The precipitate is primarily an organic material.

The precipitate hydrolysis process is conducted in the Salt Process Cell [SPC] in the Vitrification Building or Canyon of the DWPF. Precipitate hydrolysis decomposes the precipitate slurry into two components: an organic stream low in radioactivity and an aqueous stream that contains most of the radioactive material [Cs-137, Sr-90, and Pu]. The organic stream is primarily benzene which is transferred to the Organic Waste Storage Tank [OWST]. The aqueous stream is transferred to the Chemical Process Cell for further treatment before eventual transfer to the glass melter.

The principal hazard of concern in the SPC and its associated vessels is deflagration or detonation of the benzene vapors that are produced during the precipitate hydrolysis process. The approach used to prevent these events is to exclude oxygen from the vapor space in each 
vessel in the SPC by use of carbon dioxide or nitrogen purges. Each vessel is provided with a primary and backup carbon dioxide purge and a backup nitrogen purge.

\subsection{The Chemical Process Cell}

The precipitate slurry from the 200-Area tank farm is separated into two streams by the precipitate hydrolysis process in the Salt Process Cell: an organic stream low in radioactivity and an aqueous stream that contains the bulk of the radioactive material. The organic stream is primarily benzene which is eventually transferred to the Organic Waste Storage Tank [OWST]. The aqueous stream [called Precipitate Hydrolysis Aqueous or PHA] is transferred to the Precipitate Reactor Bottoms Tank [PRBT] in the Chemical Process Cell for storage and subsequent transfer to the Sludge Receipt Adjustment Tank.

The Sludge Receipt Adjustment Tank [SRAT] receives washed sludge slurry from the 200Area tank farm via the Low Point Pump Pit. Formic acid is added to the sludge slurry in the SRAT to reduce mercury to the elemental state for recovery and minimize gas evolution in the glass melter. After the sludge has been properly adjusted by addition of formic acid the aqueous solution from the Salt Process Cell [PHA] is added in controlled amounts from the PRBT. The quantity of PHA added is determined by the solids content and by the required ratio of precipitate solids to sludge solids. The adjusted sludge-precipitate slurry is transferred from the SRAT to the Slurry Mix Evaporator [SME].

In the SME the remaining operations necessary to prepare a batch of precipitate-sludge slurry for the glass melter are completed. Glass frit is added to the SME to achieve a slurry containing $28 \mathrm{wt} \%$ waste solids and $72 \mathrm{wt} \%$ glass frit. Excess liquid is evaporated to achieve a total solids content of $50 \mathrm{wt} \%$ in the concentrated slurry. After cool down the concentrated slurry is transferred to the Melter Feed Tank.

The principal hazard of concern in the CPC vessels is deflagration or detonation of the benzene and/or hydrogen vapors that are present in the vessels. The approach used to prevent these events is to provide sufficient purge flow to prevent the buildup of flammable concentrations of these vapors in the vessel vapor spaces. Each vessel is provided with a primary compressed air purge and a backup nitrogen purge. The flammability problems associated with each CPC vessel are slightly different and are discussed in more detail below.

The PRBT has continual evolution of hydrogen due to radiolysis of the water in the vessel. Some benzene is also sent to the tank from the PR. The purge flow provided to the vessel from the primary or backup purge systems is sufficient to prevent a flammable mixture from these sources. However, operator errors involving transfer of high benzene content materialfrom the PR to the PRBT could cause benzene vapor generation rates large enough to reach LFL even with the purge systems functioning. The fault tree for the PRBT explosion models all of these mechanisms.

The SRAT has a radiolysis source of hydrogen generation also. Normal amounts of benzene transferred from the PRBT are boiled off with steam during the transfer process from the PRBT. The purge flow provided to the vessel from the primary or backup purge systems is sufficient to prevent a flammable mixture from these sources. In addition, improper additions of material from the PRBT, or excess formic or nitric acid additions can cause hydrogen generation rates large enough to reach LFL even with the purge systems functioning. Propagation of benzene transfer errors from the PR or incorrect boilup of benzene during transfer from the PRBT to the SRAT could also result in benzene vapor generation rates large 
enough to reach LFL even with the purge systems functioning. The fault tree for the SRAT explosion módels all of these mechanisms.

The SME has a radiolysis source of hydrogen generation also. The purge flow provided to the vessel from the primary or backup purge systems is sufficient to prevent a flammable mixture from this source. In addition, excess addition of formic acid can cause hydrogen generation rates large enough to reach LFL even with the purge systems functioning. The fault tree for the SME explosion models all of these mechanisms.

The MFT has a radiolysis source of hydrogen generation only. The purge flow provided to the vessel from the primary or backup purge systems is sufficient to prevent a flammable mixture from these sources. The fault tree for the MFT explosion models this mechanism.

\subsection{The Melter Cell}

The sludge-frit slurry is transferred from the Melter Feed Tank [MFT] located in the Chemical Process Cell to the Melter in the Melter Cell. In the Melter the principal vitrification processes of drying, oxidation, and melting take place.

The Melter is operated continuously with a cold cap [crust]' composed of waste calcine and frit that covers most of the melt surface. The sludge-frit slurry is introduced into the melter on top of the cold cap which may be as thick as 6 inches. Water from the slurry is evaporated and drawn off into the offgas system. The glass melt beneath the cold cap is at a temperature of $1050-1150^{\circ} \mathrm{C}$. The cold cap melts at the melt-cold cap interface and forms the borosilicate glass waste. The extent to which the cold cap covers the melt is determined by the rate of feed of the sludge-frit slurry. The rate of feed is adjusted so that the cold cap covers about $90 \%$ of the surface, allowing gases to escape through the cold cap to the vapor space above.

Thermal convection and continuous flow of argon gas through a dip tube into the melt promote mixing of the molten glass. After an average residence time of about 65 hours in the Melter the molten borosilicate-waste glass is poured into a stainless steel canister using differential pressure pouring [i.e., the pressure in the canister is reduced relative to that in the melter vapor space].

The canister is transferred into the Melt Cell from the cold storage area through a transfer tunnel and placed under the Melter pour spout for filling. The level of glass in the canister and the canister weight are monitored during the filling operation. After filling an inner closure plug is placed in the throat of the canister and it is transferred out of the Melter Cell. 


\subsection{Organic Waste Storage}

Waste organic material, primarily benzene, which is a by-product of the vitrification process is transferred through an above ground pipe from the process cells to a large double walled tank outside the Vitrification Building. The Organic Waste Storage Tank or OWST stores the organics for further disposition.

\subsection{The Low Point Pump Pit}

This section includes those questions defining events that could occur in the Low Point Pump Pit. Two interarea transfer pipes are provided to transfer the radioactive sludge and the precipitate slurry from the Tank Farm to the DWPF. A third pipe is used to transfer waste from the DWPF that must be recycled through the tank farm. A fourth pipe serves as a spare. All interarea transfers are batch transfers.

Radioactive sludge is transferred from the H-Area tank farm to the Sludge Tank in the Pump Pit approximately twice each week. The tank is monitored during transfer to avoid overfilling. The sludge.is transferred from the Low Point Pump Pit to the Sludge Receipt Adjustment Tank as needed.

Precipitate slurry is transferred from the H-Area tank farm to the Precipitate Tank in the Pump Pit approximately four times each week. As for the transfer of sludge the Precipitate Tank is monitored during transfer to avoid overfilling. The precipitate slurry is transferred to the Precipitate Reactor Feed Tank in the Salt Process Cell as needed.

During the Vitrification process wastes accumulate that must be recycled through the tank farm. These wastes are collected in the Recycle Collection Tank in the Chemical Process Cell and transferred to the tank farm via the Recycle Tank in the Low Point Pump Pit.

\section{THE ACCIDENT PROGRESSION EVENT TREE}

This section presents the logic model, called the Accident Progression Event Tree [APET], for the analysis of postulated accidents in the DWPF. As previously discussed, each question in the APET is analogous to a top event in a conventional event tree. The outcome of each question or event may be dependent on events that have happened previously. Therefore, each question is generally accompanied by logic [called case structure] that [1] separates circumstances for which the outcome probabilities for a question may differ and [2] distinguishes regimes of behavior for the phenomena that may be involved.

\subsection{Outcome Probabilities}

As previously discussed the EVNTRE[2] program was used to solve the APET. A special user function (UFUN) was written specifically for the DWPF analysis to solve the accident fault tree cut sets [12]. It is therefore necessary that the structure of the APET be compatible with EVNTRE. This structure is seen in the listing of the APET in Appendix A. Also shown in the APET listing is the best estimate value of the outcome probabilities for each question. Where questions have multiple cases the outcome probabilities are shown for each case. The 
determination of outcome probabilities is a major task in quantification of the APET. The outcome probabilities may be determined by solution to fault tree cut sets which are accessed from the UFUN subroutine in EVNTRE. Outcome probabilities may simply be determined from answers of previous questions and finally they may be input directly based on mechanistic modeling of accident phenomena or expert engineering judgment. In this solution of the DWPF APET, a significant number of the outcome probabilities that an event will occur are set to 1.0. This is done not because of the known certainty that the outcome must occur but because the probability in question is expected to be close to 1.0 , it is conservative, and the technical effort required to determine the actual outcome probability is not justified.

Many of the outcome probabilities used in the EVNTRE solution of the APET were distributed variables. Outcome probabilities that were treated this way are denoted in the text of the document with a **SAMPLED VARIABLE** label. For those outcome probabilities the values discussed in the text are best estimate values used for illustration purposes only. These outcome probabilities were determined based on analysis where possible and on engineering judgment where analysis was not available [7]. During the actual APET quantification, a probability distribution was used [4] to represent these outcome probabilities and values from the distribution were sampled and supplied to the APET by running the EVNTRE program in sampling mode. Although not labeled **SAMPLED VARIABLE** in the text, it should be understood that the outcome probabilities calculated from the cut set solutions have also had important basic events sampled.

One important feature of the EVNTRE program is the way the outcome probabilities are selected for those questions with multiple cases. As previously discussed the case structure developed for each question separates circumstances for which the outcome probabilities for a question may differ based on the occurrence of prior events in the accident progression model, viz., the answers to prior questions. When the EVNTRE program is examining the cases for a particular question the first case for which the Boolean expression representing each case is 'true' [that is the sequence of events listed in the case structure has occurred] is used as the basis for determining the outcome probabilities for the question. EVNTRE then progresses to the next question. In the event the Boolean expression for none of the sequences in the case structure is 'true' [has occurred] then EVNTRE will default to the last case, which is typically referred to as the 'otherwise' case. In general the approach taken in developing the case structure was to identify, insofar as possible, all sequences having bearing on the question, avoiding use of the default case.

Of particular significance in development of the APET structure is the 'type number' assigned each question. The following information is extracted from Reference 2 to describe question types.

There are eight types of questions that can be processed by EVNTRE. These are permutations of the following:

[1] whether the answer to the question depends upon the path through the tree,

[2] whether the branch split fractions are supplied directly or must be calculated from previously supplied reference parameters, together with comparison parameters, and

[3] whether parameters are to be supplied for later use by other questions to calculate branch split fractions. 
Question types.2, 4, 6, and 8 depend upon the path through the tree; question types 5, 6, 7, and 8 use calculated probabilities rather than supplied probabilities; and question types 3, 4, 7, and 8 supply parameters for use by subsequent type 5,6,7, and 8 questions.

The following lists describe each question type, along with its attributes:

Question Type = 1; The branch point probabilities are independent of previous branches and are input by the user,

Question Type = 2; $\quad$ The branch point probabilities are dependent upon previous branches and are input by the user,

Question Type = 3; $\quad$ The branch point probabilities are independent of previous branches, the user inputs the branch point probabilities, and inputs parameters used in a later question for internal calculation of branch point probabilities [Question Types 5, 6,7, or 8]. [a]

Question Type =4; The branch point probabilities are dependent upon previous branches, the user inputs the branch point probabilities, and inputs parameters used in a later question for internal calculation of branch point probabilities [Question Types 5, 6, 7, or 8].

Question Type = 5; The branch point probabilities are independent of previous branches and are calculated internally based on user supplied data.

Question Type $=6 ; \quad$ The branch point probabilities are dependent upon previous branches and are calculated internally based on user supplied data.

Question Type = 7; $\quad$ The branch point probabilities are independent of previous branches and are calculated internally based on user supplied data. Additionally, parameters used in later questions are read.

Question Type = 8; $\quad$ The branch point probabilities are dependent upon previous branches and are calculated internally based on user supplied data. additionally, parameters used in later questions are read.

All questions in the APET for the DWPF were Question types 2, 3, 4, 6, or 8; Question types 1,5 , and 7 were not used.

The following Table 1 presents an index of the questions in the APET.

[a] The value of the parameter used is selected on the basis of the outcome probabilities. 


\section{Table 1: Accident Progression Event Tree For DWPF}

\begin{tabular}{|c|c|c|}
\hline$\overline{Q \#}$ & Question Text & Page \\
\hline 1 & What is the accident class? & 33 \\
\hline 2 & If the accident class is 'external' what is the initiating event? & 33 \\
\hline$\overline{3}$ & Is failure of a common [ie.,support] system the initiating event? & 38 \\
\hline & $\begin{array}{l}\text { If the accident class is an 'internal' event classified 'leak' what is the } \\
\text { frequency of the event? }\end{array}$ & 39 \\
\hline 5 & $\begin{array}{l}\text { If the accident class is an 'internal' event classified 'Misc' what is the } \\
\text { frequency of the event? }\end{array}$ & 40 \\
\hline 6 & If the accident class is crane drop what is the frequency of the event? & 39 \\
\hline 7 & What are the support system failure frequencies and repair times? & 41 \\
\hline 8 & Is the failure of the normal electrical power system an 'accident enabler'? & 44 \\
\hline 9 & $\begin{array}{l}\text { Is the failure of the emergency [diesel] electrical power system an } \\
\text { 'accident enabler'? }\end{array}$ & $\overline{45}$ \\
\hline 10 & Does the Instrument Air System fail due to random events? & 46 \\
\hline 11 & Does the Instrument Air System fail due to a seismic event? & 47 \\
\hline$\frac{1}{12}$ & Does the Cooling Tower Common System fail due to random events? & 47 \\
\hline 13 & Does the Cooling Tower Common System fail due to a seismic event? & 48 \\
\hline 14 & Does the Deep Well System fail due to random events? & 48 \\
\hline 15 & Does the Deep Well System fail due to seismic events? & 49 \\
\hline 16 & Does the Cooling Tower System fail due to random events? & 49 \\
\hline 17 & Does the Cooling Tower System fail due to a seismic event? & 50 \\
\hline$\frac{18}{18}$ & Is failure of the Instrument Air System an 'accident enabler'? & 50 \\
\hline 19 & $\begin{array}{l}\text { Is the failure of the Cooling Tower Common system an 'accident } \\
\text { enabler'? }\end{array}$ & 52 \\
\hline 20 & Is the failure of the Deep Well system an 'accident enabler'? & 53 \\
\hline 21 & Is failure of the Cooling Tower an 'accident enabler'? & 54 \\
\hline 22 & Is the failure of the process steam system an 'accident enabler'? & 55 \\
\hline 23 & Is the failure of the process cooling water system an 'accident enabler? & 57 \\
\hline 24 & Is the failure of the Carbon Dioxide Purge System an accident enabler? & 58 \\
\hline 25 & Is failure of the Air Purge System an 'accident enabler'? & 59 \\
\hline 26 & Is failure of the Nitrogen Storage Tank System an 'accident enabler"? & 60 \\
\hline 27 & Is failure of the Chilled Water System an 'accident enabler'? & 62 \\
\hline 28 & Is the failure of the backup $\mathrm{CO} 2$ System an 'accident enabler'? & 63 \\
\hline 29 & Is failure of the Bulk Nitrogen System an 'accident enabler'? & 64 \\
\hline 30 & $\begin{array}{l}\text { Is the failure of the CPC backup liquid N2 Storage Tank System an } \\
\text { 'accident enabler'? }\end{array}$ & 65 \\
\hline 31 & Is failure of the CPC Nitrogen System an 'accident enabler'? & 66 \\
\hline 32 & Is failure of the LPPP Backup Nitrogen System an 'accident enabler'? & 67 \\
\hline 33 & Is failure of the SPC N2 System an 'accident enabler"? & 68 \\
\hline 34 & Is the failure of the SPC Seismic trigger System an accident enabler? & 69 \\
\hline 35 & Is the failure of the SPC Isolation valve UPS an 'accident enabler'? & 70 \\
\hline 36 & Is there a randomly initiated explosion in a process tank or cell? & 71 \\
\hline 37 & $\begin{array}{l}\text { Do the cell covers over the Salt Process Cell dislodge and fall because of } \\
\text { a seismic event? }\end{array}$ & $\overline{73}$ \\
\hline 38 & Does a cell cover fall while being moved by the crane? & 73 \\
\hline
\end{tabular}




\begin{tabular}{|c|c|c|}
\hline$\overline{Q \#}$ & Question Text & Page \\
\hline 39 & Do the cell covers that fall in a crane accident strike a tank? & 74 \\
\hline 40 & Do the cell covers that fall in a seismic event strike the PR? & 75 \\
\hline 41 & $\begin{array}{l}\text { Does a Seismic event or cover crane fall rupture and spill the contents of } \\
\text { the Precipitate Reactor in the Salt Process Cell? }\end{array}$ & 76 \\
\hline$\overline{42}$ & Does an explosive concentration accumulate in Precipitate Reactor? & 76 \\
\hline 43 & Does a damaging deflagration/detonation occur in the Precipitate Reactor? & 77 \\
\hline 44 & Do the cell covers that fall in a seismic event strike the $\mathrm{OE}$ ? & 78 \\
\hline 45 & $\begin{array}{l}\text { Does a Seismic event or cover crane fall rupture and spill the contents of } \\
\text { the Organic Evaporator in the Salt Process Cell? }\end{array}$ & 79 \\
\hline 46 & Does an explosive concentration accumulate in Organic Evaporator Tank? & 79 \\
\hline 47 & Does a detonation/deflagration of the Organic Evaporator tank occur? & 80 \\
\hline 48 & Do the cell covers that fall in a seismic event strike the OECT? & 81 \\
\hline 49 & $\begin{array}{l}\text { Does a Seismic event or cover crane fall rupture and spill the contents of } \\
\text { the Organic Evaporator Condensate Tank in the Salt Process Cell? }\end{array}$ & 82 \\
\hline 50 & $\begin{array}{l}\text { Does an explosive concentration accumulate in Organic Evaporator } \\
\text { Condensate Tank? }\end{array}$ & 82 \\
\hline 51 & $\begin{array}{l}\text { Does a detonation/deflagration of the Organic Evaporator Condensate tank } \\
\text { occur? }\end{array}$ & 83 \\
\hline 52 & $\begin{array}{l}\text { Do the cell covers that fall in a seismic event or crane accident strike the } \\
\text { PRFT? }\end{array}$ & 84 \\
\hline 53 & $\begin{array}{l}\text { Does a Seismic event or cover crane fall rupture and spill the contents of } \\
\text { the Precipitate Reactor Feed Tank in the Salt Process Cell? }\end{array}$ & 85 \\
\hline 54 & $\begin{array}{l}\text { Does an explosive concentration accumulate in Precipitate Reactor Feed } \\
\text { Tank? }\end{array}$ & 86 \\
\hline 55 & $\begin{array}{l}\text { Does a detonation/deflagration occur in the Precipitate Reactor Feed } \\
\text { Tank? }\end{array}$ & 87 \\
\hline 56 & $\begin{array}{l}\text { Do the cell covers for the Chemical Process Cell dislodge and fall } \\
\text { following a Seismic event? }\end{array}$ & 87 \\
\hline 57 & Do the cell covers that fall in a seismic event strike the PRBT? & 88 \\
\hline 58 & $\begin{array}{l}\text { Does a Seismic event or cover crane fall rupture and spill the contents of } \\
\text { the Precipitate Reactor Bottoms Tank in the Chemical Process Cell? }\end{array}$ & 89 \\
\hline 59 & $\begin{array}{l}\text { Does an explosive concentration accumulate in the Precipitate Reactor } \\
\text { Bottoms Tank [PRBT]? }\end{array}$ & 89 \\
\hline 60 & $\begin{array}{l}\text { Does a detonation/deflagration occur in the Precipitate Reactor Bottoms } \\
\text { Tank [PRBT]? }\end{array}$ & 90 \\
\hline 61 & Do the cell covers that fall due to a seismic event strike the SME? & 91 \\
\hline 62 & $\begin{array}{l}\text { Does a Seismic event or cover crane fall rupture and spill the contents of } \\
\text { the Slurry Mix Evaporator Tank in the Chemical Process Cell? }\end{array}$ & 92 \\
\hline 63 & $\begin{array}{l}\text { Does an explosive concentration accumulate in the Slurry Mix Evaporator } \\
\text { Tank [SME]? }\end{array}$ & 92 \\
\hline 64 & $\begin{array}{l}\text { Does a detonation/deflagration occur in the Slurry Mix Evaporator Tank } \\
\text { [SME]? }\end{array}$ & 93 \\
\hline 65 & Do the cell covers that fall in a seismic event strike the SRAT? & 94 \\
\hline 66 & $\begin{array}{l}\text { Does a Seismic event or cover crane fall rupture and spill the contents of } \\
\text { the Sludge Receipt and Adjustment Tank in the Chemical Process Cell? }\end{array}$ & 95 \\
\hline
\end{tabular}




\section{-. Table 1: Accident Progression Event Tree For DWPF}

\begin{tabular}{|c|c|c|}
\hline Q\# & Question Text & Page \\
\hline 67 & $\begin{array}{l}\text { Does an explosive concentration accumulate in the Sludge Receipt and } \\
\text { Adjustment Tank [SRAT]? }\end{array}$ & 95 \\
\hline 68 & $\begin{array}{l}\text { Does a detonation/deflagration occur in the Sludge Receipt and } \\
\text { Adjustment Tank [SRAT]? }\end{array}$ & 96 \\
\hline 69 & Do the cell covers that fall in a seismic event strike the RCT? & 97 \\
\hline 70 & $\begin{array}{l}\text { Does a Seismic event or cover crane fall rupture and spill the contents of } \\
\text { the Recycle Collection Tank in the Chemical Process Cell? }\end{array}$ & 98 \\
\hline 71 & Do the cell covers that fall in a seismic event strike the MFT? & 98 \\
\hline 72 & $\begin{array}{l}\text { Does a Seismic event or cover crane fall rupture and spill the contents of } \\
\text { the Melter Feed Tank in the Chemical Process Cell? }\end{array}$ & 99 \\
\hline 73 & $\begin{array}{l}\text { Does an explosive concentration accumulate in the Melter Feed Tank } \\
\text { [MFT]? }\end{array}$ & 100 \\
\hline 74 & Does a detonation/deflagration occur in the Melter Feed Tank [MFT]? & 101 \\
\hline 75 & $\begin{array}{l}\text { Does a damaging explosion occur in the Salt Process Cell Vent } \\
\text { System[SPCV]? }\end{array}$ & 101 \\
\hline 76 & $\begin{array}{l}\text { Does a damaging explosion occur in the Chemical Process Cell Vent } \\
\text { System[CPCV]? }\end{array}$ & 102 \\
\hline 77 & $\begin{array}{l}\text { Does an explosive concentration accumulate in the Process Vessel Vent } \\
\text { Header [PVVH]? }\end{array}$ & 103 \\
\hline 78 & $\begin{array}{l}\text { Does a damaging detonation occur in the Process Vessel Vent Header } \\
\text { [PVVH]? }\end{array}$ & 104 \\
\hline 79 & Does an explosive concentration accumulate in Precipitate Reactor? & 105 \\
\hline 80 & Does a deflagration/detonation occur in the Precipitate Reactor? & 106 \\
\hline 81 & Does an explosive concentration accumulate in Organic Evaporator Tank? & 107 \\
\hline 82 & Does a detonation/deflagration of the Organic Evaporator tank occur? & 108 \\
\hline$\overline{83}$ & $\begin{array}{l}\text { Does an explosive concentration accumulate in Organic Evaporator } \\
\text { Condensate Tank? }\end{array}$ & 109 \\
\hline 84 & $\begin{array}{l}\text { Does a detonation/deflagration of the Organic Evaporator Condensate tank } \\
\text { occur? }\end{array}$ & 110 \\
\hline 85 & $\begin{array}{l}\text { Does an explosive concentration accumulate in Precipitate Reactor Feed } \\
\text { Tank? }\end{array}$ & 111 \\
\hline 86 & $\begin{array}{l}\text { Does a detonation/deflagration occur in the Precipitate Reactor Feed } \\
\text { Tank? }\end{array}$ & 112 \\
\hline 87 & $\begin{array}{l}\text { Does an explosive concentration accumulate in the Precipitate Reactor } \\
\text { Bottoms Tank [PRBT]? }\end{array}$ & 113 \\
\hline 88 & $\begin{array}{l}\text { Does a detonation/deflagration occur in the Precipitate Reactor Bottoms } \\
\text { Tank [PRBT]? }\end{array}$ & 114 \\
\hline 89 & $\begin{array}{l}\text { Does an explosive concentration accumulate in the Slurry Mix Evaporator } \\
\text { Tank [SME]? }\end{array}$ & 115 \\
\hline 90 & $\begin{array}{l}\text { Does a detonation/deflagration occur in the Slurry Mix Evaporator Tank } \\
\text { [SME]? }\end{array}$ & 116 \\
\hline 91 & $\begin{array}{l}\text { Does an explosive concentration accumulate in the Sludge Receipt and } \\
\text { Adjustment Tank [SRAT]? }\end{array}$ & 117 \\
\hline 92 & $\begin{array}{l}\text { Does a detonation/deflagration occur in the Sludge Receipt and } \\
\text { Adjustment Tank [SRAT]? }\end{array}$ & 118 \\
\hline
\end{tabular}


Table 1: Accident Progression Event Tree For DWPF

\begin{tabular}{|c|c|c|}
\hline$Q \#$ & Question Text & Page \\
\hline 93 & $\begin{array}{l}\text { Does an explosive concentration accumulate in the Melter Feed Tank } \\
\text { [MFT]? }\end{array}$ & 119 \\
\hline 94 & Does a detonation/deflagration occur in the Melter Feed Tank [MFT]? & 120 \\
\hline 95 & $\begin{array}{l}\text { Does a damaging explosion occur in the Salt Process Cell Vent } \\
\text { System[SPCV? }\end{array}$ & $\overline{121}$ \\
\hline 96 & $\begin{array}{l}\text { Does a damaging explosion occur in the Chemical Process Cell Vent } \\
\text { System[CPCV]? }\end{array}$ & $\overline{122}$ \\
\hline 97 & $\begin{array}{l}\text { Does an energetic event in the SPC fail the removable wall between the } \\
\text { SPC and the CPC? }\end{array}$ & $\overline{123}$ \\
\hline 98 & $\begin{array}{l}\text { Do the cell covers for the Chemical Process Cell dislodge and fall } \\
\text { following an energetic event? }\end{array}$ & 123 \\
\hline 99 & $\begin{array}{l}\text { Does splashing [spillage] of the Precipitate Reactor Bottoms Tank } \\
\text { contents occur? }\end{array}$ & 124. \\
\hline 100 & $\begin{array}{l}\text { Does splashing [spillage] of the Slurry Mix Evaporator Tank contents } \\
\text { occur? }\end{array}$ & 125 \\
\hline 101 & $\begin{array}{l}\text { Does splashing [spillage] of the Sludge Receipt and Adjustment Tank } \\
\text { contents occur? }\end{array}$ & 127 \\
\hline 102 & Does splashing [spillage] of the Recycle Collection Tank contents occur? & 128 \\
\hline 103 & Does splashing [spillage] of the Melter Feed Tank contents occur? & 129 \\
\hline 104 & $\begin{array}{l}\text { Does an energetic event in the CPC fail the removable wall between the } \\
\text { SPC and the CPC? }\end{array}$ & 130 \\
\hline 105 & $\begin{array}{l}\text { Do the cell covers for the Salt Process Cell dislodge and fall following an } \\
\text { energetic event? }\end{array}$ & 130 \\
\hline 106 & Does splashing [spillage] of the Precipitate Reactor contents occur? & 131 \\
\hline 107 & $\begin{array}{l}\text { ies splashing [spillage] of the contents of the Organic. Evaporator tank } \\
\text { ur? }\end{array}$ & $\overline{132}$ \\
\hline 108 & $\begin{array}{l}\text { Does splashing [spillage] of the contents of the Organic Evaporator } \\
\text { Condensate tank occur? }\end{array}$ & 133 \\
\hline$\sqrt{9}$ & $\begin{array}{l}\text { Does splashing [spillage] of the contents of the Precipitate Reactor Feed } \\
\text { Tank occur? }\end{array}$ & $\overline{135}$ \\
\hline 110 & Does the Vitrification Building collapse following a seismic event? & 136 \\
\hline 111 & Does the Sand Filter function following a seismic event? & 136 \\
\hline 112 & $\begin{array}{l}\text { Do the Building Exhaust Ducts from Zone } 1 \text { to the Sand Filter function } \\
\text { following a seismic event? }\end{array}$ & 137 \\
\hline 113 & What is the condition of the Zone 1 Exhaust Fan System? & 138 \\
\hline 114 & $\begin{array}{l}\text { Does a deflagratable concentration occur in the SPC due to Vessel } \\
\text { Overpressurization? }\end{array}$ & 140 \\
\hline 115 & $\begin{array}{l}\text { Does a vessel overpressurization in the Salt Process Cell result in a } \\
\text { deflagration in the Salt Process Cell? }\end{array}$ & 141 \\
\hline 116 & $\begin{array}{l}\text { Do the cell covers for the Salt Process Cell dislodge after the } \\
\text { overpressurization deflagration? }\end{array}$ & 142 \\
\hline 117 & Does splashing [spillage] of the Precipitate Reactor contents occur? & 142 \\
\hline 118 & $\begin{array}{l}\text { Does splashing [spillage] of the contents of the Organic Evaporator tank } \\
\text { occur? }\end{array}$ & 143 \\
\hline 119 & $\begin{array}{l}\text { Does splashing [spillage] of the contents of the Organic Evaporator } \\
\text { Condensate tank occur? }\end{array}$ & 143 \\
\hline
\end{tabular}


Table 1: Accident Progression Event Tree For DWPF

\begin{tabular}{|c|c|c|}
\hline Q\# & Question Text & Page \\
\hline 120 & $\begin{array}{l}\text { Does splashing [spillage] of the contents of the Precipitate Reactor Feed } \\
\text { Tank occur? }\end{array}$ & 144 \\
\hline 121 & $\begin{array}{l}\text { Does the collateral damage in the Salt Process Cell result in a deflagration } \\
\text { in the Salt Process Cell? }\end{array}$ & 145 \\
\hline 122 & $\begin{array}{l}\text { Does the collateral damage in the Salt Process Cell result in a fire in the } \\
\text { Salt Process Cell? }\end{array}$ & 147 \\
\hline 123 & Does a detonation/deflagration occur in the Precipitate Reactor? & 149 \\
\hline 124 & Does a detonation/deflagration of the Organic Evaporator tank occur? & 150 \\
\hline 125 & $\begin{array}{l}\text { Does a detonation/deflagration of the Organic Evaporator Condensate tank } \\
\text { occur? }\end{array}$ & 151 \\
\hline 126 & $\begin{array}{l}\text { Does a detonation/deflagration occur in the Precipitate Reactor Feed } \\
\text { Tank? }\end{array}$ & 152 \\
\hline 127 & $\begin{array}{l}\text { Does a damaging explosion occur in the Salt Process Cell Vent } \\
\text { System[SPCV]? }\end{array}$ & 153 \\
\hline 128 & Do the cell covers for the Salt Process Cell dislodge and fall? & 154 \\
\hline 129 & $\begin{array}{l}\text { Does a damaging detonation occur in the Process Vessel Vent Header } \\
\text { [PVVH]? }\end{array}$ & 155 \\
\hline 130 & $\begin{array}{l}\text { Does a detonation/deflagration occur in the Precipitate Reactor Bottoms } \\
\text { Tank [PRBT]? }\end{array}$ & 156 \\
\hline 131 & $\begin{array}{l}\text { Does a detonation/deflagration occur in the Slurry Mix Evaporator Tank } \\
\text { [SME]? }\end{array}$ & 157 \\
\hline 132 & $\begin{array}{l}\text { Does a detonation/deflagration occur in the Sludge Receipt and } \\
\text { Adjustment Tank [SRAT]? }\end{array}$ & 158 \\
\hline 133 & Does a detonation/deflagration occur in the Melter Feed Tank [MFT]? & 159 \\
\hline 134 & Does a detonation occur in the CPC Vent system? & 160 \\
\hline 135 & $\begin{array}{l}\text { Does an energetic event in the CPC fail the removable wall between the } \\
\text { SPC and the CPC? }\end{array}$ & 161 \\
\hline 136 & Does splashing [spillage] of the Precipitate Reactor contents occur? & 162 \\
\hline 137 & $\begin{array}{l}\text { Does splashing [spillage] of the contents of the Organic Evaporator tank } \\
\text { occur? }\end{array}$ & 163 \\
\hline 138 & $\begin{array}{l}\text { Does splashing [spillage] of the contents of the Organic Evaporator } \\
\text { Condensate tank occur? }\end{array}$ & 164 \\
\hline 139 & $\begin{array}{l}\text { Does splashing [spillage] of the contents of the Precipitate Reactor Feed } \\
\text { Tank occur? }\end{array}$ & 165 \\
\hline 140 & $\begin{array}{l}\text { Does an energetic event in the SPC fail the removable wall between the } \\
\text { SPC and the CPC? }\end{array}$ & 166 \\
\hline 141 & $\begin{array}{l}\text { Do the cell covers for the Chemical Process Cell dislodge and fall } \\
\text { following an energetic event? }\end{array}$ & 167 \\
\hline 142 & $\begin{array}{l}\text { Does splashing [spillage] of the Precipitate Reactor Bottoms Tank } \\
\text { contents occur? }\end{array}$ & 168 \\
\hline 143 & $\begin{array}{l}\text { Does splashing [spillage] of the Slurry Mix Evaporator Tank contents } \\
\text { occur? }\end{array}$ & 169 \\
\hline 144 & $\begin{array}{l}\text { Does splashing [spillage] of the Sludge Receipt and Adjustment Tank } \\
\text { contents occur? }\end{array}$ & 170 \\
\hline 145 & Does splashing [spillage] of the Recycle Collection Tank contents occur? & 171 \\
\hline
\end{tabular}


- Table 1: Accident Progression Event Tree For DWPF

\begin{tabular}{|c|c|c|}
\hline$\overline{Q \#}$ & Question Text & Page \\
\hline 146 & Does splashing [spillage] of the Melter Feed Tank contents occur? & 172 \\
\hline 147 & $\begin{array}{l}\text { Does a deflagration in the SPC lead to a deflagration in the RPC that } \\
\text { damages the building? }\end{array}$ & 173 \\
\hline 148 & $\begin{array}{l}\text { Do the cell covers over the Melter Cell dislodge and fall because of a } \\
\text { seismic event? }\end{array}$ & 174 \\
\hline 149 & Do the cell covers that fall in a seismic event strike the Melter? & 175 \\
\hline 150 & $\begin{array}{l}\text { Does a Seismic event rupture and spill the contents of the Melter in the } \\
\text { Melter Cell? }\end{array}$ & 175 \\
\hline 151 & Does a Seismic event rupture the Melter Offgas System in the Melter Cell? & 176 \\
\hline 152 & Does an explosive concentration exist in the Melter Offgas System? & 176 \\
\hline 153 & Does a damaging detonation occur in the Melter Offgas System? & 177 \\
\hline$\overline{154}$ & $\begin{array}{l}\text { Does a damaging steam explosion occur due to a salt/water reaction in the } \\
\text { Melter? }\end{array}$ & 178 \\
\hline 155 & Does a damaging reaction occur in the Melter? & 179 \\
\hline 156 & $\begin{array}{l}\text { Do the cell covers for the Melter Cell dislodge and fall following an } \\
\text { energetic event? }\end{array}$ & 180 \\
\hline 157 & $\begin{array}{l}\text { Does splashing [spillage] of the Melter contents occur as the result of } \\
\text { energetic events in the Melter Cell? }\end{array}$ & 181 \\
\hline 158 & $\begin{array}{l}\text { Does a Seismic event or a tornado rupture and spill the contents of the } \\
\text { Organic Waste Storage Tank? }\end{array}$ & 182 \\
\hline 159 & $\begin{array}{l}\text { Does an explosive concentration accumulate in the Organic Waste Storage } \\
\text { Tank? }\end{array}$ & 183 \\
\hline 160 & $\begin{array}{l}\text { Does a damaging deflagration occur in the Organic Waste Storage Tank } \\
\text { [OWST]? }\end{array}$ & 184 \\
\hline 161 & $\begin{array}{l}\text { Does splashing [spillage] of the Organic Waste Storage Tank [OWST] } \\
\text { contents occur? }\end{array}$ & 185 \\
\hline 162 & $\begin{array}{l}\text { Do the cell covers over the Low Point Pump Pit dislodge and fall because } \\
\text { of a seismic event (i.e., covers over all three cells)? }\end{array}$ & 185 \\
\hline 163 & $\begin{array}{l}\text { Do the cell covers that fall due to a seismic event strike a vessel in the } \\
\text { LPPP? }\end{array}$ & 186 \\
\hline 164 & $\begin{array}{l}\text { Does a Seismic event rupture and spill the contents of the Precipitate tank } \\
\text { in the Low Point Pump Pit? }\end{array}$ & 186 \\
\hline 165 & $\begin{array}{l}\text { Does an explosive concentration accumulate in the Precipitate tank in the } \\
\text { Low Point Pump Pit? }\end{array}$ & 187 \\
\hline 166 & $\begin{array}{l}\text { Does a damaging detonation/deflagration occur in the Precipitate tank in } \\
\text { the Low Point Pump Pit? }\end{array}$ & 188 \\
\hline 167 & $\begin{array}{l}\text { Does splashing [spillage] of the Pump Pit Precipitate Tank contents } \\
\text { occur? }\end{array}$ & 189 \\
\hline 168 & $\begin{array}{l}\text { Does an explosive concentration accumulate in Pump Pit Precipitate Tank } \\
\text { Cell? }\end{array}$ & 190 \\
\hline 169 & $\begin{array}{l}\text { Does a damaging detonation/deflagration occur in the Pump Pit } \\
\text { Precipitate Tank Cell? }\end{array}$ & 190 \\
\hline$\overline{170}$ & $\begin{array}{l}\text { Do the cell covers for the Pump Pit Precipitate Cell dislodge and fall } \\
\text { following an energetic event? }\end{array}$ & 191 \\
\hline$\overline{171}$ & $\begin{array}{l}\text { Does splashing [spillage] of the Pump Pit Precipitate Tank contents } \\
\text { occur? }\end{array}$ & 192 \\
\hline
\end{tabular}


-. Table 1: Accident Progression Event Tree For DWPF

\begin{tabular}{|l|l|l|}
\hline Q\# & - & Question Text \\
\hline 172 & $\begin{array}{l}\text { Does a Seismic event rupture and spill the contents of the Sludge tank in } \\
\text { the Low Point Pump Pit? }\end{array}$ & 193 \\
\hline 173 & Does an explosive concentration accumulate in Pump Pit Sludge Tank? & 193 \\
\hline 174 & $\begin{array}{l}\text { Does a damaging detonation/deflagration occur in the Pump Pit Sludge } \\
\text { Tank? }\end{array}$ & 194 \\
\hline 175 & Does splashing [spillage] of the Pump Pit Sludge Tank contents occur? & 195 \\
\hline 176 & Does an explosive concentration accumulate in Pump Pit Sludge Cell? & 196 \\
\hline 177 & $\begin{array}{l}\text { Does a damaging detonation/deflagration occur in the Pump Pit Sludge } \\
\text { Tank Cell? }\end{array}$ & 197 \\
\hline 178 & $\begin{array}{l}\text { Do the cell covers for the Pump Pit Sludge Cell dislodge and fall } \\
\text { following an energetic event? }\end{array}$ & 197 \\
\hline 179 & Does splashing [spillage] of the Pump Pit Sludge Tank contents occur? & 198 \\
\hline 180 & $\begin{array}{l}\text { Does a Seismic event rupture and spill the contents of the Recycle tank in } \\
\text { the Low Point Pump Pit? }\end{array}$ & 199 \\
\hline 181 & Does splashing [spillage] of the Pump Pit Recycle Tank contents occur? & 199 \\
\hline 182 & Does an uncontrolled reaction occur in the CPC? & 200 \\
\hline 183 & Does an uncontrolled reaction occur in the SPC? & 201 \\
\hline 184 & Does a vessel overflow occur in the CPC? & 201 \\
\hline 185 & Does a vessel overflow of process material occur in the SPC? & 201 \\
\hline 186 & Does a vessel overflow of benzene occur in the SPC? & 202 \\
\hline 187 & Does a vessel overflow occur in the Low Point Pump Pit? & 202 \\
\hline 188 & Does a leak occur in the Chemical Process Cell? & 203 \\
\hline 189 & Does a leak of process material occur in the Salt Process Cell? & 203 \\
\hline 190 & Does a leak of benzene occur in the Salt Process Cell? & 204 \\
\hline 191 & Does an incidental leak occur in the Pump Pits? & 204 \\
\hline 192 & Does the rupture of a filled storage canister occur? & 205 \\
\hline 193 & What is the status of the ventilation for the Low Point Pump Pit Building? & 205 \\
\hline
\end{tabular}




\subsection{Summary Outline of DWPF APET}

The following outline summarizes the major sections of the Mode C DWPF APET:

1. Questions 1-7: Selection of accident class, data input and initiating event frequencies.

2. Questions 8-35: Support systems status

3. Question 36: Probability of random explosion

4. Questions 37-78:

- Rupture question for each tank in SPC and CPC for seismic or crane drop

- First series of explosion questions for each tank for seismic or internal energetic events

- Explosion question for vent headers

5. Questions 79-96: These questions are the second series of tank explosions as a result of an initial explosion and propagation through vent headers.

6. Questions 97-109: Failed walls, fallen covers and splashed tank questions for SPC and CPC (from first and second series of explosion questions).

7. Questions 110-112: Building condition as a result of seismic event

8. Questions 113: Condition of zone 1 exhaust fan system.

9. Questions 114-115: Salt cell deflagration \#1 (due to vessel over pressurization).

10. Questions 116 - 120: SPC covers falling and tank splashing a result of cell deflagration $\# 1$.

11. Questions 121-122: Salt cell deflagration \#2 and/or fire as a result of benzene in cell from splashed tanks, leaks or overflows.

12. Questions 123-129: Third series of explosion progression questions and covers falling as a result of SPC deflagration \#2.

13. Questions 130-135: Explosion progressions in CPC and wall failure as a result of SPC deflagration \#2 (third round).

14. Questions 136-139: Tank splashing in SPC as a result of covers falling or wall failure (from third round).

15. Questions 140: SPC wall failure from third round of explosions

16. Question 141: CPC covers fall (from third round).

17. Questions 142-146: Tank splashing in CPC as a result of covers falling or wall failure (from third round).

18. Question 147: RPC deflagration. 
19. Questions 148 - 157: Melter and melter offgas accidents

20. Questions 158-161: OWST accidents

21. Questions 162-163: LPPP cover displacement as a result of seismic event.

22. Questions 164-171: Seismic or explosion damage in PPPC or PPPT.

23. Questions 172-179: Seismic or explosion damage in PPSC or PPST.

24. Questions 180-181: Seismic damage to PPRT.

25. Questions 182-193: Miscellaneous events.

The APET treats five different accident classes. These are: 1) Internal-Energetic events, 2) External events (seismic initiator only), 3) Crane load drop accidents, 4) Leaks and spills and 5) miscellaneous accident. The accident class to be considered is specified in question one. The attached flow charts illustrate the logical structure and sequence of questions for each accident class in the APET.

During operation, the DWPF is subject to equipment failures that may result in the release of radionuclides to the environment. These equipment failures may be the result of some event external to the DWPF or they may be the result of random failure or personnel error. The first : 36 questions in the APET define the events or failures that initiate the accident and the state of the plant at the time of the accident. The chart in Figure 1 shows the first portion of the APET which sets up the problem to be solved by inputting specific information required for solution and then establishes the status of the various support systems. For seismic events, data input includes seismic fragilities and $96 \mathrm{hr}$. mission time failure rates also called random failure rates. These are used by the APET in selected questions from 8 to 36 to calculate the unavailability of a system after the seismic event. The APET uses this calculation later to determine explosion frequencies. The individual fragilities are also used where the APET.specifically inquires about the probability of the failure of a individual component (e.g., a tank). For the internal energetic and other accidents, accident initiator frequencies (i.e. system failure rates) are input in questions 3-6. Support system status is also calculated for these accidents in questions 8-36.

System failures are classified as either initiators or enablers. An initiator is an event that must be included in the set of postulated failures and errors which combine in the analysis to produce the accident of concern. In a way they can be thought of as events that could start the sequence of events ending in an accident, although fault tree analysis per se does not deal with time sequences. A component initiator failure may directly result in a facility accident if a redundant or supporting component is offline at the same time. The offline component is said to have failed as an enabler because its lack of availability allows the accident to occur if an initiator has failed. Failure of an enabler alone is not sufficient to cause an accident. It is possible for a system be fail as both an initiator or an enabler on different paths through the APET. Backup systems can only fail as enablers since the failure of the primary system is also required to lose the function provided by the two systems. For example, normal electrical power can fail as an initiator or an enabler. Backup power can only fail as an enabler. Failure of backup power by itself cannot lead to an accident because normal power would still be available. Initiating failure frequencies are input directly to the APET. The status of the various systems as enablers is therefore important and is determined using either logical dependencies or, given data for the failure frequencies and repair times, the UAVAIl subroutine of EVNTRE 
calculates the frequency of failure as enabler [11]. Once the setup portion of the APET is complete, the APET begins the necessary logical questions required to determine the progression sequences that may follow an initiating accident.

Figure 2 illustrates the sequence of questions used by the APET for the various internal energetic events. Each box represents a series of questions regarding the outcome of possible events. These are in the order that the APET treats them. Explosions, which can be detonations or deflagrations, may occur randomly or as a result of a common system failure whose failure frequency is input in question 3. The UFUN subroutine [12] is used to obtain fault tree calculations giving the frequency of explosive concentrations. Explosive concentrations of both hydrogen and benzene are considered depending on the vessel [5]. UFUN determines the frequency of an explosive concentration with an ignition source and the conditional probability of an explosive concentration without an ignition source. The possibility of explosions is considered in the Salt Process Cell (from benzene vapor clouds), and the tanks of the Salt, Chemical, Meiter cells, the Low Point Pump Pit and the OWST.

Figures 3 through 5 subdivide the flow chart in Figure 2 to more easily illustrate the possible internal energetic progressions in the APET. Figure 3 shows the progressions in the SPC and the CPC. The initiator and enabler information is input and then a 1st round or series of questions is initiated to determine the frequencies of tank explosions. Both detonations and deflagrations are considered probable with deflagrations considered far more likely. The order of the questions is not important as there is no dependency among the tanks. Any individual or multiple tank explosions results in a flame front propagated into the cell vent headers and the process vent headers. This means that an ignition source is instantaneously available to other tanks in either cell and additional progression is possible. This propagation becomes the ignition source for additional explosions. if conditions in other tanks are favorable for ignition but had not exploded initially because there was no ignition source present. This is a second round of explosions. Again the order of the secondary explosion is not important as the propagation of the flame front through the headers is considered instantaneous. For accidents initiated by tank explosions, it is assumed that all possibilities for explosions have been exhausted as a result of the initial event because there are no more ignition sources. The results of SPC/CPC explosions are: 1) tank detonations rupture a tank and possibly adjacent tanks, and/or 2) falling cell covers, a collapsing wall or flying shrapnel that rupture tanks resulting in releases. As a result of benzene spills and subsequent vapor cloud formation possible in the SPC from the OEV and/or the OECT, there may be a deflagration within the cell. The cell deflagration may cause a SPC fire. There is no propagation into the CPC because at this time the vent headers have already been destroyed by the fallen salt process cell covers. The progressions may ultimately lead to a deflagration in the RPC if there had not been a fire because the initial cell deflagration pushes a cloud of benzene into the RPC. The RPC deflagration is assumed to result in significant damage to the building and to release the contents of all vessels.

Figure 4 shows the progression for the melter cell, the low point pump pit and OWST which are separate from the SPC and CPC. Progressions from the SPC/CPC to these areas are not considered feasible. In the melter cell, releases may occur from the melter vessel or the melter offgas system, either one of which may explode. The melter offgas system may accumulate an explosive concentration that may detonate in the presence of an ignition source. In the melter tank, a salt and water reaction may cause an explosion. An explosion in the melter will cause the melter cell covers to fall resulting in possible releases. Source term releases are possible from the melter offgas system and the melter. In the LPPP only the precipitate and sludge tanks can explode. A detonation or falling cell covers can release the tank contents. The 
OWST is outside the vitrification building and releases its contents as a result of a deflagration alone.

Another progression that can occur is illustrated in Figure 5. In this case, if the SPC is isolated and the nitrogen system overpressurises the tanks forcing benzene vapor into the cell, a simultaneous failure of the ventilation system allows a buildup of benzene vapor in the cell. If an explosive concentration develops and an ignition source is available, an explosion in the cell can result. This can be further propagated if tanks containing benzene are splashed leading to a second cell deflagration, SPC tank explosions and then ultimately to a possible RPC deflagration.

Figure 6 shows the modeled progressions for a seismic event. Again the APET establishes the support system status before beginning the accident progression questions. The seismic event may have several immediate outcomes: 1) cell covers fall and rupture tanks, 2) tanks are ruptured by the seismic event, 3) support system.damage leads to explosions, 4) zone 1 ventilation system failure and 5) major damage to the building. Figure 6 is subdivided in Figures 7 through 10 to illustrate the various progressions possible. In Figure 7, several progressions or combinations can occur. Tanks can be ruptured as a result of dislodged cell covers or the direct effect of the seismic event. If the OEV and/or the OECT are splashed the benzene released can lead to cell deflagrations. The more likely sequence is a tank explosion in the SPC or CPC that basically results in the same series of progressions as shown in Figure 3. All progressions may result in an RPC deflagration. In Figure 8, tanks in the melter cell, the LPPP and the OWST are subject to releases because of ruptures or explosions. Finally in Figure 9, the progression is shown where loss of zone 1 fans causes a SPC overpressure deflagration and possibly an RPC deflagration.

Figure 10 shows the progression of events considered in the accident where a heavy object (cell cover) is dropped while being moved by a crane. Operations are procedurally suspended during maintenance requiring crane operations with heavy objects but tanks are not necessarily empty. Before overhead operations with the crane are allowed to commence, the benzene tanks are de-inventoried such that the total release of these contents will not overflow the SPC sump. If a tank is struck, it is assumed that it is ruptured and the contents are released. If the SPC ventilation has failed at the same time a potential exists for a cell deflagration and/or a fire. No additional propagation is assumed beyond the cell deflagration.

Figure 11 shows the progression of events considered if a tank leak occurs. If the release and a zone 1 fan failure occur simultaneously then the possibility a SPC deflagration and/or fire exists.

Miscellaneous events include an uncontrolled reaction in a chemical process cell vessel, an uncontrolled reaction in the precipitate reactor in the salt process cell and unintentional injection of water into the melter. Figure 12 shows that the miscellaneous categories do not lead to additional progressions. 


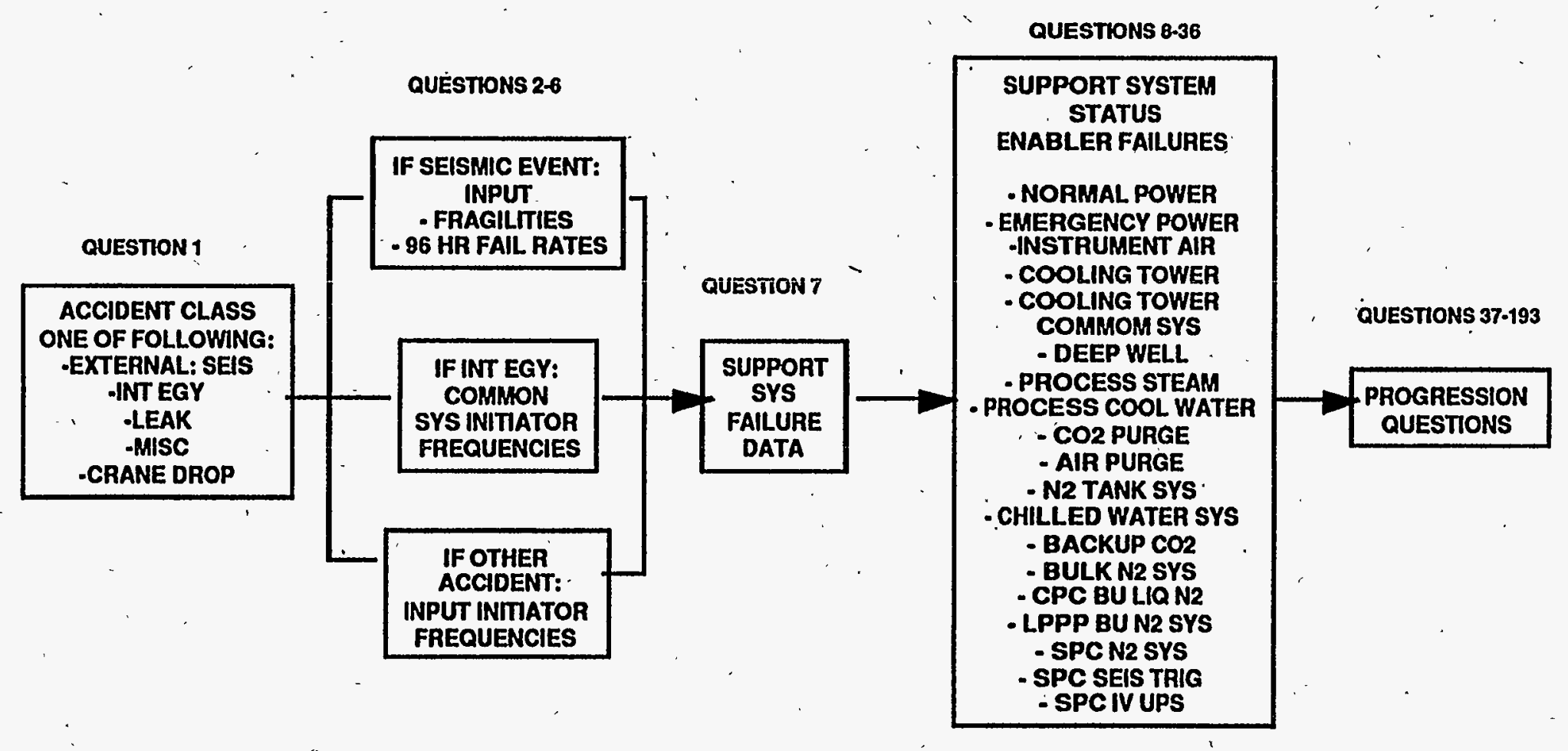

片 


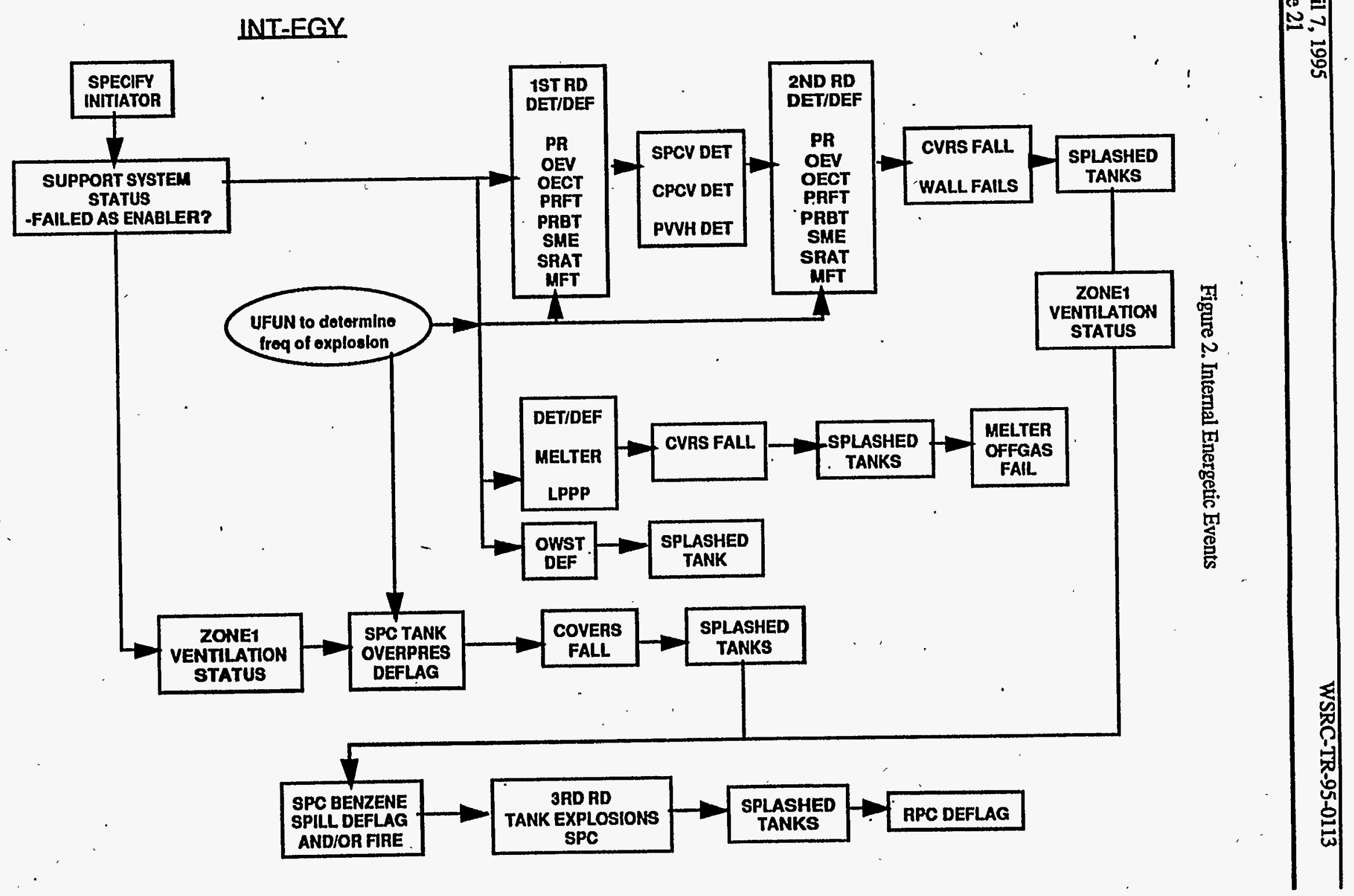




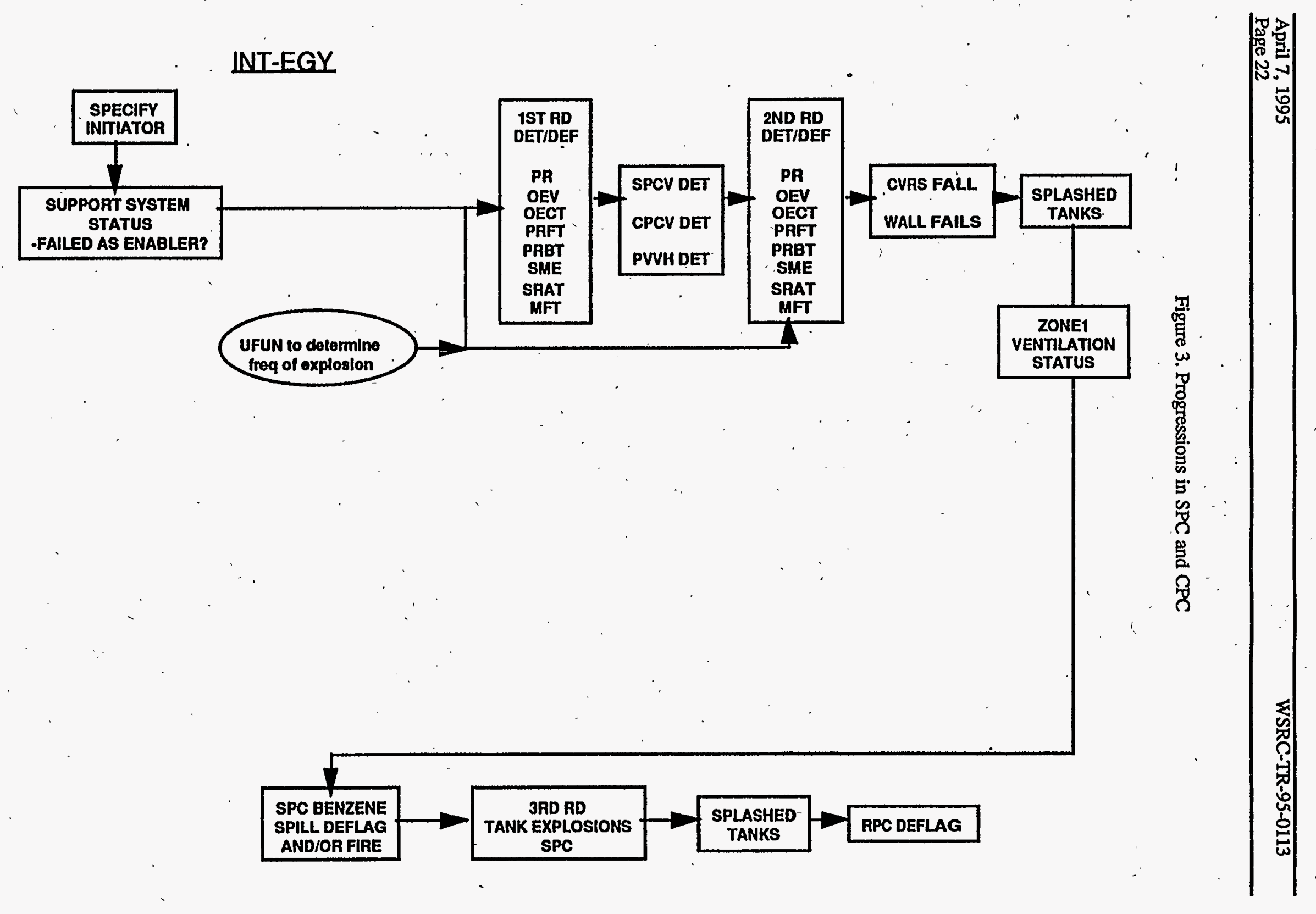




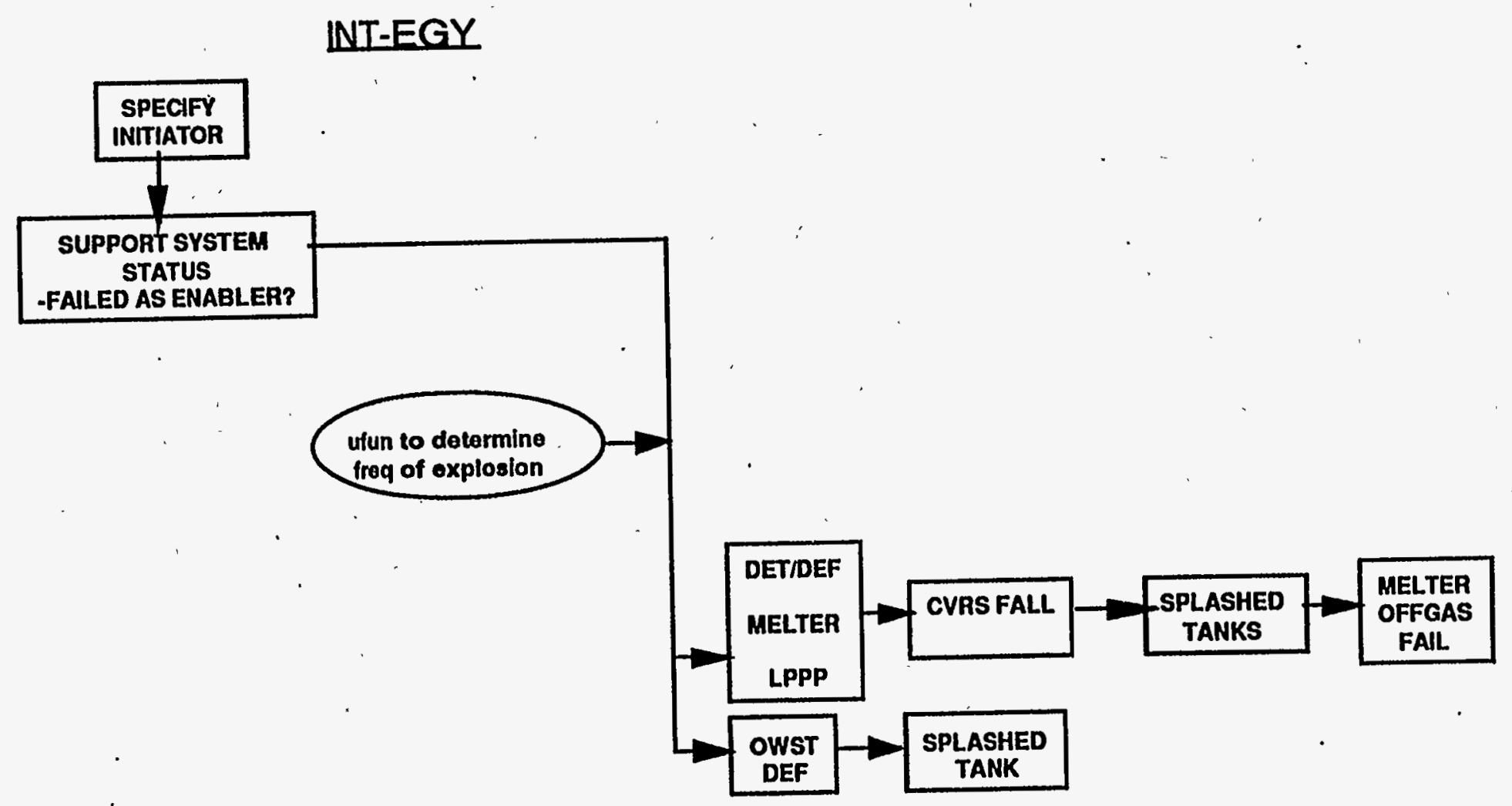


Figure 5. Zone 1 Ventilation Failure Progression

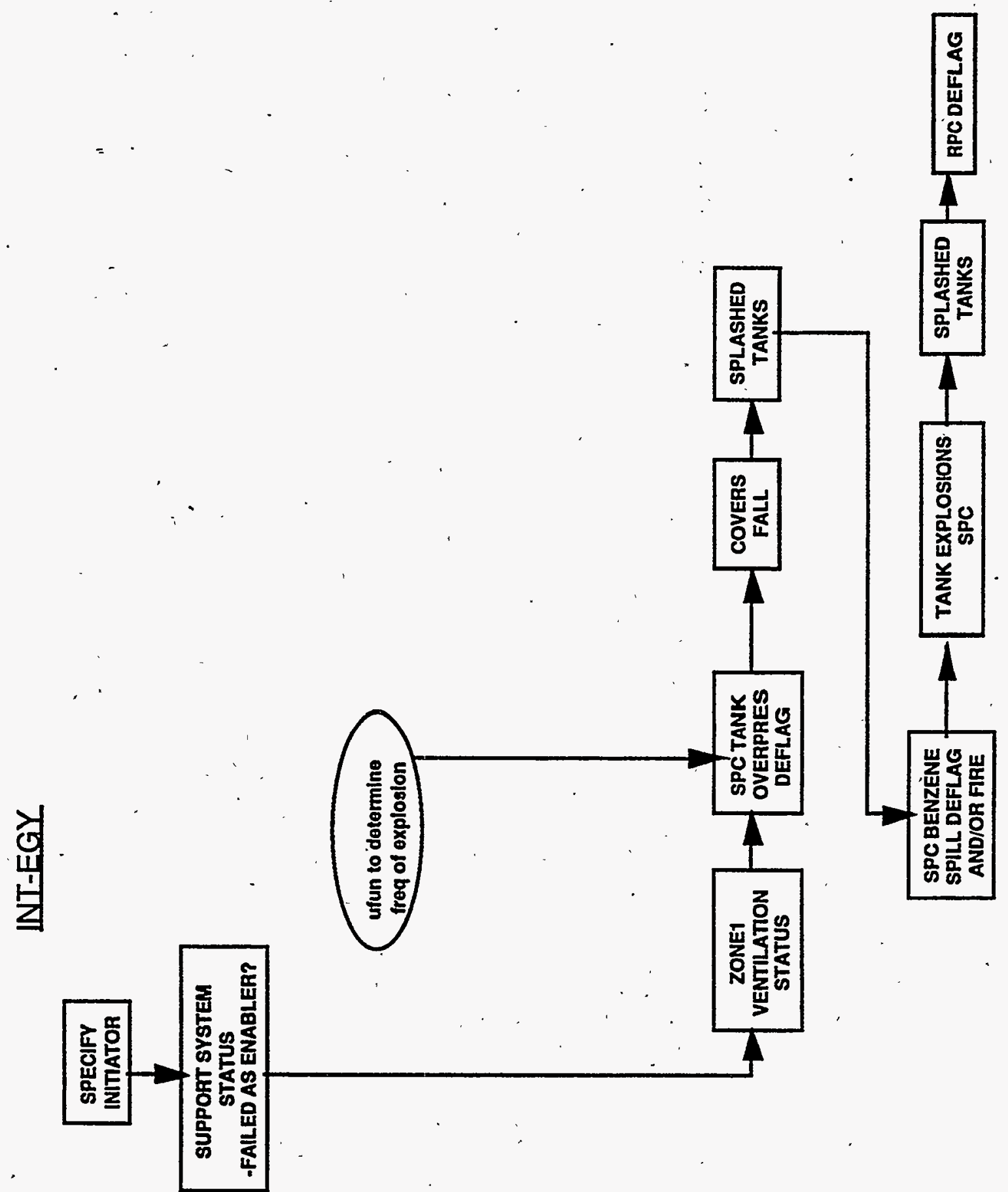




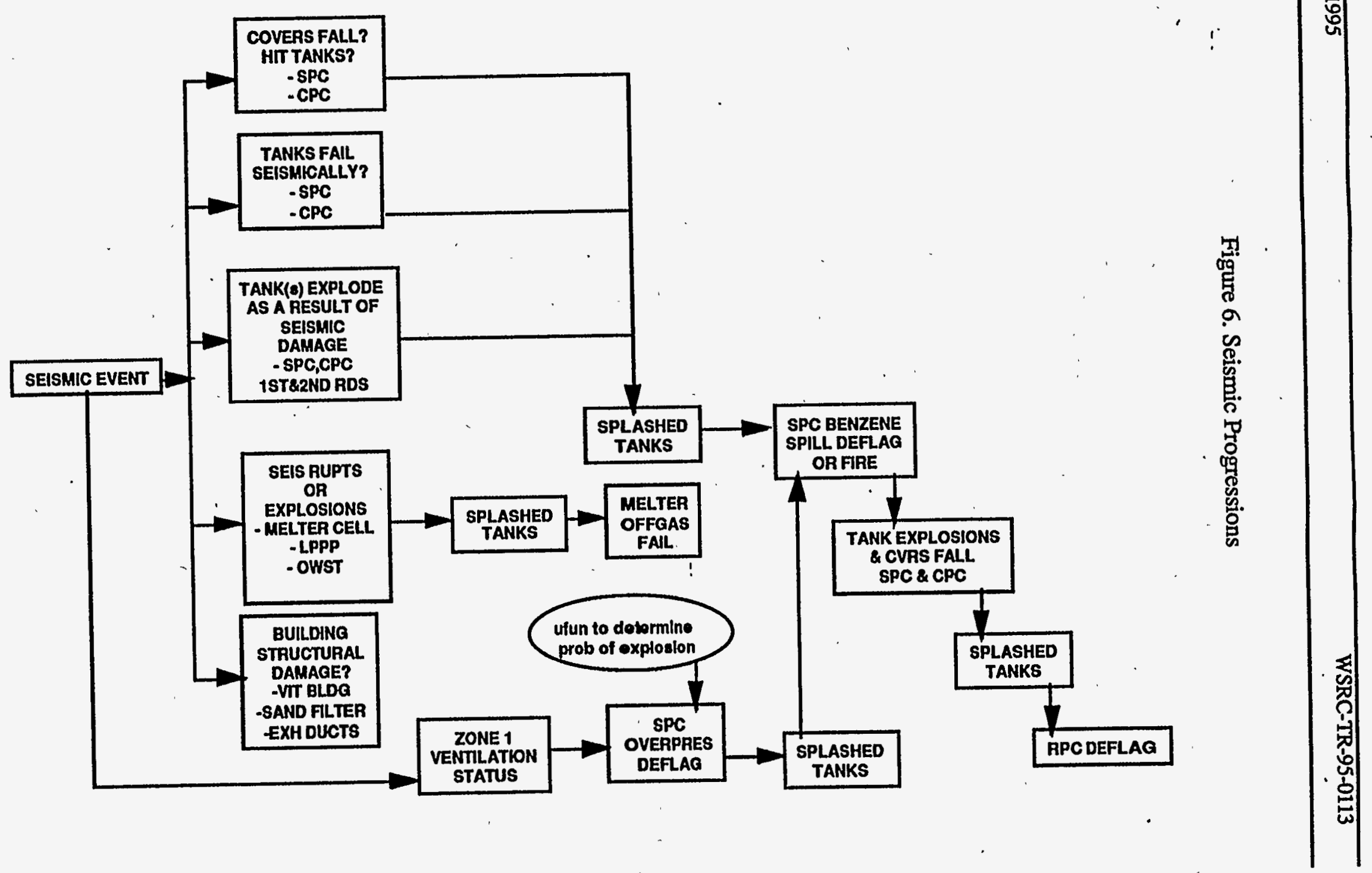


Figure 7. Seismic Progression in SPC and CPC

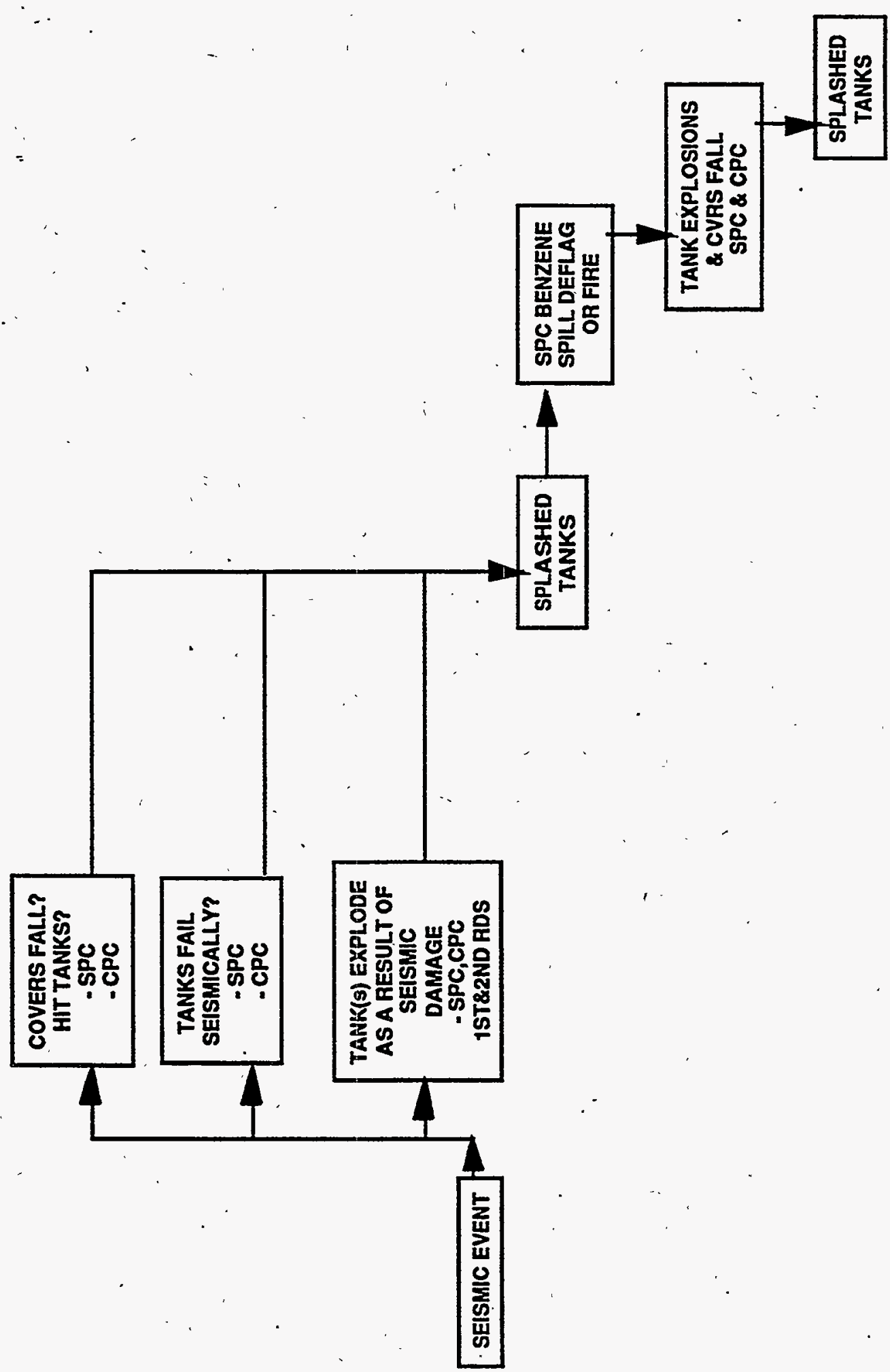




\section{SEISMIC EVENT}

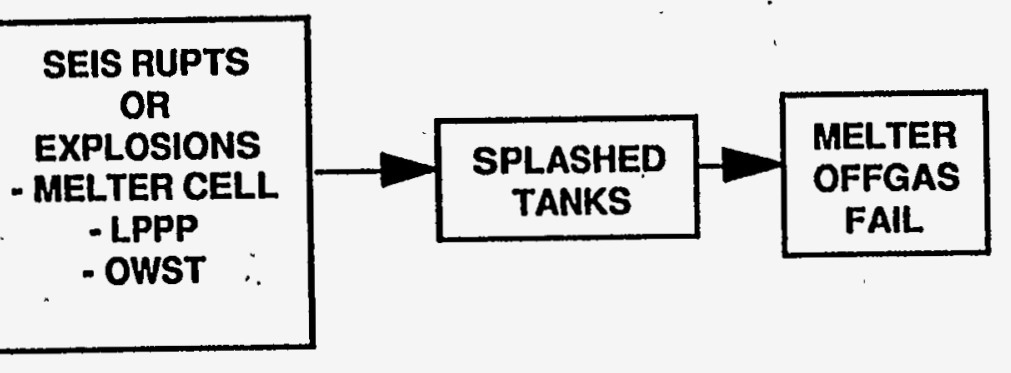



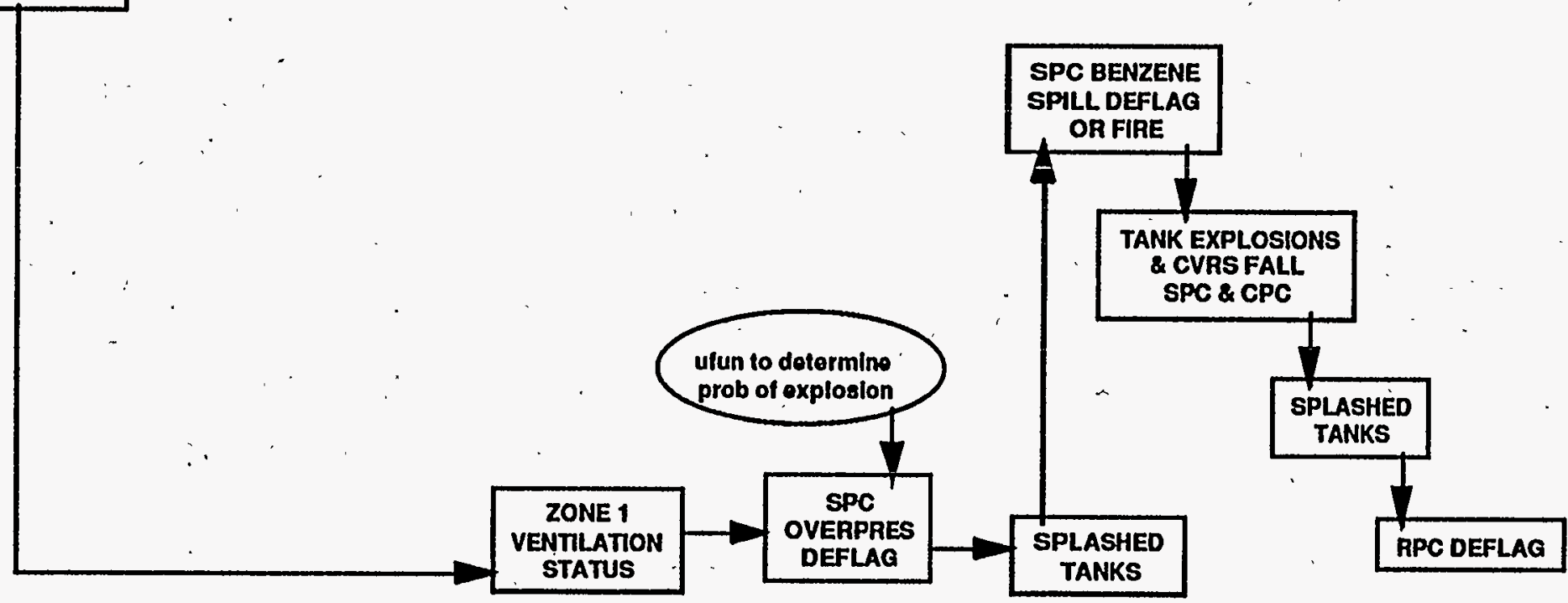

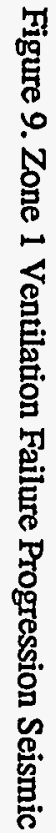




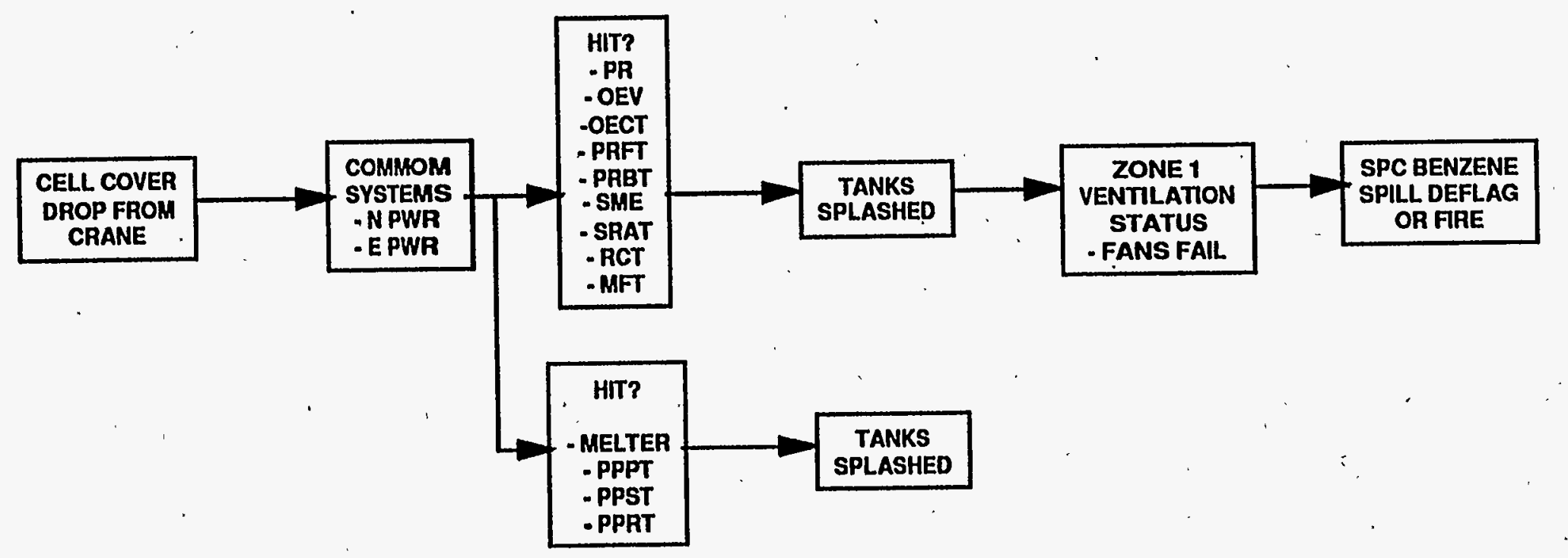

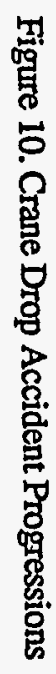




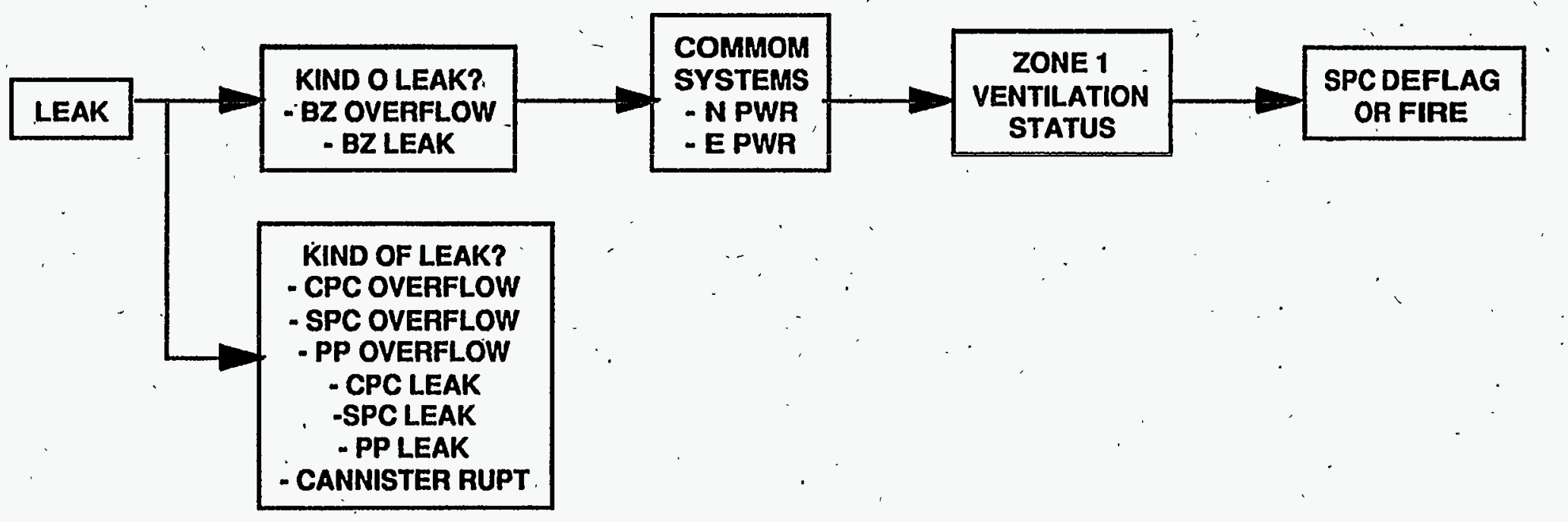


Figure 12. Miscellaneous

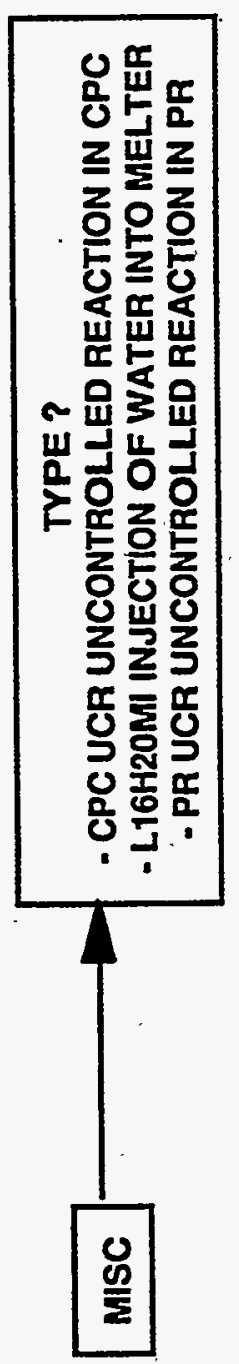




\subsection{APET Method}

The APET can be visualized as a very large event tree with 193 questions (top events). Questions are permitted to have more than 2 responses (branches). Boolean expressions utilizing the answers to previous questions in the APET can be constructed so that the path through the tree can be dependent on answers to previous questions.

Information about the frequency of explosions of the DWPF tanks and systems that have been modeled are provided to the APET from the cut sets that resulted from quantification of the internal and seismic fault trees for these systems. The DWPF User Function (UFUN) is a FORTRAN subroutine designed to be used during quantification of the DWPF APET by the EVNTRE computer code. The UFUN provides an interface between the APET and the processed individual accident fault tree solution files that are provided as input. Given the status of the various DWPF support systems, it selects and solves the appropriate cut sets from the fault tree solutions. The UFUN also has the capability to read information from the Latin Hypercube Sample (LHS) file so that events in the cut sets can be sampled as part of an uncertainty analysis.

The determination of outcome probabilities is a major task in quantification of the APET. As described above the outcome probabilities may be determined by solution of fault tree cut sets which are accessed from the UFUN subroutine in EVNTRE. Outcome probabilities may simply be determined from answers of previous questions and finally they may be input. directly based on mechanistic modeling of accident phenomena or expert engineering judgment.

Each path (accident progression sequence) through the tree is followed until either it reaches the end of the tree or until the sequence frequency drops below a truncation value (1.0E-12 per hour was used for the DWPF quantifications). Quantification of the APET can result in several hundred thousand to more than a million accident progression sequences depending on the particular accident class being modeled. The many sequences that result indicate far more detail about accident progression than can be assessed. Consequently, sequences from the APET are grouped or binned based on similar characteristics or attributes in the accident progression. The result of the sequence binning process can be visualized as a much smaller event tree whose sequences are each an accident progression bin. The cut set that corresponds to each sequence is then a listing of the bin attributes. Although the number of accident progression bins may still be extensive [tens of thousands], they represent a substantial reduction from the total number of accident progression sequences developed by solution of the APET. 


\subsection{Explanation of the APET Questions}

The following sections describe the questions and associated case structure for the APET. Reference should be made to the EVNTRE input file presented in Appendix A for additional information on the case structure.

\section{Question 1: What is the accident class?}

In the development of the APET it was beneficial to categorize the postulated accidents into different classes depending on the initiating event and the expected system response to the initiating event [e.g., potential for release of energy and for progression of the accident]. There are five defined outcomes as follows:

External: This accident class includes all initiating events that are external to the DWPF [e.g., seismic, wind, flood, aircraft crashes].

Int-Egy: . This accident class includes all initiating events that are caused by failure of systems internal to the DWPF and that are expected to result in a significant release of energy.

Ltdleak: This accident class includes all initiating events that result in a leak of process material in the DWPF whose consequences are expected to be limited.

LtdMisc: This accident class includes a number of initiating events that are not process material leaks whose consequences are expected to be limited.

LtdCD: This accident class includes events whose initiator is a drop of a load from an overhead crane in the DWPF facility. In this analysis, the load is assumed to be a cell cover that has been removed in the facility.

This is a Type 3 question and the outcome probabilities are input. An outcome probability of 1.0 is specified for the particular class of accidents to be analyzed. All other outcome probabilities are specified as 0.0 . The answer to this question is used in the logic to determine the outcome probabilities in questions throughout the event tree. This question is also used to specify a control parameter used in solution of the APET as follows:

Arg [90]: LHS, control parameter that indicates the Latin Hypercube sample being used.

The above parameter is used in determining the outcome of Questions $42,46,50,54,59,63$, $67,73,77,79,81,83,85,87,89,91,93,114,152,154,159,165,168,173$, and 176.

\section{Question 2: If the accident class is 'external' what is the initiating event?}

The answer to this question defines the type of external initiating event. There are eight outcomes defined for this question as follows: 
nExtrnl: . This outcome is selected if the initiating event is not an external event, i.e., the outcome of Question 1 is other than Branch 1.

E1-Seis: - This outcome is selected if the external initiating event is a seismic event.

E2-Wind: This outcome is selected if the external initiating event is a high wind.

E3-Flood: This outcome is selected if the external initiating event is a flood.

E4-Other: This outcome is selected if the external initiating event is an undefined external event.

E5-Heli: This outcome is selected if the external initiating event is the crash of a helicopter into the facility.

E6-Plane: This outcome is selected if the external initiating event is the crash of an airplane into the facility.

E7-Vhcle: This outcome is selected if the external initiating event is the crash of a ground vehicle into the facility.

[For the Mode C safety analysis for the DWPF only the seismic event was considered as an initiating event. The rest of the branches are included only as place holders.]

The answers to this question depend upon the outcome of previous Question 1 and are used in the logic to determine the outcome probabilities in questions throughout the event tree. Three cases are used to develop the outcome probabilities on the basis of the outcome of Question 1 as follows:

Case 1 This case includes those sequences in which the accident class is either an internal-energetic event, a limited leak, limited miscellaneous event or crane load drop accident from Question 1. The outcome probability is 1.0 in Branch 1.

Case 2 This case includes the sequences in which the accident class is an external event. The outcome probability is 1.0 in Branch 2.

Case 3 The default outcome probability is 1.0 in Branch 1.

This is a Type 4 question and is also used to define sixty three [63] parameters, primarily external event fragilities, used in the quantification of the APET. These parameters, whose value will depend on the question outcomes, are defined as follows (The source of fragility values shown in the APET in Appendix A are summarized in Reference 4.):

Arg [1]: $\quad$ MsTmEx, a constant used in calculating the failure of support systems for 96 hours after the external event. In the Mode $C$ analysis this parameter is always set equal to 1.0 because the support system fault trees were solved with a 96 hour mission time rather then on a per hour basis.

Arg [2]: NPwrFr, the external event fragility of the normal electrical power system. 
$\operatorname{Arg}[3]: \ldots \quad$ EPwrFr, the external event fragility of the emergency electrical power system.

Arg [4]: $\quad$ PStmFr, the external event fragility of the process steam system.

Arg [5]: PCWFr, the external event fragility of the process cooling water system.

$\operatorname{Arg}[6]$ : $\quad \mathrm{CO} 2 \mathrm{Fr}$, the external event fragility of the $\mathrm{SPC}$ primary $\mathrm{CO}_{2}$ purge system.

Arg [7]: $\quad$ AirFr, the external event fragility of the CPC primary air purge system.

Arg [8]: . N2TkFr, the external event fragility of the bulk liquid nitrogen storage tank system.

Arg [9]: ChWFr, the external event fragility of the chilled water system.

Arg [10]: CoTFr, the external event fragility of the cooling tower pumping system.

$\operatorname{Arg}[11]: \quad \quad B C O 2 F r$, the external event fragility of the SPC backup $\mathrm{CO}_{2}$ purge system.

Arg [12]: CanyClps, the external event fragility for collapse of the canyon.

Arg [13]: TrigFr, the external event fragility for the SPC seismic trigger.

Arg [14]: FiltFr, the external event fragility of the sand filter system.

Arg [15]: DuctFr, the external event fragility of the exhaust duct system.

Arg [16]: FanFr, the external event fragility of the exhaust fan system.

Arg [17]: $\quad$ PPPTFr, the external évent fragility of the Pump Pit Precipitate Tank.

Arg [18]: $\quad$ PPSTFr, the external event fragility of the Pump Pit Sludge Tank.

Arg [19]: PPRTFr, the external event fragility of the Pump Pit Recycle Tank.

Arg [20]: $\quad P R x-F r$, the external event fragility of the Precipitate Reactor.

Arg [21]: $\quad$ OEV-Fr, the external event fragility of the Organic Evaporator Tank.

Arg [22]: OECTFr, the external event fragility of the Organic Evaporator Condensate Tank.

Arg [23]: PRFTFr, the external event fragility of the Precipitate Reactor Feed Tank.

Arg [24]: PRBTFr, the external event fragility of the Precipitate Reactor Bottoms Tank.

Arg [25]: $\quad$ SME-Fr, the external event fragility of the Slurry Mix Evaporator Tank. 
Arg [26]: _. SPCUPSFr, the external event fragility of the uninteruptible power supply for the SPC vent header isolation valves.

Arg [27]:- $\quad$ SRATFr, the external event fragility of the Sludge Receipt and Adjustment Tank.

Arg [28]: $\quad$ RCTFr, the external event fragility of the Recycle Collection Tank.

-Arg [29]: $\quad$ MFTFr, the external event fragility of the Melter Feed Tank.

Arg [30]: MeltFr, the external event fragility of the melter.

Arg [31]: OWSTFr, the external event fragility of the Organic Waste Storage Tank.

Arg [32]: $\quad$ PPCovFr, the external event fragility of the PumF Covers.

Arg [33]: CCovFr, the external event fragility of the : .ers for the Salt and Chemical Process Cells.

Arg [34]: IAirFr, the external event fragility of the Instrument Air System.

Arg [72]: : BlkN2Fr, the external event fragility of the bulk N2 system.

Arg [73]: $\quad$ CPCN2Fr, the external event fragility of the $\mathrm{CPC}$ nitrogen purge system (backup CPC purge).

Arg [74]: $\quad$ NPwrRn, normal power system random failure probability for the 96 hour mission time assumed after a seismic event.

Arg [75]: $\quad$ EPwrRn, emergency power system random failure probability for the 96 hour mission time assumed after a seismic event.

Arg [76]: PStmRn, process steam system random failure probability for the 96 hour mission time assumed after a seismic event.

Arg [77]: $\quad$ PCWRn, process cooling water system random failure probability for the 96 hour mission time assumed after a seismic event.

Arg [78]: $\quad \mathrm{CO} 2 \mathrm{Rn}, \mathrm{SPC}$ primary $\mathrm{CO}_{2}$ purge system random failure probability for the 96 hour mission time assumed after a seismic event.

Arg [80]: AirRn, CPC primary air purge system random failure probability for the 96 hour mission time assumed after a seismic event.

Arg [81]: $\quad$ N2TkRn, bulk N2 storage tank system random failure probability for the 96 hour mission time assumed after a seismic event.

Arg [82]: $\quad$ ChWRn, chilled water system random failure probability for the 96 hour mission time assumed after a seismic event.

Arg [83]: CoTRn, cooling tower pumping system random failure probability for the 96 hour mission time assumed after a seismic event. 
Arg [84]:- $\quad$ BCO2Rn, SPC backup $\mathrm{CO}_{2}$ purge system random failure probability for the 96 hour mission time assumed after a seismic event.

Arg [85]: IAirRn, instrument air system random failure probability for the 96 hour mission time assumed after a seismic event.

Arg [86]: BlkN2Rn, bulk N2 purge system random failure probability for the 96 hour mission time assumed after a seismic event.

Arg [87]: . CPCN2Rn, CPC backup N2 purge system random failure probability for the 96 hour mission time assumed after a seismic event.

Arg [96]: FanRn1, Zone 1 ventilation system random failure probability for the 96 hour mission time assumed after a seismic event under the conditions where normal power has failed but diesel power and instrument air are available.

Arg [97]: CTCmFr, the external event fragility of the cooling tower common system.

Arg [101]: $\quad$ CTCmRn, cooling tower common system random failure probability for the 96 hour mission time assumed after a seismic event.

Arg [102]: DWeIFr, the external event fragility of the deep well cooling system.

Arg [106]: DWelRn, deep well cooling system random failure probability for the 96 hour mission time assumed after a seismic event.

Arg [107]: CPCTkFr, the external event fragility of the liquid nitrogen tank for the CPC backup purge system.

Arg [111]: - CPCTkRn, CPC backup purge system liquid nitrogen tank random failure probability for the 96 hour mission time assumed after a seismic event.

Arg [112]: PPN2Fr, the external event fragility of the Pump Pit nitrogen purge system.

Arg [116]: PPN2Rn, Pump Pit nitrogen purge system random failure probability for the 96 hour mission time assumed after a seismic event.

Arg [117]: $\quad$ SPCN2Fr, the external event fragility of the SPC nitrogen purge system.

Arg [121]: SPCN2Rn, SPC nitrogen purge system random failure probability for the 96 hour mission time assumed after a seismic event.

Arg [122]: FanRn2, Zone 1 ventilation system random failure probability for the 96 hour mission time assumed after a seismic event under the conditions where normal power and instrument air have failed but diesel power is still available. 
Arg [123]: FanRn3, Zone 1 ventilation system random failure probability for the 96 -. hour mission time assumed after a seismic event under the conditions where normal power, diesel power, and instrument air are all available.

Arg [129]: MOGFr, the external event fragility of the Melter Offgas System.

Question 3: Is failure of a common [i.e., support] system the initiating event?

The initiating event that results in damage to the DWPF may be caused by a failure of a 'support' system within the DWPF that is common to more than one process system in the DWPF. The defined outcomes are as follows. (The initiating frequencies are sampled variables, Reference 8. The ones shown in App A. are placeholders.): **SAMPLED VARIABLE**

nCoSyIn: The initiating event is other than failure of a common 'support' system.

NPowInit: Failure of the normal electrical power system is the initiating event.

PStmInit: - Failure of the process steam system is the initiating event.

IAirInit: , Failure of the instrument air system is the initiating event.

CO2Init: Failure of the SPC primary $\mathrm{CO} 2$ purge system is the initiating event.

AirInit: $\quad$ Failure of the CPC primary air purge system is the initiating event.

N2TkInit: . Failure of the bulk nitrogen storage tank system is the initiating event.

ChWInit: Failure of the chilled water system is the initiating event.

CoTInit: Failure of the cooling tower pumping system is the initiating event.

BlkN2Init: Failure of the bulk nitrogen purge system is the initiating event.

CTCmInit: - Failure of the cooling tower common system is the initiating event.

The ansivers to this question depends upon the outcome of previous Question' 1 and are used in the logic to determine the outcome probabilities in Questions $8,9,10,12,16,18,19,21$ through $29,31,32,36,59,63,67,73,77,87,89,91,93,113,152$, and 193 .

Three cases are used to develop the outcome probabilities on the basis of prior events or questions as follows:

Case 1 This case includes those sequences in which the accident class is either an external event, a limited leak, or other limited event from Question 1. The outcome probability is 1.0 in Branch 1.

Case 2 This case includes the sequences in which the accident class is an internalenergetic event. The outcome probability for Branches 2 through 11 are the hourly failure rates determined by failure analysis of the system. The outcome probability for Branch 1 is 1.0 less the sum of all the frequencies in Branches 2 through 11. 
Case 3 The default outcome probability is 1.0 in Branch 1.

This is a Type 4 question and defines a parameter used in the quantification of the APET. This parameter, whose value will depend on the question outcomes, is defined as follows:

Arg [35]: MsTmInt, the time in hours required to restore the failed support system to an operational state.

NOTE: This parameter is not used in the Mode $C$ analysis.

Question 4: If the accident class is an 'internal' event classified 'leak' what is the frequency of the event?

If the initiating event is a leak of radioactive material from a process vessel or pipe in the DWPF whose consequences are expected to be limited, this question defines the frequency of the event. Eleven outcomes have been defined as follows (The initiating frequencies shown in the APET in Appendix A are derived from Reference 10):

nIntLeak: The initiating event is not a leak in DWPF.

Mltr-Leak: The initiating event is a spill of the contents of the melter. (not used)

CPC-Oflow: The initiating event is an overflow of a vessel in the Chemical Process Cell.

PP-Oflow: The initiating event is an overflow of a vessel in the Pump Pit.

CPC-Leak: The initiating event is a leak from a vessel in the Chemical Process Cell.

SPC-Leak: The initiating event is a leak from the PR or PRFT in the Salt Process $\therefore \quad$ Cell.

PP-Leak: The initiating event is a leak in the Pump Pit.

Can-Rup: Waste canister rupture from dropped cell cover, with assumed 1E-03 conditional prob that drop occurs during cannister handling and is struck.

BZ-Oflow: The initiating event is an overflow from the OEV or the OECT in the Salt Process Cell.

BZ-Leak: The initiating event is a leak from the OEV or OECT in the Salt Process Cell.

SPC-Oflow: The initiating event is an overflow from the PR or PRFT in the Salt Process Cell.

This is a Type 2 question. The outcome probabilities for this question depend on the answer to Question 1 and are used in the logic to determine the outcome probabilities in Questions 8,9, $113,121,122,157$, and 184 through 192. Two cases are used to develop the outcome probabilities on the basis of prior events as follows: 
Case 1 This case includes those sequences in which the accident class is a limited -. leak, Branch 3, Question 1. The branch probabilities for Branches 2 through 11 are the hourly frequencies of occurrence of the respective leaks, based on experience in the 200-Area. The outcome probability for Branch 1 is 1.0 less the sum of all the frequencies in Branches 2 through . 11.

Case 2 The default outcome probability is 1.0 in Branch 1.

Question 5: If the accident class is an 'internal' event classified 'Misc.' . what is the frequency of the event?

This question defines the frequency of those events whose consequences are expected to be limited that are other than leaks. Four outcomes have been defined as follows:

nIntMisc: $\quad$ The initiating event is not a one that is classified as Misc.

CPC-UcR: The initiating event is an uncontrolled reaction in Chemical Process Cell vessel.

I16H2OMl: - The initiating event is addition of water to the melter.

PR-UcR: $\quad$ The initiating event is an uncontrolled reaction in the PR in the Salt Process Cell.

This is a Type 2 question. The outcome probabilities for this question depend on the answer to Question 1 and are used in the logic to determine the outcome probabilities in Questions 157, 182, and 183. Two cases are used to develop the outcome probabilities on the basis of prior events as follows (The initiating frequencies shown in the APET in Appendix A are derived from Reference 10. The 'water to the melter' event was included in an earlier version of the DWPF APET but is now known to be an insignificant event and is retained only as a placeholder):

Case 1

This case includes those sequences in which the accident class is a limited miscellaneous event, Branch 4, Question 1. The branch probabilities for Branches 2 through 4 are the hourly frequencies of occurrence of the respective events, based on experience in the 200-Area. The outcome probability for Branch 1 is 1.0 less the sum of all the frequencies in Branches 2 through 4.

Case 2 The default outcome probability is 1.0 in Branch 1.

\section{Question 6: If the accident class is crane drop what is the frequency of the} event?

This question defines the frequency of accidents caused by crane load drops. Two outcomes have been defined as follows:

nCraDrop: $\quad$ The initiating event is not a crane load drop. 
CraneDrop: The initiating event is a crane load drop. For the purposes of this analysis, the crane load is assumed to be a cell cover.

This is a Type 2 question. The outcome probabilities for this question depend on the answer to Question 1 and are used in the logic to determine the outcome probabilities in Question 38. Two cases are used to develop the outcome probabilities on the basis of prior events as follows (The initiating frequencies shown in the APET in Appendix A are derived from Reference 6):

Case 1

This case includes those sequences in which the accident class is a limited cranedrop, Branch 5, Question 1. The outcome probability for Branch 2 is the hourly frequency of occurrence of a crane load drop, based on SRS experience. The outcome probability for Branch 1 is 1.0 less the sum of Branch 2.

Case 2 The default outcome probability is 1.0 in Branch 1.

\section{Question 7: What are the support system failure frequencies and repair times?}

This is a Type 4 question and is used to define forty eight parameters for use in quantification of the APET. The values assigned the parameters are dependent on the outcome of Question 1. Three cases are used to define the condition for assigning values to the parameters on the basis of prior events as follows (The failure frequencies $(\lambda)$ and the repair times $(\tau)$ shown in the APET in Appendix A are derived from Reference 5):

Case 1 This case includes those sequences in which the accident class is an internal-energetic event.

Case 2 This case includes those sequences in which the accident class is an external event.

Case 3 This case includes all remaining sequences.

The parameters are as follows:

Arg [36] NPwrLmda, the hourly failure frequency of the normal electrical power system.

Arg [37] EPwrLmda, the hourly failure frequency of the emergency electrical power system.

Arg [38] PStmLmda, the hourly failure frequency of the process steam system.

Arg [39] PCWLmda, the hourly failure frequency of the process cooling water system.

$\operatorname{Arg}[40] \quad \mathrm{CO} 2 \mathrm{Lmda}$, the hourly failure frequency of the SPC primary $\mathrm{CO}_{2}$ purge system.

$\operatorname{Arg}$ [41] AirLmda, the hourly failure frequency of the CPC primary air purge system. 
Arg [42] _. N2TkLmda, the hourly failure frequency of the bulk $\mathrm{N}_{2}$ storage tank system.

Arg [43]. . ChWLmda, the hourly failure frequency of the chilled water system.

Arg [44] FanLmda1, the hourly failure frequency of the Zone 1 ventilation exhaust fans with normal power failed, diesel power available, and instrument air available.

$\operatorname{Arg}$ [45] IAirLmda, the hourly failure frequency of the instrument air system.

Arg [46] CoTLmda, the hourly failure frequency of the cooling tower pumping system.

$\operatorname{Arg}[47]^{\prime} \quad B C O 2 L m d a$, the hourly failure frequency of the SPC backup $\mathrm{CO}_{2}$ purge system.

Arg [48] NPwrTau, the repair time, in hours, of the normal electrical power system.

$\operatorname{Arg}[49]$

EPwrTau, the repair time, in hours, of the emergency electrical power system.

Arg [50] PStmTau, the repair time, in hours, of the process steam system.

Arg [51] PCWTau, the repair time, in hours, of the process cooling water system.

$\operatorname{Arg}[52] \quad \mathrm{CO}_{2} \mathrm{Tau}$, the repair time, in hours, of the SPC primary $\mathrm{CO}_{2}$ purge system.

$\operatorname{Arg}[53] \quad$. AirTau, the repair time, in hours, of the CPC primary air purge system.

$\operatorname{Arg}$ [54] . N2TkTau, the repair time, in hours, of the bulk N2 storage tank system.

$\operatorname{Arg}$ [55] ChWTau, the repair time, in hours, of the chilled water system.

Arg [56] FanTau1, the repair time, in hours, of the Zone 1 ventilation exhaust fans with normal power failed, diesel power available, and instrument air available.

Arg [57] IAirTau, the repair time, in hours, of the instrument air system.

$\operatorname{Arg}[58]$. CoTTau, the repair time, in hours, of the cooling tower pumping system.

$\operatorname{Arg}[59] \quad$ BCO2Tau, the repair time in hours of the SPC backup $\mathrm{CO}_{2}$ purge system.

Arg [79] Dummy, a logical parameter used to force specific outcomes in certain cases in some APET questions.

ATg [88] BlkN2Lmda, the hourly failure frequency of the bulk $\mathrm{N}_{2}$ purge system.

Arg [89] . BlkN2Tau, the repair time in hours of the bulk $\mathrm{N}_{2}$ purge system.

Arg [91] CPCN2Lmda, the hourly failure frequency of the CPC backup N2 purge system. 
Arg [92] ${ }^{-} \quad$ CPCN2Tau, the repair time in hours of the CPC backup N2 purge system.

Arg [99]

CTCmLmda, the hourly failure frequency of the cooling tower common system.

Arg [100] CTCmTau, the repair time in hours of the cooling tower common system.

Arg [104]

DWell mda, the hourly failure frequency of the deep well cooling system.

$\operatorname{Arg}[105]$

DWelTau, the repair time in hours of the deep well cooling system.

$\operatorname{Arg}[109]$

CPCTkLmda, the hourly failure frequency of the CPC backup purge liquid nitrogen storage tank system.

Arg [110] CPCTkTau, the repair time in hours of the CPC backup purge liquid nitrogen storage tank system.

Arg [114] PPN2Lmda, the hourly failure frequency of the Pump Pit nitrogen purge system.

Arg [115] PPN2Tau, the repair time in hours of the Pump Pit nitrogen purge system.

Arg [119] SPCN2Lmda, the hourly failure frequency of the SPC backup nitrogen purge system.

Arg [120] SPCN2Tau, the repair time in hours of the SPC backup nitrogen purge system.

$\operatorname{Arg}$ [124] FanLmda2, the hourly failure frequency of the Zone 1 ventilation exhaust fans with normal power failed, diesel power available, and instrument air failed.

$\operatorname{Arg}[125] \quad$ FanTau2, the repair time, in hours, of the Zone 1 ventilation exhaust fans with normal power failed, diesel power available, and instrument air failed.

$\operatorname{Arg}$ [126] FanLmda3, the hourly failure frequency of the Zone 1 ventilation exhaust fans with normal power available, diesel power available, and instrument air available.

Arg [127] FanTau3, the repair time, in hours, of the Zone 1 ventilation exhaust fans with normal power available, diesel power available, and instrument air available.

Arg [130] TrigLmda, the hourly failure frequency of the SPC seismic trigger.

Arg [131] TrigTau, the repair time, in hours, of the SPC seismic trigger.

Arg [132] SUPSLmda, the hourly failure frequency of the SPC isolation valve uninteruptible power supply.

Arg [133] SUPSTau, the repair time, in hours, of the SPC isolation valve uninteruptible power supply. 
Arg [134] - Zero, a logical parameter used to force specific outcomes in certain cases in some APET questions.

As previously indicated the APET for the DWPF is different from the usual accident progression tree using EVNTRE methodology. The APET is used not only to describe accident progression but the event tree and its associated FORTRAN function subroutines includes and solves fault trees for failures leading to accidents in various DWPF systems. Also the APET is organized to present a consistent approach to the analysis of accidents that may occur at the DWPF. This is accomplished in-part by treating common system events explicitly, i.e., they are defined in Questions 8 through 35. These events are necessary to represent dependencies, and consequently, dependent failures among the DWPF system fault trees.

\section{Question 8: Is the failure of the normal electrical power system an 'accident enabler'?}

Failure of the normal electric power system can contribute to the events leading to an accident, either as the initiating event [Question 3] or as one of a series of events that causes the accident [an 'enabler']. The normal power system has no dependancies on any other DWPF support systems. There are two defined outcomes as follows:

NPowEn: The normal electric power system fails but does not initiate the accident.

nNPowEn: The normal electric power system does not fail [or its failure has previously been identified in Question 3 as the failure that initiates the accident].

This is a Type 8 question and defines a parameter that is also used in calculations to determine the outcome probabilities of later Questions $42,46,50,54,59,63,67,73,77,79,81,83$, $85,87,89,91,93,114,159,168,173$, and 176. The parameter is as follows:

Arg [60] FailNPwr, a logical variable that indicates the state of the normal power system. The parameter is assigned a value of $\{-1.0\}$ if the system has failed as the 'initiator', $\{+1.0\}$ if the system is failed but not as the 'initiator', and $\{0.0\}$ if the system is available.

The outcome probabilities for this question depend primarily on the accident class and the reliability of the normal electric power system. They are used in the logic to determine the outcome probabilities in Questions 9,18,21, 22, 23, 24, 25, 27, 113, 152, and 193 and are used to determine the value assigned the above parameter. Four cases are used to develop the outcome probabilities on the basis of prior events as follows:

Case 1 This case includes those sequences in which the accident class is an internal-energetic event [Question 1, Branch 2] and failure of the normal electric power is an initiating event [Question 3]. The outcome probability assigned is 1.0 for Branch 2. The parameter, FailNPwr, is assigned a value of $\{-1.0\}$ when Branch 2 is selected as the outcome.

Case 2 This case includes those sequences in which the accident class is an internal-energetic event, a limited leak, a limited miscellaneous event, or a crane drop and failure of the normal electric power is not an initiating 
Case 3

Case 4 event [Question 3]. The outcome probability for Branch 1 is calculated as a function of NPwrLmda and NPwrTau. The outcome probability for Branch 2 is 1.0 less the probability assigned Branch 1. The parameter, FailNPwr, is assigned a value of $\{+1.0\}$ when Branch 1 is selected as the outcome and a value of $\{0.0\}$ when Branch 2 is selected as the outcome.

This case includes those sequences in which the accident class is an external event [Question 1, Branch 1]. The outcome probability for Branch 1 is calculated as a function of NPwrLmda and NPwrTau, NPwrFr, and NPwrRn and MsTmEx. The outcome probability for Branch 2 is 1.0 less the probability assigned Branch 1. The parameter, FailNPwr, is assigned a value of $\{+1.0\}$ when Branch 1 is selected as the outcome and a value of $\{0.0\}$ when Branch 2 is selected as the outcome.

The default case includes all other sequences. The outcome probability assigned is 1.0 in Branch 2, failure of the normal electric power is not an enabler. The parameter, FailNPwr, is assigned a value of $\{0.0\}$ when branch 2 is selected.

\section{Question 9: Is the failure of the emergency [diesel] electrical power system} an 'accident enabler'?

Failure of the emergency electric power system can contribute to an accident as one of a series of events. Failure of the emergency electric power system can not initiate an accident because it is a backup system and not on-line. The emergency power system has no dependancies on any other DWPF support systems. There are two defined outcomes as follows:

EPowEn: The emergency electric power fails.

nEPowEn: The emergency electric power does not fail.

This is a Type 8 question and defines a parameter that is also used in calculations to determine the outcome probabilities of later Questions $63,67,77,89,91,159,168,173$, and 176. The parameter is as follows:

Arg [61] FailEPwr, a logical variable that indicates the state of the emergency power system. The parameter is assigned a value of $\{+1.0\}$ if the system is failed and $\{0.0\}$ if the system is available.

The outcome probabilities for this question depend primarily on the accident class and the reliability of the emergency electric power system. They are used in the logic to determine the outcome probabilities in Questions 18,23,27,113, and 193 and are used to determine the value assigned the above parameter. Four cases are used to develop the outcome probabilities on the basis of prior events as follows:

Case 1

This case includes those sequences in which the accident class is an internal-energetic event [Question 1, Branch 2] and normal electric power has failed, either as an 'initiator' or an 'enabler'. The outcome probability for Branch 1 is calculated as a function of EPwrLmda and EPwrTau. The outcome probability for Branch 2 is 1.0 less the probability assigned Branch 1. The parameter, FailEPwr, is assigned a value of $\{+1.0\}$ when 

Branch 1 is selected as the outcome and a value of $\{0.0\}$ when Branch 2 -. is selected as the outcome.
Case $2^{\prime}$ - This case includes sequences in which the accident class is a limited leak, limited crane drop, or limited miscellaneous and the normal power system has failed as an enabler. The outcome probability for Branch 1 is calculated as a function of EPwrLmda and EPwrTau. The outcome probability for Branch 2 is 1.0 less the probability assigned Branch 1 . The parameter, FailEPwr, is assigned a value of $\{+1.0\}$ when Branch 1 is selected as the outcome and a value of $\{0.0\}$ when Branch 2 is selected as the outcome.
Case 3 This case includes those sequences in which the accident class is an external event [Question 1, Branch 1]. The outcome probability for Branch 1 is calculated in subroutine UAVAIL as a function of EPwrLmda and EPwrTau, EPwrFr, and EPwrRn and MsTmEx. The outcome probability for Branch 2 is 1.0 less the probability assigned Branch 1. The parameter, FailEPwr, is assigned a value of $\{+1.0\}$ when Branch 1 is selected as the outcome and a value of $\{0.0\}$ when Branch 2 is selected as the outcome.
Case 4 The default case includes all other sequences. The outcome probability assigned is 1.0 in Branch 2, failure of the emergency electric power is not an accident 'enabler'. The parameter, FailEPwr, is assigned a value of $\{0.0\}$ when branch 2 is selected.

\section{Question 10: Does the Instrument Air system fail due to random events?}

Failure of the instrument air system to function can contribute to the events leading to an accident, either as the initiating event [Question 3] or as one of a series of events that causes the accident [an 'enabler]. There are two defined outcomes as follows:

IAirRf: The instrument air system fails due to random events but does not initiate the accident.

nIAirRf : - The instrument air system does not fail due to random events.

This is a Type 6 question. The outcome probabilities for this question depend on the accident class and the reliability of the system and are used in the logic to determine the outcome probabilities in Questions 18, 19, and 21. Two cases are used to develop the outcome probabilities on the basis of prior events as follows:

Case 1 This case includes those sequences in which the accident class is an internal-energetic event [Question 1, Branch 2] and failure of the instrument air system is not the initiating event [Question 3]. The outcome probability for Branch 1 is calculated as a function of IAirLmda and IAirTau. The outcome probability for Branch 2 is 1.0 less the probability assigned Branch 1. 
Case 2 .. The default case includes all other sequences. The outcome probability assigned is 1.0 in Branch 2, failure of the instrument air system does not occur due to random events.

\section{Question 11: Does the Instrument Air system fail due to a seismic event?}

Failure of the instrument air system to function after an external event can contribute to the events leading to an accident as one of a series of events that causes the accident [an 'enabler']. There are two defined outcomes as follows:

IAirSe: The instrument air system fails due to an external event.

nIAirSe: The instrument air system does not fail due to an external event.

This is a Type 6 question. The outcome probabilities for this question depend on the accident class and the fragility of the system to the external event, and are used in the logic to determine the outcome probabilities in Questions 18,19,21. Two cases are used to develop the outcome probabilities on the basis of prior events:

Case 1 This case includes those sequences in which the accident class is an external event [Question 1, Branch 1]. The outcome probability for Branch 1 is calculated as a function of IAirLmda and IAirTau, IAirFr, and IAirRn and MsTmEx. The outcome probability for Branch 2 is 1.0 less the probability assigned Branch 1.

Case 2 The default case includes all other sequences. The outcome probability assigned is 1.0 in Branch 2, failure of the instrument air system does not occur due to an external event.

\section{Question 12: Does the Cooling Tower Common system fail due to random events?}

Failure of the cooling tower common system to function can contribute to the events leading to an accident, either as the initiating event [Question 3] or as one of a series of events that causes the accident [an 'enabler']. There are two defined outcomes as follows:

CTCmRf: The cooling tower common system fails due to random events but does not initiate the accident.

nCTCmRf : The cooling tower common system does not fail due to random events.

This is a Type 6 question. The outcome probabilities for this question depend on the accident class and the reliability of the system and are used in the logic to determine the outcome probabilities in Questions 18 and 21. Two cases are used to develop the outcome probabilities on the basis of prior events as follows:

Case 1 This case includes those sequences in which the accident class is an internal-energetic event [Question 1, Branch 2] and failure of the cooling tower common system is not the initiating event [Question 3]. The outcome probability for Branch 1 is calculated as a function of CTCmLmda 
and CTCmTau. The outcome probability for Branch 2 is 1.0 less the

$\therefore$ probability assigned Branch 1 :

Case 2 .. The default case includes all other sequences. The outcome probability assigned is 1.0 in Branch 2, failure of the cooling tower common system does not occur due to random events.

Question 13: Does the Cooling Tower Common system fail due to a seismic event?

Failure of the cooling tower common system to function after an external event can contribute to the events leading to an accident as one of a series of events that causes the accident [an 'enabler']. There are two defined outcomes as follows:

CTCmSe: The cooling tower common system fails due to an external event.

nCTCmSe: The cooling tower common system does not fail due to an external event.

This is a Type 6 question. The outcome probabilities for this question depend on the accident class and the fragility of the system to the external event, and are used in the logic to determine the outcome probabilities in Questions 18 and 21. Two cases are used to develop the outcome probabilities on the basis of prior events as follows:

Case 1 This case includes those sequences in which the accident class is an external event [Question 1, Branch 1]. The outcome probability for Branch 1 is calculated as a function of CTCmLmda and CTCmTau, CTCmFr, and CTCmRn and MsTmEx. The outcome probability for Branch 2 is 1.0 less the probability assigned Branch 1 .

Cáse 2 The default case includes all other sequences. The outcome probability assigned is 1.0 in Branch 2, failure of the cooling tower common system does not occur due to an external event.

\section{Question 14: Does the Deep Well system fail due to random events?}

Failure of the deep well cooling system to function can contribute to the events leading to an accident as one of a series of events that causes the accident [an 'enabler']. There are two defined outcomes as follows:

DWelRf: . The deep well cooling system fails due to random events.

nDWelRf: The deep well cooling system does not fail due to random events.

This is a Type 6 question. The outcome probabilities for this question depend on the accident class and the reliability of the system and are used in the logic to determine the outcome probabilities in Questions 18 and 20. Two cases are used to develop the outcome probabilities on the basis of prior events as follows:

Case 1 This case includes those sequences in which the accident class is an internal-energetic event [Question 1, Branch 2]. The outcome probability 
-. for Branch 1 is calculated as a function of DWellmda and DWelTau. The outcome probability for Branch 2 is 1.0 less the probability assigned Branch 1.

Case 2

The default case includes all other sequences. The outcome probability assigned is 1.0 in Branch 2, failure of the deep well cooling system does not occur due to random events.

\section{Question 15: Does the Deep Well system fail due to seismic events?}

Failure of the deep well cooling system to function after an external event can contribute to the events leading to an accident as one of a series of events that causes the accident [an 'enabler']. There are two defined outcomes as follows:

DWelSe: $\quad$ The deep well cooling system fails due to an external event.

nDWelSe: The deep well cooling system does not fail due to an external event.

This is a Type 6 question. The outcome probabilities for this question depend on the accident class and the fragility of the system to the external event and are used in the logic to determine the outcome probabilities in Questions 18 and 20. Two cases ate used to develop the outcome probabilities on the basis of prior events as follows:

Case 1 This case includes those sequences in which the accident class is an external event [Question 1, Branch 1]. The outcome probability for Branch 1 is calculated as a function of DWellmda and DWelTau, DWelFr, and DWelRn and MsTmEx. The outcome probability for Branch 2 is 1.0 less the probability assigned Branch 1 .

Case 2 The default case includes all other sequences. The outcome probability assigned is 1.0 in Branch 2, failure of the deep well cooling system does not occur due to an external event.

\section{Question 16: Does the Cooling Tower system fail due to random events?}

Failure of the cooling tower pumping system to function can contribute to the events leading to an accident, either as the initiating event [Question 3] or as one of a series of events that causes the accident [an 'enabler']. There are two defined outcomes as follows:

CoTRf: The cooling tower pumping system fails due to random events but does not initiate the accident.

nCoTRf : The cooling tower pumping system does not fail due to random events.

This is a Type 6 question. The outcome probabilities for this question depend on the accident class and the reliability of the system and are used in the logic to determine the outcome probabilities in Questions 18 and 21 . Two cases are used to develop the outcome probabilities. on the basis of prior events as follows: 
Case $1 \quad \ldots$ This case includes those sequences in which the accident class is an internal-energetic event [Question 1, Branch 2] and failure of the cooling tower pumping system is not the initiating event [Question 3]. The outcome probability for Branch 1 is calculated as a function of CoTLmda and CoTTau. The outcome probability for Branch 2 is 1.0 less the probability assigned Branch 1.

Case 2 - The default case includes all other sequences. The outcome probability assigned is 1.0 in Branch 2, failure of the cooling tower pumping system does not occur due to random events.

\section{Question 17: Does the Cooling Tower system fail due to a seismic event?}

Failure of the cooling tower pumping system to function after an external event can contribute to the events leading to an accident as one of a series of events that causes the accident [an 'enabler']. There are two defined outcomes as follows:

CoTSe: The cooling tower pumping system fails due to an external event.

nCoTSe: The cooling tower pumping system does not fail due to an external event.

This is a Type 6 question. The outcome probabilities for this question depend on the accident class and the fragility of the system to the external event and are used in the logic to determine the outcome probabilities in Questions 18 and 21. Two cases are used to develop the outcome probabilities on the basis of prior events as follows:

Case 1 This case includes those sequences in which the accident class is an external event [Question 1, Branch 1]. The outcome probability for Branch 1 is calculated as a function of CoTLmda and CoTTau, CoTFr, and CoTRn and MsTmEx. The outcome probability for Branch 2 is 1.0 less the probability assigned Branch 1 .

Case 2 The default case includes all other sequences. The outcome probability assigned is 1.0 in Branch 2, failure of the cooling tower pumping system does not occur due to an external event.'

\section{Question 18: Is failure of the Instrument Air system an 'accident enabler'?}

Failure of the instrument air system to function can contribute to the events leading to an accident, either as the initiating event [Question 3] or as one of a series of events that causes the accident [an 'enabler']. The instrument air system is dependant on the following DWPF support systems: normal and emergency electrical power, and the cooling tower. Total failure of power or failure of the cooling tower system causes failure of the instrument air system. There are two defined outcomes as follows:

IAirEn: The instrument air system does not function but does not initiate the accident. . 
nIAirEn: The instrument air system continues to function [or its failure to function has previously been identified in Question 3 as the failure that initiates the accident].

This is a Type 8 question and defines a parameter that is used in calculations to determine the outcome probabilities of later Questions $63,67,77,89,91$, and 152. The parameter is as follows:

Arg [93] FaillAir, a logical variable that indicates the state of the instrument air system. The parameter is assigned a value of $\{-1.0\}$ if the loss of function of the instrument air system is the 'initiator', $\{+1.0\}$ if the instrument air system is not functioning but is not the 'initiator', and $\{0.0\}$ if the instrument air system is functioning.

The outcome probabilities for this question depend on the accident class and the reliability of the system and are used in the logic to determine the outcome probabilities in Questions 22, 23, 27 , and 113 and to determine the value assigned the above parameter. Six cases are used to develop the outcome probabilities on the basis of prior events as follows:

Case 1 This case includes those sequences in which the accident class is an internal-energetic event [Question 1, Branch 2] and failure of the instrument air system is the initiating event [Question 3]. The outcome probability assigned is 1.0 for Branch 2 . The parameter, FaillAir, is assigned a value of $\{-1.0\}$ when Branch 2 is selected as the outcome.

Case 2 This case includes those sequences in which the accident class is an internal-energetic event [Question 1, Branch 2] and failure of the cooling tower common system is the initiating event [Question 3]. The outcome probability assigned is 1.0 for Branch 1 . The parameter, FaillAir, is assigned a value of $\{1.0\}$ when Branch 1 is selected as the outcome.

Case 3 This case includes those sequences in which the normal and emergency electric power systems are failed. The instrument air system cannot fulfill its function in the event the normal and emergency electric power systems fail. Therefore, an outcome probability of 1.0 is assigned to Branch 1 . The parameter, FaillAir, is assigned a value of $\{+1.0\}$ when Branch 1 is selected as the outcome.

Case 4 This case includes those sequences in which the accident class is an internal-energetic event [Question 1, Branch 2] and either the cooling water system has failed due to any of its three modeled subsystems (cooling tower common, cooling tower pumping, deep well) or the instrument air system has failed randomly. An outcome probability of 1.0 is assigned to Branch 1. The parameter, FaillAir, is assigned a value of $\{+1.0\}$ when Branch 1 is selected as the outcome.

Case 5 This case includes those sequences in which the accident class is an external event [Question 1, Branch 1] and either the cooling water system has failed due to any of its three modeled subsystems being failed by the external event (cooling tower common, cooling tower pumping, deep well) or the instrument air system has failed due to the external event. An outcome probability of 1.0 is assigned to Branch 1 . The parameter, 
FaillAir, is assigned a value of $\{+1.0\}$ when Branch 1 is selected as the outcome.

Case 6 .. The default case includes all other sequences. The outcome probability assigned is 1.0 in Branch 2, failure of the instrument air system is not an accident 'enabler'. The parameter, FaillAir, is assigned a value of $\{0.0\}$ when branch 2 is selected.

\section{Question 19: Is the failure of the Cooling Tower Common system an 'accident enabler'?}

Failure of the cooling tower common system to function can contribute to the events leading to an accident, either as the initiating event [Question 3] or as one of a series of events that causes the accident [an 'enabler']. The cooling tower common system is dependant on the instrument air system. Failure of the instrument air system causes the cooling tower common system to fail. There are two defined outcomes as follows:

CTCmEn: The cooling tower common system does not function but does not initiate the accident.

nCTCmEn: The cooling tower common system continues to function [or its failure to function has previously been identified in Question 3 as the failure that initiates the accident].

This is a Type 8 question and defines a parameter that is used in calculations to determine the outcome probabilities of later Questions $63,67,77,89,91$, and 152 . The parameter is as follows:

$\operatorname{Arg}[98]$

FailCTCm, a logical variable that indicates the state of the cooling tower common system. The parameter is assigned a value of $\{-1.0\}$ if the loss of function of the cooling tower common system is the 'initiator', $\{+1.0\}$ if the cooling tower common system is not functioning but is not the 'initiator', and $\{0.0\}$ if the cooling tower common system is functioning.

The outcome probabilities for this question depend on the accident class and the reliability of the system and are used in the logic to determine the outcome probabilities in Questions 23 and 27 and to determine the value assigned the above parameter. Five cases are used to develop the outcome probabilities on the basis of prior events as follows:

Case 1 This case includes those sequences in which the accident class is an internal-energetic event [Question 1, Branch 2] and failure of the cooling tower common system is the initiating event [Question 3]. The outcome probability assigned is 1.0 for Branch 2. The parameter, FailCTCm, is assigned a value of $\{-1.0\}$ when Branch 2 is selected as the outcome.

Case 2 This case includes those sequences in which the accident class is an internal-energetic event [Question 1,.Branch 2] and failure of the instrument air system is the initiating event [Question 3]. The cooling tower common system cannot perform its function if the instrument air system has failed. The outcome probability for Branch 1 is assigned a 
value of 1.0. The parameter, FailCTCm, is assigned a value of $\{+1.0\}$ when Branch 1 is selected as the outcome.

Case 3 - This case includes those sequences in which the accident class is an internal-energetic event [Question 1, Branch 2] and failure of the cooling tower common system or the instrument air system due to random events occurs. The outcome probability assigned is 1.0 for Branch 1 . The parameter, FailCTCm, is assigned a value of $\{1.0\}$ when Branch 1 is selected as the outcome.

Case 4

This case includes those sequences in which the accident class is an external event [Question 1, Branch 1] and the cooling tower common system or the instrument air system have failed due to the external event. The outcome probability for Branch 1 is assigned a value of 1.0. The parameter, FailCTCm, is assigned a value of $\{+1.0\}$ when Branch 1 is selected as the outcome.

Case 5 The default case includes all other sequences. The outcome probability assigned is 1.0 in Branch 2, failure of the cooling tower common system is not an accident 'enabler'. The parameter, FailCTCm, is assigned a value of $\{0.0\}$ when branch 2 is selected.

\section{Question 20: Is the failure of the Deep Well system an 'accident enabler'?}

Failure of the deep well system to function can contribute to the events leading to an accident as one of a series of events that causes the accident [an 'enabler]. The deep well system has no dependancies on any other DWPF support systems. There are two defined outcomes as follows:

DWeiEn: The deep well system does not function.

nDWelEn: The deep well system continues to function.

This is a Type 8 question and defines a parameter that is used in calculations to determine the outcome probabilities of later Questions $63,67,77,89,91$, and 152 . The parameter is as follows:

Arg [103] FailDWel, a logical variable that indicates the state of the deep well system. The parameter is assigned a value of $\{+1.0\}$ if the deep well system is not functioning and $\{0.0\}$ if the deep well system is functioning.

The outcome probabilities for this question depend on the accident class and the reliability of the system and are used in the logic to determine the outcome probabilities in Questions 23 and 27 and to determine the value assigned the above parameter. Three cases are used to develop the outcome probabilities on the basis of prior events as follows:

Case 1

This case includes those sequences in which the accident class is an internal-energetic event [Question 1, Branch 2] and the deep well system has failed randomly. An outcome probability of 1.0 is assigned to Branch 
1. The parameter, FailDWel, is assigned a value of $\{+1.0\}$ when Branch 1 is selected as the outcome.

Case 2. : This case includes those sequences in which the accident class is an external event [Question 1, Branch 1] and the deep well system has failed due to the external event. An outcome probability of 1.0 is assigned to Branch 1. The parameter, FaildWel, is assigned a value of $\{+1.0\}$ when Branch 1 is selected as the outcome.

Case 3 = The default case includes all other sequences. The outcome probability assigned is 1.0 in Branch 2, failure of the deep well system is not an accident 'enabler'. The parameter, FailDWel, is assigned a value of $\{0.0\}$ when branch 2 is selected.

\section{Question 21: Is failure of the Cooling Tower an 'accident enabler'?}

Failure of the cooling tower system to function can contribute to the events leading to an accident, either as the initiating event [Question 3] or as one of a series of events that causes the accident [an 'enábler']. The cooling tower system is dependant on the following DWPF support systems:- normal electrical power, and instrument air. Failure of normal power or failure of the instrument air system causes failure of the cooling tower system. There are two defined outcomes as follows:

CoTEn: The cooling tower common system does not function but does not initiate the accident.

nCoTEn: The cooling tower common system continues to function [or its failure to function has previously been identified in Question 3 as the failure that initiates the accident].

This is a Type 8 question and defines a parameter that is used in calculations to determine the outcome probabilities of later Questions $63,67,77,89,91$, and 152 . The parameter is as follows:

Arg [62] FailCoT, a logical variable that indicates the state of the cooling tower system. The parameter is assigned a value of $\{-1.0\}$ if the loss of function of the cooling tower is the 'initiator', $\{+1.0\}$ if the cooling tower is not functioning but is not the 'initiator', and $\{0.0\}$ if the cooling tower is functioning.

The outcome probabilities for this question depend on the accident class and the reliability of the system and are used in the logic to determine the outcome probabilities in Questions 23 and 27 and to determine the value assigned the above parameter. Seven cases are used to develop the outcome probabilities on the basis of prior events as follows:

Case 1 - This case includes those sequences in which the accident class is an internal-energetic event [Question 1, Branch 2] and failure of the cooling tower is the initiating event [Question 3]. The outcome probability assigned is 1.0 for Branch 2. The parameter, FailCoT, is assigned a value of $\{-1.0\}$ when Branch 2 is selected as the outcome. 
Case 2 This case includes those sequences in which the cooling tower common system is failed. The cooling tower cannot fulfill its function in the event the cooling tower common system fails. Therefore, an outcome probability of 1.0 is assigned to Branch 1 . The parameter, FailCoT, is assigned a value of $\{+1.0\}$ when Branch 1 is selected as the outcome.

Case 3

This case includes those sequences in which the normal electric power system is failed. The cooling tower cannot fulfill its function in the event the normal electric power system fails. Therefore, an outcome probability of 1.0 is assigned to Branch 1. The parameter, FailCoT, is assigned a value of $\{+1.0\}$ when Branch 1 is selected as the outcome.

Case 4

This case includes those sequences in which the instrument air system is failed. The cooling tower cannot fulfill its function in the event the instrument air system fails. Therefore, an outcome probability of 1.0 is assigned to Branch 1. The parameter, FailCoT, is assigned a value of $\{+1.0\}$ when Branch 1 is selected as the outcome.

Case 5 This case includes those sequences in which the accident class is an internal-energetic event [Question 1, Branch 2] and failure of the cooling tower is not the initiating event [Question 3] but does fail randomly. The outcome probability for Branch 1 is assigned a value of 1.0. The parameter, FailCoT, is assigned a value of $\{+1.0\}$ when Branch 1 is selected as the outcome.

Case 6

This case includes those sequences in which the accident class is an external event [Question 1, Branch 1] and the cooling tower system, the cooling tower common system, or the instrument air system have failed due to the external event: The outcome probability for Branch 1 is assigned a value of 1.0. The parameter, FailCoT, is assigned a value of $\{+1.0\}$ when Branch 1 is selected as the outcome.

Case 7 The default case includes all other sequences. The outcome probability assigned is 1.0 in Branch 2, failure of the cooling tower function is not an accident 'enabler'. The parameter, FailCoT, is assigned a value of $\{0.0\}$ when branch 2 is selected.

\section{Question 22: Is the failure of the process steam system an 'accident enabler'?}

Failure of the process steam system to function can contribute to the events leading to an accident, either as the initiating event [Question 3] or as one of a series of events that causes the accident [an 'enabler']. The process steam system is dependant on the following DWPF support systems: normal electrical power and instrument air. Failure of normal power or the instrument air system causes failure of the process steam system. There are two defined outcomes as follows:

PStmEn: The process steam system fails but does not initiate the accident. 
nPStmEn: The process steam system continues to function [or its failure to function has previously been identified in Question 3 as the failure that initiates the accident].

This is a Type 8 question and defines a parameter that is used in calculations to determine the outcome probabilities of later Questions 67,77 and 91. The parameter is as follows:

Arg [63] FailPStm, a logical variable that indicates the state of the process steam system. The parameter is assigned a value of $\{-1.0\}$ if the loss of function of the process steam system is the 'initiator', $\{+1.0\}$ if the process steam system is not functioning but is not the 'initiator', and $\{0.0\}$ if the process steam system is functioning.

The outcome probabilities for this question depend on the accident class and the reliability of the system and are used to determine the value assigned the above parameter. Six cases are used to develop the outcome probabilities on the basis of prior events as follows:

Case 1 This case includes those sequences in which the accident class is an internal-energetic event [Question 1, Branch 2] and failure of the process steam is the initiating event [Question 3]. The outcome probability assigned is 1.0 for Branch 2. The parameter, FailPStm, is assigned a value of $\{-1.0\}$ when Branch 2 is selected as the outcome.

Case 2 This case includes those sequences in which the instrument air system is failed. The process steam system is inoperative if the instrument air system fails. Therefore, an outcome probability of 1.0 is assigned to Branch 1. The parameter, FailPStm, is assigned a value of $\{+1.0\}$ when Branch 1 is selected as the outcome.

Case 3 This case includes those sequences in which the normal electric power system is failed. The process steam system is inoperative if the normal electric power system fails. Therefore, an outcome probability of 1.0 is assigned to Branch 1. The parameter, FailPStm, is assigned a value of $-\{+1.0\}$ when Branch 1 is selected as the outcome.

Case 4 . This case includes those sequences in which the accident class is an internal-energetic event [Question 1, Branch 2] and failure of the process steam is not the initiating event [Question 3]. The outcome probability for Branch 1 is calculated as a function of PStmLmda and PStmTau. The outcome probability for Branch 2 is 1.0 less the probability assigned Branch 1. The parameter, FailPStm, is assigned a value of $\{+1.0\}$ when Branch 1 is selected as the outcome and a value of $\{0.0\}$ when Branch 2 is selected as the outcome.

Case 5 . This case includes those sequences in which the accident class is an external event [Question 1, Branch 1]. The outcome probability for Branch 1 is calculated as a function of PStmLmda and PStmTau, PStmFr, and PStmRn and MsTmEx. The outcome probability for Branch 2 is 1.0 less the probability assigned Branch 1 . The parameter, FailPStm, is assigned a value of $\{+1.0\}$ when Branch 1 is selected as the outcome and a value of $\{0.0\}$ when Branch 2 is selected as the outcome. - 
Case 6 The default case includes all other sequences. The outcome probability assigned is 1.0 in Branch 2, failure of the process steam is not an accident 'enabler'. The parameter, FailPStm, is assigned a value of $\{0.0\}$ when branch 2 is selected.

\section{Question 23: Is the failure of the process cooling water system an 'accident enabler'?}

Failure of the process cooling water system to function can contribute to the events leading to an accident as one of a series of events that causes the accident [an 'enabler]. The process cooling water system is dependant on the following DWPF support systems: normal and emergency electrical power, and the cooling tower. Total failure of power or failure of the cooling tower system causes failure of the process cooling water system. There are two defined outcomes as follows:

PCWEn: The process cooling water system fails.

nPCWEn: The process cooling water system continues to function.

This is a Type 8 question and defines a parameter that is used in calculations to determine the outcome probabilities of later Questions $63,67,77,89$, and 91 . The parameter is as follows:

Arg [64]

FailPCW, a logical variable that indicates the state of the process cooling water system. The parameter is assigned a value of $\{+1.0\}$ if the process cooling water system is not functioning and $\{0.0\}$ if the process cooling water system is functioning.

The outcome probabilities for this question depend on the accident class and the reliability of the system and are used to determine the value assigned the above parameter. Five cases are used to develop the outcome probabilities on the basis of prior events as follows:

Case 1 This case includes those sequences in which both the normal electric power system and the emergency electrical power system are failed. Because the process cooling water system fails in the event of loss of electric power, an outcome probability of 1.0 is assigned to Branch 1 . The parameter, FailPCW, is assigned a value of $\{+1.0\}$ when Branch 1 is selected as the outcome.

Case 2 This case includes those sequences in which the cooling tower common system, the instrument air system, or the cooling tower pumping/deep well systems are failed. The process cooling water system cannot fulfill its function in the event the cooling tower system fails. Therefore, an outcome probability of 1.0 is assigned to Branch 1 . The parameter, FailPCW, is assigned a value of $\{+1.0\}$ when Branch 1 is selected as the outcome.

Case 3 This case includes those sequences in which the accident class is an internal-energetic event [Question 1, Branch 2]. The outcome probability for Branch 1 is calculated as a function of PCWLmda and PCWTau. The outcome probability for Branch 2 is 1.0 less the probability assigned Branch 1. The parameter, FailPCW, is assigned a value of $\{+1.0\}$ when 
Branch 1 is selected as the outcome and a value of $\{0.0\}$ when Branch 2 is selected as the outcome.

Case 4 - This case includes those sequences in which the accident class is an external event [Question 1, Branch 1]. The outcome probability for Branch 1 is calculated as a function of PCWLmda and PCWTau, PCWFr, and PCWRn and MsTmEx. The outcome probability for Branch 2 is 1.0 less the probability assigned Branch 1 . The parameter, FailPCW, is assigned a value of $\{+1.0\}$ when Branch 1 is selected as the outcome and a value of $\{0.0\}$ when Branch 2 is selected as the outcome.

Case 5

The default case includes all other sequences. The outcome probability assigned is 1.0 in Branch 2, failure of the process cooling water function is not an accident 'enabler'. The parameter, FailPCW, is assigned a value of $\{0.0\}$ when branch 2 is selected.

\section{Question 24: Is the failure of the Carbon Dioxide Purge System an 'accident enabler'?}

Failure of the SPC primary carbon dioxide purge system to function can contribute to the events leading to an accident, either as the initiating event [Question 3] or as one of a series of events that causes the accident [an 'enabler']. The primary carbon dioxide purge system is dependant on the following DWPF support systems: normal electrical power. Failure of normal power causes failure of the primary carbon dioxide purge system. There are two defined outcomes as follows:

CO2En: The SPC primary $\mathrm{CO} 2$ purge system fails but does not initiate the accident.

nCO2En: $\quad$ The SPC primary $\mathrm{CO} 2$ purge system continues to function [or its failure to function has previously been identified in Question 3 as the failure that initiates the accident].

This is a Type 8 question and defines a parameter that is used in determining outcome probabilities of later Questions $42,46,50,54,79,81,83$, and 85 . The parameter is as follows:

$\operatorname{Arg}[65]$

FailCO2, a logical variable that indicates the state of the $\mathrm{CO} 2$ purge system. The parameter is assigned a value of $\{-1.0\}$ if the loss of function of the $\mathrm{CO}_{2}$ purge system is the 'initiator', $\{+1.0\}$ if the $\mathrm{CO}_{2}$ purge system is not functioning but is not the 'initiator', and $\{0.0\}$ if the $\mathrm{CO}_{2}$ purge system is functioning.

The outcome probabilities for this question depend on the accident class and the reliability of the system and are used in the logic to determine the outcome probabilities in Question 28 and to determine the value assigned the above parameter. Five cases are used to develop the outcome probabilities on the basis of prior events as follows:

Case 1 This case includes those sequences in which the accident class is an internal-energetic event [Question 1, Branch 2] and failure of the $\mathrm{CO}_{2}$ purge system is the initiating event [Question 3]. The outcome probability 
assigned is 1.0 for Branch 2. The parameter, FailCO2, is assigned a value of $\{-1.0\}$ when Branch 2 is selected as the outcome.

Case 2 - This case includes those sequences in which the normal electric power system is in a failed state. Because the $\mathrm{CO} 2$ purge system fails in the event of loss of electric power an outcome probability of 1.0 is assigned to Branch 1. The parameter, FailCO2, is assigned a value of $\{+1.0\}$ when Branch 1 is selected as the outcome.

Case 3 This case includes those sequences in which the accident class is an internal-energetic event [Question 1, Branch 2] and failure of the $\mathrm{CO} 2$ purge system is not the initiating event [Question 3]. The outcome probability for Branch 1 is calculated as a function of CO2Lmda and CO2Tau. The outcome probability for Branch 2 is 1.0 less the probability assigned Branch 1. The parameter, FailCO2, is assigned a value of $\{+1.0\}$ when Branch 1 is selected as the outcome and a value of $\{0.0\}$ when Branch 2 is selected as the outcome.

Case 4 This case includes those sequences in which the accident class is an external event [Question 1, Branch 1]. The outcome probability for Branch 1 is calculated as a function of CO2Lmda and CO2Tau, CO2Fr, and CO2Rn and MsTmEx. The outcome probability for Branch 2 is 1.0 less the probability assigned Branch 1 . The parameter, FailCO2, is assigned a value of $\{+1.0\}$ when Branch 1 is selected as the outcome and a value of $\{0.0\}$ when Branch 2 is selected as the outcome.

Case 5 The default case includes all other sequences. The outcome probability assigned is 1.0 in Branch 2, failure of the $\mathrm{CO}_{2}$ purge system is not an accident 'enabler'. The parameter, FailCO2, is assigned a value of $\{0.0\}$ when branch 2 is selected.

\section{Question 25: Is failure of the Air Purge System an 'accident enabler'?}

Failure of the CPC primary air purge system to function can contribute to the events leading to an accident, either as the initiating event [Question 3] or as one of a series of events that causes the accident [an 'enabler']. The primary air purge system is dependant on the following DWPF support system: normal electrical power. Failure of normal power causes failure of the primary air purge system. There are two defined outcomes as follows:

AirEn: $\quad$ The CPC primary air purge system fails but does not initiate the accident.

nAirEn: $\quad$ The CPC primary air purge system continues to function [or its failure to function has previously been identified in Question 3 as the failure that initiates the accident].

This is a Type 8 question and defines a parameter that is used in determining outcome probabilities of later Questions 59,63,67,73,77,87,89, 91, and 93. The parameter is as follows:

Arg [66] FailAir, a logical variable that indicates the state of the air purge system. The parameter is assigned a value of $\{-1.0\}$ if the loss of function of the 
air purge system is the "initiator', $\{+1.0\}$ if the air purge system is not functioning but is not the 'initiator', and $\{0.0\}$ if the air purge system is functioning.

The outcome probabilities for this question depend on the accident class and the reliability of the system and are used to determine the value assigned the above parameter. Five cases are used to develop the outcome probabilities on the basis of prior events as follows:

Case 1 - This case includes those sequences in which the accident class is an internal-energetic event [Question 1, Branch 2] and failure of the air purge system is the initiating event [Question 3]. The outcome probability assigned is 1.0 for Branch 2. The parameter, FailAir, is assigned a value of $\{-1.0\}$ when Branch 2 is selected as the outcome.

Case 2 This case includes those sequences in which the normal electric power system is in a failed state. Because the air purge system fails in the event of loss of electric power an outcome probability of 1.0 is assigned to Branch 1. The parameter, FailAir, is assigned a value of $\{+1.0\}$ when Branch 1 is selected as the outcome.

Case 3 This case includes those sequences in which the accident class is an internal-energetic event [Question 1, Branch 2] and failure of the air purge system is not the initiating event [Question 3]. The outcome probability for Branch 1 is calculated as a function of Airlmda and AirTau. The outcome probability for Branch 2 is 1.0 less the probability assigned Branch 1. The parameter, FailAir, is assigned a value of $\{+1.0\}$ when Branch 1 is selected as the outcome and a value of $\{0.0\}$ when Branch 2 is selected as the outcome.

Case 4 This case includes those sequences in which the accident class is an external event [Question 1, Branch 1]. The outcome probability for Branch 1 is calculated as a function of AirLmda and AirTau, AirFr, and AirRn and MsTmEx. The outcome probability for Branch 2 is 1.0 less the probability assigned Branch 1. The parameter, FailAir, is assigned a value of $\{+1.0\}$ when Branch 1 is selected as the outcome and a value of $\{0.0\}$ when Branch 2 is selected as the outcome.

Case 5 The default case includes all other sequences. The outcome probability assigned is 1.0 in Branch 2, failure of the air purge system is not an accident 'enabler'. The parameter, FailAir, is assigned a value of $\{0.0\}$ when branch 2 is selected.

\section{Question 26: Is failure of the Nitrogen Storage Tank System an 'accident enabler'?}

The nitrogen storage tank system (also known as bulk nitrogen storage) supplies nitrogen to the bulk N2 system and is one of the supplies to the CPC backup N2 system. Failure of the nitrogen storage tank system to function can contribute to the events leading to an accident, either as the initiating event [Question 3] or as one of a series of events that causes the accident [an 'enabler']. The nitrogen storage tank system is not dependant on any other DWPF support systems. 'There are two defined outcomes as follows: 
N2TkEn: _. The N2 storage tank system fails but does not initiate the accident.

nN2TkEn: The N2 storage tank system continues to function [or its failure to function has previously been identified in Question 3 as the failure that initiates the accident]:

This is a Type 8 question and defines a parameter that is used in determining outcome probabilities of later Questions $59,63,67,73,77,87,89,91,93,159,165,168,173$, and 176. The parameter is as follows:

Arg [67] FailN2Tk, a logical variable that indicates the state of the N2 storage tank system. The parameter is assigned a value of $\{-1.0\}$ if the loss of function of the $\mathrm{N}_{2}$ storage tank system is the 'initiator', $\{+1.0\}$ if the $\mathrm{N}_{2}$ storage tank system is not functioning but is not the 'initiator', and $\{0.0\}$ if the $\mathrm{N}_{2}$ storage tank system is functioning.

The outcome probabilities for this question depend on the accident class and the reliability of the system and are used in the logic to determine the outcome probabilities in Questions 29,31, and 33 and to determine the value assigned the above parameter. Four cases are used to develop the outcome probabilities on the basis of prior events as follows:

Case 1

This case includes those sequences in which the accident class is an internal-energetic event [Question 1, Branch 2] and failure of the N2 storage tank system is the initiating event [Question 3]. The outcome probability assigned is 1.0 for Branch 2 . The parameter, FailN2Tk, is assigned a value of $\{-1.0\}$ when Branch 2 is selected as the outcome.

Case 2

This case includes those sequences in which the accident class is an internal-energetic event [Question 1, Branch 2] and failure of the N2 storage tank system is not the initiating event [Question 3]. The outcome probability for Branch 1 is calculated as a function of N2TkLmda and N2TkTau. The outcome probability for Branch 2 is 1.0 less the probability assigned Branch 1 . The parameter, FailN2Tk, is assigned a value of $\{+1.0\}$ when Branch 1 is selected as the outcome and a value of $\{0.0\}$ when Branch 2 is selected as the outcome.

Case 3

This case includes those sequences in which the accident class is an external event [Question 1, Branch 1]. The outcome probability for Branch 1 is calculated as a function of N2TkLmda and N2TkTau, N2TkFr, and N2TkRn and MsTmEx. The outcome probability for Branch 2 is 1.0 less the probability assigned Branch 1. The parameter, FailN2Tk, is assigned a value of $\{+1.0\}$ when Branch 1 is selected as the outcome and a value of $\{0.0\}$ when Branch 2 is selected as the outcome.

Case 4 The default case includes all other sequences. The outcome probability assigned is 1.0 in Branch 2, failure of the N2 storage tank system is not an accident 'enabler'. The parameter, FailN2Tk, is assigned a value of $\{0.0\}$ when branch 2 is selected. 


\section{Question 27: . Is failure of the Chilled Water System an 'accident enabler'?}

Failure of the:chilled water system to function can contribute to the events leading to an accident, either as the initiating event [Question 3] or as one of a series of events that causes the accident [an 'enabler']. The chilled water system is dependant on the following DWPF support systems: normal and emergency electrical power and the cooling tower. Total failure of power or failure of the cooling tower system causes failure of the chilled water system. There are two defined outcomes as follows:

ChWEn: $\quad$ The chilled water system fails but does not initiate the accident.

nChWEn: The chilled water system continues to function [or its failure to function has previously been identified in Question 3 as the failure that initiates the accident].

This is a Type 8 question and defines a parameter that is used in determining outcome probabilities of later Question 77. The parameter is as follows:

Arg [68] FailChW, a logical variable that indicates the state of the chilled water system. The parameter is assigned a value of $\{-1.0\}$ if the loss of function of the chilled water system is the 'initiator', $\{+1.0\}$ if the chilled water system is not functioning but is not the 'initiator', and $\{0.0\}$ if the chilled water system is functioning.

The outcome probabilities for this question depend on the accident class and the reliability of the system and are used to determine the value assigned the above parameter. Six cases are used to develop the outcome probabilities on the basis of prior events as follows:

Case 1.

This case includes those sequences in which the accident class is an internal-energetic event [Question 1, Branch 2] and failure of the chilled water is the initiating event [Question 3]. The outcome probability assigned is 1.0 for Branch 2. The parameter, FailChW, is assigned a value of $\{-1.0\}$ when Branch 2 is selected as the outcome.

Case 2 This case includes those sequences in which the normal electric power system and the emergency electrical power systems are failed. Because the chilled water system fails in the event of loss of electric power, an outcome probability of 1.0 is assigned to Branch 1 . The parameter, FailChW, is assigned a value of $\{+1.0\}$ when Branch 1 is selected as the outcome.

Case 3 This case includes those sequences in which the cooling tower common system, the instrument air system, or the cooling tower pumping/deep well systems are failed. The chilled water system cannot fulfill its function in the event the cooling tower system fails. Therefore, an outcome probability of 1.0 is assigned to Branch 1. The parameter, FailChW, is assigned a value of $\{+1.0\}$ when Branch 1 is selected as the outcome.

Case 4 - This case includes those sequences in which the accident class is an internal-energetic event [Question 1, Branch 2] and failure of the chilled water system is not the initiating event [Question 3]. The outcome 
probability for Branch 1 is calculated as a function of ChWLmda and ChWTau. The outcome probability for Branch 2 is 1.0 less the probability assigned Branch 1 . The parameter, FailChW, is assigned a value of $\{+1.0\}$ when Branch 1 is selected as the outcome and a value of $\{0.0\}$ when Branch 2 is selected as the outcome.

Case 5 This case includes those sequences in which the accident class is an external event [Question 1, Branch 1]. The outcome probability for Branch 1 is calculated as a function of ChWLmda and ChWTau, ChWFr, and ChWRn and MsTmEx. The outcome probability for Branch 2 is 1.0 less the probability assigned Branch 1 . The parameter, FailChW, is assigned a value of $\{+1.0\}$ when Branch 1 is selected as the outcome and a value of $\{0.0\}$ when Branch 2 is selected as the outcome.

Case 6 The default case includes all other sequences. The outcome probability assigned is 1.0 in Branch 2, failure of the chilled is not an accident 'enabler'. The parameter, FailChW, is assigned a value of $\{0.0\}$ when branch 2 is selected.

\section{Question 28: Is the failure of the backup $\mathrm{CO} 2$ System an 'accident enabler'?}

Failure of the SPC backup carbon dioxide purge system to function can contribute to the events leading to an accident as one of a series of events that causes the accident [an 'enabler']. The backup carbon dioxide system is not dependant on any of the other DWPF support systems. There are two defined outcomes as follows:

BCO2En: The SPC backup $\mathrm{CO} 2$ purge system fails.

nBCO2En: The SPC backup $\mathrm{CO} 2$ purge system continues to function.

This is a Type 8 question and defines a parameter that is used in determining outcome probabilities of later Questions $42,46,50,54,79,81,83$, and 85 . The parameter is as follows:

Arg [69] FailBCO2, a logical variable that indicates the state of the backup $\mathrm{CO} 2$ purge system. The parameter is assigned a value of $\{+1.0\}$ if the backup $\mathrm{CO}_{2}$ purge system is failed and $\{0.0\}$ if the backup $\mathrm{CO}_{2}$ purge system is available.

The outcome probabilities for this question depend on the accident class and the reliability of the system and are used to determine the value assigned the above parameter. Three cases are used to develop the outcome probabilities on the basis of prior events as follows:

Case 1 This case includes those sequences in which the accident class is an internal-energetic event [Question 1, Branch 2] and the SPC primary $\mathrm{CO} 2$ purge system fails either as an initiator or an enabler. The outcome probability for Branch 1 is calculated as a function of BCO2Lmda and BCO2Tau. The outcome probability for Branch 2 is 1.0 less the probability assigned Branch 1 . The parameter, FailBCO2, is assigned a value of $\{+1.0\}$ when Branch 1 is selected as the outcome and a value of $\{0.0\}$ when Branch 2 is selected as the outcome. 
Case 2 This case includes those sequences in which the accident class is an external event [Question 1, Branch 1] and the SPC primary $\mathrm{CO}_{2}$ purge ; system fails as an enabler. The outcome probability for Branch 1 is calculated as a function of BCO2Lmda and BCO2Tau, BCO2Fr, and BCO2Rn and MsTmEx. The outcome probability for Branch 2 is 1.0 less the probability assigned Branch 1. The parameter, FailBCO2, is assigned a value of $\{+1.0\}$ when Branch 1 is selected as the outcome and a value of $\{0.0\}$ when Branch 2 is selected as the outcome.

Casie 3 The default case includes all other sequences. The outcome probability assigned is 1.0 in Branch 2 , failure of the backup $\mathrm{CO}_{2}$ purge system is not an accident 'enabler'. The parameter, FailBCO2, is assigned a value of $\{0.0\}$ when either branch is selected.

\section{Question 29: Is failure of the Bulk Nitrogen System an 'accident enabler'?}

Failure of the bulk nitrogen system to function can contribute to the events leading to an accident, either as the initiating event [Question 3] or as one of a series of events that causes the accident [an 'enabler']. The bulk nitrogen system is dependant on the following DWPF support systems: nitrogen storage tank. Failure of the nitrogen storage tank system causes failure of the bulk nitrogen system. There are two defined outcomes as follows:.

BlkN2En: The bulk N2 system fails but does not initiate the accident.

nBlkN2En: The bulk N2 system continues to function [or its failure to function has previously been identified in Question 3 as the failure that initiates the accident].

This is a Type 8 question and defines a parameter that is used in determining outcome probabilities of later Questions 159,165,168,173, and 176. The parameter is as follows:

Arg [94] FailBlkN2, a logical variable that indicates the state of the bulk N2 system. The parameter is assigned a value of $\{-1.0\}$ if the loss of function of the bulk $\mathrm{N}_{2}$ system is the 'initiator', $\{+1.0\}$ if the bulk $\mathrm{N}_{2}$ system is not functioning but is not the 'initiator', and $\{0.0\}$ if the bulk $\mathrm{N}_{2}$ system is functioning.

The outcome probabilities for this question depend on the accident class and the reliability of the system and are used in the logic to determine the outcome probabilities in Question 32 and to determine the value assigned the above parameter. Five cases are used to develop the outcome probabilities on the basis of prior events as follows:

Case 1 This case includes those sequences in which the accident class is an internal-energetic event [Question 1, Branch 2] and failure of the bulk $\mathrm{N}_{2}$ system is the initiating event [Question 3]. The outcome probability assigned is 1.0 for Branch 2. The parameter, FailBlkN2, is assigned a value of $\{-1.0\}$ when Branch 2 is selected as the outcome.

Case 2 This case includes those sequences in which the accident class is an internal-energetic event [Question 1, Branch 2] and the N2 storage tank 
system has failed as initiator or enabler. The bulk N2 system cannot -. function without a supply of nitrogen. The outcome probability for value of $\{+1.0\}$ when Branch 1 is selected as the outcome.

\section{Case 3}

This case includes those sequences in which the accident class is an internal-energetic event [Question 1, Branch 2] and the bulk N2 system did not fail as the initiator. The outcome probability for Branch 1 is calculated as a function of BlkN2Lmda and BlkN2Tau. The outcome probability for Branch 2 is 1.0 less the probability assigned Branch 1 . The parameter, FailBIkN2, is assigned a value of $\{+1.0\}$ when Branch 1 is selected as the outcome and a value of $\{0.0\}$ when Branch 2 is selected as the outcome.

Case 4

This case includes those sequences in which the accident class is an external event [Question 1, Branch 1]. The outcome probability for Branch 1 is calculated as a function of BlkN2Lmda and BlkN2Tau, BlkN2Fr, and BlkN2Rn and MsTmEx. The outcome probability for Branch 2 is 1.0 less the probability assigned Branch 1. The parameter, FailBlkN2, is assigned a value of $\{+1.0\}$ when Branch 1 is selected as the outcome and a value of $\{0.0\}$ when Branch 2 is selected as the outcome.

Case 5 The default case includes all other sequences. The outcome probability assigned is 1.0 in Branch 2, failure of the bulk N2 system is not an accident 'enabler'. The parameter, FailBlkN2, is assigned a value of $\{0.0\}$ when branch 2 is selected.

\section{Question 30: Is the failure of the CPC backup liquid N2 Storage Tank System an 'accident enabler'?}

Failure of the CPC backup $\mathrm{N}_{2}$ storage tank system to function can contribute to the events leading to an accident as one of a series of events that causes the accident [an 'enabler']. The backup liquid nitrogen storage tank system is not dependant on any other DWPF support systems. There are two defined outcomes as follows:

CPCTkEn: $\quad$ The CPC backup $\mathrm{N}_{2}$ storage tank system fails.

nCPCTkEn: The CPC backup $\mathrm{N}_{2}$ storage tank system continues to function.

This is a Type 8 question and defines a parameter that is used in determining outcome probabilities of later Questions 59, 63, 67,73, 77, 87, 89, 91, and 93. The parameter is as follows:

Arg [108] FailCPCTk, a logical variable that indicates the state of the CPC backup $\mathrm{N}_{2}$ storage tank system. The parameter is assigned a value of $\{+1.0\}$ if the CPC backup $\mathrm{N}_{2}$ storage tank system is not functioning and $\{0.0\}$ if the CPC backup $\mathrm{N}_{2}$ storage tank system is functioning.

The outcome probabilities for this question depend on the accident class and the reliability of the system and are used in the logic to determine the outcome probabilities in Question 31 and 
to determine the value assigned the above parameter. Three cases are used to develop the outcome probabilities on the basis of prior events as follows:

Case 1 This case includes those sequences in which the accident class is an internal-energetic event [Question 1, Branch 2]. The outcome probability for Branch 1 is calculated as a function of CPCTkLmda and CPCTkTau. The outcome probability for Branch 2 is 1.0 less the probability assigned Branch 1. The parameter, FailCPCTk, is assigned a value of $\{+1.0\}$ when Branch 1 is selected as the outcome and a value of $\{0.0\}$ when Branch 2 is selected as the outcome.

Case 2 This case includes those sequences in which the accident class is an external event [Question 1, Branch 1]. The outcome probability for Branch 1 is calculated as a function of CPCTkLmda and CPCTkTau, CPCTkFr, and CPCTkRn and MsTmEx. The outcome probability for Branch 2 is 1.0 less the probability assigned Branch 1 . The parameter, FailCPCTk, is assigned a value of $\{+1.0\}$ when Branch 1 is selected as the outcome and a value of $\{0.0\}$ when Branch 2 is selected as the outcome.

Case 3 The default case includes all other sequences. The outcome probability assigned is 1.0 in Branch 2, failure of the CPC backup N2 storage tank system is not an accident 'enabler'. The parameter, FailCPCTk, is assigned a value of $\{0.0\}$ when branch 2 is selected.

\section{Question 31: Is failure of the CPC Nitrogen System an 'accident enabler'?}

Failure of the CPC N2 backup purge system to function can contribute to the events leading to an accident as one of a series of events that causes the accident [an 'enabler']. The CPC nitrogen backup purge system is dependant on the following DWPF support systems: the CPC backup liquid nitrogen storage tank and bulk liquid nitrogen storage tank. Failure of both of the nitrogen storage tank systems causes failure of the CPC nitrogen backup purge system. There are two defined outcomes as follows:

CPCN2En: The CPC N2 backup purge :ystem fails.

nCPCN2En: The CPC N2 backup purge system continues to function.

This is a Type 8 question and defines a parameter that is used in determining outcome probabilities of later Questions $59,63,67,73,77,87,89,91$, and 93 . The parameter is as follows:

Arg [95] FailCPCN2, a logical variable that indicates the state of the CPC N2 backup purge system. The parameter is assigned a value of $\{+1.0\}$ if the CPC N2 backup purge system is not functioning and $\{0.0\}$ if the CPC $\mathrm{N}_{2}$ backup purge system is functioning.

The outcome probabilities for this question depend on the accident class and the reliability of the system and are used in the logic to determine the value assigned the above parameter. Four cases are used to develop the outcome probabilities on the basis of prior events as follows: 
Case $1 \quad \therefore \quad$ This case includes those sequences in which the accident class is an internal-energetic event [Question 1, Branch 2] and the CPC N2 storage tank system and the bulk nitrogen storage tank system have failed. The CPC $\mathrm{N}_{2}$ backup purge system cannot function without a supply of nitrogen. The outcome probability for Branch 1 is assigned to be 1.0 . The parameter, FailCPCN2, is assigned a value of $\{+1.0\}$ when Branch 1 is selected as the outcome.

Case 2 This case includes those sequences in which the accident class is an internal-energetic event [Question 1, Branch 2] and the CPC primary air purge system has failed. The outcome probability for Branch 1 is calculated as a function of CPCN2Lmda and CPCN2Tau. The outcome probability for Branch 2 is 1.0 less the probability assigned Branch 1 . The parameter, FailCPCN2, is assigned a value of $\{+1.0\}$ when Branch 1 is selected as the outcome and a value of $\{0.0\}$ when Branch 2 is selected as the outcome.

Case 3 This case includes those sequences in which the accident class is an external event [Question 1, Branch 1]. The outcome probability for Branch 1 is calculated as a function of CPCN2Lmda and CPCN2Tau, CPCN2Fr, and CPCN2Rn and MsTmEx. The outcome probability for Branch 2 is 1.0 less the probability assigned Branch 1 . The parameter, FailCPCN2, is assigned a value of $\{+1.0\}$ when Branch 1 is selected as the outcome and a value of $\{0.0\}$ when Branch 2 is selected as the outcome.

Case 4 The default case includes all other sequences. The outcome probability assigned is 1.0 in Branch 2, failure of the CPC $\mathrm{N}_{2}$ backup purge system is not an accident 'enabler'. The parameter, FailCPCN2, is assigned a value of $\{0.0\}$ when branch 2 is selected.

\section{Question 32: Is failure of the LPPP Backup Nitrogen System an 'accident}

Failure of the LPPP backup nitrogen purge system to function can contribute to the events leading to an accident as one of a series of events that causes the accident [an 'enabler']. The LPPP backup nitrogen system is not dependant on any other DWPF support systems. There are two defined outcomes as follows:

PPN2En: $\quad$ The LPPP backup nitrogen purge system fails.

nPPN2En: The LPPP backup nitrogen purge system continues to function.

This is a Type 8 question and defines a parameter that is used in determining outcome probabilities of later Questions 165 and 173. The parameter is as follows:

$\operatorname{Arg}$ [113] FailPPN2, a logical variable that indicates the state of the LPPP backup nitrogen purge system. The parameter is assigned a value of $\{+1.0\}$ if the LPPP backup nitrogen purge system is failed and $\{0.0\}$ if the LPPP backup nitrogen purge system is available. 
The outcome probabilities for this question depend on the accident class and the reliability of the system and are used to determine the value assigned the above parameter. Three cases are used to develop the outcome probabilities on the basis of prior events as follo

Case 1 This case includes those sequences in which the accident class is an internal-energetic event [Question 1, Branch 2] and the bulk nitrogen system fails either as an initiator or an enabler. The outcome probability for Branch 1 is calculated as a function of PPN2Lmda and PPN2Tau. The outcome probability for Branch 2 is 1.0 less the probability assigned Branch 1. The parameter, FailPPN2, is assigned a value of $\{+1.0\}$ when Branch 1 is selected as the outcome and a value of $\{0.0\}$ when Branch 2 is selected as the outcome.

Case 2 This case includes those sequences in which the accident class is an external event [Question 1, Branch 1]. The outcome probability for Branch 1 is calculated as a function of PPN2Lmda and PPN2Tau, PPN2Fr, and PPN2Rn and MsTmEx. The outcome probability for Branch 2 is 1.0 less the probability assigned Branch 1. The parameter, FailPPN2, is assigned a value of $\{+1.0\}$ when Branch 1 is selecteci as the outcome and a value of $\{0.0\}$ when Branch 2 is selected as the outcome.

Case 3 The default case includes all other sequences. The outcome probability assigned is 1.0 in Branch 2, failure of the LPPP backup nitrogen purge system is not an accident 'enabler'. The parameter, FailPPN2, is assigned a value of $\{0.0\}$ when branch 2 is selected.

\section{Question 33: Is failure of the SPC N2 System an 'accident enabler'?}

Failure of the SPC N2 purge system to function can contribute to the events leading to an accident as one of a series of events that causes the accident [an 'enabler']. The SPC nitrogen system is dependant on the following DWPF support systems: bulk nitrogen storage system. Failure of the bulk nitrogen storage system causes failure of the SPC N2 purge system. There are two defined outcomes as follows:

SPCN2En: The SPC N2 purge system fails.

nSPCN2En: The SPC N2 purge system continues to function.

This is a Type 8 question and defines a parameter that is used in determining outcome probabilities of later Questions 42,46,50,54,79, 81, 83, and 85. The parameter is as follows:

Arg [118] FailSPCN2, a logical variable that indicates the state of the SPC N2 purge system. The parameter is assigned a value of $\{+1.0\}$ if the SPC $\mathrm{N}_{2}$ purge system is failed and $\{0.0\}$ if the SPC $N_{2}$ purge system is available.

The outcome probabilities for this question depend on the accident class and the reliability of the system and are used to determine the value assigned the above parameter. Four cases are used to develop the outcome probabilities on the basis of prior events as follows: 
Case 1 This case includes those sequences in which the accident class is an internal-energetic event [Question 1; Branch 2] and the N2 storage tank system has failed. The SPC N2 purge system cannot function without a supply of nitrogen. The outcome probability for Branch 1 is assigned to be 1.0. The parameter, FailSPCN2, is assigned a value of $\{+1.0\}$ when Branch 1 is selected as the outcome.

Case 2

This case includes those sequences in which the accident class is an internal-energetic event [Question 1, Branch 2]. The outcome probability for Branch 1 is calculated as a function of SPCN2Lmda and SPCN2Tau. The outcome probability for Branch 2 is 1.0 less the probability assigned Branch 1. The parameter, FailsPCN2, is assigned a value of $\{+1.0\}$ when Branch 1 is selected as the outcome and a value of $\{0.0\}$ when Branch 2 is selected as the outcome.

Case 3 This case includes those sequences in which the accident class is an external event [Question 1, Branch 1]. The outcome probability for Branch 1 is calculated as a function of SPCN2Lmda and SPCN2Tau, SPCN2Fr, and SPCN2Rn and MsTmEx. The outcome probability for Branch 2 is 1.0 less the probability assigned Branch 1. The parameter, FailSPCN2, is assigned a value of $\{+1.0\}$ when Branch 1 is selected as the outcome and a value of $\{0.0\}$ when Branch 2 is selected as the outcome.

Case 4. The default case includes all other sequences. The outcome probability assigned is 1.0 in Branch 2, failure of the SPC N2 purge system is not an accident 'enabler'. The parameter, FailsPCN2, is assigned a value of $\{0.0\}$ when branch 2 is selected.

\section{Question 34: Is the failure of the SPC Seismic trigger System an 'accident enabler'?}

Failure of the SPC seismic trigger system to function can contribute to the events leading to an accident as one of a series of events that causes the accident [an 'enabler]. The SPC seismic trigger is not dependant on any other DWPF support systems. There are two defined outcomes as follows:

STrigEn: $\quad$ The SPC seismic trigger system fails.

nSTrigEn: The SPC seismic trigger system continues to function.

This is a Type 8 question and defines a parameter that is used in determining outcome probabilities of later Questions $42,46,50,54,77,79,81,83$, and 85 . The parameter is as follows:

Arg [135] FailTrig, a logical variable that indicates the state of the SPC seismic trigger system. The parameter is assigned a value of $\{+1.0\}$ if the SPC seismic trigger system is failed and $\{0.0\}$ if the SPC seismic trigger system is available. 
The outcome probabilities for this question depend on the accident class and the reliability of the system and are used to determine the value assigned the above parameter. Two cases are used to develop the outcome probabilities on the basis of prior eve: as follows:

Case 1 - This case includes those sequences in which the accident class is an external event [Question 1, Branch 1]. The outcome probability for Branch 1 is calculated as a function of TrigLmda and TrigTau, TrigFr, and Zero and MsTmEx. The outcome probability for Branch 2 is 1.0 less the probability assigned Branch 1 . The parameter, FailTrig, is assigned a value of $\{+1.0\}$ when Branch 1 is selected as the outcome and a value of $\{0.0\}$ when Branch 2 is selected as the outcome.

Case 2 The default case includes all other sequences. The outcome probability assigned is 1.0 in Branch 2, failure of the SPC seismic trigger system is not an accident 'enabler'. The parameter, FailTrig, is assigned a value of $\{0.0\}$ when branch 2 is selected.

\section{Question 35: Is the failure of the SPC Isolation valve UPS an 'accident enabler!?}

Failure of the SPC Isolation valve UPS system to function can contribute to the events leading to an accident as one of a series of events that causes the accident [an 'enabler']. The SPC isolation valve UPS system is not dependant on any other DWPF support systems. There are two defined outcomes as follows:

SUPSEn: $\quad$ The SPC Isolation valve UPS system fails.

nSUPSEn: The SPC Isolation valve UPS system continues to function.

This is a Type 8 question and defines a parameter that is used in determining outcome probabilities of later Questions $42,46,50,54,77,79,81,83$, and 85 . The parameter is as follows:

Arg [136] FailUPS, a logical variable that indicates the state of the SPC Isolation valve UPS system. The parameter is assigned a value of $\{+1.0\}$ if the SPC Isolation valve UPS system is failed and $\{0.0\}$ if the SPC Isolation valve UPS system is available.

The outcome probabilities for this question depend on the accident class and the reliability of the system and are used to determine the value assigned the above parameter. Two cases are used to develop the outcome probabilities on the basis of prior events as follows:

Case 1 This case includes those sequences in which the accident class is an external event [Question 1, Branch 1]. The outcome probability for Branch 1 is calculated as a function of SUPSLmda and SUPSTau, SPCUPSFr, and Zero and MsTmEx. The outcome probability for Branch 2 is 1.0 less the probability assigned Branch 1 . The parameter, FailUPS, is assigned a value of $\{+1.0\}$ when Branch 1 is selected as the outcome and a value of $\{0.0\}$ when Branch 2 is selected as the outcome. 
Case 2 The default case includes all other sequences. The outcome probability assigned is 1.0 in Branch 2, failure of the SPC Isolation valve UPS system is not an accident 'enabler'. The parameter, FailUPS, is assigned a value of $\{0.0\}$ when branch 2 is selected.

\section{Question 36: Is there a randomly initiated explosion in a process tank or cell?}

As disçussed above, the common system events are handled in Questions 8-35. Each of the fault trees developed may include hundreds of other independent events that can cause failure in addition to the common system events. Therefore, it is necessary to treat properly the portions of the accident fault trees which do not involve common system events as initiating events and in fact may be completely independent of the state of the common systems. That is the purpose of this question. There are sixteen possible outcomes to this question as follows:

nRndExp: There has been no random explosion.

Ex-SRAT: A random explosion [i.e., not caused by failure of a common system] has occurred in the Sludge Receipt and Adjustment Tank [SRAT].

Ex-SME: A random explosion has occurred in the Slurry Mix Evaporator [SME].

Ex-PVVH: A random explosion has occurred in the Process Vessel Vent Header [PVVH].

Ex-PRBT: A random explosion has occurred in the Precipitate Reactor Bottoms Tank [PRBT].

Ex-MFT: $\quad$ A random explosion has occurred in the Melter Feed Tank [MFT].

Ex-OWST: A random explosion has occurred in the Organic Waste Storage Tank [OWST].

Ex-PR: $\quad$ A random explosion has occurred in the Precipitate Reactor [PR].

Ex-MOG: A random explosion has occurred in the Melter Offgas System [MOG].

Ex-OEV A random explosion has occurred in the Organic Evaporator [OEV].

Ex-OECT A random explosion has occurred in the Organic Evaporator Condensate Tank [OECT].

Ex-PRFT A random explosion has occurred in the Precipitate Reactor Feed Tank [PRFT].

Ex-PPST A random explosion has occurred in the Low Point Pump Pit Sludge Tank [PPST].

Ex-PPPT A random explosion has occurred in the Low Point Pump Pit Precipitate Tank [PPPT]. 
Ex-SMEL A random steam explosion has occurred in the Melter.

Ex-SPC - A random explosion due to overpressurization has occurred in the Salt - Process Cell [SPCC].

This is a Type 4 question and defines parameters used in calculations to determine the outcome probabilities in later Questions $42,46,50,54,59,63,67,73,77,79,81,83,85,87,89,92$, $93,114,152,154,159,165,168,173$, and 176. The parameters are as follows:

$\operatorname{Arg}[70]: \quad$ MissTime, the repair time of the affected system. Note: this parameter is a place holder and is not used in the Mode C analysis.

Arg [71]: - nCommI, a parameter whose value indicates whether or not a random explosion [i.e., not caused by failure of a common system] has occurred. If the value of the parameter is 0.0 a random explosion has not occurred. If the value of the parameter is $>1.0$ then a random explosion has occurred, the value of the parameter defines the location of the explosion.

The answer to this question, and the values assigned the parameters. depends upon the outcome of previous Questions 1 and 3. Two cases are used to: elop the outcome probabilities on the basis of prior events as follows:

Case 1

This case includes those sequences in which the accident class is an internal initiating event and failure of a common system is not an initiating event. The outcome probability assigned to Branch 1 is 0.0 , i.e., no explosion was caused by failure of a common system. Each of the remaining branches are assigned the same outcome probability of $1 / 15$. Although the outcome probabilities assigned are arbitrary the frequency of random explosions in the various tanks and systems will be renormalized to eliminate the arbitrary nature of these assigned probabilities. The UFUN performs this re-normalization by multiplying the calculated results by 15 during a UFUN call that came when the APET was on one of the branches of this question other then branch 1 .

The parameters MissTime and nCommI are assigned values of 0.0 for Branch 1. When Branches 2 through 16 are selected the parameter MissTime is assigned a value of 0.0 ; the parameter $n$ CommI is assigned a value of the branch number minus 1.0.

Case 2 The default case includes all other sequences and assumes that failure of a common system is designated as the initiating event or that the initiating event is a seismic event. The outcome probability assigned is 1.0 in Branch 1. The parameter MissTime is assigned values of 0.0 , and the parameter nCommI is assigned dummy values that are the same as the values assigned in Case 1 except for branch 1 which is assigned the value of -10 . The -10 value is a control parameter used to tell the UFUN how to calculate the seismic accident probabilities. 


\section{Question 37: Do the cell covers over the Salt Process Cell dislodge and fall because of a seismic event?}

The process cells within the main canyon of the DWPF are 'roofed' by removable steel covers. These covers provide limited isolation of the cell in the event of an in-cell release, assist in maintaining proper ventilation flow, and protect the equipment within the cell from falling objects. However, if the cell covers are dislodged and fall into the cell, either through a seismic-event or an energetic event within the cell, they may damage the equipment in the cell. This question examines the impact of a seismic event on the covers for the.Salt Process Cell. There are two possible outcomes as follows:

fSCvSeis: $\quad$ The Salt Cell Covers are dislodged by the Seismic event and falls on the equipment [tanks, piping, etc.] in the cell.

nfSCvSei: The Salt Cell Covers remain in place following the Seismic event.

This is a Type 6 question. The answer to this question depends upon the outcome of previous Question 2 and the value specified for the parameter CCovFr, the seismic fragility of the covers for the Salt and Chemical Process Cells. The answers to this question are used in the logic to determine the outcome probabilities in Questions $40,41,44,45,48,49,52,53,75,95,105$, 127 and 128.

Two cases are used to develop the outcome probabilities on the basis of prior events:

Case 1 This case includes those sequences in which the initiating event is a seismic event. The seismic fragility of the covers for the Salt and Chemical Process Cells [the probability the cell covers will fall] is placed in Branch 1. The outcome probability for Branch 2 is 1.0 less the probability assigned Branch 1.

Case 2 The default case includes all other sequences. The outcome probability assigned is 1.0 in Branch 2, there is no seismic event and the cell covers do not fall.

\section{Question 38: Does a cell cover fall while being moved by the crane?}

Another possible way to drop a cell cover into a cell is for it to fall while suspended from an overhead crane. The process cell covers may be dropped during maintenance operations while being moved using the overhead crane. Crane load drops are a documented occurrence at SRS (Reference 6) and therefore are to be considered in the DWPF. Again, the falling covers may strike a tank causing it to be ruptured and spilling the contents into the cell. There are two defined outcomes as follows:

CDCvSPC: $\quad$ A cell cover is dropped by the overhead crane into a cell.

nCDCvSPC: A cell cover is not dropped by the overhead crane a cell.

This is a Type 2 question. The answers to this question are dependent on the outcome of previous Questions 1 and 6 and are used in the logic to determine the outcome of Question 39. Two cases are used to develop the outcome probabilities as follows: 
Case 1 This case includes all events defined by Question 1, Branch 5 [accident - class - Ltd-CD] and Question 6, Branch 2 [Crane Drop]. An outcome - probability of 1.0 is assigned Branch 1.

Case 2 The default case includes all sequences remaining. An outcome probability of 1.0 is assigned Branch 2.

\section{Question 39: Do the cell covers that fall in a crane accident strike a tank?}

There are fourteen defined outcomes for a crane load drop:

No_Hit: A cell cover is dropped by the overhead crane but does not strike any process tank.

CD_Prx: A cell cover is dropped by the overhead crane into the Salt Process Cell and strikes the PR.

CD_Oev: A cell cover is dropped by the overhead crane into the Salt Process Cell and strikes the OEV.

CD_OECT: : A cell cover is dropped by the overhead crane into the Salt Process Cell and strikes the OECT.

CD_PRFT: A cell cover is dropped by the overhead crane into the Salt Process Cell and strikes the PRFT.

CD_PRBT: - A cell cover is dropped by the overhead crane into the Chemical Process Cell and strikes the PRBT.

CD_SME: A cell cover is dropped by the overhead crane into the Chemical Process Cell and strikes the SME.

CD_SRAT: A cell cover is dropped by the overhead crane into the Chemical Process Cell and strikes the SRAT.

CD_RCT: A cell cover is dropped by the overhead crane into the Chemical Process Cell and strikes the RCT.

CD_MFT: A cell cover is dropped by, the overhead crane into the Chemical Process Cell and strikes the MFT.

CD_MEL: A cell cover is dropped by the overhead crane into the Melter Cell and strikes the Melter.

CD_PPPT: A cell cover is dropped by the overhead crane into the Low Point Pump Pit Precipitate Cell and strikes the PPPT.

CD_PPST: A cell cover is dropped by the overhead crane into the Low Point Pump Pit Sludge Cell and strikes the PPST. 
CD_PPRT: A cell cover is dropped by the overhead crane into the Low Point Pump -. Pit Recycle Cell and strikes the PPRT.

This is a Type 2 question. The answers to this question are dependent on the outcome of previous Question 38 and are used in the logic to determine the outcome of Questions 41, 45, $49,53,58,62,66,70,72,157,171,179$, and 181 . Two cases are used to develop the outcome probabilities as follows:

Case 1 This case includes all events defined by Question 38, Branch 1 [a cell cover has been dropped by a crane]. The selection of the outcome probabilities for each branch are based on the number of cell covers available in the facility that may be dropped and the probability of striking and damaging $a$ individual tank. The calculational details are discussed in Reference 7. Branch 1 is assigned a probability of 1.0 minus the sum of the other branch probabilities.

Case 2 The default case includes all sequences remaining. An outcome probability of 1.0 is assigned Branch 1.

\section{Question 40: Do the cell covers that fall in a seismic event strike the PR?}

Falling cell covers during a seismic event can rupture and spill the contents of the Precipitate Reactor. There are two possible outcomes as follows:

SCv-PR: The Salt Cell Covers are dislodged by the Seismic event and fall on the Precipitate Reactor.

nSCv-PR: The Salt Cell Covers are dislodged by the Seismic event and do not fall on the Precipitate Reactor.

This is a Type 2 question. The answer to this question depends upon the outcome of previous Questions 2 and 37. The answers to this question are used in the logic to determine the outcome probabilities in Question 41. Two cases are used to develop the outcome probabilities on the basis of prior events as follows:

Case 1 This case includes those sequences in which the initiating event is a seismic event and the Salt Process Cell covers fell due to the seismic event. The outcome probability for Branch 1 is 0.01 [7], the outcome probability for Branch 2 is 1.0 less the probability assigned Branch 1 . **SAMPLED VARIABLE**.

Case 2 The default case includes all other sequences. The outcome probability assigned is 1.0 in Branch 2, there is no seismic event and the cell covers do not fall. 


\section{Question 41: Does a Seismic event or cover crane fall rupture and spill the contents of the Precipitate Reactor in the Salt Process Cell?}

Falling cell covers during a seismic event or from a crane drop accident can rupture and spill the contents of the Precipitate Reactor. Shaking and overturning of the tank during a seismic event can also cause spillage of the contents. There are two defined outcomes to this question as follows:

Rpt-PRx: . The Precipitate Reactor is ruptured and the contents spilled as a result of a seismic event or a crane drop accident.

nRpt-PRx: The Precipitate Reactor is intact following a seismic event or crane drop accident.

This is a Type 6 question. The answer to this question depends upon the outcome of previous Questions 2, 37, 39, and 40 and the value specified for the parameter PRx-Fr, the seismic fragility of the Precipitate Reactor. The answers to this question are used in the logic to determine the outcome probabilities in Questions 43, 80,106,123, and 136. Three cases are used to develop the outcome probabilities on the basis of prior events as follows:

Case 1 This case includes all sequences in which a seismic event or crane drop accident has caused the cell covers over the Salt Process Cell to fall and strike the PR. The falling cell covers are assumed to rupture and spill the contents of the Precipitate Reactor, i.e., an outcome probability of 1.0 is assigned Branch 1.

Case 2 . This case includes all sequences in which a seismic event has occurred but the cell covers do not fall or the cell covers fall but do not strike the PR. The seismic fragility of the Precipitate Reactor [the probability that a seismic event will cause a structural failure of the Precipitate Reactor] is placed in Branch 1. The outcome probability for Branch 2 is 1.0 less the probability assigned Branch 1.

Case 3 The default case includes all remaining sequences. The outcome probability assigned is 1.0 in Branch 2 and the Precipitate Reactor is undamaged.

Questions 42 to 78 contain the first round of explosion questions for tanks in the SPC and the CPC. The questions consider the possibility of an explosive concentration being present in a tank along with the probability of an ignition source being present. Reference 5 is the common source for the fault tree calculations to determine the frequency of explosions.

\section{Question 42: Does an explosive concentration accumulate in Precipitate Reactor?}

A fault tree was constructed to calculate the frequency of explosions in the PR for all credible initiators. The solution of this fault tree (cut sets) is used to determine the frequency (or probability) of an explosive concentration in the Precipitate Reactor. The two outcomes defined for this question are as follows:

Con-PRx: An explosive concentration accumulates in the Precipitate Reactor. 
nCon-PRx: . An explosive concentration does not accumulate in the Precipitate Reactor.

This is a Type 6 question and uses previously quantified parameters $[60,65,69,70,71,90$, 118,135 , and 136] and a user defined function to determine the probability that an explosive concentration will occur in the Precipitate Reactor. The user function includes information from solution of the fault tree for the Precipitate Reactor Explosion. The function used to calculate the answer depends on the answer to previous Question 1 (i.e., different fault trees are used for internal-energetic events and seismic events). The answers to this question are used in the logic to determine the outcome probabilities in Question 43.

The case structure is simplified by not considering the possibility of prior rupture of the Precipitate Reactor [Question 41] which is assumed to prevent the buildup of an explosive concentration in the Precipitate Reactor. The possibility of prior rupture of the Precipitate Reactor is included in Question 43. Four cases are used to develop the outcome probabilities as follows:

Case 1 This case is used to exclude those sequences in the classes of accidents [Question 1] which are neither external nor internal-energetic. The outcome probability assigned is 1.0 in Branch 2, there is no explosive concentration.

Case 2 This case includes all sequences in which the class of accidents is internalenergetic [Branch 2, Question 1]. The outcome probability is calculated using FUN-PR and assigned to Branch 1 . The outcome probability for Branch 2 is 1.0 less the probability assigned Branch 1

Case 3 This case includes all sequences in which the class of accidents is external [Branch 1, Question 1]. The outcome probability is calculated using FUN-PRS and assigned to Branch 1 . The outcome probability for Branch 2 is 1.0 less the probability assigned Branch 1

Case 4 The default case assumes that all sequences in which an explosive concentration can occur are included in Cases 2 and 3 . An outcome probability of 1.0 is assigned Branch 2.

\section{Question 43: Does a damaging deflagration/detonation occur in the Precipitate Reactor?}

If an explosive concentration occurs in the Precipitate Reactor and an ignition source is available it is assumed that a damaging detonation or deflagration will follow. The fault tree for the Precipitate Reactor Explosion includes the probability of an ignition source. Therefore, if Question 42 indicates that an explosive concentration is available then a detonation/deflagration will follow. A detonation/deflagration in the Precipitate Reactor can spill the contents of the vessel to the Salt Process Cell, blow the cell covers, and damage adjacent vessels. There are three defined outcomes as follows:

PRx-Det: $\quad$ A damaging detonation occurs in the Precipitate Reactor.

PRx-Def: $\quad$ A damaging deflagration occurs in the Precipitate Reactor. 
nPRx-Exp: No damaging explosion occurs in the Precipitate Reactor.

This is a Type 2 question. The answer is dependent on the outcome of previous Questions 41 and 42. The answers to this question are used in the logic to determine the outcome probabilities in Questions 75, 78, 80,105, 106, 107, and 123. Four cases are used to develop the outcome probabilities as follows:

Case 1 This case includes all sequences in which an explosive concentration does not accumulate in the Precipitate Reactor. The outcome probability assigned is 1.0 in Branch 3, there is no explosion.

Case 2 This case includes the sequences in which a prior event has ruptured the Precipitate Reactor precluding the accumulation of an explosive concentration. The outcome probability assigned is 1.0 in Branch 3 , there is no explosion.

Case 3 - This case includes the sequences in which a prior event has not ruptured the Precipitate Reactor and an explosive concentration has accumulated in the vessel. The outcome probability assigned is 0.001 in Branch 1, there is a damaging detonation and 0.999 in Branch 2 , there is a damaging deflagration [7]. **SAMPLED VARIABLE**

Case 4 The default case includes any remaining sequences. It is assumed that all sequences resulting in a damaging explosion are included in previous cases. The outcome probability assigned is 1.0 in Branch 3, there is no explosion.

\section{Question 44: Do the cell covers that fall in a seismic event strike the OEV?}

Falling cell covers during a seismic event can rupture and spill the contents of the Organic Evaporator. There are two possible outcomes as follows:

SCv-OEV: The Salt Cell Covers are dislodged by the Seismic event and fall on the Organic Evaporator.

nSCV-OEV: The Salt Cell Covers are dislodged by the Seismic event and do not fall on the Organic Evaporator.

This is a Type 2 question. The answer to this question depends upon the outcome of previous Questions 2 and 37. The answers to this question are used in the logic to determine the outcome probabilities in Question 45. Two cases are used to develop the outcome probabilities on the basis of prior events as follows:

.Case 1 This case includes those sequences in which the initiating event is a seismic event and the Salt Process Cell covers fell due to the seismic event. The outcome probability for Branch 1 is 0.01 [7], the outcome probability for Branch 2 is 1.0 less the probability assigned Branch 1 . **SAMPLED VARLABLE**. 
Page 79

Case 2 The default case includes all other sequences. The outcome probability assigned is 1.0 in Branch 2, there is no seismic event and the cell covers do not fall.

Question 45: Does a Seismic event or cover crane fall rupture and spill the contents of the Organic Evaporator in the Salt Process Cell?

Falling cell covers during a seismic event or from a crane drop accident can rupture and spill the contents of the Organic Evaporator. Shaking and overturning of the tank during a seismic event can also cause spillage of the contents. There are two defined outcomes to this question as follows:

Rpt-OEV: - The Organic Evaporator is ruptured and the contents spilled as a result of a seismic event or a crane drop accident.

nRpt-OEV: The Organic Evaporator is intact following a seismic event or crane drop accident.

This is a Type 6 question. The answer to this question depends upon the outcome of previous Questions 2,37,39, arid 44 and the value specified for the parameter OEV-Fr, the seismic fragility of the Organic Evaporator. The answers to this question are used in the logic to determine the outcome probabilities in Questions 47, 82, 107, 124, and 137. Three cases are used to develop the outcome probabilities on the basis of prior events as follows:

Case 1 This case includes all sequences in which a seismic event or crane drop accident has caused the cell covers over the Salt Process Cell to fall and strike the OEV. The falling cell covers are assumed to rupture and spill the contents of the Organic Evaporator, i.e., an outcome probability of 1.0 is assigned Branch 1.

Case 2 This case includes all sequences in which a seismic event has occurred but the cell covers do not fall or the cell covers fall but do not strike the OEV. The seismic fragility of the Organic Evaporator [the probability that a seismic event will cause a structural failure of the Organic Evaporator] is placed in Branch 1. The outcome probability for Branch 2 is 1.0 less the probability assigned Branch 1.

Case 3 The default case includes all-remaining sequences. The outcome probability assigned is 1.0 in Branch 2 and the Organic Evaporator is undamaged.

\section{Question 46: Does an explosive concentration accumulate in the Organic Evaporator Tank?}

A fault tree was constructed to calculate the frequency of explosions in the OEV for all credible initiators. The solution of this fault tree (cut sets) is used to determine the frequency (or probability) of an explosive concentration in the Organic Evaporator. The two outcomes defined for this question are as follows:

Con-OEV: An explosive concentration accumulates in the Organic Evaporator. 
nCon-OEV: $\cdots$ An explosive concentration does not accumulate in the Organic Evaporator.

This is a Type 6 question and uses previously quantified parameters $[60,65,69,70,71,90$, 118,135 , and 136] and a user defined function to determine the probability that an explosive concentration will occur in the Organic Evaporator. The user function includes information from solution of the fault tree for the Organic Evaporator Explosion. The function used to calculate the answer depends on the answer to previous Question 1. The answers to this question are used in the logic to determine the outcome probabilities in Question.47.

The case structure is simplified by not considering the possibility of prior rupture of the Organic Evaporator [Question 45] which is assumed to prevent the buildup of an explosive concentration in the Organic Evaporator. The possibility of prior rupture of the Organic Evaporator is included in Question 47. Four cases are used to develop the outcome probabilities as follows:

Case 1 This case is used to exclude those sequences in the classes of accidents [Question 1] which are neither external nor internal-energetic. The outcome probability assigned is 1.0 in Branch 2, there is no explosive concentration.

Case 2 This case includes all sequences in which the class of accidents is internalenergetic [Branch 2, Question 1]. The outcome probability is calculated using FUN-OEV and assigned to Branch 1. The outcome probability for Branch 2 is 1.0 less the probability assigned Branch 1.

Case 3 This case includes all sequences in which the class of accidents is external [Branch 1, Question 1]. The outcome probability is calculated using FUN-OEVS and assigned to Branch 1. The outcome probability for Branch 2 is 1.0 less the probability assigned Branch 1 .

Case 4 The default case assumes that all sequences in which an explosive concentration can occur are included in Cases 2 and 3. An outcome probability of 1.0 is assigned Branch 2. Questi. 47: Does a detonation/deflagration of the Organic Evaporator tank
occur?

If an explosive concentration occurs in the Organic Evaporator and an ignition source is available it is assumed that a damaging detonation or deflagration will follow. The fault tree for the Organic Evaporator Explosion includes the probability of an ignition source. Therefore, if Question 46 indicates that an explosive concentration is available then a detonation/deflagration will follow. A detonation/deflagration in the Organic Evaporator can spill the contents of the vessel to the Salt Process Cell, blow the cell covers, and damage adjacent vessels. There are three defined outcomes as follows:

OEV-Det: . A damaging detonation occurs in the Organic Evaporator.

JEV-Def: A damaging deflagration occurs in the Organic Evaporator. 
nOEV-Exp: No damaging explosion occurs in the Organic Evaporator.

This is a Type 2 question. The answer is dependent on the outcome of previous Questions 45 and 46. The answers to this question are used in the logic to determine the outcome probabilities in Questions $75,78,82,105,106,107,108$, and 124. Four cases are used to develop the outcome probabilities as follows:

Case 1 This case includes all sequences in which an explosive concentration does not accumulate in the Organic Evaporator. The outcome probability assigned is 1.0 in Branch 3, there is no explosion.

Case 2 This case includes the sequences in which a prior event has ruptured the Organic Evaporator precluding the accumulation of an explosive concentration. The outcome probability assigned is 1.0 in Branch 3 , there is no explosion.

Case 3 This case includes the sequences in which a prior event has not ruptured the Organic Evaporator and an explosive concentration has accumulated in the vessel. The outcome probability assigned is 0.001 in Branch 1, there is a damaging detonation and 0.999 in Branch 2, there is a damaging deflagration [7]. **SAMPLED VARIABLE**

Case 4 The default case includes any remaining sequences. It is assumed that all sequences resulting in a damaging explosion are included in previous cases. The outcome probability assigned is 1.0 in Branch 3, there is no explosion.

\section{Question 48: Do the cell covers that fall in a seismic event strike the OECT?}

Falling cell covers during a seismic event can rupture and spill the contents of the Organic Evaporator Condensate Tank. There are two possible outcomes as follows:

SCV-OECT: The Salt Cell Covers are dislodged by the Seismic event and fall on the Organic Evaporator Condensate Tank.

nSCV-OECT: The Salt Cell Covers are disiodged by the Seismic event and do not fall on the Organic Evaporator Condensate Tank.

This is a Type 2 question. The answer to this question depends upon the outcome of previous Questions 2 and 37. The answers to this question are used in the logic to determine the outcome probabilities in Question 49. Two cases are used to develop the outcome probabilities on the basis of prior events as follows:

Case 1 This case includes those sequences in which the initiating event is a seismic event and the Salt Process Cell covers fell due to the seismic event. The outcome probability for Branch 1 is 0.01 [7], the outcome probability for Branch 2 is 1.0 less the probability assigned Branch 1 . **SAMPLED VARIABLE**. 
April 7, 1995

WSRC-TR-95-0113

Page 82

Case 2 The default case includes all other sequences. The outcome probability assigned is 1.0 in Branch 2, there is no seismic event and the cell covers do not fall.

Question 49: Does a Seismic event or cover crane fall rupture and spill the contents of the Organic Evaporator Condensate Tank in the Salt Process Cell?

Falling celf covers during a seismic event or from a crane drop accident can rupture and spill the contents of the Organic Evaporator Condensate Tank. Shaking and overturning of the tank during a seismic event can also cause spillage of the contents. There are two defined outcomes to this question as follows:

RptOECT: The Organic Evaporator Condensate Tank is ruptured and the contents spilled as a result of a seismic event or a crane drop accident.

nRptOECT: The Organic Evaporator Condensate Tank is intact following a seismic event or crane drop accident.

This is a Type 6 question. The answer to this question depends upon the outcome of previous Questions 2, 37, 39, and 48 and the value specified for the parameter OECTFr, the seismic fragility of the Organic Evaporator Condensate Tank. The answers to this question are used in the logic to determine the outcome probabilities in Questions 51, 84, 108, 125, and 138. 'Three cases are used to develop the outcome probabilities on the basis of prior events as follows:

Case 1 This case includes all sequences in which a seismic event or crane drop accident has caused the cell covers over the Salt Process Cell to fall and strike the OECT. The falling cell covers are assumed to rupture and spill the contents of the Organic Evaporator Condensate Tank, i.e., an outcome probability of 1.0 is assigned Branch 1.

Case 2 This case includes all sequences in which a seismic event has occurred but the cell covers do not fall or the cell covers fall but do not strike the OECT. The seismic fragility of the Organic Evaporator Condensate Tank [the probability that a seismic event will cause a structural failure of the Organic Evaporator Condensate Tank] is placed in Branch 1. The outcome probability for Branch 2 is 1.0 less the probability assigned Branch 1.

Case 3 The default case includes all remaining sequences. The outcome probability assigned is 1.0 in Branch 2. and the Organic Evaporator Condensate Tank is undamaged.

Question 50: Does an explosive concentration accumulate in Organic Evaporator Condensate Tank?

A fault tree was constructed to calculate the frequency of explosions in the OECT for all credible initiators. The solution of this fault tree (cut sets) is used to determine the frequency (or probability) of an explosive concentration in the Organic Evaporator Condensate Tank. The two outcomes defined for this question are as follows: 
Con-OECT: An explosive concentration accumulates in the Organic Evaporator Condensate Tank.

nCon-OECT: An explosive concentration does not accumulate in the Organic Evaporator Condensate Tank.

This is a Type 6 question and uses previously quantified parameters $[60,65,69,70,71,90$, 118,135 , and 136] and a user defined function to determine the probability that an explosive concentration will occur in the Organic Evaporator Condensate Tank. The user function includes information from solution of the fault tree for the Organic Evaporator Condensate Tank Explosion. The function used to calculate the answer depends on the answer to previous Question 1. The answers to this question are used in the logic to determine the outcome probabilities in Question 51.

The case structure is simplified by not considering the possibility of prior rupture of the Organic Evaporator Condensate Tank [Question 49] which is assumed to prevent the buildup of an explosive concentration in the Organic Evaporator Condensate Tank. The possibility of prior rupture of the Organic Evaporator Condensate Tank is included in Question 51. Four cases are used to develop the outcome probabilities as follows:

Case 1 This case is used to exclude those sequences in the classes of accidents [Question 1] which are neither external nor internal-energetic. The outcome probability assigned is 1.0 in Branch 2 , there is no explosive concentration.

Case 2 This case includes all sequences in which the class of accidents is internalenergetic [Branch 2, Question 1]. The outcome probability is calculated using FUN-OECT and assigned to Branch 1 . The outcome probability for Branch 2 is 1.0 less the probability assigned Branch 1.

Case 3 This case includes all sequences in which the class of accidents is external [Branch 1, Question 1]. The outcome probability is calculated using FUN-OECTS and assigned to Branch 1 . The outcome probability for Branch 2 is 1.0 less the probability assigned Branch 1 .

Case 4

The default case assumes that all sequences in which an explosive concentration can occur are included in Cases 2 and 3. An outcome probability of 1.0 is assigned Branch 2 .

\section{Question 51: Does a detonation/deflagration-of the Organic Evaporator Condensate tank occur?}

If an explosive concentration occurs in the Organic Evaporator Condensate Tank and an ignition source is available it is assumed that a damaging detonation or deflagration will follow. The fault tree for the Organic Evaporator Condensate Tank Explosion includes the probability of an ignition source. Therefore, if Question 50 indicates that an explosive concentration is available then a detonation/deflagration will follow. A detonation/deflagration in the Organic Evaporator Condensate Tank can spill the contents of the vessel to the Salt Process Cell, blow the cell covers, and damage adjacent vessels. There are three defined outcomes as follows: 
OECT-Det: A damaging detonation occurs in the Organic Evaporator Condensate -. Tank.

OECT-Def: A damaging deflagration occurs in the Organic Evaporator Condensate Tank.

nOECT-Exp: No damaging explosion occurs in the Organic Evaporator Condensate Tank.

This is a Type 2 question. The answer is dependent on the outcome of previous Questions 49 and 50. The answers to this question are used in the logic to determine the outcome probabilities in Questions 75, 78, 84, 105, 107, 108, 109, and 125. Four cases are used to develop the outcome probabilities as follows:

Case 1 This case includes all sequences in which an explosive concentration does not accumulate in the Organic Evaporator Condensate Tank. The outcome probability assigned is 1.0 in Branch 3 , there is no explosion.

Case 2 This case includes the sequences in which a prior event has ruptured the Organic Evaporator Condensate Tank precluding the accumulation of an explosive concentration. The outcome probability assigned is 1.0 in Branch 3, there is no explosion.

Case 3 This case includes the sequences in which a prior event has not ruptured the Organic Evaporator Condensate Tank and an explosive concentration has accumulated in the vessel. The outcome probability assigned is 0.001 in Branch 1, there is a damaging detonation and 0.999 in Branch 2, there is a damaging deflagration [7]. **SAMPLED VARIABLE**

Case 4. The default case includes any remaining sequences. It is assumed that all sequences resulting in a damaging explosion are included in previous cases. The outcome probability assigned is 1.0 in Branch 3 , there is no explosion.

\section{Question 52: Do the cell covers that fall in a seismic event or crane accident} strike the PRFT?

Falling cell covers during a seismic event can rupture and spill the contents of the Precipitate Reactor Feed Tank. There are two possible outcomes as follows:

SCv-PRFT: The Salt Cell Covers are dislodged by the Seismic event and fall on the Precipitate Reactor Feed Tank.

nSCV-PRFT: The Salt Cell Covers are dislodged by the Seismic event and do not fall on the Precipitate Reactor Feed Tank.

- This is a Type 2 question. The answer to this question depends upon the outcome of previous Questions 2 and 37. The answers to this question are used in the logic to determine the outcome probabilities in Question 53. 
Two cases are used to develop the outcome probabilities on the basis of prior events as follows:

Case 1. This case includes those sequences in which the initiating event is a seismic event and the Salt Process Cell covers fell due to the seismic event. The outcome probability for Branch 1 is 0.01 [7], the outcome probability for Branch 2 is 1.0 less the probability assigned Branch 1 . **SAMPLED VARIABLE**.

Case 2 The default case includes all other sequences. The outcome probability assigned is 1.0 in Branch 2, there is no seismic event and the cell covers do not fall.

Question 53: Does a Seismic event or cover crane fall rupture and spill the contents of the Precipitate Reactor Feed Tank in the Salt Process Cell?

Falling cell covers during a seismic event or from a crane dróp accident can rupture and spill the contents of the Precipitate Reactor Feed Tank. Shaking and overturning of the tank during a seismic event can also cause spillage of the contents. There are two defined outcomes to this question as follows:

RptPRFT: The Precipitate Reactor Feed Tank is ruptured and the contents spilled as a result of a seismic event or a crane drop accident.

nRptPRFT: The Precipitate Reactor Feed Tank is intact following a seismic event or crane drop accident.

This is a Type 6 question. The answer to this question depends upon the outcome of previous Questions 2, 37, 39, and 52 and the value specified for the parameter PRFTFr, the seismic fragility of the Precipitate Reactor Feed Tank. The answers to this question are used in the logic to determine the outcome probabilities in Questions 55, 86, 109, 126, and 139. Three cases are used to develop the outcome probabilities on the basis of prior events as follows:

Case 1 This case includes all sequences in which a seismic event or crane drop accident has caused the cell covers over the Salt Process Cell to fall and strike the PRFT. The falling cell covers are assumed to rupture and spill the contents of the Precipitate Reactor Feed Tank, i.e., an outcome probability of 1.0 is assigned Branch 1.

Case 2 This case includes all sequences in which a seismic event has occurred but the cell covers do not fall or the cell covers fall but do not strike the PRFT. The seismic fragility of the Precipitate Reactor Feed Tank the probability that a seismic event will cause a structural failure of the Precipitate Reactor Feed Tank] is placed in Branch 1. The outcome probability for Branch 2 is 1.0 less the probability assigned Branch 1 .

Case 3 The default case includes all remaining sequences. The outcome probability assigned is 1.0 in Branch 2 and the Precipitate Reactor Feed Tank is undamaged. 


\section{Question 54: _. Does an explosive concentration accumulate in Precipitate Reactor Feed Tank?}

A fault tree was constructed to calculate the frequency of explosions in the PRFT for all credible initiators. The solution of this fault tree (cut sets) is used to determine the frequency (or probability) of an explosive concentration in the Precipitate Reactor Feed Tank. The two outcomes defined for this question are as follows:

Con-PRFT: . An explosive concentration accumulates in the Precipitate Reactor Feed Tank.

nCon-PRFT: An explosive concentration does not accumulate in the Precipitate Reactor Feed Tank.

This is a Type 6 question and uses previously quantified parameters $[60,65,69,70,71,90$, $118,135$, and 136$]$ and a user defined function to determine the probability that an explosive concentration will occur in the Precipitate Reactor Feed Tank. The user function includes information from solution of the fault tree for the Precipitate Reactor Feed Tank Explosion. The function used to calculate the answer depends on the answer to previous Question 1. The answers to this question are used in the logic to determine the outcome probabilities in Question 55.

The case structure is simplified by not considering the possibility of prior rupture of the Precipitate Reactor Feed [Question 53] which is assumed to prevent the buildup of an explosive concentration in the Precipitate Reactor Feed Tank. The possibility of prior rupture of the Precipitate Reactor Feed Tank is included in Question 55. Four cases are used to develop the outcome probabilities as follows:

Case 1 This case is used to exclude those sequences in the classes of accidents [Question 1] which are neither external nor internal-energetic. The outcome probability assigned is 1.0 in Branch 2 , there is no explosive concentration.

Case 2 This case includes all sequences in which the class of accidents is internalenergetic [Branch 2, Question 1]. The outcome probability is calculated using FUN-PRFT and assigned to Branch 1. The outcome probability for Branch 2 is 1.0 less the probability assigned Branch 1

Case 3 This case includes all sequences in which the class of accidents is external [Branch 1, Question 1]. The outcome probability is calculated using FUN-PRFTS and assigned to Branch 1. The outcome probability for Branch 2 is 1.0 less the probability assigned Branch 1

Case 4 . The default case assumes that all sequences in which an explosive concentration can occur are included in Cases 2 and 3. An outcome probability of 1.0 is assigned Branch 2 . 


\section{Question 55: Does a detonation/deflagration occur in the Precipitate Reactor Feed tank?}

If an explosive concentration occurs in the Precipitate Reactor Feed Tank and an ignition source is available it is assumed that a damaging detonation or deflagration will follow. The fault tree for the Precipitate Reactor Feed Tank Explosion includes the probability of an ignition source. Therefore, if Question 54 indicates that an explosive concentration is available then a detonation/deflagration will follow. A detonation/deflagration in the Precipitate Reactor Feed Tank can spill the contents of the vessel to the Salt Process Cell, blow the cell covers, and damage adjacent vessels. There are three defined outcomes as follows:

PRFT-Det: A damaging detonation occurs in the Precipitate Reactor Feed Tank.

PRFT-Def: A damaging deflagration occurs in the Precipitate Reactor Feed Tank.

nPRFT-Exp: No damaging explosion occurs in the Precipitate Reactor Feed Tank.

This is a Type 2 question. The answer is dependent on the outcome of previous Questions 53 and 54. The answers to. this question are used in the logic to determine the outcome probabilities in Questions 75, 78, 86, 97, 105, 108, 109, and 126. Four cases are used to develop the outcome probabilities as follows:

Case 1 This case includes all sequences in which an explosive concentration does not accumulate in the Precipitate Reactor Feed Tank. The outcome probability assigned is 1.0 in Branch 3, there is no explosion.

Case 2 This case includes the sequences in which a prior event has ruptured the Precipitate Reactor Feed Tank precluding the accumulation of an explosive concentration. The outcome probability assigned is 1.0 in Branch 3 , there is no explosion.

Case 3 This case inciudes the sequences in which a prior event has not ruptured the Precipitate Reactor Feed Tank and an explosive concentration has accumulated in the vessel. The outcome probability assigned is 0.001 in Branch 1, there is a damaging detonation and 0.999 in Branch 2, there is a damaging deflagration [7]. **SAMPLED VARIABLE**

Case 4 The default case includes any remaining sequences. It is assumed that all sequences resulting in a damaging explosion are included in previous cases. The outcome probability assigned is 1.0 in Branch 3; there is no explosion.

Question 56: Do the cell covers for the Chemical Process Cell dislodge and fall following a sëismic event?

The process cells-within the main canyon of the DWPF are 'roofed' by removable steel covers. These covers provide limited isolation of the cell in the event of an in-cell release, assist in maintaining proper ventilation flow, and protect the equipment within the cell from falling objects. However, if the cell covers are dislodged and fall into the cell, either through a seismic event or an energetic event within the cell, they may damage the equipment in the cell. 
This question examines the impact of a seismic event on the covers for the Chemical Process Cell. There are two possible outcomes as follows:

fCCvSeis: - The Chemical Cell Covers are dislodged by the Seismic event and fall on the equipment [tanks, piping, etc.] in the cell.

nfCCvSei: The Chemical Cell Covers remain in place following the Seismic event.

This is a Type 6 question. The answer to this question depends upon the outcome of previous Question 2 and the value specified for the parameter CCovFr, the seismic fragility of the covers for the Salt and Chemical Process Cells. The answers to this question are used in the logic to determine the outcome probabilities in Questions $57,58,61,62,65,66,69,70,71,72,76$, $78,96,98,129$, and 141 . Two cases are used to develop the outcome probabilities on the basis of prior events as follows:

Case 1 This case includes those sequences in which the initiating event is a seismic event. The seismic fragility of the covers for the Salt and Chemical Process Cells [the probability the cell covers will fall] is placed in Bre-.ch 1. The outcome probability for Branch 2, is 1.0 less the probas:iity assigned Branch 1 .

Case 2 The default case includes all other sequences. The outcome probability assigned is 1.0 in Branch 2, there is no seismic event and the cell covers do not fall.

\section{Question 57: Do the cell covers that fall in a seismic event strike the PRBT?}

Falling cell covers during a seismic event can rupture and spill the contents of the Precipitate Reactor Bottoms Tank. There are two possible outcomes as follows:

CCV-PRBT: The Chemical Cell Covers are dislodged by the Seismic event and fall on the Precipitate Reactor Bottoms Tank. .

nCCV-PRBT: The Chemical Cell Covers are dislodged by the Seismic event and do not Fall on the Precipitate Reactor Bottoms Tank.

This is a Type $2 \mathrm{c} \quad$ :on. The answer to this question depends upon the outcome of previous Questions 2 and. The answers to this question are used in the logic to determine the outcome probabilitit : in Question 58. Two cases are used to develop the outcome probabilities on the basis of prior events as follows:

Case 1

This case includes those sequences in which the initiating event is a seismic event and the Chemical Process Cell covers fell due to the seismic event. The outcome probability for Branch 1 is 0.01 [7], the outcome probability for Branch 2 is 1.0 less the probability assigned Branch 1 . **SAMPLED VARIABLE**.

Case 2 The default case includes all other sequences. The outcome probability assigned is 1.0 in Branch 2, there is no seismic event and the cell covers do not fall. 


\section{Question 58: Does a Seismic event or cover crane fall rupture and spill the contents of the Precipitate Reactor Bottoms Tank in the Chemical Process Cell?}

Falling cell covers during a seismic event or a crane load drop can rupture and spill the contents of the Precipitate Reactor Bottoms Tank [PRBT]. Shaking and overturning of the tank during a seismic event can also cause spillage of the contents. There are two defined outcomes to this question as follows:

RptPRBT: The Precipitate Reactor Bottoms Tank is ruptured and the contents spilled as a result of the seismic event or a crane drop accident.

nRptPRBT: The Precipitate Reactor Bottoms Tank is intact.

This is a Type 6 question. The answer to this question depends upon the outcome of previous Question 2, 39, 56, and 57 and the value specified for the parameter PRBTFr, the seismic fragility of the Precipitate Reactor Bottoms Tank. The answers to this question are used in the logic to determine the outcome probabilities in Questions 60,88,99,130, and 142: Three cases are used to develop the outcome probabilities on the basis of prior events as follows:

Case 1 This case includes all sequences in which a seismic event or crane drop accident has caused the cell covers over the Chemical Process Cell to fall and strike the PRBT. The falling cell covers are assumed to rupture and spill the contents of the PRBT, i.e., an outcome probability of 1.0 is assigned Branch 1, RptPRBT.

Case 2 This case includes all sequences in which a seismic event has occurred but the cell covers do not fall: The seismic fragility of the PRBT [the probability that a seismic event will cause a structural failure of the PRBT] is placed in Branch 1. The outcome probability for Branch 2, nRptPRBT, is 1.0 less the probability assigned Branch 1.

Case 3 The default case includes all remaining sequences. The outcome probability assigned is 1.0 in Branch 2 , there is no seismic event and the PRBT is undamaged.

\section{Question 59: Does an explosive concentration accumulate in the Precipitate} Reactor Bottoms Tank [PRBT]?

A fault tree was constructed to calculate the frequency of explosions in the PRBT for all credible initiators. The solution of this fault tree (cut sets) is used to determine the frequency (or probability) of an explosive concentration in the Precipitate Reactor Bottoms Tank. The two outcomes defined for this question are as follows:

ConPRBT: An explosive concentration accumulates in the PRBT.

nConPRBT: An explosive concentration does not accumulate in the PRBT.

This is a Type 6 question and uses previously quantified parameters $[60,66,67,70,71,90$, 95 , and 108] and a user defined function to determine the probability that an explosive 
Page 90

concentration will occur in the PRBT. The user function includes information from solution of the fault tree for the PRBT explosion. The function used to calculate the answer depends on the answer to previous Question 1. The outcome probabilities are used in the logic to determine the outcome probabilities in Question 60.

The following case structure is simplified by not considering the possibility of prior rupture of the PRBT [Question 58]. Rupture of the PRBT would prevent the buildup of an explosive concentration in the PRBT. The possibility of prior rupture of the PRBT is included in Question 60. Five cases are used to develop the outcome probabilities as follows:

Case 1 This case is used to exclude those sequences in the classes of accidents - [Question 1] which are neither external nor internal-energetic. The outcome probability assigned is 1.0 in Branch 2 , there is no explosive concentration.

Case 2 This case is used to exclude those sequences where the bulk $\mathrm{N}_{2}$ tank fails as an initiator. The bulk $\mathrm{N}_{2}$ tank supplies nitrogen to the CPC backup nitrogen system. Since the system is a backup, its failure cannot initiate accidents in the CPC. The outcome probability assigned is 1.0 in Branch 2 , there is no explosive concentration.

Case 3 This case includes all sequences in which the class of accidents is internalenergetic [Branch 2, Question 1]. The outcome probability is calculated using FUN-PRBT and assigned to Branch 1 . The outcome probability for Branch 2 is 1.0 less the probability assigned Branch 1.

Case 4 This case includes all sequences in which the class of accidents is external [Branch 1, Question 1]. The outcome probability is calculated using FUN-PRBTS and assigned to Branch 1 . The outcome probability for Branch 2 is 1.0 less the probability assigned Branch 1.

Case 5. The default case assumes that all sequences in which an explosive concentration can occur are included in Cases 3 and 4. An outcome probability of 1.0 is assigned Branch 2.

\section{Question 60: Does a detonation/deflagration occur in the Precipitate Reactor Bottoms Tank [PRBT]?}

If an explosive concentration occurs in the Precipitate Reactor Bottoms Tank and an ignition source is available it is assumed that a damaging detonation or deflagration will follow. The fault tree for the explosion includes the probability of an-ignition source. Therefore, if Question 59 indicates that an explosive concentration is available then a detonation/deflagration will follow. A detonation or deflagration in the Precipitate Reactor Bottoms Tank can spill the contents of the vessel to the Chemical Process Cell, blow the cell covers, and damage adjacent vessels. There are three defined outcomes as follows:

PRBT-Det: A damaging detonation occurs in the Precipitate Reactor Bottoms Tank.

PRBT-Def: $\quad$ A damaging deflagration occurs in the Precipitate Reactor Bottoms Tank.

nPRBT-Ex: No damaging explosion occurs in the Precipitate Reactor Bottoms Tank. 
This is a Type 2 question. The answer is dependent on the outcome of previous Questions 58 and 59. The answers to this question are used in the logic to determine the outcome probabilities in Questions $76,78,88,98,99,100,104$, and 130. Four cases are used to develop the outcome probabilities as follows:

Case 1 This case includes all sequences in which an explosive concentration does not accumulate in the PRBT. The outcome probability assigned is 1.0 in Branch 3, there is no explosion.

Case 2 This case includes the sequences in which a prior event has ruptured the PRBT precluding the accumulation of an explosive concentration. The . outcome probability assigned is 1.0 in Branch 3, there is no explosion.

Case 3 This case includes the sequences in which a prior event has not ruptured the Precipitate Reactor Bottoms Tank and an explosive concentration has accumulated in the vessel. The outcome probability assigned is 0.001 in Branch 1, there is a damaging detonation and 0.999 in Branch 2, there is a damaging deflagration [7]. **SAMPLED VARIABLE**

Case 4 The default case includes any remaining sequences. It is assumed that all sequences resulting in a damaging explosion are included in previous cases. The outcome probability assigned is 1.0 in Branch 3 , there is no explosion.

Question 61: Do the cell covers that fall due to a seismic event strike the SME?

Falling cell covers during a seismic event can rupture and spill the contents of the Slurry Mix Evaporator. There are two possible outcomes as follows:

CCv-SME: The Chemical Cell Covers are dislodged by the Seismic event and fall on the Slurry Mix Evaporator.

nCCV-SME: The Chemical Cell Covers are dislodged by the Seismic event and do not fall on the Slurry Mix Evaporator.

This is a Type 2 question. The answer to this question depends upon the outcome of previous Questions 2 and 56. The answers to this question are used in the logic to determine the outcome probabilities in Question 62. Two cases are used to develop the outcome probabilities on the basis of prior events as follows:

Case 1 This case includes those sequences in which the initiating event is a seismic event and the Chemical Process Cell covers fell due to the seismic event. The outcome probability for Branch 1 is 0.01 [7], the outcome probability for Branch 2 is 1.0 less the probability assigned Branch 1 . **SAMPLED VARIABLE**.

Case 2 The default case includes all other sequences. The outcome probability assigned is 1.0 in Branch 2, there is no seismic event and the cell covers do not fall. 
Question 62: " Does a Seismic event or cover crane fall rupture and spill the contents of the Slurry Mix Evaporator Tank in the Chemical Process Cell?

Falling cell covers during a seismic event or a crane load drop can rupture and spill the contents of the Slurry Mix Evaporator. Shaking and overturning of the tank during a seismic event can also cause spillage of the contents. There are two defined outcomes to this question as follows:

RptSME: The Slurry Mix Evaporator is ruptured and the contents spilled as a result of the seismic event or a crane drop accident.

nRptSME: The Slurry Mix Evaporator is intact.

This is a Type 6 question. The answer to this question depends upon the outcome of previous Question 2, 39, 56, and 61 and the value specified for the parameter SME-Fr, the seismic fragility of the Slurry Mix Evaporator. The answers to this question are used in the logic to determine the outcome probabilities in Questions 64,90,100,131, and 143. Three cases are used to develop the outcome probabilities on the basis of prior events as follows:

Case 1. This case includes all sequences in which a seismic event or crane drop accident has caused the cell covers over the Chemical Process Cell to fall and strike the SME. The falling cell covers are assumed to rupture and spill the contents of the SME, i.e., an outcome probability of 1.0 is assigned Branch 1.

Case 2 . This case includes all sequences in which a seismic event has occurred but the cell covers do not fall." The seismic fragility of the SME [the probability that a seismic event will cause a structural failure of the SME] is placed in Branch 1. The outcome probability for Branch 2, nRptSME, is 1.0.less the probability assigned Branch 1 .

Case 3 The default case includes all remaining sequences. The outcome probability assigned is 1.0 in Branch 2, there is no seismic event and the SME is undamaged.

Question 63: Does an explosive concentration accumulate in the Slurry Mix Evaporator Tank [SME]?

A fault tree was constructed to calculate the frequency of explosions in the SME for all credible initiators. The solution of this fault tree (cut sets) is used to determine the frequency (or probability) of an explosive concentration in the Slurry Mix Evaporator. The two outcomes defined for this question are as follows:

ConSME: An explosive concentration accumulates in the SME.

nConSME: An explosive concentration does not accumulate in the SME.

This is a Type 6 question and uses previously quantified parameters $[60,61,62,64,66,67$, $70,71,90,93,95,98,103$, and 108$]$ and a user defined function to determine the probability 
that an explosive concentration will occur in the SME. The user function includes information from solution of the fault tree for the SME explosion. The function used to calculate the answer depends on the answer to previous Question 1. The outcome probabilities are used in the logic to determine the outcome probabilities in Question 64.

The following case structure is simplified by not considering the possibility of prior rupture of the SME [Question 62]. Rupture of the SME would prevent the buildup of an explosive concentration in the SME. The possibility of prior rupture of the SME is included in Question 64. Five cases are used to develop the outcome probabilities as follows:

Case 1 This case is used to exclude those sequences in the classes of accidents [Question 1] which are neither external nor internal-energetic. The outcome probability assigned is 1.0 in Branch 2, there is no explosive concentration.

Case 2 This case is used to exclude those sequences where the bulk $\mathrm{N}_{2}$ tank fails as an initiator. The bulk $\mathrm{N}_{2}$ tank supplies nitrogen to the CPC backup nitrogen system. Since the system is a backup, its failure cannot initiate accidents in the CPC. The outcome probability assigned is 1.0 in Branch 2 , there is no explosive concentration.

Case 3 This case includes all sequences in which the class of accidents is internalenergetic [Branch 2, Question 1]. The outcome probability is calculated using FUN-SME and assigned to Branch 1 . The outcome probability for Branch 2 is 1.0 less the probability assigned Branch 1.

Case 4 This case includes all sequences in which the class of accidents is external [Branch 1, Question 1]. The outcome probability is calculated using FUN-SMES and assigned to Branch 1. The outcome probability for Branch 2 is 1.0 less the probability assigned Branch 1.

Case 5 The default case assumes that all sequences in which an explosive concentration can occur are included in Cases 3 and 4. An outcome probability of 1.0 is assigned Branch 2.

\section{Question 64: Does a detonation/deflagration occur in the Slurry Mix Evaporator Tank [SME]?}

If an explosive concentration occurs in the Slurry Mix Evaporator and an ignition source is available it is assumed that a damaging detonation or deflagration will follow. The fault tree for the explosion includes the probability of an ignition source. Therefore, if Question 63 indicates that an explosive concentration is available then a detonation/deflagration will follow. A detonation or deflagration in the Slurry Mix Evaporator can spill the contents of the vessel to the Chemical Process Cell, blow the cell covers, and damage adjacent vessels. There are three defined outcomes as follows:

SME-Det: A damaging detonation occurs in the Slurry Mix Evaporator.

SME-Def: A damaging deflagration occurs in the Slurry Mix Evaporator.

nSME-Ex: No damaging explosion occurs in the Slurry Mix Evaporator. 
This is a Type 2 question. The answer is dependent on the outcome of previous Questions 62 and 63. The answers to this question are used in the logic to determine the outcome probabilities in Questions 76, 78, 90, 98, 99, 100, and 131. Four cases are used to develop the outcome probabilities as follows:

Case 1 This case includes all sequences in which an explosive concentration does not accumulate in the SME. The outcome probability assigned is 1.0 in Branch 3, there is no explosion.

Case 2. This case includes the sequences in which a prior event has ruptured the SME precluding the accumulation of an explosive concentration. The outcome probability assigned is 1.0 in Branch 3, there is no explosion.

Case 3 This case includes the sequences in which a prior event has not ruptured the Slurry Mix Evaporator and an explosive concentration has accumulated in the vessel. The outcome probability assigned is 0.001 in Branch 1, there is a damaging detonation and 0.999 in Branch 2, there is a damaging deflagration [7]. **SAMPLED VARIABLE**

Case 4 The default case includes any remaining sequences. It is assumed that all sequences resulting in a damaging explosion are included in previous cases. The outcome probability assigned is 1.0 in Branch 3 , there is no explosion.

\section{Question 65: Do the cell covers that fall in a seismic event strike the SRAT?}

Falling cell covers during a seismic event can rupture and spill the contents of the Sludge Receipt Adjustment Tank. There are two possible outcomes as follows:

CCV-SRAT: The Chemical Cell Covers are dislodged by the seismic event and fall on the Sludge Receipt Adjustment Tank.

nCCv-SRAT: The Chemical Cell Covers are dislodged by the seismic event and do not fall on the Sludge Receipt Adjustment Tank.

This is a Type 2 question. The answer to this question depends upon the outcome of previous Questions 2 and 56. The answers to this question are used in the logic to determine the outcome probabilities in Question 66. Two cases are used to develop the outcome probabilities on the basis of prior events as follows:

Case 1. This case includes those sequences in which the initiating event is a seismic event and the Chemical Process Cell covers fell due to the seismic event. The outcome probability for Branch 1 is 0.01 [7], the outcome probability for Branch 2 is 1.0 less the probability assigned Branch 1. **SAMPLED VARIABLE**.

Case 2 The default case includes all other sequences. The outcome probability assigned is 1.0 in Branch 2, there is no seismic event and the cell covers do not fall. 


\section{Question 66: Does a Seismic event or cover crane fall rupture and spill the contents of the Sludge Receipt And Adjustment Tank in the Chemical Process Cell?}

Falling cell covers during a seismic event or a crane load drop can rupture and spill the contents of the Sludge Receipt Adjustment Tank. Shaking and overtuming of the tank during a seismic event can also cause spillage of the contents. There are two defined outcomes to this question as follows:

RptSRAT: The Sludge Receipt Adjustment Tank is ruptured and the contents spilled as a result of the seismic event or a crane drop accident.

nRptSRAT: The Sludge Receipt Adjustment Tank is intact.

This is a Type 6 question. The answer to this question depends upon the outcome of previous Question 2,39,56, and 65 and the value specified for the parameter SRATFr, the seismic fragility of the Sludge Receipt Adjustment Tank. The answers to this question are used in the logic to determine the outcome probabilities in Questions 68, 92, 101, 132, and 144. Three cases are used to develop the outcome probabilities on the basis of prior events as follows:

Case 1 This case includes all sequences in which a seismic event or crane drop accident has caused the cell covers over the Chemical Process Cell to fall and strike the SRAT. The falling cell covers are assumed to rupture and spill the contents of the SRAT, i.e., an outcome probability of 1.0 is assigned Branch 1.

Case 2 This case includes all sequences in which a seismic event has occurred but the cell covers do not fall. The seismic fragility of the SRAT [the probability that a seismic event will cause a structural failure of the SRAT] is placed in Branch 1. The outcome probability for Branch 2, nRptSRAT, is 1.0 less the probability assigned Branch 1 .

Case 3 The default case includes all remaining sequences. The outcome probability assigned is 1.0 in Branch 2, there is no seismic event and the SRAT is undamaged.

Question 67: Does an explosive concentration accumulate in the Sludge Receipt And Adjustment Tank [SRAT]?

A fault tree was constructed to calculate the frequency. of explosions in the SRAT for all credible initiators. The solution of this fault tree (cut sets) is used to determine the frequency (or probability) of an explosive concentration in the Sludge Receipt Adjustment Tank. The two outcomes defined for this question are as follows:

ConSRAT: An explosive concentration accumulates in the SRAT.

nConSRAT: An explosive concentration does not accumulate in the SRAT.

This is a Type 6 question and uses previously quantified parameters $[60,61,62,63,64,66$, $67,70,71,90,93,95,98,103$, and 108] and a user defined function to determine the 
probability that an explosive concentration will occur in the SRAT. The user function includes information from solution of the fault tree for the SRAT explosion. The function used to calculate the answer depends on the answer to previous Question 1. The outcome probabilities are used in the logic to determine the outcome probabilities in Question 68.

The following case structure is simplified by not considering the possibility of prior rupture of the SRAT [Question 66]. Rupture of the SRAT would prevent the buildup of an explosive concentration in the SRAT. The possibility of prior rupture of the SRAT is included in Question 68. Five cases are used to develop the outcome probabilities as follows:

Case 1 This case is used to exclude those sequences in the classes of accidents [Question 1] which are neither external nor internal-energetic. The outcome probability assigned is 1.0 in Branch 2 , there is no explosive concentration.

Case 2 This case is used to exclude those sequences where the bulk $\mathrm{N}_{2}$ tank fails as an initiator. The bulk $\mathrm{N}_{2}$ tank supplies nitrogen to the CPC backup nitrogen system. Since the system is a backup, its failure cannot initiate accidents in the CPC. The outcome probability assigned is 1.0 in Branch 2 , there is no explosive concentration.

Case 3 This case includes all sequences in which the class of accidents is internalenergetic [Branch 2, Question 1]. The outcome probability is calculated using FUN-SRAT and assigned to Branch 1. The outcome probability for Branch 2 is 1.0 less the probability assigned Branch 1.

Case 4 This case includes all sequences in which the class of accidents is external [Branch 1, Question 1]. The outcome probability is calculated using FUN-SRATS and assigned to Branch 1 . The outcome probability for Branch 2 is 1.0 less the probability assigned Branch.1.

Case 5 The default case assumes that all sequences in which an explosive concentration can occur are included in Cases 3 and 4. An outcome probability of 1.0 is assigned Branch 2.

\section{Question 68: - Does a detonation/deflagration occur in the Sludge Receipt} Adjustment Tank [SRAT]?

If an explosive concentration occurs in the Sludge Receipt Adjustment Tank and an ignition source is available it is assumed that a damaging detonation or deflagration will follow. The fault tree for the explosion includes the probability of an.ignition source. Therefore, if Question 67 indicates that an explosive concentration is available then a detonation/deflagration will follow. A detonation or deflagration in the Sludge Receipt Adjustment Tank can spill the contents of the vessel to the Chemical Process Cell, blow the cell covers, and damage adjacent vessels. There are three defined outcomes as follows:

SRAT-Det: A damaging detonation occurs in the Sludge Receipt Adjustment Tank.

SRAT-Def: A damaging deflagration occurs in the Sludge Receipt Adjustment Tank.

nSRAT-Ex: No damaging explosion occurs in the Sludge Receipt Adjustment Tank. 
This is a Type 2 question. The answer is dependent on the outcome of previous Questions 66 and 67. The answers to this question are used in the logic to determine the outcome probabilities in Questions 76, 78,92,98,101,102, and 132. Four cases are used to develop the outcome probabilities as follows:

Case 1 . This case includes all sequences in which an explosive concentration does not accumulate in the SRAT. The outcome probability assigned is 1.0 in Branch 3, there is no explosion.

Case 2 This case includes the sequences in which a prior event has ruptured the SRAT precluding the accumulation of an explosive concentration. The outcome probability assigned is 1.0 in Branch 3 , there is no explosion.

Case 3 This case includes the sequences in which a prior event has not ruptured the Sludge Receipt Adjustment Tank and an explosive concentration has accumulated in the vessel. The outcome probability assigned is 0.001 in Branch 1, there is a damaging detonation and 0.999 in Branch 2, there is a damaging deflagration [7]. **SAMPLED VARIABLE**

Case 4 The default case includes any remaining sequences. It is assumed that all sequences resulting in a damaging explosion are included in previous cases. The outcome probability assigned is 1.0 in Branch 3 , there is no explosion.

\section{Question 69: Do the cell covers that fall in a seismic event strike the RCT?}

Falling cell covers during a seismic event can rupture and spill the contents of the Recycle Collection Tank. There are two possible outcomes as follows:

CCv-RCT: The Chemical Cell Covers are dislodged by the seismic event and fall on the Recycle Collection Tank.

nCCV-RCT: The Chemical Cell Covers are dislodged by the seismic event and do not fall on the Recycle Collection Tank.

This is a Type 2 question. The answer to this question depends upon the outcome of previous Questions 2 and 56. The answers to this question are used in the logic to determine the outcome probabilities in Question 70. Two cases are used to develop the outcome probabilities on the basis of prior events as follows:

Case 1 This case includes those sequences in which the initiating event is a seismic event and the Chemical Process Cell covers fell due to the seismic event. The outcome probability for Branch 1 is 0.01 [7], the outcome probability for Branch 2 is 1.0 less the probability assigned Branch 1. **SAMPLED VARIABLE**.

Case 2. The default case includes all other sequences. The outcome probäbility assigned is 1.0 in Branch 2, there is no seismic event and the cell covers do not fall. 
Question 70: Does a Seismic event or cover crane fall rupture and spill the contents of the Recycle Collection Tank in the Chemical = Process Cell?

Falling cell covers during a seismic event or a crane load drop can rupture and spill the contents of the Recycle Collection Tank. Shaking and overturning of the tank during a seismic event can also cause spillage of the contents. There are two defined outcomes to this question as follows:

Rpt-RCT: The Recycle Collection Tank is ruptured and the contents spilled as a result of the seismic event or a crane drop accident.

nRpt-RCT: : The Recycle Collection Tank is intact.

This is a Type 6 question. The answer to this question depends upon the outcome of previous Question 2, 39, 56, and 69 and the value specified for the parameter RCTFr, the seismic fragility of the Recycle Collection Tank. The answers to this question are used in the logic to determine the outcome probabilities in Questions 102 and 145. Three cases are used to develop the outcome probabilities on the basis of prior events as follows:

Case 1 This case includes all sequences in which a seismic event or crane drop accident has caused the cell covers over the Chemical Process Cell to fall and strike the RCT. The falling cell covers are assumed to rupture and spill the contents of the RCT, i.e., an outcome probability of 1.0 is assigned Branch 1.

Case 2

This case includes all sequences in which a seismic event has occurred but the cell covers do not fall. The seismic fragility of the RCT [the probability that a seismic event will cause a structural failure of the RCT] is placed in Branch 1. The outcome probability for Branch 2, nRptRCT, is 1.0 less the probability assigned Branch 1.

Case 3 The default case includes all remaining sequences. The outcome probability assigned is 1.0 in Branch 2, there is no seismic event and the RCT is undamaged.

Question 71: Do the cell covers that fall in a seismic event strike the MFT?

Falling cell covers during a seismic event can rupture and spill the contents of the Melter-Feed Tank. There are two possible outcomes as follows:

CCV-MFT: The Chemical Cell Covers are dislodged by the seismic event and fall on the Melter Feed Tank.

nCCV-MFT: The Chemical Cell Covers are dislodged by the seismic event and do not fall on the Melter Feed Tank.

This is a Type 2 question. The answer to this question depends upon the outcome of previous Questions 2 and 56. The answers to this question are used in the logic to determine the outcome probabilities in Question 72. 
Two cases are used to develop the outcome probabilities on the basis of prior events as follows:

Case 1

This case includes those sequences in which the initiating event is.a seismic event and the Chemical Process Cell covers fell due to the seismic event. The outcome probability for Branch 1 is 0.01 [7], the outcome probability for Branch 2 is 1.0 less the probability assigned Branch 1. **SAMPLED VARIABLE**.

Case 2 The default case includes all other sequences. The outcome probability assigned is 1.0 in Branch 2, there is no seismic event and the cell covers do not fall.

Question 72: , Does a Seismic event or cover crane fall rupture and spill the contents of the Melter Feed Tank in the Chemical Process Cell?

Falling cell covers during a seismic event or a crane load drop can rupture and spill the contents of the Melter Feed Tank. Shaking and overturning of the tank during a seismic event can also cause spillage of the contents. There are two defined outcomes to this question as follows:

Rpt-MFT: The Melter Feed Tank is ruptured and the contents spilled as a result of the seismic event or a crane drop accident.

nRpt-MFT: The Melter Feed Tank is intact.

This is a Type 6 question. The answer to this question depends upon the outcome of previous Question 2, 39, 56, and 71 and the value specified for the parameter MFTFr, the seismic fragility of the Melter Feed Tank. The answers to this question are used in the logic to determine the outcome probabilities in Questions 74, 94,103,133, and 146. Three cases are used to develop the outcome probabilities on the basis of prior events as follows:

Case 1 This case includes all sequences in which a seismic event or crane drop accident has caused the cell covers over the Chemical Process Cell to fall and strike the MFT. The falling cell covers are assumed to rupture and spill the contents of the MFT, i.e., an outcome probability of 1.0 is assigned Branch 1.

Case 2 This case includes all sequences in which a seismic event has occurred but the cell covers do not fall. The seismic fragility of the MFT [the probability that a seismic event will cause a structural failure of the MFT] is placed in Branch 1. The outcome probability for Branch 2, nRpt-MFT, is 1.0 less the probability assigned Branch 1 .

Case 3 The default case includes all remaining sequences. The outcome probability assigned is 1.0 in Branch 2, there is no seismic event and the MFT is undamaged. 
Question 73: Does an explosive concentration accumulate in the Melter Feed Tank [MFT]?

A fault tree was constructed to calculate the frequency of explosions in the MFT for all credible initiators. The solution of this fault tree (cut sets) is used to determine the frequency (or probability) of an explosive concentration in the Melter Feed Tank. The two outcomes defined for this question are as follows:

ConMFT: An explosive concentration accumulates in the MFT.

nConMFT: An explosive concentration does not accumulate in the MFT:

This is a Type 6 question and uses previously quantified parameters $[60,66,67,70,71,90$, and 108] and a user defined function to determine the probability that an explosive concentration will occur in the MFT. The user function includes information from solution of the fault tree for the MFT explosion. The function used to calculate the answer depends on the answer to previous Question 1. The outcome probabilities are used in the logic to determine the outcome probabilities in Question 74.

The following case structure is simplified by not considering the possibility of prior rupture of the MFT [Question 72]. Rupture of the MFT would prevent the buildup of an explosive concentration in the MFT. The possibility of prior rupture of the MFT is included in Question 74. Five cases are used to develop the outcome probabilities as follows:

Case 1 This case is used to exclude those sequences in the classes of accidents [Question 1] which are neither external nor internal-energetic. The outcome probability assigned is 1.0 in Branch 2 , there is no explosive concentration.

Case 2 This case is used to exclude those sequences where the bulk $\mathrm{N}_{2}$ tank fails as an initiator. The bulk $\mathrm{N}_{2}$ tank supplies nitrogen to the CPC backup nitrogen system. Since the system is a backup, its failure cannot initiate accidents in the CPC. The outcome probability assigned is 1.0 in Branch 2 , there is no explosive concentration.

Case 3 This case includes all sequences in which the class of accidents is internalenergetic [Branch 2, Question 1]. The outcome probability is calculated using FUN-MFT and assigned to Branch 1. The outcome probability for Branch 2 is 1.0 less the probability assigned Branch 1.

Case 4 This case includes all sequences in which the class of accidents is external [Branch 1, Question 1]. The outcome probability is calculated using FUN-MFTS and assigned to Branch 1 . The outcome probability for Branch 2 is 1.0 less the probability assigned Branch 1.

Case 5 The default case assumes that all sequences in which an explosive concentration can occur are included in Cases. 3 and 4. An outcome probability of 1.0 is assigned Branch 2. 


\section{Question_74: Does a detonation/deflagration occur in the Melter Feed Tank [MFT]?}

If an explosive concentration occurs in the Melter Feed Tank and an ignition source is available it is assumed that a damaging detonation or deflagration will follow. The fault tree for the explosion includes the probability of an ignition source. Therefore, if Question 73 indicates that an explosive concentration is available then a detonation/deflagration will follow. A detonation or deflagration in the Melter Feed Tank can spill the contents of the vessel to the Chemical Process Cell, blow the cell covers, and damage adjacent vessels. There are three defined outcomes as follows:

MFT-Det: A damaging detonation occurs in the Melter Feed Tank.

MFT-Def: · A damaging deflagration occurs in the Melter Feed Tank.

nMFT-Ex: $\quad$ No damaging explosion occurs in the Melter Feed Tank.

This is a Type 2 question. The answer is dependent on the outcome of previous Questions 72 and 73. The answers to this question are used in the logic to determine the outcome probabilities in Questions 76, 78,94, 98, 102, 103, and 133. Four cases are used to develop the outcome probabilities as follows:

Case 1 This case includes all sequences in which an explosive concentration does not accumulate in the MFT. The outcome probability assigned is 1.0 in Branch 3, there is no explosion.

Case 2 This case includes the sequences in which a prior event has ruptured the MFT precluding the accumulation of an explosive concentration. The outcome probability assigned is 1.0 in Branch 3 , there is no explosion.

Case 3 This case includes the sequences in which a prior event has not ruptured the Meiter Feed Tank and an explosive concentration has accumulated in the vessel. The outcome probability assigned is 0.001 in Branch 1 , there is a damaging detonation and 0.999 in Branch 2, there is a damaging deflagration [7]. **SAMPLED VARIABLE**

Case 4 The default case includes any remaining sequences. It is assumed that all sequences resulting in a damaging explosion are included in previous cases. The outcome probability assigned is 1.0 in Branch 3 , there is no explosion.

\section{Question 75: Does a damaging explosion occur in the Salt Process Cell Vent System [SPCV]?}

Conditions in the Salt Process Cell Vent System [SPCV] are expected to be the same as the tanks exhausting into the header. Therefore an explosion in any of the SPC tanks will propagate into the header causing the header to also explode. Because of the size of the SPCV, it is expected to detonate (not deflagrate) which would produce shrapnel that could cause venting of cell tanks. There are two possible outcomes as follows:

SPCV-Exp: A damaging explosion occurs in the Salt Process Cell Vent System. 
nSPCV-Ex: A damaging explosion does not occur in the Salt Process Cell Vent System.

This is a Type 2 question. The answer is dependent on the outcome of previous Questions 37, $43,47,51$, and 55 . The answers to this question are used in the logic to determine the outcome probabilities in Questions 78 and 95. Four cases are used to develop the outcome probabilities as follows:

Case 1 This case includes all sequences in which the SPC cell covers have fallen due to a seismic event. If the cell covers fall, the SPCV piping is assumed to be damged to the point that no accumulation of explosive mixtures is possible. An outcome probability of 1.0 is assigned to Branch 2 .

Case 2 This case includes all sequences in which one of the SPC vessels has detonated. A detonation in any SPC vessel is assumed to cause the SPCV to explode. An outcome probability of 1.0 is assigned Branch 1.

Case 3 This case includes all sequences in which one of the SPC vessels has deflagrated. A deflagration in any SPC vessel is assumed to cause the SPCV to explode. An outcome probability of 1.0 is assigned Branch 1.

Case 4 The default case includes all sequences remaining and assumes that there is no other mechanism for an explosion in the SPCV. An outcome probability of 1.0 is assigned Branch 2 .

\section{Question 76: Does a damaging explosion occur in the Chemical Process Cell Vent System [CPCV]?}

Conditions in the Chemical Process Cell Vent System [CPCV] are expected to be the same as the tanks exhausting into the header. Therefore an explosion in any of the CPC tanks will propagate into the header causing the header to also explode. Because of the size of the CPCV, it is expected to detonate (not deflagrate) which would produce shrapnel that could cause venting of cell tanks. There are two possible outcomes as follows:

CPCV-Exp: A damaging explosion occurs in the Chemical Process Cell Vent System.

nCPCV-Ex: No damaging explosion occurs in the Chemical Process Cell Vent System.

This is a Type 2 question. The answer is dependent on the outcome of previous Question 56, $60,64,68$, and 74 . The answers to this question are used in the logic to determine the outcome probabilities in Questions 78 and 96. Four cases are used to develop the outcome probabilities as follows:

Case 1 This case includes all sequences in which the CPC cell covers have fallen due to a seismic event. If the cell covers fall, the CPCV piping is assumed to be damged to the point that no accumulation of explosive mixtures is possible. An outcome probability of 1.0 is assigned to Branch 2. 
Case $2 \ldots$ This case includes all sequences in which one of the CPC vessels has detonated. A detonation in any CPC vessel is assumed to cause the CPCV to explode. An outcome probability of 1.0 is assigned Branch 1.

Case 3

This case includes all sequences in which one of the CPC vessels has deflagrated. A deflagration in any CPC vessel is assumed to cause the CPCV to explode. An outcome probability of 1.0 is assigned Branch 1.

Case 4

The default case includes all sequences remaining and assumes that there is no other mechanism for an explosion in the CPCV. An outcome probability of 1.0 is assigned Branch 2.

\section{Question 77: Does an explosive concentration accumulate in the Process Vessel Vent Header [PVVH]?}

The Process Vessel Vent Header [PVVH] consists of all piping from the base load air inlet to the PVVH blowers, including inlet HEPA filter, flow control valve, PVVH filter, preheater, and blower suction dampers. Potential sources of explosive gases to the PVVH are benzene from the vessels in the Salt Process Cell and hydrogen and benzene from the process vessels in the Chemical Process Cell. There are two defined outcomes as follows:

ConPVVH: An explosive concentration accumulates in the Process Vessel Vent Header.

nConPVVH: An explosive concentration does not accumulate in the Process Vessel Vent Header.

This is a Type 6 question and uses previously quantified parameters $[60,61,62,63,64,66$, $67,68,70,71,90,93,95,98,103,108,135$, and 136] and a user defined function to determine the probability that an explosive concentration will occur in the PVVH. The user function includes information from solution of the fault tree for a PVVH explosion. The function used to calculate the answer depends on the answer to previous Question 1. The outcome probabilities are used in the logic to determine the outcome probabilities in Question 78. Five cases are used to develop the outcome probabilities as follows:

Case 1 This case is used to exclude those sequences in the classes of accidents [Question 1] which are neither external nor internal-energetic. The outcome probability assigned is 1.0 in Branch 2 , there is no explosive concentration.

Case 2 This case is used to exclude those sequences where the bulk $\mathrm{N}_{2}$ tank fails as an initiator. The bulk $\mathrm{N}_{2}$ tank supplies nitrogen to the CPC backup nitrogen system. Since the system is a backup, its failure cannot initiate accidents in the CPC. The outcome probability assigned is 1.0 in Branch 2 , there is no explosive concentration.

Case 3 This case includes all sequences in which the class of accidents is internalenergetic [Branch 2, Question 1]. The outcome probability is calculated using FUN-PVVH and assigned to Branch 1 . The outcome probability for Branch 2 is 1.0 less the probability assigned Branch 1. 
Case 4 _. This case includes all sequences in which the class of accidents is external [Branch 1, Question 1]. The outcome probability is calculated using FUN-PVVHS and assigned to Branch 1 . The outcome probability for Branch 2 is 1.0 less the probability assigned Branch 1 .

Case 5

The default case assumes that all sequences in which an explosive concentration can occur are included in Cases 3 and 4. An outcome probability of 1.0 is assigned Branch 2.

\section{Question 78: Does a damaging detonation occur in the Process Vessel Vent Header [PVVH]?}

If an explosive concentration occurs in the Process Vessel Vent Header [PVVH] and an ignition source is available it is, assumed that a damaging detonation will follow. The fault tree for the PVVH detonation includes the probability of an ignition source. Therefore, if Question 77 indicates that an explosive concentration is available then a detonation will follow. Also, conditions in the [PVVH] are expected to be the same as the tanks exhausting into the header. Therefore an explosion in any of the SPC or CPC tanks will propagate into the header causing the header to also explode. A detonation in the PVVH can cause cell covers in the Chemical Process Cell to fall. There are two defined outcomes:

PVVH-Det: A damaging detonation occurs in the Process Vessel Vent Header.

nPVVH-Ex: No damaging explosion occurs in the Process Vessel Vent Header.

This is a Type 2 question. The answer is dependent on the outcome of previous Questions 43, $47,51,55,56,60,64,68,74,75,76$, and 77 . The answers to this question are used in the logic to determine the outcome probabilities in Questions 80, 82, 84, 86, 88, 90, 92, 94, and 98. Six cases are used to develop the outcome probabilities as follows:

Case 1 This case includes all sequences in which the CPC cell covers have fallen due to a seismic event. If the cell covers fall, the PVVH piping is. assumed to be damged to the point that no accumulation of explosive mixtures is possible. An outcome probability of 1.0 is assigned to Branch 2.

Case 2 This case includes all sequences in which a damaging detonation or deflagration has occurred in the SPC. The outcome probability àssigned is 1.0 in Branch 1 . If there is an explosion in one of the process tanks the explosion is assumed to propagate to the PVV.H.

Case 3 This case includes all sequences in which a damaging detonation or deflagration has occurred in the CPC. The outcome probability assigned is 1.0 in Branch 1. If there is an explosion in one of the process tanks the explosion is assumed to propagate to the PVVH.

Case 4 This case includes the sequences in which an explosive concentration has not accumulated in the PVVH. The outcome probability assigned is 1.0 in Branch 2, there is not a damaging explosion. 
Case 5 .. This case includes the sequences in which an explosive concentration has accumulated in the PVVH. The outcome probability assigned is 1.0 in Branch 1, there is a damaging explosion.

Case 6 The default case includes any remaining sequences. It is assumed that all sequences resulting in a damaging explosion are included in previous cases. The outcome probability assigned is 1.0 in Branch 2 , there is no explosion.

Question 78 is the last question in the first round of possible energetic events considered in the APET. Questions 79-96 consider the possibility that another vessel has an explosive concentration built up (but no ignition source) at the same time another vessel deflagrates or detonates. This begins the second round of explosion questions. Since the process vessels are all cross-connected via the cell ventilation systems (SPCV, CPCV, PVVH), the vessel that deflagrates or detonates provides an ignition source to any other vessel that has an explosive concentration.

\section{Question 79: Does an explosive concentration accumulate in Precipitate Reactor?}

The cut sets solved by the UFUN in this question have been modified to calculate the probability of an explosive concentration in the PR without the presence of an ignition source. The two outcomes defined for this question are as follows:

Con-PRx2: An explosive concentration accumulates in the Precipitate Reactor.

nCon-PRx: An explosive concentration does not accumulate in the Precipitate Reactor.

This is a Type 6 question and uses previously quantified parameters $[60,65,69,70,71,90$, 118,135 , and 136] and a user defined function to determine the probability that an explosive concentration will occur in the Precipitate Reactor. The function used to calculate the answer depends on the answer to previous Question 1. The answers to this question are used in the logic to determine the outcome probabilities in Questions 80 and 123.

The case structure is simplified by not considering the possibility of any prior events with the Precipitate Reactor which would prevent the buildup of an explosive concentration in the Precipitate Reactor. The possibility of prior events with the Precipitate Reactor is included in Question 80. Four cases are used to develop the outcome probabilities as follows:

Case 1 This case is used to exclude those sequences in the classes of accidents [Question 1] which are neither external nor internal-energetic. The outcome probability assigned is 1.0 in Branch 2, there is no explosive concentration.

Case 2 This case includes all sequences in which the class of accidents is internalenergetic [Branch 2, Question 1]. The outcome probability is calculated using FUN-PRIIII and assigned to Branch 1 . The outcome probability for Branch 2 is 1.0 less the probability assigned Branch 1. 
Case 3 This case includes all sequences in which the class of accidents is external -. [Branch 1, Question 1]. The outcome probability is calculated using FUN-PRSI and assigned to Branch 1. The outcome probability for Branch 2 is 1.0 less the probability assigned Branch 1 .

Case 4 The default case assumes that all sequences in which an explosive concentration can occur are included in Cases 2 and 3. An outcome probability of $1: 0$ is assigned Branch 2 .

\section{Question 80: Does a deflagration/detonation occur in the Precipitate} Reactor?

If an explosive concentration occurs in the Precipitate Reactor and an ignition source is available it is assumed that a damaging detonation or deflagration will follow. A detonation/deflagration in the Precipitate Reactor can spill the contents of the vessel to the Salt Process Cell, blow the cell covers, and damage adjacent vessels. There are three defined outcomes as follows:

PRx@2Det: A damaging detonation occurs in the Precipitate Reactor following a detonation or deflagration in another process vessel that provides. an ignition source.

PRx@2Def: A damaging deflagration occurs in the Precipitate Reactor following a detonation or deflagration in another process vessel that provides an ignition source.

nPRx-Exp: No damaging explosion occurs in the Precipitate Reactor.

This is a Type 2 question. The answer is dependent on the outcome of previous Questions 41, 43,78 , and 79 . The answers to this question are used in the logic to determine the outcome probabilities in Questions 95,105, 106, 107 and 123. Six cases are used to develop the outcome probabilities as follows:

Case 1

Case 2

Case 3

Case 4
This case includes all sequences in which a previous detonation occurred in the Precipitate Reactor. The outcome probability assigned is 1.0 in Branch 3, there is no explosion.

This case includes all sequences in which a previous deflagration occurred in the Precipitate Reactor. The outcome probability assigned is 1.0 in Branch 3, there is no explosion.

This case includes all sequences in which an explosive concentration does not accumulate in the Precipitate Reactor. The outcome probability assigned is 1.0 in Branch 3, there is no explosion.

This case includes the sequences in which a prior event has ruptured the Precipitate Reactor precluding the accumulation of an explosive concentration. The outcome probability assigned is 1.0 in Branch 3, there is no explosion. 
Case 5 .. This case includes the sequences in which a prior event has not occurred in the Precipitate Reactor and an explosive concentration has accumulated in the vessel with a detonation in the PVVH (provides the ignition source). The outcome probability assigned is 0.001 in Branch 1 , there is a damaging detonation and 0.999 in Branch 2, there is a damaging deflagration [7].

Case 6

The default case includes any remaining sequences. It is assumed that all sequences resulting in a damaging explosion are included in previous cases. The outcome probability assigned is 1.0 in Branch 3 , there is no explosion.

\section{Question 81: Does an explosive concentration accumulate in Organic Evaporator Tank?}

The cut sets solved by the UFUN in this question have been modified to calculate the probability of an explosive concentration in the OEV without the presence of an ignition source. The two outcomes defined for this question are as follows:

Con-OEV2: An explosive concentration accumulates in the Organic Evaporator.

nCon-OEV: An explosive concentration does not accumulate in the Organic Evaporator.

This is a Type 6 question and uses previously quantified parameters $[60,65,69,70,71,90$, 118,135 , and 136] and a user defined function to determine the probability that an explosive concentration will occur in the Organic Evaporator. The function used to calculate the answer depends on the answer to previous Question 1. The answers to this question are used in the logic to determine the outcome probabilities in Questions 82 and 124.

The case structure is simplified by not considering the possibility of any prior events with the Organic Evaporator which would prevent the buildup of an explosive concentration in the Organic Evaporator. The possibility of prior events with the Organic Evaporator is included in Question 82. Four cases are used to develop the outcome probabilities as follows:

Case 1 This case is used to exclude those sequences in the classes of accidents [Question 1] which are neither external nor internal-energetic. The outcome probability, assigned is 1.0 in Branch 2 , there is no explosive concentration.

Case 2 This case includes all sequences in which the class of accidents is internalenergetic [Branch 2, Question 1]. The outcome probability is calculated using FUN-OEVIII and assigned to Branch 1 . The outcome probability for Branch 2 is 1.0 less the probability assigned Branch 1.

Case 3 This case includes all sequences in which the class of accidents is external [Branch 1, Question 1]. The outcome probability is calculated using FUN-OEVSI and assigned to Branch 1 . The outcome probability for Branch 2 is 1.0 less the probability assigned Branch 1. 
Case 4 _. The default case assumes that all sequences in which an explosive concentration can occur are included in Cases 2 and 3. An outcome probability of 1.0 is assigned Branch 2.

\section{Question 82: Does a deflagration/detonation of the Organic Evaporator tank occur?}

If an explosive concentration occurs in the Organic Evaporator and an ignition source is available it is assumed that a damaging detonation or deflagration $i$ follow. A detonation/deflagration in the Organic Evaporator can spill the contents of the vessel to the Salt Process Cell, blow the cell covers, and damage adjacent vessels. There are three defined outcomes as follows:

OEV@2Det A damaging detonation occurs in the Organic Evaporator following a detonation or deflagration in another process vessel that provides an ignition source.

OEV@2Def: A damaging deflagration occurs in the Organic Evaporator following a detonation or deflagration in another process vessel that provides an ignition source.

nOEV-Exp: No damaging explosion occurs in the Organic Evaporator.

This is a Type 2 question. The answer is dependent on the outcome of previous Questions 45, 47,78 , and 81 . The answers to this question are used in the logic to determine the outcome probabilities in Questions $95,105,106,107,108$, and 124. Six cases are used to develop the outcome probabilities as follows:

Case 1 This case includes all sequences in which a previous detonation occurred in the Organic Evaporator. The outcome probability assigned is 1.0 in Branch 3, there is no explosion.

Case 2 This case includes all sequences in which a previous deflagration occurred in the Organic Evaporator. The outcome probability assigned is 1.0 in Branch 3, there is no explosion.

Case 3 This case includes all sequences in which an explosive concentration does not accumulate in the Organic Evaporator. The outcome probability assigned is 1.0 in Branch 3, there is no explosion.

Case 4 This case includes the sequences in which a prior event has ruptured the Organic Evaporator precluding the accumulation of an explosive concentration. The outcome probability assigned is 1.0 in Branch 3 , there is no explosion.

Case 5 This case includes the sequences in which a prior event has not occurred in the Organic Evaporator and an explosive concentration has accumulated in the vessel with a detonation in the PVVH (provides the ignition source). The outcome probability assigned is 0.001 in Branch 1, there is a damaging detonation and 0.999 in Branch 2 , there is a damaging deflagration [7]. 
Case $6^{-} \quad$ The default case includes any remaining sequences. It is assumed that all sequences resulting in a damaging explosion are included in previous cases. The outcome probability assigned is 1.0 in Branch 3 , there is no explosion.

\section{Question 83: Does an explosive concentration accumulate in Organic Evaporator Condensate Tank?}

The cut sets solved by the UFUN in this question have been modified to calculate the probability of an explosive concentration in the OECT without the presence of an ignition source. The two outcomes defined for this question are as follows:

ConOECT2: An explosive concentration accumulates in the Organic Evaporator Condensate Tank.

nCon-OECT: An explosive concentration does not accumulate in the Organic Evaporator Condensate Tank.

This is a Type 6 question and uses previously quantified parameters $[60,65,69,70,71,90$, 118,135 , and 136] and a user defined function to determine the probability that an explosive concentration will occur in the Organic Evaporator Condensate Tank. The function used to calculate the answer depends on the answer to previous Question 1. The answers to this question are used in the logic to determine the outcome probabilities in Questions 84 and 125.

The case structure is simplified by not considering the possibility of any prior events with the Organic Evaporator Condensate Tank which would prevent the buildup of an explosive concentration in the Organic Evaporator Condensate Tank. The possibility of prior events with the Organic Evaporator Condensate Tank is included in Question 84. Four cases are used to develop the outcome probabilities as follows:

Case 1 This case is used to exclude those sequences in the classes of accidents [Question 1] which are neither external nor internal-energetic. The outcome probability assigned is 1.0 in Branch 2 , there is no explosive concentration.

Case 2 . This case includes all sequences in which the class of accidents is internalenergetic [Branch 2, Question 1]. The outcome probability is calculated using FUN-OECTII and assigned to Branch 1 . The outcome probability for Branch 2 is 1.0 less the probability assigned Branch 1.

Case 3 This case includes all sequences in which the class of accidents is external [Branch 1, Question 1]. The outcome probability is calculated using FUN-OECTSI and assigned to Branch 1. The outcome probability for Branch 2 is 1.0 less the probability assigned Branch 1.

Case 4 The default case assumes that all sequences in which an explosive concentration can occur are included in Cases 2 and 3. An outcome probability of 1.0 is assigned Branch 2. 


\section{Question 84: Does a deflagration/detonation of the Organic Evaporator} Condensate tank occur?

If an explosive concentration occurs in the Organic Evaporator Condensate Tank and an ignition source is available it is assumed that a damaging detonation or deflagration will follow. A detonation/deflagration in the Organic Evaporator Condensate Tank can spill the contents of the vessel to the Salt Process Cell, blow the cell covers, and damage adjacent vessels. There are three defined outcomes as follows:

OECT@2Det: A damaging detonation occurs in the Organic Evaporator Condensate Tank following a detonation or deflagration in another process vessel that provides an ignition source.

OECT@2Def: A damaging deflagration occurs in the Organic Evaporator Condensate Tank following a detonation or deflagration in another process vessel that provides an ignition source.

nOECT-Ex: No damaging explosion occurs in the Organic Evaporator Condensate Tank.

This is a Type 2 question. The answer is dependent on the outcome of previous Questions 49, 51,78 , and 83. The answers to this question are used in the logic to determine the outcome probabilities in Questions $95,105,107,108,109$, and 125 . Six cases are used to develop the outcome probabilities as follows:

Case 1 This case includes all sequences in which a previous detonation occurred in the Organic Evaporator Condensate Tank. The outcome probability assigned is 1.0 in Branch 3, there is no explosion.

Case 2 : This case includes all sequences in which a previous deflagration occurred in the Organic Evaporator Condensate Tank. The outcome probability assigned is 1.0 in Branch 3, there is no explosion.

Case 3 This case includes all sequences in which an explosive concentration does not accumulate in the Organic Evaporator Condensate Tank. The outcome probability assigned is 1.0 in Branch 3 , there is no explosion.

Case 4 This case includes the sequences in which a prior event has ruptured the Organic Evaporator Condensate Tank precluding the accumulation of an explosive concentration. The outcome probability assigned is 1.0 in Branch 3, there is no explosion.

Case 5 This case includes the sequences in which a prior event has not occurred in the Organic Evaporator Condensate Tank and an explosive concentration has accumulated in the vessel with a detonation in the PVVH (provides the ignition source): The outcome probability assigned is 0.001 in Branch 1, there is a damaging detonation and 0.999 in Branch 2 , there is a damaging deflagration [7].

Case 6 The default case includes any remaining sequences. It is assumed that all sequences resulting in a damaging explosion are included in previous 
cases. The outcome probability assigned is 1.0 in Branch 3 , there is no explosion.

\section{Question 85: Does an explosive concentration accumulate in Precipitate Reactor Feed Tank?}

The cut sets solved by the UFUN in this question have been modified to calculate the probability of an explosive concentration in the PRFT without the presence of an ignition source. The two outcomes defined for this question are as follows:

ConPRFT2: An explosive concentration accumulates in the Precipitate Reactor Feed Tank.

nConPRFT: An explosive concentration does not accumulate in the Precipitate Reactor Feed Tank.

This is a Type 6 question and uses previously quantified parameters $[60,65,69,70,71,90$, $118,135$, and 136$]$ and a user defined function to determine the probability that an explosive concentration will occur in the Precipitate Reactor Feed Tank. The function used to calculate the answer depends on the answer to previous Question 1. The answers to this question are used in the logic to determine the outcome probabilities in Questions 86 and 126.

The case structure is simplified by not considering the possibility of any prior events with the Precipitate Reactor Feed Tank which would prevent the buildup of an explosive concentration in the Precipitate Reactor Feed Tank. The possibility of prior events with the Precipitate Reactor Feed Tank is included in Question 86. Four cases are used to develop the outcome probabilities as follows:

Case 1 This case is used to exclude those sequences in the classes of accidents [Question 1] which are neither external nor internal-energetic. The outcome probability assigned is 1.0 in Branch 2, there is no explosive concentration.

Case 2 This case includes all sequences in which the class of accidents is internalenergetic [Branch 2, Question 1]. The outcome probability is calculated using FUN-PRFTII and assigned to Branch 1. The outcome probability for Branch 2 is 1.0 less the probability assigned Branch 1.

Case 3 This case includes all sequences in which the class of accidents is external [Branch 1, Question 1]. The outcome probability is calculated using FUN-PRFTSI and assigned to Branch 1. The outcome probability for Branch 2 is 1.0 less the probability assigned Branch 1.

Case 4 The default case assumes that all sequences in which an explosive concentration can occur are included in Cases 2 and 3. An outcome probability of 1.0 is assigned Branch 2. 


\section{Question 86: . Does a deflagration/detonation occur in the Precipitate Reactor Feed Tank?}

If an explosive concentration occurs in the Precipitate Reactor Feed Tank and an ignition source is available it is assumed that a damaging detonation or deflagration will follow. A detonation/deflagration in the Precipitate Reactor Feed Tank can spill the contents of the vessel to the Salt Process Cell, blow the cell covers, and damage adjacent vessels. There are three defined outcomes as follows:

PRFT@2Det: A damaging detonation occurs in the Precipitate Reactor Feed Tank following a detonation or deflagration in another process vessel that provides an ignition source.

PRFT@2Def: A damaging deflagration occurs in the Precipitate Reactor Feed Tank following a detonation or deflagration in another process vessel that provides an ignition source.

nPRFT-Exp: No damaging explosion occurs in the Precipitate Reactor Feed Tank.

This is a Type 2 question. The answer is dependent on the outcome of previous Questions 53, 55,78 , and 85 . The answers to this question are used in the logic to determine the outcome probabilities in Questions $95,97,105,108,109$, and 126. Six cases are used to develop the outcome probabilities as follows:

Case 1 This case includes all sequences in which a previous detonation occurred in the Precipitate Reactor Feed Tank. The outcome probability assigned is 1.0 in Branch 3, there is no explosion.

Case 2 This case includes all sequences in which a previous deflagration occurred in the Precipitate Reactor Feed Tank. The outcome probability assigned is 1.0 in Branch 3, there is no explosion.

Case 3 This case includes all sequences in which an explosive concentration does not accumulate in the Precipitate Reactor Feed Tank. The outcome probability assigned is 1.0 in Branch 3 , there is no explosion.

Case 4 This case includes the sequences in which a prior event has ruptured the Precipitate Reactor Feed Tank precluding the accumulation of an explosive concentration. The outcome probability assigned is 1.0 in Branch 3 , there is no explosion.

Case 5 This case includes the sequences in which a prior event has not occurred in the Precipitate Reactor Feed Tank and an explosive concentration has accumulated in the vessel with a detonation in the PVVH (provides the ignition source). The outcome probability assigned is 0.001 in Branch 1, there is a damaging detonation and 0.999 in Branch 2, there is a damaging deflagration [7].

Case 6 The default case includes any remaining sequences. It is assumed that all sequences resulting in a damaging explosion are included in previous cases. The outcome probability assigned is 1.0 in Branch 3, there is no explosion. 


\section{Question 87: Does an explosive concentration accumulate in the Precipitate Reactor Bottoms Tank?}

The cut sets solved by the UFUN in this question have been modified to calculate the probability of an explosive concentration in the PRBT without the presence of an ignition source. The two outcomes defined for this question are as follows:

ConPRBT2: An explosive concentration accumulates in the Precipitate Reactor Bottoms Tank.

nConPRBT: An explosive concentration does not accumulate in the Precipitate Reactor Bottoms Tank.

This is a Type 6 question and uses previously quantified parameters $[60,66,67,70,71,90$, 95 , and 108] and a user defined function to determine the probability that an explosive concentration will occur in the Precipitate Reactor Bottoms Tank. The function used to calculate the answer depends on the answer to previous Question 1. The answers to this question are used in the logic to determine the outcome probabilities in Questions 88 and 130.

The case structure is simplified by not considering the possibility of any prior events with the Precipitate Reactor Bottoms Tank which would prevent the buildup of an explosive concentration in the Precipitate Reactor Bottoms Tank. The possibility of prior events with the Precipitate Reactor Bottoms Tank is included in Question 88. Five cases are used to develop the outcome probabilities as follows:

Case 1 This case is used to exclude those sequences in the classes of accidents [Question 1] which are neither external nor internal-energetic. The outcome probability assigned is 1.0 in Branch 2 , there is no explosive concentration.

Case 2 This case includes all sequences in which the class of accidents is internalenergetic and the bulk nitrogen tank has failed as an initiator. Since the CPC nitrogen is a backup system, failure of the system supply as an initiator cannot lead to an explosive concentration in the PRBT. The outcome probability assigned in 1.0 in Branch 2, there is no explosive concentration.

Case 3 This case includes all sequences in which the class of accidents is internalenergetic [Branch 2, Question 1]. The outcome probability is calculated using FUN-PRBTII and assigned to Branch 1 . The outcome probability for Branch 2 is 1.0 less the probability assigned Branch 1.

Case 4 This case includes all sequences in which the class of accidents is external [Branch 1, Question 1]. The outcome probability is calculated using FUN-PRBTSI and assigned to Branch 1 . The outcome probability for Branch 2 is 1.0 less the probability assigned Branch 1.

Case 5

The default case assumes that all sequences in which an explosive concentration can occur are included in Cases 3 and 4. An outcome probability of 1.0 is assigned Branch 2 . 
Question 88: Does a deflagration/detonation occur in the Precipitate Reactor Bottoms Tank [PRBT]?

If an explosive concentration occurs in the Precipitate Reactor Bottoms Tank and an ignition source is available it is assumed that a damaging detonation or deflagration will follow. A detonation/deflagration in the Precipitate Reactor Bottoms Tank can spill the contents of the vessel to the Chemical Process Cell, blow the cell covers, and damage adjacent vessels. There are three defined outcomes as follows:

PRBT@2Det: A damaging detonation occurs in the Precipitate Reactor Bottoms Tank following a detonation or deflagration in another process vessel that provides an ignition source.

PRBT@2Def: A damaging deflagration occurs in the Precipitate Reactor Bottoms Tank following a detonation or deflagration in another process vessel that provides an ignition source.

nPRBT-Ex: . No damaging explosion occurs in the Precipitate Reactor Bottoms Tank.

This is a Type 2 question. The answer is dependent on the outcome of previous Questions 58, 60,78 , and 87 . The answers to this question are used in the logic to determine the outcome probabilities in Questions 96, 98, 99, 100, 104, and 130. Six cases are used to develop the outcome probabilities as follows:"

Case 1 This case includes all sequences in which a previous detonation occurred in the Precipitate Reactor Bottoms Tank. The outcome probability assigned is 1.0 in Branch 3, there is no explosion.

Case 2 This case includes all sequences in which a previous deflagration occurred in the Precipitate Reactor Bottoms Tank. The outcome probability assigned is 1.0 in Branch 3, there is no explosion.

Case 3 This case includes all sequences in which an explosive concentration does not accumulate in the Precipitate Reactor Bottoms Tank. The outcome probability assigned is 1.0 in Branch 3 , there is no explosion.

Case 4 . This case includes the sequences in which a prior event has ruptured the Precipitate Reactor Bottoms Tank precluding the accumulation of an explosive concentration. The outcome probability assigned is 1.0 in Branch 3, there is no explosion.

Case 5 This case includes the sequences in which a prior event has not occurred in the Precipitate Reactor Bottoms Tank and an explosive concentration has accumulated in the vessel with a detonation in the PVVH (provides the ignition source). The outcome probability assigned is 0.001 in Branch 1 , there is a damaging detonation and 0.999 in Branch 2, there is a damaging deflagration [7].

Case 6 The default case includes any remaining sequences. It is assumed that all sequences resulting in a damaging explosion are included in previous 
cases. The outcome probability assigned is 1.0 in Branch 3 , there is no explosion.

Question 89: Does an explosive concentration accumulate in the Slurry Mix Evaporator Tank [SME]?

The cut sets solved by the UFUN in this question have been modified to calculate the probability of an explosive concentration in the SME without the presence of an ignition source. The two outcomes defined for this question are as follows:

ConSME2: An explosive concentration accumulates in the Slurry Mix Evaporator Tank.

nConSME: An explosive concentration does not accumulate in the Slurry Mix Evaporator Tank.

This is a Type 6 question and uses previously quantified parameters $[60,61,62,64,66,67$, $70,71,90,93,95,98,103$, and 108] and a user defined function to determine the probability that an explosive concentration will occur in the Slurry Mix Evaporator Tank. The function used to calculate the answer depends on the answer to previous.Question 1. The answers to this question are used in the logic to determine the outcome probabilities in Questions 90 and 131.

The case structure is simplified by not considering the possibility of any prior events with the Slurry Mix Evaporator Tank which would prevent the buildup of an explosive concentration in the Slurry Mix Evaporator Tank. The possibility of prior events with the Slurry Mix Evaporator Tank is included in Question 90. Five cases are used to develop the outcome probabilities as follows:

Case 1 This case is used to exclude those sequences in the classes of accidents [Question 1] which are neither external nor internal-energetic. The outcome probability assigned is 1.0 in Branch 2 , there is no explosive concentration.

Case 2 This case includes all sequences in which the class of accidents is internalenergetic and the bulk nitrogen tank has failed as an initiator. Since the CPC nitrogen is a backup system, failure of the system supply as an initiator cannot lead to an explosive concentration in the SME. The outcome probability assigned in 1.0 in Branch 2, there is no explosive concentration.

Case 3 This case includes all sequences in which the class of accidents is internalenergetic [Branch 2, Question 1]. The outcome probability is calculated using FUN-SMEIII and assigned to Branch 1. The outcome probability for Branch 2 is 1.0 less the probability assigned Branch 1.

Case 4 This case includes all sequences in which the class of accidents is external [Branch 1, Question 1]. The outcome probability is calculated using FUN-SMESI and assigned to Branch 1. The outcome probability for Branch 2 is 1.0 less the probability assigned Branch 1. 
Case $5 \quad \because$ The default case assumes that all sequences in which an explosive concentration can occur are included in Cases 3 and 4. An outcome probability of 1.0 is assigned Branch 2.

\section{Question 90: Does a deflagration/detonation occur in the Slurry Mix Evaporator Tank [SME]?}

If an explosive concentration occurs in the Slurry Mix Evaporator Tank and an ignition source is available it is assumed that a damaging detonation or deflagration will follow. A detonation/deflagration in the Slurry Mix Evaporator Tank can spill the contents of the vessel to the Chemical Process Cell, blow the cell covers, and damage adjacent vessels. There are three defined outcomes as follows:

SME@2Det: A damaging detonation occurs in the Slurry Mix Evaporator Tank following a detonation or deflagration in another process vessel that provides an ignition source.

SME@2Def: A damaging deflagration occurs in the Slurry.Mix Evaporator Tank following a detonation or deflagration in another process vessel that provides an ignition source.

nSME-Ex: No damaging explosion occurs in the Slurry Mix Evaporator Tank.

This is a Type 2 question. The answer is dependent on the outcome of previous Questions 62, 64,78 , and 89 . The answers to this question are used in the logic to determine the outcome probabilities in Questions 96, 98, 99, 100, and 131. Six cases are used to develop the outcome probabilities as follows:

Case 1 This case includes all sequences in which a previous detonation occurred in the Slurry Mix Evaporator Tank. The outcome probability. assigned is 1.0 in Branch 3, there is no explosion.

Case 2 This case includes all sequences in which a previous deflagration occurred in the Slurry Mix Evaporator Tank. The outcome probability assigned is 1.0 in Branch 3, there is no explosion.

Case 3. This case includes all sequences in which an explosive concentration does not accumulate in the Slurry Mix Evaporator Tank. The outcome probability assigned is 1.0 in Branch 3 , there is no explosion.

Case 4 . This case includes the sequences in which a prior event has ruptured the Slurry Mix Evaporator Tank precluding the accumulation of an explosive concentration. The outcome probability assigned is 1.0 in Branch 3 , there is no explosion.

Case 5 This case includes the sequences in which a prior event has not occurred in the Slurry Mix Evaporator Tank and an explosive concentration has accumulated in the vessel with a detonation in the PVVH (provides the ignition source). The outcome probability assigned is 0.001 in Branch 1, there is a damaging detonation and 0.999 in Branch 2, there is a damaging deflagration [7]. 
Case $6{ }^{-\cdots}$ The default case includes any remaining sequences. It is assumed that all sequences resulting in a damaging explosion are included in previous cases. The outcome probability assigned is 1.0 in Branch 3 , there is no explosion.

\section{Question 91: Does an explosive concentration accumulate in the Sludge Receipt and Adjustment Tank [SRAT]?}

The cut sets solved by the UFUN in this question have been modified to calculate the probability of an explosive concentration in the SRAT without the presence of an ignition source. The two outcomes defined for this question are as follows:

ConSRAT2: An explosive concentration accumulates in the Sludge Receipt Adjustment Tank.

nConSRAT: - An explosive concentration does not accumulate in the Sludge Receipt Adjustment Tank.

This is a Type 6 question and uses previously quantified parameters $[60,61,62,63,64,66$, $67,70,71,90,93,95,98,103$, and 108] and a user defined function to determine the probability that an explosive concentration will occur in the Sludge Receipt Adjustment Tank. The function used to calculate the answer depends on the answer to previous Question 1. The. answers to this question are used in the logic to determine the outcome probabilities in Questions 92 and 132.

The case structure is simplified by not considering the possibility of any prior events with the Sludge Receipt Adjustment Tank which would prevent the buildup of an explosive concentration in the Sludge Receipt Adjustment Tank. The possibility of prior events with the Sludge Receipt Adjustment Tank is included in Question 92. Five cases are used to develop the outcome probabilities as follows:

Case 1 This case is used to exclude those sequences in the classes of accidents [Question 1] which are neither external nor internal-energetic. The outcome probability assigned is 1.0 in Branch 2 , there is no explosive concentration.

Case.2 This case includes all sequences in which the class of accidents is internalenergetic and the bulk nitrogen tank has failed as an initiator. Since the CPC nitrogen is a backup system, failure of the system supply as an initiator cannot lead to an explosive concentration in the SRAT. The outcome probability assigned in 1.0 in Branch 2, there is no explosive concentration.

Case 3 This case includes all sequences in which the class of accidents is internalenergetic [Branch 2, Question 1]. The outcome probability is calculated using FUN-SRATII and assigned to Branch 1 . The outcome probability for Branch 2 is 1.0 less the probability assigned Branch 1

Case 4 This case includes all sequences in which the class of accidents is external [Branch 1, Question 1]. The outcome probability is calculated using 
Case 5 .. The default case assumes that all sequences in which an explosive concentration can occur are included in Cases 3 and 4. An outcome probability of 1.0 is assigned Branch 2.

\section{Question 92: Does a deflagration/detonation occur in the Sludge Receipt and Adjustment Tank [SRAT]?}

If an explosive concentration occurs in the Sludge Receipt Adjustment Tank and an ignition source is available it is assumed that a damaging detonation or deflagration will follow. A detonation/deflagration in the Sludge Receipt Adjustment Tank can spill the contents of the vessel to the Chemical Process Cell; blow the cell covers, and damage adjacent vessels. There are three defined outcomes as follows:

SRAT@2Det: A damaging detonation occurs in the Sludge Receipt Adjustment Tank following a detonation or deflagration in another process vessel that provides an ignition source.

SRAT@2Def: A damaging deflagration occurs in the Sludge Receipt Adjustment Tank following a detonation or deflagration in another process vessel that provides an ignition source.

nSRAT-Ex: No damaging explosion occurs in the Sludge Receipt Adjustment Tank.

This is a Type 2 question. The answer is dependent on the outcome of previous Questions 66, 68,78 , and 91 . The answers to this question are used in the logic to determine the outcome probabilities in Questions $96,98,101,102$, and 132. Six cases are used to develop the outcome probabilities as follows:

Case 1 This case includes all sequences in which a previous detonation occurred in the Sludge Receipt Adjustment Tank. The outcome probability assigned is 1.0 in Branch 3, there is no explosion.

Case 2 This case includes all sequences in which a previous deflagration occurred in the Sludge Receipt Adjustment Tank. The outcome probability assigned is 1.0 in Branch 3, there is no explosion.

Case 3 This case includes all sequences in which an explosive concentration does not accumulate in the Sludge Receipt Adjustment Tank. The outcome probability assigned is 1.0 in Branch 3, there is no explosion.

Case $4 \quad$ This case includes the sequences in which a prior event has ruptured the Sludge Receipt Adjustment Tank precluding the accumulation of an explosive concentration. The outcome probability assigned is 1.0 in Branch 3, there is no explosion.

Case 5 This case includes the sequences in which a prior event has not occurred in the Sludge Receipt Adjustment Tank and an explosive concentration has accumulated in the vessel with a detonation in the PVVH (provides the 
.. ignition source). The outcome probability assigned is 0.001 in Branch 1, there is a damaging detonation and 0.999 in Branch 2, there is a damaging deflagration [7].

Case 6 . The default case includes any remaining sequences. It is assumed that all sequences resulting in a damaging explosion are included in previous cases. The outcome probability assigned is 1.0 in Branch 3 , there is no explosion.

\section{Question 93: Does an explosive concentration accumulate in the Melter Feed Tank [MFT]?}

The cut sets solved by the UFUN in this question have been modified to calculate the probability of an explosive concentration in the MFT without the presence of an ignition source. The two outcomes defined for this question are as follows:

ConMFT2: An explosive concentration accumulates in the Melter Feed Tank.

nConMFT: An explosive concentration does not accumulate in the Melter Feed Tank.

This is a Type 6 question and uses previously quantified parameters $[60,66,67,70,71,90$, 95 , and 108] and a user defined function to determine the probability that an explosive concentration will occur in the Melter Feed Tank. The function used to calculate the answer depends on the answer to previous Question 1. The answers to this question are used in the logic to determine the outcome probabilities in Questions 94 and 133.

The case structure is simplified by not considering the possibility of any prior events with the Melter Feed Tank which would prevent the buildup of an explosive concentration in the Melter Feed Tank. The possibility of prior events with the Melter Feed Tank is included in Question 94. Five cases are used to develop the outcome probabilities as follows:

Case 1 This case is used to exclude those sequences in the classes of accidents [Question 1] which are neither external nor internal-energetic. The outcome probability assigned is 1.0 in Branch 2 , there is no explosive concentration.

Case 2 This case includes all sequences in which the class of accidents is internalenergetic and the bulk nitrogen tank has failed as an initiator. Since the CPC nitrogen is a backup system, failure of the system supply as an initiator cannot lead to an explosive concentration in the MFT. The outcome probability assigned in 1.0 in Branch 2 , there is no explosive concentration.

Case 3 This case includes all sequences in which the class of accidents is internalenergetic [Branch 2, Question 1]. The outcome probability is calculated using FUN-MFTIII and assigned to Branch 1. The outcome probability for Branch 2 is 1.0 less the probability assigned Branch 1

Case 4 This case includes all sequences in which the class of accidents is external [Branch 1, Question 1]. The outcome probability is calculated using 


\section{.. FUN-MFTSI and assigned to Branch 1 . The outcome probability for} Branch 2 is 1.0 less the probability assigned Branch 1

Case $5^{\cdots} \quad$ The default case assumes that all sequences in which an explosive concentration can occur are included in Cases 3 and 4. An outcome probability of 1.0 is assigned Branch 2.

\section{Question 94:- Does a deflagration/detonation occur in the Melter Feed Tank [MFT]?}

If an explosive concentration occurs in the Melter Feed Tank and an ignition source is available it is assumed that a damaging detonation or deflagration will follow. A detonation/deflagration in the Melter Feed Tank can spill the contents of the vessel to the Chemical Process Cell. blow the cell covers, and damage adjacent vessels. There are three defined outcomes as follows:

MFT@2Det: A damaging detonation occurs in the Melter Feed Tank following a detonation or deflagration in another process vessel that provides an ignition source.

MFT@2Def: A damaging deflagration occurs in the Melter Feed Tank following a detonation or deflagration in another process vessel that provides an ignition source.

nMFT-Ex: No damaging explosion occurs in the Melter Feed Tank.

This is a Type 2 question. The answer is dependent on the outcome of previous Questions 72, 74,78 , and 93 . The answers to this question are used in the logic to determine the outcome probabilities in Questions $96,98,102,103$, and 133. Six cases are used to develop the outcome probabilities as follows:

Case 1 This case includes all sequences in which a previous detonation occurred in the Melter Feed Tank. The outcome probability assigned is 1.0 in Branch 3, there is no explosion.

Case 2 This case includes all sequences in which a previous deflagration occurred in the Melter Feed Tank. The outcome probability assigned is 1.0 in Branch 3, there is no explosion.

Case 3 This case includes all sequences in which an explosive concentration does not accumulate in the Melter Feed Tank. The outcome probability assigned is 1.0 in Branch 3, there is no explosion.

Case 4. This case includes the sequences in which a prior event has ruptured the Melter Feed Tank precluding the accumulation of an explosive concentration. The outcome probability assigned is 1.0 in Branch 3, there is no explosion.

Case 5 This case includes the sequences in which a prior event has not occurred in the Melter Feed Tank and an explosive concentration has accumulated in the vessel with a detonation in the PVVH (provides the ignition source). The outcome probability assigned is 0.001 in Branch 1 , there is 

sequences resulting in a damaging explosion are included in previous cases. The outcome probability assigned is 1.0 in Branch 3, there is no explosion.

\section{Question 95: Does a damaging explosion occur in the Salt Process Cell Vent System [SPCV]?}

Conditions in the Salt Process Cell Vent System [SPCV] are expected to be the same as the tanks exhausting into the header. Therefore an explosion in any of the SPC tanks will propagate into the header causing the header to also explode. Because of the size of the SPCV, it is expected to detonate (not deflagrate) which would produce shrapnel that could cause venting of cell tanks. There are two possible outcomes as follows:

SPCV-Exp2: A damaging explosion occurs in the Salt Process Cell Vent System following the second round of potential explosions.

nSPCV-Ex: A damaging explosion does not occur in the Salt Process Cell Vent System following the second round of potential explosions.

This is a Type 2 question. The answer is dependent on the outcome of previous Questions.37, $75,80,82,84$, and 86 . The answers to this question are not used in the remaining logic but are instead used in some of the output binners to designate that tank venting has occurred thus resulting in source term release.

Five cases are used to develop the outcome probabilities as follows:

Case 1 This case includes all sequences in which the SPC cell covers have fallen due to a seismic event. If the cell covers fall, the SPCV piping is assumed to be damged to the point that no accumulation of explosive mixtures is possible. An outcome probability of 1.0 is assigned to Branch 2.

Case 2

This case includes all sequences in which the SPCV has previously exploded. A previous explosion is assumed to damage the piping to the point that no accumulation of explosive mixtures is possible. An outcome probability of 1.0 is assigned to Branch 2 .

Case 3 .This case includes all sequences in which one of the SPC vessels has detonated in the second round. A detonation in any SPC vessel is assumed to cause the SPCV to explode. An outcome probability of 1.0 is assigned Branch 1.

Case $4 \quad$ This case includes all sequences in which one of the SPC vessels has deflagrated in the second round. A deflagration in any SPC vessel is assumed to cause the SPCV to explode. An outcome probability of 1.0 is assigned Branch 1. 
Page 122

Case 5. - The default case includes all sequences remaining and assumes that there is no other mechanism for an explosion in the SPCV. An outcome probability of 1.0 is assigned Branch 2.

Question 96: Does a damaging explosion occur in the Chemical Process Cell Vent System [CPCV]?

Conditions in the Chemical Process Cell Vent System [CPCV] are expected to be the same as the tanks exhausting into the header. Therefore an explosion in any of the CPC tanks will propagate into the header causing the header to also explode. Because of the size of the CPCV, it is expected to detonate (not deflagrate) which would produce shrapnel that could cause venting of cell tanks. There are two possible outcomes as follows:

CPCV-Exp2: A damaging explosion occurs in the Chemical Process Cell Vent System following the second round of potential explosions.

nCPCV-Ex: No damaging explosion occurs in the Chemical Process Cell Vent System following the second round of potential explosions.

This is a Type 2 question. The answer is dependent on the outcome of previous Question 56, $76,88,90,92$, and 94 . The answers to this question are not used in the remaining logic but are instead used in some of the output binners to designate that tank venting has occurred thus resulting in release of radioactive material. Five cases are used to develop the outcome probabilities as follows:

Case 1 This case includes all sequences in which the CPC cell covers have fallen due to a seismic event. If the cell covers fall, the CPCV piping is assumed to be damged to the point that no accumulation of explosive mixtures is possible. An outcome probability of 1.0 is assigned to Branch 2.

Case 2 This case includes all sequences in which the CPCV has previously exploded. A previous explosion is assumed to damage the piping to the point that no accumulation of explosive mixtures is possible. An outcome probability of 1.0 is assigned to Branch 2.

Case 3 This case includes all sequences in which one of the CPC vessels has detonated in the second round. A detonation in any CPC vessel is assumed to cause the CPCV to explode. An outcome probability of 1.0 is assigned Branch 1.

Case 4 This case includes all sequences in which one of the CPC vessels has deflagrated in the second round. A deflagration in any CPC vessel is assumed to cause the CPCV to explode. An outcome probability of 1.0 is assigned Branch 1.

Case 5 The default case includes all sequences remaining and assumes that there is no other mechanism for an explosion in the CPCV. An outcome probability of 1.0 is assigned Branch 2. 
Question 97: Does an energetic event in the SPC fail the removable wall -. between the SPC and the CPC?

The Salt Process Cell and the Chemical Process Cell are separated by a removable [from above] partition. Some events in the SPC are assumed to be sufficiently energetic to displace the partition from its supports, possibly causing damage to the tanks, piping, etc. in the CPC. There are two defined outcomes as follows:

FailWall: The wall separating the Salt Process Cell and the Chemical Process Cell is failed.

nFailWal: The wall separating the Salt Process Cell and the Chemical Process Cell is not failed.

This is a Type 2 question. The answers to this question are dependent on the outcomes of Questions 55 and 86 and are used in the logic to determine the outcome probabilities for Questions 99, 100,101,102,103,135, and 140. Two cases are used to develop the outcome probabilities as follows:

Case 1 This case includes all sequences in which a detonation has occurred in the Precipitate Reactor Feed Tank which is adjacent to the partition. An outcome probability of 0.5 is assigned to Branch 1, FailWall and an outcome probability of 0.5 is assigned to Branch 2, $\mathrm{nFailWal} \mathrm{[7].}$

Case 2 The default case includes all sequences remaining. It is expected that all . sequences in which the wall could be failed are included in previous cases. An outcome probability of 1.0 is assigned Branch $2, n$ FailWal.

Question 98: Do the cell covers for the Chemical Process Cell dislodge and fall following an energetic event?

The cell covers in the CPC can be dislodged and fall as a result of an energetic event in the $\mathrm{CPC}$. The falling cell covers can damage equipment and piping in the cell. There are two defined outcomes as follows:

CPCovfl: The cell covers in the Chemical Process Cell dislodge and fall due to an in-cell energetic event.

nCPCovFl: $\quad$ The cell covers in the Chemical Process Cell are in place.

This is a Type 2 question. The answer is dependent on the outcome of previous Questions 56, $60,64,68,74,78,88,90,92$, and 94 . The answers to this question are used in the logic to determine the outcome probabilities in Questions 99, 100,101,102,103,134, and 141. Five cases are used to develop the outcome probabilities as follows:

Case 1 This case includes all sequences in which no energetic event has occurred in the CPC and a seismic event has not dislodged the cell covers. An outcome probability of 1.0 is assigned Branch 2 , the cell covers are not dislodged due to an energetic event. 
Case 2 _. This case includes all sequences in which a seismic event has caused the cell covers to be dislodged. An outcome probability of 1.0 is assigned Branch 2, the cell covers are not dislodged due to an energetic event.

Case 3 This case includes all sequences in which a detonation or deflagration has occurred in a CPC vessel or piping during the first round of energetic events. An outcome probability of 1.0 is assigned Branch 1 , the cell covers are dislodged due to an energetic event.

Case 4

This case includes all sequences in which a detonation or deflagration has occurred in a CPC vessel or piping during the second round of energetic events. An outcome probability of 1.0 is assigned Branch 1, the cell covers are dislodged due to an energetic event.

Case 5

The default case includes all sequences remaining and assumes that all means by which the cell covers can fall have been included in the previous cases. An outcome probability of 1.0 is assigned Branch 2.

\section{Question 99: Does splashing [spillage] of the Precipitate Reactor Bottoms Tank contents occur?}

If the Precipitate Reactor Bottoms Tank [PRBT] is damaged some or all of the contents of the tank may be spilled to the floor. Damage to the PRBT can be caused by falling cell covers, by shaking during a Seismic event, by a crane load drop accident, by an explosion associated with the PRBT, or by damage from a failed SPC/CPC removable wall. The PRBT is also located next to the Slurry Mix Evaporator [SME]. A detonation or deflagration in the SME may generate shrapnel which can damage the PRBT. Spilling the contents of the PRBT is of concern because the contents are radioactive. There are two defined outcomes as follows:

PRBT-Spl: $\quad$ The contents of the Precipitate Reactor Bottoms Tank are spilled into the Chemical Process Cell.

nPRBT-Spl: The contents of the Precipitate Reactor Bottoms Tank are not spilled into the Chemical Process Cell.

This is a Type 2 question. The answers to this question are dependent on the outcome of previous Questions 58,60,64,88,90, and 98 and are used in the logic to determine the outcome probabilities in Questions 130 and 142. Eight cases are used to develop the outcome probabilities as follows (The outcome probabilities are from Reference 7):

Case 1 This case includes all sequences in which an energetic event that could cause possible damage to the PRBT has not occurred. Spillage of the tank contents will not occur. An outcome probability of 1.0 is assigned Branch 2.

Case 2 This case includes all sequences in which the PRBT has been ruptured due to a seismic event or a crane load drop accident. Spillage of the tank contents will occur. An outcome probability of 1.0 is assigned Branch 1. 
Case 3 .. This case includes all sequences in which there is a detonation in the Precipitate Reactor Bottoms Tank. Spillage of the tank contents will occur. An outcome probability of 1.0 is assigned Branch 1.

Case 4

This case includes all sequences in which the removable wall between the CPC and the SPC has failed. Spillage of the tank contents is possible. An outcome probability of 0.5 is assigned Branch 1 and Branch 2 . **SAMPLED VARIABLE**

Case 5 This case includes all sequences in which the cell covers are displaced and fall and there is a detonation of the Slurry Mix Evaporator, which is adjacent to the PRBT. The falling cell covers or shrapnel from the detonating SME can penetrate the PRBT causing spillage. An outcome probability of 0.5 is assigned Branch 1 and Branch 2 . **SAMPLED VARIABLE**

Case 6 This case includes all sequences in which the cell covers are displaced and fall and there is a deflagration of the Slurry Mix Evaporator, which is adjacent to the PRBT. The falling cell covers or shrapnel from the vent piping above the deflagrating SME can penetrate the PRBT causing spillage. An outcome probability of 0.2 is assigned Branch 1 and an outcome probability of 0.8 is assigned Branch 2 .

**SAMPLED VARIABLE**

Case 7 This case includes all sequences in which the cell covers are displaced and fall. An outcome probability of 0.1 is assigned Branch 1 and an outcome probability of 0.9 is assigned Branch $2 . * *$ SAMPLED VARIABLE**

Case 8 The default case includes all sequences remaining and assumes that all means by which spillage of the contents of the Precipitate Reactor Bottoms Tank can occur have been included in the previous cases. An outcome probability of 1.0 is assigned Branch 2 .

\section{Question 100: Does splashing [spillage] of the Slurry Mix Evaporator Tank contents occur?}

If the Slurry Mix Evaporator [SME] is damaged some or all of the contents of the tank may be spilled to the floor. Damage to the SME can be caused by falling cell covers, by shaking during a Seismic event, by a crane load drop accident, by an explosion associated with the SME, or by damage from a failed SPC/CPC removable wall. The SME is also located next to the PRBT. An energetic event in the PRBT may generate shrapnel and cause damage to the SME. Spilling the contents of the SME is of concern because the contents are radioactive. There are two defined outcomes as follows:

SME-Spl: $\quad$ The contents of the Slurry Mix Evaporator are spilled into the Chemical Process Cell.

nSME-Spl: The contents of the Slurry Mix Evaporator are not spilled into the Chemical Process Cell. 
This is a Type 2 question. The answers to this question are dependent on the outcome of previous Questions 60,62,64,88,97, and 98 and are used in the logic to determine the outcome probabilities in Questions 131 and 143. Eight cases are used to develop the outcome -probabilities as follows (The outcome probabilities are from Reference 7):

Case 1

This case includes all sequences in which an energetic event that could cause possible damage to the SME has not occurred. Spillage of the tank contents will not occur. An outcome probability of 1.0 is assigned Branch 2.

Case 2

This case includes all sequences in which there was a seismic initiated rupture of the SME or the SME is ruptured due to a crane load drop accident. An outcome probability of 1.0 is assigned Branch 1.

Case 3 : $\quad$ This case includes all sequences in which there is a detonation of the Slurry Mix Evaporator. Spillage of the tank contents will occur. An outcome probability of 1.0 is assigned Branch 1 .

Case 4 This case includes all sequences in which the cell covers are displaced and fall and a detonation occurs in the Precipitate Reactor Bottoms Tank, which is adjacent to the SME. The falling cell covers or shrapnel from the exploding PRBT can penetrate the SME causing spillage. An outcome probability of 0.5 is assigned Branch 1 and Branch 2 . **SAMPLED VARIABLE**.

Case 5 This case includes all sequences in which the cell covers are displaced and fall and a deflagration occurs in the Precipitate Reactor Bottoms Tank, which is adjacent to the SME. The falling cell covers or shrapnel from the vent piping above the deflagrating PRBT can penetrate the SME causing spillage. An outcome probability of 0.2 is assigned Branch 1 and an outcome probability of 0.8 is assigned Branch 2 .

**SAMPLED VARTABLE**

Case 6 This case includes all sequences in which the wall separating the SPC and the CPC is displaced into the CPC. The SME is separated from this wall by the PRBT. An outcome probability of 0.1 is assigned Branch 1 and an outcome probability of 0.9 is assigned Branch 2 . **SAMPLED VARIABLE**

Case 7 This case includes all sequences in which the cell covers are displaced and fall. The falling cell covers can damage the SME. An outcome probability of 0.1 is assigned Branch 1 and an outcome probability of 0.9 is assigned Branch 2. **SAMPLED VARIABLE**

Case 8 The default case includes all sequences remaining and assumes that all means by which spillage of the contents of the Slurry Mix Evaporator can occur have been included in the previous cases. An outcome probability of 1.0 is assigned Branch 2. 


\section{Question-101: Does splashing [spillage] of the Sludge Receipt and Adjustment Tank contents occur?}

If the Sludge Receipt Adjustment Tank [SRAT] is damaged some or all of the contents of the tank may be spilled to the floor. Damage to the SRAT can be caused by falling cell covers, by shaking during a Seismic event, by a crane load drop accident, by an explosion associated with the SRAT, or by damage from a failed SPC/CPC removable wall. Spilling the contents of the SRAT is of concern because the contents are radioactive. There are two defined outcomes as follows:

SRAT-Spl: The contents of the SRAT are spilled into the Chemical Process Cell.

nSRAT-Spl: The contents of the SRAT are not spilled into the Chemical Process Cell.

This is a Type 2 question. The answers to this question are dependent on the outcome of previous Questions 66, 68, 92,97, and 98 and are used in the logic to determine the outcome probabilities in Questions 132 and 144. Six cases are used to develop the outcome probabilities as follows (The outcome probabilities are from Reference 7):

Case 1 This case includes all sequences in which an energetic event that could cause possible damage to the SRAT has not occurred. Spillage of the tank contents will not occur. An outcome probability of 1.0 is assigned Branch 2.

Case 2 This case includes all sequences in which there was a seismic initiated rupture of the SRAT or the SRAT is ruptured due to a crane load drop accident. An outcome probability of 1.0 is assigned Branch 1.

Case 3 This case includes all sequences in which there is a detonation in the SRAT. Spillage of the tank contents will occur. An outcome probability of 1.0 is assigned Branch 1.

Case 4 This case includes all sequences in which the cell covers are displaced and fall. An outcome probability of 0.1 is assigned Branch 1 and an outcome probability of 0.9 is assigned Branch 2. **SAMPLED VARIABLE**

Case 5 This case includes all sequences in which the wall separating the SPC and the CPC is displaced into the CPC. The SRAT is separated from this wall by three other tanks. An outcome probability of 0.1 is assigned Branch 1 and an outcome probability of 0.9 is assigned Branch 2.

$$
\text { **SAMPLED VARIABLE** }
$$

Case 6 The default case includes all sequences remaining and assumes that all means by which spillage of the contents of the SRAT can occur have been included in the previous cases. An outcome probability of 1.0 is assigned Branch 2. 
April 7, 1995

WSRC-TR-95-0113

Page 128

\section{Question 102: Does splashing [spillage] of the Recycle Collection Tank contents occur?}

If the Recycle Collection Tank [RCT] is damaged some or all of the contents of the tank may be spilled to the floor. Damage to the RCT can be caused by falling cell covers, by shaking during a Seismic event, by a crane load drop accident and by explosion driven shrapnel from adjacent tanks. Spilling the contents of the RCT is of concern because the contents are radioactive. There are two defined outcomes as follows:

RCT-Spl: The contents of the RCT are spilled into the Chemical Process Cell.

nRCT-Spl: The contents of the RCT are not spilled into the Chemical Process Cell.

This is a Type 2 question. The answers to this question are dependent on the outcome of previous Questions 68, 70,74, 92,94, 97, and 98 and are used in the logic to determine the outcome probabilities in Question 145. 'Seven cases are used to develop the outcome probabilities as follows (The outcome probabilities are from Reference 7):

Case 1 This case includes all sequences in which an energetic event that could cause possible damage to the RCT has not occurred. Spillage of the tank contents will not occur. An outcome probability of 1.0 is assigned Branch 2.

Case 2 This case includes all sequences in which there was a seismic initiated rupture of the RCT or the RCT is damaged due to a crane load drop accident. An outcome probability of 1.0 is assigned Branch 1.

Case 3 This case includes all sequences in which the cell covers are displaced and fall and there is a detonation in the MFT or the SRAT, which are adjacent - to the RCT. The falling cell covers or shrapnel from the exploding MFT or SRAT can penetrate the RCT causing spillage. An ourcome probability of 0.5 is assigned Branch 1 and Branch 2 .

Case 4 This case includes all sequences in which the cell covers a isplaced anc fall and there is a deflagration in the MFT or the SE. adjacent to the RCT. The falling cell covers or shrapnei from the vent piping above the deflagrating MFT or SRAT can penetrate the RCT causing spillage. An outcome probability of 0.2 is assigned Branch 1 and an outcome probability of 0.8 is assigned Branch 2 . **SAMPLED VARIABLE**

Case 5 This case includes all sequences in which the cell covers are displaced and fall. The falling cell covers can damage the RCT. An outcome probability of 0.1 is assigned Branch 1 and an outcome probability of 0.9 is assigned Branch 2.

**SAMPLED VARIABLE**

Case 6. This case includes all sequences in which the wall separating the SPC and the CPC is displaced into the CPC. The RCT is separated from this wall by four other tanks. An outcome probability of 0.1 is assigned Branch 1 and an outcome probability of 0.9 is assigned Branch 2 .

**SAMPLED VARIABLE** 
Case $7^{-} \quad$ The default case includes all sequences remaining and assumes that all means by which spillage of the contents of the RCT can occur have been included in the previous cases. An outcome probability of 1.0 is assigned Branch 2.

\section{Question 103: Does splashing [spillage] of the Melter Feed Tank contents occur?}

If the Melter Feed Tank [MFT] is damaged some or all of the contents of the tank may be spilled to the floor. Damage to the MFT can be caused by falling cell covers, by shaking during a Seismic event, by a crane load drop accident, by an explosion associated with the MFT, or by damage from a failed SPC/CPC removable wall. Spilling the contents of the MFT is of concern because the contents are radioactive. There are two defined outcomes as follows:

MFT-Spl: The contents of the MFT are spilled into the Chemical Process Cell.

nMFT-Spl: The contents of the MFT are not spilled into the Chemical Process Cell.

This is a Type 2 question. The answers to this question are dependent on the outcome of previous Questions 72, 74, 94,97, and 98 and are used in the logic to determine the outcome probabilities in Questions 133 and 146. Six cases are used to develop the outcome probabilities as follows (The outcome probabilities are from Reference 7):

Case 1 This case includes all sequences in which an energetic event that could cause possible damage to the MFT has not occurred. Spillage of the tank contents will not occur. An outcome probability of 1.0 is assigned Branch 2.

Case 2 This case includes all sequences in which there was a seismic initiated rupture of the MFT or a rupture due to a crane drop accident. An outcome probability of 1.0 is assigned Branch 1.

Case 3 This case includes all sequences in which there is a detonation in the MFT. Spillage of the tank contents will occur. An outcome probability of 1.0 is assigned Branch 1.

Case $4 \quad$ This case includes all sequences in which the cell covers are displaced and fall. An outcome probability of 0.1 is assigned Branch 1 and an outcome probability of 0.9 is assigned Branch 2. **SAMPLED VARIABLE**

Case 5 This case includes all sequences in which the wall separating the SPC and the CPC is displaced into the CPC. The MFT is separated from this wall by five other tanks. An outcome probability of 0.1 is assigned Branch 1 and an outcome probability of 0.9 is assigned Branch 2. **SAMPLED VARIABLE**'

Case 6 The default case includes all sequences remaining and assumes that all means by which spillage of the contents of the MFT can occur have been included in the previous cases. An outcome probability of 1.0 is assigned Branch 2. 
Question 104: Does an energetic event in the CPC fail the removable wall between the SPC and the CPC?

The Salt Process Cell and the Chemical Process Cell are separated by a removable [from above] partition. Some events in the CPC are assumed to be sufficiently energetic to displace the partition from its supports, possibly causing damage to the tanks, piping, etc. in the SPC. There are-two defined outcomes as follows:

FailWall: The wall separating the Salt Process Cell and the Chemical Process Cell is failed.

nFailWal: The wall separating the Salt Process Cell and the Chemical Process Cell is not failed.

This is a Type 2 question. The answers to this question are dependent on the outcomes of Questions 60 and 88 and are used in the logic to determine the outcome probabilities for Questions 106, 107, 108,109,135, and 140. Two cases are used to develop the outcome probabilities as follows:

Case 1 This case includes all sequences in which a detonation has occurred in the PRBT which is adjacent to the partition. An outcome probability of 0.5 is assigned to Branch 1 and an outcome probability of 0.5 is assigned to Branch 2 [7].

Case 2 The default case includes all sequences remaining. It is expected that all sequences in which the wall could be failed are included in previous cases. An outcome probability of 1.0 is assigned Branch 2.

Question 105: Do the cell covers for the Salt Process Cell dislodge and fall following an energetic event?

The cell covers in the SPC can be dislodged and fall as a result of an energetic event in the SPC. The falling cell covers can damage equipment and piping in the cell. There are two defined outcomes as follows:

SPCovFl: The cell covers dislodge and fall due to an in-cell energetic event.

nSPCovFl: The cell covers do not fall as a result of an energetic event.

This is a Type 2 question. The answers to this question are dependent on the outcome of previous Questions 37, 43, 47,51,55,80,82;84, and 86 and are used in the logic to determine the outcome probabilities in Questions 106, 107, 108,109,116, 127, 128,129, $131,132,133$, and 134. Five cases are used to develop the outcome probabilities as follows:

Case 1 This case includes all sequences in which there were no energetic events in the SPC and the cell covers were not dislodged previously by a seismic event. An outcome probability of 1.0 is assigned Branch 2. 
Case 2 .. This case includes all sequences in which the cell covers were dislodged previously by a seismic event. An outcome probability of 1.0 is assigned Branch 2.

Case 3

This case includes all sequences in which there was a detonation in one of the SPC vessels. An outcome probability of 1.0 is assigned Branch 1.

Cașe 4 This case includes all sequences in which there was a deflagration in one of the SPC vessels. An outcome probability of 1.0 is assigned Branch 1.

Case 5 The default case includes all sequences remaining and assumes that all means by which the cell covers can fall have been included in the previous cases. An outcome probability of 1.0 is assigned Branch 2.

\section{Question 106: Does splashing [spillage] of the Precipitate Reactor contents occur?}

If the Precipitate Reactor is damaged some or all of the contents of the tank may be spilled to the floor. Damage to the PR can be caused by falling cell covers, by shaking during a Seismic event, by a crane load drop accident, by an explosion associated with the PR, or by damage from a failed SPC/CPC removable wall. The PR is located next to the OEV. An energetic event in the OEV may generate shrapnel and cause damage to the PR. Spilling the contents of the Precipitate Reactor is of concern because the contents are radioactive. There are two defined outcomes as follows:

PRx-Spl: . The contents of the Precipitate Reactor are spilled into the Salt Process Cell.

nPRx-Spl: The contents of the Precipitate Reactor are not spilled into the Salt Process Cell.

This is a Type 2 question. The answers to this question are dependent on the outcome of previous Questions 41, 43, 47, 80,82,104, and 105 and are used in the logic to determine the outcome probabilities in Questions 115, 117,123, and 136. Eight cases are used to develop the outcome probabilities as follows (The outcome probabilities are from Reference 7):

Case 1 This case includes all sequences in which there was no explosion of the PR or the OEV, the cell covers were not dislodged previously by an energetic event or seismic event; the PR was not damaged by a crane load drop accident, and the PR was not ruptured by a seismic event. An outcome probability of 1.0 is assigned Branch 2 .

Case 2 This case includes all sequences in which there was a rupture of the Precipitate Reactor due to a seismic cell cover strike, seismic damage to the PR, or a crane load drop accident. An outcome probability of 1.0 is assigned Branch 1.

Case 3 This case includes all sequences in which there was a detonation of the Precipitate Reactor. An outcome probability of 1.0 is assigned Branch 1. 
Case $4 \quad$-. This case includes all sequences in which the cell covers are displaced and fall and there is a detonation in the OEV, which is adjacent to the PR. The falling cell covers or shrapnel from the exploding OEV can penetrate the PR causing spillage. An outcome probability of 0.5 is assigned Branch 1 and Branch 2.

** SAMPLED VARIABLE**

Case 5. This case includes all sequences in which the cell covers are displaced and fall and there is a deflagration in the OEV which is adjacent to the PR. The falling cell covers or shrapnel from the vent piping above the deflagrating OEV can penetrate the PR causing spillage. An outcome probability of 0.2 is assigned Branch 1 and an outcome probability of 0.8 is assigned Branch 2.

\section{**SAMPLED VARIABLE**}

Case 6 This case includes all sequences in which the cell covers are dislodged and fall. An outcome probability of 0.1 is assigned Branch 1 and an outcome probability of 0.9 is assigned Branch 2 . **SAMPLED VARIABLE**

Case 7 This case includes all sequences in which the wall separating the SPC and the CPC is displaced into the SPC. The PR is separated from this wall by three other tanks. An outcome probability of 0.1 is assigned Branch 1 and an outcome probability of 0.9 is assigned Branch 2.

**SAMPLED VARIABLE***

Case 8 The default case includes all sequences remaining and assumes that all means by which the PR can. be damaged have been included in the previous cases. An outcome probability of 1.0 is assigned Branch 2.

\section{Question 107: Does splashing [spillage] of the contents of the Organic Evaporator tank occur?}

If the OEV is damaged some or all of the contents of the tank may be spilled to the floor. Damage to the OEV can be caused by falling cell covers, by shaking during a seismic event, by a crane load drop accident, by an explosion associated with the OEV, or by damage from a failed SPC/CPC removable wall. The OEV is located between the PR and OECT. An energetic event in the PR or OECT may generate shrapnel and cause damage to the OEV. Spilling the contents of the Organic Evaporator is of concern primarily because part of the spilled benzene will evaporate and may lead to a deflagration in the Salt Process Cell. There are two defined outcomes as follows:

OEV-Spl: $\quad$ The contents of the OEV are spilled into the Salt Process Cell.

nOEV-Spl: . The contents of the OEV are not spilled into the Salt Process Cell.

This is a Type 2 question. The answers to this question are dependent on the outcome of previous Questions 43, 45, 47, 51, 80, 82, 104, and 105 and are used in the logic to determine the outcome probabilities in Questions 115, 118, 121, 122, 124, 137, and 147. Eight cases are used to develop the outcome probabilities as follows (The outcome probabilities are from Reference 7): 
Case 1 This case includes all sequences in which there was no explosion of the PR , OECT, or the OEV, the cell covers were not dislodged previously by an energetic event or seismic event, the OEV was not damaged by a crane load drop accident, and the OEV was not ruptured by a seismic event. An outcome probability of 1.0 is assigned Branch 2 .

Case 2 This case includes all sequences in which there was a rupture of the OEV due to a seismic cell cover strike, seismic damage to the OEV, or a crane load drop accident. An outcome probability of 1.0 is assigned Branch 1.

Case 3 This case includes all sequences in which there was a detonation of the OEV. An outcome probability of 1.0 is assigned Branch 1.

Case $4 \quad$ This case includes all sequences in which the cell covers are displaced and fall and there is a detonation in the PR or OECT which are adjacent to the OEV. The falling cell covers or shrapnel from the exploding PR or OECT can penetrate the OEV causing spillage. An outcome probability of 0.5 is assigned Branch 1 and Branch 2. ** SAMPLED VARIABLE**

Case 5 This case includes all sequences in which the cell covers are displaced and fall and there is a deflagration in the PR or OECT which are adjacent to the OEV. The falling cell covers or shrapnel from the vent piping above the deflagrating PR or OECT can penetrate the OEV causing spillage. An outcome probability of 0.2 is assigned Branch 1 and an outcome probability of 0.8 is assigned Branch 2 . **SAMPLED VARIABLE**

Case 6 This case includes all sequences in which the cell covers are dislodged and fall. An outcome probability of 0.1 is assigned Branch 1 and an outcome probability of 0.9 is assigned Branch 2. **SAMPLED VARIABLE**

Case 7 This case includes all sequences in which the wall separating the SPC and the CPC is displaced into the SPC. The OEV is separated from this wall by two other tanks. An outcome probability of 0.1 is assigned Branch 1 and an outcome probability of 0.9 is assigned Branch 2. **SAMPLED VARIABLE**.

Case 8 The default case includes all sequences remaining and assumes that all means by which the OEV can be damaged have been included in the previous cases. An outcome probability of 1.0 is assigned Branch 2.

\section{Question 108: Does splashing [spillage] of the contents of the Organic Evaporator Condensate tank occur?}

If the OECT is damaged some or all of the contents of the tank may be spilled to the floor. Damage to the OECT can be caused by falling cell covers, by shaking during a seismic event, by a crane load drop accident, by an explosion associated with the OECT, or by damage from a failed SPC/CPC removable wall. The OECT is located between the PRFT and OEV. An energetic event in the PRFT or OEV may generate shrapnel and cause damage to the OECT. Spilling the contents of the OECT is of concern primarily because part of the spilled benzene 
will evaporate and may lead to a deflagration in the Salt Process Cell. There are two defined outcomes as follows:

OECT-Spi: The contents of the OECT are spilled into the Salt Process Cell.

nOECT-Spl: The contents of the OECT are not spilled into the Salt Process Cell.

This is a. Type 2 question. The answers to this question are dependent on the outcome of previous Questions 47, 49, 51, 55, 82, 84, 86, 104, and 105. and are used in the logic to determine the outcome probabilities in Questions 115, 119, 121, 122, 125, 138, and 147. Eight cases are used to develop the outcome probabilities as follows (The outcome probabilities are from Reference 7):

Case 1 This case includes all sequences in which there was no explosion of the PRFT, OECT, or the OEV, the cell covers were not dislodged previously by an energetic event or seismic event, the OECT was not damaged by a crane load drop accident, and the OECT was not ruptured by a seismic event. An outcome probability of 1.0 is assigned Branch 2.

Case 2 This case includes all sequences in which there was a rupture of the OECT due to a seismic cell cover strike, seismic damage to the OECT, or a crane load drop accident. An outcome probability. of 1.0 is assigned Branch 1.

Case 3 This case includes all sequences in which there was a detonation of the OECT. An outcome probability of 1.0 is assigned Branch 1.

Case 4 This case includes all sequences in which the cell covers are displaced and fall and there is a detonation in the PRFT or OEV which are adjacent to the OECT. The falling cell covers or shrapnel from the exploding PRFT or OEV can penetrate the OECT causing spillage. An outcome probability of 0.5 is assigned Branch 1 and Branch 2.

** SAMPLED VARIABLE**

Case 5. This case includes all sequences in which the cell covers are displaced and fall and there is a deflagration in the PRFT or OEV which are adjacent to the OECT. The falling cell covers or shrapnel from the vent piping above the deflagrating PRFT or OEV can penetrate the OECT causing spillage. An outcome probability of 0.2 is assigned Branch 1 and an outcome probability of $0: 8$ is assigned Branch 2 . **SAMPLED VARIABLE**

Case 6 This case includes all sequences in which the wall separating the SPC and the CPC is displaced into the SPC. The OECT is separated from this wall by one other tank: An outcome probability of 0.1 is assigned Branch 1 and an outcome probability of 0.9 is assigned Branch 2. **SAMPLED VARIABLE**

Case 7 This case includes all sequences in which the cell covers are dislodged and fall. An outcome probability of 0.1 is assigned Branch 1 and an outcome probability of 0.9 is assigned Branch 2. **SAMPLED VARIABLE** 
Case 8 The default case includes all sequences remaining and assumes that all means by which the OECT can be damaged have been included in the previous cases. An outcome probability of 1.0 is assigned Branch 2 .

\section{Question 109: Does splashing [spillage] of the contents of the Precipitate Reactor Feed Tank occur?}

If the PRFT is damaged some or all of the contents of the tank may be spilled to the floor. Damage to the PRFT can be caused by falling cell covers, by shaking during a seismic event, by a crane load drop accident, by an explosion associated with the PRFT, or by damage from a failed SPC/CPC removable wall. The PRFT is located adjacent to the OECT. An energetic event in the OECT may generate shrapnel and cause damage to the PRFT. Spilling the contents of the PRFT is of concern because the contents are radioactive. There are two defined outcomes as follows:

PRFT-Spl: The contents of the PRFT are spilled into the Salt Process Cell.

nPRFT-Spl: The contents of the PRFT are not spilled into the Salt Process Cell.

This is a Type 2 question. The answers to this question are dependent on the outcome of previous Questions 51, 53, 55, 84, 86, 104, and 105 and are used in the logic to determine the outcome probabilities in Questions 115, 120,126, and 139. Eight cases are used to develop the outcome probabilities as follows (The outcome probabilities are from Reference 7):

Case 1 This case includes all sequences in which there was no explosion of the PRFT or the OECT, the cell covers were not dislodged previously by an energetic event or seismic event, the PRFT was not damaged by a crane load drop accident, and the PRFT was not ruptured by a seismic event. An outcome probability of 1.0 is assigned Branch 2 .

Case 2 This case includes all sequences in which there was a rupture of the PRFT due to a seismic cell cover strike, seismic damage to the PRFT, or a crane load drop accident. An outcome probability of 1.0 is assigned Branch 1.

Case 3 This case includes all sequences in which there was a detonation of the PRFT. An outcome probability of 1.0 is assigned Branch 1.

Case 4 This case includes all sequences in which the wall separating the SPC and the CPC is displaced into the SPC. The PRFT is adjacent to this wall. An outcome probability of 0.5 is assigned Branch 1 and Branch 2. **SAMPLED VARIABLE**

Case 5 This case includes all sequences in which the cell covers are displaced and fall and there is a detonation in the OECT which is adjacent to the PRFT. The falling cell covers or shrapnel from the exploding OECT can penetrate the PRFT causing spillage. An outcome probability of 0.5 is assigned Branch 1 and Branch 2. ** SAMPLED VARIABLE**

Case 6 This case includes all sequences in which the cell covers are displaced and fall and there is a deflagration in the OECT which is adjacent to the PRFT. 
The falling cell covers or shrapnel from the vent piping above the deflagrating OECT can penetrate the PRFT causing spillage. An outcome probability of 0.2 is assigned Branch 1 and an outcome probability of 0.8 is assigned Branch 2 . **SAMPLED VARIABLE**

Case $7 \quad$ This case includes all sequences in which the cell covers are dislodged and fall. An outcome probability of 0.1 is assigned Branch 1 and an outcome probability of 0.9 is assigned Branch 2. **SAMPLED VARIABLE**

Case 8

The default case includes all sequences remaining and assumes that all means by which the PRFT can be damaged have been included in the previous cases. An outcome probability of 1.0 is assigned Branch 2 .

\section{Question 110: Does the DWPF Vitrification Building collapse following a seismic event?}

The DWPF Vitrification Building houses most of the equipment and process cells required for the solidification of the high level waste. Collapse of the Vitrification Building is extremely unlikely at the ground acceleration of the design basis earthquake. Such collapse, however, will result in complete bypass of the filter system for any released radionuclides and will damage equipment and fail processes within the building. There are two defined outcomes as follows:

VB-Clpse: The Vitrification Building collapses as the result of a seismic event.

nVB-Clps: The Vitrification Building does not collapse as the result of a seismic event.

This is a Type 6 question. The answers to this question are dependent only on Question 2 and are used in the logic to determine the outcome probabilities in Question 112 and 147.

Two cases are used to develop the outcome probabilities as follows:

Case 1 This case includes all sequences in which a seismic event is the initiating event [external class]. The fragility of the Canyon [CanyClps] is placed in Branch 1. The outcome probability for Branch 2 is 1.0 less the probability assigned Branch 1 .

Case 2. The default case includes all sequences remaining. An outcome probability of 1.0 is assigned Branch 2.

\section{Question 111: Does the Sand Filter function following a seismic event?}

A Sand Filter, separated from the Vitrification Building, is provided to filter the exhaust air and offgases from the Canyon. Proper operation of the filter is essential in preventing the release of radionuclides to the environment, particularly in the event of a process upset or accident that results in the release of radioactive material from the process vessels or piping. Damage to the Sand Filter could occur during a Seismic event. There are two defined outcomes as follows: 
fSndFIt: . The Sand Filter is failed as the result of a seismic event.

nfSndFlt: The Sand Filter is not failed as the result of a seismic event.

This is a Type 6 question. The answers to this question, which are dependent on the outcome of Question 2 are not used in the logic to determine the outcome probabilities for other questions but are used in the source term binning file. Two cases are used to develop the outcome probabilities as follows:

Case 1 This case includes all sequences in which a seismic event is the initiating event [external class]. The fragility of the Sand Filter [FiltFr] is placed in Branch 1. The outcome probability for Branch 2 is 1.0 less the probability assigned Branch 1 .

Case 2 The default case includes all sequences remaining. An outcome probability of 1.0 is assigned Branch 2 .

\section{Question 112: Do the Building Exhaust Ducts from Zone 1 to the Sand Filter function following a seismic event?}

The Zone 1 exhaust ducts collect offgas and ventilation air from the canyon process areas, process vessels and enclosures, and the melter and directs the exhaust to the Sand Filter. Collapse of the building during a Seismic event could cause collapse and blockage of the exhaust ducts or the ducts could collapse directly during a Seismic event. In either event exhaust flow to the Sand Filter is stopped. Any radionuclides released from primary confinement will leak from the building. There are two defined outcomes as follows:

failDuct: The exhaust ducts are failed as the result of a seismic event.

nfailDct: The exhaust ducts are not failed as the result of a seismic event.

This is a Type 6 question. The answers to this question, which are dependent on the outcomes of Questions 2 and 110, are not used in the logic to determine the outcome probabilities for other questions but are used in the source term binning file. Three cases are used to develop the outcome probabilities as follows:

Case 1 This case includes all sequences in which a seismic event is the initiating event [external class] and the Canyon has collapsed. If the building has collapsed then the exhaust ducts are assumed to collapse. The outcome probability for Branch 1 is 1.0 .

Case 2 This case includes all sequences in which a seismic event is the initiating event [extemal class] and the Canyon has not collapsed. The fragility of the exhaust ducts [DuctFr] is placed in Branch 1. The outcome probability for Branch 2 is 1.0 less the probability assigned Branch 1.

Case 3 The default case includes all sequences remaining. An outcome probability of 1.0 is assigned Branch 2. 


\section{Question 113: What is the condition of the Zone 1 Exhaust Fan System?}

The operation of the Zone 1 exhaust fans is essential during normal or off normal operation to [1] maintain a negative pressure within the Canyon and [2] transport any radionuclides released from primary confinement to the Sand Filter. Failure of the exhaust fans in conjunction with a failure of primary confinement would result in the release of radionuclides to the environment. There are two defined outcomes as follows:
Fan-Fail:
The Zone 1 exhaust fans are failed.
nFanFail:
The Zone 1 exhaust fans are not failed.

This is a Type 8 question and defines a parameter that is also used in calculations to determine the outcome probabilities of later Question 114. The parameter is as follows:

Arg [128] FailFan, a logical variable that indicates the state of the Zone 1 exhaust fan system. The parameter is assigned a value $\{+1.0\}$ if the system is failed and $\{0.0\}$ if the system is available.

The outcome probabilities for this question are dependent on the outcomes of Questions 1, 3, 8,9 , and 18 and are used in the logic to determine the outcome probabilities in Questions 121 and 122 and are used to determine the value assigned the above parameter. Twelve cases are used to develop the outcome probabilities on the basis of prior events as follows:

Case 1 This case includes all sequences in which the accident is an internalenergetic scenario and both the normal and the emergency power systems have failed. The fans will not operate without power and the outcome probability specified is 1.0 for Branch 1. The parameter, FailFan, is assigned a value of $\{+1.0\}$ when Branch 1 is selected as the outcome.

Case 2 This case includes all sequences in which the accident is either a limited leak, a crane load drop, or a limited miscellaneous scenario and both the normal and the emergency power systems have failed. The fans will not operate without power and the outcome probability specified is 1.0 for Branch 1. The parameter, Failfan, is assigned a value of $\{+1.0\}$ when Branch 1 is selected as the outcome.

Case 3 This case includes all sequences in which an internal-energetic event has occurred, normal electric power has failed, but emergency electrical power and the instrument air system are available. The outcome probability for Branch 1 is calculated as a function of FanLmda1 and FanTau1. The outcome probability for Branch 2 is 1.0 less the probability assigned Branch 1. The parameter, Failfan, is assigned a value of $\{+1.0\}$ when Branch 1 is selected as the outcome and a value of $\{0.0\}$ when Branch 2 is selected as the outcome.

Case 4 This case includes all sequences in which the accident is either a limited leak, a crane load drop, or a limited miscellaneous scenario and the normal power system has failed. The emergency power system and the instrument air system are still available. The outcome probability for Branch 1 is calculated as a function of FanLmda1 and FanTau1. The outcome probability for Branch 2 is 1.0 less the probability assigned 
Branch 1. The parameter, FailFan, is assigned a value of $\{+1.0\}$ when Branch 1 is selected as the outcome and a value of $\{0.0\}$ when Branch 2 is selected as the outcome.

Case 5

This case includes all sequences in which an internal-energetic event has occurred, normal electric power and the instrument air system have failed, but emergency electrical power is still available. The outcome probability for Branch 1 is calculated as a function of FanLmda2 and FanTau2. The outcome probability for Branch 2 is 1.0 less the probability assigned Branch 1. The parameter, FailFan, is assigned a value of $\{+1.0\}$ when Branch 1 is selected as the outcome and a value of $\{0.0\}$ when Branch 2 is selected as the outcome.

Case 6 This case includes all sequences in which the accident is either a limited leak, a crane load drop, or a limited miscellaneous scenario and the normal power system and the instrument air system have failed. The emergency power system is still available. The outcome probability for Branch 1 is calculated as a function of FanLmda2 and FanTau2. The outcome probability for Branch 2 is 1.0 less the probability assigned Branch 1 . The parameter, FailFan, is assigned a value of $\{+1.0\}$ when Branch 1 is selected as the outcome and a value of $\{0.0\}$. when Branch 2 is selected as the outcome.

Case 7 This case includes all sequences in which the accident is either an internalenergetic scenario, a limited leak, a crane load drop, or a limited miscellaneous scenario and the normal power system, the instrument air system, and the emergency power system are all available. The outcome probability for Branch 1 is calculated as a function of Fanlmda3 and FanTau3. The outcome probability for Branch 2 is 1.0 less the probability assigned Branch 1 . The parameter, FailFan, is assigned a value of $\{+1.0\}$ when Branch 1 is selected as the outcome and a value of $\{0.0\}$ when Branch 2 is selected as the outcome.

Case 8 This case includes all sequences in which an external event has occurred and both normal and emergency electric power have failed. The fans will not operate without power and the outcome probability specified is 1.0 for Branch 1. The parameter, Failfan, is assigned a value of $\{+1.0\}$ when Branch 1 is selected as the outcome.

Case 9 This case includes all sequences in which an external event has occurred and normal electric power has failed. Emergency electrical power and the instrument air system are available. The outcome probability for Branch 1 is calculated as a function of FanLmdal and FanTau1, FanFr, and FanRn1 and MsTmEx. The outcome probability for Branch 2 is 1.0 less the probability assigned Branch 1 . The parameter, FailFan, is assigned a value of $\{+1.0\}$ when Branch 1 is selected as the outcome and a value of $\{0.0\}$ when Branch 2 is selected as the outcome.

Case 10 This case includes all sequences in which an external event has occurred and normal electric power and the instrument air system have failed. Emergency, electrical power is available. The outcome probability for Branch 1 is calculated as a function of FanLmda2 and FanTau2, FanFr, 
and FanRn2 and MsTmEx. The outcome probability for Branch 2 is 1.0 less the probability assigned Branch 1. The parameter, FailFan, is assigned a value of $\{+1.0\}$ when Branch 1 is selected as the outcome and a value of $\{0.0\}$ when Branch 2 is selected as the outcome.

Case 11

This case includes all sequences in which an external event has occurred and the normal and emergency electrical power systems and the instrument air system are avaiable. The outcome probability for Branch 1 is calculated as a function of FanLmda3 and FanTau3, FanFr, and FanRn3 and MsTmEx. The outcome probability for Branch 2 is 1.0 less the probability assigned Branch 1 . The parameter, FailFan, is assigned a value of $\{+1.0\}$ when Branch 1 is selected as the outcome and a value of $\{0.0\}$ when Branch 2 is selected as the outcome.

Case 12. The default case includes all sequences remaining. An outcome probability of 1.0 is assigned Branch 2. The parameter, Failfan, is assigned a value of $\{0.0\}$ when branch 2 is selected as the outcome.

\section{Question 114: Does a deflagratable concentration occur in the SPC due to Vessel Overpressurization?}

As indicated previously benzene may be present in any or all of the tanks in the Salt Process Cell. Overpressurization of these vessels will cause release of benzene vapors into the cell which if coupled with a loss of ventilation flow (failure to sweep vapors from the cell) and an ignition source could cause a cell deflagration. A cell deflagration will raise the cell covers and could lead to damaged process equipment in the cell. A fault tree was constructed to calculate the frequency of this SPC overpressurization deflagration for all credible initiators [5]. The solution of this fault tree (cut sets) is used to determine the frequency (or probability) of a deflagratable concentration. There are two defined outcomes as follows:

SPCDefOP: A deflagratable concentration occurs in the SPC due to vessel overpressurization.

nSPCDefOP: A deflagratable concentration does not occur in the SPC due to vessel overpressurization.

This is a Type 6 question and uses previously quantified parameters $[60,70,71,90$, and 128] and a user defined function to determine the probability that a deflagratable concentration will occur in the SPC. The user function includes information from solution of the fault tree for the SPC overpressure deflagration. The function used to calculate the answer depends on the answer to previous Question 1. The answers to this question are used in the logic to determine the outcome probabilities in Question 115.

The case structure is simplified by not considering the possibility of prior rupture of items in the SPC that would prevent overpressurization. The possibility of prior rupture of SPC items assumed to prevent overpressurization are included in Question 115. Four cases are used to develop the outcome probabilities as follows:

Case 1 This case includes all sequences in which the accident is not an internalenergetic or external (seismic) scenario. A SPC overpressure deflagration 
cannot occur for any other accident type. An outcome probability of 1.0 is assigned Branch 2 .

Case 2 This case includes all sequences in which the class of accidents is internalenergetic [Branch 2, Question 1]. The outcome probability is calculated using FUN-SPC and assigned to Branch 1 . The outcome probability for Branch 2 is 1.0 less the probability assigned Branch 1

Case 3

This case includes all sequences in which the class of accidents is external [Branch 1, Question 1]. The outcome probability is calculated using FUN-SPCS and assigned to Branch 1. The outcome probability for Branch 2 is 1.0 less the probability assigned Branch 1

Case 4 The default case includes all sequences remaining. It is expected that all sequences in which a deflagratable concentration from vessel overpressurization could occur are included in previous cases. An outcome probability of 1.0 is assigned Branch 2.

\section{Question 115: Does a vessel overpressurization in the Salt Process Cell result in a deflagration in the Salt Process Cell?}

If a deflagratable concentration occurs in the SPC and an ignition source is available it is assumed that a damaging deflagration will follow. The fault tree for the SPC. overpressurization deflagration includes the probability of an ignition source. Therefore, if Question 114 indicates that an explosive concentration is available then a deflagration will follow. A deflagration in the SPC can blow the cell covers and damage SPC vessels. There are two defined outcomes as follows:

SPC-Def1: A damaging deflagration occurs in the SPC.

nSPCDef1: No damaging deflagration occurs in the SPC.

This is a Type 2 question. The answer is dependent on the outcome of previous Questions $106,107,108,109$, and 114. The answers to this question are used in the logic to determine the outcome probabilities in Question 116. Three cases are used to develop the outcome probabilities as follows:

Case 1 This case includes all sequences in which a vessel in the SPC has been previously damaged. An overpressurization deflagration cannot occur with any of the SPC vessels damaged. The outcome probability assigned is 1.0 in Branch 2.

Case 2 This case includes the sequences in which a deflagratable concentration has accumulated in the SPC. The outcome probability assigned is 1.0 in Branch 1.

Case 3 The default case includes any remaining sequences. It is assumed that all sequences resulting in a damaging deflagration due to overpressurization are included.in previous cases. The outcome probability assigned is 1.0 in Branch 2. 


\section{Question 116: Do the cell covers for the Salt Process Cell dislodge after the overpressurization deflagration?}

The cell covers in the SPC can be dislodged and fall as a result of an overpressure deflagration event in the SPC. The falling cell covers can damage equipment and piping in the cell. There are two defined outcomes as follows:

SPCvFi@2: The cell covers dislodge and fall due to an overpressure deflagration.

nSPCovFl: The cell covers do not fall as a result of an overpressure deflagration.

This is a Type 2 question. The answers to this question are dependent on the outcome of previous Questions 105 and 115 and are used in the logic to determine the outcome probabilities in Questions 117, 118, 119, 120,127, 128, 129,130,131, 132, 133, and 134. Three cases are used to develop the outcome probabilities as follows:

Case 1 This case includes all sequences in which the ce!: covers were dislodged previously. An outcome probability of 1.0 is assigned Branch 2 .

Case 2 This case includes all sequences in which there was an overpressurization deflagration in the SPC. An outcome probability of 1.0 is assigned Branch 1.

Case 3 The default case includes all sequences remaining and assumes that all means by which the cell covers can fall have been included in the previous cases. An outcome probability of 1.0 is assigned Branch 2.

\section{Question 117: Does splashing [spillage] of the Precipitate Reactor contents occur?}

If the Precipitate Reactor is damaged some or all of the contents of the tank may be spilled to the floor. This question is concerned only with damage to the PR caused by falling cell covers after a SPC overpressurization deflagration. There are two defined outcomes as follows:

PRx-Spl2: $\quad$ The contents of the Precipitate Reactor are spilled into the Salt Process Cell after an overpressurization deflagration.

nPRx-Spl2: The contents of the Precipitate Reactor are not spilled into the Salt Process Cell after an overpressurization deflagration.

This is a Type 2 question. The answers to this question are dependent on the outcome of previous Questions 106 and 116 and are used in the logic to determine the outcome probabilities in Questions 123 and 136. Three cases are used to develop the outcome probabilities as follows (The outcome probabilities are from Reference 7):

Case 1 This case includes all sequences in which the PR has been previously splashed. An outcome probability of 1.0 is assigned Branch 2 .

Case 2 This case includes all sequences in which the cell covers are displaced and fall as a result of an overpressurization deflagration. The falling cell 
covers can damage the PR causing spillage. An outcome probability of 0.1 is assigned Branch 1 and an outcome probability of 0.9 is assigned Branch 2. ** SAMPLED VARIABLE**

Case 3

The default case includes all sequences remaining and assumes that all means by which the PR can be damaged have been included in the previous cases. An outcome probability of 1.0 is assigned Branch 2.

\section{Question 118: Does splashing [spillage] of the contents of the Organic Evaporator tank occur?}

If the OEV is damaged some or all of the contents of the tank may be spilled to the floor. This question is concerned only with damage to the OEV caused by falling cell covers after a SPC overpressurization deflagration. Spilling the contents of the Organic Evaporator is of concern primarily because part of the spilled benzene will evaporate and may lead to a deflagration in the Salt Process Cell. There are two defined outcomes as follows:

OEV-Sp12: The contents of the OEV are spilled into the Salt Process Cell after an overpressurization deflagration.

nOEV-Sp12: The contents of the OEV are not spilled into the Salt Process Cell after an overpressurization deflagration.

This is a Type 2 question. The answers to this question are dependent on the outcome of previous Questions 107 and 116 and are used in the logic to determine the outcome probabilities in Questions 121,122,124,137, and 147. Three cases are used to develop the outcome probabilities as follows (The outcome probabilities are from Reference 7):

Case 1 This case includes all sequences in which the OEV has been previously splashed. An outcome probability of 1.0 is assigned Branch 2.

Case 2 This case includes all sequences in which the cell covers are displaced and fall as a result of an overpressurization deflagration. The falling. cell covers can damage the OEV causing spillage. An outcome probability of 0.1 is assigned Branch 1 and an outcome probability of 0.9 is assigned Branch 2. ** SAMPLED VARIABLE**

Case 3 The default case includes all sequences remaining and assumes that all means by which the OEV can be damaged have been included in the previous cases. An outcome probability of 1.0 is assigned Branch 2 .

\section{Question 119: Does splashing [spillage] of the contents of the Organic Evaporator Condensate tank occur?}

If the OECT is damaged some or all of the contents of the tank may be spilled to the floor. This question is concerned only with damage to the OECT caused by falling cell covers after a SPC overpressurization deflagration. Spilling the contents of the OECT is of concern primarily because part of the spilled benzene will evaporate and may lead to a deflagration in the Salt Process Cell. There are two defined outcomes as follows: 
OECT-Spl2: . The contents of the OECT are spilled into the Salt Process Cell after an overpressurization deflagration.

nOECT-Sp12: The contents of the OECT are not spilled into the Salt Process Cell after an overpressurization deflagration.

This is a Type 2 question. The answers to this question are dependent on the outcome of previous Questions 108 and 116 and are used in the logic to determine the outcome probabilities in Questions 121,122,125,138, and 147. Three cases are used to develop the outcome probabilities as follows (The outcome probabilities are from Reference 7):

Case 1 This case includes all sequences in which the OECT has been previously splashed. An outcome probability of 1.0 is assigned Branch 2.

Case 2 This case includes all sequences in which the cell covers are displaced and fall as a result of an overpressurization deflagration. The falling cell covers can damage the OECT causing spillage. An outcome probability of 0.1 is assigned Branch 1 and an outcome probability of 0.9 is assigned Branch 2. ** SAMPLED VARIABLE**

Case 3 The default case includes all sequences remaining and assumes that all means by which the OECT can be damaged have been included in the previous cases. An outcome probability of 1.0 is assigned Branch 2.

\section{Question 120: Does splashing [spillage] of the contents of the Precipitate Reactor Feed Tank occur?}

If the PRFT is damaged some or all of the contents of the tank may be spilled to the floor. This question is concerned only with damage to the OECT caused by falling cell covers after a SPC overpressurization deflagration. There are two defined outcomes as follows:

PRFT-Sp12: The contents of the PRFT are spilled into the Salt Process Cell after an overpressurization deflagration.

nPRFT-Spl: The contents of the PRFT are not spilled into the Salt Process Cell after an overpressurization deflagration.

This is a Type 2 question. The answers to this question are dependent on the outcome of previous Questions 109 and 116 and are used in the logic to determine the outcome probabilities in Questions 126 and 139. Three cases are used to develop the outcome probabilities as follows (The outcome probabilities are from Reference 7):

Case 1 This case includes all sequences in which the PRFT has been previously splashed. An outcome probability of 1.0 is assigned Branch 2 .

Case 2 This case includes all sequences in which the cell covers are displaced and fall as a result of an overpressurization deflagration. The falling cell covers can damage the PRFT causing spillage. An outcome probability of 0.1 is assigned Branch 1 and an outcome probability of 0.9 is assigned Branch 2. ** SAMPLED VARIABLE** 
Case 3 .. The default case includes all sequences remaining and assumes that all means by which the PRFT can be damaged have been included in the previous cases. An outcome probability of 1.0 is assigned Branch 2.

\section{Question 121: Does the collateral damage in the Salt Process Cell result in a deflagration in the Salt Process Cell?}

Spillage of benzene into the Salt Process Cell from the OEV or OECT could result in a deflagration. The PR and PRFT do not contain enough benzene to be of concern even if their entire contents are spilled onto the floor of the SPC. A deflagration occurring in the Salt Process Cell will be very energetic, blowing the cell covers. There are two defined outcomes as follows:

SPC-Def2: $\quad$ A deflagration occurs in the Salt Process Cell.

nSPCDef2: No deflagration occurs in the Salt Process Cell.

This is a Type 2 question. The answers to this question are dependent on the outcomes of Questions 1, 4,107, 108,113,118, and 119 and are used in the logic to determine the outcome probabilities for Questions 122, 123, 124, 125, 126, 128, and 147. Fifteen cases are used to develop the outcome probabilities as follows (The outcome probabilities are from Reference 7):

Case 1

This case includes all sequences in which the accident type is external, internal-energetic, or a crane load drop accident and there is no spillage from the OEV or the OECT. If there is no spillage of benzene there is no potential for a deflagration and the outcome probability is specified as 1.0 for Branch 2.

Case 2 This case includes all sequences in which the accident type is external or internal-energetic and spillage of benzene from the OEV or the OECT after the first or second round of energetic events has occurred. The zone 1 ventilation system has failed. An outcome probability of 1.0 is assigned to Branch 1.

Case 3 This case includes all sequences in which the accident type is external or internal-energetic and spillage of benzene from the OEV or the OECT after a SPC overpressure deflagration has occurred. Since the zone 1 ventilation system must fail for a SPC overpressure deflagration to occur, it is not explicitly asked about. An outcome probability of 1.0 is assigned to Branch 1.

Case 4 This case includes all sequences in which the accident type is external or internal-energetic and spillage of benzene from the OEV after the first or second round of energetic events has occurred. The zone 1 ventilation system has not failed and the OEV contains hot material no more than $10 \%$ of the time. An outcome probability of 0.1 is assigned to Branch 1 and an outcome probability of 0.9 is assigned to Branch 2. **SAMPLED VARIABLE** 
Case 5 - . This case includes all sequences in which the accident type is external or internal-energetic and spillage of benzene from the OECT after the first or second round of energetic events has occurred. The zone 1 ventilation system has not failed and the OECT may contain hot material although not like the OEV. An outcome probability of 0.01 is assigned to Branch 1 and an outcome probability of 0.99 is assigned to Branch 2 . **SAMPLED VARIABLE**

Case 6

This case includes all sequences in which the accident type is a limited leak from the PR or the PRFT and the exhaust fans have failed. The PR and PRFT do not contain enough benzene to be of concern even if their entire contents are spilled onto the floor of the SPC and ventilation has failed. An outcome probability of 1.0 is assigned to Branch 2 .

Case 7 This case includes all sequences in which the accident type is a limited leak from the PR or the PRFT and the exhaust fans have not failed. An outcome probability of 1.0 is assigned to Branch 2 .

Case 8 This case includes all sequences in which the accident type is a limited leak due to an overflow from the PR or the PRFT and the exhaust fans have failed. The PR and PRFT do not contain enough benzene to be of concern even if their entire contents are spilled onto the floor of the SPC and ventilation has failed. An outcome probability of 1.0 is assigned to Branch 2.

Case 9 This case includes all sequences in which the accident type is a limited leak due to an overflow from the PR or the PRFT and the exhaust fans have not failed. An outcome probability of 1.0 is assigned to Branch 2.

Case 10 This case includes all sequences in which the accident type is a limited leak due to an overflow from the OEV or the OECT and the exhaust fans have failed. The liklihood of a deflagration is less likely for this case because the amount of benzene assumed to be spilled during a overflow - scenario is less than the entire contents of the tanks. An outcome probability of 0.01 is assigned to Branch 1 and an outcome probabiliyt of 0.99 is assigned to Branch 2. **SAMPLED VARIABLE**

Case 11 This case includes all sequences in which the accident type is a limited leak due to an overflow from the OEV or the OECT and the exhaust fans have not failed. An outcome probability of 1.0 is assigned to Branch 2 .

Case 12 This case includes all sequences in which the accident type is a crane load drop which results in spillage from the OEV or the OECT and the exhaust fans have failed. An outcome probability of 0.01 is assigned to Branch 1 and an outcome probability of 0.99 is assigned to Branch 2. **SAMPLED VARIABLE**

Case 13 This case includes all sequences in which the accident type is a limited leak from the OEV or the OECT and the exhaust fans have failed. The liklihood of a deflagration is less likely for this case because the amount of benzene assumed to be spilled during a leak scenario is less then the amount from either the total spillage or an overlfow. An outcome 
probability of 0.001 is assigned to Branch 1 and an outcome probability of 0.999 is assigned to Branch $2 .{ }^{* *}$ SAMPLED VARIABLE**

Case 14 This case includes all sequences in which the accident type is a limited leak from the OEV or the OECT and the exhaust fans have not failed. An outcome probability of 1.0 is assigned to Branch 2 .

Case 15 The default case includes all sequences remaining. It is expected that all sequences in which a deflagration in the cell could occur are included in previous cases. An outcome probability of 1.0 is assigned Branch 2.

\section{Question 122: Does the collateral damage in the Salt Process Cell result in a fire in the Salt Process Cell?}

As indicated previously benzene may be present in any or all of the tanks in the Salt Process Cell. Spillage of this benzene into the cell could result in a fire. The PR and PRFT do not contain enough benzene to be of concern even if their entire contents are spilled onto the floor of the SPC. A fire will increase the transport of released radionuclides from the cell. There are two defined outcomes as follows:

SPC-Fire: $\quad$ A fire occurs in the Salt Process Cell.

nSPCFire: . A fire does not occur in the Salt Process Cell.

This is a Type 2 question. The answers to this question are dependent on the outcomes of Questions $1,4,107,108,113,118,119$, and 121 and are used in the logic to determine the outcome probabilities for Question 147. Ten cases are used to develop the outcome probabilities as follows (The outcome probabilities are from Reference 7):

Case 1 This case includes all sequences in which there is no spillage from the $\mathrm{OEV}$ or the OECT. If there is no spillage of benzene there is no potential for fire and the outcome probability is specified as 1.0 for Branch 2 .

Case 2 This case includes all sequences in which the accident type is external or internal-energetic, benzene has spilled from either the OEVor the OECT, and a SPC deflagration has occurred. An outcome probability of 0.9 is assigned to Branch 1 and an outcome probability of 0.1 is assigned Branch 2. **SAMPLED VARIABLE**

Case 3 This case includes all sequences in which the accident type is external or internal-energetic, benzene has spilled from either the OEVor the OECT, and a SPC deflagration has not occurred. An outcome probability of 0.1 is assigned to Branch 1 and an outcome probability of 0.9 is assigned Branch 2. **SAMPLED VARIABLE**

Case $4 \quad$ This case includes all sequences in which the accident type is a limited leak due to overflow from the OEVor the OECT and a SPC deflagration has occurred. An outcome probability of 0.9 is assigned to Branch 1 and an outcome probability of 0.1 is assigned Branch 2 . 


..
Case 5AMPLED VARIABLE**
This case includes all sequences in which the accident type is a limited
leak due to overflow from the OEVor the OECT, a SPC deflagration has
not occurred, and the Zone 1 exhaust fans have not failed (if fans failed,
and no deflagration occurs in question 121 , it is implicitly assumed there
is no ignition source for a fire either, if fans have not failed there may be
no deflagration as is seen in case 11 of question 121 but an ignition source
may still be present which could ignite a fire; this also applies to cases 7
and 9). An outcome probability of 0.05 is assigned to Branch 1 and an
outcome probability of 0.95 is assigned Branch 2. * SAMPLED
VARIABLE**

Case 6

This case includes all sequences in which the accident type is a crane load drop, benzene has spilled from either the OEVor the OECT, and a SPC deflagration has occurred. An outcome probability of 0.9 is assigned to Branch 1 and an outcome probability of 0.1 is assigned Branch 2. **SAMPLED VARIABLE**

Case 7 This case includes all sequences in which the accident type is a crane load drop, benzene has spilled from the OEVor the OECT, a SPC deflagration has not occurred, and the Zone 1 exhaust fans have not failed. An outcome probability of 0.05 is assigned to Branch 1 and an outcome probability of 0.95 is assigned Branch 2 . **SAMPLED VARIABLE**

Case 8 This case includes all sequences in which the accident type is a limited leak from the OEVor the OECT and a SPC deflagration has occurred. An outcome probability of 0.9 is assigned to Branch 1 and an outcome probability of 0.1 is assigned Branch 2 . **SAMPLED VARIABLE**

Case 9 This case includes all sequences in which the accident type is a limited leak from the OEVor the OECT, a SPC deflagration has not occurred, and the Zone 1 exhaust fans have not failed. Since the amount of benzene spilled in a leak is less than the amount from an overflow, the chance of a fire is slightly lower for this case then for an overflow accident. An outcome probability of 0.01 is assigned to Branch 1 and an outcome probability of 0.99 is assigned Branch 2.

**SAMPLED VARIABLE**

Case 10 The default case includes all sequences remaining. It is expected that all sequences in which a fire in the cell could occur are included in previous cases. An outcome probability of 1.0 is assigned Branch 2 .

Questions 123-126 consider the possibility that another vessel has an explosive concentration built up (but no ignition source) at the same time the SPC deflagration occurs. This is the third round of tank explosions. The flame from the SPC deflagration provides the ignition source for a tank deflagration in the SPC. Since the process vessels are all cross-connected via the cell ventilation systems (SPCV, CPCV, PVVH), the vessel that deflagrates or detonates in the SPC provides an ignition source to vessels in the CPC that have an explosive concentration provided the ventilation headers are still intact. Note, for the internal energetic accidents initiated by a tank explosion, all tank explosions are complete at the end of round 2 because any tank with a 
explosive concentration will have been exposed to an ignition source at that time. A "3rd round" exp losion can only occur on a sequence where the SPC overpressurization deflagration (question 115) initiates a propagation leading to a second SPC deflagration thus providing an ignition source for this round of tank explosions. Propagation into the CPC is not possible because the SPC covers will have fallen and opened the SPCV preventing the flame front from further propagation. For the seismic event, propagation to the CPC is only possible if the SPC covers have not fallen prior to the SPC deflagration.

\section{Question 123: Does a detonation/deflagration occur in the Precipitate Reactor?}

If an explosive concentration occurs in the Precipitate Reactor and an ignition source is available (in this case the deflagration in the SPC), it is assumed that a damaging detonation or deflagration will follow. A detonation/deflagration in the Precipitate Reactor can spill the contents of the vessel to the Salt Process Cell, blow the cell covers, and damage adjacent vessels. There are three defined outcomes as follows:

PRx@3Det: A damaging detonation occurs in the Precipitate Reactor following a SPC deflagration that provides an ignition source.

PRx@3Def: A damaging deflagration occurs in the Precipitate Reactor following a SPC deflagration that provides an ignition source.

nPRx-Exp: No damaging explosion occurs in the Precipitate Reactor.

This is a Type 2 question. The answer is dependent on the outcome of previous Questions 41, $43,79,80,106,117$, and 121 . The answers to this question are used in the logic to determine the outcome probabilities in Questions 127, 128,136, and 137. Seven cases are used to develop the outcome probabilities as follows:

Case 1 This case includes all sequences in which a previous detonation occurred in the Precipitate Reactor. The outcome probability assigned is 1.0 in Branch 3.

Case 2 This case includes all sequences in which a previous deflagration occurred in the Precipitate Reactor. The outcome probability assigned is 1.0 in Branch 3.

Case 3 This case includes all sequences in which an explosive concentration does not accumulate in the Precipitate Reactor. The outcome probability assigned is 1.0 in Branch 3.

Case 4 This case includes the sequences in which a seismic event or crane load drop accident has ruptured the Precipitate Reactor precluding the accumulation of an explosive concentration. The outcome probability assigned is 1.0 in Branch 3.

Case 5 This case includes the sequences in which a prior energetic event has ruptured the Precipitate Reactor precluding the accumulation of an explosive concentration. The outcome probability assigned is 1.0 in Branch 3. 
Case 6 - This case includes the sequences in which a prior event has not occurred in the Precipitate Reactor and an explosive concentration has accumulated in the vessel with a deflagration in the SPC (provides the ignition source). The outcome probability assigned is 0.001 in Branch 1 and 0.999 in Branch 2.

Case 7 = The default case includes any remaining sequences. It is assumed that all sequences resulting in a damaging explosion are included in previous cases. The outcome probability assigned is 1.0 in Branch 3.

\section{Question 124: Does a detonation/deflagration of the Organic Evaporator tank occur?}

If an explosive concentration occurs in the Organic Evaporator and an ignition source is available it is assumed that a damaging detonation or deflagration will follow. A detonation/deflagration in the Organic Evaporator can spill the contents of the vessel to the Salt Process Cell, blow the cell covers, and damage adjacent vessels. There are three defined outcomes as follows:

OEV@3Det. . A damaging detonation occurs in the Organic Evaporator following a SPC deflagration that provides an ignition source.

OEV@3Def: A damaging deflagration occurs in the Organic Evaporator following a SPC deflagration that provides an ignition source.

nOEV-Exp: No damaging explosion occurs in the Organic Evaporator.

This is a Type 2 question. The answer is dependent on the outcome of previous Questions 45, $47,81,82,107,118$, and 121 . The answers to this question are used in the logic to determine the outcome probabilities in Questions 127, 128,136,137, and 138. Seven cases are used to develop the outcome probabilities as follows:

Case 1 This case includes all sequences in which a previous detonation occurred in the Organic Evaporator. The outcome probability assigned is 1.0 in Branch 3.

Case 2 . This case includes all sequences in which a previous deflagration occurred in the Organic Evaporator. The outcome probability assigned is 1.0 in Branch 3.

Case 3 This case includes all sequences in which an explosive concentration does not accumulate in the Organic Evaporator. The outcome probability assigned is 1.0 in Branch 3.

Case $4 \quad$ This case includes the sequences in which a seismic event or crane load drop accident has ruptured the Organic Evaporator precluding the accumulation of an explosive concentration. The outcome probability assigned is 1.0 in Branch 3. 
Case 5 .. This case includes the sequences in which a prior energetic event has ruptured the Organic Evaporator precluding the accumulation of an explosive concentration. The outcome probability assigned is 1.0 in Branch 3.

Case 6 This case includes the sequences in which a prior event has not occurred in the Organic Evaporator and an explosive concentration has accumulated in the vessel with a deflagration.in the SPC (provides the ignition source). The outcome probability assigned is 0.001 in Branch 1 and 0.999 in Branch 2 [7].

Case 7 The default case includes any remaining sequences. It is assumed that all sequences resulting in a damaging explosion are included in previous cases. The outcome probability assigned is 1.0 in Branch 3.

\section{Question 125: Does a detonation/deflagration of the Organic Evaporator Condensate tank occur?}

If an explosive concentration occurs in the Organic Evaporator Condensate tank and an ignition source is available it is assumed that a damaging detonation or deflagration will follow. A detonation/deflagration in the Organic Evaporator Condensate tank can spill the contents of the vessel to the Salt Process Cell, blow the cell covers, and damage adjacent vessels. There are three defined outcomes as follows:

OECT@3Det: A damaging detonation occurs in the Organic Evaporator Condensate tank following a SPC deflagration that provides an ignition source.

OECT@3Def: A damaging deflagration occurs in the Organic Evaporator Condensate tank following a SPC deflagration that provides an ignition source.

nOECT-Ex: No damaging explosion occurs in the Organic Evaporator Condensate tank.

This is a Type 2 question. The answer is dependent on the outcome of previous Questions 49, $51,83,84,108,119$, and 121 . The answers to this question are used in the logic to determine the outcome probabilities in Questions 127, 128,137, 138, and 139. Seven cases are used to develop the outcome probabilities as follows:

Case 1 This case includes all sequences in which a previous detonation occurred in the Organic Evaporator Condensate tank. The outcome probability assigned is 1.0 in Branch 3.

Case $2^{\circ} \quad$ This case includes all sequences in which a previous deflagration occurred in the Organic Evaporator Condensate tank. The outcome probability assigned is 1.0 in Branch 3.

Case 3

This case includes all sequences in which an explosive concentration does not accumulate in the Organic Evaporator Condensate tank. The outcome probability assigned is 1.0 in Branch 3. 
Case $4 \quad$ This case includes the sequences in which a seismic event or crane load drop accident has ruptured the Organic Evaporator Condensate tank precluding the accumulation of an explosive concentration. The outcome probability assigned is 1.0 in Branch 3.

Case 5 This case includes the sequences in which a prior energetic event has ruptured the Organic Evaporator Condensate tank precluding the accumulation of an explosive concentration. The outcome probability assigned is 1.0 in Branch 3.

Case 6

This case includes the sequences in which a prior event has not occurred in the Organic Evaporator Condensate tank and an explosive concentration has accumulated in the vessel with a deflagration in the SPC (provides the ignition source). The outcome probability assigned is 0.001 in Branch 1 and 0.999 in Branch 2 [7].

Case 7 The default case includes any remaining sequences. It is assumed that all sequences resulting in a damaging explosion are included in previous cases. The outcome probability assigned is 1.0 in Branch 3.

\section{Question 126: Does a detonation/deflagration occur in the Precipitate Reactor Feed Tank?}

If an explosive concentration occurs in the Precipitate Reactor Feed Tank and an ignition source is available it is assumed that a damaging detonation or deflagration will follow. A detonation/deflagration in the Precipitate Reactor Feed Tank can spill the contents of the vessel to the Salt Process Cell, blow the cell covers, and damage adjacent vessels. There are three defined outcomes as follows:

PRFT@3Det: A damaging detonation occurs in the Precipitate Reactor Feed Tank following a SPC deflagration that provides an ignition source.

PRFT@3Def: A damaging deflagration occurs in the Precipitate Reactor Feed Tank following a SPC deflagration that provides an ignition source.

nPRFT-Exp: No damaging explosion occurs in the Precipitate Reactor Feed Tank.

This is a Type 2 question. The answer is dependent on the outcome of previous Questions 53, $55,85,86,109,120$, and 121 . The answers to this question are used in the logic to determine the outcome probabilities in Questions 127, 128,138,139, and 140. Seven cases are used to develop the outcome probabilities as follows:

Case 1 This case includes all sequences in which a previous detonation occurred in the Precipitate Reactor Feed Tank. The outcome probability assigned is 1.0 in Branch 3.

Case 2 This case includes all sequences in which a previous deflagration occurred in the Precipitate Reactor Feed Tank. The outcome probability assigned is 1.0 in Branch 3. 
Case 3 .. This case includes all sequences in which an explosive concentration does not accumulate in the Precipitate Reactor Feed Tank. The outcome probability assigned is 1.0 in Branch 3.

Case 4 This case includes the sequences in which a seismic event or crane load drop accident has ruptured the Precipitate Reactor Feed Tank precluding the accumulation of an explosive concentration. The outcome probability assigned is 1.0 in Branch 3.

Case 5

This case includes the sequences in which a prior energetic event has ruptured the Precipitate Reactor Feed Tank precluding the accumulation of an explosive concentration. The outcome probability assigned is 1.0 in Branch 3.

Case 6 This case includes the sequences in which a prior event has not occurred in the Precipitate Reactor Feed Tank and an explosive concentration has accumulated in the vessel with a deflagration in the SPC (provides the ignition source). The outcome probability assigned is 0.001 in Branch 1 and 0.999 in Branch 2 [7].

Case 7 The default case includes any remaining sequences. It is assumed that all sequences resulting in a damaging explosion are included in previous cases. The outcome probability assigned is 1.0 in Branch 3.

\section{Question 127: Does a damaging explosion occur in the Salt Process Cell Vent System [SPCV]?}

Conditions in the Salt Process Cell Vent System [SPCV] are expected to be the same as the tanks exhausting into the header. Therefore an explosion in any of the SPC tanks will propagate into the header causing the header to also explode. Because of the size of the SPCV, it is expected to detonate (not deflagrate) which would produce shrapnel that could cause venting of cell tanks. There are two possible outcomes as follows:

SPCV-Exp3: A damaging explosion occurs in the Salt Process Cell Vent System following the third round of potential explosions.

nSPCV-Ex: A damaging explosion does not occur in the Salt Process Cell Vent System following the third round of potential explosions.

This is a Type 2 question. The answer is dependent on the outcome of previous Questions 37, $105,116,123,124,125$, and 126 . The answers to this question are not used in the remaining logic but are instead used in some of the output binners. Five cases are used to develop the outcome probabilities as follows:

Case 1 This case includes all sequences in which the SPC cell covers have fallen due to a seismic event. If the cell covers fall, the SPCV piping is assumed to be damaged to the point that no accumulation of explosive mixtures is possible. An outcome probability of 1.0 is assigned to Branch 2.

Case 2

This case includes all sequences in which the SPC cell covers have fallen due to a previous energetic event. If the cell covers fall, the SPCV piping 
is assumed to be damaged to the point that no accumulation of explosive mixtures is possible. An outcome probability of 1.0 is assigned to Branch 2.

Case 3 This case includes all sequences in which one of the SPC vessels has detonated in the third round. A detonation in any SPC vessel is assumed to cause the SPCV to explode. (For internal energetic events, has already been destroyed and cannot explode.) An outcome probability of 1.0 is assigned Branch 1.

Case 4. This case includes all sequences in which one of the SPC vessels has deflagrated in the third round. A deflagration in any SPC vessel is assumed to cause the SPCV to explode. (For internal energetic events, has already been destroyed and cannot explode.) An outcome probability of 1.0 is assigned Branch 1.

Case 5 The default case includes all sequences remaining and assumes that there is no other mechanism for an explosion in the SPCV. An outcome probability of 1.0 is assigned Branch 2.

\section{Question 128: Do the cell covers for the Salt Process Cell dislodge and fall?}

The cell covers in the SPC can be dislodged and fall as a result of an energetic event in the SPC. The falling cell covers can damage equipment and piping in the cell. This will only occur for a seismic event where the cell covers have not already fallen. There are two defined outcomes as follows:

SPCvF1@3: The cell covers dislodge and fall due to an energetic event in the third round of explosions.

nSPCovFl: The cell covers do not fall as a result of an energetic event in the third round of explosions.

This is a Type 2 question. The answers to this question are dependent on the outcome of previous Questions 37, 105,116,121,123,124, 125,126, and 127 and are used in the logic to determine the outcome probabilities in Questions 136,137, 138, and 139. Six cases are used to develop the outcome probabilities as follows:

Case 1 This case includes all sequences in which the cell covers were dislodged previously. An outcome probability of 1.0 is assigned Branch 2.

Case 2 This case includes all sequences in which there was no explosion in any SPC vessel, the SPCV did not explode, the cell covers did not fall due to a seismic event, and no SPC deflagration occurred. An outcome probability of 1.0 is assigned Branch 2 .

Case 3 This case includes all sequences in which there was a deflagration in the SPC. An outcome probability of 1.0 is assigned Branch 1 . 
Case 4 -. This case includes all sequences in which there was a detonation in any SPC vessel during the third round of explosions. An outcome probability of 1.0 is assigned Branch 1.

Case 5 This case includes all sequences in which there was a deflagration in any SPC vessel during the third round of explosions. An outcome probability of 1.0 is assigned Branch 1.

Case 6 The default case includes all sequences remaining and assumes that all means by which the cell covers can fall have been included in the previous cases. An outcome probability of 1.0 is assigned Branch 2 .

\section{Question 129: Does a damaging detonation occur in the Process Vessel Vent} Header [PVVH]?

The PVYH is assumed to explode if an explosion occurs in one of the headers that connect to it (SPCV or CPCV). Therefore, if Question 127 indicates that an explosion occurred in the SPCV then a detonation in the PVVH will follow. A detonation in the PVVH can cause cell covers in the Chemical Process Cell to fall. There are two defined outcomes as follows:

PVVH-DT2: A damaging detonation occurs in the Process Vessel Vent Header following the third round of explosions.

nPVVH-Ex: No damaging explosion occurs in the Process Vessel Vent Header.

This is a Type 2 question. The answer is dependent on the outcome of previous Questions 56, 105,116 , and 127. The answers to this question are used in the logic to determine the outcome probabilities in Questions 130,131,132, 133, and 141. Four cases are used to develop the outcome probabilities as follows:

Case 1 This case includes all sequences in which the CPC cell covers have fallen due to a seismic event. If the cell covers fall, the PVVH piping is assumed to be damged to the point that no accumulation of explosive mixtures is possible. An outcome probability of 1.0 is assigned to Branch 2.

Case 2 This case includes all sequences in which the SPC cell covers were dislodged previously. If the SPC cell covers were dislodged previously, the SPCV piping is assumed to be damaged to the point that it would be unable to transmit an explosion to the PVVH. An outcome probability of 1.0 is assigned Branch 2.

Case 3 This case includes all sequences in which a damaging detonation has occurred in the SPCV after the third round of explosions. The outcome probability assigned is 1.0 in Branch 1.

Case 4 The default case includes any remaining sequences. It is assumed that all sequences resulting in a damaging explosion are included in previous cases. The outcome probability assigned is 1.0 in Branch 2. 


\section{Question 130: Does a detonation/deflagration occur in the Precipitate Reactor Bottoms Tank [PRBT]?}

If an explosive concentration occurs in the Precipitate Reactor Bottoms Tank and an ignition source is available it is assumed that a damaging detonation or deflagration will follow. A detonation/deflagration in the Precipitate Reactor Bottoms Tank can spill the contents of the vessel to the Chemical Process Cell, blow the cell covers, and damage adjacent vessels. There are three defined outcomes as follows:

PRBT@3Det: A damaging detonation occurs in the Precipitate Reactor Bottoms Tank following a detonation or deflagration in another process vessel during the third round of explosions that provides an ignition source.

PRBT@3Def: A damaging deflagration occurs in the Precipitate Reactor Bottoms Tank following a detonation or deflagration in another process vessel during the third round of explosions that provides an ignition source.

nPRBT-Ex: No damaging explosion occurs in the Precipitate Reactor Bottoms Tank.

This is a Type 2 question. The answer is dependent on the outcome of previous Questions 58, $60,87,88,99,105,116$, and 129 . The answers to this question are used in the logic to determine the outcome probabilities in Questions 134,135, 141,142, and 143. Eight cases are used to develop the outcome probabilities as follows:

Case 1 This case includes all sequences in which a previous detonation occurred in the Precipitate Reactor Bottoms Tank. The outcome probability assigned is 1:0 in Branch 3.

Case 2 This case includes all sequences in which a previous deflagration occurred in the Precipitate Reactor Bottoms Tank. The outcome probability assigned is 1.0 in Branch 3.

Case 3 This case includes all sequences in which an explosive concentration does not accumulate in the Precipitate Reactor Bottoms Tank. The outcome probability assigned is 1.0 in Branch 3.

Case 4 This case includes the sequences in which a seismic event or crane drop accident has ruptured the Precipitate Reactor Bottoms Tank precluding the accumulation of an explosive concentration. The outcome probability assigned is 1.0 in Branch 3.

Case 5 This case includes all sequences in which the PRBT has been splashed as a result of a prior energetic event. Splashing of the tank precludes the accumulation of an explosive concentration. The outcome probability assigned is 1.0 in Branch 3.

Case 6 This case includes all sequences in which the SPC cell covers have previously fallen. If the cell covers have fallen there is no path to conduct the ignition source from the SPC to the CPC. As a result, no explosion can occur. The outcome probability assigned is 1.0 in Branch 3. 
Case 7 ... This case includes the sequences in which a prior event has not occurred in the Precipitate Reactor Bottoms Tank and an explosive concentration has accumulated in the vessel with a detonation in the PVVH (provides the ignition source). The outcome probability assigned is 0.001 in Branch 1 and 0.999 in Branch 2 [7].

Case 8 The default case includes any remaining sequences. It is assumed that all sequences resulting in a damaging explosion are included in previous cases. The outcome probability assigned is 1.0 in Branch 3 , there is no explosion.

\section{Question 131: Does a detonation/deflagration occur. in the Slurry Mix Evaporator Tank [SME]?}

If an explosive concentration occurs in the Slurry Mix Evaporator Tank and an ignition source is available it is assumed that a damaging detonation or deflagration will follow. A detonation/deflagration in the Slurry Mix Evaporator Tank can spill the contents of the vessel to the Chemical Process Cell, blow the cell covers, and damage adjacent vessels. There are three defined outcomes as follows:

SME@3Det: A damaging detonation, occurs in the Slurry Mix Evaporator Tank following a detonation or deflagration in another process vessel during the third round of explosions that provides an ignition source.

SME@3Def: A damaging deflagration occurs in the Slurry Mix Evaporator Tank following a detonation or deflagration in another process vessel during the third round of explosions that provides an ignition source.

nSME-Ex: - No damaging explosion occurs in the Slurry Mix Evaporator Tank.

This is a Type 2 question. The answer is dependent on the outcome of previous Questions 62, $64,89,90,100,105,116$, and 129 . The answers to this question are used in the logic to determine the outcome probabilities in Questions 134, 141, 142, and 143. Eight cases are used to develop the outcome probabilities as follows:

Case 1 This case includes all sequences in which a previous detonation occurred in the Slurry Mix Evaporator Tank. The outcome probability assigned is 1.0 in Branch 3.

Case 2 This case includes all sequences in which a previous deflagration occurred in the Slurry Mix Evaporator Tank. The outcome probability assigned is 1.0 in Branch 3.

Case 3 This case includes all sequences in which an explosive concentration does not accumulate in the Slurry Mix Evaporator Tank. The outcome probability assigned is 1.0 in Branch 3.

Case 4 This case includes the sequences in which a seismic event or crane load drop accident has ruptured the Slurry Mix Evaporator Tank precluding the accumulation of an explosive concentration. The outcome probability assigned is 1.0 in Branch 3. 
Case 5 - This case includes all sequences in which the SPC cell covers have previously fallen. If the cell covers have fallen there is no path to conduct the ignition source from the SPC to the CPC. As a result, no explosion can occur. The outcome probability assigned is 1.0 in Branch 3 .

Case 6 This case includes all sequences in which the SME has been splashed as a result of a prior energetic event. Splashing of the tank precludes the accumulation of an explosive concentration. The outcome probability assigned is 1.0 in Branch 3.

Case 7 This case includes the sequences in which a prior event has not occurred in the Slurry Mix Evaporator Tank and an explosive concentration has accumulated in the vessel with a detonation in the PVVH (provides the ignition source). The outcome probability assigned is 0.001 in Branch 1 and 0.999 in Branch 2 [7].

Case 8 The default case includes any remaining sequences. It is assumed that all sequences resulting in a damaging explosion are included in previous cases. The outcome probability assigned is 1.0 in Branch 3.

\section{Question 132: Does a detonation/deflagration occur in the Sludge Receipt and Adjustment Tank [SRAT]?}

If an explosive concentration occurs in the Sludge Receipt Adjustment Tank and an ignition source is available it is assumed that a damaging detonation or deflagration will follow. A detonation/deflagration in the Sludge Receipt Adjustment Tank can spill the contents of the vessel to the Chemical Process Cell, blow the cell covers, and damage adjacent vessels. There are three defined outcomes as follows:

SRAT@3Det: A damaging detonation occurs in the Sludge Receipt Adjustment Tank following a detonation or deflagration in another process vessel during the third round of explosions that provides an ignition source.

SRAT@3Def: A damaging deflagration occurs in the Sludge Receipt Adjustment Tank following a detonation or deflagration in another process vessel during the third round of explosions that provides an ignition source.

nSRAT-Ex: No damaging explosion occurs in the Sludge Receipt Adjustment Tank.

This is a Type 2 question. The answer is dependent on the outcome of previous Questions 66, $68,91,92,101,105,116$, and 129. The answers to this question are used in the logic to determine the outcome probabilities in Questions 134, 141,144, and 145. Eight cases are used to develop the outcome probabilities as follows:

Case 1 This case includes all sequences in which a previous detonation occurred in the Sludge Receipt Adjustment Tank. The outcome probability assigned is 1.0 in Branch 3. 
Case 2 .. This case includes all sequences in which a previous deflagration occurred in the Sludge Receipt Adjustment Tank. The outcome probability assigned is 1.0 in Branch 3.

Case 3 This case includes all sequences in which an explosive concentration does not accumulate in the Sludge Receipt Adjustment Tank. The outcome probability assigned is 1.0 in Branch 3.

Case 4 This case includes the sequences in which a seismic event or crane load drop accident has ruptured the Sludge Receipt Adjustment Tank precluding the accumulation of an explosive concentration. The outcome probability assigned is 1.0 in Branch 3.

Case 5 This case includes all sequences in which the SRAT has been splashed as a result of a prior energetic event. Splashing of the tank precludes the accumulation of an explosive concentration. The outcome probability assigned is 1.0 in Branch 3.

Case 6 This case includes all sequences in which the SPC cell covers have previously fallen. If the cell covers have fallen there is no path to conduct the ignition source from the SPC to the CPC. As a result, no explosion can occur. The outcome probability assigned is 1.0 in Branch 3.

Case 7 This case includes the sequences in which a prior event has not occurred in the Sludge Receipt Adjustment Tank and an explosive concentration has accumulated in the vessel with a detonation in the PVVH (provides the ignition source). The outcome probability assigned is 0.001 in Branch 1 and 0.999 in Branch 2 [7]:

Case 8 The default case includes any remaining sequences. It is assumed that all sequences resulting in a damaging explosion are included in previous cases. The outcome probability assigned is 1.0 in Branch 3 .

\section{Question 133: Does a detonation/deflagration occur in the Melter Feed Tank [MFT]?}

If an explosive concentration occurs in the Melter Feed Tank and an ignition source is available it is assumed that a damaging detonation or deflagration will follow. A detonation/deflagration in the Melter Feed Tank can spill the contents of the vessel to the Chemical Process Cell, blow the cell covers, and damage adjacent vessels. There are three defined outcomes as follows:

MFT@3Det: A damaging detonation occurs in the Melter Feed Tank following a . detonation or deflagration in another process vessel during the third round of explosions that provides an ignition source.

MFT@3Def: A damaging deflagration occurs in the Melter Feed Tank following a detonation or deflagration in another process vessel during the third round of explosions that provides an ignition source.

nMFT-Ex: No damaging explosion occurs in the Melter Feed Tank. 
This is a Type 2 question. The answer is dependent on the outcome of previous Questions 72, $74,93,94,103,105,116$, and 129 . The answers to this question are used in the logic to determine the outcome probabilities in Questions 134, 141, 145, and 146. Eight cases are used to develop the outcome probabilities as follows:

Case 1. This case includes all sequences in which a previous detonation occurred in the Melter Feed Tank. The outcome probability assigned is 1.0 in Branch 3.

Case 2 This case includes all sequences in which a previous deflagration occurred in the Melter Feed Tank. The outcome probability assigned is 1.0 in Branch 3.

Case 3 This case includes all sequences in which an explosive concentration does not accumulate in the Melter Feed Tank. The outcome probability assigned is 1.0 in Branch 3.

Case 4 This case includes the sequences in which a seismic event or crane load drop accident has ruptured the Melter Feed Tank precluding the accumulation of an explosive concentration. The outcome probability assigned is 1.0 in Branch 3.

Case 5 This case includes all sequences in which the MFT has been splashed as a result of a prior energetic event. Splashing of the tank precludes the accumulation of an explosive concentration. The outcome probability assigned is 1.0 in Branch 3.

Case 6 This case includes all sequences in which the SPC cell covers have previously fallen. If the cell covers have fallen there is no path to conduct the ignition source from the SPC to the CPC. As a result, no explosion can occur. The outcome probability assigned is 1.0 in Branch 3.

Case $7 \quad$ This case includes the sequences in which a prior event has not occurred in the Melter Feed Tank and an explosive concentration has accumulated in the vessel with a detonation in the PVVH (provides the ignition source). The outcome probability assigned is 0.001 in Branch 1 and 0.999 in Branch 2 [7].

Case 8 The default case includes any remaining sequences. It is assumed that all sequences resulting in a damaging explosion are included in previous cases. The outcome probability assigned is 1.0 in Branch 3.

\section{Question 134: Does a detonation occur in the CPC Vent system?}

An explosion in the CPCV can cause spillage of the contents of some of the tanks in the CPC due to lifting of the cell covers. There are two defined outcomes as follows:

CPCV-Exp1: A damaging explosion occurs in the Chemical Process Cell Vent System following the third round of potential explosions. 
nCPCV-Ex: No damaging explosion occurs in the Chemical Process Cell Vent System following the third round of potential explosions.

This is a Type 2 question. The answer is dependent on the outcome of previous Questions 98, $105,116,130,131,132$, and 133. The answers to this question are used in the logic to determine the outcome probabilities in Question 141. Four cases are used to develop the outcome probabilities as follows:

Case 1 This case includes all sequences in which the CPC cell covers have previously fallen. If the cell covers fall, the CPCV piping is assumed to be damged to the point that no accumulation of explosive mixtures is possible. An outcome probability of 1.0 is assigned to Branch 2 .

Case 2 This case includes all sequences in which the SPC cell covers have previously fallen. If the cell covers have fallen there is no path to conduct the ignition source from the SPC to the CPC. As a result, no explosion can occur. The outcome probability assigned is 1.0 in Branch 2.

Case 3 This case includes all sequences in which one of the CPC vessels has detonated or deflagrated in the third round. A detonation in any CPC vessel is assumed to cause the CPCV to explode. An outcome probability of 1.0 is assigned Branch 1.

Case 4 The default case includes all sequences remaining and assumes that there is no other mechanism for an explosion in the CPCV. An outcome probability of 1.0 is assigned Branch 2.

\section{Question 135: Does an energetic event in the CPC fail the removable wall between the SPC and the CPC?}

The Salt Process Cell and the Chemical Process Cell are separated by a removable [from above] partition. Some events in the CPC are assumed to be sufficiently energetic to displace the partition from its supports, causing damage to the tanks, piping, etc. in the SPC. There are two defined outcomes as follows:

FailWall2: The wall separating the Salt Process Cell and the Chemical Process Cell is failed due to an energetic event in the third round of explosions.

nFailWal: The wall separating the Salt Process Cell and the Chemical Process Cell is not failed.

This is a Type 2 question. The answers to this question are dependent on the outcomes of Questions 97, 104, and 130 and are used in the logic to determine the outcome probabilities for Questions 136, 137, 138, and 139. Three cases are used to develop the outcome probabilities. as follows:

Case 1 This case includes all sequences in which the removable wall has been failed in a previous energetic event. An outcome probability of 1.0 is assigned Branch 2. 
Case 2 - This case includes all sequences in which a detonation has occurred in the PRBT during the third round of explosions. An outcome probability of 0.5 is assigned to Branch 1 and Branch 2 [7].

Case 3 The default case includes all sequences remaining. It is expected that all sequences in which the wall could be failed are included in previous cases. An outcome probability of 1.0 is assigned Branch 2.

\section{Question 136: Does splashing [spillage] of the Precipitate Reactor contents occur?}

If the Precipitate Reactor is damaged some or all of the contents of the tank may be spilled to the floor. This question is concerned only with damage to the PR caused by falling cell covers, a detonation in the PR during the third round of explosions, shrapnel from an adjacent tank generated during the third round of explosions or failure of the wall between the CPC and SPC. There are two defined outcomes as follows:

PRx-Spl3: The contents of the Precipitate Reactor are spilled into the Salt Process Cell after an energetic event during the third round of explosions.

nPRx-Spl: The contents of the Precipitate Reactor are not spilled into the Salt Process Cell.

This is a Type 2 question. The answers to this question are dependent on the outcome of previous Questions 41, 106,117, 123,124, 128, and 135. The answers to this question are used in the source term binner. Nine cases are used to develop the outcome probabilities as follows (The outcome probabilities are from Reference 7):

Case 1

This case includes all sequences in which the PR has been previously ruptured by a seismic event or crane load drop accident. An outcome probability of 1.0 is assigned Branch 2 .

Case 2 This case includes all sequences in which the PR has been splashed by a previous energetic event. An outcome probability of 1.0 is assigned Branch 2.

Case 3 This case includes all sequences in which no event that could damage the PR has occurred. An outcome probability of 1.0 is assigned Branch 2.

Case 4 This case includes all sequences in which there was a detonation of the Precipitate Reactor during the third round of explosions. An outcome probability of 1.0 is assigned Branch 1 :

Case 5 This case includes all sequences in which the cell covers are displaced and fall and there is a detonation in the OEV, which is adjacent to the PR, during the third round of explosions. The falling cell covers or shrapnel from the exploding OEV can penetrate the PR causing spillage. An outcome probability of 0.5 is assigned Branch 1 and Branch 2.

Case 6 This case includes all sequences in which the cell covers are displaced and fall and there is a deflagration in the OEV, which is adjacent to the PR, 
during the third round of explosions. The falling cell covers or shrapne causing spillage. An outcome probability of 0.2 is assigned Branch 1 and an outcome probability of 0.8 is assigned Branch 2 .

Case 7 This case includes all sequences in which the cell covers are dislodged and fall. An outcome probability of 0.1 is assigned Branch 1 and an outcome probability of 0.9 is assigned Branch 2.

Case 8

This case includes all sequences in which the wall separating the SPC and the CPC is displaced into the SPC. The PR is separated from this wall by three other tanks. An outcome probability of 0.1 is assigned Branch 1 and an outcome probability of 0.9 is assigned Branch 2.

Case 9 The default case includes all sequences remaining and assumes that all means by which the PR can be damaged have been included in the previous cases. An outcome probability of 1.0 is assigned Branch 2 .

\section{Question 137: Does splashing [spillage] of the contents of the Organic Evaporator tank occur?}

If the OEV is damaged some or all of the contents of the tank may be spilled to the floor. This question is concerned only with damage to the OEV caused by falling cell covers, a detonation in the OEV during the third round of explosions, shrapnel from an adjacent tank generated during the third round of explosions or failure of the wall between the CPC and SPC. There are two defined outcomes as follows:

OEV-Spl3: The contents of the OEV are spilled into the Salt Process Cell after an energetic event during the third round of explosions.

nOEV-Spl: The contents of the OEV are not spilled into the Salt Process Cell.

This is a Type 2 question. The answers to this question are dependent on the outcome of previous Questions $45,107,118,123,124,125,128$, and 135. The answers to this question are used in the source term binner. Nine cases are used to develop the outcome probabilities as follows (The outcome probabilities are from Reference 7):

Case 1 This case includes all sequences in which the OEV has been previously ruptured by a seismic event or crane load drop accident. An outcome probability of 1.0 is assigned Branch-2.

Case 2 This case includes all sequences in which the OEV has been splashed by a previous energetic event. An outcome probability of 1.0 is assigned Branch 2.

Case 3 This case includes all sequences in which no event that could damage the OEV has occurred. An outcome probability of 1.0 is assigned Branch 2.

Case 4 This case includes all sequences in which there was a detonation of the OEV during the third round of explosions. An outcome probability of 1.0 is assigned Branch 1. 
Case $5 \quad-$ This case includes all sequences in which the cell covers are displaced and fall and there is a detonation in the PR or OECT which are adjacent to the OEV. The falling cell covers or shrapnel from the exploding PR or OECT. can penetrate the OEV causing spillage. An outcome probability of 0.5 is assigned Branch 1 and Branch 2.

Case 6. This case includes all sequences in which the cell covers are displaced and fall and there is a deflagration in the PR or OECT which are adjacent to the OEV. The falling cell covers or shrapnel from the vent piping above the deflagrating PR or OECT can penetrate the OEV causing spillage. An outcome probability of 0.2 is assigned Branch 1 and an outcome probability of 0.8 is assigned Branch 2 .

Case $7 \quad$ This case includes all sequences in which the cell covers are dislodged and fall. An outcome probability of 0.1 is assigned Branch 1 and an outcome probability of 0.9 is assigned Branch 2.

Case 8 , This case includes all sequences in which the wall separating the SPC and the CPC is displaced into the SPC. 'The OEV is separated from this wall by two other tanks. An outcome probability of 0.1 is assigned Branch 1 and an outcome probability of 0.9 is assigned Branch 2.

Case 9 The default case includes all sequences remaining and assumes that all means by which the OEV can be damaged have been included in the previous cases. An outcome probability of 1.0 is assigned Branch 2 .

\section{Question 138: Does splashing [spillage] of the contents of the Organic Evaporator Condensate tank occur?}

If the OECT is damaged some or all of the contents of the tank may be spilled to the floor. This question is concerned only with damage to the OECT caused by falling cell covers, a detonation in the OECT during the third round of explosions, shrapnel from an adjacent tank generated during the third round of explosions or failure of the wall between the CPC and SPC. There are two defined outcomes as follows:

OECT-Spl3: The contents of the OECT are spilled into the Salt Process Cell after an energetic event during the third round of explosions.

nOECT-Spl: The contents of the OECT are not spilled into the Salt Process Cell.

This is a Type 2 question. The answers to this question are dependent on the outcome of previous Questions 49,108,119,124,125,126,128, and 135. The answers to this question are used in the source term binner. Nine cases are used to develop the outcome probabilities as follows (The outcome probabilities are from Reference 7):

Case 1 This case includes all sequences in which the OECT has been previously ruptured by a seismic event or crane load drop accident. An outcome probability of 1.0 is assigned Branch 2. 
Case 2 .. This case includes all sequences in which the OECT has been splashed by a previous energetic event. An outcome probability of 1.0 is assigned Branch 2.

Case 3 This case includes all sequences in which no event that could damage the OECT has occurred. An outcome probability of 1.0 is assigned Branch 2.

Case 4 This case includes all sequences in which there was a detonation of the OECT during the third round of explosions. An outcome probability of 1.0 is assigned Branch 1.

Case 5 This case includes all sequences in which the cell covers are displaced and fall and there is a detonation in the PRFT or OEV which are adjacent to the OECT. The falling cell covers or shrapnel from the exploding PRFT or OEV can penetrate the OECT causing spillage. An outcome probability of 0.5 is assigned Branch 1 and Branch 2.

Case 6 This case includes all sequences in which the cell covers are displaced and fall and there is a deflagration in the PRFT or OEV which are adjacent to the OECT. The falling cell covers or shrapnel from the vent piping above the deflagrating PRFT or OEV can penetrate the OECT causing spillage. An outcome probability of 0.2 is assigned Branch 1 and an outcome probability of 0.8 is assigned Branch 2.

Case 7 This case includes all sequences in which the cell covers are dislodged and fall. An outcome probability of 0.1 is assigned Branch 1 and an outcome probability of 0.9 is assigned Branch 2.

Case 8 This case includes all sequences in which the wall separating the SPC and the CPC is displaced into the SPC. The OECT is separated from this wall by one other tank. An outcome probability of 0.1 is assigned Branch 1 and an outcome probability of 0.9 is assigned Branch 2 .

Case 9 The default case includes all sequences remaining and assumes that all means by which the OECT can be damaged have been included in the previous cases. An outcome probability of 1.0 is assigned Branch 2 .

\section{Question 139: Does splashing [spillage] of the contents of the Precipitate Reactor Feed Tank occur?}

If the Precipitate Reactor Feed Tank is damaged some or all of the contents of the tank may be spilled to the floor. This question is concerned only with damage to the PRFT caused by falling cell covers, a detonation in the PRFT during the third round of explosions, shrapnel from an adjacent tank generated during the third round of explosions or failure of the wall between the CPC and SPC. There are two defined outcomes as follows:

PRFT-Spl3: The contents of the PRFT are spilled into the Salt Process Cell after an energetic event during the third round of explosions.

nPRFT-Spl: The contents of the PRFT are not spilled into the Salt Process Cell. 
This is a Type 2 question. The answers to this question are dependent on the outcome of previous Questions 53,109,120,125, 126, 128, and 135. The answers to this question are used in the source term binner. Nine cases are used to develop the outcome probabilities as follows (The outcome probabilities are from Reference 7):

Case 1 This case includes all sequences in which the PRFT has been previously ruptured by a seismic event or crane load drop accident. An outcome probability of 1.0 is assigned Branch 2.

Case 2

This case includes all sequences in which the PRFT has been splashed by a previous energetic event. An outcome probability of 1.0 is assigned Branch 2.

Case 3 This case includes all sequences in which no event that could damage the PRFT has occurred. An outcome probability of 1.0 is assigned Branch 2.

Case 4 This case includes all sequences in, which there was a detonation of the PRFT during the third round of explosions. An outcome probability of 1.0 is assigned Branch 1.

Case 5 This case includes all sequences in which the wall separating the SPC and the CPC is displaced into the SPC. The PRFT is adjacent to this wall. An outcome probability of 0.5 is assigned Branch 1 and Branch 2.

Case 6

This case includes all sequences in which the cell covers are displaced and fall and there is a detonation in the OECT which is adjacent to the PRFT. The falling cell covers or shrapnel from the exploding OECT can penetrate the PRFT causing spillage. An outcome probability of 0.5 is assigned Branch 1 and Branch 2.

Case 7 This case includes all sequences in which the cell covers are displaced and fall and there is a deflagration in the OECT which is adjacent to the PRFT. The falling cell covers or shrapnel from the vent piping above the deflagrating OECT can penetrate the PRFT causing spillage. An outcome probability of 0.2 is assigned Branch 1 and an outcome probability of 0.8 is assigned Branch 2.

Case 8 - This case includes all sequences in which the cell covers are dislodged and fall. An outcome probability of 0.1 is assigned Branch 1 and an outcome probability of 0.9 is assigned Branch 2 .

Case 9 The default case includes all sequences remaining and assumes that all means by which the PRFT can be damaged have been included in the previous cases. An outcome probability of 1.0 is assigned Branch 2.

\section{Question 140: Does an energetic event in the SPC fail the removable wall} between the SPC and the CPC?

The Salt Process Cell and the Chemical Process Cell are separated by a removable [from above] partition. Some events in the SPC are assumed to be sufficiently energetic to displace 
the partition from its supports, causing damage to the tanks, piping, etc. in the CPC. There are two defined outcomes as follows:

FailWall: The wall separating the Salt Process Cell and the Chemical Process Cell is failed due to an energetic event in the third round of explosions.

nFailWal: The wall separating the Salt Process Cell and the Chemical Process Cell is not failed due to an energetic event in the third round of explosions.

This is a Type 2 question. The answers to this question are dependent on the outcomes of Questions 97,104, and 126 and are used in the logic to determine the outcome probabilities for Questions 142,143,144, 145, and 146. Three cases are used to develop the outcome probabilities as follows:

Case 1 This case includes all sequences in which the removable wall has been failed in a previous energetic event. An outcome probability of 1.0 is assigned Branch 2.

Case 2 This case includes all sequences in which a detonation has occurred in the PRFT during the third round of explosions. An outcome probability of 0.5 is assigned to Branch 1 and Branch 2 [7].

Case 3 The default case includes all sequences remaining. It is expected that all sequences in which the wall could be failed are included in previous cases. An outcome probability of 1.0 is assigned Branch 2.

\section{Question 141: Do the cell covers for the Chemical Process Cell dislodge and fall following an energetic event?}

The cell covers in the CPC can be dislodged and fall as a result of an energetic event in the CPC. The falling cell covers can damage equipment and piping in the cell. There are two defined outcomes as follows:

CPCovF1@2: The cell covers in the Chemical Process Cell dislodge and fall due to an energetic event during the third round of explosions.

nCPCovFl: The cell covers in the Chemical Process Cell do not dislodge and fall due to an energetic event during the third round of explosions.

This is a Type 2 question. The answer is dependent on the outcome of previous Questions 56 , $98,129,130,131,132,133$, and 134. The answers to this question are used in the logic to determine the outcome probabilities in Questions 142,143, 144, 145, and 146. Four cases are used to develop the outcome probabilities as follows:

Case 1 This case includes all sequences in which a previous event has caused the cell covers to be dislodged. An outcome probability of 1.0 is assigned Branch 2. 
Case 2 _ This case includes all sequences in which no energetic event has occurred in the CPC and a seismic event has not dislodged the cell covers. An outcome probability of 1.0 is assigned Branch 2 .

Case 3 This case includes all sequences in which a detonation or deflagration has occurred in a CPC vessel or piping during the third round of energetic events. An outcome probability of 1.0 is assigned Branch 1.

Case 4

The default case includes all sequences remaining and assumes that all means by which the cell covers can fall have been included in the previous cases. An outcome probability of 1.0 is assigned Branch 2.

\section{Question 142: Does splashing [spillage] of the Precipitate Reactor Bottoms Tánk contents occur?}

If the PRBT is damaged some or all of the contents of the tank may be spilled to the floor. This question is concerned only with damage to the PRBT caused by falling cell covers, a detonation in the PRBT during the third round of explosions, shrapnel from an adjacent tank generated during the third round of explosions or failure of the wall between the CPC and SPC. There are two defined outcomes as follows:

PRBT-Sp12: The contents of the Precipitate Reactor Bottoms Tank are spilled into the Chemical Process Cell after an energetic event during the third round of explosions.

nPRBT-Spl: The contents of the Precipitate Reactor Bottoms Tank are not spilled into the Chemical Process Cell.

This is a Type 2 question. The answers to this question are dependent on the outcome of previous Questions 58, 99,130,131,140, and 141. The answers to this question are used in the source term binner. Nine cases are used to develop the outcome probabilities as follows (The outcome probabilities are from Reference 7):

Case 1 This case includes all sequences in which the PRBT has been previously ruptured by a seismic event or crane load drop accident. An outcome probability of 1.0 is assigned Branch 2.

Case 2 This case includes all sequences in which the PRBT has been splashed by a previous energetic event. An outcome probability of 1.0 is assigned Branch 2.

Case 3 This case includes all sequences in which no event that could damage the PRBT has occurred. An outcome probability of 1.0 is assigned Branch 2 .

Case 4 This case includes all sequences in which there is a detonation in the Precipitate Reactor Bottoms Tank during the third round of explosions. Spillage of the tank contents will occur. An outcome probability of 1.0 is assigned Branch 1. 
Case 5 This case includes all sequences in which the removable wall betweed the CPC and the SPC has failed. Spillage of the tank contents is possible. An outcome probability of 0.5 is assigned Branch 1 and Branch 2 .

Case 6

This case includes all sequences in which the cell covers are displaced and fall and there is a detonation of the Slurry Mix Evaporator, which is adjacent to the PRBT. The falling cell covers or shrapnel from the detonating SME can penetrate the PRBT causing spillage. An outcome probability of 0.5 is assigned Branch 1 and Branch 2.

Case 7 This case includes all sequences in which the cell covers are displaced and fall and there is a deflagration of the Slurry Mix Evaporator, which is adjacent to the PRBT. The falling cell covers or shrapnel from the vent piping above the deflagrating SME can penetrate the PRBT causing spillage. An outcome probability of 0.2 is assigned Branch 1 and an outcome probability of 0.8 is assigned Branch 2 .

Case 8 This case includes all sequences in which the cell covers are displaced and fall. An outcome probability of 0.1 is assigned Branch 1 and an outcome probability of 0.9 is assigned Branch 2.

Case 9

The default case includes all sequences remaining and assumes that all means by which spillage of the contents of the Precipitate Reactor Bottoms Tank can occur have been included in the previous cases. An outcome probability of 1.0 is assigned Branch 2.

\section{Question 143: Does splashing [spillage] of the Slurry Mix Evaporator Tank contents occur?}

If the SME is damaged some or all of the contents of the tank may be spilled to the floor. This question is concerned only with damage to the SME caused by falling cell covers, a detonation in the SME during the third round of explosions, shrapnel from an adjacent tank generated during the third round of explosions or failure of the wall between the CPC and SPC. There are two defined outcomes as follows:

SME-Spl2: The contents of the Slurry Mix Evaporator are spilled into the Chemical Process Cell Cell after an energetic event during the third round of explosions.

nSME-Spl: The contents of the Slurry Mix Evaporator are not spilled into the Chemical Process Cell.

This is a Type 2 question. The answers to this question are dependent on the outcome of previous Questions 62,100,130,131,140, and 141. The answers to this question are used in the source term binner. Nine cases are used to develop the outcome probabilities as follows (The outcome probabilities are from Reference 7):

Case 1 This case includes all sequences in which the SME has been previously ruptured by a seismic event or crane load drop accident. An outcome probability of 1.0 is assigned Branch 2. 
April 7, 1995

WSRC-TR-95-0113

Page 170

Case 2 _. This case includes all sequences in which the SME has been splashed by a previous energetic event. An outcome probability of 1.0 is assigned Branch 2.

Case 3

This case includes all sequences in which no event that could damage the SME has occurred. An outcome probability of 1.0 is assigned Branch 2.

Case 4= This case includes all sequences in which there is a detonation of the Slurry Mix Evaporator during the third round of explosions. Spillage of the tank contents will occur. An outcome probability of 1.0 is assigned Branch 1.

Case 5

This case includes all sequences in which the cell covers are displaced and fall and a detonation occurs in the Precipitate Reactor Bottoms Tank, which is adjacent to the SME. The falling cell covers or shrapnel from the exploding PRBT can penetrate the SME causing spillage. An outcome probability of 0.5 is assigned Branch 1 and Branch 2 .

Case 6 This case includes all sequences in which the cell covers are displaced and fall and a deflagration occurs in the Precipitate Reactor Bottoms Tank, which is adjacent to the SME. The falling cell covers or shrapnel from the vent piping above the deflagrating PRBT can penetrate the SME causing spillage. An outcome probability of 0.2 is assigned Branch 1 and an outcome probability of 0.8 is assigned Branch 2 .

Case 7. This case includes all sequences in which the cell covers are displaced and fall. The falling cell covers can damage the SME. An outcome probability of 0.1 is assigned Branch 1 and an outcome probability of 0.9 is assigned Branch 2.

Case 8 This case includes all sequences in which the wall separating the SPC and the CPC is displaced into the CPC. The SME is separated from this wall by the PRBT. An outcome probability of 0.1 is assigned Branch 1 and an outcome probability of 0.9 is assigned Branch 2 .

Case 9 The default case includes all sequences remaining and assumes that all means by which spillage of the contents of the Slurry Mix Evaporator can occur have been included in the previous cases. An outcome probability of 1.0 is assigned Branch 2.

\section{Question 144: Does splashing [spillage] of the Sludge Receipt and Adjustment Tank contents occur?}

If the SRAT is damaged some or all of the contents of the tank may be spilled to the floor. This question is concerned only with damage to the SRAT caused by falling cell covers, a detonation in the SRAT during the third round of explosions, shrapnel from an adjacent tank generated during the third round of explosions or failure of the wall between the CPC and SPC. There are two defined outcomes as follows:

SRAT-Spl2: The contents of the SRAT are spilled into the Chemical Process Cell after an energetic event during the third round of explosions. 
nSRAT-Spl: The contents of the SRAT are not spilled into the Chemical Process Cell.

This is a Type 2 question. The answers to this question are dependent on the outcome of previous Questions 66,101,132,140, and 141. The answers to this question are used in the source term binner. Seven cases are used to develop the outcome probabilities as follows (The outcome probabilities are from Reference 7):

Case 1 This case includes all sequences in which the SRAT has been previously ruptured by a seismic event or crane load drop accident. An outcome probability of 1.0 is assigned Branch 2.

Case 2 This case includes all sequences in which the SRAT has been splashed by a previous energetic event. An outcome probability of 1.0 is assigned Branch 2.

Case 3 This case includes all sequences in which no event that could damage the SRAT has occurred. An outcome probability of 1.0 is assigned Branch 2.

Case 4 This case includes all sequences in which there is a detonation in the SRAT during the third round of explosions. Spillage of the tank contents will occur. An outcome probability of 1.0 is assigned Branch 1.

Case 5 This case includes all sequences in which the cell covers are displaced and fall. An outcome probability of 0.1 is assigned Branch 1 and an outcome probability of 0.9 is assigned Branch 2.

Case 6 This case includes all sequences in which the wall separating the SPC and the CPC is displaced into the CPC. The SRAT is separated from this wall by three other tanks. An outcome probability of 0.1 is assigned Branch 1 and an outcome probability of 0.9 is assigned Branch 2 .

Case 7 The default case includes all sequences remaining and assumes that all means by which spillage of the contents of the SRAT can occur have been included in the previous cases. An outcome probability of 1.0 is assigned Branch 2.

\section{Question 145: Does splashing [spillage] of the Recycle Collection Tank contents occur?}

If the RCT is damaged some or all of the contents of the tank may be spilled to the floor. This question is concerned only with damage to the RCT caused by falling cell covers, shrapnel from an adjacent tank generated during the third round of explosions or failure of the wall between the CPC and SPC.or shrapnel from an adjacent tank generated during the third round of explosions. There are two defined outcomes as follows:

RCT-Sp12: The contents of the RCT are spilled into the Chemical Process Cell after an energetic event during the third round of explosions.

nRCT-Spl: The contents of the RCT are not spilled into the Chemical Process Cell. 
This is a Type 2 question. The answers to this question are dependent on the outcome of previous Questions 70,102,132,133,140, and 141. The answers to this question are used in the source term binner. Eight cases are used to develop the outcome probabilities as follows (The outcome probabilities are from Reference 7):

Case 1 This case includes all sequences in which the RCT has been previously ruptured by a seismic event or crane load drop accident. An outcome probability of 1.0 is assigned Branch 2.

Case 2 This case includes all sequences in which the RCT has been splashed by a previous energetic event. An outcome probability of 1.0 is assigned Branch 2.

Case 3 This case includes all sequences in which no event that could damage the RCT has occurred. An outcome probability of 1.0 is assigned Branch 2.

Case 4 This case includes all sequences in which the cell covers a: Hisplaced and fall and there is a detonation in the MFT or the SRAT, wi ti are adjacent to the RCT. 'The falling cell covers or shrapnel from the exploding MFT or SRAT can penetrate the RCT causing spillage. An outcome probability of 0.5 is assigned Branch 1 and Branch 2 .

Case 5 This case includes all sequences in which the cell covers are displaced and fall and there is a deflagration in the MFT or the SRAT, which are adjacent to the RCT. The falling cell covers or shrapnel from the vent piping above the deflagrating MFT or SRAT can penetrate the RCT causing spillage. An outcome probability of 0.2 is assigned Branch 1 and an outcome probability of 0.8 is assigned Branch 2 .

Case 6 This case includes all sequences in which the cell covers are displaced and fall. The falling cell covers can damage the RCT. An outcome probability of 0.1 is assigned Branch 1 and an outcome probability of 0.9 is assigned Branch 2.

Case 7 This case includes all sequences in which the wall separating the SPC and the CPC is displaced into the CPC. The RCT is separated from this wall by four other tanks. An outcome probability of 0.1 is assigned Branch 1 and an outcome probability of 0.9 is assigned Branch 2 .

Case 8 The default case includes all sequences remaining and assumes that all means by which spillage of the contents of the RCT can occur have been included in the previous cases. An outcome probability of 1.0 is assigned Branch 2.

\section{Question 146: Does splashing [spillage] of the Melter Feed Tank contents occur?}

If the MFT is damaged some or all of the contents of the tank may be spilled to the floor. This question is concerned only with damage to the MFT caused by falling cell covers, a detonation in the MFT during the third round of explosions, shrapnel from an adjacent tank generated during the third round of explosions or failure of the wall between the CPC and SPC. or 
shrapnel from an adjacent tank generated during the third round of explosions. There are two defined outcomes as follows:

MFT-Sp12: The contents of the MFT are spilled into the Chemical Process Cell after an energetic event during the third round of explosions.

nMFT-Spl: The contents of the MFT are not spilled into the Chemical Process Cell.

This is a Type 2 question. The answers to this question are dependent on the outcome of previous Questions 72,103,133, and 141. The answers to this question are used in the source term binner. Seven cases are used to develop the outcome probabilities as follows (The outcome probabilities are from Reference 7):

Case 1 This case includes all sequences in which the MFT has been previously ruptured by a seismic event or crane load drop accident. An outcome probability of 1.0 is assigned Branch 2.

Case 2 This case includes all sequences in which the MFT has been splashed by a previous energetic event: An outcome probability of 1.0 is assigned Branch 2.

Case 3 This case includes all sequences in which no event that could damage the MFT has occurred. An outcome probability of 1.0 is assigned Branch 2.

Case 4 This case includes all sequences in which there is a detonation in the MFT during the third round of explosions. Spillage of the tank contents will occur. An outcome probability of 1.0 is assigned Branch 1.

Case 5 This case includes all sequences in which the cell covers are displaced and fall. An outcome probability of 0.1 is assigned Branch 1 and an outcome probability of 0.9 is assigned Branch 2.

Case 6 This case includes all sequences in which the wall separating the SPC and the CPC is displaced into the CPC. The MFT is separated from this wall by five other tanks. An outcome probability of 0.1 is assigned Branch 1 and an outcome probability of 0.9 is assigned Branch 2.

Case 7 The default case includes all sequences remaining and assumes that all means by which spillage of the contents of the MFT can occur have been included in the previous cases. An outcome probability of 1:0 is assigned Branch 2.

Question 147: Does a deflagration in the SPC lead to a deflagration in the RPC that damages the building?

A deflagration that occurs in the SPC due to spillage of large quantities of benzene from the OEV or OECT will blow the cell covers that separate the SPC from the Remote Process Cell (RPC). If the SPC deflagration is not followed by a fire that consumes the remainder of the benzene, it is possible for benzene vapors to migrate from the SPC to the RPC in sufficient quantity to reach LFL in the RPC. If an ignition source subsequently ignites these vapors, the resulting deflagration is assumed to cause severe damage to the vitrification building. For 
source term calculations, as a result of a RPC deflagration and the resultant building damage, all of the tanks in the building are assumed to have their contents spilled. There are two defined outcomes as follows:

VB-Clpse2: The Vitrification Building is damaged by a RPC deflagration.

nVB-Clps2: The Vitrification Building is not damaged by a RPC deflagration.

This is a Type 2 question. The answers to this question are dependent on the outcomes of Questions $1,107,108,110,118,119,121$, and 122 . The answers to this question are used in the source term binner. Three cases are used to develop the outcome probabilities as follows:

Case 1 This case includes all sequences in which the Vitrification Building has been collapsed by a seismic event. The outcome probability is specified as 1.0 for Branch 2 .

Case 2 This case includes all sequences in which the accident type is external or internal-energetic, the OEV or OECT have been splashed, and a SPC deflagration without a fire has occurred. An outcome probability of 0.9 is assigned to Branch 1 and an outcome probability of 0.1 is assigned to Branch 2 [7]. **SAMPLED VARIABLE**

Case 3 The default case includes all sequences remaining. It is expected that all sequences in which the vitrification building could be breached are included in previous cases. An outcome probability of 1.0 is assigned to Branch 2.

\section{Question 148: Do the cell covers over the Melter Cell dislodge and fall because of a seismic event?}

The process cells within the main canyon of the DWPF are 'roofed' by removable steel covers. These covers provide limited isolation of the cell in the event of an in-cell release, assist in maintaining proper ventilation flow, and protect the equipment within the cell from falling objects. However, if the cell covers are dislodged and fall into the cell, either through a seismic event or an energetic event within the cell, they may damage the equipment in the cell. This question examines the impact of a seismic event on the covers for the Melter Cell. There are two possible outcomes as follows:

fMCvSeis: The Melter Cell covers are dislodged by the Seismic event and fall into the cell.

nfMCvSei: The Melter Cell covers remain in place following the Seismic event.

This is a Type 6 question. The answer to this question depends upon the outcome of previous Question 2 and the value specified for the parameter CCovFr, the seismic fragility of the covers for the Salt and Chemical Process Cells and the Melter Cells. The answers to this question are used in the logic to determine the outcome probabilities in Questions 149 and 156. Two cases are used to develop the outcome probabilities on the basis of prior events as follows:

Case 1 This case includes those sequences in which the initiating event is a seismic event. The seismic fragility of the covers for the Melter Cell [the 


\section{probability the cell covers will fall] is placed in Branch 1. The outcome} probability for Branch 2 is 1.0 less the probability assigned Branch 1.

Case'2

The default case includes all other sequences. The outcome probability assigned is 1.0 in Branch 2, there is no seismic event and the cell covers do not fall.

\section{Question 149: Do the cell covers that fall in a seismic event strike the Melter?}

Falling cell covers during a seismic event can rupture and spill the contents of the Melter. There are two possible outcomes as follows:
MCv-Melt:
The Melt Cell Covers are dislodged by the Seismic event and fall on the Melter.

nMCv-Mel: $\quad$ The Melt Cell Covers do not fall on the Melter.

This is a Type 2 question. The answer to this question depends upon the outcome of previous Questions 2 and 148. The answers to this question are used in the logic to determine the outcome probabilities in Question 150 and 151. Two cases are used to develop the outcome probabilities on the basis of prior events as follows:

Case 1 This case includes those sequences in which the initiating event is a seismic event and the Melt Cell covers fell due to the seismic event. The outcome probability for Branch 1 is 0.01 , the outcome probability for Branch 2 is 0.99 [7].

Case 2 The default case includes all other sequences. The outcome probability assigned is 1.0 in Branch 2.

\section{Question 150: Does a Seismic event rupture and spill the contents of the Melter in the Melter Cell?}

Falling cell covers during a seismic event can rupture and spill the contents of the Melter. Shaking and overturning of the tank during a seismic event can also cause spillage of the contents. There are two defined outcomes to this question as follows:

RptMelt: The Melter is ruptured and the contents spilled as a result of the seismic event.

nRptMelt: The Melter is intact following the seismic event.

This is a Type 6 question. The answer to this question depends upon the outcome of previous Questions 2, 148, and 149 and the value specified for the parameter MeltFr, the seismic fragility of the Melter. The answers to this question are used in the logic to determine the outcome probabilities in Questions 153,155, and 157. Three cases are used to develop the outcome probabilities on the basis of prior events as follows:

Case 1 This case includes all sequences in which a seismic event has caused the cell covers over the Melter Cell to fall and strike the Melter. The falling 
cell covers are assumed to rupture and spill the contents of the Melter. An

-. outcome.probability of 1.0 is assigned Branch 1 .

Case 2 . This case includes all sequences in which a seismic event has occurred but the cell covers do not fall or if they fall they do not strike the Melter. The · seismic fragility of the Melter [the probability that a seismic event will cause a structural failure of the Melter] is placed in Branch 1. The outcome probability for Branch 2 is 1.0 less the probability assigned Branch 1.

Case 3

The default case includes all remaining sequences. The outcome probability assigned is 1.0 in Branch 2.

\section{Question 151: Does a Seismic event rupture the Melter Offgas System in the Melter Cell?}

Falling cell covers during a seismic event can rupture the Melter Offgas system. Shaking during a seismic event can also damage the Melter Offgas system. There are two defined outcomes to this question as follows:

RptMog: ' The Melter Offgas. System is ruptured as a result of the seismic event.

nRptMog: The Melter Offgas System is intact following the seismic event.

This is a Type 6 question. The answer to this question depends upon the outcome of previous Questions 2, 148, and 149 and the value specified for the parameter MOG_Fr, the seismic fragility of the Melter Offgas System. The answers to this question are used in the logic to determine the outcome probabilities in Questions 153 and 157. Three cases are used to develop the outcome probabilities on the basis of prior events as follows:

Case 1 This case includes all sequences in which a seismic event has caused the cell covers over the Melter Cell to fall and strike the Melter Offgas System. The falling cell covers are assumed to rupture the Melter Offgas System. An outcome probability of 1.0 is assigned Branch 1.

Case 2 This case includes all sequences in which a seismic event has occurred but the cell covers do not fall. The seismic fragility of the Melter Offgas System is placed in Branch 1 . The outcome probability for Branch 2 is 1.0 less the probability assigned Branch 1 .

Case 3 The default case includes all remaining sequences. The outcome probability assigned is 1.0 in Branch 2.

Question 152: Does an explosive concentration exist in the Melter Offgas System?

Chemical reactions in the Meirar produce combustible gases including hydrogen and carbon monoxide. The melter vapor ce is maintained above $650^{\circ} \mathrm{C}$ by four groups of heaters in the dome of the Melter. This tes. :erature assures that the combustible gases burn as produced 
rather than accumulating to an explosive concentration. The two outcomes defined for this question are as follows:

Con-MOG: An explosive concentration accumulates in the Melter Offgas System.

nConMOG: An explosive concentration does not accumulate in the Melter Offgas System.

This is a Type 6 question and uses previously quantified parameters $[62,70,71,90,93$, and 103] and a user defined function to determine the probability that an explosive concentration will occur in the MOG. The user function includes information from solution of the fault tree for a MOG explosion. The function used to calculate the answer depends on the answer to previous Question 1. The outcome probabilities are used in the logic to determine the outcome probabilities in Question 153.

The following case structure is simplified by not considering the possibility of prior rupture of the Melter or Melter Offgas System during a seismic event. Rupture of the Melter or Melter Offgas System would prevent the buildup of an explosive concentration in the MOG. The possibility of prior rupture of the Melter or Melter Offgas System is included in Question 153. Five cases are used to develop the outcome probabilities as follows:

Case 1 This case is used to exclude those sequences in the classes of accidents which are neither external nor internal-energetic. The outcome probability assigned is 1.0 in Branch 2.

Case 2 This case includes all sequences in which the accident class is internalenergetic and the normal power system has failed. Failure of normal power stops the melter process and precludes the formation of an explosive concentration. An outcome probability of 1.0 is assigned Branch 2.

Case 3 This case includes all sequences in which the class of accidents is internalenergetic [Branch 2, Question 1]. The outcome probability is calculated using FUN-OGAS and assigned to Branch 1 . The outcome probability for Branch 2 is 1.0 less the probability assigned Branch 1 .

Case $4 \quad$ This case includes all sequences in which the class of accidents is external [Branch 1, Question 1]. The outcome probability is calculated using FUN-OGASS and assigned to Branch 1 . The outcome probability for Branch 2 is 1.0 less the probability assigned Branch 1 .

Case 5 The default case assumes that all sequences in which an explosive concentration can occur are included in Cases 3 and 4. An outcome probability of 1.0 is assigned Branch 2 .

\section{Question 153: Does a damaging detonation occur in the Melter Offgas System?}

If an explosive concentration occurs in the Melter Offgas System and an ignition source is available it is assumed that a damaging explosion will follow. The fault tree for the MOG explosion includes the probability of an ignition source. Therefore, if Question 152 indicates 
that an explosive concentration is available then an explosion will follow. An explosion in the Melter Offgas System can release the contents of the Melter Offgas into the Melter Cell. There are two definied outcomes as follows:

MOG-Det: A damaging detonation occurs in the Melter Offgas System.

nMOG-Ex: No damaging detonation occurs in the Melter Offgas System.

This is a Type 2 question. The answer is dependent on the outcome of previous Questions 150,151 , and 152. The answers to this question are used in the logic to determine the outcome probabilities in Questions 156 and 157. Five cases are used to develop the outcome probabilities as follows:

Case 1 This case includes all sequences in which an explosive concentration does not accumulate in the MOG. The outcome probability assigned is 1.0 in Branch 2.

Case 2 This case includes the sequences in which a prior event has ruptured the Melter, therefore, venting the offgas system and precluding the accumulation of an explosive concentration. The outcome probability assigned is 1.0 in Branch 2.

Case 3 This case includes all sequences in which the Melter Offgas System has been ruptured, precluding the accumulation of an explosive concentration. An outcome probability of 1.0 is assigned Branch 2.

Case 4 This case includes the sequences in which a prior event has not ruptured the MOG and an explosive concentration has accumulated in the system. The outcome probability assigned is 1.0 in Branch 1.

Case 5 The default case includes any remaining sequences. It is assumed that all sequences resulting in a damaging explosion are included in previous cases. The outcome probability assigned is 1.0 in Branch 2.

\section{Question 154: Does a damaging steam explosion occur due to a salt/water reaction in the Melter?}

Conditions that could lead to a steam explosion in the melter require the presence of both water and partially melted excess salt. A fault tree was constructed to calculate the frequency of steam explosions in the Melter due to a salt/water reaction [5]. There are no major support systems modeled in the Melter steam explosion fault tree. The events in the tree include only human errors and failures of sampling equipment used to verify the salt content of each melter batch. The solution of this fault tree (cut sets) is used to determine the frequency of a steam explosion in the Melter due to a salt/water reaction. Such an explosion could damage the Melter and spill the contents or release melter offgas into the Melter Cell. The two outcomes defined for this question are as follows:

Con-MEL: A damaging salt/water reaction takes place in the Melter.

nConMEL: A damaging salt/water reaction does not take place in the Melter. 
This is a Type 6 question and uses previously quantified parameters $[70,71,90]$ and a user defined function to determine the probability that an salt/water reaction will occur in the Melter. The user function includes information from solution of the fault tree for the Melter salt/water reaction. The function used to calculate the answer depends on the answer to previous Question 1. The answers to this question are used in the logic to determine the outcome probabilities in Question 155. Four cases are used to develop the outcome probabilities on the basis of prior events as follows:

Case 1

This case includes all sequences in which the accident type is not external or internal-energetic. A salt/water reaction cannot happen for the other accident types. An outcome probability of 1.0 is assigned Branch 2.

Case 2 This case includes all sequences in which the class of accidents is internalenergetic [Branch 2, Question 1]. The outcome probability is calculated using FUN-SMEL and assigned to Branch 1 . The outcome probability for Branch 2 is 1.0 less the probability assigned Branch 1

Case 3 This case includes all sequences in which the class of accidents is external [Branch 1; Question 1]. The outcome probability is calculated using FUN-SMELS and assigned to Branch 1 . The outcome probability for Branch 2 is 1.0 less the probability assigned Branch 1

Case 4 The default case includes all remaining sequences. It is expected that all sequences that would result in a steam explosion have been identified in the previous cases. The outcome probability assigned is 1.0 in Branch 2 .

\section{Question 155: Does a damaging reaction occur in the Melter?}

The fault tree for the Melter Explosion includes all factors necessary for a salt/water reaction to occur. Therefore, if Question 154 indicates that an reaction occurs, then a steam explosion will follow. A steam explosion in the Melter can spill the contents of the vessel, damage the Melter Offgas system, and blow the cell covers. There are two defined outcomes as follows:

MEL-Exp: . A damaging steam explosion occurs in the Melter.

nMEL-Ex: No damaging explosion occurs in the Melter.

This is a Type 2 question. The answer is dependent on the outcome of previous Questions 150 and 154. The answers to this question are used in the logic to determine the outcome probabilities in Questions 156 and 157. Four cases are used to develop the outcome probabilities as follows:

Case 1

This case includes all sequences in which an salt/water reaction does'not take place in the Melter. The outcome probability assigned is 1.0 in Branch 2.

Case 2 This case includes the sequences in which a prior event has ruptured the Melter precluding a steam explosion. The outcome probability assigned is 1.0 in Branch 2. 
Case $3 \quad \cdots \quad$ This case includes the sequences in which a prior event has not ruptured the Melter and a salt/water reaction has taken place in the vessel. The outcome probability assigned is 1.0 in Branch 1.

Case 4

The default case includes any remaining sequences. It is assumed that all sequences resulting in a damaging explosion are included in previous cases. The outcome probability assigned is 1.0 in Branch 2.

\section{Question 156: Do the cell covers for the Melter Cell dislodge and fall following an energetic event?}

The previous Question : seismic event has not occ $\because d$ it is assumed that the cell covers are in place prior to any in-cell energetic event. If a seisi: $;:$ event has occurred then the cell covers may have been dislodged. The assumed effect of disiciged cell covers on the tanks and equipment in the cell is essentially the same, whether dislodged by a seismic event or an in-cell explosion. Falling cell covers can rupture and spill the contents of the Melter or damage the Melter Offgas System. There are two defined outcomes as follows:

MCovFal: The cell covers in the Melter Cell dislodge and fall, either the consequence of a seismic event or an in-cell explosion.

nMCovFal: The cell covers in the Melter Cell are in place.

This is a Type 2 question. The answer is dependent on the outcome of previous Questions 2, 148,153 , and 155. The answers to this question are used in the logic to determine the outcome probabilities in Question .157. Five cases are used to develop the outcome probabilities as follows:

Case 1 This case includes all sequences in which the there is no explosion in the Melter Offgas System, no expiosion in the Melter, and no Seismic event. An outcome probability of $1 . \hat{0}$ is assigned Branch 2 .

Case 2 This case includes all sequences in which the cell covers were dislodged by a seismic event. An outcome probability of 1.0 is assigned Branch 1 .

Case 3 This case includes all sequences in which there is an explosion in the Melter Offgas System. The explosions are not sufficiently energetic to dislodge the cell covers [13]. An outcome probability of 1.0 is assigned Branch 2.

Case 4 This case inciudes all sequences in which there is an explosion in the Melter. It is assumed that these explosions are sufficiently energetic to dislodge the cell covers. An outcome probability of 1.0 is assigned Branch 1.

Case 5 The default case includes all sequences remaining and assumes that all means by which the cell covers can fall have been included in the previous cases. An outcome probability of 1.0 is assigned Branch 2. 


\section{Question 157: Does splashing [spillage] of the Melter contents occur as the} result of energetic events in the Melter Cell?

If the Melter or the Melter Offgas System is damaged some or all of the contents of the Melter may be spilled to the floor and/or the offgases released to the cell. Damage to the Melter can be caused by falling cell covers (including crane drops), by shaking during a Seismic event and by an explosion associated with the Melter. Spilling the contents of the Melter is of concern primarily because the contents are radioactive. Failure of the Offgas System may release combustible gases and volatized radioactive material to the cell. There are four defined outcomes as follows:

tMSpl/OG: All of the contents of the Melter and the Melter Offgas System are released to the Melter Cell.

pMSpl/OG: Part of the contents of the Melter and the Melter Offgas System are released to the Melter Cell.

Mlt-OG: The Melter Offgas System is damaged and its contents released to the Melter Cell.

nMltRlse: There is no release of material from the Melter or the Melter Offgas System.

This is a Type 2 question. The answers to this question are dependent on the outcome of previous Questions 1,2, 4, 5, 39,150,151,153,155, and 156. The answers are not used in the logic to determine the outcome probabilities in other questions but are used in the source term binning file. Nine cases are used to develop the outcome probabilities as follows (The outcome probabilities are from Reference 9):

Case 1 . This case includes all sequences in which there is a limited leak in the Melter. Some spillage of the tank contents will occur. An outcome probability of 0.01 is assigned Branch 2; an outcome probability of 0.99 is assigned Branch 3. **SAMPLED VARIABLE**

Case 2 This case includes all sequences in which there was a seismic initiated rupture of the Melter, either from falling cell covers or from shaking. An outcome probability of 1.0 is assigned Branch 1 .

Case 3 This case includes all sequences in which there was a seismic initiated rupture of the Melter Offgas System, either from falling cell covers or from shaking. An outcome probability of 1.0 is assigned Branch 3.

Case 4 This case includes all sequences in which the covers of the Melter Cell fall from an energetic event in the cell. An outcome probability of 0.01 is assigned Branch 2 and an outcome probability of 0.99 is assigned Branch 3. ${ }^{* *}$ SAMPLED VARIABLE**

Case 5 This case includes all sequences in which the covers of the Melter Cell fall due to a crane load drop accident. An outcome probability of 0.01 is 
assigned Branch 2 and an outcome probability of 0.99 is assigned Branch 3. **SAMPLED VARIABLE**

Case 6 . This case includes all sequences in which there is a steam explosion in the Melter. An outcome probability of 0.01 is assigned Branch 2 and an outcome probability of 0.99 is assigned Branch 3. **SAMPLED VARIABLE**

Case 7 This case includes all sequences in which there is an explosion in the Melter Offgas System. An outcome probability of 1.0 is assigned Branch 3.

Case 8 This case includes all sequences in which there is injection of water to the Melter. A steam explosion does not occur and water injection continues until all the Melter contents are displaced and discharged to the cell floor. An outcome probability of 1.0 is assigned Branch 1 .

Case 9 The default case includes all sequences remaining and assumes that all means by which spillage of the contents of the Melter and the Melter Offgas System can occur have been included in the previous cases. An outcome probability of 1.0 is assigned Branch 4 .

Question 158: Does a Seismic event or tornado rupture and spill the contents of the Organic Waste Storage Tank?

A Seismic event or a tornado can damage the Organic Waste Storage Tank [OWST] causing spillage of the stored organic material, primarily benzene. Spillage of the material is of concern because benzene is a carcinogen and highly flammable. There are two defined outcomes to this question as follows:

RptOWST: The OWST is ruptured and the contents spilled as a result of the Seismic event or tornado.

nRptOWST: The OWST is intact following the seismic event or tomado.

This is a Type 6 question. The answer to this question depends upon the outcome of previous Question 2 and the value specified for the parameter OWSTFr, the fragility of the OWST to a seismic event or tornado. The answers to this question are used in the logic to determine the outcome probabilities in Questions 160 and 161. Three cases are used to develop the outcome probabilities on the basis of prior events as follows:

Case 1. This case includes all sequences in which a seismic event has occurred. The seismic fragility of the OWST [the probability that a seismic event will cause a structural failure of the OWST] is placed in Branch 1. The outcome probability for Branch 2 is 1.0 less the probability assigned Branch 1.

Case 2 This case includes all sequences in which a tornado has impacted the OWST [Question 2, Branch 3]. The tornado fragility of the OWST [the probability that a tomado will cause a structural failure of the OWST] is 
placed in Branch 1. The outcome probability for Branch 2 is 1.0 less the probability assigned Branch 1.

Case 3

The default case includes all remaining sequences. The outcome probability assigned is 1.0 in Branch 2.

NOTE: The tornado portion of the above question was included as a place holder. Only the seismic portion of this question was used for the current analysis. It is recognized that the tornado fragility would be different than the seismic fragility.

\section{Question 159: Does an explosive concentration accumulate in the Organic Waste Storage Tank?}

Nitrogen is provided to the inner tank of the OWST to protect against combustion of the benzene vapors. An explosion can occur in the inner tank if the amount of oxygen in the vapor space reaches the minimum required for combustion and the vapor is ignited. An explosion can occur in the annulus between the inner and outer tanks if sufficient benzene lẹaks from the inner tank and the benzene vapors are ignited. A fault tree was constructed to calculate the frequency of explosions in the OWST for all credible initiators [5]. The solution of this fault tree (cut sets) is used to determine the frequency ( or probability) of an explosive concentration in the OWST. The two outcomes defined for this question are as follows:

ConOWST: An explosive concentration accumulates in the OWST.

nConOWST: An explosive concentration does not accumulate in the OWST.

This is a Type 6 question and uses previously quantified parameters $[60,61,67,70,71,90$, and 94] and a user defined function to determine the probability that an explosive concentration will occur in the OWST. The user function includes information from solution of the fault tree for an OWST explosion. The function used to calculate the answer depends on the answer to previous Question 1. The outcome probabilities are used in the logic to determine the outcome probabilities in Question 160.

The following case structure is simplified by not considering the possibility of prior rupture of the OWST during a Seismic event or a tornado [Question 158]. Rupture of the OWST would prevent the buildup of an explosive concentration. The possibility of prior rupture of the OWST is inciuded in Question 160. Four cases are used to develop the outcome probabilities as follows:

Case 1 This case is used to exclude those sequences in the classes of accidents [Question 1] which are neither external nor internal-energetic. The outcome probability assigned is 1.0 in Branch 2.

Case 2 This case includes all sequences in which the class of accidents is internalenergetic [Branch 2, Question 1]. The outcome probability is calculated using FUN-OWST and assigned to Branch 1 . The outcome probability for Branch 2 is 1.0 less the probability assigned Branch 1. 
Case 3 This case includes all sequences in which the class of accidents is external - [Branch 1, Question 1]. The outcome probability is calculated using FUN-OWSTS and assigned to Branch 1 . The outcome probability for Branch 2 is 1.0 less the probability assigned Branch 1.

Case 4

The default case assumes that all sequences in which an explosive concentration can occur are included in Cases 2 and 3. An outcome probability of 1.0 is assigned Branch 2.

\section{Question 160: Does a damaging deflagration occur in the Organic Waste Storage Tank.[OWST]?}

If an explosive concentration occurs in the Organic Waste Storage Tank [OWST] and an ignition source is available it is assumed that a damaging explosion will follow. The fault tree for the OWST deflagration includes the probability of an ignition source. Therefore, if Question 159 indicates that an explosive concentration is available then a deflagration will follow. A deflagration in the OWST will rupture the tank spilling the inventory. There are two defined outcomes as follows:

OWST-Def: A damaging deflagration occurs in the OWST.

nOWST-Ex: No damaging explosion occurs in the OWST.

This is a Type 2 question. The answer is dependent on the outcome of previous Questions 158 and 159. The answers to this question are used in the logic to determine the outcome probabilities in Question 161. Four cases are used to develop the outcome probabilities as follows:

Case 1 This case includes all sequences in which an explosive concentration does not accumulate in the OWST. The outcome probability assigned is 1.0 in Branch 2.

Case 2 This case includes the sequences in which a prior event has ruptured the OWST, therefore, spilling the contents and precluding the accumulation of an explosive concentration. The outcome probability assigned is 1.0 in Branch 2.

Case 3 This case includes the sequences in which a prior event has not ruptured the OWST and an explosive concentration has accumulated in the system. The outcome probability assigned is 1.0 in Branch 1.

Case 4 The default case includes any remaining sequences. It is assumed that all sequences resulting in a damaging deflagration are included in previous cases. The outcome probability assigned is 1.0 in Branch 2. 
Case 3 This case includes the sequences in which a prior event has not ruptured the PPPT and an explosive concentration has accumulated in the vessel. An outcome probability of 0.001 is assigned Branch 1 and an outcome probability of 0.999 is assigned Branch 2 [7]. **SAMPLED VARIABLE**

Case 4

The default case includes any remaining sequences. It is assumed that all sequences resulting in a damaging explosion are included in previous cases. The outcome probability assigned is 1.0 in Branch 3 , there is no explosion.

\section{Question 167: Does splashing [spillage] of the Pump Pit Precipitate Tank contents occur?}

If the Pump Pit Precipitate Tank [PPPT] is damaged some or all of the contents of the tank may be spilled to the floor. Damage to the PPPT can be caused by falling cell covers, by shaking during a Seismic event and by an explosion associated with the PPPT. Spilling the contents of the PPPT is of concern because the contents are radioactive. There are two defined outcomes as follows:

PPPTSpl: The contents of the PPPT are spilled into the Low Point Pump Pit.

nPPPTSpl: The contents of the PPPT are not spilled into the Low Point Pump Pit.

This is a Type 2 question. The answers to this question are dependent on the outcome of previous Questions 164 and 166 and are used in the logic to determine the outcome probabilities in Questions 169 and 171. Four cases are used to develop the outcome probabilities as follows:

Case 1 This case includes all sequences in which there was no explosion of the PPPT and there was no seismic initiated rupture of the PPPT. An outcome probability of 1.0 is assigned Branch 2 .

Case 2 This case includes all sequences in which there was a prior rupture of the PPPT. Spillage of the tank contents will occur. An outcome probability of 1.0 is assigned Branch 1.

Case 3 This case includes all sequences in which there is a detonation of the PPPT. Spillage of the tank contents will occur. An outcome probability of 1.0 is assigned Branch 1.

Case 4 The default case includes all sequences remaining and assumes that all means by which spillage of the contents of the PPPT can occur have been included in the previous cases. An outcome probability of 1.0 is assigned Branch 2. 
Question 168: Does an explosive concentration accumulate in the Pump Pit Precipitate Tank Cell?

This question was included as a place holder during APET development. Subsequent analysis indicates that it is not possible to develop an explosive mixture in the PPPC. Since no cutset file is provided to the APET for this. accident during the quantification of the tree, the UFUN returns a value of 0 for the probability that an explosive concentration exists.

The two outcomes defined for this question are as follows:

ConPPPC: An explosive concentration accumulates in the Pump Pit Precipitate Tank Cell.

nConPPPC: An explosive concentration does not accumulate in the Pump Pit Precipitate Tank Cell.

This is a Type 6 question and would use previously quantified parameters and a user defined function to determine the probability that an explosive concentration will occur in the PPPC if it was possible for one to occur. The function used to calculate the answer depends on the answer to previous Question 1. The outcome probabilities are used in the logic to determine the outcome probabilities in Question 169.

The following case structure does not consider leakage from the tank but only extracts information from the fault tree on loss of veritilation to the cell and an ignition source. Leakage from the tank is considered in Question 167. Four cases are used to develop the outcome probabilities as follows:

Case 1 This case is used to exclude those sequences in the classes of accidents [Question 1] which are neither external nor internal-energetic. The outcome probability assigned is 1.0 in Branch 2 .

Case 2 This case includes all sequences in which the class of accidents is internalenergetic [Branch 2, Question 1]: The outcome probability is calculated using FUN-PPPC and assigned to Branch 1 . The outcome probability for Branch 2 is 1.0 less the probability assigned Branch 1.

Case 3 This case includes all sequences in which the class of accidents is extemal [Branch 1, Question 1]. The outcome probability is calculated using FUN-PPPCS and assigned to Branch 1 . The outcome probability for Branch 2 is 1.0 less the probability assigned Branch 1 .

Case 4. The default case assumes that all sequences in which an explosive concentration can occur are included in Cases 2 and 3. An outcome probability of 1.0 is assigned Branch 2.

Question 169: Does a damaging explosion occur in the Pump Pit Precipitate Tank Cell?

This question was included as a place holder during APET development. Subsequent analysis indicates that it is not possible to develop an explosive 
mixture in the PPPC. Since no cutset file is provided to the APET for this accident during the quantification of the tree, the UFUN returns a value of 0 for the probability that an explosive concentration exists. The outcome probabilities for each case in this question have been set to 1.0 in Branch 3, there is no explosion.

There are three defined outcomes as follows:

PPṔCDet: A damaging detonation occurs in the Pump Pit Precipitate Tank Cell.

PPPCDef: A damaging deflagration occurs in the Pump Pit Precipitate Tank Cell.

nPPPCExp: No damaging explosion occurs in the Pump Pit Precipitate Tank Cell.

This is a Type 2 question. The answer is dependent on the outcome of previous Questions 167 and 168. The answers to this question are used in the logic to determine the outcome probabilities in Questions 170 and 193. Three cases are used to develop the outcome probabilities as follows:

Case 1 This case includes all sequences in which an explosive concentration does not accumulate in the PPPC [i.e., ventilation is not lost, an ignition source is not available, or no spillage into the cell has occurred]. As stated above, the outcome probability assigned is 1.0 in Branch 3.

Case 2 This case includes the sequences in which a prior event has ruptured the PPPT and an explosive concentration does not accumulate in the PPPC. As stated above, the outcome probability assigned is 1.0 in Branch 3.

Case 3 The default case includes any remaining sequences. It is assumed that all sequences resulting in a damaging explosion are included in previous cases. As stated above, the outcome probability assigned is 1.0 in Branch 3.

Question 170: Do the cell covers for the Pump Pit Precipitate Cell dislodge and fall following an energetic event?

A previous question determined the impact of a seismic event on the cell covers. If a seismic event has not occurred it is assumed that the cell covers are in place prior to any in-cell energetic event. If a seismic event has occurred then the cell covers may have been dislodged. The assumed effect of dislodged cell covers on the tanks.and equipment in the cell is essentially the same, whether dislodged by a seismic event or an in-cell explosion. Falling cell covers can rupture and spill the contents of the tanks in the Pump Pit Precipitate Cell. There are two defined outcomes as follows:

fPPPCov: The cell covers dislodge and fall, either the consequence of a seismic event or an in-cell explosion.

nfPPPCov: The cell covers are in place.

This is a Type 2 question. The answers to this question are dependent on the outcome of previous Questions 162,166, and 169 and are used in the logic to determine the outcome 
probabilities in Questions 171and 193. Five cases are used to develop the outcome probabilities as follows:

Case 1 This case includes all sequences in which there was no explosion of the Pump Pit Precipitate Tank, no explosion in the Pump Pit Precipitate Tank Cell, and the cell covers were not dislodged previously by a seismic event. An outcome probability of 1.0 is assigned Branch 2 .

Case 2 This case includes all sequences in which the cell covers were dislodged previously by a seismic event. An outcome probability of 1.0 is assigned Branch 1.

Case 3 This case includes all sequer ?s in which there was an explosion of the Pump Pit Precipitate Tank 11. An outcome probability of 1.0 is assigned Branch 1.

Case 4 This case includes all sequences in which there was an explosion of the Pump Pit Precipitate Tank. An outcome probability of 1.0 is assigned Branch 1.

Case 5. The default case includes all sequences remaining and assumes that all means by which the cell covers can fall have been included in the previous cases. An outcome probability of 1.0 is assigned Branch 2.

\section{Question 171: Does splashing [spillage] of the Pump Pit Precipitate Tank contents occur?}

If the Pump Pit Precipitate Tank [PPPT] is damaged some or all of the contents of the tank may be spilled to the floor. Damage to the PPPT can be caused by falling cell covers, by shaking during a Seismic event and by an explosion associated with the PPPT. Spilling the contents of the PPPT is of concern because the contents are radioactive. There are two defined outcomes as follows:

PPPTSpl@2: The contents of the PPPT are spilled into the Low Point Pump Pit.

nPPPTSpl: The contents of the PPPT are not spilled into the Low Point Pump Pit.

This is a Type 2 question. The answers to this question are dependent on the outcome of previous Questions 164 and 166 and are used in the logic to determine the outcome probabilities in Question 169 and 171. Four cases are used to develop the outcome probabilities as follows:

Case 1 This case includes all sequences in which there was prior rupture of the PPPT. Spillage of the tank contents will occur. An outcome probability of 1.0 is assigned Branch 1.

Case 2 This case includes all sequences in which the LPPP cell covers have fallen. An outcome probability of 0.9 is assigned Branch 1 and an outcome probability of 0.1 is assigned Branch 2 [7]. **SAMPLED VARIABLE** 
Case 3 .. This case includes all sequences in which a crane load drop accident occurs. Spillage of the tank contents will occur. An outcome probability of 1.0 is assigned Branch 1.

Case 4 The default case includes all sequences remaining and assumes that all means by which spillage of the contents of the PPPT can occur have been included in the previous cases. An outcome probability of 1.0 is assigned Branch 2.

Question 172: Does a Seismic event rupture and spill the contents of the Sludge Tank in the Low Point Pump Pit?

Falling cell covers during a seismic event can rupture and spill the contents of the Pump Pit Sludge Tank [PPST]. Shaking and overturning of the tank during a seismic event can also cause spillage of the contents. There are two defined outcomes to this question as follows:

RptPPST: The Pump Pit Sludge Tank is as a result of the seismic event.

nRptPPST: The Pump Pit Sludge Tank is intact following the seismic event.

This is a Type 6 question. The answer to this question depends upon the outcome of previous Question 2, 162, and 163 and the value specified for the parameter PPSTFr, the seismic fragility of the Pump Pit Sludge Tank. The answers to this question are used in the logic to determine the outcome probabilities in Questions 174 and 175. Three cases are used to develop the outcome probabilities on the basis of prior events as follows:

Case 1 This case includes all sequences in which a seismic event has caused the cell covers over the Low Point Pump Pit to fall and strike the tank. The falling cell covers are assumed to rupture and spill the contents of the PPST. An outcome probability of 1.0 is assigned Branch 1.

Case 2 This case includes all sequences in which a seismic event has occurred but the cell covers do not fall or if they fall they do not strike the tank. The seismic fragility of the PPST [the probability that a seismic event will cause a structural failure of the PPST] is placed in Branch 1. The outcome probability for Branch 2 is 1.0 less the probability assigned Branch 1.

Case 3 The default case includes all remaining sequences. The outcome probability assigned is 1.0 in Branch 2.

\section{Question 173: Does an explosive concentration accumulate in Pump Pit Sludge Tank?}

A fault tree was constructed to calculate the frequency of explosions in the PPST for all credible initiators [5]. The solution of this fault tree (cut sets) is used to determine the frequency (or probability) of an explosive concentration in the PPST. The two outcomes defined for this question are as follows:

ConPPST: An explosive concentration accumulates in the Pump Pit Sludge Tank. 


\section{nConPPST: An explosive concentration does not accumulate in the Pump Pit Sludge} Tank.

This is a Type 6 question and uses previously quantified parameters $[60,61,67,70,71,90$, 94 , and 113] and a user defined function to determine the probability that an explosive concentration will occur in the PPST. The user function includes information from solution of the fault tree for a PPST explosion. The function used to calculate the answer depends on the answer to previous Question 1. The outcome probabilities are used in the logic to determine the outcome probabilities in Question 174.

The following case structure is simplified by not considering the possibility of prior rupture of the PPST during a seismic event [Question 172]. Rupture of the PPST would prevent the buildup of an explosive concentration in the PPST. The possibility of prior rupture of the PPST is included in Question 174. Four cases are used to develop the outcome probabilities as follows:

Case 1 This case is used to exclude those sequences in the classes of accidents [Question 1] which are neither external nor internal-energetic. The outcome probability assigned is 1.0 in Branch 2.

Case 2 This case includes all sequences in which the class of accidents is internalenergetic [Branch 2, Question 1]. The outcome probability is calculated using FUN-PPST and assigned to Branch 1 . The outcome probability for Branch 2 is 1.0 less the probability assigned Branch 1.

Case 3 This case includes all sequences in which the class of accidents is external [Branch 1, Question 1]. The outcome probability is calculated using FUN-PPSTS and assigned to Branch 1 . The outcome probability for Branch 2 is 1.0 less the probability assigned Branch 1.

Case 4 The default case assumes that all sequences in which an explosive concentration can occur are included in Cases 2 and 3 . An outcome probability of 1.0 is assigned Branch 2.

\section{Question 174: Does a damaging detonation/deflagration occur in the Pump Pit Sludge Tank?}

If an explosive concentration occurs in the Pump Pit Sludge Tank and an ignition source is available it is assumed that a damaging explosion will follow. The fault tree for the PPST explosion includes the probability of an ignition source. Therefore, if Question 173 indicates that an explosive concentration is available then an explosion will follow. An explosion in Pump Pit Sludge Tank will spill the contents of the vessel to the Pump Pit and possibly blow the cell covers. There are three defined outcomes as follows:

PPSTDet: A damaging detonation occurs in the Pump Pit Precipitate Tank.

PPSTDef: A damaging deflagration occurs in the Pump Pit Precipitate Tank.

nPPSTExp: . No damaging explosion occurs in the Pump Pit Precipitate Tank. 
RuptPPT: $\quad$ The Pump Pit Precipitate Tank is ruptured and the contents spilled as a result of the seismic event.

nRuptPPT: The Pump Pit Precipitate Tank is intact following the seismic event.

This is a Type 6 question. The answer to this question depends upon the outcome of previous Question 2,162, and 163 and the value specified for the parameter PPPTFr, the seismic fragility of the Pump Pit Precipitate Tank. The answers to this question are used in the logic to deterimine the outcome probabilities in Questions 166 and 167. Three cases are used to develop the outcome probabilities on the basis of prior events as follows:

Case 1 This case includes all sequences in which a seismic event has caused the cell covers over the Low Point Pump Pit to fall and strike the tank. The falling cell covers are assumed to rupture and spill the contents of the PPPT. An outcome probability of 1.0 is assigned Branch 1.

Case 2 This case includes all sequences in which a seismic event has occurred but the cell covers do not fall or if they fall they do not strike the PPPT. The seismic fragility of the PPPT [the probability that a seismic event will cause a structural failure of the PPPT] is placed in Branch 1. The outcome probability for Branch 2 is 1.0 less the probability assigned Branch 1.

Case 3 The default case includes all remaining sequences. The outcome probability assigned is 1.0 in Branch 2.

\section{Question 165: Does an explosive concentration accumulate in the Precipitate Tank in the Low Point Pump Pit?}

The precipitate slurry transferred from the 200-Area tank farm to the Pump Pit Precipitate Tank [PPPT] is composed primarily of cesium/potassium tetraphenylborate. Benzene is continuously formed in this slurry by radiolytic decomposition. A fault tree was constructed to calculate the frequency of explosions in the PPPT for all credible initiators [5]. The solution of this fault tree (cut sets) is used to determine the frequency (or probability) of an explosive concentration in the PPPT. The two outcomes defined for this question are as follows:

ConPPPT: An explosive concentration accumulates in the Pump Pit Precipitate Tank.

nConPPPT: An explosive concentration does not accumulate in the Pump Pit Precipitate Tank.

This is a Type 6 question and uses previously quantified parameters $[67,70$, and $71,90,94$, and 113] and a user defined function to determine the probability that an explosive concentration will occur in the PPPT. The user function includes information from solution of the fault tree for a PPPT explosion. The function used to calculate the answer depends on the answer to previous Question 1. The outcome probabilities are used in the logic to determine the outcome probabilities in Question 166.

The following case structure is simplified by not considering the possibility of prior rupture of the PPPT during a seismic event [Question 164]. Rupture of the PPPT would prevent the buildup of an explosive concentration in the PPPT. The possibility of prior rupture of the 
PPPT is included in Question 166. Four cases are used to develop the outcome probabilities as follows:

Case 1 This case is used to exclude those sequences in the classes of accidents. [Question 1] which are neither external nor internal-energetic. The outcome probability assigned is 1.0 in Branch 2.

Case 2.

This case includes all sequences in which the class of accidents is internalenergetic [Branch 2, Question 1]. The outcome probability is calculated using FUN-PPPT and assigned to Branch 1. The outcome probability for Branch 2 is 1.0 less the probability assigned Branch 1.

Case 3

This case includes all sequences in which the class of accidents is external [Branch 1, Question 1]. The outcome probability is calculated using FUN-PPPTS and assigned to Branch 1. The outcome probability for Branch 2 is 1.0 less the probability assigned Branch 1 .

Case 4 The default case assumes that all sequences in which an explosive concentration can occur are included in Cases 2 and 3. An outcome probability of 1.0 is assigned Branch 2.

Question 166: Does a damaging detonation/deflagration occur in the Precipitate Tank in the Low Point Pump Pit?

If an explosive concentration occurs in the Pump Pit Precipitate Tank and an ignition source is available it is assumed that a damaging explosion will follow. The fault tree for the PPPT explosion includes the probability of an ignition source. Therefore, if Question 165 indicates that an explosive concentration is available then an explosion will follow. An explosion in Pump Pit Precipitate Tank will spill the contents of the vessel to the Pump Pit and sossibly blow the cell covers. There are three defined outcomes as follows:

PPPTDet: A damaging detonation occurs in the Pump Pit Precipitate Tank.

PPPTDef: A damaging deflagration occurs in the Pump Pit Precipitate Tank.

nPPPTExp: No damaging explosion occurs in the Pump Pit Precipitate Tank.

This is a Type 2 question. The answer is dependent on the outcome of previous Questions 164 and 165. The answers to this question are used in the logic to determine the outcome probabilities in Questions 167, 170, and 193. Four cases-are used to develop the outcome probabilities as follows:

Case 1 This case includes all sequences in which an explosive concentration does not accumulate in the PPPT. The outcome probability assigned is 1.0 in Branch 3.

Case 2 This case includes the sequences in which a prior event has ruptured the PPPT precluding the accumulation of an explosive concentration. The outcome probability assigned is 1.0 in Branch 3. 


\section{Question_161: Does splashing [spillage] of the Organic Waste Storage Tank [OWST] contents occur?}

If the Organic Waste Storage Tank [OWST] is ruptured some or all of the contents of the contents will be spilled. Damage to the OWST can be caused by shaking during a Seismic event, by tornadic winds, or by a deflagration associated with the OWST. Spilling the contents of the OWST is of concern because benzene is a carcinogen and is highly flammable. There. are two defined outcomes as follows:

OWST-Spl: The OWST is ruptured and its contents spilled.

nOWSTSpl: The OWST is not ruptured and no spillage occurs.

This is a Type 2 question. The answers to this question are dependent on the outcome of previous Questions 158 and 160. The answers to this question are used in the source term binner. Four cases are used to develop the outcome probabilities as follows:

Case 1 This case includes all sequences in which there is no explosion associated with the OWST and there was no external event to damage the tank. An outcome probability of 1.0 is assigned Branch 2 .

Case 2 This case includes all sequences in which a prior event has ruptured the tank. An outcome probability of 1.0 is assigned Branch 1.

Case 3 This case includes all sequences in which there is a deflagration in the OWST. An outcome probability of 1.0 is assigned Branch 1 .

Case 4 The default case includes all sequences remaining and assumes that all means by which spillage of the contents of the OWST can occur have been included in the previous cases. An outcome probability of 1.0 is assigned Branch 2.

Question 162: Do the cell covers over the Low Point Pump Pit dislodge and fall because of a seismic event (i.e., covers over all three cells)?

The process cells within the main canyon of the DWPF are 'roofed' by removable steel covers. The Pump Pit cells are also 'roofed' by removable covers but constructed of reinforced concrete rather than steel and are, therefore, much heavier. These covers provide limited isolation of the cell in the event of an in-cell.release, assist in maintaining proper ventilation flow, and protect the equipment.within the cell from falling objects. If the cell covers are dislodged and fall into the cell, either through a seismic event or an energetic event within the cell, they will damage the equipment in the cell. This question examines the impact of a seismic event on the covers for the Low Point Pump Pit. There are two possible outcomes as follows:

fPCvSeis: The Low Point Pump Pit Covers are dislodged by the Seismic event and fall on the equipment [tanks, piping, etc.] in the cell.

nfPCvSei: The Low Point Pump Pit Covers remain in place following the Seismic event. 
This is a Type ${ }^{-} \dot{6}$ question. The answer to this question depends upon the outcome of previous Question 2 and the value specified for the parameter PPCovFr, the seismic fragility of the covers for the Low Point Pump Pit. The answers to this question are used in the logic to determine the outcome probabilities in Questions 163, 170, and 178. Two cases are used to develop the outcome probabilities on the basis of prior events as follows:

Case 1. This case includes those sequences in which the initiating event is a seismic event. The seismic fragility of the covers for the Low Point Pump Pit [the probability the cell covers will fall] is placed in Branch 1 . The outcome probability for Branch 2 is 1.0 less the probability assigned Branch 1.

Case 2 The default case includes all other sequences. The outcome probability assigned is 1.0 in Branch 2.

Question 163: Do the cell covers that fall due to a seismic event strike a vessel in the LPPP?

Falling cell covers during a seismic event can rupture and spill the contents of vessels in the LPPP. There are two possible outcomes as follows:

PCV-LPPP: The LPPP Cell Covers are dislodged by the Seismic event and strike vessels in the LPPP.

nPCV-LPPP: The LPPP Cell Covers are dislodged by the Seismic event and do not strike vessels in the LPPP.

This is a Type 2 question. The answer to this question depends upon the outcome of previous Questions 2 and 162 . The answers to this question are used in the logic to determine the outcome probabilities in Que: on 164,172 , and 180. Two cases are used to develop the outcome probabilities on the $t$. . of prior events as follows:

Case 1 This case includes those sequences in which the initiating event is a seismic event and the LPPP cell covers fell due to the seismic event. The outcome probability for Branch 1 is 0.9 , the outcome probability for Branch 2 is 0.1 [7]. **SAMPLED VARIABLE**.

Case 2 The default case includes all other sequences. The outcome probability assigned is 1.0 in Branch 2.

\section{Question 164: 'Does a Seismic event rupture and spill the contents of the Precipitate Tank in the Low Point Pump Pit?}

Falling cell covers during a seismic event can rupture and spill the contents.of the Pump Pit Precipitate Tank [PPPT]. Shaking and overturning of the tank during a seismic event can also cause spillage of the contents. There are two defined outcomes to this question as follows: 
This is a Type 2 question. The answer is dependent on the outcome of previous Questions 172 and 173. The answers to this question are used in the logic to determine the outcome probabilities in Questions 175, 178, and 193. Four cases are used to develop the outcome probabilities as follows:

Case 1 This case includes all sequences in which an explosive concentration does not accumulate in the PPST. The outcome probability assigned is 1.0 in Branch 3.

Case 2 This case includes the sequences in which a prior event has ruptured the PPST precluding the accumulation of an explosive concentration. The outcome probability assigned is 1.0 in Branch 3.

Case 3 This case includes the sequences in which a prior event has not ruptured the PPST and an explosive concentration has accumulated in the vessel. An outcome probability of 0.001 is assigned Branch 1 and an outcome probability of 0.999 is assigned Branch 2 [7]. **SAMPLED VARIABLE**

Case 4 The default case includes any remaining sequences. It is assumed that all sequences resulting in a damaging explosion are included in previous cases. The outcome probability assigned is 1.0 in Branch 3.

\section{Question 175: Does splashing [spillage] of the Pump Pit Sludge Tank contents occur?}

If the PPST is damaged some or all of the contents of the tank may be spilled to the floor. Damage to the PPST can be caused by falling cell covers, shaking during a Seismic event, or from a detonation occurring in the tank. Spilling the contents of the PPST is of concern because the contents are radioactive. There are two defined outcomes as follows:

PPSTSpl: The contents of the PPST are spilled into the Low Point Pump Pit.

nPPSTSpl: The contents of the PPST are not spilled into the Low Point Pump Pit.

This is a Type 2 question. The answers to this question are dependent on the outcome of previous Question 172 and 174 and the answers to this question are used in the logic to determine the outcome probabilities in Questions 177 and 179. Four cases are used to develop the outcome probabilities as follows:

Case 1 This case includes all sequences in which there was no energetic event in the tank and no seismic initiated rupture of the PPST. An outcome probability of 1.0 is assigned Branch 2 .

Case 2 This case includes all sequences in which there was a seismic initiated rupture of the PPST. Spillage of the tank contents will occur. An outcome probability of 1.0 is assigned Branch 1 .

Case 3 This case includes all sequences in which there is a detonation in the PPST. Spillage of the tank contents will occur. An outcome probability of 1.0 is assigned Branch 1 . 
Case 4 The default case includes all sequences remaining and assumes that all means by which spillage of the contents of the PPST can occur have been included in the previous cases. An outcome probability of 1.0 is assigned Branch 2.

Question 176: Does an explosive concentration accumulate in Pump Pit Sludge Cell?

This question was included as a place holder during APET development. Subsequent analysis indicates that it is not possible to develop an explosive mixture in the PPSC. Since no cutset file is provided to the APET for this accident during the quantification of the tree, the UFUN returns a value of 0 for the probability that an explosive concentration exists.

The two outcomes defined for this question are as follows:

ConPPSC: An explosive concentration accumulates in the Pump Pit Sludge Cell.

nConPPSC: An explosive concentration does not accumulate in the Pump Pit Sludge Cell.

This is a Type 6 question and would use previously quantified parameters and a user defined function to determine the probability that an explosive concentration will occur in the PPSC if it was possible for one to occur. The function used to calculate the answer depends on the answer to previous Question 1. The outcome probabilities are used in the logic to determine the outcome probabilities in Question 177. Four cases are used to develop the outcome probabilities as follows:

Case 1 This case is used to exclude those sequences in the classes of accidents [Question 1] which are neither external nor internal-energetic. The outcome probability assigned is 1.0 in Branch 2.

Case 2 This case includes all sequences in which the class of accidents is internalenergetic [Branch 2, Question 1]. The outcome probability is calculated using FUN-PPSC and assigned to Branch 1 . The outcome probability for Branch 2 is 1.0 less the probability assigned Branch 1.

Case 3 This case includes all sequences in which the class of accidents is external [Branch 1, Question 1]. The outcome probability is calculated using FUN-PPSCS and assigned to Branch 1 . The outcome probability for Branch 2 is 1.0 less the probability assigned Branch 1.

Case 4 The default case assumes that all sequences in which an explosive concentration can occur are included in Cases 2 and 3 . An outcome probability of 1.0 is assigned Branch 2. 
Question 177: Does a damaging detonation/deflagration occur in the Pump Pit Sludge Tank Cell?

This question was included as a place holder during APET development. Subsequent analysis indicates that it is not possible to develop an explosive mixture in the PPSC. Since no cutset file is provided to the APET for this accident during the quantification of the tree, the UFUN returns a value of 0 for the probability that an explosive concentration exists. The outcome probabilities for each case in this question have been set to. 1.0 in Branch 3, there is no explosion.

There are three defined outcomes as follows:

PPSCDet: A damaging detonation occurs in the Pump Pit Sludge Tank Cell.

PPSCDef: A damaging deflagration occurs in the Pump Pit Sludge Tank Cell.

nPPSCExp: No damaging explosion occurs in the Pump Pit Sludge Tank Cell.

This is a Type 2 question. The answer is dependent on the outcome of previous Questions 175 and 176. The answers to this question are used in the logic to determine the outcome probabilities in Questions 178 and 193. Three cases are used to develop the outcome probabilities as follows:

Case 1 This case includes all sequences in which an explosive concentration does not accumulate in the PPSC. The outcome probability assigned is 1.0 in Branch 3.

Case 2 This case includes the sequences in which a prior event has ruptured the PPST and an explosive concentration does not accumulate in the PPSC. The outcome probability assigned is 1.0 in Branch 3.

Case 3 The default case includes any remaining sequences. It is assumed that all sequences resulting in a damaging explosion are included in previous cases. The outcome probability assigned is 1.0 in Branch 3.

\section{Question 178: Do the cell covers for the Pump Pit Sludge Cell dislodge and fall following an energetic event?}

A previous question determined the impact of a seismic event on the cell covers. If a seismic event has not occurred it is assumed that the cell covers are in place prior to any in-cell energetic event. If a seismic event has occurred then the cell covers may have been dislodged. The assumed effect of dislodged cell covers on the tanks and equipment in the cell is essentially the same, whether dislodged by a seismic event or an in-cell explosion. Falling cell covers can rupture and spill the contents of the tanks in the Pump Pit Sludge Cell. There are two defined outcomes as follows:

fPPSCov: The cell covers dislodge and fall, either the consequence of a seismic event or an in-cell explosion.

nfPPSCov: The cell covers are in place. 
This is a Type 2 question. The answers to this question are dependent on the outcome of previous Questions 162,174, and 177 and are used in the logic to determine the outcome probabilities in Questions 179 and 193. Five cases are used to develop the outcome probabilities as follows:

Case 1 This case includes all sequences in which there was no explosion of the Pump Pit Sludge Tank, no explosion in the Pump Pit Sludge Tank Cell, and the cell covers were not dislodged previously by a seismic event. An outcome probability of 1.0 is assigned Branch 2 .

Case 2 This case includes all sequences in which the cell covers were dislodged previously by a seismic event. An outcome probability of 1.0 is assigned Branch 1.

Case 3 This case includes all sequences in which there was an explosion of the Pump Pit Sludge Tank Cell. An outcome probability of 1.0 is assigned Branch 1.

Case 4 This case includes all sequences in which there was an explosion of the Pump Pit Sludge Tank. An outcome probability of 1.0 is assigned Branch 1.

Case 5 The default case includes all sequences remaining and assumes that all means by which the cell covers can fall have been included in the previous cases. An outcome probability of 1.0 is assigned Branch 2.

\section{Question 179: Does splashing [spillage] of the Pump Pit Sludge Tank contents occur?}

If the PPST is damaged some or all of the contents of the tank may be spilled to the floor. Damage to the PPST can be caused by falling cell covers, by shaking during a seismic event and by an explosion associated with the PPST. Spilling the contents of the PPST is of concern because the contents are radioactive. There are two defined outcomes as follows:

PPSTSpl@2: The contents of the PPST are spilled into the Low Point Pump Pit.

nPPSTSpl: The contents of the PPST are not spilled into the Low Point Pump Pit.

This is a Type 2 question. The answers to this question are dependent on the outcome of previous Questions 39, 175, and 178. The answers to this question are used in the source term binner. Four cases are used to develop the outcome probabilities as follows:

Case 1 This case includes all sequences in which there was prior rupture of the PPST. Spillage of the tank contents will occur. An outcome probability of 1.0 is assigned Branch 1.

Case 2 This case includes all sequences in which the LPPP cell covers have fallen. An outcome probability of 0.9 is assigned Branch 1 and an outcome probability of 0.1 is assigned Branch 2 [7]. **SAMPLED VARIABLE** 
Case $3^{--} \quad$ This case includes all sequences in which a crane load drop accident occurs. Spillage of the tank contents will occur. An outcome probability of 1.0 is assigned Branch 1.

Case 4 The default case includes all sequences remaining and assumes that all means by which spillage of the contents of the PPST can occur have been included in the previous cases. An outcome probability of 1.0 is assigned Branch 2.

Question 180: Does a Seismic event rupture and spill the contents of the Recycle Tank in the Low Point Pump Pit?

Falling cell covers during a seismic event can rupture and spill the contents of the Pump Pit Recycle Tank [PPRT]. Shaking and overturning of the tank during a seismic event can also cause spillage of the contents. There are two defined outcomes to this question as follows:

RptPPRT: The Pump Pit Recycle Tank is ruptured and the contents spilled as a result of the seismic event.

nRptPPRT: The Pump Pit Recycle Tank is intact following the seismic event.

This is a Type 6 question. The answer to this question depends upon the outcome of previous Question 2, 162, and 163 and the value specified for the parameter PPRTFr, the seismic fragility of the Pump Pit Recycle Tank. The answers to this question are used in the logic to determine the outcome probabilities in Questions 181. Three cases are used to develop the outcome probabilities on the basis of prior events as follows:

Case 1 This case includes all sequences in which a seismic event has caused the cell covers over the Low Point Pump Pit to fall. The falling cell covers are assumed to rupture and spill the contents of the PPRT. An outcome probability of 1.0 is assigned Branch 1.

Case 2 This case includes all sequences in which a seismic event has occurred but the cell covers do not fall or if they fall they do not strike the tank. The seismic fragility of the PPRT [the probability that a seismic event will cause a structural failure of the PPRT] is placed in Branch 1. The outcome probability for Branch 2 is 1.0 less the probability assigned Branch 1.

Case 3 The default case includes all remaining sequences. The outcome probability assigned is 1.0 in Branch 2, there is no seismic event and the PPRT is undamaged.

\section{Question 181: Does splashing [spillage] of the Pump Pit Recycle Tank contents occur?}

If the PPRT is damaged some or all of the contents of the tank may be spilled to the floor. Damage to the PPRT can be caused by falling cell covers or by shaking during a seismic event. 
Spilling the contents of the PPRT is of concern because the contents are radioactive and also may include some residual benzene. There are two defined outcomes as follows:

PPRTSpl: The contents of the PPRT are spilled into the Low Point Pump Pit.

nPPRTSpl: $\quad$ The contents of the PPRT are not spilled into the Low Point Pump Pit.

This is a Type 2 question. The answers to this question are dependent on the outcome of previous Questions 2, 39, and 180. The answers to this question are used in the source term binner. Four cases are used to develop the outcome probabilities as follows:

Case 1 This case includes all sequences in which there was no seismic initiated rupture of the PPRT. An outcome probability of 1.0 is assigned Branch 2.

Case 2 : This case includes all sequences in which there was a seismic rupture of the PPRT. Spillage of the tank contents will occur. An outcome probability of 1.0 is assigned Branch 1.

Case 3 This case includes all sequences in which there is a crane load drop accident that damates the PPRT. Spillage of the tank contents will occur. An outcome probability of 1.0 is assigned Branch 1 .

Case 4 The default case includes all sequences remaining and assumes that all means by which spillage of the contents of the PPRT can occur have been included in the previous cases. An outcome probability of 1.0 is assigned Branch 2.

\section{Question 182: Does an uncontrolled reaction occur in the CPC?}

Uncontrolled reactions are defined as the sudden loss of part of the contents of a vessel [eructation], foaming, boilover, gassing, or an undesirable high temperature causing material decomposition with evolution of hazardous vapors. There are two defined outcomes as follows:

UcRx-CPC: An uncontrolled reaction occurs in the CPC.

$\mathrm{nUcRx}$-CPC: No uncontrolled reaction occurs in the CPC.

This is a Type 2 question. The answers to this question are dependent on the outcome of previous Questions 1 and 5 and are referenced in the source term binning file. Two cases are used to develop the outcome probabilities as follows:

Case 1 This case includes all events defined by Question 1, Branch 4 [accident class -LtdMisc] and Question 5, Branch 2 [CPC-UcR]. An outcome probability of 1.0 is assigned Branch 1 .

Case 2 The default case includes all sequences remaining. An outcome probability of 1.0 is assigned Branch 2 . 


\section{Question 183: Does an uncontrolled reaction occur in the SPC?} -.

Uncontralled reactions are defined as the sudden loss of part of the contents of a vessel [eructation], foaming, boilover, gassing, or an undesirable high temperature causing material decomposition with evolution of hazardous vapors. There are two defined outcomes as follows:

UcRx-SPC: An uncontrolled reaction occurs in the SPC.

nUcRx-SPC: No uncontrolled reaction occurs in the SPC.

This is a Type 2 question. The answers to this question are dependent on the outcome of previous Questions 1 and 5 and are referenced in the source term binning file. Two cases are used to develop the outcome probabilities as follows:

Case 1 This case includes all events defined by Question 1, Branch 4 [accident class -LtdMisc] and Question 5, Branch 4 [PR-UcR]. An outcome probability of 1.0 is assigned Branch 1.

Case 2 The default case includes all sequences remaining. An outcome probability of 1.0 is assigned Branch 2.

\section{Question 184: Does a vessel overflow occur in the CPC?}

Overflow occurs when the volumetric capacity of a vessel is exceeded. The result may be the loss of process liquid to the cell floor or sump, the vessel vent header, or another vessel. There are two defined outcomes as follows:

CPCOvfl: $\quad$ A vessel overflow occurs in the CPC.

nCPCOvfl: No vessel overflow occurs in the CPC.

This is a Type 2 question. The answers to this question are dependent on the outcome of previous Questions 1 and 4 and are referenced in the source term binning file. Two cases are used to develop the outcome probabilities as follows:

Case 1 This case includes all events defined by Question 1, Branch 3 [accident class -LtdLeak] and Question 4, Branch 3 [CPC-Oflow]. An outcome probability of 1.0 is assigned Branch 1.

Case 2 The default case includes all sequences remaining. An outcome probability of 1.0 is assigned Branch 2.

\section{Question 185: Does a vessel overflow of process material occur in the SPC?}

Overflow occurs when the volumetric capacity of a vessel is exceeded. The result may be the loss of process liquid to the cell floor or sump, the vessel vent header, or another vessel. In the SPC, the PR and PRFT contain process material. This question is concerned with a PR or PRFT overflow. There are two defined outcomes as follows: 
SPCOvfl: _. A vessel overflow of process material occurs in the SPC.

nSPCOvfl:: No vessel overflow of process material occurs in the SPC.

This is a Type 2 question. The answers to this question are dependent on the outcome of previous Questions 1 and 4 and are referenced in the source term binning file. Two cases are used to develop the outcome probabilities as follows:

Case 1 This case includes all events defined by Question 1, Branch 3 [accident class -LtdLeak] and Question 4, Branch 1.1 [SPC-Oflow]. An outcome probability of 1.0 is assigned Branch 1.

Case 2 The default case includes all sequences remaining. An outcome probability of 1.0 is assigned Branch 2 .

\section{Question 186: Does a vessel overflow of benzene occur in the SPC?}

Overflow occurs when the volumetric capacity of a vessel is exceeded. The result may be the loss of process liquid to the cell floor or sump, the vessel vent header, or another vessel. In the SPC, the OEV and OECT contain process material. This question is concerned with a OEV or OECT overflow. There are two defined outcomes as follows:

- Bnżovfl: A vessel overflow of benzene occurs in the SPC.

nBnzOvfl: - No vessel overflow of benzene occurs in the SPC.

This is a Type 2 question. The answers to this question are dependent on the outcome of previous Questions 1 and 4 and are referenced in the source term binning file. Two cases are used to develop the outcome probabilities as follows:

Case 1 This case includes all events defined by Question 1, Branch 3 [accident class -LtdLeak] and Question 4, Branch 9 [BZ-Oflow]. An outcome probability of 1.0 is assigned Branch 1.

Case 2 The default case includes all sequences remaining. An outcome probability of 1.0 is assigned Branch 2.

\section{Question 187: Does a vessel overflow occur in the Low Point Pump Pit?}

Overflow occurs in the Pump Pit when the volumetric capacity of a tank is exceeded. The result may be the loss of process liquid to the cell floor or sump or the vessel vent header. There are two defined outcomes as follows:

LPP-OvFl: . A vessel overflow occurs in the Low Point Pump Pit.

nLPPOvFl: . No vessel overflow occurs in the Low Point Pump Pit.

This is a Type 2 question. The answers to this question are dependent on the outcome of previous Questions 1 and 4 and are referenced in the source term binning file. Two cases are used to develop the outcome probabilities as follows: 
Case 1 -. This case includes all events defined by Question 1, Branch 3 [accident class -LtdLeak] and Question 4, Branch 4 [PP-Oflow]. An outcome probability of 1.0 is assigned Branch 1 .

Case 2 The default case includes all sequences remaining. An outcome probability of 1.0 is assigned Branch 2.

\section{Question 188: Does a leak occur in the Chemical Process Cell?}

Leaks permit process fluids to penetrate the primary confinement structures such as vessels and piping. The consequences are generally small but can range from the loss of a few milliliters of solution to all of the material in holdup. Leaks can involve significant amounts of radioactive material. There are two defined outcomes as follows:

CPCLeak: A leak occurs in the Chemical Process Cell.

nCPCLeak: No leak occurs in the Chemical Process Cell.

This is a Type 2 question. The answers to this question are dependent on the outcome of previous Questions 1 and 4 and are referenced in the source term binning file. Two cases are used to develop the outcome probabilities as follows:

Case 1 This case includes all events defined by Question 1, Branch 3 [accident class -LtdLeak] and Question 4, Branch 5 [CPC-Leak]. An outcome probability of 1.0 is assigned Branch 1.

Case 2 The default case includes all sequences remaining. An outcome probability of 1.0 is assigned Branch 2.

\section{Question 189: Does a leak of process material occur in the Salt Process Cell?}

Leaks permit process fluids to penetrate the primary confinement structures such as vessels and piping. The consequences are generally small but can range from the loss of a few milliliters of solution to all of the material in holdup. Leaks can involve significant amounts of radioactive material. There are two defined outcomes as follows:

SPCLeak: A leak of process material occurs in the Salt Process Cell.

nSPCLeak: No leak of process material occurs in the Salt Process Cell.

This is a Type 2 question. The answers to this question are dependent on the outcome of previous Questions 1 and 4 and are referenced in the source term binning file. Two cases are used to develop the outcome probabilities as follows:

Case 1 This case includes all events defined by Question 1, Branch 3 [accident class -LtdLeak] and Question 4, Branch 6 [SPC-Leak]. An outcome probability of 1.0 is assigned Branch 1. 
Case $2 \quad \therefore$ The default case includes all sequences remaining. An outcome probability of 1.0 is assigned Branch 2.

\section{Question 190: Does a leak of benzene occur in the Salt Process Cell?}

Leaks permit process fluids to penetrate the primary confinement structures such as vessels and piping. The consequences are generally small but can range from the loss of a few milliliters of solution to all of the material in holdup. Leaks can involve significant amounts of radioactive material. Leaks of benzene are of particular concern due to the possibility of subsequent deflagrations in the SPC. There are two defined outcomes as follows:

BnzLeak: $\quad$ A leak of benzene occurs in the Salt Process Cell.

nBnzLeak: No leak of benzene occurs in the Salt Process Cell.

This is a Type 2 question. The answers to this question are dependent the outcome of previous Questions 1 and 4 and are referenced in the source term binning: $\quad$ Two cases are used to develop the outcome probabilities as follows:

Case 1 This case includes all events defined by Question 1, Branch 3 [accident class -LtdLeak] and Question 4, Branch 10 [BZ-Leak]. 'An outcome probability of 1.0 is assigned Branch 1.

Case 2 The default case includes all sequences remaining. An outcome probability of 1.0 is assigned Branch 2 .

\section{Question 191: Does an incidental leak occur in the Pump Pits?}

Leaks permit process fluids to penetrate the primary confinement structures such as vessels and piping. The consequences are generally small but can range from the loss of a few milliliters of sol:ition to all of the material in holdup. Leaks can involve significant amounts of radioactive $\mathrm{m}$ : rial. There are two defined outcomes as follows:

LPPLeak: A leak occurs in the Low Point Pump Pit.

nLPPLeak: No leak occurs in the Low Point Pump Pit.

This is a Type 2 question. The answers to this question are dependent on the outcome of previous Questions 1 and 4 and are referenced in the source term binning file. Two cases are used to develop the outcome probabilities as follows:

Case 1 This case includes all events defined by Question 1, Branch 3 [accident class -LtdLeak]. and Question 4, Branch 7 [PP-Leak]. An outcome probability of 1.0 is assigned Branch 1.

Case 2. The default case includes all sequences remaining. An outcome probability of 1.0 is assigned Branch 2. 


\section{Question 192: Does the rupture of a filled storage canister occur?}

Fully encapsulated canisters are resistant to rupture if dropped or impacted by most objects. Tests show that fully encapsulated canisters can be dropped from heights of up to 30 feet without rupturing. However, canisters can be breached during processing and prior to completion of encapsulation although a breach is unlikely. There are two defined outcomes as follows:

CaniRpt: Rupture of a storage canister occurs.

nCanRpt: No rupture of a storage canister occurs.

This is a Type 2 question. The answers to this question are dependent on the outcome of previous Questions 1 and 4 and are referenced in the source term binning file. Two cases are used to develop the outcome probabilities as follows:

Case 1 This case includes all events defined by Question 1, Branch 3 [accident class -LtdLeak] and Question 4, Branch 8 [Can-Rpt]. An outcome probability of 1.0 is assigned Branch 1.

Case 2 The default case includes all sequences remaining. An outcome probability of 1.0 is assigned Branch 2.

\section{Question 193: What is the status of the ventilation for the Low Point Pump Pit Building?}

There are four defined outcomes as follows:

PPnFn/Br: The cells in Low Point Pump Pit Building are breached [cell covers blown or ventilation system breached] and the exhaust fans are not operating.

PPnF/nBr: The cells in Low Point Pump Pit Building are not breached [cell covers not blown] and the exhaust fans are not operating.

PPFan/Br: The cells in Low Point Pump Pit Building are breached [cell covers blown] and the exhaust fans are operating.

LPPVent: The ventilation system for the Low Point Pump Pit Building is functioning

This is a Type 2 question. The answers to this question are dependent on the outcome of previous Questions 2, 3, 8, 9,166,169,170,174,177, and 178. The outcomes are used in the source term binning file. Seven cases are used to develop the outcome probabilities as follows:

Case 1 This case includes all sequences in which a seismic event or tornadic winds has occurred. It is assumed that the building housing the Pump Pits has been destroyed, the vent system damaged and the exhaust fans damaged. Cell covers may or may not be in place. An outcome probability of 1.0 is assigned Branch 1. 
Case 2 .. This case includes all sequences in which electrical power failure causes failure of the exhaust fans and an explosion in the PPPT or PPPC has caused dislodging of the cell covers. An outcome probability of 1.0 is assigned Branch 1 .

Case 3

This case includes all sequences in which electrical power failure causes failure of the exhaust fans and an explosion in the PPST or PPSC has caused dislodging of the cell covers. An outcome probability of 1.0 is assigned Branch 1.

Case 4

This case includes all sequences in which electrical power failure causes failure of the exhaust fans and the cell covers are in place. An outcome probability of 1.0 is assigned Branch 2 .

Case 5 This case includes all sequences in which the exhaust fans are operating and an explosion in the PPPT or PPPC has caused dislodging of the cell covers. An outcome probability of 1.0 is assigned Branch 3.

Case 6 This case includes all sequences in which the exhaust fans are operating and an explosion in the PPST or PPSC has caused dislodging of the cell covers. An outcome probability of 1.0 is assigned Branch 3.

Case 7 The default case includes all sequences remaining. It is assumed that all sequences in which the Ventilation System for the Pump Pit is not functioning normally are included in the above cases. An outcome probability of 1.0 is assigned Branch 4. 


\section{THE SOURCE TERM BINNER}

For this analysis the information of primary concern was the radionuclide source term that could result from damage to one or more of the process vessels in the DWPF. The primary binning of the APET sequences, therefore, serves as a source term binner. APET sequences are binned into groups that are expected to yield similar radionuclide source terms. Of particular interest is the severity of damage to each of the vessels (e.g., is a tank destroyed by detonation or is it vented by flying shrapnel). This information establishes the initial size and mode of release. Also, of interest is the condition of the building ventilation ducts and fans which may or may not be available to mitigate the release. The binner is discussed in the following paragraphs.

Nineteen characteristics or dimensions are used in the source term binner and characterize the accident in terms of damage to the various process vessels within the DWPF and the status of barriers to release and mitigation systems. For detailed descriptions of each vessel and contents see Reference 1. The EVNTRE software ensures that every accident progression sequence identified as having significance [i.e., above a specified cutoff frequency] is assigned to a primary bin. It is the frequencies of these bins that are reported in the SAR. Each sequence that is not truncated based on frequency is placed into a bin according to the values for each dimension. The bin frequency is the sum of the frequencies for all accident progression sequences that had the same end state for each of the 19 dimensions in the bin. For every dimension there is an attribute defined that is appropriate for every accident progression sequence. If the user fails to define the attributes for each dimension such that this is true, the EVNTRE software generates an error condition and flags the accident progression sequence that cannot be binned. Any sequence characteristic that is potentially significant to understanding the accident progression or determining the source term is included in the list of attributes considered in developing the accident progression bins. [These attributes are similar to the branch points of a top event or question.]

The results of the source term binner are used by the DWPF Algorithm for Source Terms (DWPFAST) which reads the accident progression bins, and calculates an individual radiological source term for each [14].

The dimensions used in the DWPF analysis are:

OWST STATUS

LOW POINT PUMP PIT SLUDGE PUMP TANK STATUS

LOW POINT PUMP PIT PRECIPITATE PUMP TANK STATUS

LOW POINT PUMP PIT RECYCLE WASTE PUMP TANK STATUS

PRECIPITATE REACTOR STATUS

PRECIPITATE REACTOR FEED TANK STATUS

PRECIPITATE REACTOR BOTTOMS TANK STATUS

SLURRY MIX EVAPORATOR TANK STATUS

SLUDGE RECEIPT AND ADIUSTMENT TANK STATUS

MELTER FEED TANK STATUS

RECYCLE COLLECTION TANK STATUS

MELT CELL STATUS

DEFLAGRATION IN SPC

FIRE IN SPC

CPC CELL COVER STATUS

SPC CELL COVER STATUS

MELT CELL COVER STATUS 


\section{ZONE 1 VENTILATION STATUS}

LPPP VENTILATION STATUS

The EXCEL Spreadsheet for the source term binner is shown at the end of the APET in Appendix A. Each element or dimension of the binner is discussed in the paragraphs that follow.

\section{OWST}

The first dimension addresses the condition of the OWST. The OWST contains small amounts of radionucliedes which could be released if the tank deflagrates and material is spilled to the ground. The OWST is considered to have three end states characteristics:

Deflagration, splash or no release, (these are listed on the first line of the dimension data for the OWST)

(The OWST dimension statements then reference the following cases in the APET questions to determine the total frequency of the outcome.)

- Accident sequences in which the OWST has deflagrated are binned in the OWSTDef attribute. Deflagration of the OWST is assigned to the first branch of APET question 160. Each sequence has some frequency calculation for this case. The sum of the frequencies from all sequences is put here.

- Accident sequences in which the OWST has splashed are binned in the OWST/Sp attribute. Splashing of the OWST is assigned to the first branch of APET question 161.

- The frequency that nothing has happened (i.e., no release) to the OWST is determined based on sum of the frequencies calculated by the second branches of questions 160 and 161.

\section{PPST}

The second dimension addresses the condition of the low point pump pit sludge tank (LPPP PPST) and release mechanisms of radioactive materials. The PPST has six possible characteristics:

Detonation, deflagration, splash, leak, overflow or no release

- Accident sequences in which the PPST has detonated are binned in the PPSDet attribute. Detonation of the PPST is assigned to the first branch of APET question 174.

- Accident sequences in which the PPST has deflagrated are binned in the PPSDef attribute. Deflagration of the PPST is assigned to the second branch of APET question 174. 
- Accident sequences in which the PPST has splashed are binned in the PPSSpl attribute. Splashing of the PPST is assigned to the first branch of APET question $=179$.

- Accident sequences in which the PPST has leaked are binned in the PPSLeak attribute. Leaking of the PPST is assigned to the first branch of APET question 191.

- Accident sequences in which the PPST has overflowed are binned in the PPSOvFI attribute. Overflowing of the PPST is assigned to the first branch of APET question 187.

- The frequency for no release is determined by the outcome frequencies of the branches of questions $174,179,187$ and 191 on which nothing happens.

3. PPPT

The third dimension addresses the condition of the low point pump pit precipitate tank (LPPP PPPT) and release mechanisms of radioactive materials. The PPPT has six possible characteristics:

Detonation, deflagration, splash, leak, overflow or no release

- Accident sequences in which the PPPT has detonated are binned in the PPPDet attribute. Detonation of the PPPT is assigned to the first branch of APET question 166.

- Accident sequences in which the PPPT has deflagrated are binned in the PPPDef attribute. Deflagration of the PPPT is assigned to the second branch of APET question 166.

- Accident sequences in which the PPPT has splashed are binned in the PPPSpl attribute. Splashing of the PPPT is assigned to the first branch of APET question 171.

- Accident sequences in which the PPPT has leaked are binned in the PPPLeak attribute. Leaking of the PPPT is assigned to the first branch of APET question 191.

- Accident sequences in which the PPPT has overflowed are binned in the PPPOvFI attribute. Overflowing of the PPPT is assigned to the first branch of APET question 187.

- The frequency for no release is determined by the outcome frequencies of the branches of questions $166,169,171,187$ and 191 on which nothing happens.

\section{PPRT}

The fourth dimension addresses the condition of the low point pump pit recycle tank (LPPP PPRT) and release mechanisms of radioactive materials. The PPRT has four possible characteristics: 
Splash, leak, overflow or no release

- Accident sequences in which the PPRT has splashed are binned in the PPRSpl attribute. Splashing of the PPRT is assigned to the first branch of APET question 181.

- Accident sequences in which the PPRT has leaked are binned in the PPRLeak = attribute. Leaking of the PPRT is assigned to the first branch of APET question 191.

- Accident sequences in which the PPRT has overflowed are binned in the PPROvFI attribute. Overflowing of the PPRT is assigned to the first branch of APET question 187.

- The frequency for no release is determined by the outcome frequencies of the branches of questions 181, 187 and 191 on which nothing happens.

5. $P R$

The fifth dimension addresses the condition of the salt process cell precipitate reactor tank (SPC PR) and release mechanisms of radioactive materials. The PR has eight possible characteristics: release

Detonation, deflagration, splash, leak, vented, uncontrolled reaction, overflow or no

- Accident sequences in which the PR has detonated are binned in the PRxDet attribute. Detonation of the PR is assigned to the first branches of APET questions 43,80 and 123 . On a given sequence, only one case can contribute frequency. For a complete APET run, contributions will come from all of the above cases.

- Accident sequences in which the PR has deflagrated are binned in the PRxDef attribute. Deflagration of the PR is assigned to the second branches of APET questions 43, 80 and 123.

- Accident sequences in which the PR has splashed are binned in the PRx-Spl attribute. Splashing of the PR is assignied to the first branches of APET questions $106,117,136$ and 147. An RPC deflagration (Branch 1, question 147) is postulated to cause sufficient building damage to crush the tanks.

- Accident sequences in which the PR has leaked are binned in the PRx-Leak attribute. Leaking of the PR is assigned to the first branch of APET question 189.

- Accident sequences in which the PR has vented are binned in the PRx-Vent attribute. Venting of the PR is assigned to the first branches of APET questions 75, 95 and 127. Venting is assumed to occur if the salt process cell vent header detonates.

- Accident sequences in which the PR has an uncontrolled reaction are binned in the PRx-UcR attribute. Uncontrolled reaction of the PR is assigned to the first branch of APET question 183. 
- Accident sequences in which the PR has overflowed are binned in the PRx-OvFl attribute. Overflow of the PR is assigned to the first branch of APET question 185.

- The frequency for no release is determined by the outcome frequencies of the branches of questions $43,75,80,106,117,123,127,136,147,185,183$, and 189 on which nothing happens.

\section{PRFT}

The sixth dimension addresses the condition of the salt process cell precipitate reactor tank (SPC PRFT) and release mechanisms of radioactive materials. The PRFT has seven possible characteristics:

Detonation, deflagration, splash, leak, vented, overflow or no release

- Accident sequences in which the PRFT has detonated are binned in the PRFTDet attribute. Detonation of the PRFT is assigned to the first branches of APET questions 55,86 and 126 . On a given sequence, only one case can contribute frequency. For a complete APET run, contributions will come from all of the above cases.

- Accident sequences in which the PRFT has deflagrated are binned in the PRFTDef attribute. Deflagration of the PRFT is assigned to the second branches of APET questions 55, 86 and 126.

- Accident sequences in which the PRFT has splashed are binned in the PRFTSpl attribute. Splashing of the PRFT is assigned to the first branches of APET questions 109,120,139 and 147. An RPC deflagration is postulated to cause sufficient building damage to crush the tanks.

- Accident sequences in which the PRFT has leaked are binned in the PRFTLeak attribute. Leaking of the PRFT is assigned to the first branch of APET question 189.

- Accident sequences in which the PRFT has vented are binned in the PRFTVent attribute. Venting of the PRFT is assigned to the first branches of APET questions 75,95 and 127 . Venting is assumed to occur if the salt process cell vent header detonates.

- Accident sequences in which the PRFT has overflowed are binned in the PRFTOvFl attribute. Overflow of the PRFT is assigned to the first branch of APET question 185.

- The frequency for no release is determined by the outcome frequencies of the branches of questions 55, 75, 86, 109,120,126,127, 139,147, 185, and 189 on which nothing happens.

\section{PRBT}

The sixth dimension addresses the condition of the chemical process cell precipitate reactor bottoms tank (CPC PRBT) and release mechanisms of radioactive materials. The PRBT has seven possible characteristics: 
Detonation, deflagration, splash, leak, vented, overflow or no release

- Accident sequences in which the PRBT has detonated are binned in the PRBT-Det attribute. Detonation of the PRBT is assigned to the first branches of APET questions 60,88 and 130 . On a given sequence, only one case can contribute frequency. For a complete APET run, contributions will come from all of the - above cases.

- Accident sequences in which the PRBT has deflagrated are binned in the PRBT-Def attribute. Deflagration of the PRBT is assigned to the second branches of APET questions 60,88 and 130 .

- Accident sequences in which the PRBT has splashed are binned in the PRBT-Spl attribute. Splashing of the PRBT is assigned to the first branches of APET questions 99,142 and 147. An RPC deflagration is postulated to cause sufficient building damage to crush the tanks.

- Accident sequences in which the PRBT has leaked are binned in the PRBTLeak attribute. Leaking of the PRBT is assigned to the first branch of APET question 188.

- Accident sequences in which the PRBT has vented are binned in the PRBTVent attribute. Venting of the PRBT is assigned to the first branches of APET questions 76,96 and 134. Venting is assumed to occur if the chemical process cell vent header detonates.

- Accident sequences in which the PRBT has overflowed are binned in the PRBTOvFl attribute. Overflow of the PRBT is assigned to the first branch of APET question 184.

- The frequency for no release is determined by the outcome frequencies of the branches of questions $60,76,88,99,130,134,142,147,184$, and 188 on which nothing happens.

\section{SME}

The eighth dimension addresses the condition of the chemical process cell slurry mix evaporator tank (CPC SME) and release mechanisms of radioactive materials. The SME has eight possible characteristics: release

Detonation, deflagration, splash, leak, vented, uncontrolled reaction, overflow or no

- Accident sequences in which the SME has detonated are binned in the SME_Det attribute. Detonation of the SME is assigned to the first branches of APET questions 64,90 and 131 . On a given sequence, only one case can contribute frequency. For a complete APET run, contributions will come from all of the above cases. 
- Accident sequences in which the SME has deflagrated are binned in the SME_Def - attribute. Deflagration of the SME is assigned to the second branches of APET - questions 64, 90 and 131.

- Accident sequences in which the SME has splashed are binned in the SME_Spl attribute. Splashing of the SME is assigned to the first branches of APET questions 100,143 and 147. An RPC deflagration is postulated to cause sufficient building damage to crush the tanks.

- Accident sequences in which the SME has leaked are binned in the SME_Leak attribute. Leaking of the SME is assigned to the first branch of APET question 188.

- Accident sequences in which the SME has vented are binned in the SME_Vent attribute. Venting of the SME is assigned to the first branches of APET questions 76, 96 and 134. Venting is assumed to occur if the salt process cell vent header detonates.

- Accident sequences in which the SME has an uncontrolled reaction are binned in the SME_UcR attribute. Uncontrolled reaction of the SME is assigned to the first branch of APET question 182.

- Accident sequences in which the SME has overflowed are binned in the SME_OvFl attribute. Overflow of the SME is assigned to the first branch of APET question 184.

- The frequency for no release is determined by the outcome frequencies of the branches of questions $64,76,90,100,131,134,143,147,182,184$ and 188 on which nothing happens.

\section{SRAT}

The ninth dimension addresses the condition of the chemical process cell sludge receipt and adjustment tank (CPC SRAT) and release mechanisms of radioactive materials. The SRAT has eight possible characteristics: release

Detonation, Deflagration, Splash, leak, vented, uncontrolled reaction, overflow or no

- Accident sequences in which the SRAT has detonated are binned in the SRATDet attribute. Detonation of the SRAT is assigned to the first branches of APET questions 68,92 and 132 . On a given sequence, only one case can contribute frequency. For a complete APET run, contributions will come from all of the above cases.

- Accident sequences in which the SRAT has deflagrated are binned in the SRATDef attribute. Deflagration of the SRAT is assigned to the second branches of APET questions 68, 92 and 132 .

- Accident sequences in which the SRAT has splashed are binned in the SRATSpl attribute. Splashing of the SRAT is assigned to the first branches of APET 
questions 101,144 and 147. An RPC deflagration is postulated to cause sufficient building damage to crush the tanks.

- Accident sequences in which the SRAT has leaked are binned in the SRATLeak attribute. Leaking of the SRAT is assigned to the first branch of APET question 188.

$\because$ Accident sequences in which the SRAT has vented are binned in the SRATVent attribute. Venting of the SRAT is assigned to the first branches of APET questions 76, 96 and 134. Venting is assumed to occur if the salt process cell vent header detonates.

- Accident sequences in which the SRAT has an uncontrolled reaction are binned in the SRATUcRx attribute. Uncontrolled reaction of the SRAT is assigned to the first branch of APET question 182.

- Accident sequences in which the SRAT has overflowed are binned in the SRATOvFl attribute.' Overflow of the SRAT is assigned to the first branch of APET question 184.

- The frequency for no releàse is determined by the outcome frequencies of the branches of questions $68,92,132,101,144,147,76,134,188,184$ and 182 on which nothing happens.

10. MFT

The tenth dimension addresses the condition of the chemical process cell melter feed tank (CPC MFT) and release mechanisms of radioactive materials. The MFT has seven possible characteristics:

Detonation, deflagration, splash, leak, vented, overflow or no release

- Accident sequences in which the MFT has detonated are binned in the MFT-Det attribute. Detonation of the MFT is assigned to the first branches of APET questions 74,94 and 133 . On a given sequence, only one case can contribute frequency. For a complete APET run, contributions will come from all of the above cases.

- Accident sequences in which the MFT has deflagrated are binned in the MFT-Def attribute. Deflagration of the MFT is assigned to the second branches of APET questions 74, 94 and 133.

- Accident sequences in which the MFT has splashed are binned in the MFT-Spl attribute. Splashing of the MFT is assigned to the first branches of APET questions 103,146 and 147. An RPC deflagration is postulated to cause sufficient building damage to crush the tanks.

- Accident sequences in which the MFT has leaked are binned in the MFT-Leak attribute. Leaking of the MFT is assigned to the first branch of APET question 188. 
- Accident sequences in which the MFT has vented are binned in the MFT-Vent attribute. Venting of the MFT is assigned to the first branches of APET questions 76,96 and 134. Venting is assumed to occur if the salt process cell vent header detonates.

- Accident sequences in which the MFT has overflowed are binned in the MFT_OvFI attribute. Overflow of the MFT is assigned to the first branch of APET question $=184$.

- The frequency for no release is determined by the outcome frequencies of the branches of questions $74,76,94,103,133,134,146,147,184$, and 188 on which nothing happens.

\section{RCT}

The eleventh dimension addresses the condition of the chemical process cell recycle collection tank (CPC RCT) and release mechanisms of radioactive materials. The RCT has six possible characteristics:

Splash, leak, vented, uncontrolled reaction, overflow or no release

- Accident sequences in which the RCT has splashed are binned in the RCT_Spl attribute. Splashing of the RCT is assigned to the first branches of APET questions 102,145 and 147. An RPC deflagration is postulated to cause sufficient building damage to crush the tanks.

- Accident sequences in which the RCT has leaked are binned in the RCT_Leak attribute. Leaking of the RCT is assigned to the first branch of APET question 188.

- Accident sequences in which the RCT has vented are binned in the RCT_Vent attribute. Venting of the RCT is assigned to the first branches of APET questions 76,96 and 134. Venting is assumed to occur if the salt process cell vent header detonates.

- Accident sequences in which the RCT has an uncontrolled reaction are binned in the RCT_UcRx attribute. Uncontrolled reaction of the RCT is assigned to the first branch of APET question 182.

- Accident sequences in which the RCT has overflowed are binned in the RCT_OvFI attribute. Overflow of the RCT. is assigned to the first branch of APET question 184.

- The frequency for no release is determined by the outcome frequencies of the branches of questions $102,76,134,145,147,182,184$ and 188 on which nothing happens.

\section{Melter and Melter Offgas system}

The twelfth dimension addresses the condition of the melter cell tank and the melter offgas system and release mechanisms of radioactive materials. This dimension has five possible characteristics: 
Total melter release, partial melter release, melter offgas release, glass cannister rupture or no release.

- Accident sequences in which the melter contents are completely released are binned in the TMltRlse attribute. Contents release of the melter is assigned to the first branches of APET questions 157, and 147.

- Accident sequences in which the melter contents are partially released are binned in the pMltRlse attribute. Partial contents release of the melter is assigned to the second branch of APET question 157.

- Accident sequences in which the melter offgas contents are completely released are binned in the MitOGRls attribute. Release of the melter offgas is assigned to the third branch of APET question 157.

- Accident sequences in which the cannister contents are completely released are binned in the Can_Rupt attribute. Release of the cannister contents is assigned to the first branch of APET question 192.

- The frequency for no release is determined by the outcome frequencies of the branches of questions 147,192 , and 157 on which nothing happens.

\section{Salt Process Cell Deflagration}

The thirteenth dimension addresses the condition of the salt process cell with respect to damage occurred from a deflagration and release mechanisms of radioactive materials. This dimension has two possible characteristics:

SPC deflagration or no deflagration (i.e., no release)

- Accident sequences in which a deflagration occurs in the SPC are binned into the SPC-Def attribute. Deflagration of the SPC is assigned to the first branch of question 121.

- The frequency at which no salt process cell deflagration occurs is determined by the - outcome frequencies of the second branch of question 121 .

\section{Salt Process Cell Fire}

The fourteenth dimension addresses the condition of the salt process cell with respect to damage occurred from a fire and release mechanisms of radioactive materials. This dimension has two possible characteristics:

SPC fire or no fire

- Accident sequences in which a fire occurs in the SPC are binned into the SPC-Fire attribute. Fire in the SPC is assigned to the first branch of question 122.

- The frequency at which no salt process cell fire occurs is determined by the outcome frequency of the second branch of question 122.

\section{Chemical Process Cell Covers}


The fifteenth dimension addresses the condition of the chemical process cell covers and release mechanisms of radioactive materials. This dimension has two possible characteristics:

CPC covers fall or do not fall

- Accident sequences in which the cell covers in the CPC fall are binned into the CPCCvfl attribute. Fallen covers in the CPC is assigned to the first branches of questions 98,141 and 147.

- The frequency at which the chemical process cell covers do not fall is based on the sum of the frequencies calculated by the second branches of questions 98,141 or 147.

\section{Salt Process Cell Covers}

The sixteenth dimension addresses the condition of the salt process cell covers and release mechanisms of radioactive materials. This dimension has two possible characteristics:

SPC covers fall or do not fall

- Accident sequences in which the cell covers in the SPC fall are binned into the SPCCvfl attribute. Fallen covers in the SPC is assigned to the first branches of questions 105, 116 and 128.

- The frequency at which the salt process cell covers do not fall is based on the sum of the frequencies calculated by the second branches of questions 105,116 or 128 .

\section{Melter Cell Covers}

The seventeenth dimension addresses the condition of the melter cell covers and release mechanisms of radioactive materials. This dimension has two possible characteristics:

Melter cell covers fall or do not fall

- Accident sequences in which the cell covers in the Melter Cell fall are binned into the MCBlown attribute. Fallen covers in the Melter cell is assigned to the first branch of question 156.

- The frequency at which the melter cell covers do not fall is based on the sum of the frequencies calculated by the second branch of question 156 .

\section{Zone 1 Ventilation}

The eighteenth dimension addresses the condition of the zone 1 ventilation and possible reduced mitigation of the ventilation system. This dimension has five possible characteristics:

- Vitrification building collapsed

- Vitrification building collapsed, fans fail, sand filter failed and ventilation duct failed

- Virrification building collapsed, fans okay, sand filter okay and ventilation duct okay

- Vitrification building okay, fans fail, sand filter failed and ventilation duct failed

- no failures 
- Accident sequences in which the vitrification building collapses are binned into the Collapse attribute. Building collapse is assigned to the first branches of questions 110 and 147.

- Accident sequences in which the vitrification building collapses, the fans fail, the sand filter fails and the ventilation duct fails are binned into the $\mathrm{nFanBrch}$ attribute. This combination of events is assigned to the first branches of questions 110,113 , 111 , and 112.

- Accident sequences in which the vitrification building collapses, the fans do not fail, the sand filter does not fail and the ventilation duct does not fail are binned into the FrFn/Fit attribute. This combination of events is assigned to the first branch of question 110 and the second branches of questions 113,111, and 112 .

- Accident sequences in which the vitrification building does no sllapse, but the fans fail, the sand filter fails and the ventilation duct fails ar. jinned into the $\mathrm{nFn} / \mathrm{nFit}$ attribute. This combination of events is assigned to the second branch of question 110 and the first branches of questions 113,111, and 112.

- The frequency of no failure to the zone 1 ventilation system is determined based on the sum of the frequencies calculated by the second branches of questions 110 , $111,112,113$ and 147.

19. Low point pump pit ventilation

The nineteenth dimension addresses the condition of the low point pump pit ventilation system and possible reduced mitigation of the ventilation system. This dimension has four possible characteristics:

- Fans fail and building breached

- Fans fail and building okay

- Fans okay and building breached

- No failures

- Accident sequences in which the fans fail and the building is breached are binned into the $\mathrm{nFanBrch}$ attribute. This combination of events is assigned to the first branch of question 193.

- Accident sequences in which which the fans fail and the building is not breached is binned into the $\mathrm{nFn} / \mathrm{nBrch}$ attribute. This combination of events is assigned to the second branch of question 193.

- Accident sequences in which the fans do not fail and the building is breached is binned into the Fan/Brch attribute. This combination of events is assigned to the third branch of question 193.

- The frequency for no release is determined based on the sum of the frequencies calculated by the fourth branch of question 193. 


\section{REFERENCES:}

$\sim$

1. Safety Analysis-200-S Area, Savannah River Site Defense Waste Processing Facility Operations, WSRC-SA-6, Rev. 12.

2. A Reference Manual for the Event Progression Analysis Code [EVNTRE], NUREG/CR5174, SAND88-1607, Sandia National Laboratories, Albuquerque, NM, September 1989.

3. S. T. Gough, "DWPFASTXL: Defense Waste Processing Facility Algorithm for Source Terms for EXCEL", WSRC-TR-94-0532, November, 1994.

4. W. M. Massey, "DWPF LHS Calculation Methodology and Results", S-CLC-S-00028, April, 1995.

5. A. G. Sarrack, Accident Fault Trees for Defense Waste Processing Facility (U), S-ESRS-00001, Rev. 0, February, 1995.

6. A. G. Sarrack, "DWPF Crane Load Drop Frequency", S-CLC-S-00028, January, 1995.

7. J. K. Thomas and D. A. Kalinich, "DWPF Accident Progression Event Tree Energetic Event Split Fractions", M-CLC-S-00530, March, 1995.

8. A. G. Sarrack, Preparation of CAFTA Output Data for LHS Matrix Input, S-CLC-S00044, WSRC, Aiken, SC, March, 1995.

9. S. T. Gough, "Melter Split Fractions", N-CLC-S-00024, December, 1994.

10. J. H. Weber, Statistical Analysis of Frequency of DWPF Accident Scenarios, WSRCTR-93-335, June 1993.

11. S. T. Gough, "Software Quality Assurance Package for EVNTRE", N-SVR-S-00003, Westinghouse Savannah River Company, January 11, 1995.

12. S. T. Gough; "Software Quality Assurance Package for DWPF UFUN", N-SVR-S00009, Westinghouse Savannah River Company, February 16, 1995.

13. S: T. Gough; "Cell Cover Lifting Following a MOG Explosion", N-CLC-S-00014, August, 1994.

14. S. T. Gough, "User's Manual for DWPFAST: Defense Waste Processing Facility Algorithm for Source Terms", WSRC-TR-94-0504, Westinghouse Savannah River Company, August, 1994. 
APPENDIX A: EXCEL Spreadsheet of the Accident Progression Event Tree for Defense Waste Processing Facility: MODE C 


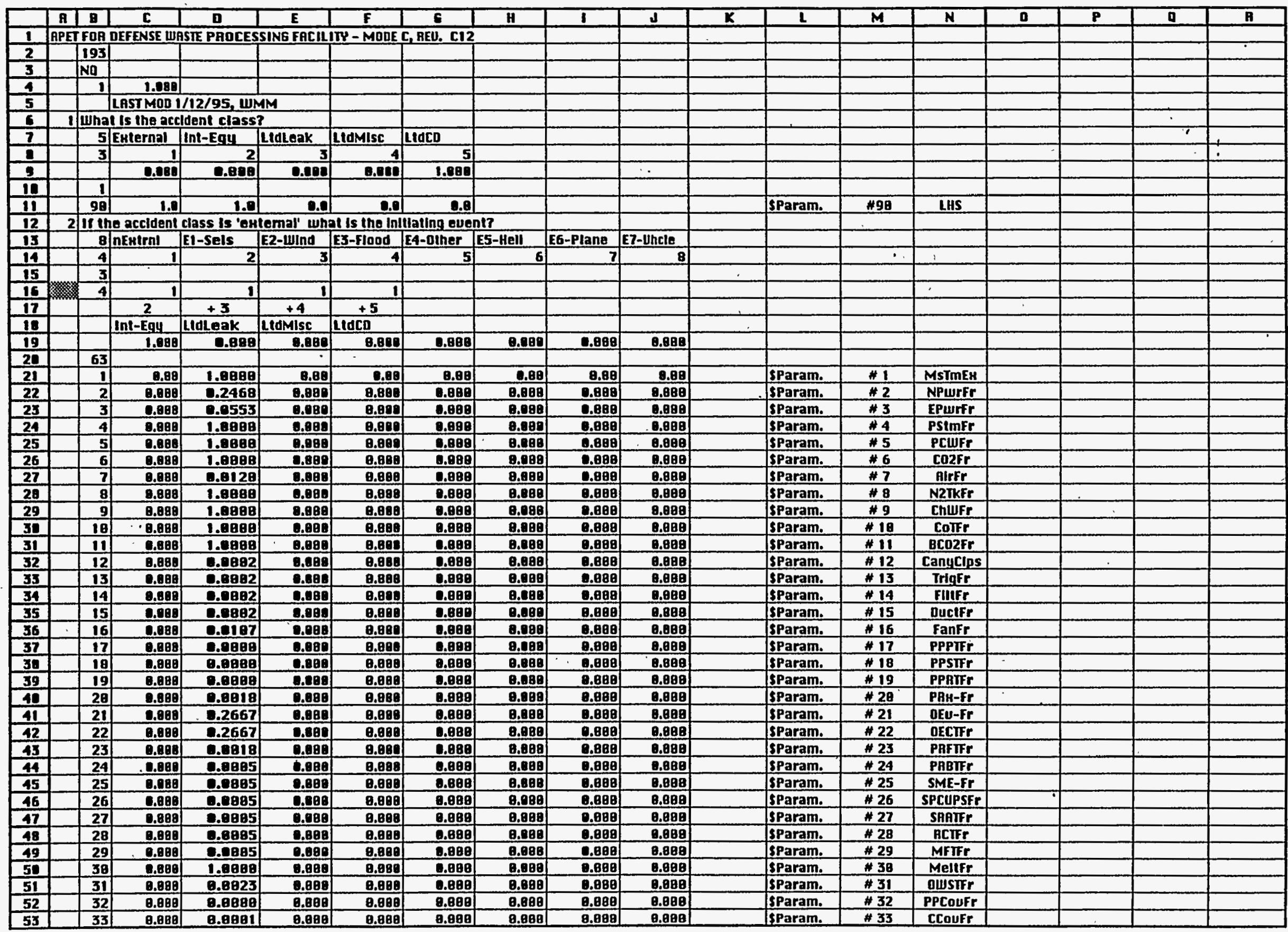




\begin{tabular}{|c|c|c|c|c|c|c|c|c|c|c|c|c|c|c|c|c|c|c|}
\hline & A & $\bar{B}$ & 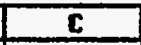 & a & $\mathbf{E}$ & $F$ & G & H & $I$ & $J$ & $\bar{K}$ & 1 & $M$ & $\mathbf{N}$ & D & $P$ & $\therefore 0$ & 8 \\
\hline 54 & & 34 & 0.080 & 1.0080 & 0.080 & 0.008 & 0.808 & 0.098 & 0.808 & 0.080 & & sparam. & $\# 34$ & IAlirfr & & & & \\
\hline 55 & & 72 & 0.000 & 1.9808 & 0.090 & 0.008 & 0.000 & 0.018 & 0.000 & 0.080 & & sparam. & $\$ 72$ & BlkN2Fr & & & & . \\
\hline 56 & & 73 & 0.000 & 0.0120 & 0.080 & 0.008 & 8.080 & 0.0100 & 0.60 & 0.980 & & sparam. & 473 & CPCN2Fr & & & & \\
\hline 57 & & 74 & 0.080 & 0.0000 & 0.000 & 8.000 & 0.080 & 0.0118 & 0.800 & 0.880 & & sparam. & $\$ 74$ & NPwrAn & & & & \\
\hline 50 & & 75 & 0.000 & 8.0900 & 0.090 & 8.088 & e.pag & 0.009 & 0.08 & Q.988 & & sparam. & $\# 75$ & EPurAn & & & & \\
\hline 59 & & 76 & 0.008 & 0.0000 & 0.000 & 8.000 & 0.080 & 0.000 & 0.808 & 0.080 & & sparam. & $\# 76$ & Pstmin & & & & \\
\hline 50 & & 77 & 0.000 & 0.0000 & 0.080 & 0.000 & 0.080 & 0.000 & 0.080 & 0.088 & & sparam. & 477 & PCWAn & & & I & \\
\hline 61 & & 78 & 0.090 & 0.2889 & 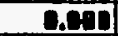 & 0.010 & 0.080 & 0.0108 & 0.008 & 0.098 & & sparam. & $\$ 78$ & CO2An & & & & 1 \\
\hline 62 & & 80 & 0.000 & 0.0000 & 0.010 & 0.000 & 0.000 & 0.000 & $0.00 \mathrm{~B}$ & 0.088 & & sparam. & 180 & AlrRn & & & & \\
\hline 6 & & 81 & 0.000 & 0.0990 & D.201 & 0.000 & 0.000 & 0.208 & $0.8 \mathrm{BO}$ & 0.080 & & sparam. & 481 & N2IKRn & & & & $\therefore$ \\
\hline 6 & & 82 & 0.000 & 0.0008 & 0.000 & 0.000 & 0.080 & 0.000 & 0.080 & 8.898 & & sparam. & $\# 82$ & ChWAn & & & & \\
\hline 5 & & 83 & 0.080 & 0.0909 & 0.080 & 0.010 & 0.090 & 0.000 & 0.808 & 8.808 & & sparam. & 483 & CoInn & & & & \\
\hline 55 & & 84 & 0.000 & 0.0800 & 0.000 & 0.000 & 0.080 & 0.000 & 0.800 & 0.089 & & sparam. & $\$ 84$ & BCo2n & & & & \\
\hline 67 & & 85 & 0.090 & 0.0980 & 6.090 & 0.010 & e.080 & 0.010 & Q.068 & 0.080 & & sparam. & 485 & I Birfin & & & & \\
\hline 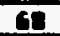 & & 86 & 0.080 & 0.0800 & 0.010 & 0.00 & 0.080 & 0.000 & 0.008 & 0.098 & & sparam. & $\$ 86$ & BlkN2Rn & & & & \\
\hline 69 & & 87 & 0.080 & 0.0028 & 0.080 & 0.010 & 0.000 & 0.000 & 0.080 & 0.890 & & sparam. & 487 & CPCN2Rn & & & & \\
\hline 70 & & 96 & 0.080 & 0.0080 & 0.000 & 0.000 & Q.080 & 0.000 & 0.000 & Q.988 & & sparam. & $\# 96$ & Fanini & & & & \\
\hline$\pi$ & & 92 & 0.080 & 1.0890 & 0.080 & 0.000 & 0.080 & 0.000 & 0.000 & 8.980 & & SParam. & 497 & cTrmfr & & & & \\
\hline 72 & & 181 & 0.080 & 0.0020 & 0.808 & 0.010 & 0.080 & 8.090 & 0.800 & 8.680 & & sparam. & $\# 181$ & cremBn & & & & \\
\hline 73 & & 182 & 0.000 & 1.0080 & 0.080 & 0.000 & 0.800 & 2088 & 0.000 & 8.080 & & sparam. & $\because 102$ & DWelfr & & & & \\
\hline 74 & & 186 & 0.880 & 0.0880 & 0.080 & 0.008 & 0.080 & 0.000 & 0.080 & 8.880 & & sparam. & \#186 & Duelin & & & & \\
\hline 75 & & 187 & 8.080 & 8.0889 & 0.080 & 8.008 & 9.080 & 0.098 & 0.089 & 8.800 & & sparam. & $\# 107$ & CPCIKFr & & & & \\
\hline 76 & & 111 & 0.08 & 0.0889 & 0.080 & 0.008 & 0.888 & 8.088 & 0.080 & 8.680 & & sparam: & $\# 111$ & CPCIKAn & & & & \\
\hline 77 & & 112 & 0.080 & 8.8031 & 0.080 & 0.088 & 0.080 & 0.008 & $0.0 \mathrm{Bg}$ & 0.880 & & sparam. & $\# 112$ & PPN2Fr & & & & \\
\hline 70 & & 116 & 0.000 & 0.0088 & 0.009 & 0.000 & Q.080 & D.eng & 0.808 & 8.880 & & SParam. & *116 & PPN2An & & & & \\
\hline 79 & & 117 & Q.808 & 0.0831 & Q.8Be & 0.808 & 0.080 & 0.000 & 0.089 & Q.880 & & SParam. & \#117 & SPCNZFr & & & & \\
\hline 10 & & 121 & 8.080 & 0.6808 & 0.088 & 0.080 & 0.080 & 0.000 & 0.809 & 0.880 & & sparam. & $\# 121$ & SPCN2Bn & & & & \\
\hline 81 & & 122 & $0.0 \mathrm{BO}$ & 0.9800 & 0.080 & 0.080 & 0.080 & 0.009 & 8.080 & 8.080 & & sparam. & $\# 122$ & Fanin2 & & $\therefore$ & & \\
\hline 12 & & 123 & 0.808 & 0.8888 & 0.080 & 0.008 & 0.088 & 0.020 & 0.080 & D.808 & & sparam. & $\# 123$ & FanRn3 & & & & \\
\hline 13 & & 129 & 0.800 & 1.0000 & 0.000 & 0.080 & 0.808 & . $\mathbf{0 . 0 0 8}$ & 0.000 & 8.800 & & sparam. & $\$ 129$ & MOG_Fr & & & & \\
\hline 84 & & 1 & 1 & & & & & & & & & & & & & & & \\
\hline 15 & & & 1 & & & & & & & & & & & & & & & \\
\hline 10 & & & Esternal & & & & & & & & & & & & & & & \\
\hline 97 & & & 0.68080 & 1.00800 & 2.08000 & 8.08988 & Q.8B日80 & 0.00000 & e.09080 & D.a8e8g & & & & & & & & \\
\hline 98 & & 63 & & & & & & & & & & & & & & & & \\
\hline 89 & & 1 & 0.00 & 1.0888 & 0.80 & 0.08 & 0.80 & 0.00 & 0.68 & 0.80 & & sparam. & $\# 1$ & MsTmEK & & & & \\
\hline 90 & & 2 & 0.080 & 0.2468 & 0.080 & 0.080 & 0.080 & 0.028 & 2.880 & 8.808 & & sparam. & 42 & NPurFr & & & & \\
\hline 91 & & 3 & 0.000 & 0.0553 & 0.000 & 0.000 & 0.880 & 8.000 & 0.080 & 8.080 & & sparam. & 43 & EPWurfr & & & & \\
\hline 92 & & 4 & 0.080 & $1.00 \mathrm{Bg}$ & 0.080 & 0.008 & Q.8B0 & 0.008 & 0.080 & 0.080 & & sParam. & $\# 4$ & PStmfr & & & & \\
\hline 93 & & 5 & 0.080 & 1.0880 & 0.080 & 0.800 & 0.680 & 0.020 & 0.090 & 8.080 & & \$Param. & 45 & PCWFr & & & & \\
\hline 94 & & 6 & 0.000 & 1.0000 & 0.080 & 0.000 & 0.080 & 0.000 & 0.880 & D.000 & & sparam. & $\# 6$ & CO2Fr & & & & \\
\hline 95 & & 7 & 8.980 & 0.0120 & 9.080 & 8.000 & 0.888 & 8.928 & 0.880 & 8.080 & & sparam. & $\# 7$ & Alirfr & & & & \\
\hline 95 & & 8 & 0.060 & 1.0808 & 0.080 & 0.008 & 0.080 & 8.088 & 0.090 & 8.808 & & sparam. & $\# 8$ & N2TkFr & & & & \\
\hline 97 & & 9 & 0.080 & 1.0008 & 0.080 & 8.890 & Q.0BA & 0.000 & 0.880 & 9.088 & & sparam. & 49 & chwfr & & & & \\
\hline 32 & & 10 & 0.080 & $1.008 \mathrm{~B}$ & 0.080 & 8.000 & 0.080 & 0.008 & 0.880 & 0.886 & & sparam. & $\# 10$ & CoTrr & & & & \\
\hline 99 & & 11 & 0.980 & 1.8808 & 0.000 & 0.000 & 0.080 & 0.080 & 0.000 & 0.800 & & sparam. & $\# 11$ & BCO2fr & & & & \\
\hline 10 & & 12 & 0.080 & 0.0882 & 0.000 & 8.080 & e.080 & 0.080 & 0.808 & 0.880 & & sparam. & $\# 12$ & CanyCIps & & & & \\
\hline In! & & 13 & D.080 & 0.0802 & $0.08 \mathrm{~g}$ & 0.080 & 0.080 & 0.088 & 0.888 & 0.808 & & sparam. & $\# 13$ & Trigfr & & & & \\
\hline 102 & & 14 & 0.000 & 0.0802 & 0.080 & 9.808 & $0.0 \mathrm{Bg}$ & 0.008 & 2.888 & 0.880 & & sparam. & $\$ 14$ & Flltir & & & & \\
\hline 103 & & 15 & 8.680 & 0.0802 & 0.080 & 8.800 & 0.080 & 0.080 & 0.880 & 0.080 & & sparam. & $\# 15$ & Ducifr & & & & \\
\hline 184 & & 16 & 8.080 & 0.0187 & 0.088 & 0.888 & 0.898 & 8.000 & 2.088 & 0.880 & & sparam. & $\# 16$ & Fanfr & & & & \\
\hline 115 & & 17 & 8.800 & 0.0888 & 0.088 & 8.808 & 0.888 & 0.088 & 0.080 & 8.888 & & sparam. & $\# 17$ & PPPIFr & & & & \\
\hline 106 & & 18 & 0.080 & 0.8888 & 0.008 & 8.080 & 8.080 & 8.89B & 8.880 & $8.8 B 8$ & & sparam. & $\# 18$ & PPSTr & & & & \\
\hline
\end{tabular}




\begin{tabular}{|c|c|c|c|c|c|c|c|c|c|c|c|c|c|c|c|c|c|c|}
\hline & $\overline{8}$ & B & $E$ & $\bar{D}$ & $E$ & $F$ & $E$ & H & 1 & $J$ & $\underline{K}$ & 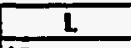 & $M$ & $N$ & D & $P$ & 으 & A \\
\hline 127 & & 19 & 0.808 & $\begin{array}{l}0.0888 \\
\end{array}$ & 0.889 & e.089 & 0.898 & 2.889 & 8.e88 & 0.800 & & SParam. & \#19 & PPATFr & & & & \\
\hline 108 & & 20 & 0.090 & 0.0018 & Q.8B日 & 0.800 & 0.088 & 0.020 & 0.088 & 0.080 & & sparam. & $\# 20$ & PAR-Fr & & & & $\cdot$ \\
\hline 109 & & 21 & 0.289 & 0.2667 & 9.098 & 8.000 & 0.080 & 0.800 & 0.088 & 8.88g & & SParam. & $\# 21$ & OEU-Fr & & & & \\
\hline 110 & & 22 & 0.010 & 0.2657 & 0.088 & 0.208 & e.080 & 0.028 & 0.088 & 8.088 & & SParam. & $\# 22$ & OECTIFr & & & & \\
\hline III & & 23 & 0.080 & 0.0018 & 0.008 & 0.080 & 0.089 & Q.89g & 8.088 & 8.889 & & sparam. & 423 & PAFIFr & & $\pi$ & & \\
\hline 112 & & 24 & 8.810 & 0.0185 & 0.090 & e.182 & D.089 & 0.001 & 0.098 & 0.889 & & SParam. & $\# 24$ & PABTFr & & & & \\
\hline 113 & & 25 & 0.008 & 0.0185 & e.end & 8.020 & 0.080 & 0.800 & E.088 & 0.989 & & sparam. & $\# 25$ & SME-Fr & & & $\because$ & \\
\hline 114 & & 26 & .000 & 0.0185 & 0.000 & 2.080 & 0.080 & 0.000 & 0.098 & 0.898 & & sparam. & $\# 26$ & SPCUPSFr & & & & \\
\hline 115 & & 27 & 0.080 & 0.0105 & 0.080 & e.1010 & 0.080 & 0.808 & 0.089 & Q.838 & & sparam. & $\# 27$ & SAATFr & & & & \\
\hline 116 & & 28 & 0.090 & 0.0195 & 0.28 & 0.00 & $0.0 \mathrm{BO}$ & 0.009 & 0.098 & 8.890 & & sparam. & $\# 28$ & ACTr & & & & \\
\hline 117 & & 29 & c.008 & 0.0185 & 0.08 & 0.189 & E.8e日 & 0.808 & 0.080 & 8.08B & & sparam. & $\$ 29$ & MFIFr & & & & \\
\hline 118 & & 30 & 8.890 & 1.0010 & 0.080 & 2.000 & 0.080 & 0.000 & 2.898 & 9.808 & & sparam. & \#B & Meltfr & & & & \\
\hline 119 & & 31 & 0.080 & 0.0123 & 0.088 & 0.080 & 0.000 & 0.028 & 0.080 & $0.8 \mathrm{Bg}$ & & sparam. & $\# 31$ & DWSIFr & & & & - \\
\hline 120 & & 32 & 0.000 & 9.9189 & e.088 & 0.000 & 0.080 & 0.800 & 0.088 & 8.089 & & sparam. & $\$ 32$ & PPCoufr & & & & \\
\hline 121 & & 33 & 0.080 & 0.0081 & 2.000 & 2.080 & 0.080 & 8.000 & 0.080 & Q.808 & & sparam. & H33 & CCoufr & & & & \\
\hline 122 & & 34 & 0.000 & 1.0280 & 0.000 & 0.009 & e.080 & 0.909 & 0.880 & 8.888 & & sParam. & 434 & IAlufr & & & & \\
\hline 123 & & 72 & 0.080 & 1.0800 & 8.089 & 8.080 & e.089 & 0.029 & D.08B & 8.898 & & SParam. & 172 & BlkN2Fr & & & & \\
\hline 124 & & 73 & 0.009 & .0120 & D.eBg & Q.080 & 0.080 & 0.000 & 0.089 & Q.8B日 & & sparam. & $\because 73$ & CPCN2Fr & & & & \\
\hline 125 & & 74 & 0.090 & 0.0080 & 8.8Be & D.880 & 0.889 & 0.000 & 0.088 & 0.8B日 & & sparam. & $\# 74$ & NPwrAn & & & & \\
\hline 126 & & 75 & 2.008 & 0.0008 & 0.088 & 0.880 & 0.080 & 0.800 & $0.0 \mathrm{BO}$ & $0.8 B 8$ & & sparam. & $\# 75$ & EPwrAn & & & & \\
\hline 127 & & 26 & 0.800 & $0.088 \mathrm{~B}$ & 8.080 & Q.8B0 & Q.889 & 0.008 & $0.08 \mathrm{~B}$ & 0.088 & & sparam. & $\# 76$ & PStman & & & & \\
\hline 128 & & 77 & 0.008 & C.8888 & $0.8 \mathrm{Bg}$ & 0.988 & 0.080 & e.989 & 0.088 & 6.888 & & sParam. & 477 & PCWRn & & & & \\
\hline 129 & & 78 & 0.098 & 0.0808 & 8.080 & $0.8 \mathrm{BB}$ & 8.890 & 0.889 & 0.088 & 0.080 & & sparam. & $\# 78$ & CO2Rn & & & & \\
\hline 130 & & 80 & 0.088 & 0.8889 & 0.080 & 8.880 & Q.898 & 0.088 & Q.08B & B.888 & & sParam. & $\# 88$ & Birnn & & & & \\
\hline 131 & & 81 & 0.088 & 8.8888 & $0.0 \mathrm{ge}$ & Q.689 & 9.898 & 8.808 & E.0BO & 0.080 & & sparam. & \#81 & N2IKAin & & & & \\
\hline 132 & & 82 & 0.000 & 8.0889 & 0.880 & 0.808 & B.680 & 8.880 & 0.089 & $0.80 \mathrm{a}$ & - & sParam. & $\# 82$ & ChIuRn & & & & \\
\hline 133 & & 83 & 8.008 & 0.8880 & 0.080 & 0.088 & 8.889 & Q.800 & Q.'AB & Q.808 & & sparam. & $\# 83$ & Comn & & & & \\
\hline 134 & & 84 & Q.08B & D.8888 & 0.000 & 0.800 & 0.080 & 0.888 & D.088 & 0.808 & & Sparam. & $\# 84$ & 8co2nn & & & & \\
\hline 135 & & 85 & 2.028 & 0.0889 & 0.080 & 0.888 & 8.8BB & D.808 & E.080 & 0.080 & & SParam. & $\# 85$ & IAirinn & & & & \\
\hline 136 & & 86 & 8.089 & 0.6989 & 0.080 & 0.088 & 0.689 & 0.080 & 0.080 & 0.098 & & sparam. & $\# 86$ & BIKN2Bn & & & & \\
\hline 137 & & 87 & 0.000 & Q.8888 & D.008 & e日e日 & Q.808 & .000 & 0.000 & 0,088 & & sparam. & $\$ 87$ & CPCN2An & & & & \\
\hline 138 & & 96 & 0.088 & 0.0000 & 0.089 & 0.088 & 0.080 & 0.808 & 0.808 & 0.089 & & sparam. & \#96 & FanfnI & & & & \\
\hline 139 & & 97 & 0.080 & 1.0080 & 0.088 & 0.000 & Q.8B8 & 8.8OB & 0.080 & 8.809 & & sparam. & $\$ 97$ & CTCmFr & & & & \\
\hline 141 & & 101 & 0.680 & 0.1100 & 0.080 & 0.088 & $0.9 \mathrm{ag}$ & Q.080 & D.eng & Q.08B & & sparam. & 4181 & creman & & & & \\
\hline 141 & & 182 & 0.089 & 1.0880 & e.8Bg & 0,080 & e.e8g & Q.880 & 0.009 & 0.080 & 1 & sParam. & $\# 102$ & DLelfr & & & & \\
\hline 142 & & 106 & 0.000 & Q.98098 & $0.8 B 8$ & 0.088 & 0.808 & 0.088 & 0.080 & 0.89g & & sparam. & $\$ 186$ & DWelAn & & & & \\
\hline 143 & & 107 & 2.000 & 0.0899 & 0.000 & 0.688 & 9.08B & 0.800 & 0.009 & 0.890 & & sparam. & $\$ 187$ & CPCTKFr & & & & \\
\hline 144 & & III & 0.680 & e.8age & 0.088 & 0.000 & Q.8B日 & D.600 & D.89g & Q.889 & & sparam. & $\$ 111$ & CPCTkAn & & & & \\
\hline 145 & & 112 & 0.009 & 0.0031 & 0.888 & 0.808 & 0.888 & 8.989 & 0.088 & 0.080 & & sparam. & $\# 112$ & PPN2Fr & & & & \\
\hline 146 & & 116 & 0.089 & 0.0880 & 0.080 & 0.080 & e.080 & 0.800 & 0.000 & 8.800 & & sParam. & $\# 116$ & PPN2Rn & & & & \\
\hline 147 & & 117 & 0.800 & 0.0031 & E.0Be & 0.089 & 0.080 & 0.800 & e.009 & 0.800 & & sparam. & $\# 117$ & SPCN2Fr & & & & \\
\hline 148 & & 121 & 0.080 & 0.0808 & 0.008 & 0.800 & 8.608 & 0.608 & 0.890 & 0.800 & & Sparam. & $\# 121$ & SPCN2An & & & & \\
\hline 149 & & 122 & 0.800 & $0.00 \mathrm{ad}$ & E.0Bg & 0.089 & 0.880 & 0.908 & 0.089 & $0.08 B$ & & sparam. & 1122 & Fannn2 & & & & \\
\hline 151) & & 123 & 2.080 & 0.0080 & 0.080 & $0.8 \mathrm{Bg}$ & Q.880 & 0.880 & 0.009 & 0.088 & & sparam. & 123 & Fanin3 & & & & \\
\hline 151 & & 129 & 0.000 & 1.0808 & 0.080 & Q.800 & Q.880 & 0.080 & 0.098 & 0.080 & & sparam. & $\# 129$ & MOG_Fr & & & & \\
\hline 152 & 8 & & Dlherwise- & opath & & & & & & & & & & & & & & \\
\hline 153 & & & 1.080 & 0.080 & 0.008 & 0.880 & Q.808 & 0.089 & 0.098 & 0.800 & & & & & & & & \\
\hline 154 & & 63 & & & & & & & & & & & & & & & & \\
\hline 155 & & 1 & 0.0 & 1.0880 & Q.06 & 8.80 & 0.09 & 8.89 & Q.60 & 9.88 & & SParam. & $\# 1$ & MsTmEH & & & - & \\
\hline 156 & & 2 & 0.880 & 6.2468 & 0.809 & 0.080 & 0.088 & $0.8 \mathrm{Bg}$ & 0,080 & 9.080 & & sparam. & $\# 2$ & NPwrFr & & & & \\
\hline 157 & & 3 & 2.688 & 0.8553 & 8.080 & 0.889 & 0.800 & Q.898 & 0.808 & 9.88g & & sparam. & $\# 3$ & EPwrfr & & & & \\
\hline 158 & & 4 & 0.000 & 1.8080 & 0.080 & 0.889 & Q.80日 & B.008 & 0.888 & 8.080 & & sparam. & $\# 4$ & Pstmfr & & & & \\
\hline 159 & & 5 & 8.880 & $1.808 \mathrm{~B}$ & 0.8Ba & 8.880 & B.889 & B.989 & $\theta . \theta 8 \theta$ & 0.08B & & sparam. & $\# 5$ & Pcufr & & & & \\
\hline
\end{tabular}




\begin{tabular}{|c|c|c|c|c|c|c|c|c|c|c|c|c|c|c|c|c|c|c|}
\hline & A & $B$ & $\mathbf{c}$ & D & $\bar{E}$ & $F$ & C & $\mathrm{H}$ & 1 & J & $\mathbf{K}$ & $\mathbf{L}$ & $M$ & $N$ & 0 & $P$ & a & n \\
\hline 160 & & 6 & 0.000 & 1.0000 & 0.080 & 0.098 & 0.080 & $0.88 \mathrm{~B}$ & 0.880 & 0.089 & & SParam. & $\# 6$ & Co2fr & & & & \\
\hline 161 & & 7 & .000 & 0.0120 & 0.080 & 0.000 & 0.080 & 8.888 & 0.880 & 0.088 & & sparam. & 17 & AlrFr & & & & $\cdot$ \\
\hline 162 & & 8 & 0.000 & 1.0008 & 0.090 & 2.000 & 0.080 & 0.088 & 0.080 & 8.090 & & sparam. & $\# 8$ & N2IKFr & & & & \\
\hline 163 & & 9 & 0.000 & 1.0089 & 0.080 & 0.889 & 0.000 & Q.888 & 0.880 & 0.080 & & sparam. & $\$ 9$ & ChWFr & & & & \\
\hline 164 & & 10 & 0.00 & 1.0000 & 0.000 & 0.009 & 0.080 & $0.0 B B$ & 0.090 & 8.000 & & sparam. & $\# 10$ & CoIfr & & & & $\cdot$ \\
\hline 165 & & 11 & e.eng & 1.0009 & 0.090 & 0.010 & e.eng & Q.AB日 & 0.980 & 0.080 & & sparam. & $\# 11$ & $\mathrm{BCO} F \mathrm{Fr}$ & & & & \\
\hline 166 & & 12 & 0.00 & 0.0022 & 2.000 & 0.0018 & D.000 & 0.800 & 0.880 & 8.080 & & sparam. & $\# 12$ & Canyclps & & & 1 & \\
\hline 167 & & 13 & 0.200 & 0.0012 & 0.019 & 0.008 & 0.01 & 0.880 & 0.080 & 0.089 & & sparam. & $\# 13$ & Irgfr & & & & \\
\hline 168 & & 14 & 0.00 & 0.0012 & 0.000 & 0.010 & 0.000 & 0.008 & 0.000 & 0.080 & & sparam. & $\# 14$ & Flitfr & & & & \\
\hline 169 & & 15 & 0.00 & 0.0002 & 0.080 & e.010 & 0.000 & e.eeg & 0.080 & 8.280 & & sparam. & $\# 15$ & Ductrr & & & & \\
\hline 170 & & 16 & 0.00 & 0.0107 & 0.080 & 0.000 & 0.000 & 0.000 & 0.080 & 0.088 & & SParam. & $\# 16$ & Fanfr & & & & \\
\hline 171 & & 17 & Q.e00 & e.enge & Q.010 & 0.00 & e.nge & 0.088 & 0.88 & 0.089 & & SParam. & $\# 17$ & PPPTFr & & & & \\
\hline 172 & & 18 & e.enge & e.0200 & 0.090 & enan & 0.080 & Q.8B8 & 8.088 & 0.080 & & sParam. & $\# 18$ & PPSIFr & & & & \\
\hline 173 & & 19 & 0.02 & 0.000 & 0.090 & 0.000 & e.201 & 0.080 & .0 .000 & 8.090 & & sparam. & $* 19$ & PPAIFr & & & & \\
\hline 174 & & 20 & 0.188 & 0.0110 & 0.080 & 0.000 & 0.090 & 0.8B8 & .0 .090 & 0.098 & & SParam. & $\# 28$ & PAH-Fr & & & & \\
\hline 175 & & 21 & 0.000 & 0.2667 & 2.000 & 0.000 & D.eng & Q.6us & $0.0 B 8$ & D.080 & & SParam. & $\# 21$ & OEU-Fr & & & & \\
\hline 176 & & 22 & 0.000 & 0.2667 & 0.080 & 0.008 & 0.018 & 0.888 & 0.080 & Q.982 & & sparam. & $\$ 22$ & DECIF & & & & \\
\hline 177 & & 23 & 0.000 & 0.0918 & 0.090 & 0.000 & 0.000 & Q.8B日 & E.098 & O.0Bg & & SParam. & $\# 23$ & PBFTFr & & & & \\
\hline 178 & & 24 & $0.0 \mathrm{BO}$ & e.enes & 0.088 & 0.040 & 0.010 & 8.880 & 0.880 & 8.280 & & SParam. & $\# 24$ & PABIFr & & & & \\
\hline 179 & & 25 & 0.19 & 0.0285 & 8.480 & 0.989 & C.eng & Q.8Be & Q.8BE & 8.680 & & sparam. & $\# 25$ & SME-Fr & & & & \\
\hline 188 & & 26 & 0.800 & D.2085 & 8.4日0 & 0.800 & 0.080 & 8.080 & 2.880 & 0.988 & & sParam. & $\# 26$ & SPCUPSFr & & & & \\
\hline 181 & & 27 & 0.000 & E.0885 & 0.088 & 0.800 & 0.080 & 8.8B8 & 0.880 & 0.080 & & sParam. & $\# 27$ & SAATFr & & & & \\
\hline 182 & & 28 & 0.888 & 0.0925 & 0.080 & 8.890 & Q.ene & 8.860 & 0.880 & 0.080 & & SParam. & $\# 28$ & ACIFr & & & & \\
\hline 105 & & 29 & 0.000 & 0.0095 & 0.080 & 2.800 & e.080 & 8.880 & 0.800 & 0.080 & & \$Param. & $\# 29$ & MFTFr & & & & \\
\hline 104 & & 38 & 0.880 & 1.0098 & 0.189 & C.8B日 & $0.08 \mathrm{~g}$ & 8.880 & C.B88 & 8.680 & & SParam. & \#38 & Mellfr & & & & \\
\hline 185 & & 31 & 0.000 & 0.0923 & 0.000 & 0.880 & 0.080 & 0.080 & 0.008 & 0.080 & & sparam. & $\# 31$ & DLUSTFr & & & & \\
\hline 185 & & 32 & 8.000 & 8.0808 & 8.880 & 0.680 & e.ese & 0.890 & 9.8BO & 0.889 & & sParam. & 432 & PPCoufr & & & & \\
\hline 187 & & 33 & 0.080 & 0.0981 & 0.180 & 0.089 & Q.008 & 8.880 & 0.800 & 0.080 & & sParam. & $\# \mathbf{3 3}$ & CCoufr & & & & \\
\hline 188 & & 34 & 0.080 & 1.0898 & e.080 & 0.000 & Q.0B日 & 9.880 & 0.880 & Q.8B日 & & SParam. & $\# 34$ & Iflinfr & & & & \\
\hline 189 & & 72 & 8.008 & 1.2010 & 0.280 & 0.080 & 0.008 & 0.888 & 0.000 & Q.080 & & SParam. & $\# 72$ & BlkN2Fr & & & & \\
\hline 198 & & 73 & 8.000 & 0.012 & e.ene & 0.080 & 0.000 & 2.808 & 0.000 & 0.808 & & sparam. & $\# 73$ & CPCN2Fr & & & & \\
\hline 191 & & 74 & 0.800 & 0.0018 & 0.089 & 0.080 & Q.0pa & Q.8B日 & 0.880 & 8.080 & & Sparam. & $\# 74$ & NPwrAn & & & & $\therefore$ \\
\hline 192 & & 75 & 8.888 & 0.0880 & 0.080 & 0.000 & e.180 & 0.080 & 0.080 & 0.080 & & \$Param. & $\# 75$ & EPErtin & & & & \\
\hline 193 & & 76 & $0.08 B$ & 0.0980 & C.08B & 2.890 & 0.080 & Q.8B日 & e.980 & Q.8B & & sparam. & $\# 76$ & PStman & & & & \\
\hline 194 & & 77 & 9.080 & e.egne & 0.000 & 0.098 & e.080 & 0.680 & Q.8BO & $0.08 B$ & & SParam. & $\$ 77$ & PCWAn & & & & \\
\hline 195 & & 78 & 0.000 & 0.0000 & 0.080 & 0.098 & 0.080 & 0.888 & 0.800 & 0.008 & & SParm. & $\# 78$ & $\mathrm{CO} R \mathrm{Rn}$ & & & & \\
\hline 196 & & 80 & 0.080 & 0.090 & 0.080 & E.008 & 0.080 & 8.880 & -0.880 & 8.080 & & sparam. & $\# 8 B$ & Allan & & & & \\
\hline 197 & & 81 & 0.080 & 2.0898 & e.e98 & 0.000 & 0.090 & 0.808 & 0.080 & 0.000 & & sParam. & $\# 81$ & N2IKRn & & & $\therefore$ & \\
\hline 198 & & 82 & 8.080 & 0.0000 & 0.080 & 8.080 & e.e8g & 9.888 & 0.088 & 0.800 & & SParam. & $\# 82$ & CnERn & & & & \\
\hline 199 & & 83 & O.ABQ & 0.0880 & 0.089 & 0.809 & 0.080 & 0.089 & 0.880 & 0.800 & & SParam. & $\# 83$ & Collin & & & & \\
\hline 200 & & 84 & 8.000 & 0.0900 & 0.080 & 0.980 & 0.080 & 8.880 & 0.880 & 8.280 & & sparam. & $\# 84$ & BCO2An & & & & \\
\hline 201 & & 85 & 0.080 & 0.0880 & 0.088 & 8.889 & 0.098 & 0.880 & 0.180 & 0.100 & & sparam. & $\# 85$ & Inirkn & & & & \\
\hline 202 & & 86 & 0.000 & 0.0988 & 0.289 & 0.808 & 0.080 & 0.880 & 0.880 & B.880 & & SParam. & \#86 & BlkN2An & & & & \\
\hline 203 & & 87 & 8.080 & 0.0898 & 0.000 & 0.088 & 0.088 & 0.808 & 0.888 & 8.880 & & sparam. & \# 87 & CPCN2Rn & & & & \\
\hline 204 & & 96 & 8.898 & 0.0000 & 0.081 & 0.020 & Q.080 & 0.088 & 0.080 & 0.080 & & sparam. & $\# 96$ & Fanfn1 & & & & \\
\hline 205 & & 97 & 0.080 & 1.0880 & 0.088 & 0.080 & 8.089 & 0.080 & 0.890 & 0.080 & & SParam. & \#97 & cTCmfr & & & & \\
\hline 206 & & 101 & 0.889 & 0.0880 & 0.800 & 8.880 & 2.880 & 0.880 & 0.88B & 0.888 & & \$param. & $\# 101$ & creman & & & & \\
\hline 207 & & 102 & 0.898 & 1.8889 & 0.080 & 2.898 & 8.880 & 0.808 & 0.888 & 0.8B日 & & SParam. & \#102 & DWelfr & & & & \\
\hline 208 & & 186 & 0.080 & 0.0890 & 0.080 & 8.080 & 9.088 & 0.800 & e.e日8 & 0.089 & & SParam. & +186 & DEelAn & & & & \\
\hline 209 & & 187 & 8.880 & 0.0889 & 0.098 & 0.800 & 2.800 & 0.080 & 0.898 & D.80日 & & SParam. & $\# 187$ & CPCIKFr & & & & \\
\hline 210 & & 111 & 0.090 & 0.0898 & 0.089 & 0.080 & 8.880 & 8.000 & 0.088 & Q.088 & & SParam. & $\# 111$ & CPCTKRn & & & & \\
\hline 211 & & 112 & 8.880 & 0.0831 & 0.889 & 8.080 & 9.088 & 0.080 & 0.088 & 0.080 & & sparam. & $* 112$ & PPN2Fr & & & & \\
\hline 212 & & 116 & 8.898 & O.80日a日 & 6.8B日 & Q.8B日 & 8.88日 & 8.880 & 8.088 & $0.8 \mathrm{Bg}$ & & SParam. & $\# 116$ & PPN2An & & & & \\
\hline
\end{tabular}


APPENDIH A

WSAC-TR-95-8113

\begin{tabular}{|c|c|c|c|c|c|c|c|c|c|c|c|c|c|c|c|c|c|c|}
\hline & n & 2 & c & \pm & $\bar{E}$ & $\bar{F}$ & E & H & $I$ & J & $\underline{K}$ & $L$ & $M$ & $N$ & 0 & $P$ & a & H \\
\hline 213 & & 117 & C.888 & 0.1831 & 0.080 & 0.898 & 0.098 & 9.08B & . .888 & 0.080 & & sparam. & $\# 117$ & SPCN2Fr & & & & \\
\hline 214 & & 121 & 0.080 & 0.2010 & . .80 & 8.200 & 0.000 & $0.0 \mathrm{Bg}$ & 8.889 & e.ege & & sparam. & $\# 121$ & SPCN2Rn & & & & ${ }^{\circ}$ \\
\hline 215 & & 122 & 0.082 &. $.18 \mathrm{Bg}$ & 0.800 & 0.010 & 1.280 & 8.089 & 0.898 & 0.080 & & sparam. & $\$ 122$ & Fanfnz & & & & \\
\hline 216 & & 123 & 0.010 & 0.0000 & 0.090 & 0.089 & 0.090 & 8.089 & Q.8989 & 0.080 & & sparam. & $\$ 123$ & FanAn3 & & & & \\
\hline 217 & & 129 & e.ene & 1.0010 &. .890 & 0.028 & ..280 & 0.098 & 0.090 & 0.080 & & SParam. & \#129 & M06_fr & & & & \\
\hline 218 & 3 & Is ta & allure of $a c$ & common lle. & sypportl s & system the I & Inlliating eu & vent? & & & & & & & & & & \\
\hline 219 & & 11 & ncosyln & NPowinli & PSImInit & Ifourinll & Co2Inil & Firinglt & N2IKInII & chwinls & Colinit & BIKN2InIt & cTeminit & & & & 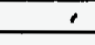 & \\
\hline 220 & & 4 & & & & 4 & 5 & 6 & 7 & & 9 & 10 & 11 & & & & & \\
\hline 221 & & 3 & & & & & & & & & & & & & & & & \\
\hline 222 & & (4) & 1 & 1 & 1 & 1 & & & & & & & & & & & & \\
\hline 223 & & & 1 & +3 & +4 & +5 & & & & & & & & & & & & \\
\hline 224 & & & Exlernal & Lfoleak & LIAMISE & LIACD & & & & & & & & & & & & \\
\hline 225 & & & 1.00898 & 0.01000 & Q.99000 & 8.00009 & 2.18089 & Q.8B日80 & C.08e日ge & 8.00980 & 8.89820 & 0.08889 & 8.8B日BQ & & & & & \\
\hline 226 & & 1 & & & & & & & & & & & & & & & & \\
\hline 227 & & 35 & 0.08 & 0.09 & 0.00 & 0.08 & 8.20 & 0.08 & 8.88 & 0.80 & 0.88 & $\theta .89$ & 0.00 & & SPar* 35 & MsTmInt & & \\
\hline 228 & & 1 & & & & & & & & & & & & & & & & \\
\hline 229 & & & 2 & & & & & & & & & & & & & & & \\
\hline 238 & & & Int-Eq4 & & & & & & & & & & & & & & & \\
\hline 231 & & & 0.99949 & $3.45 E-05$ & $2.13 E-04$ & $1.89 \mathrm{E}-\mathrm{-s5}$ & 1.16E-B4 & $2.31 E-85$ & $2.98 E-85$ & $1.47 E-85$ & $2.47 E-86$ & $4.38 E-85$ & $1.05 E-05$ & & & & & $\dot{-}$ \\
\hline 232 & & 1 & & & & & & & & & & & & & & & & \\
\hline 233 & & 35 & 1.08 & 0.80 & 0.09 & 0.08 & 8.08 & 1.80 & 8.88 & 0.00 & 8.08 & 8.80 & 0.80 & & & & & \\
\hline 234 & 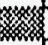 & & Otherwise- & -nopalih & & & & & & & & & & & & & & \\
\hline 235 & & & 1.08090 & D.08Bge & 0.08800 & 0.88808 & 0.08800 & 8.08880 & 0.088880 & B.88898 & E.88800 & 8.80808 & 8.08889 & & & & & \\
\hline 236 & & 1 & & & & & & & & & & & & & & & & \\
\hline 237 & & 35 & 0.80 & 8.80 & 0.08 & 0.80 & 8.80 & 0.80 & 0.88 & 0.80 & 8.88 & 8.80 & 8.88 & & & & 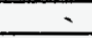 & \\
\hline 238 & 4 & ifth & he acclotent & class is an & 'Intemal' e & event class! & Ifled 'leak' & what is the & frequencyo & of the even & $n 18$ & & & & & & & \\
\hline 239 & & 11 & InIntleak & Mllir-Leak & CPC-0Ilou & I]PP-Oflow & CPC-Leak & SPC-LeaK & PP-LEak & Can-Aup & BZ-0flow & BZ-Leak & SPC-0Ilow & & & & & \\
\hline 240 & & 2 & 1 & 2 & 3 & 4 & 5 & & & 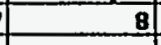 & 9 & 10 & 11 & & & & & \\
\hline 241 & & 2 & & & & & & & & & & & & & & & & \\
\hline 242 & 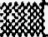 & 1 & & & & & & & & & & & & & & & & \\
\hline 243 & & 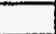 & 3 & & & & & & & & & & & & & & & \\
\hline 244 & & & Litaleak & & & & & & & & & & & & & & & \\
\hline 245 & & & 0.998281 & $0.09 E+8 \mathrm{~B}$ & $9.74 E-86$ & $9.74 E-96$ & $4.2 \mathrm{gE}-04$ & $4.20 \mathrm{E}-89$ & $4.28 E-84$ & $1.48 \mathrm{E}-8 \mathrm{~B}$ & $9.74 E-86$ & 4.28E- -84 & $9.74 E-16$ & & & & & \\
\hline 246 & . & & Diherwise & no leak & & & & & & & & & & & & & & \\
\hline 217 & & & 1.080 & 0.808 & 0.080 & 0.098 & 8.080 & 8.098 & 8.880 & 0.000 & 0.880 & 0.980 & 8.809 & & & & & \\
\hline 248 & 5 & IIth & ne accident & classis an & internal' a & yent clessl & ed 'Misc' & what is the & Prequency & of the even & int? & & & & & & & \\
\hline 249 & & 4 & $|n \ln 1 \mathrm{M}| \mathrm{sc}$ & CPC-UCA & $116420 \mathrm{MI}$ & PA-UCA & & & & & & & & & & & & \\
\hline 258 & - & 2 & 1 & & 3 & 4 & & & & & & & & & & & & \\
\hline 251 & 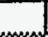 & 2 & & & & & & & & & & & & & & & & \\
\hline 252 & 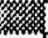 & 1 & 1 & & & & & & & & & & & & & & & \\
\hline 253 & 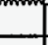 & & 4 & & & & & & & & & & & & & & & \\
\hline 254 & & & LIOMISE & & & & & & & & & & & & & & & \\
\hline 255 & & & 0.999989 & $5.09 \varepsilon-86$ & 1.00E-106 & 5.09E- 86 & & & & & & & & & & & & \\
\hline 256 & & 6 & Jotherwlse & - not Mlsc. & Inlliator & & & & & & & & & & & & & \\
\hline 257 & & & 1.890 & $8.89 \mathrm{~g}$ & I 8.08 a & 0.999 & & & & & & & & & & & & \\
\hline 258 & 6 & Inth & ne accldent & class is cra & ane drop wh & hat is the ir & requency of & the event? & & & & & & & & & & \\
\hline 259 & & 2 & InCraprop & Cranedrop & & & & & & & & & & & & & & \\
\hline 260 & & 2 & 1 & & & & & & & & & & & & & & & \\
\hline 261 & & 2 & & & & & & & & & & & & & & & & \\
\hline 262 & 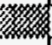 & 1 & 1 & & & & & & & & & & & & & & & \\
\hline 263 & 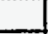 & & & & & & & & & & & & & & & & & \\
\hline 264 & & E & Ltaco & & & & & & & & & & & & & & & \\
\hline 265 & & E & 8.999851 & $1.49 \mathrm{E}-84$ & & & & & & & & & & & & & & \\
\hline
\end{tabular}


APPENDIH :

ACCIDENT PROGRESSION EUENT TAEE FOA DEFENSE WASTE PROCESSING FACILITY - MODE C - PRODUCTION UERSION

\begin{tabular}{|c|c|c|c|c|c|c|c|c|c|c|c|c|c|c|c|c|c|c|}
\hline & n & B & C & D & $E$ & $F$ & s & $\mathrm{H}$ & I. & $J$ & $K$ & $L$ & $\bar{M}$ & $\bar{N}$ & 0 & $\bar{P}$ & a & A \\
\hline 266 & & & Dtherulse & not co & & & & & & & & & & & & & & \\
\hline 267 & & & 1.090 & 0.098 & & & & & & & & & $\therefore$ & & & & & \\
\hline 268 & 7 & Wha & If are the st & ppoort syste & em fallure & Trequencle & is and repal & Ir IImes? & & & & & & & & & & \\
\hline 269 & & 1 & Lam/Tau & & & & & & & & & & & & & & & \\
\hline 270 & & 4 & 1 & & & & & & & & & & & & & & & \\
\hline 271 & & 3 & & & & & & & & & & $E$ & & & & & $\cdots$ & \\
\hline 272 & & 1 & 1 & & & & & & & & & & & & & & & \\
\hline 273 & & & 2 & & & & & & & & & . & & & & & & \\
\hline 274 & & & Int-Egy & & & & & & & & & & & & & & & $\therefore$ \\
\hline 275 & & & 1.000 & & & & & & & & & & & & & - & & \\
\hline 276 & & 48 & & & & & & & & & & & & & & & & \\
\hline 277 & & 36 & $3.48 E-85$ & & & & & & & & & sparam. & $\$ 36$ & NPErLmda & & & & \\
\hline 278 & & 37 & $1.09 \mathrm{E}-02$ & & & & & & & & & sparam. & $\$ 37$ & EPwrimda & $\therefore$ & & & \\
\hline 279 & & 38 & $2.22 \mathrm{E}-94$ & & & & . & & & & & sparam. & $\# 38$ & PStmLmua & & & & \\
\hline 200 & & 39 & $2,39 E-123$ & & & & & & & & & sparam. & $\# 39$ & PCULmde & & & & \\
\hline 221 & & 42 & $6.76 E-05$ & & & & & & & & & sparam. & $\# 48$ & \begin{tabular}{|c|} 
C02Lmda \\
\end{tabular} & & & & \\
\hline 282 & & 41 & $1.85 E-05$ & & & & & & & & & sparam. - & $\# 41$ & Girlmda & & & & \\
\hline 283 & & 42 & $7.92 E-86$ & & & & & & & & & \$Param. & $\# 42$ & N2TKLImda & & & & \\
\hline 294 & & 43 & $2.29 \mathrm{E}-\mathrm{C5}$ & & & & & & & & & sParam. & $\# 43$ & Chulmoa & & & & \\
\hline 225 & & 44 & $1.08 E-03$ & & & & & & & & & sparam. & $\# 44$ & FanLmda I & & & & \\
\hline 206 & & 45 & $1.11 E-05$ & & & & & & & & & sparam. & $\# 45$ & Ifintmoa & & & & \\
\hline 287 & & 46 & $6.21 E-86$ & & & & & & & 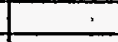 & & sparam. & $\# 46$ & Collmda & & & & \\
\hline 288 & & 47 & $6.23 \mathrm{E}-\mathrm{B3}$ & & & & & & & & & SParam. & $\# 47$ & BCO2Limda & & & & \\
\hline 289 & & 48 & $8.58 E+80$ & & & & & & & & & SParam. & 448 & NPurTau & & & & \\
\hline 298 & & 49 & $1.89 E+80$ & & & & & & & & & sparam. & $\# 49$ & EPwrTau & & & & \\
\hline 291 & & 50 & $5.49 E+11$ & & & & & & & & & sparam. & 450 & PStmTau & & & & \\
\hline 292 & & 51 & $1.08 E+80$ & & & & & & & & & sparam. & $\# 51$ & . PCWIau & & & & \\
\hline 293 & & 52 & $8.88 E+80$ & & & & & & & & & sparam. & $\# 52$ & C02Tau & & $\overline{-}$ & & \\
\hline 294 & & 53 & $5.12 E+11$ & & & & & & & & & sparam. & $\$ 53$ & AlirTau & & & & \\
\hline 295 & & 54 & $1.72 E+81$ & . & & & & & & & & sparam. & $\$ 54$ & N2TkTau & & & & \\
\hline 296 & & 55 & $1.43 E+01$ & & & & & & & & & sparam. & $\# 55$ & ChWTau & & & & \\
\hline 297 & & 56 & $1.09 E+80$ & & & $\dot{-}$ & & & & & & sparam. & $\$ 56$ & FanTaul & & & & \\
\hline 298 & & 57 & $2.65 \mathrm{E}+01$ & & & & & & & & & sparam. & $\$ 57$ & IfilrTau & & & & \\
\hline 299 & & 58 & $9.02 \mathrm{E}+21$ & & & & & & & & & sparam. & $\$ 58$ & Corran & & & & \\
\hline 308 & & 59 & $1.0 B E+00$ & & & & & & & & $!$ & sparam. & $\$ 59$ & BCo2Tau & $=$ & & & \\
\hline 301 & & 79 & $1.08 E+80$ & & & & & & & & & sparam. & $\$ 79$ & Dummy & & & & \\
\hline 302 & & 88 & $5.41 E-06$ & & & & & & & $\therefore$ & & sparam. & 488 & BIkN2Lmda & & & & \\
\hline 303 & & 89 & $1.96 E+81$ & & & & & & & & & sparam. & $\$ 89$ & BIKN2Tau & & & & \\
\hline 304 & & 91 & $3.27 \mathrm{E}-83$ & & & & & & & & & sparam. & $\$ 91$ & CPCN2Lmda & & & & \\
\hline 305 & & 92 & 1.BBE+8B & & & & & & & & & sparam. & $\$ 92$ & CPCN2Tau & & & & \\
\hline 326 & & 99 & $1.08 E-85$ & & & & & & & & & sparam. & 499 & cTrmlmua & & & & \\
\hline 307 & & 100 & $5.58 E+80$ & & & & & & & & & sparam. & $\# 108$ & cremiau & & & & \\
\hline 318 & & 104 & $5.29 E-03$ & & & & & & & & & sparam. & $\# 104$ & DWellmoa & & & & \\
\hline 309 & & 105 & $1.00 E+00$ & & & & & & & & & sparam. & $\# 185$ & Dueltau & & & & \\
\hline 310 & & 189 & $3.82 \mathrm{E}-05$ & & & & & $=$ & & & & sparam. & $\# 109$ & CPCIKLmde & & & & \\
\hline 311 & & 110 & $1.08 E+B 8$ & & & & & & & & & sparam. & $\$ 110$ & CPETKTаU & & & & \\
\hline 312 & & 114 & $5.16 E-84$ & $=$ & & & & & & & & sparam. & $\# 114$ & PPN2Lmda & & & & \\
\hline 313 & & 115 & 1.9BE+80 & & & & & & & & & sparam. & $\# 115$ & PPN2Tau & & & & \\
\hline 314 & & 119 & $4.11 E-84$ & & & & & & & & & sparam. & $\# 119$ & SPCN2Lmda & & & & \\
\hline 315 & & 120 & $1.00 \mathrm{E}+8 \mathrm{\theta}$ & & & & & & & & & sparam. & $\# 128$ & SPCN2TaU & 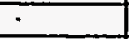 & & & \\
\hline 316 & & 124 & $7.42 E-83$ & & & & & & & & & sparam. & $\# 124$ & Fanlmda2 & & & & \\
\hline 317 & & 125 & $1.0 \mathrm{EE}+8 \mathrm{\theta}$ & & & & & & & & & sparam. & $\# 125$ & FanTau2 & & & & \\
\hline 318 & & 126 & $3.51 E-85$ & & & & & & & & & Sparam. & $\# 126$ & Fankmoa3 & & & & \\
\hline
\end{tabular}




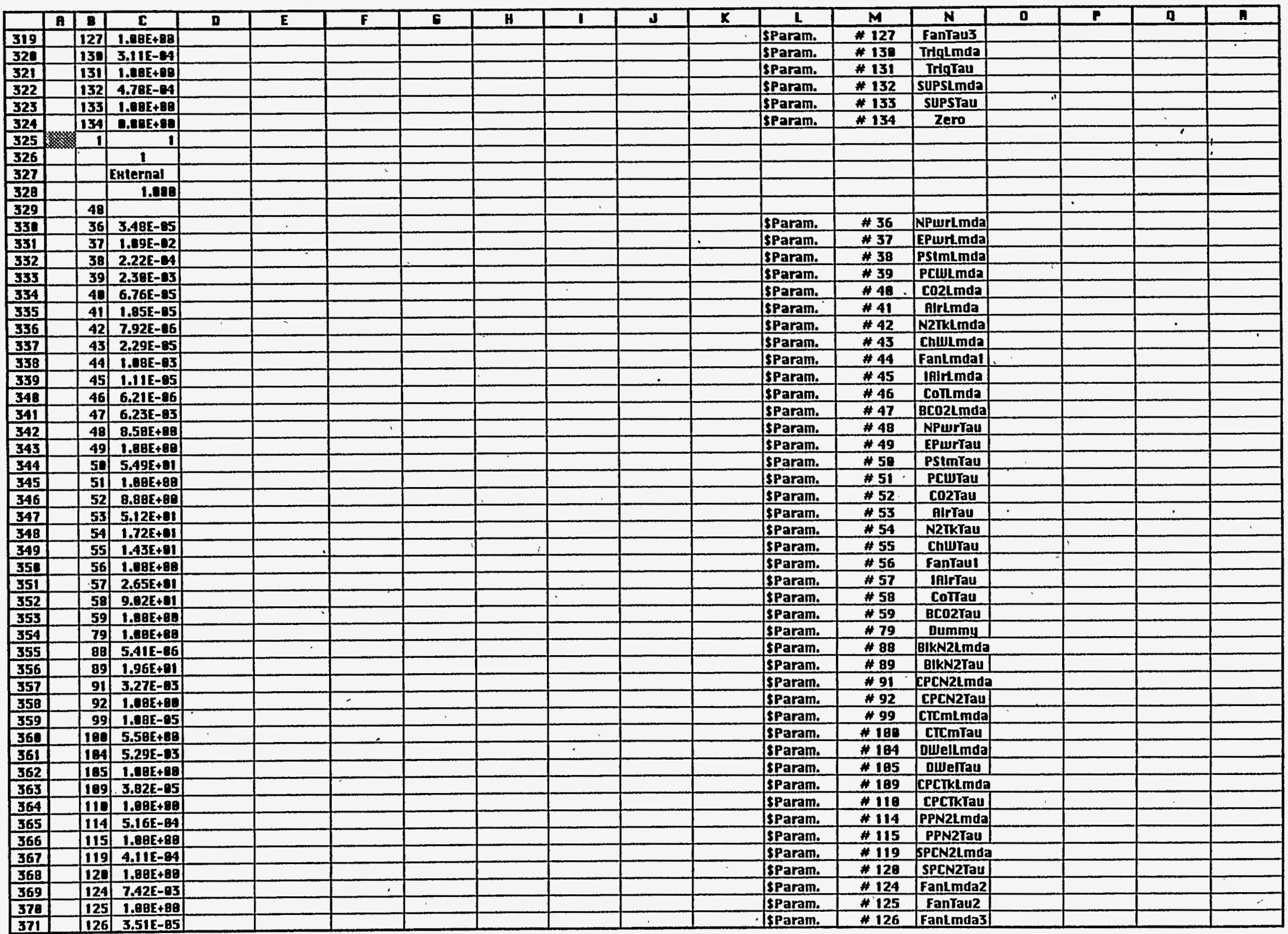


APPENDIH A

ACCIDENT PAOGRESSION EUENT TREE FOR BEFENSE UUSTE PROCESSING FACILITY - MODE C - PRODUCTION UERSION

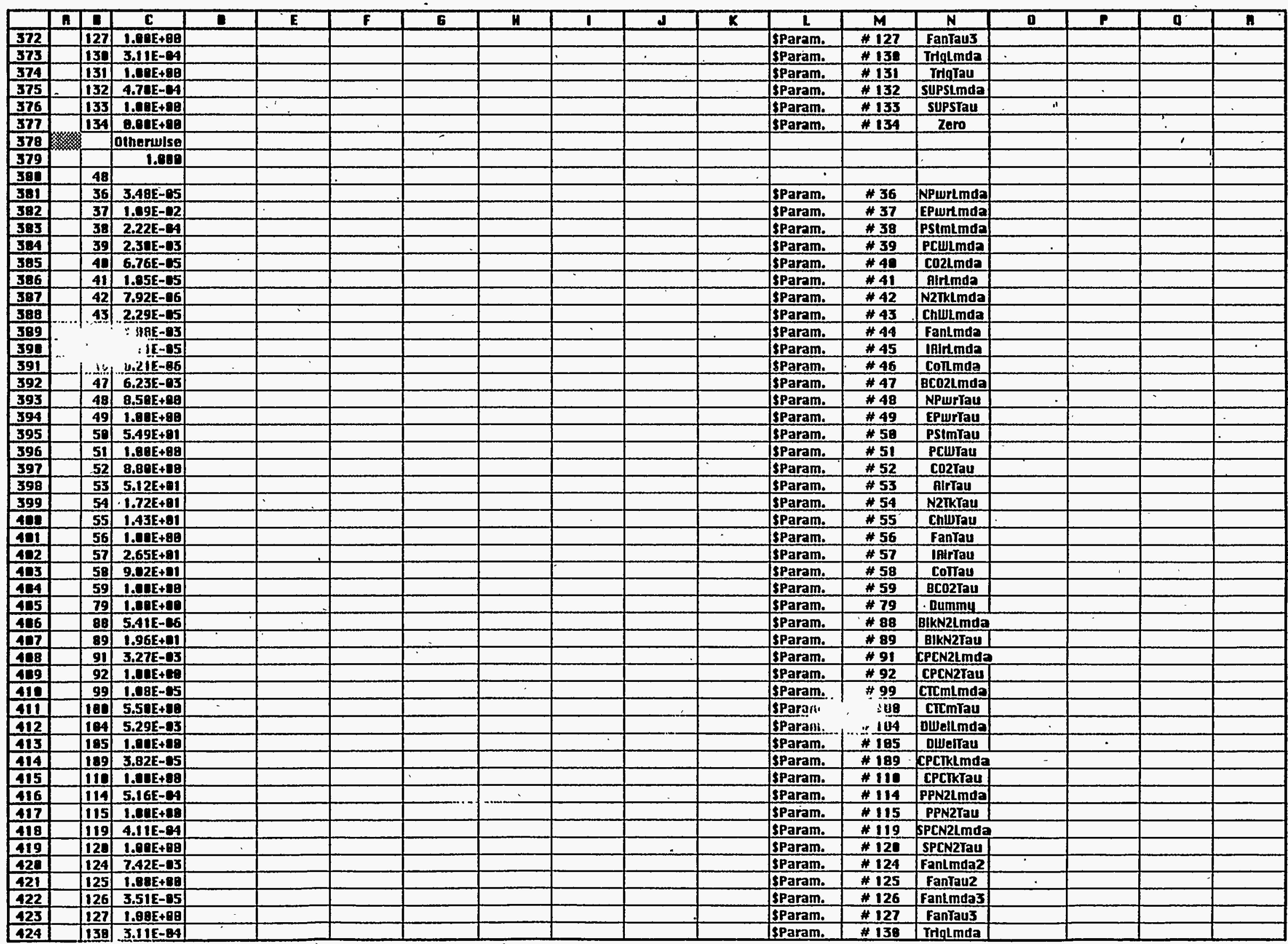


APPENDIH A

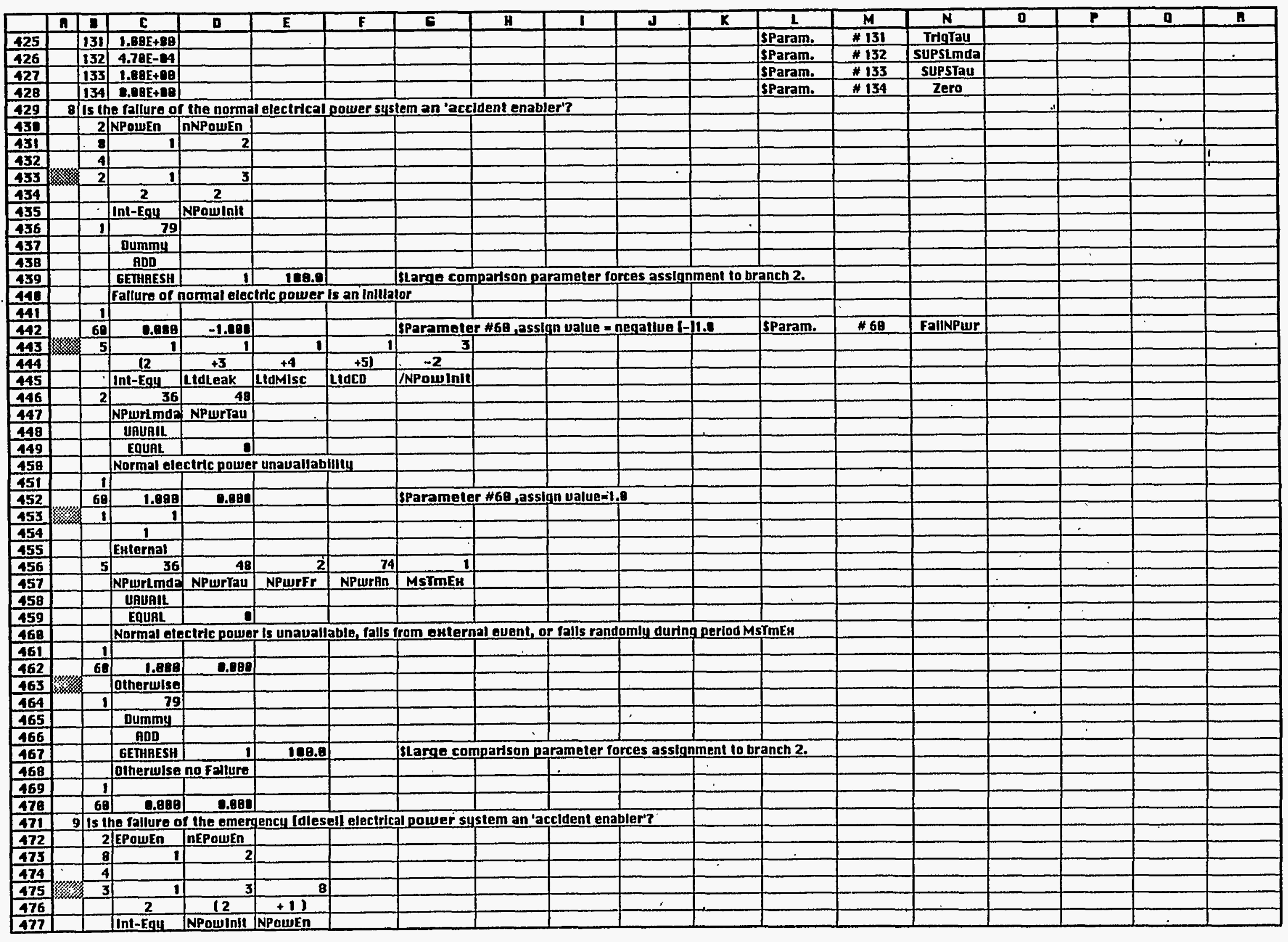




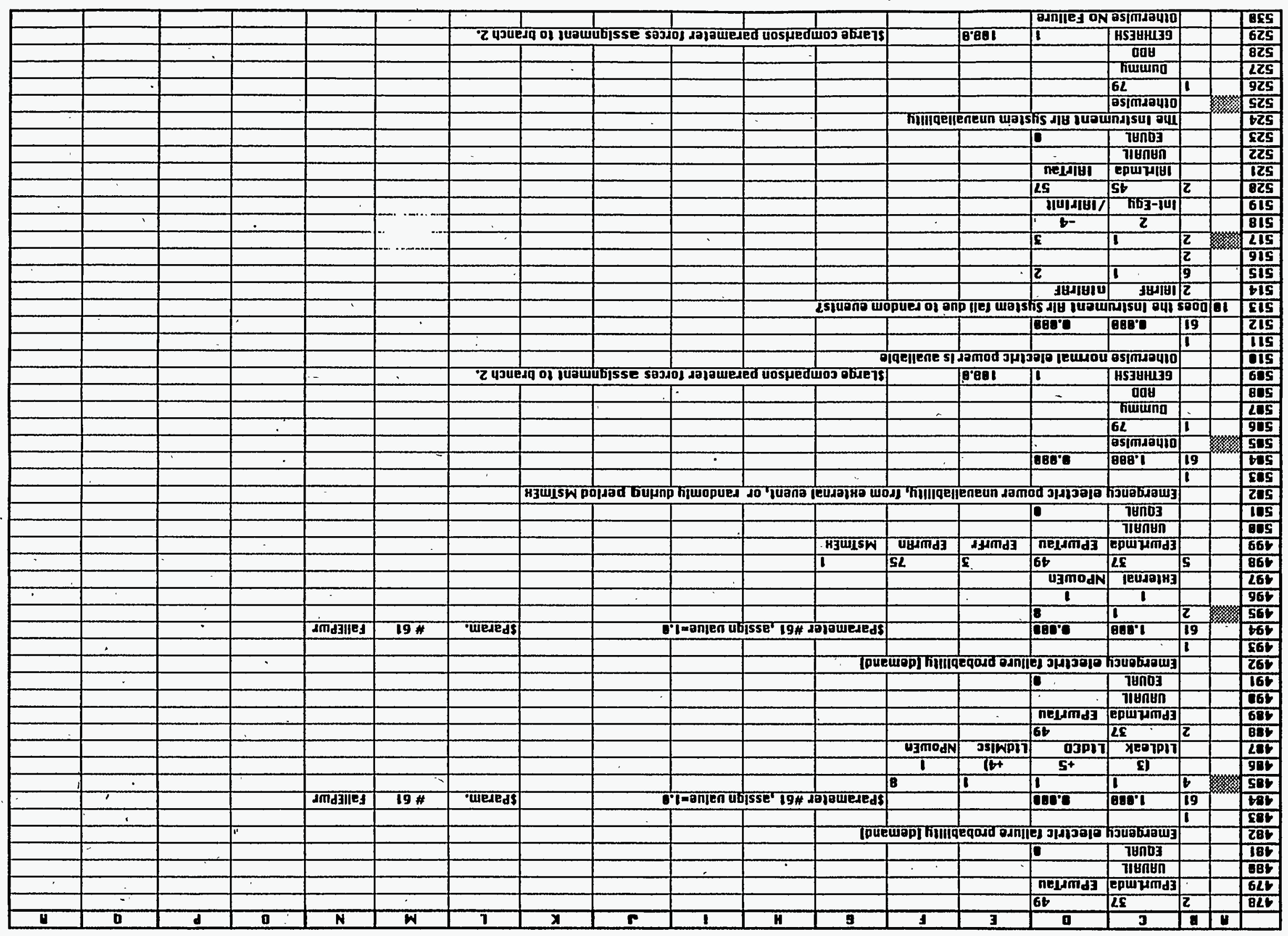

ocz obed

ELB-56-HL-3usm

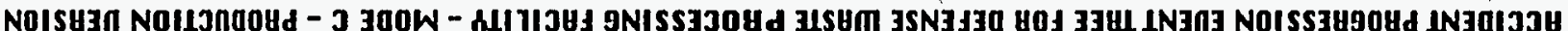
B HION3ddU 


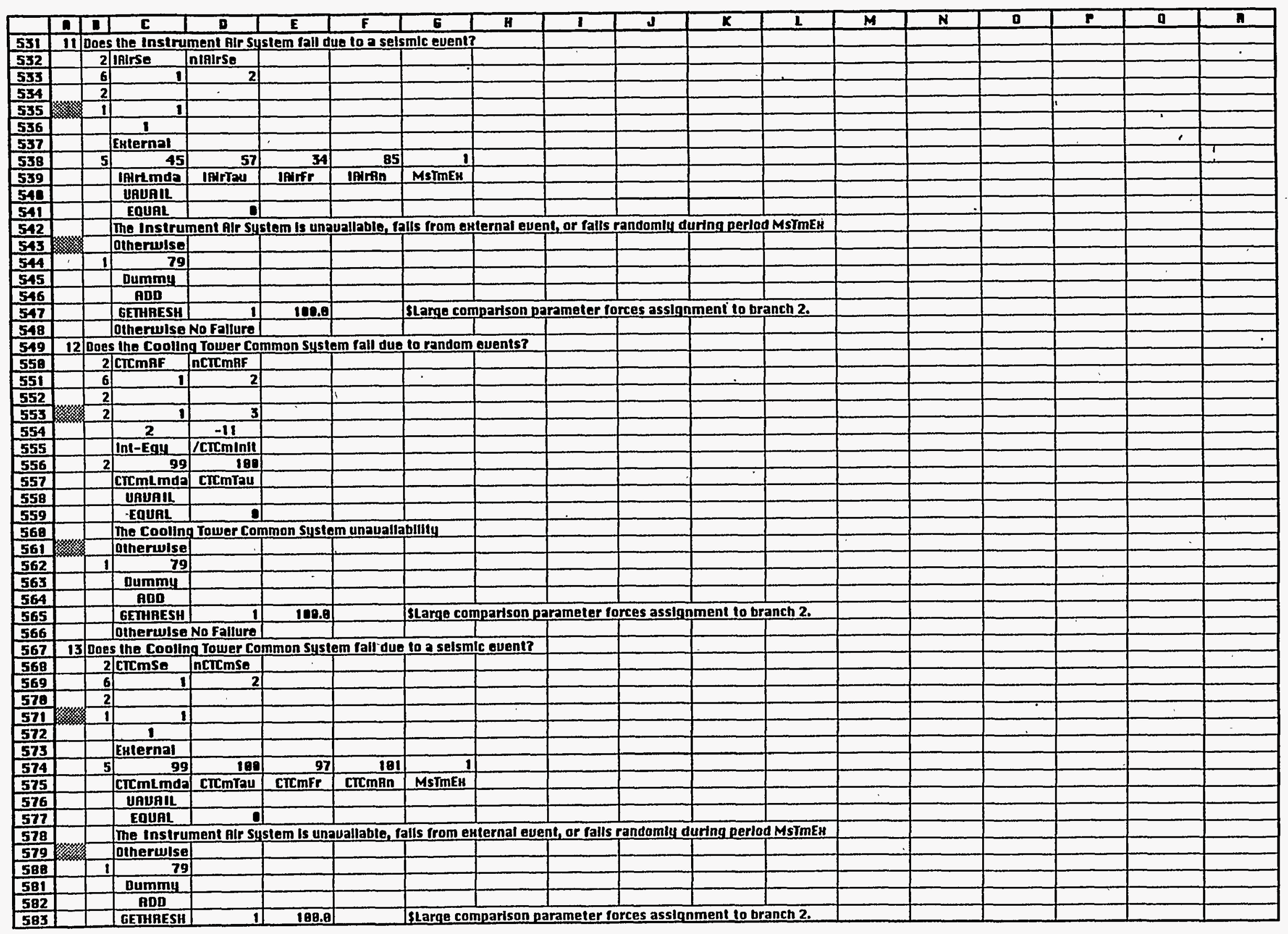




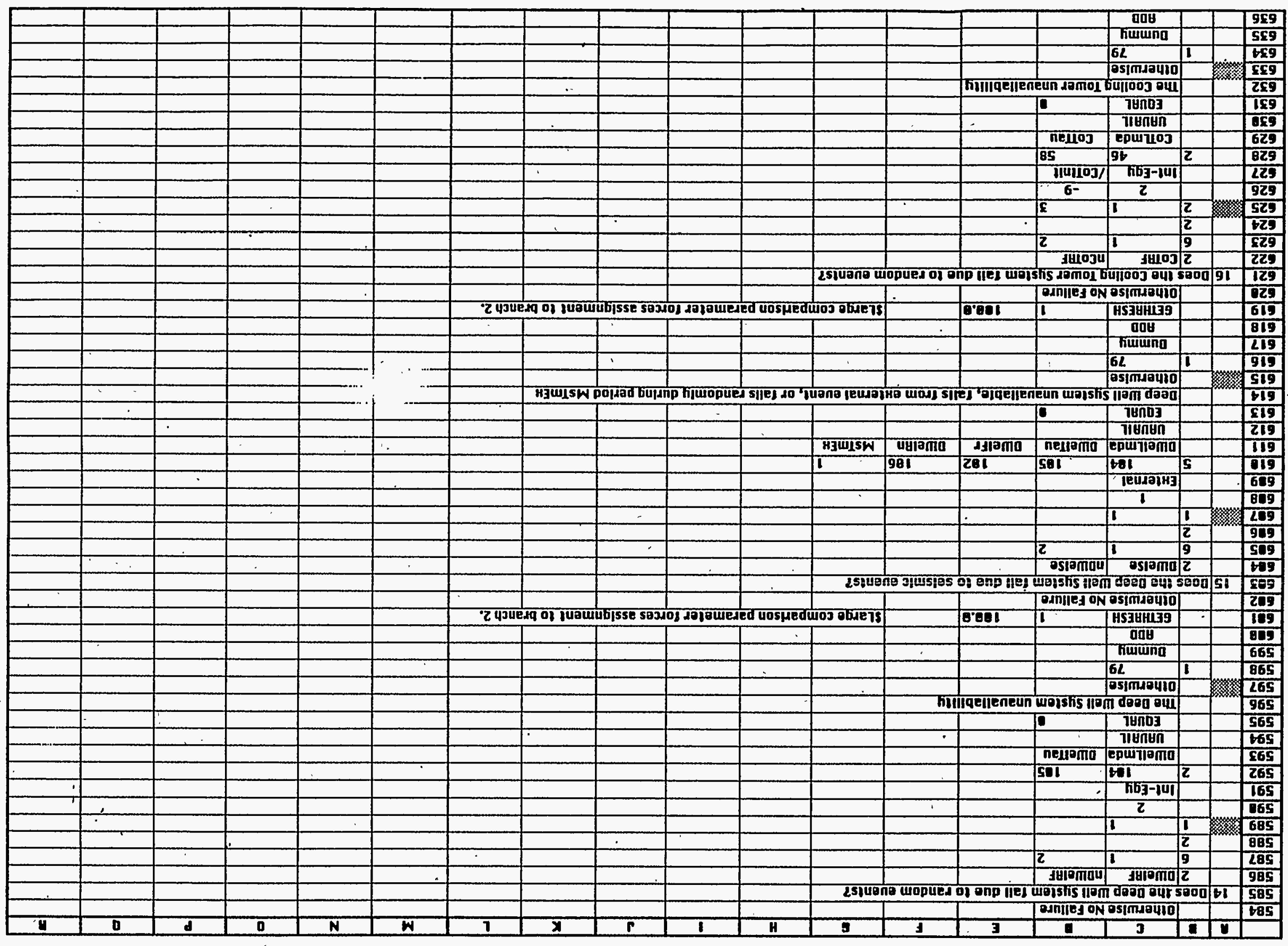

Z\&Z จfied

S118-56-H1-J8Sm

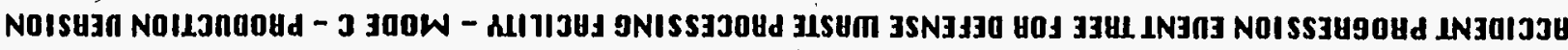

H HIONBddU 


\begin{tabular}{|c|c|c|c|c|c|c|c|c|c|c|c|c|c|c|c|c|c|c|}
\hline & A & E & C & D & $E$ & $F$ & $E$ & H & 1 & 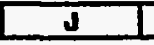 & $\mathbf{K}$ & 1 & $M$ & $N$ & 0 & $\mathbf{P}$ & a & A \\
\hline 637 & & & \begin{tabular}{|l|} 
GETHAESH \\
\end{tabular} & II & 180.0 & & sLarge com & partson p? & rameter for & rrces asslgn & ment to br: & anth 2. & & & & & & \\
\hline 638 & & & otherwlse & No Fallure & & & & & & & & & & & & & & \\
\hline 639 & 17 & Does & the coolln & a rower Sys & tem foll du & to o sels & mic eventry & & & & & & & & & & & \\
\hline 648 & & 2 & Corse & ncolse & & & & & & & & & & & & & & \\
\hline 641 & & 6 & 1 & 2 & & & & & & & & & & & . & & & \\
\hline G42 & & 2 & & & & & & & & & & & & & & & 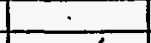 & \\
\hline 643 & 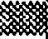 & 1 & 1 & & & & & & & & & & & & & & 2 & \\
\hline 644 & & & 1 & & & & & & & & & & & & & & & \\
\hline 645 & & & Esternal & & & & & & & & & & & & & & & \\
\hline 646 & & 5 & 46 & 58 & 10 & 83 & 1 & & & $\therefore$ & & & & & & & & \\
\hline 647 & & & \begin{tabular}{|c|} 
Corlmda \\
\end{tabular} & ColTau & CoIfr & CoIfn & MSIIIEH & & & & & & & & & & & \\
\hline 648 & & & URUARIL & & & & & & & & & & & & & & & \\
\hline 649 & & & EQUAL & 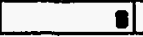 & & & & & & & & & & & & & & \\
\hline 658 & & & Coolling Tou & wer System & unavalleble & fells Iran & n estemals & vent, or 1 & alls random & dy during pe & erjod Msim & & & & & & & \\
\hline 651 & & & otherwise & & & & & & & & & & & & & & & \\
\hline 652 & & 1 & 79 & & & & & & & & & & & & & & $\cdot$ & \\
\hline 653 & & & Dummy & & & & & & & & & & & & & & & \\
\hline 654 & & & ADD & & & & & & & & & & & & & & & \\
\hline 655 & & & GETHAESH & 1 & 180.0 & & SLarae com & parison pa & rameter fo & arces assign & Iment to br & anch 2. & & & & & & \\
\hline 656 & & & otherulse & No Fallure & & & & & & & & & & & & & & \\
\hline 657 & 18 & 1512 & allure of the & Instrumen & It gir Syster & an 'accid & ent enabler & & & & & & & & & & & \\
\hline 658 & & 2 & IAIrEn & nipiren & & & & & & & & & & & & & & \\
\hline 659 & & 8 & 1 & 2 & & & & & & & & & & & & & & \\
\hline 868 & & 6 & & & & & & & & & & & & & & & & \\
\hline 661 & 纱 & 2 & 1 & 3 & & & & & & & & & & & & & & \\
\hline 662 & & - & 2 & 4 & & & & & & & & & & & & & 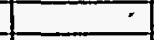 & \\
\hline 663 & & & Int-Eg4 & |firinte & & & & & & & & & & & & & & \\
\hline 664 & & 1 & 79 & & & & & & & & & & & & & & & \\
\hline 665 & & & Dummy & & & & & & & & & & & & & & & \\
\hline 666 & & & ADD & & & & & & & & & & & & & & & \\
\hline 667 & & & GETHAESH & 1 & 180.9 & & SLarge com & parison pa & rameter fo & arces assign & ament to br & anch 2. & & & & & & \\
\hline 668 & & & fallure of & the Instrum & ent fir Sys & em an Init & lator & & & & & & & & & & & \\
\hline 669 & & 1 & & & & & & & & & & & & & & & & \\
\hline 678 & & 93 & 0.080 & -1.000 & & & sparamete & r 193 , assi & gn value $=1$ & negatlue 1-1 & 11.8 & SParam. & $\# 93$ & Fallifir & & & & \\
\hline 671 & 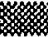 & 2 & 1 & 3 & & & & & & & & & & & & & & \\
\hline 672 & & & 2 & 11 & & & & & & & & & & & & & & \\
\hline 673 & & & Int-Egy & CTCmlnit & & & & & & & & & & & & & & \\
\hline 674 & & 1 & 79 & & & & & & & & & & & & & & & \\
\hline 675 & & & Dummy & & & & & & & & & & & & & & & \\
\hline 676 & & & ADD & & & & & & & & & & & & & & & \\
\hline 677 & & & THHESH & 1 & 0.800 & & scompariso & on parames & ter forces a & asslgnment & to branch 1 & & & & & & & \\
\hline 678 & & & Instrumen & I Ale falls b & ecause of 1 & llupe of Co & olling tower & comman & system as a & an Inillator & & & & & & & & \\
\hline 679 & & 1 & & & & & & & & & & & & & & & & \\
\hline 689 & & 93 & 1.080 & 8.888 & & & SParamele & \#93,assi & gn value-1. & & & & & & & & & \\
\hline 681 & & 3 & 3 & 8 & 9 & & & & & & & & & & & & & \\
\hline 682 & & & 12 & +11 & 1 & & & & & & & & & & & & & \\
\hline 683 & & & NPowInlt & NPOWEN & EPOWEn & & & & & & & & & & & & & \\
\hline 684 & & 1 & 79 & & & & & & & & & & & & & & & \\
\hline 685 & & & Dummy & & & & & & & & & & & & & & & \\
\hline 686 & & & $\mathrm{BDD}$ & & & & & & & & & & & & & & & \\
\hline 687 & & & THAESH & 1 & 0.890 & & scompartso & on paramet & ler forces a & assignment & 10 branch 1 & & & & & & & \\
\hline 6日8 & & & Insirumen & If Ale falls b & ecause of 8 & lliure of el & ectric polue & & & & & & & & & & & \\
\hline 689 & & i) & E & & & & & & & & & & & & & & & \\
\hline
\end{tabular}




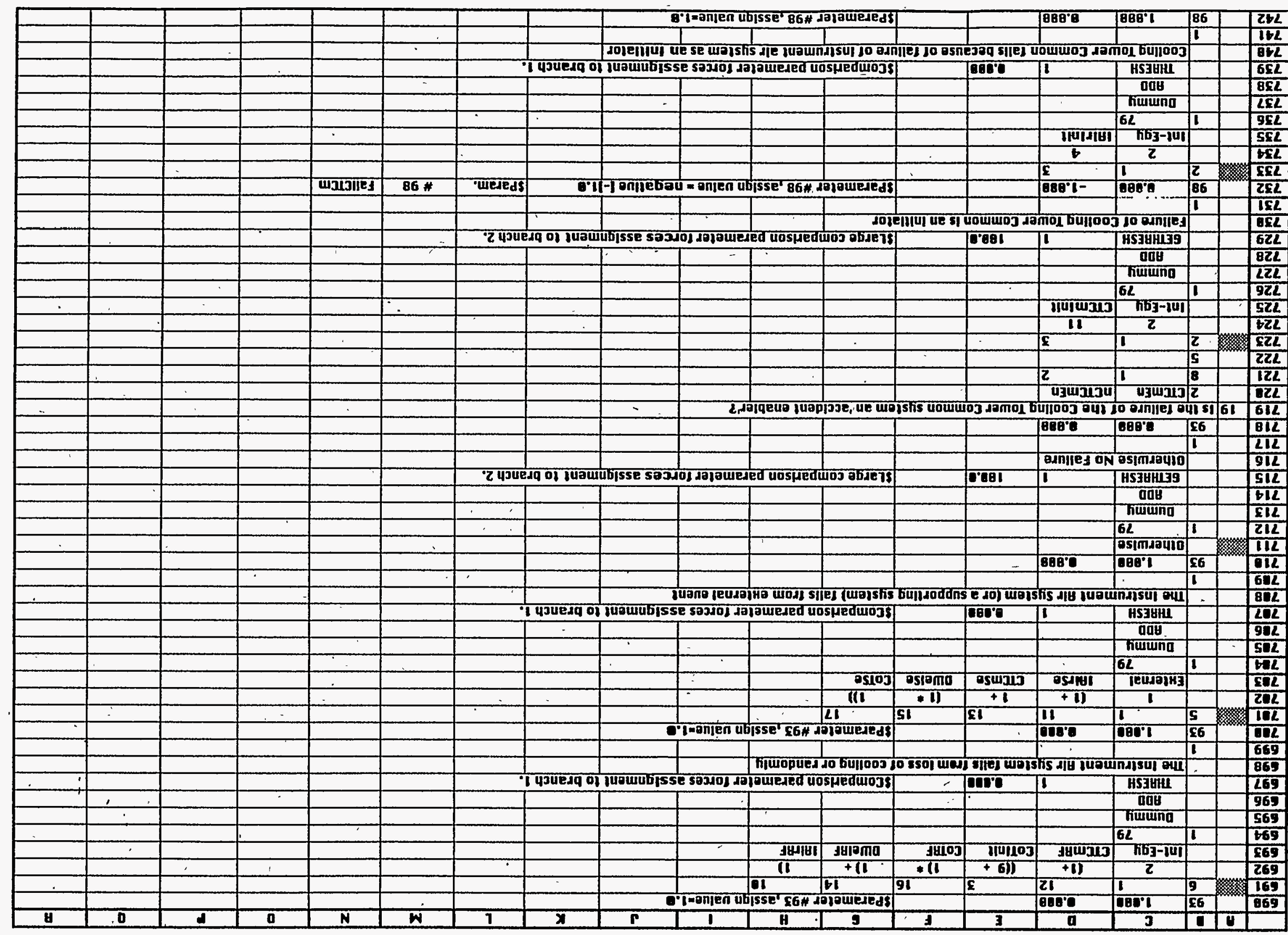

tEZ abed

c118-56-4L-38sm

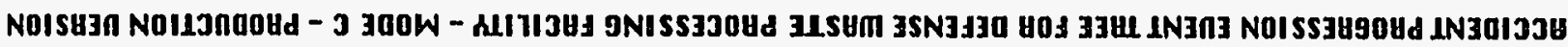

G IIONZAdt 
APPENDIH ด

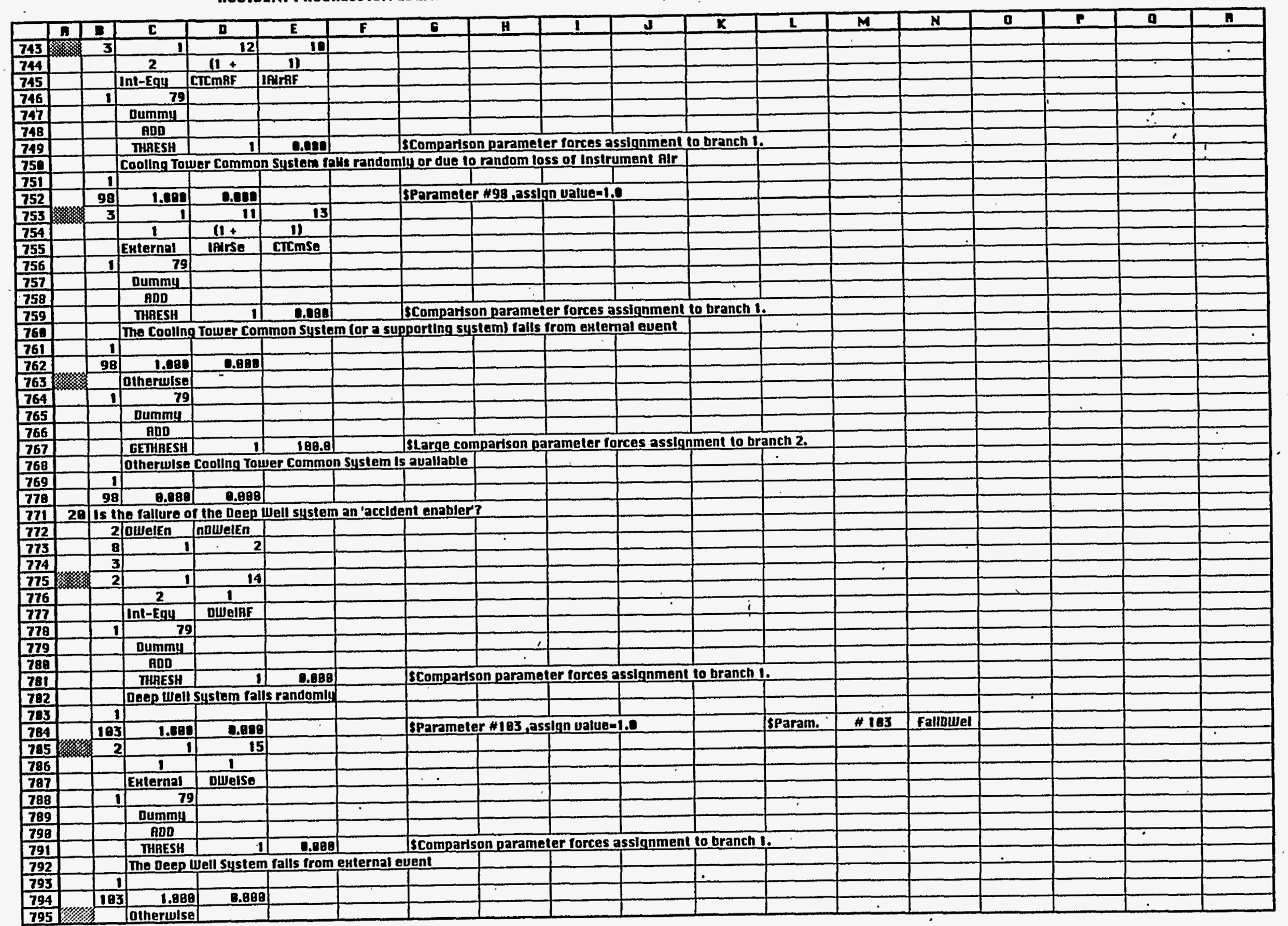




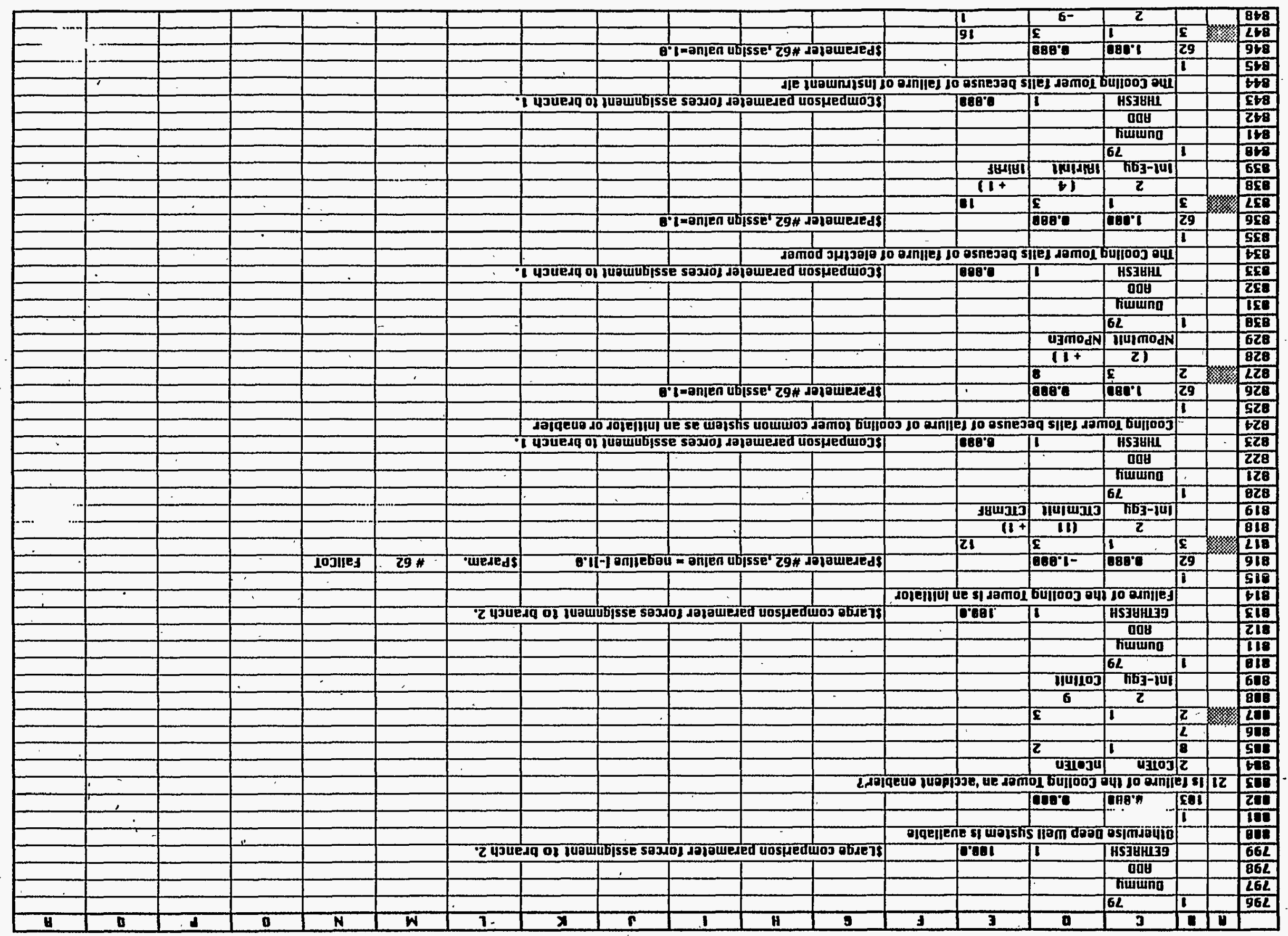

9\&2 o6ed

cLtด-s6-HL-Jusm

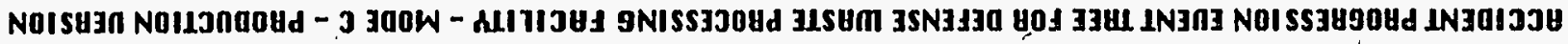

H HIONIdAU 


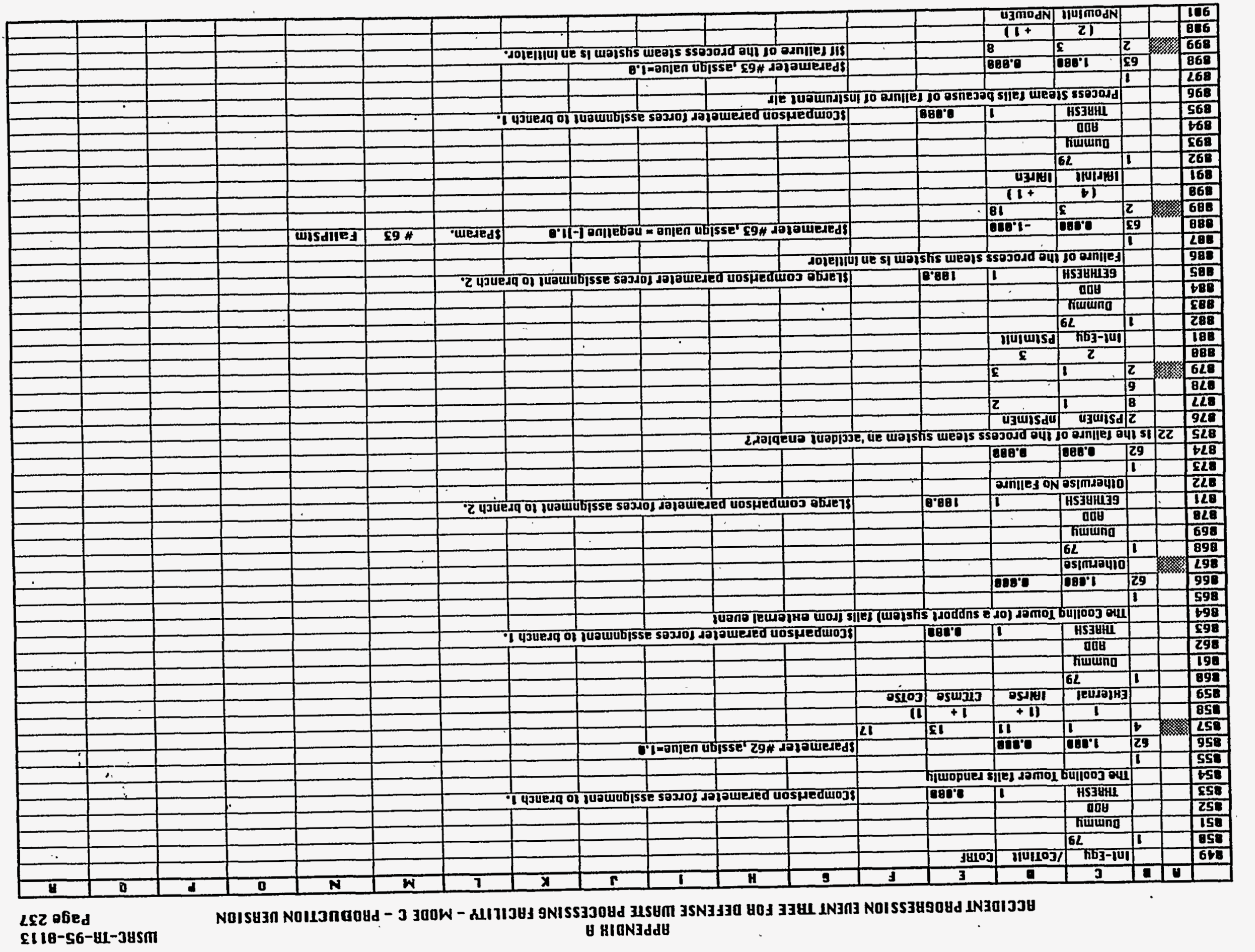




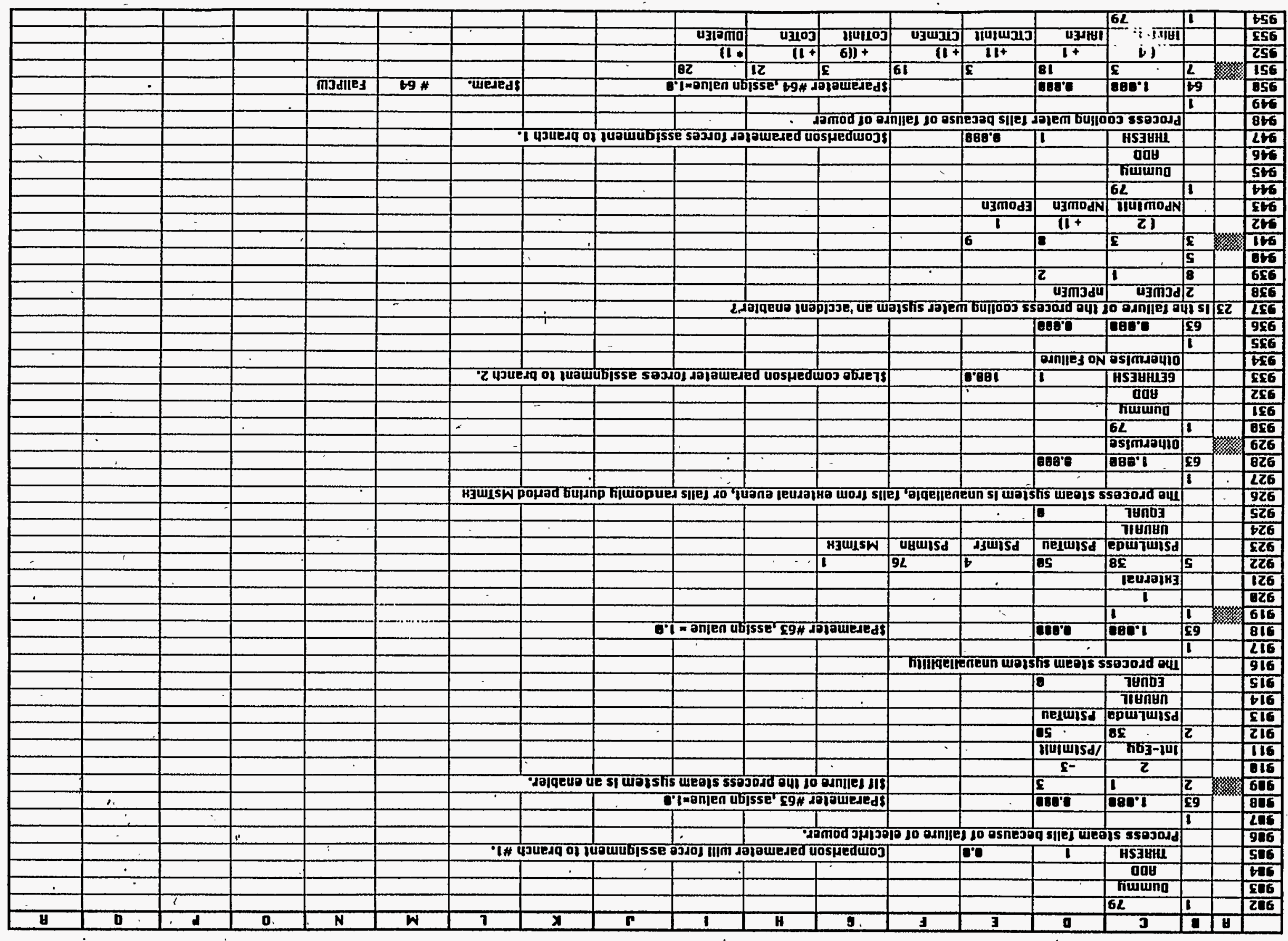

8\&z abed

c110-56-uh-34sm

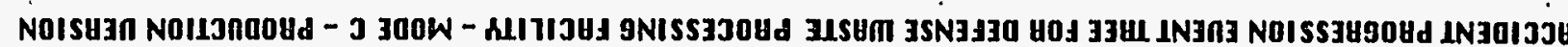

HHaNidd 


\begin{tabular}{|c|c|c|c|c|c|c|c|c|c|c|c|c|c|c|c|c|c|c|}
\hline & $n$ & 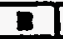 & C & D & $\bar{E}$ & $F$ & E & H & $I$ & $d$ & $\bar{x}$ & t & $M$ & $\mathbf{N}$ & 0 & $P$ & 0 & $n$ \\
\hline 955 & & & Dummy & & & & & & & & & & & & & & & \\
\hline 956 & & & ADD & & & & & & & & & & & & & & & 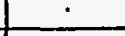 \\
\hline 957 & & & THRESH & II & 0.000 & & Scomparts & on paramel & ter forces a & sslgnment & 10 branch & & & & & & & \\
\hline 958 & & & The process & coollng we & ater system & falls beca & ause of rall & ure of Instr & rument alr & or coolling 1 & lower & & & & & & & \\
\hline 959 & & 1 & & & & & & & & & & & & & & & & \\
\hline 968 & & 64 & 1.010 & 0.090 & & & sparamete & \%64,assl & Inn uplue $=1$. & & & & & & & & & \\
\hline 961 & & .7 & 1) & & & & sif process & cooling w & ater talls. & & & & & & & & $\because$ & \\
\hline 962 & & & 2 & & & & & & & & & & & & & & & \\
\hline 963 & & & $\ln 1$-Equ & & & & & & & & & & & & & & & \\
\hline 964 & & 2 & 39 & 51 & & & & & & & & & & & & & & \\
\hline 965 & & & \begin{tabular}{|l|} 
PCWLmda \\
\end{tabular} & РсшTан & & & & & & & & & & & & & & \\
\hline 966 & & & UAUAIL & & & & & & & & & & & & & & & \\
\hline 967 & & & EOUAL & a & & & & & & & & & & & & & & \\
\hline 968 & & & The proces: & 5 cooling we & ater system & unauallab & bilite & & & & & & & & & & & \\
\hline 969 & & 1 & & & & & & & & & & & & & & & & \\
\hline 978 & & 69 & 1.089 & 0.020 & & & sparamete & \#64, ass & Ign value-1 & & & & & & & & & \\
\hline 971 & & 11 & 1 & & & & sir fallure & of the proc & cess cooling & water sys & stem is the & nabler. & & & & & & \\
\hline 972 & & & 1 & & & & & & & & & & & & & & & \\
\hline 973 & & & Esternal & & & & & & & & & & & & & & & \\
\hline 974 & & 5) & 39 & 5i & 5 & 77 & 1 & & & & & & & & & & & \\
\hline 975 & & & PCWLLmda & PCWTau & PCWFr & PCWAn & MsimEH & & & & & & & & & & & \\
\hline 976 & & & UAUALL & & & & & & & & & & & & & & & \\
\hline 977 & & & EQUAR & -1 & & & & & & & & & & & & & & \\
\hline 978 & & & The proces & 5 coolling we & ater systen & Is unaual & llable, falls & trom arter & mal event, & or falls ran & domly durin & geperiod $M$ & SImEH & & & & & \\
\hline 979 & & I & & & & & & & & & & & & & & & & \\
\hline 988 & & 64 & 1.290 & 0.800 & & & & & & & & & & & & & & \\
\hline 981 & & & Ditherwise & & & & & & & & & & & & & & & \\
\hline 982 & & 1 & 79 & & & & & & & & & & & & & & 1 & \\
\hline 983 & & & Dummy & & & & & & & & & & & & $\dot{-}$ & & & \\
\hline 984 & & & And & & & & & & & & & & & & & & & \\
\hline 985 & & & GETHRESH & II & 180.0 & & SLarge con & nparison pr & arameler 10 & arces assig & nment to br & anch 2. & & & & & & \\
\hline 986 & & & otherwise & No fallure & & & & & & $\therefore$ & & & & & & & & \\
\hline 987 & & 1 & & & & & & & & & $\therefore$ & & & & & & & \\
\hline 988 & & 64 & 8.000 & 0.091 & & & & & & & & & & & & & & \\
\hline 989 & 24 & Is in & he fallure o & the Carbon & a bloside P & rge Suste & im an 'accid & ent enabler & & & & & & & & & & \\
\hline 998 & & 2 & CO2En & nCO2En & & & & & & & & & & & & & & \\
\hline 991 & & B & 1 & 2 & & & & & & & & & & & & & & \\
\hline 992 & & 5 & & & & & & & & & & & & & & & & \\
\hline 993 & 㽝聯 & 2 & 1 & 3 & & & & & & & & & & & & & & \\
\hline 994 & & & 2 & 5 & & & & & & & & & & & & & & \\
\hline 995 & & & $\ln 1$-Eg4 & CO2Init & & & & & & & . & & & & & & $E$ & \\
\hline 996 & & 1 & 79 & & & & & & & & & & & & & & & \\
\hline 997 & & & Durnmy & & & & & & & - & & & & & & & & \\
\hline 998 & & & ADn & & & & & & & & & & & & & & & \\
\hline 999 & & & GETHAESH & II & 180.0 & & SLarge con & nparison pa & arameter to & arces assign & inment to br & $\operatorname{anch} 2$. & & & & & & \\
\hline 1080 & & & Fallure of & the carbon & dloside pur & sustem & Is an inilla & & & & & & & & & & & \\
\hline 1081 & & 1 & & & & & & & & & & & & & & & & \\
\hline 1002 & & 65 & 0.080 & -1.080 & & & sparamete & a65, assi & gn value $=1$ & negallue I- & -11.8 & sparam. & $\# 65$ & Fallic02 & & & & \\
\hline 1093 & 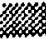 & 2 & 3 & 8 & & & sif fallure & of the cart & Ion dlouide & purge syst & Iem is the In & iflator. & & & & & & \\
\hline 1194 & & & 12 & +11 & & & & & & & & & & & & & & \\
\hline 1005 & & & NPOEInit & NPDiWEn & & & & & & & & & & & & & & \\
\hline 1086 & - & 1 & 79 & & & & & & & & & & & & & & & \\
\hline 1867 & . & & Dummu & & & & & & & & & & & & & & & \\
\hline
\end{tabular}


APPENDIH A

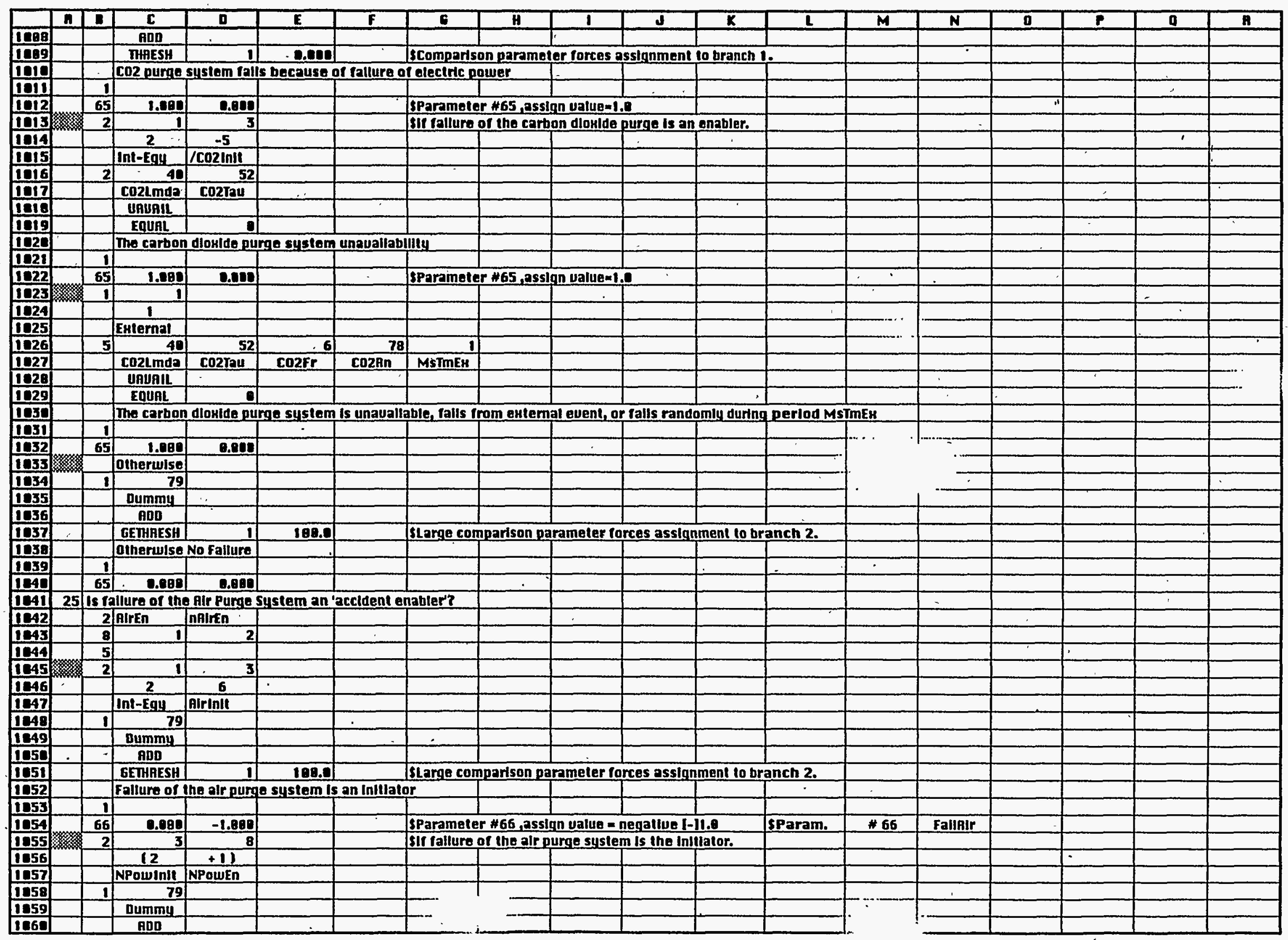




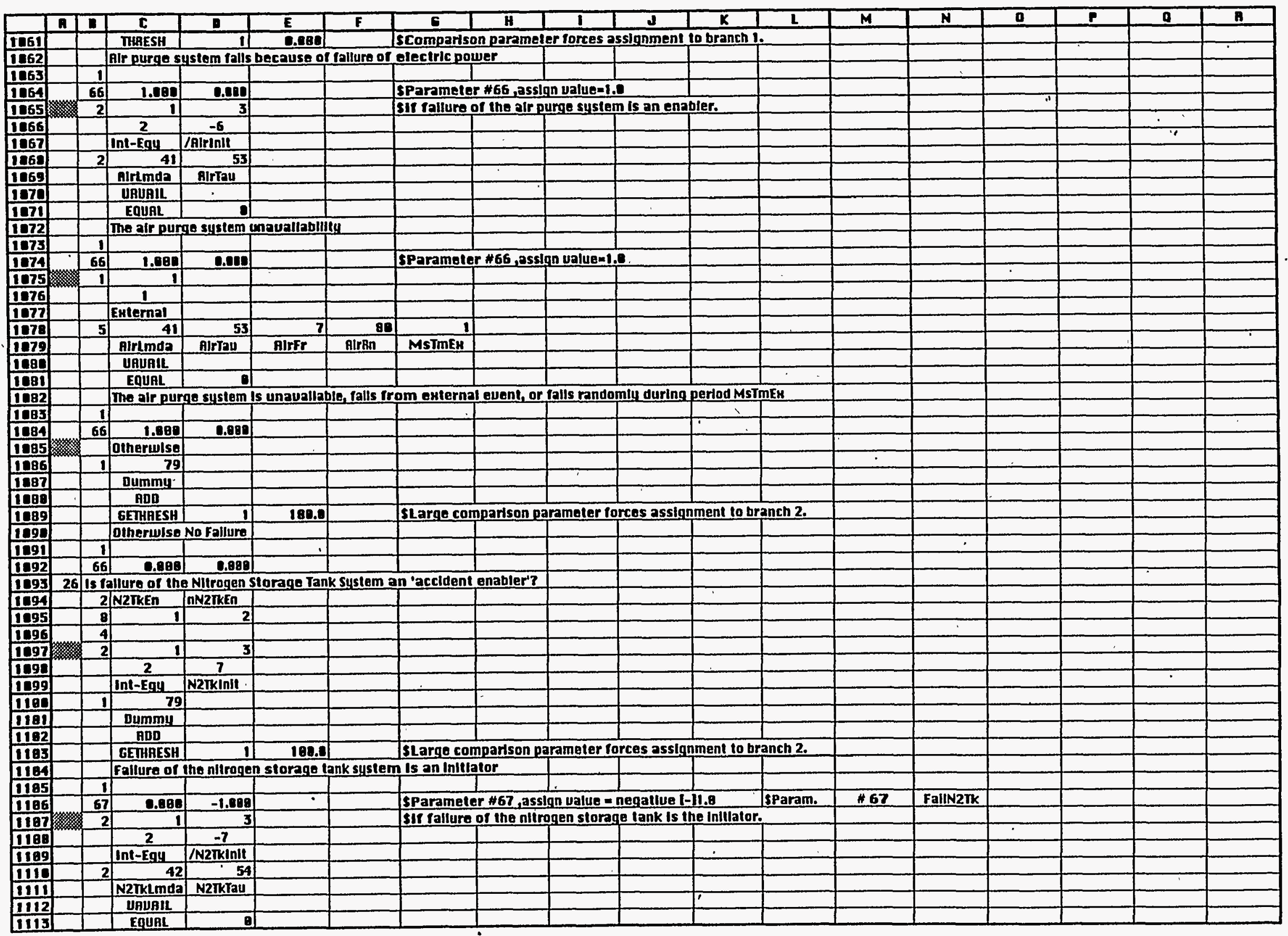




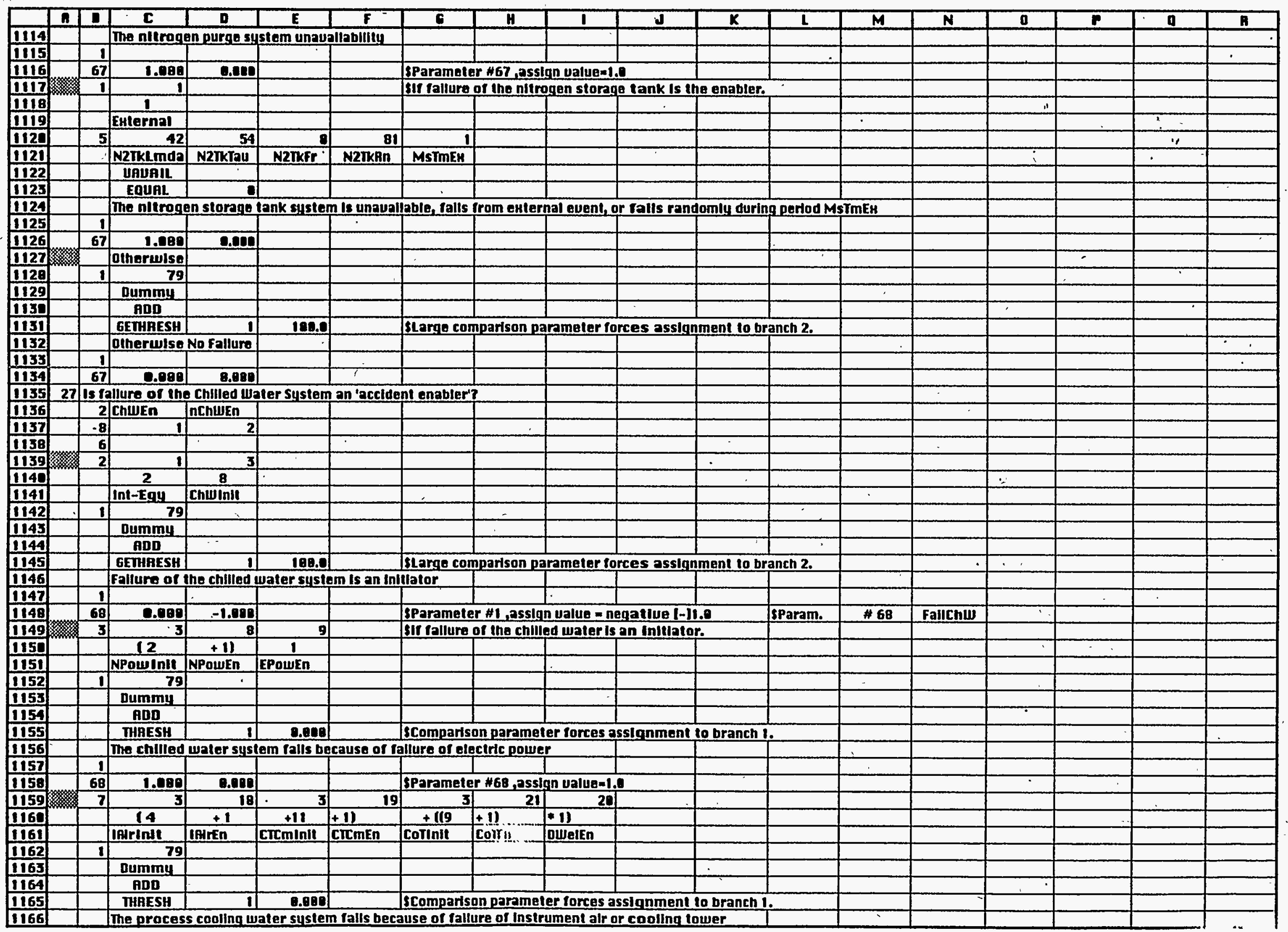


APPENDIH a

RCCIDENT PROGRESSION EUENT TREE FOR DEFENSE UASTE PROCESSING FACILITY - MODE C - PRODUCTION UERSION

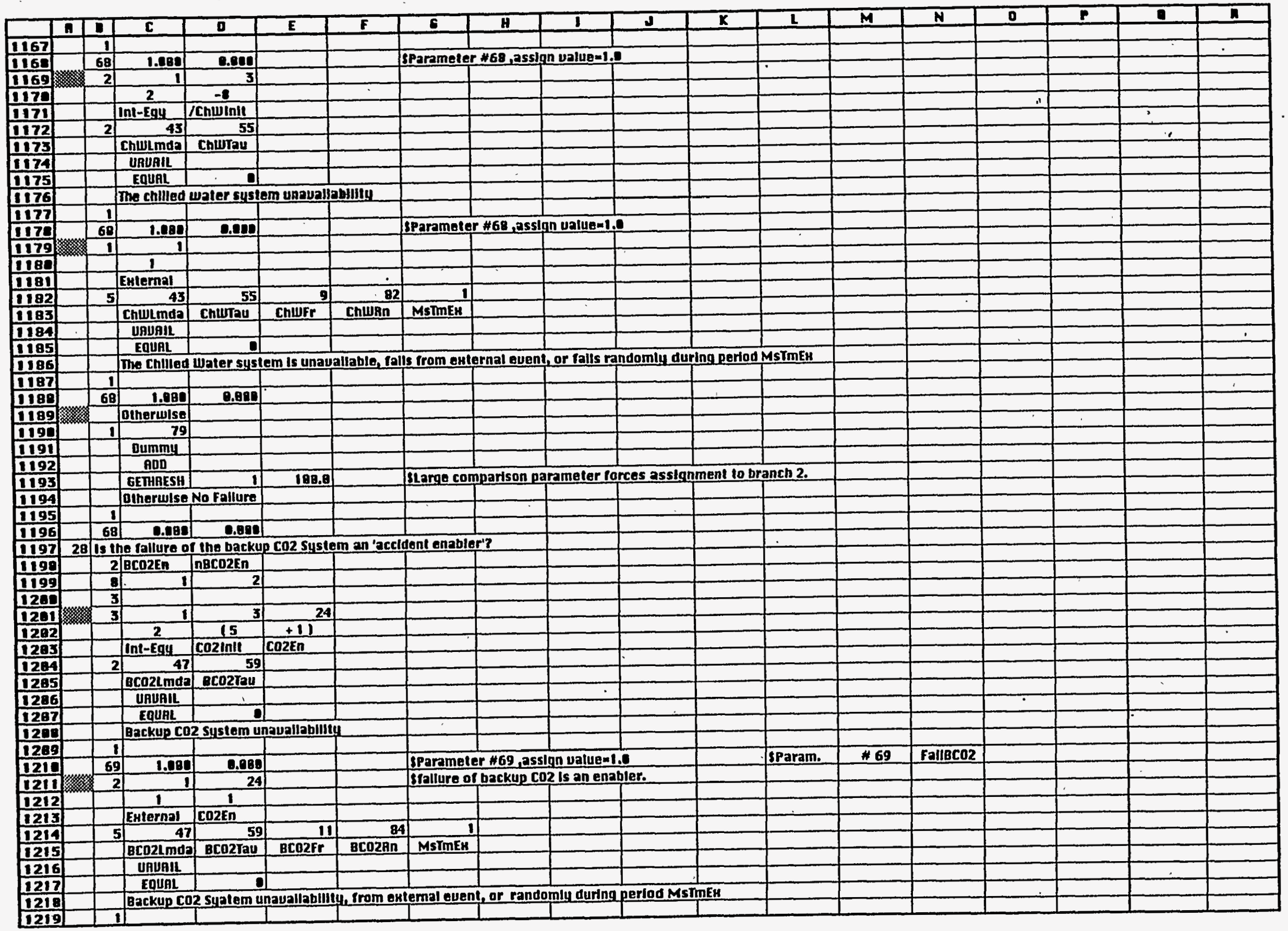




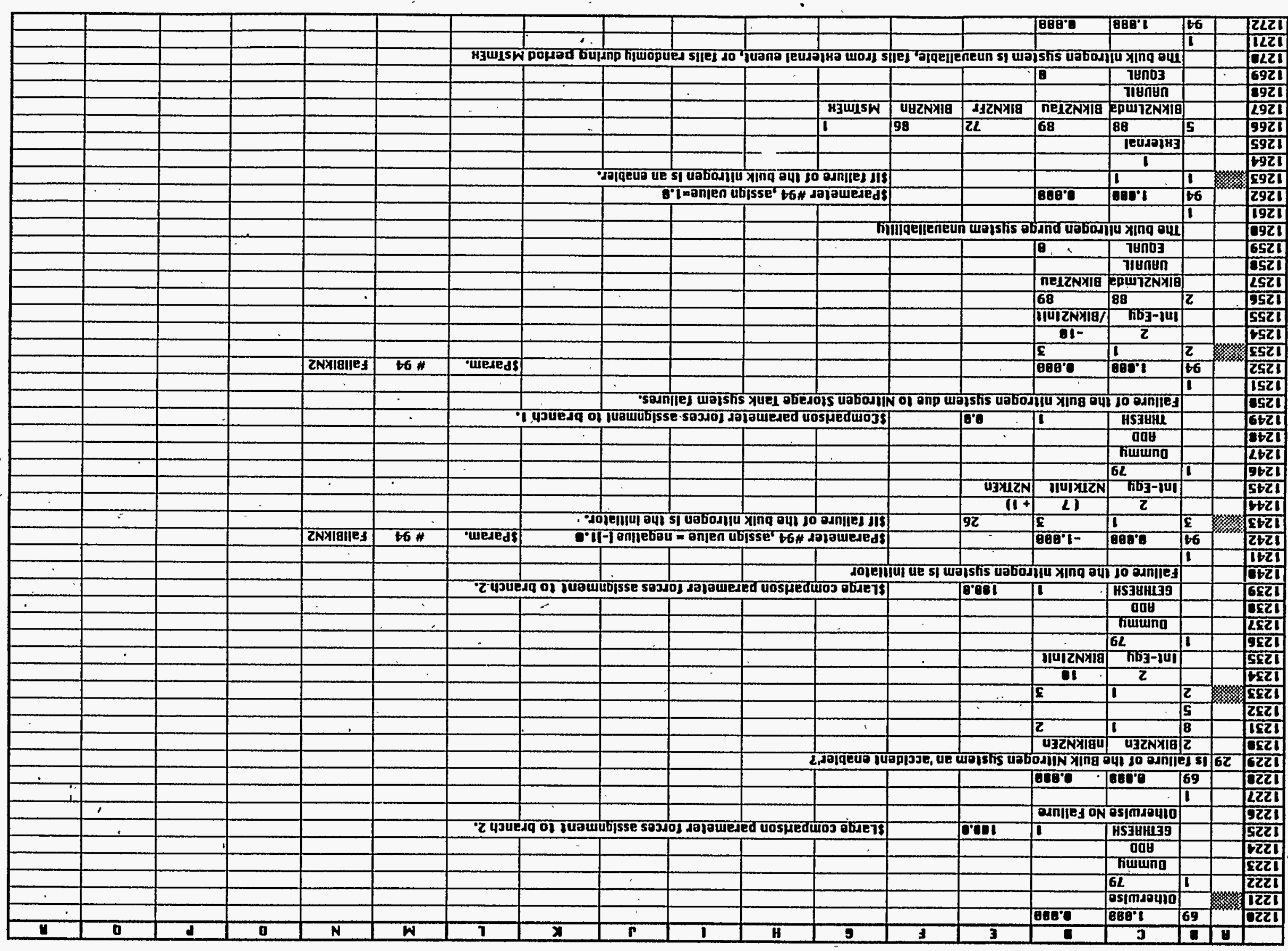

662 o6ed

C116-56- HII-34Sm

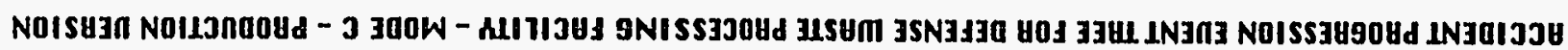

H HIONBdit 
APPENDIH 9

ACCIDENT PROGAESSION EUENT JAEE FOR DEFENSE WRSTE PROCESSING FRCILITY - MODE C - PRODUCTION UERSION

UUSRC-TR-95-0113

Page 245

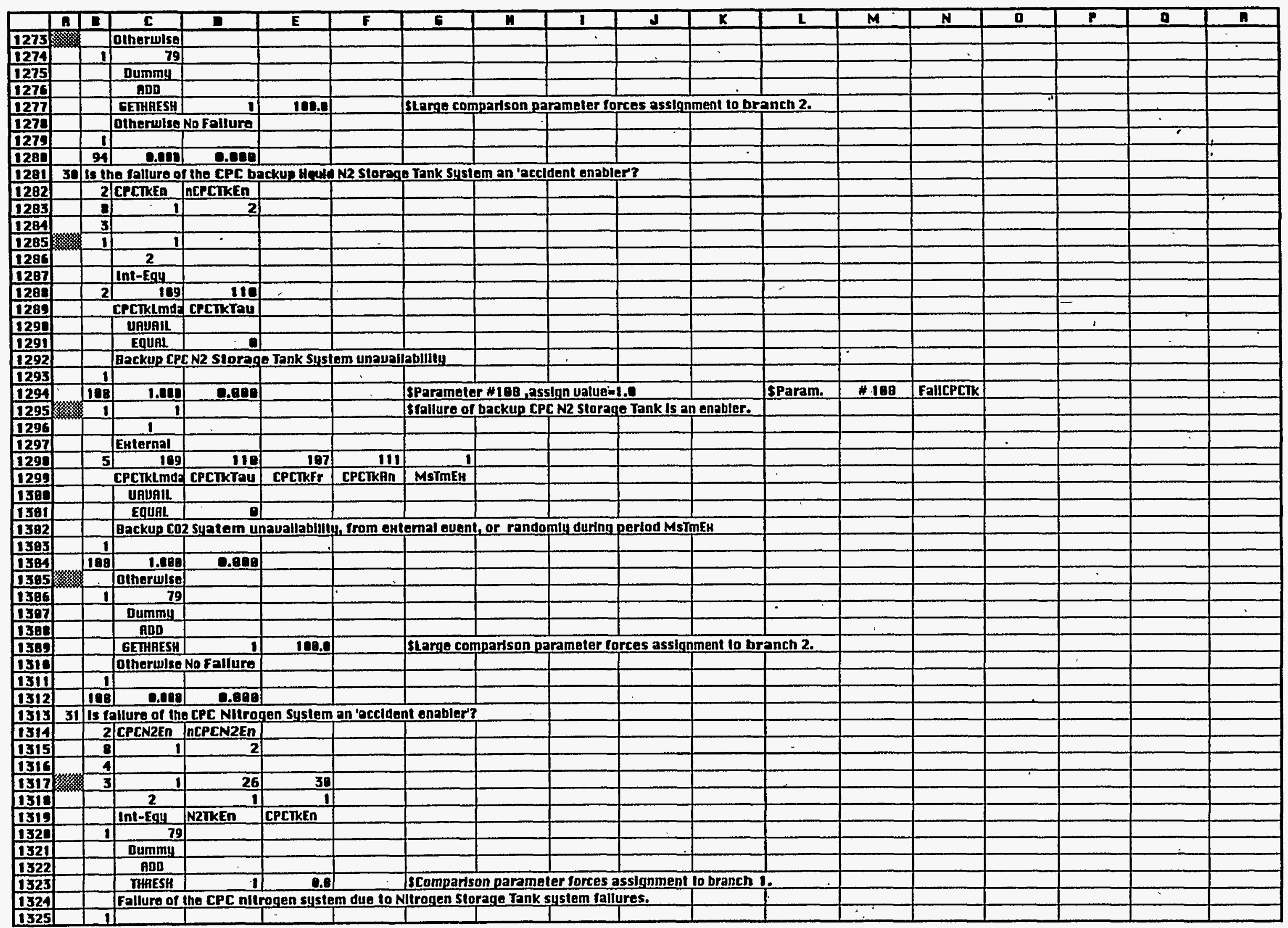




\begin{tabular}{|c|c|c|c|c|c|c|c|c|c|c|c|c|c|c|c|c|c|c|}
\hline & n & $B$ & $\mathbf{C}$ & $\bar{D}$ & $E$ & $F$ & E & H & 1 & $J$ & $\bar{k}$ & $\mathbf{L}$ & $\bar{M}$ & $\bar{N}$ & o & $\bar{P}$ & a & A \\
\hline 1326 & & 95 & 1.000 & 0.000 & & & & & & & & sparam. & $\$ 95$ & FallCPCN2 & & & & \\
\hline 1327 & & 3 & 1 & 3 & 25 & & & & & & & & & & & & & $\because$ \\
\hline 1328 & & & 2 & 16 & $+\mid 1$ & & & & & & & $\dot{0}$ & & & & & & \\
\hline 1329 & & & $\ln 1-E q 4$ & Alrinli & Alren & & & & & & & & & & & & & \\
\hline 1330 & & 2 & 91 & 92 & & & & & & & & & & & & & & \\
\hline 1331 & & & CPCNZLLmda & CPCN2TaU & & & & & & $\dot{\square}$ & & & & & & & & \\
\hline 1332 & & & UAUAIL & & & & & & & & $=$ & & & & & & 1 & \\
\hline 1533 & & & EQUAL & ] & & & & & & 6 & & & 1 & & & & & $?$ \\
\hline 1334 & & & The cec nit & Iragen purg & to systam $y$ & navallabline & & & & & & & & & & & & \\
\hline 1335 & & 1 & & & & & & & & & & & & & & & & \\
\hline 1336 & & 95 & 1.008 & 0.00 & & & sparamete & \#95, assis & Lnn value $=1$. & & & Sparam. & $\# 95$ & FallCPCN2 & & & & \\
\hline 1537 & & 1 & 1 & & & & sil fellurec & of the CPC & nitragen is & the enable & er. & & & & & & & \\
\hline 1530 & & & 1 & & & & & & & & $\dot{\square}$ & & & & & & & \\
\hline 1339 & & & Esternal & & & & & & & & & & & & & & & \\
\hline 1340 & & 5 & 91 & 92 & 73 & 87 & 1 & , & 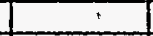 & & & & & & & & & \\
\hline 1341 & & & CPCN2LImad & CPCN2T2U & CPCN2Fr & \begin{tabular}{|c|c|} 
CPCN2月n \\
\end{tabular} & MsimEн & & & & & & & & & & & \\
\hline 1342 & & & UAUALI & & & & & & & & & & & & & & & \\
\hline 1343 & & & EQUAL & 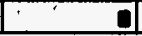 & & & & & & & & & & & & & & \\
\hline 1544 & & & The CPC nit & rogen syste & em Is unau & allable, falts & s trom erte & rnal event & 4 or falls ra & ndomly du & ring period & MSTMEH & & & & & & ; \\
\hline 1345 & & 1 & & & & & & & & & & & & & & & & \\
\hline 1346 & & 95 & 1.000 & 0.000 & & & & & & & & & & & & & & \\
\hline 1347 & & & Detherwlse & & & & & & & & & & & & & & & \\
\hline 1348 & & 1 & 79 & & & & & & & & & & & & & & & \\
\hline 1349 & & & Dummy & & & & & & & & & & & & & & & \\
\hline 135 & & & ADD & & & & & & & & & D. & & & & & & \\
\hline 1351 & & & GETHAESH & 1 & 109.0 & & SLarge com & ppartson pa & arameter Io & urces assig & Inment to b & branch 2. & & & & & & \\
\hline 1352 & & & otherwise & No fallure & & & & & & & & & & & & 2 & & \\
\hline 1353 & & 1 & & & & & & & & - & & & & & & & & \\
\hline 1354 & & 95 & 2.880 & 0.000 & & & & & & & & & & $\dot{5}$ & & & & \\
\hline 1355 & 32 & Is 18 & allure of the & L LPP Back & Sup Nliroger & n System an & $n$ accldent & enabler? & & & & & & & & & & \\
\hline 1355 & & 2 & PPN2En & nPPN2En & & & & & & & & & & & & & & \\
\hline 1357 & & 8 & $\therefore 1$ & 2 & & & $\therefore$ & & & & 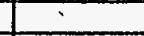 & & & & & &. & \\
\hline 1350 & & 3 & & & & & & & & & & & & & & & & \\
\hline 1359 & 㬎 & 3 & 1 & 29 & 3 & & & & & & & & & & & & & \\
\hline 1360 & & & 2 & (1) & $+10)$ & & & & & & & 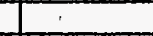 & & & & & & \\
\hline 1561 & & & Int-Egy & BIKN2En & $B|\mathrm{kN} 2 \mathrm{n}| \mathrm{t}$ & & & & & . & & & & & & & . & \\
\hline 1362 & & 2 & 114 & 115 & & & & & & & & & & & & & & \\
\hline 1363 & & & PPN2Lmda & PPN2TEU & & & & & & & & & & & & & & \\
\hline 1364 & & & UAUAIL & & & & & & & & & & & & & & & \\
\hline 1365 & & & EQUAL & al & & & & $=$ & & & & & & & & & & \\
\hline 1366 & & & The cPC nit & Irogen purg & e system u & nauallablitt & & & & & & & & & & & & \\
\hline 1367 & & 1 & & & & & & & & & & & & & 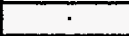 & & & \\
\hline 1360 & & 113 & 1.000 & 0.090 & & & SParamete & r $\$ 95$, asslo & lgn ualue $=1$. & & & sparam. & 1113 & FallPPN2 & & & & \\
\hline 1369 & & 1 & 1 & & & & Sif rallure & of the CPC & niltrogen is & the enable & & & & & & & & \\
\hline 137 & & & 1 & & & & & & & & - & & & & & & & \\
\hline 1371 & & & Emternal & & & & & & & & & & & & & & . & \\
\hline 1372 & & 5 & 114 & 115 & 112 & 116 & 1 & & & & & & & & & & & \\
\hline 1373 & & & PPN2LInde & \begin{tabular}{|l|} 
PPN2TaU \\
\end{tabular} & PPN2Fr & PPN2An & MsTmEr & & & & & & & & & & & \\
\hline 1374 & & & UAUAIL & & & & & & & & & & & & & & & \\
\hline 1375 & & & EQUAL & ef & & & & & & & & & & & & & & \\
\hline 1376 & & & The CPC nit & Irogen suste & em Is unave & allable, falls & s from este & rnal euent, & or ralls ran & ndomly dur & ring period & MsImEH & & & & & & \\
\hline 1372 & & 1 & & & & & & & & & & & & & & & & \\
\hline 1378 & & 113 & $i$ ing & ..88日 & & & & & & & & & & & & & & \\
\hline
\end{tabular}


APPENDIH A

RCCIDENT PROGRESSION EUENT TREE FOR DEFENSE WASTE PROCESSING FACILITY - MODE C - PRODUCTION UERSION

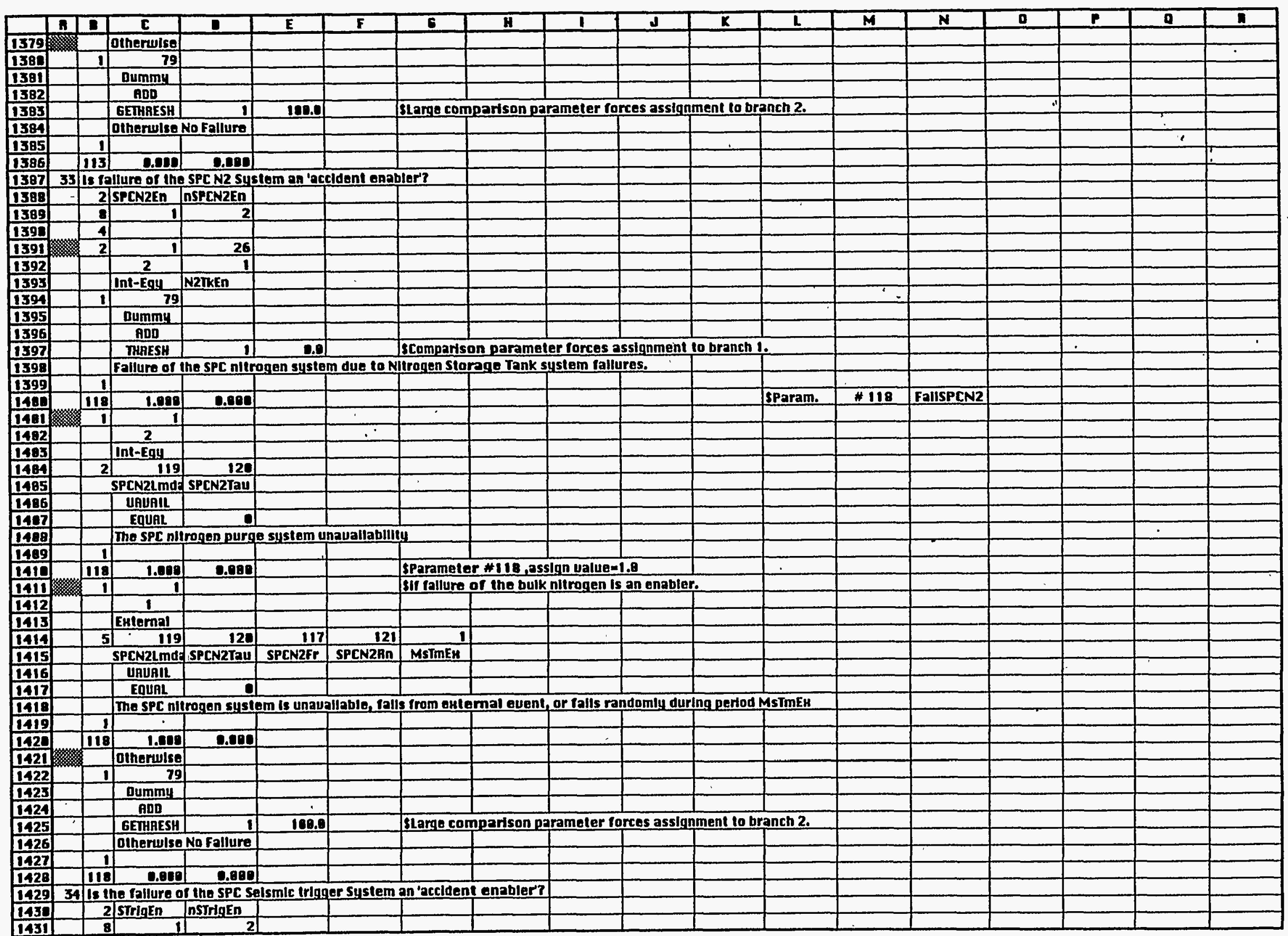

-95-8113

Page 247 
APPENDIK

WSAC-Th-95-8113

ACCIDENT PAOGRESSION EUENT TREE FOR DEFENSE WASTE PROCESSING FACILITY - MODE C - PRODUCTION UERSION

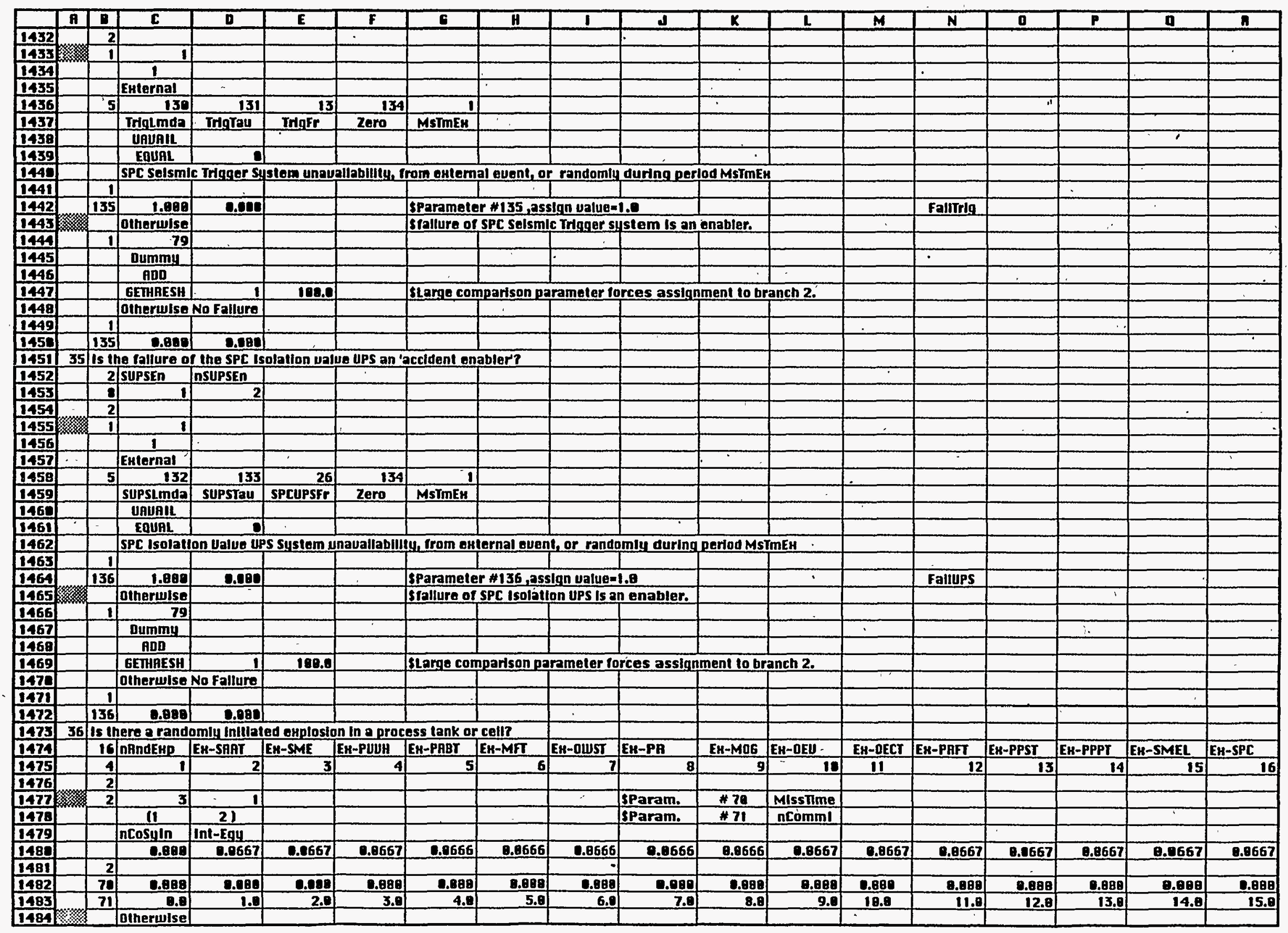


APPENDIK

\begin{tabular}{|c|c|c|c|c|c|c|c|c|c|c|c|c|c|c|c|c|c|c|}
\hline & 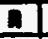 & 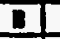 & $\mathbf{c}$ & $\bar{D}$ & $E$ & $F$ & $E$ & $H$ & 1 & J & $\mathbf{K}$ & 1 & $M$ & $N$ & 0 & $F$ & a & I \\
\hline 1485 & & & I.eng & 0.000 & 0.019 & 0.020 & 0.010 & 0.000 & D.980 & e.000 & 2.080 & 0.880 & 0.180 & 8.8B日 & 0.020 & Q.88日 & 0.080 & 0.000 \\
\hline 1486 & & 2 & & & & & & & & & & & & & & & & \\
\hline 1487 & & 70 & D.010 & 0.000 & 0.000 & e.ent & 0.090 & 0.000 & 0.080 & 0.000 & 0.080 & B.888 & 0.082 & 808 & 0.098 & Q.880 & 0.800 & $0.10 \mathrm{~B}$ \\
\hline 148 & & 7il & -10.0 & 1.0 & 2.0 & 3.01 & 4.0 & 5.0 & 6.0 & 7.8 & 8.0 & 9.8 & 10.0 & 11.0 & 12.8 & 13.2 & 14.0 & 15.0 \\
\hline 1489 & 37 & Bothin & he cell couse & rs ouer the & Sall Proces & ss Cell disio & adge and fo & Ill because & of a selsmic & c event? & & & & & & & & \\
\hline 149 & & 21 & Iscusels & niscusel & & & & & & & & & & & & & & \\
\hline 1491 & & 6 & 1 & 2 & & & & & & & & & & & & & $\therefore$ & \\
\hline \multicolumn{19}{|l|}{$\frac{1992}{1003}$} \\
\hline 1493 & & 1 & 2 & & & & & & & & & & & & & & & \\
\hline 149 & & & 2 & & & & & & & & & & & & & & & \\
\hline 1495 & & & EI-Sels & & & & & & & & & & & & & & & \\
\hline 1496 & & 1 & 33 & & & & & & & & & & & & & & & \\
\hline 1497 & & & CCoufr & & & & & & & & & & & & & & & \\
\hline 1498 & & & AnD & & & & & & & & & & & & & & & \\
\hline 1499 & & & EQUAL & 0 & & $\dot{-}$ & & & & & & & & & & & & \\
\hline 150 & & \multicolumn{17}{|c|}{ Probabillty of Sall Process Coll couers falling Is placed in branch 1 , remainder in branch 2.} \\
\hline 150 & & & Ounerulse & & & & & & & & & & & & & & & \\
\hline 1512 & & 1 & 79 & & & & & & & & & & & & & & & \\
\hline 1503 & & & Dummy & & & & & & & & & & & & & & & $\because$ \\
\hline 154 & & & ADD & & & & & & & & & & & & & & & \\
\hline I5es & & & GETHAESH & 1 & 108.0 & & & & & & & & & & & & & \\
\hline 1510 & & \\
\hline 1597 & \multirow{2}{*}{\multicolumn{15}{|c|}{\begin{tabular}{|l|l|l|}
38 Does a cell couer rall while belng moued by the crane? & & \\
\end{tabular}}} & & & \\
\hline 150 & & & & & & & + & & & & & & & & & & & \\
\hline 1589 & & 2 & 1 & 2 & & & & & & & & & & & & & & \\
\hline 1510 & & 2 & & & & & & & & & & & & & & & & \\
\hline 1511 & & 2 & 1 & 6 & & & & & & 1 & & & & & & & & \\
\hline 1512 & & & 5 & 2 & & & & & & & & & & & & & & \\
\hline 1513 & & & Ltaco & CreneDrop & & & & & & & & & & & & & & \\
\hline 1514 & & & 1.000 & 0.000 & & & & & & & & & & & & & & \\
\hline 1515 & & & olheruise & & & & & & & & & & & & & & & \\
\hline 1516 & & & 0.000 & 1.080 & & & & & & & & & & & & & & \\
\hline 1517 & \multicolumn{18}{|c|}{39 Do the coll cousers that ball in a crane accldent strike a tank? } \\
\hline 1510 & & 1418 & No_HII & CDPrA & CD,OED & CD_OECT & CD_PAFT & CO_PBAI & $\mathrm{CO}_{\mathrm{S} S M E}$ & CD_SART & CD_ACT & CO_MFT & CD,MEL & CO_PPPI & CD_PPST & CO_PPAI & & \\
\hline 1519 & & 2 & 1 & 2. & $\mathbf{3}$ & 4 & 5 & 6 & 7 & 8 & 19 & 18 & 11 & 12 & 13 & 14 & & \\
\hline 1520 & & 2 & & & & & & & & & & & & & & & & \\
\hline 1521 & 維 & 1 & 38 & & & & & & & & & & & & & & & \\
\hline 1522 & & & 1 & & & & & & & & & & & & & & & \\
\hline 1523 & & & CoCuSPC & & & & & & & & & & & & & & & \\
\hline 1524 & & & 0.874609 & 0.09339 & 0.01339 & 0.09339 & 0.01339 & 0.09339 & 0.0339 & E.00359 & 0.09339 & 0.00339 & 0.09339 & 0.03858 & 0.03058 & 0.03050 & & \\
\hline 1525 & & & Dinerwise & & & & & & & & & & & & & & & \\
\hline 1526 & & & 1.000 & 0.0 & 0.1 & 0.0 & 0.0 & 0.0 & 0.0 & 8.9 & 8.0 & 0.0 & 0. & 0.0 & 0.8 & 0.1 & & \\
\hline 1527 & \multirow{2}{*}{\multicolumn{18}{|c|}{$\begin{array}{l}40 \text { Do the cell couers that fell in a selemic event strike the PA? } \\
2|5 C u-P R| \operatorname{lnsCu-PA} \mid\end{array}$}} \\
\hline 1520 & & 25 & SCU-PR & & & & & & & & & & & & & & & \\
\hline 1529 & & 2 & 1 & 2 & & & & & & & & & & & & & & \\
\hline 1530 & & 2 & & & & & & & & & & & 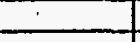 & & & & & \\
\hline 1531 & & 2 & 2 & 37 & & & & & & & & & & & & & & $\therefore$ \\
\hline \begin{tabular}{|l|}
532 \\
\end{tabular} & & & 2 & 1 & & & & & & & & & & & & & & \\
\hline 1533 & & & E1-Seis & ISCuSels & & & & & & & & & & & & & $\therefore$ & \\
\hline 1534 & & & 0.010 & 0.990 & & & & & & & & & & & & & & \\
\hline 1535 & Sk & & Dinerwise & & & & & & & & & & & & & & & \\
\hline 1536 & & & 0.800 & 1.080 & & & & & & & & & $\therefore$ & & & & & \\
\hline 1537 & 41 & DoRs & sa Selsmic e & event or cal & Uer crane if & all rupture & and splli th & he contents & of the Prec & Cjpifale Rea & actor In the & Sall Proce & s Cell? & & & & & \\
\hline
\end{tabular}




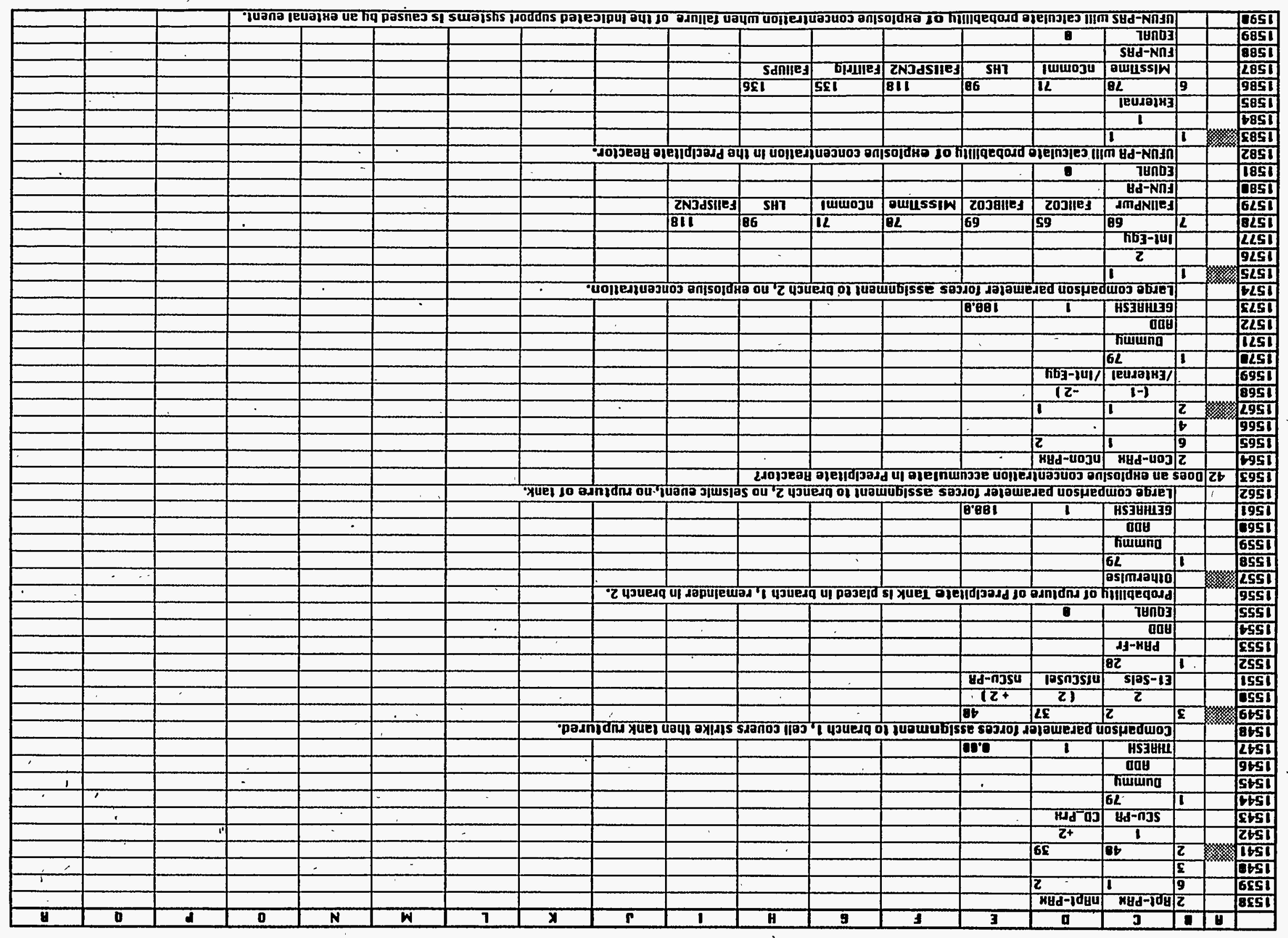

OSz obed

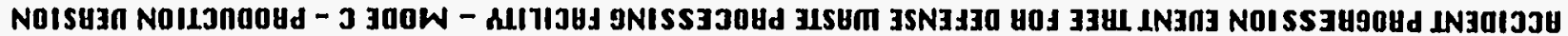




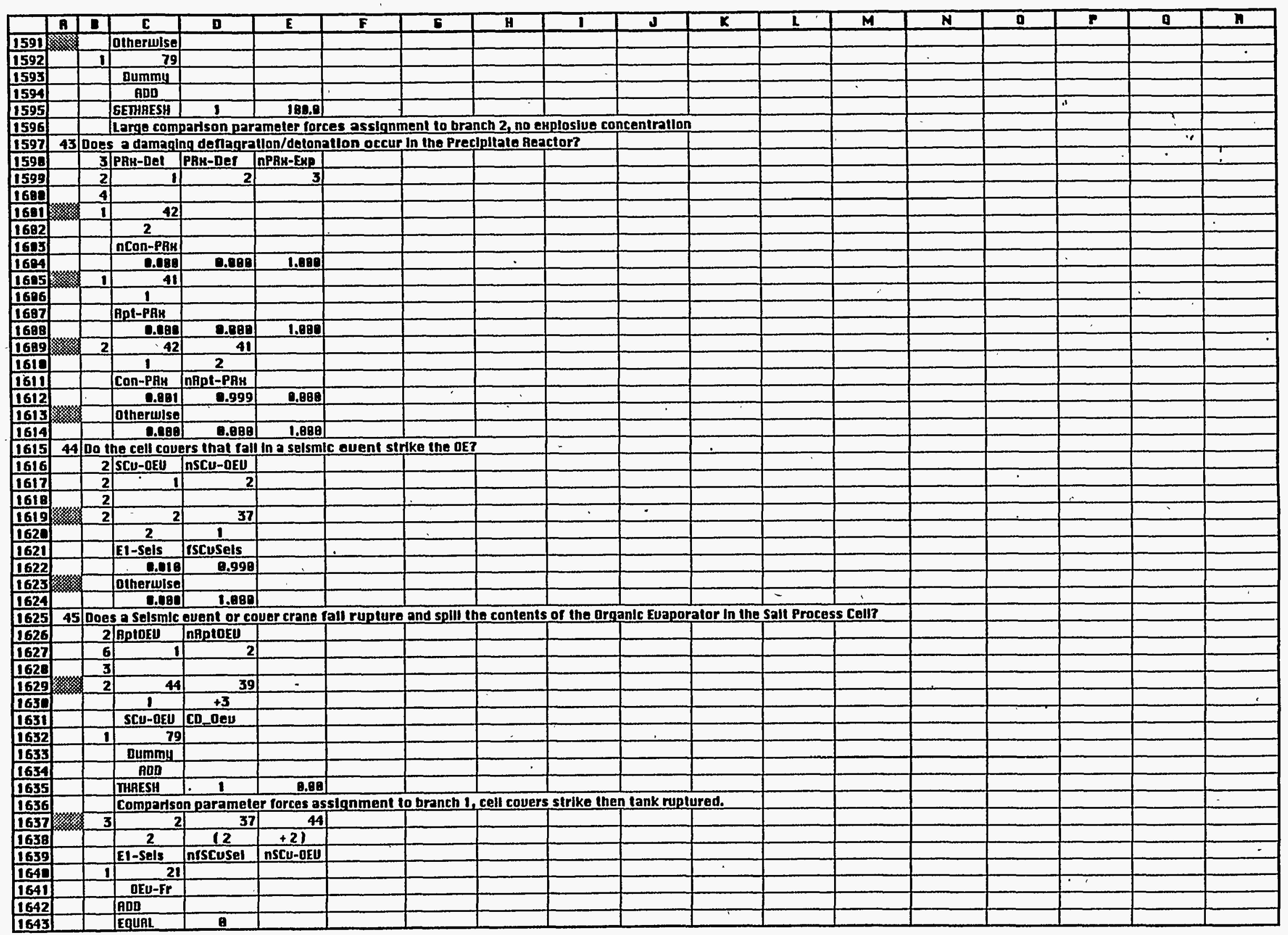




\begin{tabular}{|c|c|c|c|c|c|c|c|c|c|c|c|c|c|c|c|c|c|c|}
\hline & & $\therefore$ & & & & & & & & & & & 68. & 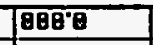 & $680^{\circ}$ & & & 9691 \\
\hline & & & & & & & 5 & & & & & & & & n30idy & & & 5691 \\
\hline & & & & & & & & & & & & & & & I & & & F69 \\
\hline & & & & & $\cdot$ & & & & & & & & & & st & It & 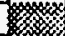 & 569 \\
\hline & & & & & & & & & & & & & 60001 & कि口0 & $\sqrt{600}$ & & & 269 \\
\hline & & & & & & & & & & & & & & & 1030-4034 & & & 169 \\
\hline & & & & & & & & & & & & & & & 2 & & & 10991 \\
\hline & & & & & & & & & & & & & & & 96 & $t$ & 森 & 6891 \\
\hline & & & & & & & & & & & & & & & & 6 & & B891 \\
\hline & & & & & & & & & & & & & $\varepsilon$ & 2 & $t$ & $\sqrt{2}$ & & L891 \\
\hline & & & & & & & & & & & & & dR3-njou & 100-010 & $100-n 30$ & $\bar{\varepsilon}$ & & 989 \\
\hline & & & & & & & & & & 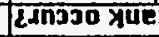 & का गणधारणत्त & 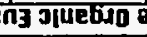 & बपा 30 uणाए & Edie/Jap/uo & गोंटuopep e & sona & 26 & SBg \\
\hline & & & & & & & & uollediuos & duoj эnjsoldh & 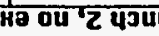 & मеगव ol lüu & mubisse sa3 & 3.0) dojoute & Eded uosped & 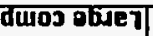 & & & 689 \\
\hline & & & & & & & & & & & & & J081 & $T$ & HSJUHIIJ9 & & & |co9| \\
\hline & & & & & & & & & & & & & & & वर्ष & & & 2891 \\
\hline & & & & & & & & & & & & & & & 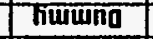 & & & 1891 \\
\hline & 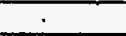 & & & & & & & & & & . & & & & 62 & t & & 1891 \\
\hline & & & & & & & & & & & & & & & DS[máno & & & 6291 \\
\hline & & & Tuena feu & 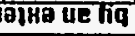 & pQSnES SIS & 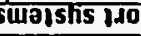 & Jddns pojes & गेगण 0पा 10 & 8dnाle पर्य & 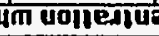 & 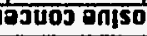 & वावस् 80 bा & IIIqEqodd ofe & 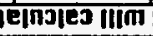 & i snjo-Nn]त & & & BL9 \\
\hline & & & & & T. & & & & & & & & & 7 & TषחOS & & & $\angle 29$ \\
\hline & & & $\div$ & & & & & . & - & & & & & & \begin{tabular}{|c|} 
SnIJ-Nns \\
\end{tabular} & & & 929 \\
\hline 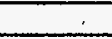 & & & & & & & & & & Sdniled & брमानड & ZNJdSIIEJ & SHT & Tưro30 & DUIISSIW & & & SLOT \\
\hline & & & & & & & & & & 95 & SE: & 811 & 106 & $\overline{\boldsymbol{L}}$ & 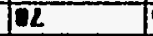 & 9 & & 629 \\
\hline & & & & 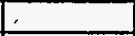 & & & & & & & & & & & TEUREIH] & & & ELTा \\
\hline & & & & & & & & & & & & & & & $T$ & & & 2221 \\
\hline & & & & & & & & & & & & & & & $t$ & t. & 㓌 & 1291 \\
\hline & & & & & & & ग्रापeI & dolexoden] & गुपebs ou & if uा uolnes\}0 & deJu03 On!s & soldHe jo kif & 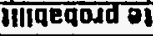 & 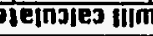 & 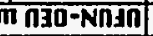 & & & 029 \\
\hline & & & & & & & & & $=$ & & & & & $\overline{0}$ & 7tyno3 & & & 6991 \\
\hline & & & & & & & & & & & & & & & 030-Nn1 & & & 899 \\
\hline & & & & & & & & & ZNJdSIE] & SHT & गயயण & DUIISSIN & Z0J8ाIEd & Z0IIIE] & dmaNIIE] & & & L990 \\
\hline & & & & & & & & & 8. & 66 & $\overline{L L}$ & 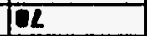 & 69 & 59 & 69 & 12 & & 9991 \\
\hline & & & & & & & & & & & & & & & $76]_{3-101}$ & & & 5990 \\
\hline & & & 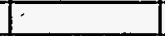 & & & & & & & & & & & & 2 & & & EgפI \\
\hline & & & & & & & & & & & & & & & 1 & 1 & 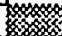 & 2391 \\
\hline & & & & 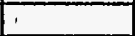 & & & & 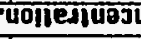 & ưJ बnjsoldh & Bg ou ' 2 पउu & sedq of jugu & untojsse soj & 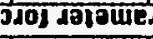 & Eded uos $\mu \mathrm{ed}$ & dü03 abse? & & & 2991 \\
\hline & & & & & & & & & & & & & E'EI & I & HS38HIJG & & & 1991 \\
\hline & & & & & & & & & & & & & & & ताप & & & |1991 \\
\hline & & & & & & & & & & & & & & & Timung & & & 699 \\
\hline & & & & & & & & & & & & & & & $\sqrt{62}$ & 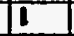 & & 859 \\
\hline & & & & & & & $\therefore$ & & & & & & - & Tibj-lut/ & Jएयगम & & & LS91 \\
\hline & & & & & & & & & & & & & & $72-$ & $|-|$ & & & gS90 \\
\hline & & & & & & & & & & & & & & t & $T$ & $\overline{2}$ & 㱞 & S59 \\
\hline & & & & & & & & & & & & & & & & 6 & & HS9O \\
\hline & & & & & & & & & & & & . & & 2 & $t$ & 9 & ? & कS90 \\
\hline & & & & & & & & & & & & & & A30-40Ju & A30-403 & $\bar{z}$ & & 259 \\
\hline & & & & & & & & & & guel dojesod & dent JuयEdo & 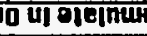 & 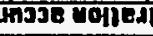 & 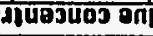 & 115070HD UQ S & soou & 96 & |S9, \\
\hline & , & & & & & & क्रयदा 100 & AnIdम ou ' & tuano j]usfa & बड कu ' 2 पगय & 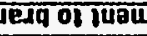 & unubisse so3 & 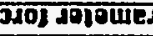 & Eded uosphed & 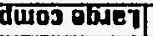 & & & I593 \\
\hline & & & & & & & & & & & & & Jotol & $T$ & HSIBHIFS & & & 659 \\
\hline & & & 1 & & & & & & & & & $\therefore$ & & & ant & & & क69 \\
\hline & & 1 & & & & & & & & & & & & & Tiumina & & & 269 \\
\hline & & & & & & & & & & & & & & & $\overline{6 L}$ & ti & & 9691 \\
\hline & & & & & & & & & & & & & & & Dडाmגauाण & & & SEg \\
\hline & - & & & & & & & द पग्णायव पा & 120pu|eugn & 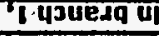 & I pDJE/d SI L & LOJedoden\} & 1 गयदूरण 500 & Odnzando & fiगाIवeqad & & & (169) \\
\hline 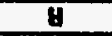 & 0 & $d$ & $\mathbf{0}$ & $\mathbf{N}$ & $\boldsymbol{W}$ & $T$ & $x$ & $\Gamma$ & I & H & 9 & $I$ & 3 & a & $\overline{3}$ & ] & $\overline{0}$ & \\
\hline
\end{tabular}

ZSz abed

ร118-56-HL-Jusm

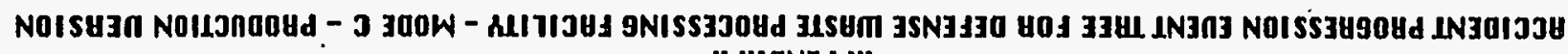

G HIONYddH 
APPENDIH A

\begin{tabular}{|c|c|c|c|c|c|c|c|c|c|c|c|c|c|c|c|c|c|c|}
\hline & A & $\bar{E}$ & $\mathbf{C}$ & D & $\bar{E}$ & $F$ & 5 & $\mathrm{H}$ & 1 & $J$ & $x$ & L & $M$ & $N$ & $\mathbf{0}$ & $\mathbf{P}$ & 0 & n \\
\hline 1697 & & 2 & 46 & 45 & & & & & & & & & & & & & & \\
\hline 1698 & & & 1 & 2 & & & & & & & & & & & & & & $\dot{-}$ \\
\hline 1699 & & & COn-DEU & ngpiofU & & & & & & & & & & & & & & \\
\hline 1728 & & & 0.001 & 0.999 & 0.000 & & & & & & & & & & & & & \\
\hline 1701 & 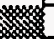 & & Dtherulse & & & & & & & & & & & & .1 & & & \\
\hline 1702 & & & 0.000 & 0.090 & 1.000 & & & & & & & & & & & & & \\
\hline 1703 & 48 & Do in & 10 cell cous & irs that fall & In a selsml & c guent sir & ike the OEC & & & & & & & & & & \pm & \\
\hline 174 & & 2 & SCU-0ECT & ASCU-OECT & & & & & & & & & & & & & & \\
\hline 1705 & & 2 & 1 & 2 & & & & & & & & & & & & & & \\
\hline 1786 & & 2 & & & & & & & & & & & & & & & & \\
\hline 1707 & 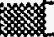 & 2 & 2 & 37 & & & & & & & & & & & & & & \\
\hline 1708 & & & 2 & 1 & & & & & & & $\dot{\square}$ & & & & & & & \\
\hline 1709 & & & EI-Sels & ISCuSels & & & & & & & & & & & & & & \\
\hline 1710 & & & 0.010 & 0.990 & & & & & & & & & & & & & & \\
\hline 1711 & 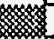 & & olherwlse & & & & & & & & & & & & & & & \\
\hline 1712 & & & 0.000 & 1.580 & & & & & & & & & & & & & & \\
\hline 1713 & 49 & Doos & a Selsmic & event or co & Uer crane fe & all ruplure & and splll in & he contents & of the org & anlc Euapor & ator Conde & insate Tank & In the salt & Process ce & & & & \\
\hline 1214 & & 2 & BPIOECT & nAptoECT & & & & & & & & & & & & & & \\
\hline 1715 & & 6 & 1 & 2 & & & & & & & & & & & & & & 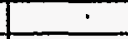 \\
\hline 1716 & & 3 & & & & & & & & & & & & & & & & \\
\hline 1712 & 纱 & 2 & 48 & 39 & & & & & & & & & & & & & & \\
\hline 1718 & & & 1 & +4 & & & & & & & & & & & & & & \\
\hline 1719 & & & \begin{tabular}{|l|} 
SCU-OECT \\
\end{tabular} & CD_DECT & & & & & & & & & & & & & & \\
\hline 1720 & & 1 & 79 & & & & & & & &. & & & & & & & \\
\hline 1721 & & & Dummy & & & & & & & & & & & & & & & \\
\hline 1722 & & & ADD. & & & & & & & & & & & & & & & \\
\hline 1723 & & & THAESH & 1 & 8.98 & & & & & & & & & & & & & \\
\hline 1724 & . & & Compariso & n paramete & Torces ass & slanment I & o branch 1 , & coll couers & strike the & n tank rupt & ured. & & & & & & & \\
\hline 1725 & 煓 & 3 & 2 & 37 & 48 & & & & & & & & & & & & & \\
\hline 1726 & 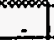 & & 2 & 12 & +21 & & & & & & & & & & & & & \\
\hline 1727 & & & EI-Sels & niscusel & nSCU-OECT & & & & & & & & & & & & & \\
\hline 1728 & & 1 & 22 & & & & & & & & & & & & & & & \\
\hline 1729 & & & DECTIF & & & & & & & & & & & & & & & \\
\hline 173 & & & ADD & & & & & & & & & & & & & & & \\
\hline 1731 & & & EQUAL & $a$ & & & & & & & & & & & & & & \\
\hline 1732 & & & Probobillil! & of rupture & of Organic & Euaporato & $r$ Condensal & te Tank Is & laced in br: & anch 1, rem & alnder In br & $\operatorname{ranch} 2$. & & & & & & \\
\hline 1733 & 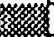 & & otherwise & & & & & & & & & & & & & & & \\
\hline 1734 & & I & 79 & & & & & & & & & & & & & & & \\
\hline 1735 & & & Bumm! & & & & & & & & & & & & & & & \\
\hline 1736 & & & fan & & & & & & & & & & & & & & & \\
\hline 1737 & & & GETHBESH & $I$ & 180.0 & & & & & & & & & & & & & \\
\hline 1738 & & & Large com & perison par & ameter forc & ces assignn & nent to bra & nch 2 , nos & elsmic euen & th, no ruptu & ire of tonk. & & & & & & & \\
\hline 1739 & 50 & Does & an esplosi & Ue concent & allon accun & mulate $\ln 0$ & rrganlc Euap & porator Cor & idensate Ta & Ink? & & & & & & & & \\
\hline 1740 & & 2 & CON-DECT & InCon-DECT & & & & & & & & & & & & & & \\
\hline 1241 & & 6 & I & 2 & & & & & : & & & & & & & & & \\
\hline 1742 & 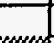 & 4 & & & & & & & & & & & & & & & & \\
\hline 1743 & 恀 & 2 & 1 & 1 & & & & & & & & & & & & & & \\
\hline 1744 & & & $(-1$ & -21 & & & & & & & & & & & & & & \\
\hline 1745 & & & /EHternal & /Int-Equ & & & & $i$ & & & & & & & & & & \\
\hline 1746 & & 1 & 79 & & & & & & & & & & & & & & & \\
\hline 1747 & & & Dummy & & & & & & & & & & & & & & & \\
\hline 1748 & & & AnD & & & & & & & & 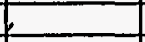 & & & & & & & \\
\hline 1749 & & - & GETHRESH & 1 & 188.8 & & & & & & & & & & & & & \\
\hline
\end{tabular}




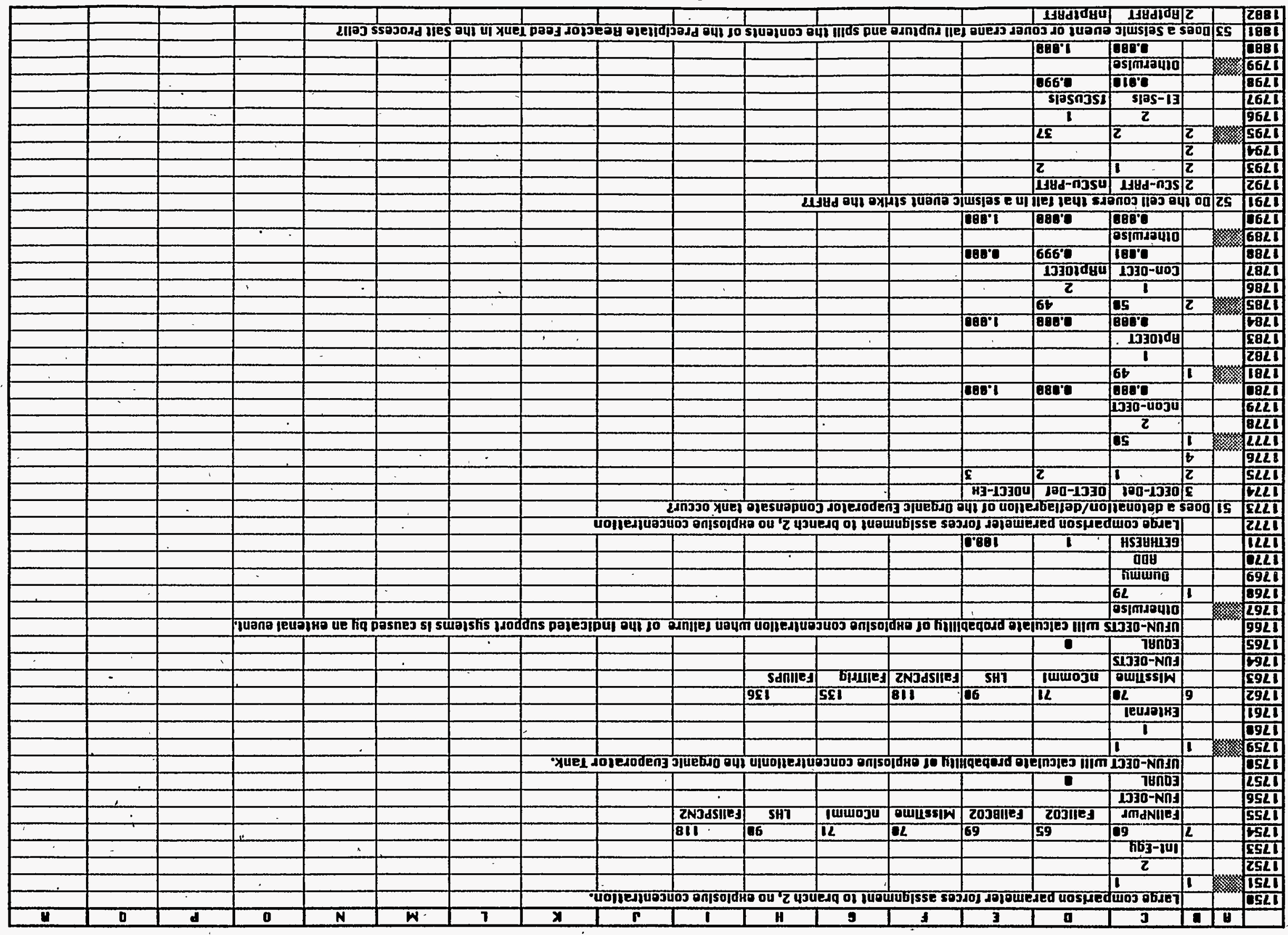


APPENDIK A

WUSRC-TA-95-8113

ACCIDENT PROGRESSION EUENT TREE FOR DEFENSE WASTE PROCESSING FACILITY - MODE C - PRODUCTION UERSION

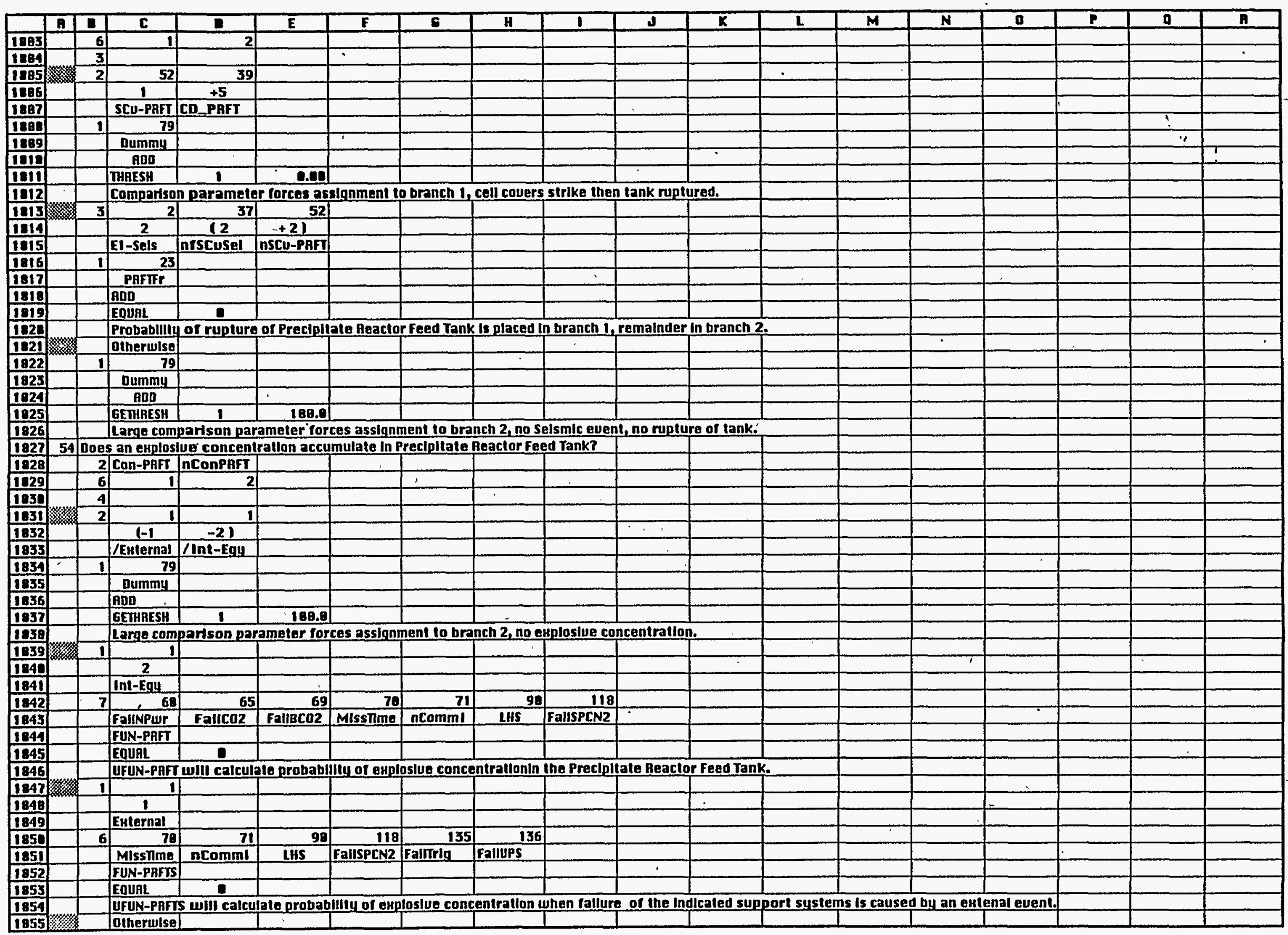




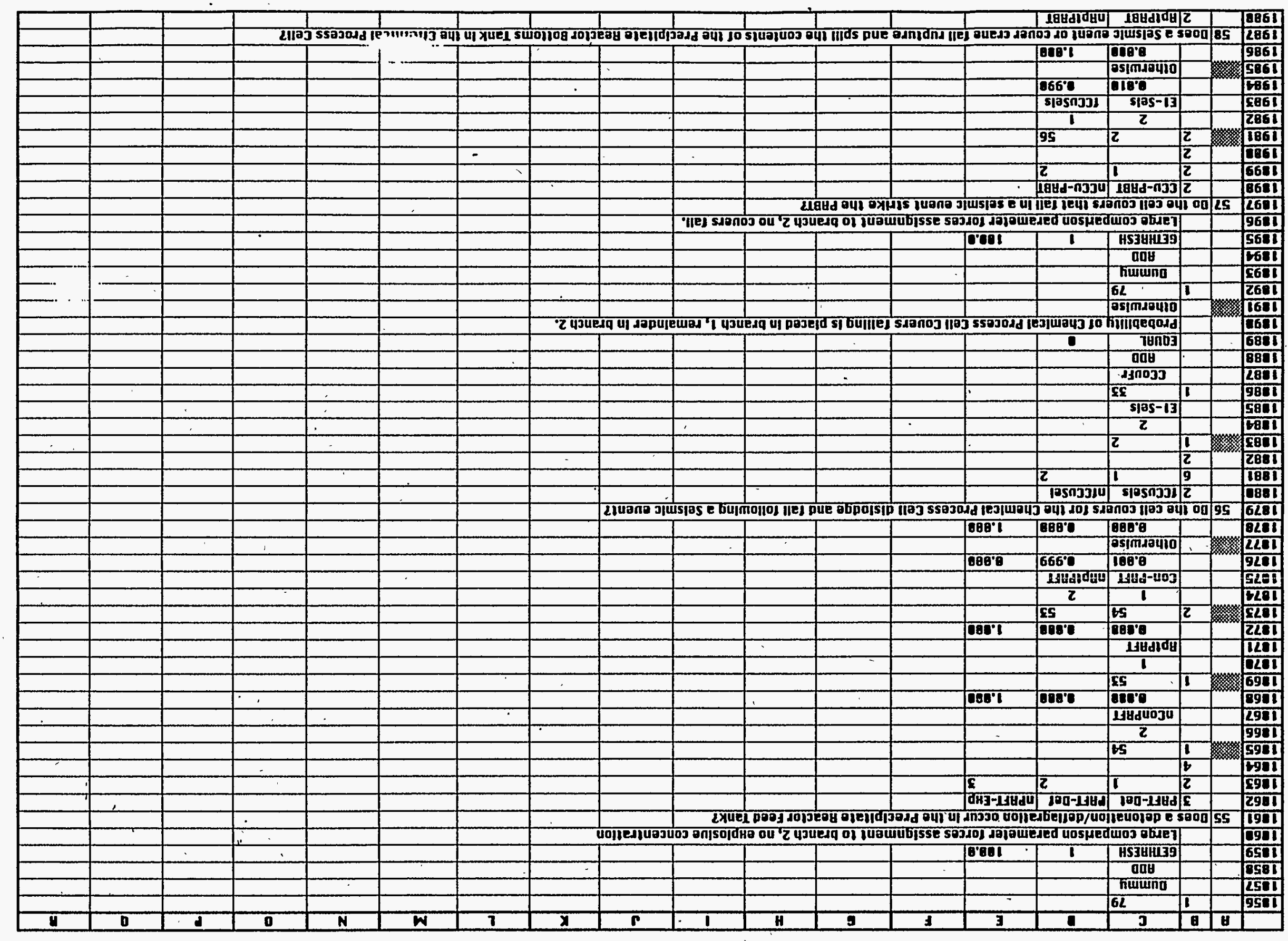

gsz abed

CLIB-S6-UL-JuSm

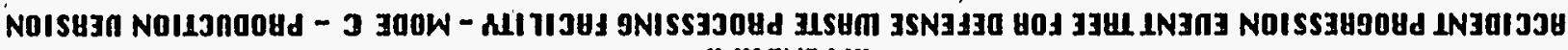

H HIONIddU 
APPENDIH R

ACCIDENT PROGRESSION EUENT TAEE FOR DEFENSE WASTE PROCESSING FACILITY - MODE C - PRODUCTION UERSION

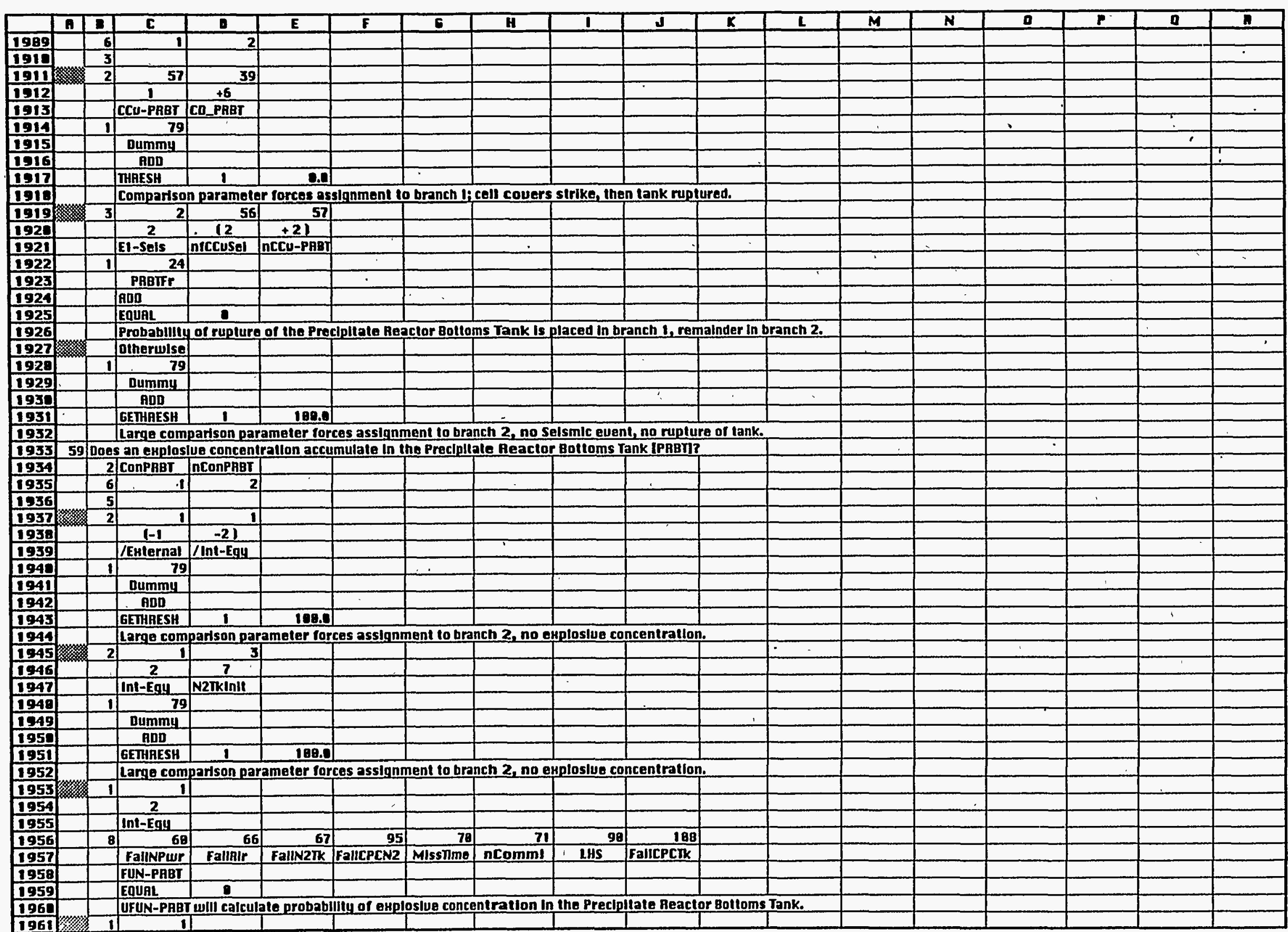




\begin{tabular}{|c|c|c|c|c|c|c|c|c|c|c|c|c|c|c|c|c|c|c|}
\hline & $\mathbf{n}$ & D & C & E & $E$ & $F$ & c & H & $I$ & $J$ & K & $\mathbf{L}$ & $M$ & $N$ & 0 & $P$ & a & $\pi$ \\
\hline 1962 & & & 1 & & & & & & & & & & & & & & & \\
\hline 1963 & & & Euternal & & & $=$ & . & . & & $\dot{5}$ & & & & & & & & $\dot{5}$ \\
\hline 1964 & & 2 & 60 & 66 & 67 & 95 & 70 & 71 & 90 & 108 & & - & & & & & & \\
\hline 1965 & & & FallNPur & Fallinir & FallN2IK & FallCPCN2 & Mlssinme & ncomml & LHS & FalicPCTK & & & & & & & & \\
\hline 1965 & & & FUN-PRBIS & & & & & - & & & & & & & $\pi$ & & $\mp$ & \\
\hline 1967 & & & EquAL & 드 & & & & & & & & & & & & & & \\
\hline 1968 & & & UFUN-PABT & S wlll calcu & late probat & jilley of eнp & loslue conc & :entrallan & when fallum & re of the in & dicateds & upport syst & tems is caus & ised by an eH & tenal euent. & & 5 & \\
\hline 1969 & & & Otherulse & & 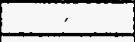 & & & & & & & & & & & & & 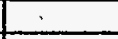 \\
\hline 1970 & & 1 & 79 & & & & & & & & & & & & & & & \\
\hline 1971 & & & Dummy & & & & & & & & & & & & & & & \\
\hline 1972 & & & ADO & & & & & & & & & & & & & & & \\
\hline 1923 & & & GETHRESH & 1 & 109.0 & & & & & & - & & & & & & & \\
\hline 1974 & & & Large com & parison par & amoter for & ces assignn & ment to bra & nch 2, no o & Hploslye co & oncentration & & & & & & & & \\
\hline 1975 & 68 & Doss & se detona! & $\operatorname{lon} / d a f l e g r$ & atlan occur & In the Prec & clpllate Aes & actor Botio & ms Tank IPA & B8n? & & & & & & & & \\
\hline 1976 & & 3 & PABT-Det & |PABT-DOT & APABT-EM & & & & & & & & & & & & & \\
\hline 1977 & & 2 & 1 & 2 & 3 & & & & 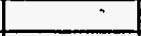 & & & & & & & & & \\
\hline 1970 & & 4 & & & & & & & & $\therefore$ & & & & & & & & \\
\hline 1979 & & 1 & 59 & & & & & & & & & & & & & & & \\
\hline 1980 & & & 2 & & & $\therefore$ & & & $=$ & & & & & & & & & \\
\hline 1981 & & & nConPABT & & & & & & & & & & & & & & & \\
\hline 1982 & & & 0.008 & 0.080 & 1.001 & & & - & $\therefore$ & $\therefore$ & & & - & & & & & \\
\hline 1983 & & 1 & 58 & & & & & & & & & & & & & 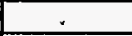 & 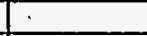 & \\
\hline I9BA & & & 1 & & & & & $\therefore$ & & & & & & & 二 & & & \\
\hline 1985 & & & APIPABT & & & & & & & & & & & & & & & \\
\hline 1986 & & & e.eng & 0.000 & 1.990 & & & & & . & & & & & & & & \\
\hline 1987 & 孟 & 2 & 59 & 58 & & & & & & & & & & & & & & \\
\hline 1988 & & & 1 & 2 & & & & & $\dot{-}$ & & & $\therefore$ & & & & & & \\
\hline 1989 & & & ConPRBT & nApIPABT & & & & & & & & 1 & & & & & & \\
\hline 1990 & & & 0.801 & $\begin{array}{r}0.999 \\
\end{array}$ & 0.000 & & & & & & & & & & & & & \\
\hline 1991 & 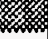 & & Otherwise & & & & & & & & & & & & & & & \\
\hline 1992 & & & 0.000 & 0.080 & 1.020 & & & & & & & & & & & & & \\
\hline 1993 & 61 & Do If & he cell coue & ers that rall & due to as & eismic even & it sirlke the & A SME? & & & & & & & & & & \\
\hline 1999 & & 2 & CCU-SME & nCCU-SME & & & & & & & & 5 & & & & & & \\
\hline 1995 & & 2 & 1 & 2 & & & $\therefore$ & & & & & & - & & 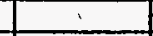 & & & \\
\hline 1996 & & 2 & & & & & & & & & & & & & & & & - \\
\hline 1997 & 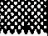 & 2 & 2 & 56 & & & & & & & & & & & & & & \\
\hline 1999 & & & 2 & 1 & & & & & & & & & & 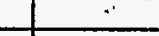 & & & & \\
\hline 1999 & & & Ei-Sels & IccuSels & & & & & & & & & & & & & & \\
\hline 2010 & & & 0.010 & 0.998 & & & & & & & & & & & & & & \\
\hline 2091 & 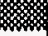 & & Olherwise & & & & & & & & & & & & & & & \\
\hline 2082 & & & 0.080 & 1.000 & & & & & & & & & & & & & & \\
\hline 2083 & 62 & Does & 5 a Selsmic & event or co & wer crane & all rupture & and spill th & e contents & of the Slur & rry Мiн Eua & parator T & nk in the $C$ & Chemical Pro & ocess Cell? & & & & \\
\hline 2081 & & 2 & BpISME & nApISME & & & & & & & & & & & & & & \\
\hline 2085 & & 6) & 1 & 2 & & & & . & & & & & & & 1 & & & \\
\hline 2096 & & 3 & & & & & & & & & & & & & & & & \\
\hline 2097 & & 2 & 61 & 39 & & & & & & & & & & & & & & \\
\hline 2089 & & & 1 & +7 & & & & & & & & & & & & & & \\
\hline 2089 & & & CCU-SME & CD_SME & & & & & & & & & & $\therefore$ & & $\therefore$ & & \\
\hline 2010 & & 1 & 79 & & & & & & & & & & & & & & & \\
\hline 2011 & & & Dummy & & & & & & & & & & & & & & & \\
\hline 2012 & & & ADD & & & & & & & & & & & & & & & \\
\hline 2013 & & & THAESH & 1 & 0.0 & & & & & & & & & & & & & \\
\hline 2014 & & & Comparisor & n paramete & forces as & slgnment to & o branch li: & cell covers & strike, the & en iank rup! & ured. & & & & & & & \\
\hline
\end{tabular}


APPENDIH A

ACCIDENT PAOGRESSION EUENT TAEE FOR DEFENSE WASTE PROCESSING FACILITY - MODE C - PAODUCTION UERSION

USAC-TR-95-9113

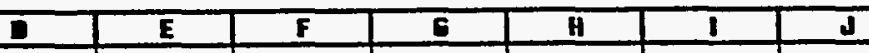

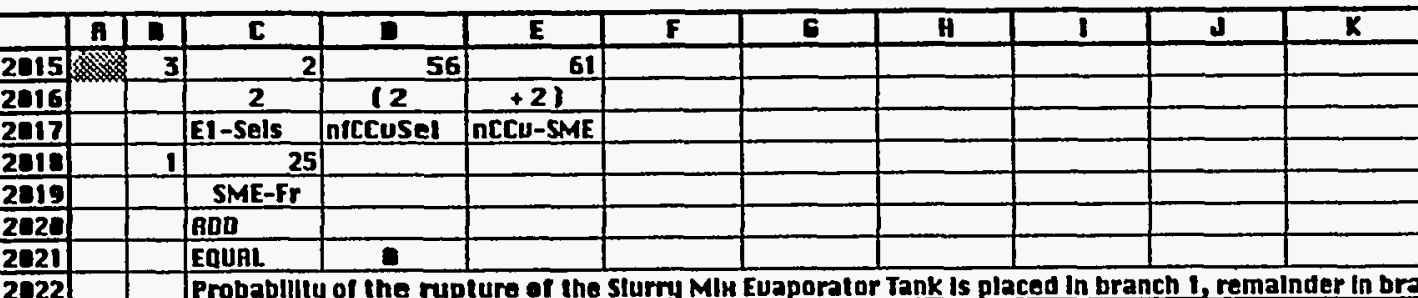

2022 Probablitty of the ruplure of the Slurry Mlu Euaporator Tank is placed in branch 1 , remainder in branch 2.

2023 Otheriuis:

$2221+-1$

2225

GETHRESH

2028 Lame comparison parameler forces

2030 2 ConSME 1 nConSME

$2031+\frac{6}{2032}$

2032

2033

2035

2836

2037

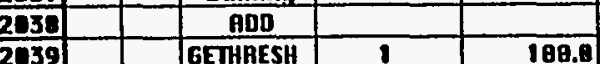

2040

2041.

\begin{tabular}{l|l}
\hline & 1 \\
\hline
\end{tabular}

$(-1)-2)$ 1 (-1)ernal $/ \ln t-E q 4$

2043

2044

2045

\begin{tabular}{r|}
2046 \\
\hline 2047 \\
\hline 2546
\end{tabular}

\begin{tabular}{|l|}
2047 \\
\hline 2048 \\
\hline 2049 \\
\hline 2058 \\
\hline
\end{tabular}

2050

2051

\begin{tabular}{r|r|r|r|r|r|r}
\hline Int-Egy & & & & & & \\
\hline
\end{tabular}

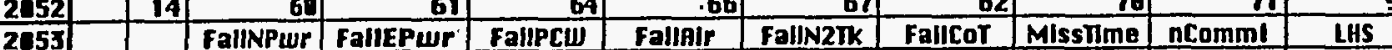

2054

2055

2056

2057

$\frac{2058}{2059}$

2069

2061

2062

2063

2069

2065

2065 Large comparison parameter forces

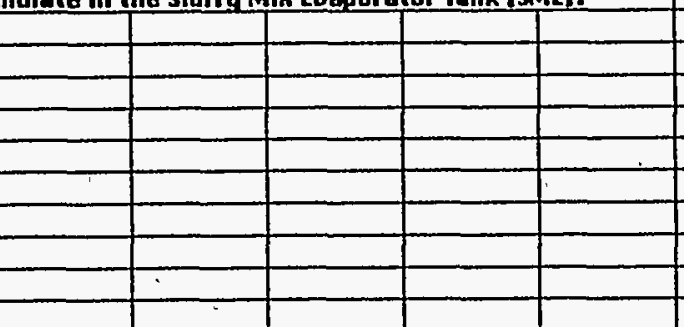

e of tank

206 Int-Egh N2IkInIt Dummy Dummy

GITnESH 1

Large comparison parameter forces asslgnment to branch 2 , no euploslue concentratlon FUN-SME

\begin{tabular}{l|l} 
EQUAL & $\mathbf{a}$ \\
\hline
\end{tabular}

UFUN-SME will calculpte probabillty of eHplosiue concentralion in the SIUrry Mik Euaporator Tank. \begin{tabular}{l|l}
\hline & UFUN \\
\hline &
\end{tabular}

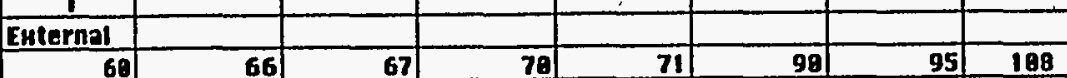

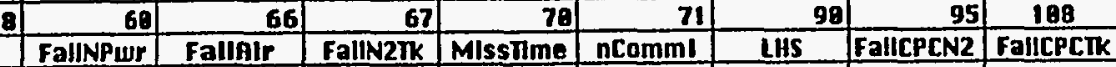
FUN-SMES

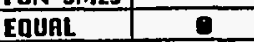
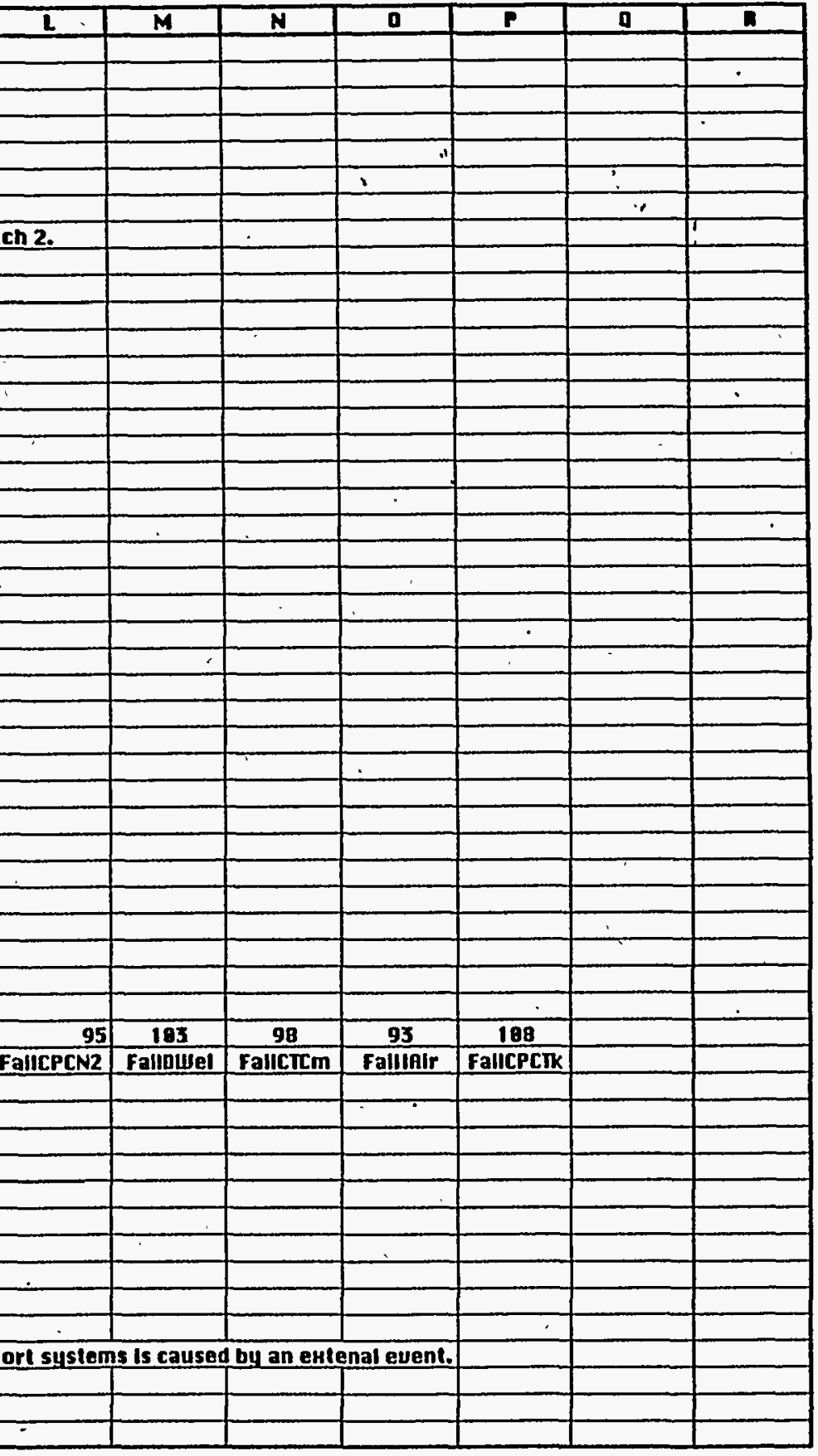

Page 259 
APPENDIH A

UUSRC-Th-95-6113

ACCIDENT PROGRESSION EUENT THEE FOR DEFENSE WASIE PROCESSING FACILITY - MODE C - PRODUCTION UERSION

Page 260

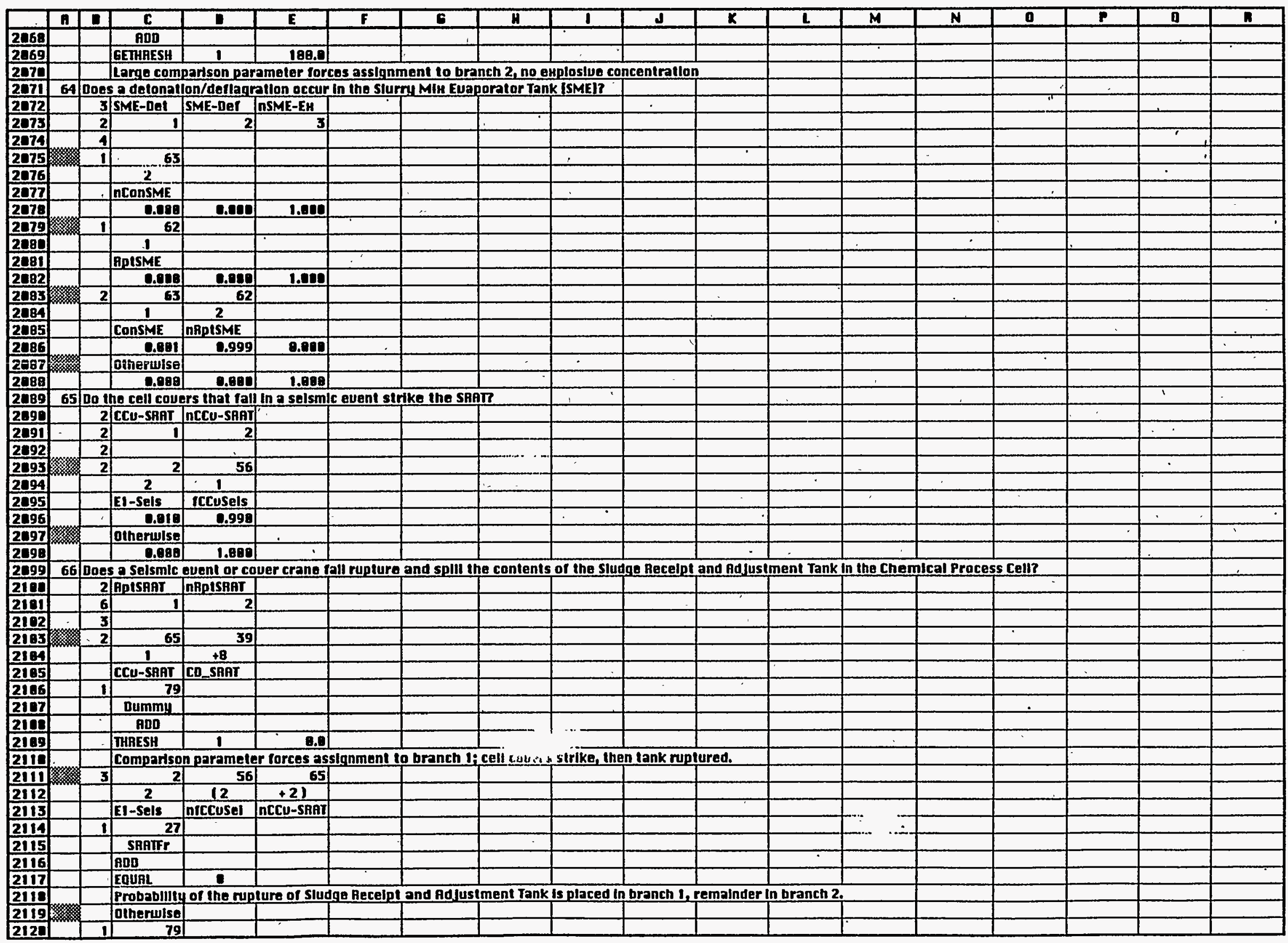


APPENDIH A

\begin{tabular}{|c|c|c|c|c|c|c|c|c|c|c|c|c|c|c|c|c|c|c|}
\hline & A & $\mathbf{E}$ & $\bar{C}$ & $\overline{8}$ & $\bar{E}$ & $\mathbf{F}$ & F & H & $I$ & $\mathrm{~J}$ & $\mathbf{K}$ & 2 & $\bar{M}$ & $N$ & 0 & $\mathbf{P}$ & a & n \\
\hline 2121 & & & Dummy & & & & & & & & & & & & & & & \\
\hline 2122 & & & ADo & & & & & & & & & & & & & & & $\dot{-}$ \\
\hline 2123 & & & GETHAESH & 1 & 100.0 & & & & & & $\div$ & & & & & & $\dot{\square}$ & \\
\hline 2124 & & & Larqe comp & parson parr & meter forc & ces assignm & Ient lo bran & nch 2 , no Se & elsmic even & ino ruplun & of tank. & & & & & & & \\
\hline 2125 & 67 & Does & an esplosl & Ue concentr & rallon aecun & mulate in 11 & e sludge $\mathrm{B}$ & ecelpt and & Adjustmen & I Tank ISAAT & & & & &. & & & \\
\hline 2126 & & 2 & ConSAAT & nConSBAT & & & & & & & & & & & & & $\dot{2}$ & \\
\hline 2127 & & 6 & 1 & 2 & & & & & & & & & & & & & 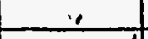 & \\
\hline 2120 & & 5 & & & & & & & & & & & & & & & & \\
\hline 2129 & & 2 & 1 & 1 & & & & & & & & & & & & & & \\
\hline 213 & & & $1-1$ & -21 & & & & & & & & & & & & & & \\
\hline 2131 & & & /Esternal & $\ln \mid-E g y$ & & & & & & & & & & & & & & \\
\hline 2132 & & II & 79 & & & & & & & & & & & & & & & \\
\hline 2133 & & & Dummy & & & & & & & & & & & & & & & \\
\hline 2134 & & & ADD & & & & & & & & & & & & & & & - \\
\hline 2135 & & & GETHAESH & 1 & 100.0 & & & & & & & & & & & & & \\
\hline 2136 & & & Lange com & partsan par: & ameter sorc & ces assignn & nent to bran & $\mathrm{nch} 2, \mathrm{no}$ eH & uploslue can & ncentratlon. & & & & & & & & \\
\hline 2137 & & 2 & 1 & 3 & & & & & & & & & & & & & & \\
\hline 2138 & & & 2 & 7 & & & & & & & & & & & & & & \\
\hline 2139 & & & Int-Egy & N2IKInII & & & $\therefore$ & & & & & & & & & & & $\therefore$ \\
\hline 2140 & & 1 & 79 & & & & & & & & & & & & & & & \\
\hline 2141 & & & Dummy & & & & & & & & & & & & & & & \\
\hline 2142 & & & $\mathrm{ADD}$ & & & & & & & & & & & & & & & \\
\hline 2143 & & & GETHAESH & 1 & 180.0 & & & & & & & 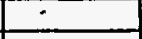 & & & & & & \\
\hline 2144 & & & Large com & parison par & ameter forc & ces assignn & nent to bra & $\mathrm{nch} 2, \mathrm{no}$ ef & uploslue con & ncentratlon. & & & & & & & & \\
\hline 2145 & 织怚 & 1 & 1 & & & & & & & & & & & & & & & \\
\hline 2146 & 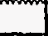 & & 2 & & & & & & 7 & & & & & & & & & \\
\hline 2147 & & & Int-Egy & & & & & & & & & & & & & & & \\
\hline 2148 & & 15 & 60 & 61 & 63 & 64 & 66 & 67 & 62 & 70 & 71 & 98 & 95 & 183 & 98 & 93 & 108 & \\
\hline 2149 & & & FaliNpwr & FallePUr & FallPSIm & FollpCW & Fallialr & \begin{tabular}{|l|} 
Falin2IK \\
\end{tabular} & Falicor & Missnime & \begin{tabular}{|l} 
ncommin \\
\end{tabular} & LAS & FallCPCN2 & Fallowel & Fallcrem & Falliair & FallcPCTK & \\
\hline 2150 & & & FUN-SAAT & & & & - & & & & & & & & & & & \\
\hline 2151 & & & EQUAL & - & & & & & & & & & & & & & & \\
\hline 2152 & & & UFUN-SART & ifili calcula & Ie prababill & IIty af aspl & slue cancel & niratlon In & the vapors & space of the & - Sludge Be & ecelpt and p & idjustmen! & Tant. & & & & \\
\hline 2153 & 嬨 & 1 & 1 & & & & & & & & & & & & & & & \\
\hline 2154 & & & 1 & & & & & & & & & & & & & & & \\
\hline 2155 & & & Esternal & & & & & & & & & & & & & & & \\
\hline 2158 & & 8 & 61 & 66 & 67. & 70 & 71) & 98 & 95 & 188 & & & & & & & & \\
\hline 2157 & & & FallNPwr & Falliair & FallN2IK & MissTIme & ncomml & LHS & FaliCPCN2 & Fallcpctk & & & & & & & & \\
\hline 2158 & & & FUN-SARTS & & & & & & & & & & $\dot{-}$ & & & & & \\
\hline 2159 & & & EQUAR & 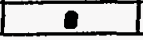 & & & & & & & & & & & & & & \\
\hline 2160 & & & UFUN-SBAT & 5 will calcul & Dete probabl & Illity of eHp & losiug conce & & When failure & of the ind & Ilcated supp & part systen & ns is caused & $\mathrm{d} \mathrm{by}$ an emt & enal event. & & & \\
\hline 2161 & 穪 & & Dinerwlse & & & & & & & & & & & & & & & \\
\hline 2162 & & 1 & 79 & & & & & & & & & & & & & & & \\
\hline 2163 & & & Dummy & & & & & & & & & & & & & & & \\
\hline 2164 & & & ADD & & & & & 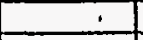 & & & & & & & & & & \\
\hline 2165 & & & GETHAESH & 1 & 108.0 & & & & & & & & $\therefore$ & & & & & \\
\hline 2166 & & & Large com & pentson par & ameter forc & ces asslgnn & nent lo bral & nch 2, no es & Hplostue co & ncentrollon & & & & & & & & \\
\hline 2167 & 68 & Does & s a detonall & lon/defliagr & allon occur & in the slud & ge hecelpt & and AdJustr & ment Tank & ISAATI? & & & & & & & & \\
\hline 2169 & & 3 & SRAT-Det & SAAT-Der & ПSPAT-EH & & & & & & & & & & & & & \\
\hline 2169 & & 2 & 1 & 2 & 3 & & & & & & & & & & & & 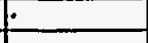 & \\
\hline 2170 & 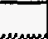 & (4) & & & & & & & & & & & & & & & & \\
\hline 217 & 㹡 & 1 & 67 & & & & & & & & & & & & & & & \\
\hline 2172 & $m$ & & 2 & & & & & & & & & & & & & & & \\
\hline 2173 & & & nConSABT & & & & & & & & & & & & & & & \\
\hline
\end{tabular}


APPENDIH $\mathrm{a}$

ACCIDENT PAOGRESSION EUENT THEE FOR DEFENSE WASTE PROCESSING FACILITY - MODE C - PHODUCTION UERSION

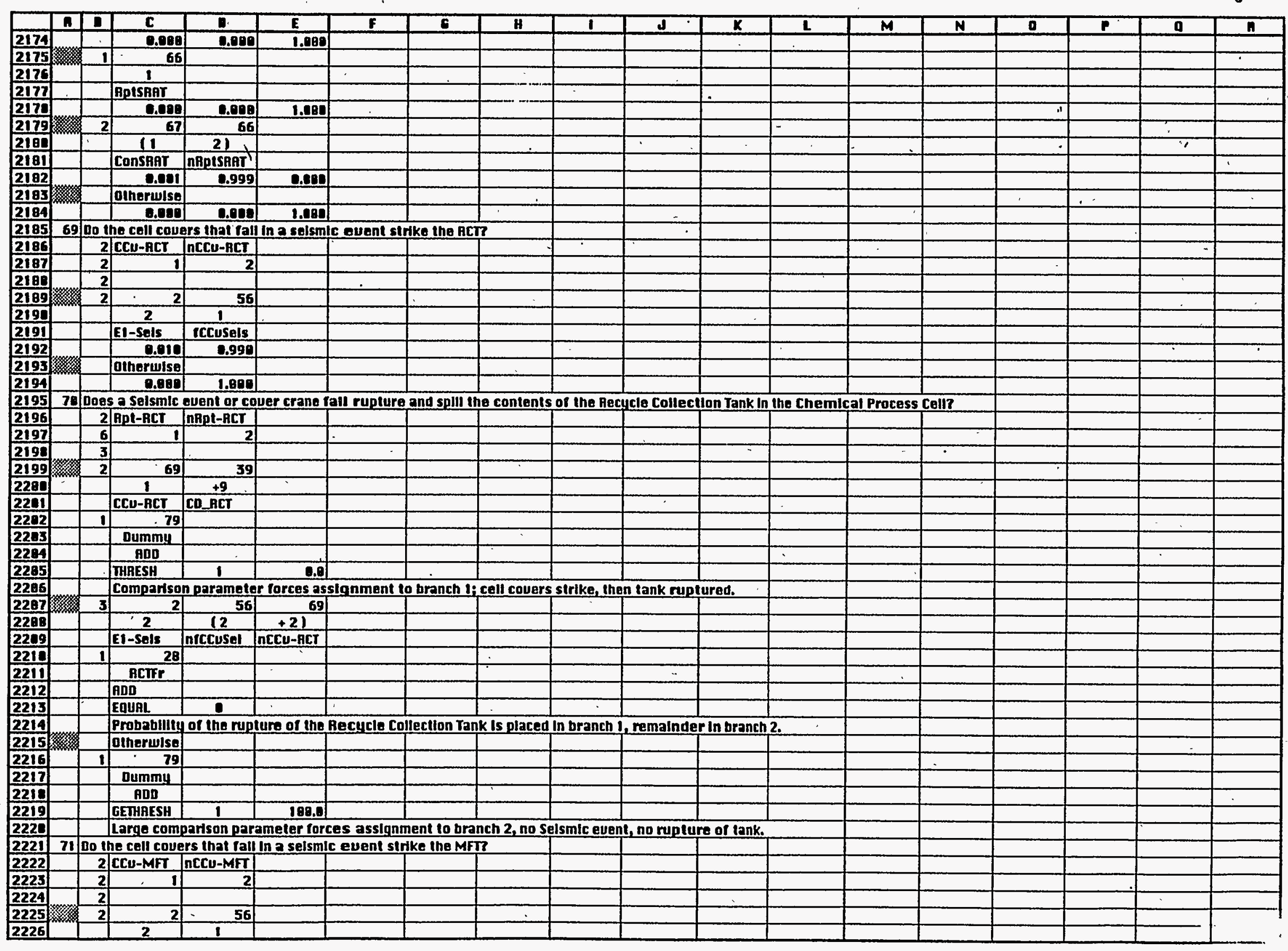




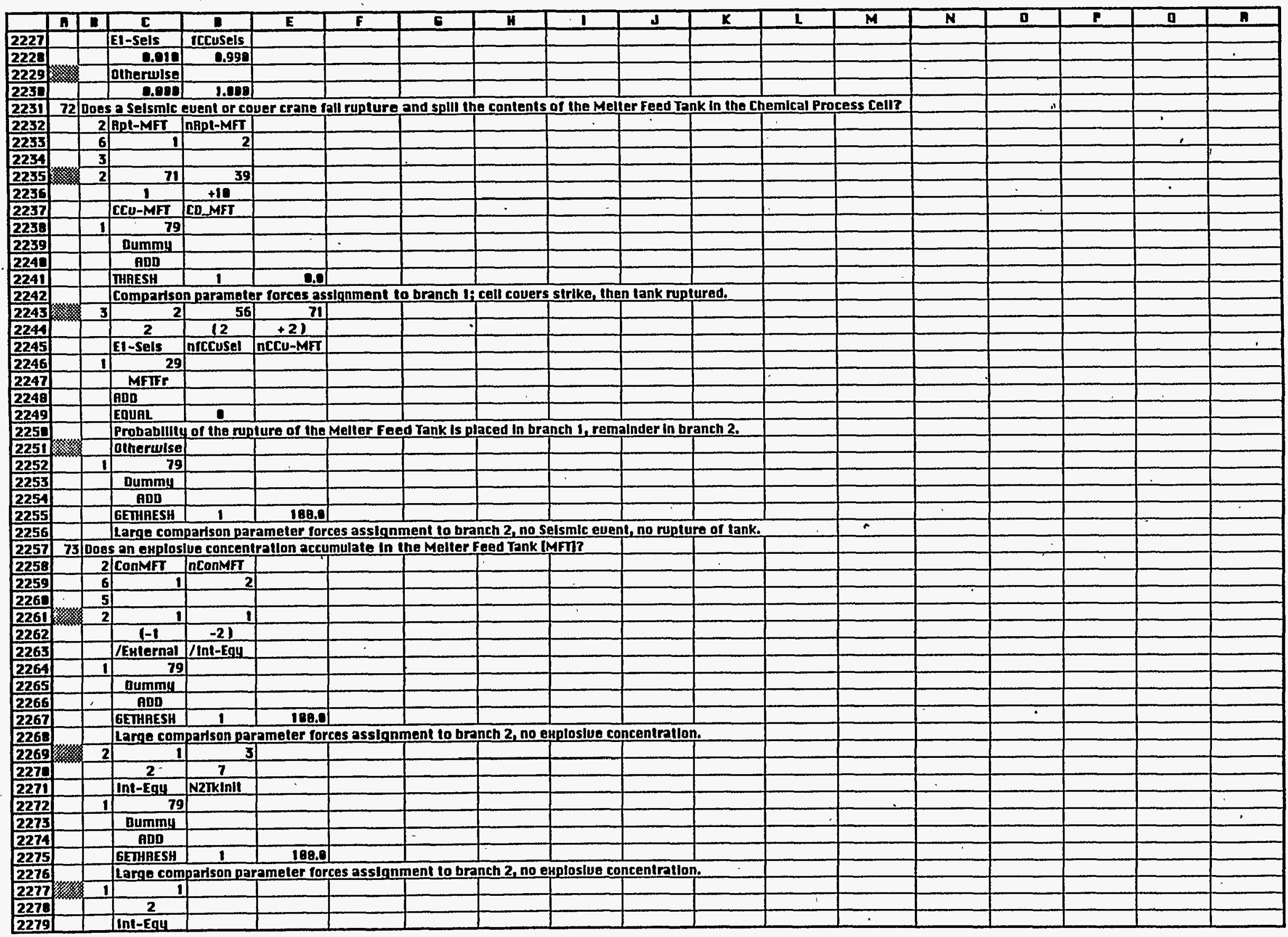


APPENDIK A

ACCIDENT PAOGAESSION EUENT TAEE FOA DEFENSE UUASTE PAOCESSING FACILITY - MODE C - PAODUCTION UERSION

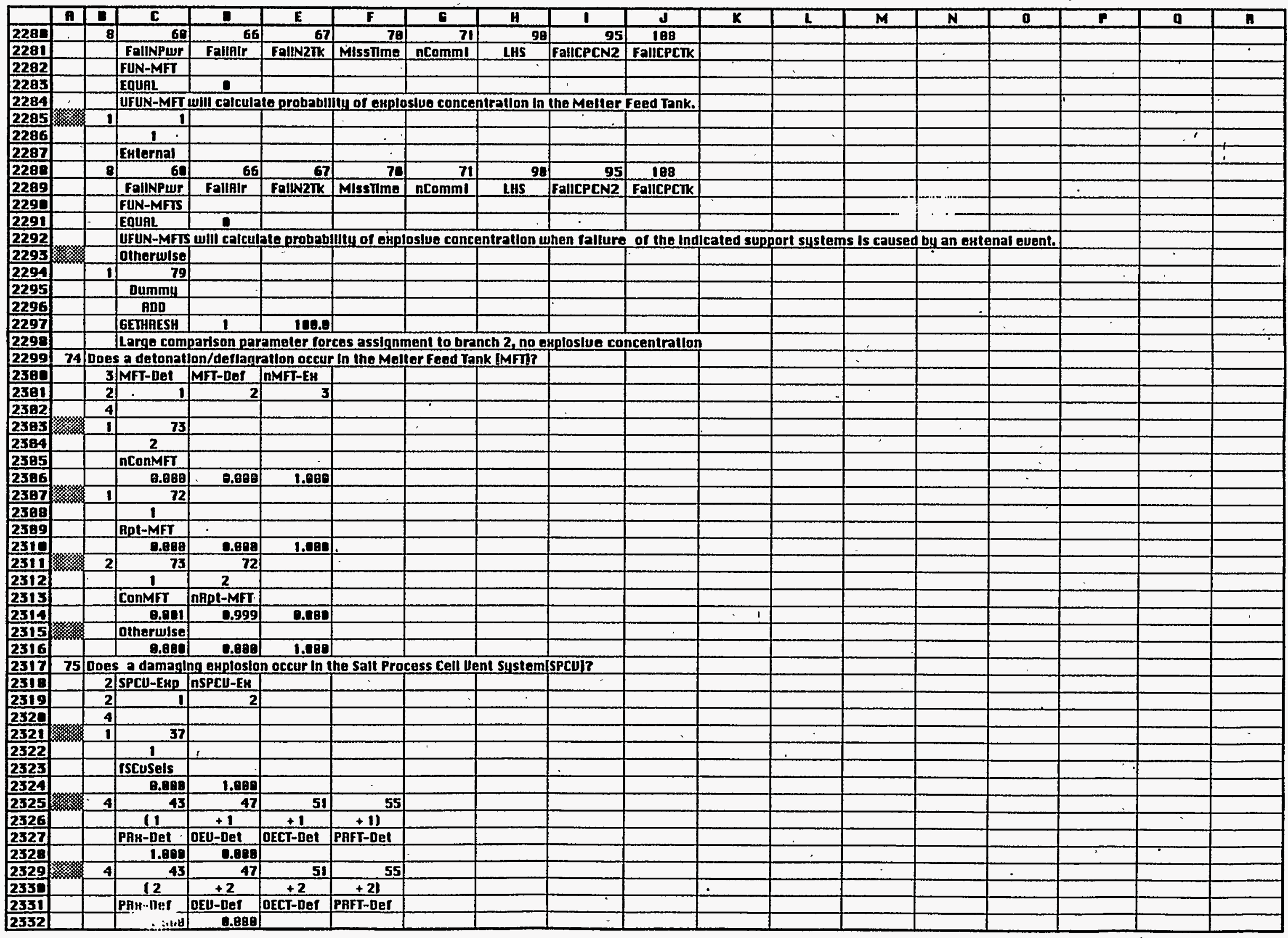




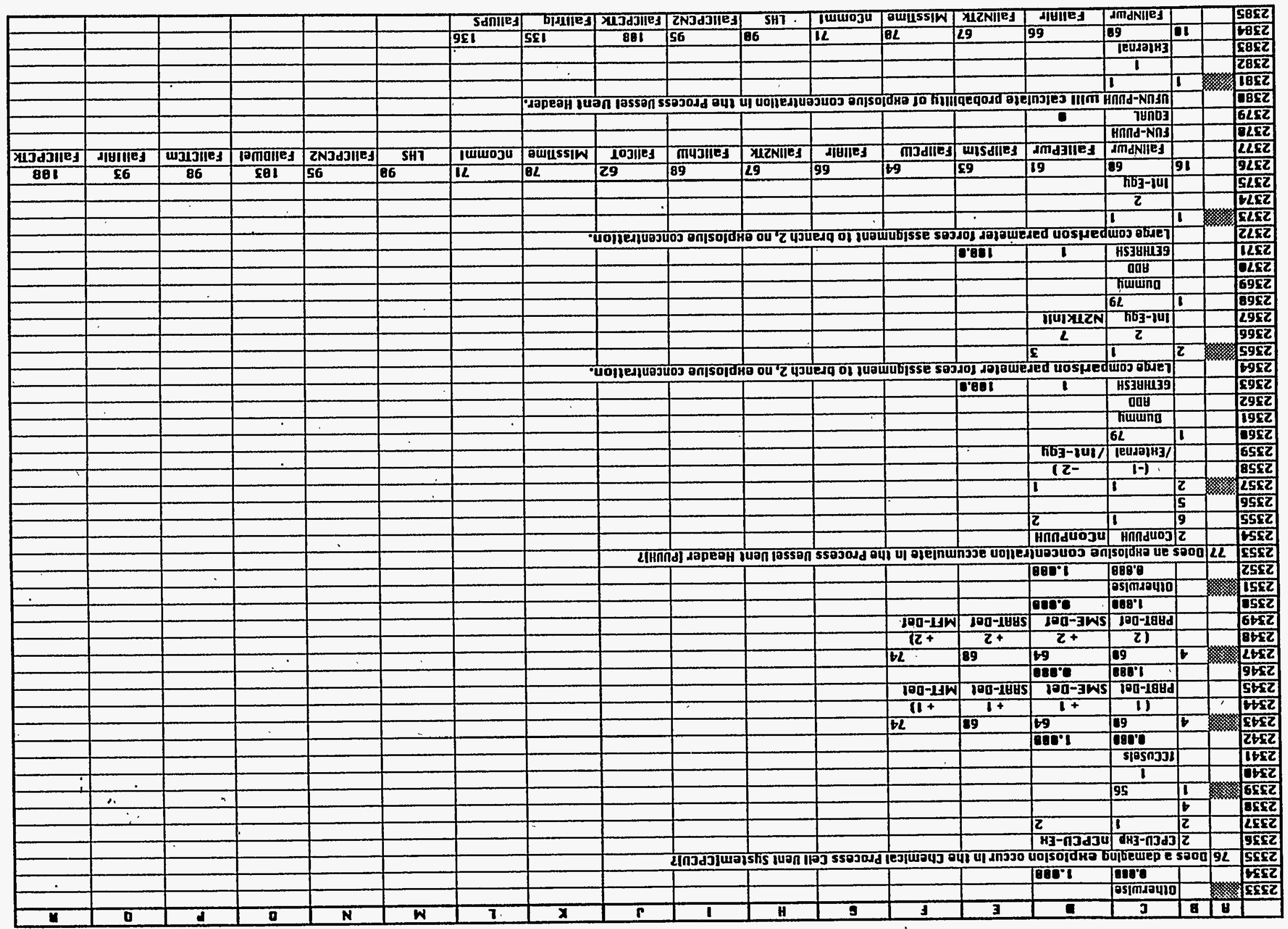

s9z ofied

c1 10-56-41-345m

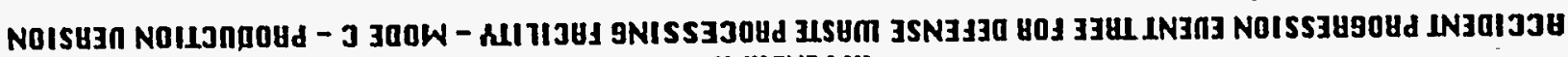

Y RIQN3ddU 


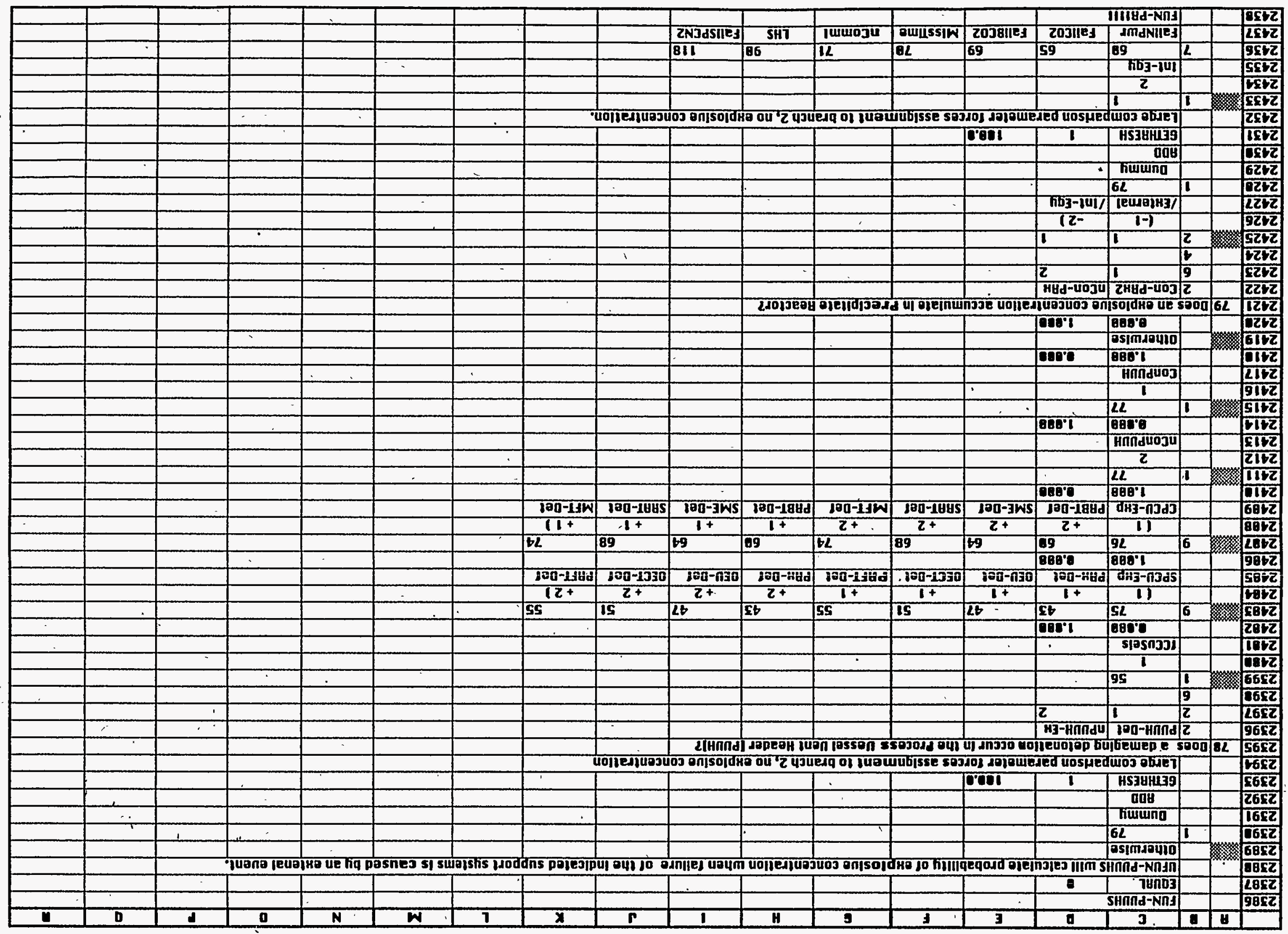


APPENDIH A

ACCIDENT PROGRESSION EUENT TAEE FOR DEFENSE WASTE PROCESSING FACILITY - MODE C - PRODUCTION UEASION

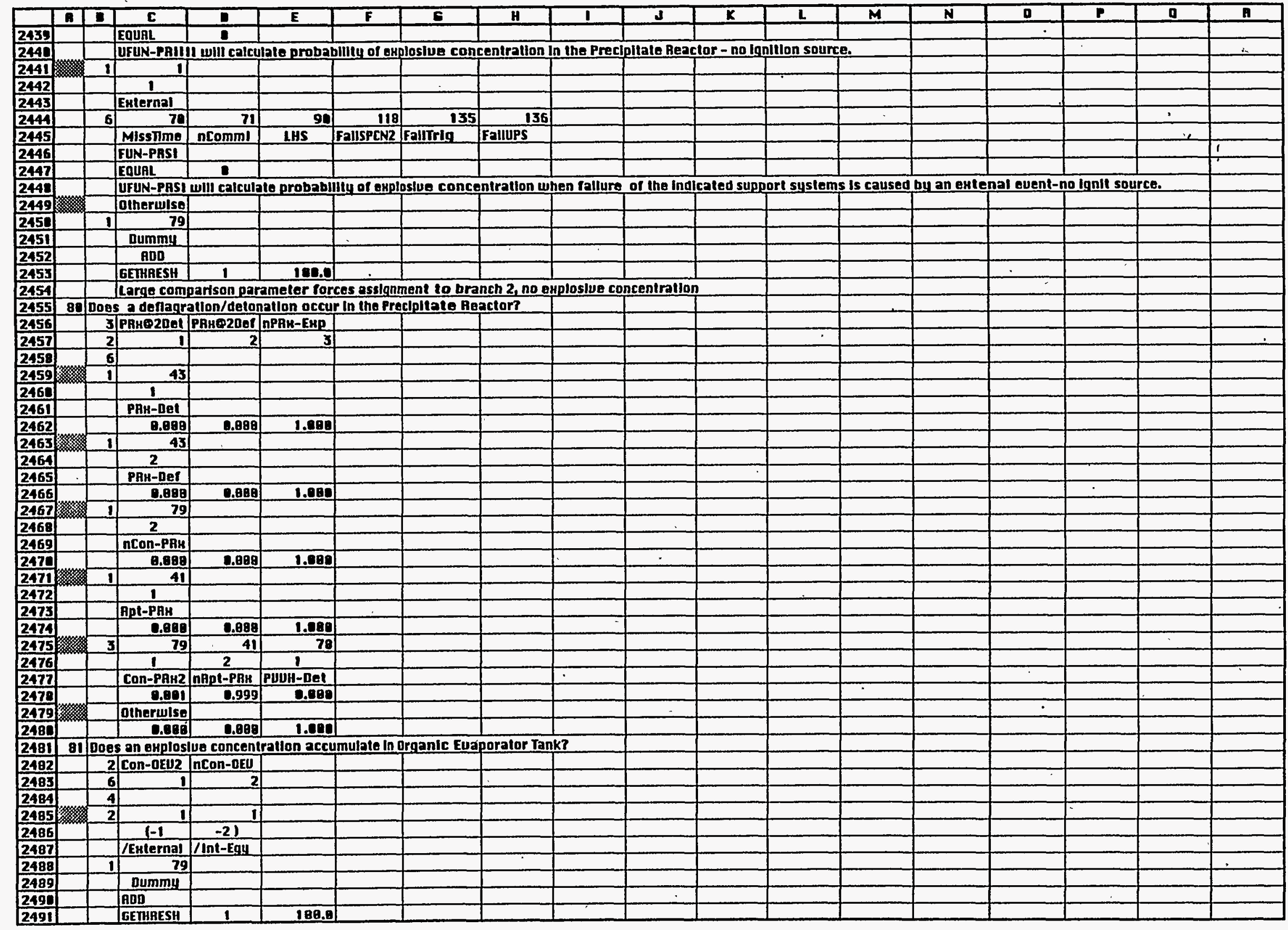




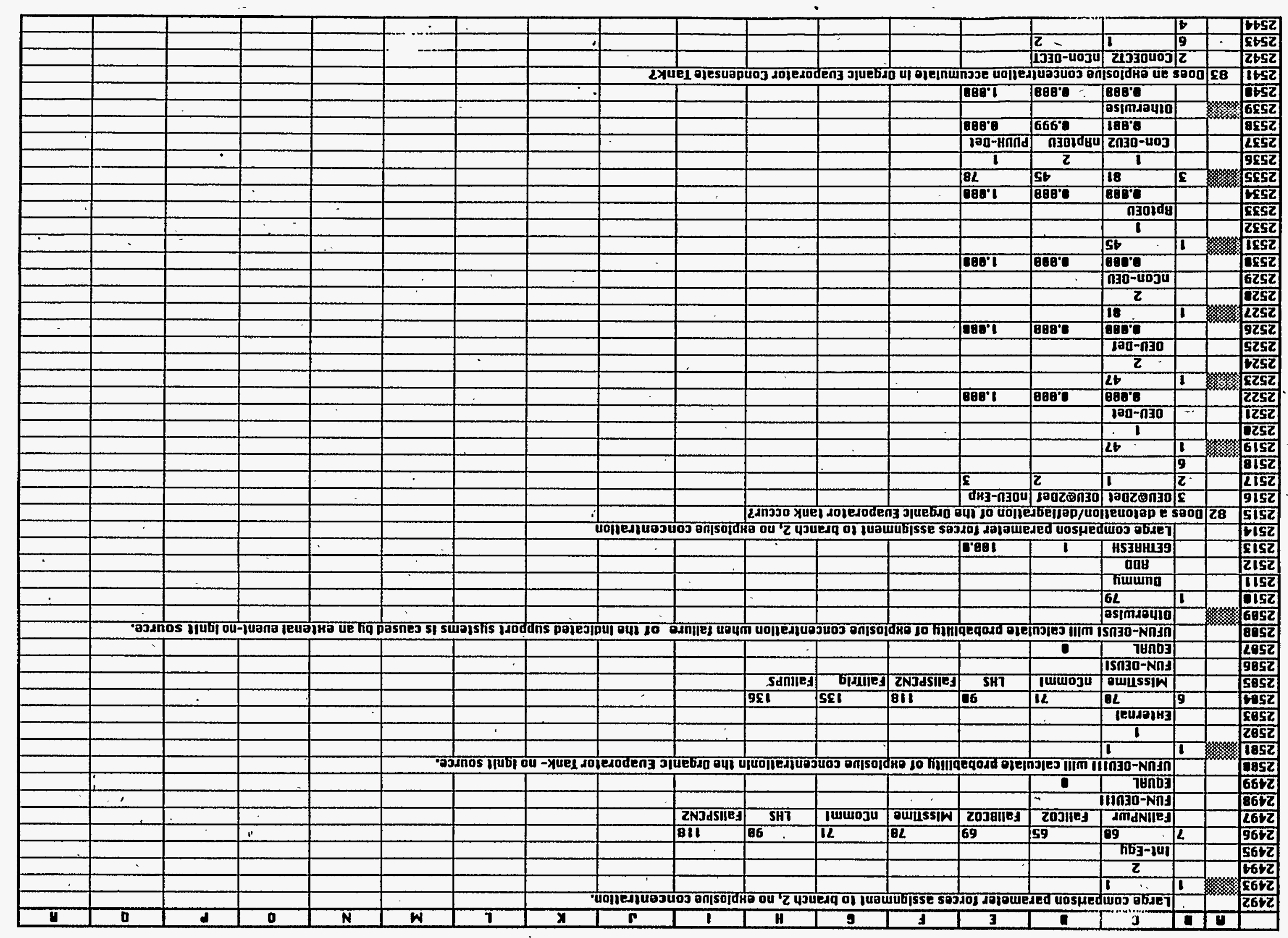

Bgz o6ed

E110-56-4I-JusN

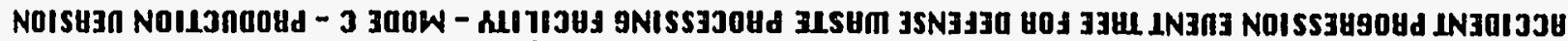

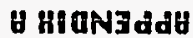


APPENDIK A

ACCIDENT PROGRESSION EUENT THEE FOR DEFENSE WASTE PRDCESSING FACILITY - MODE C - PRODUCTION UERSION

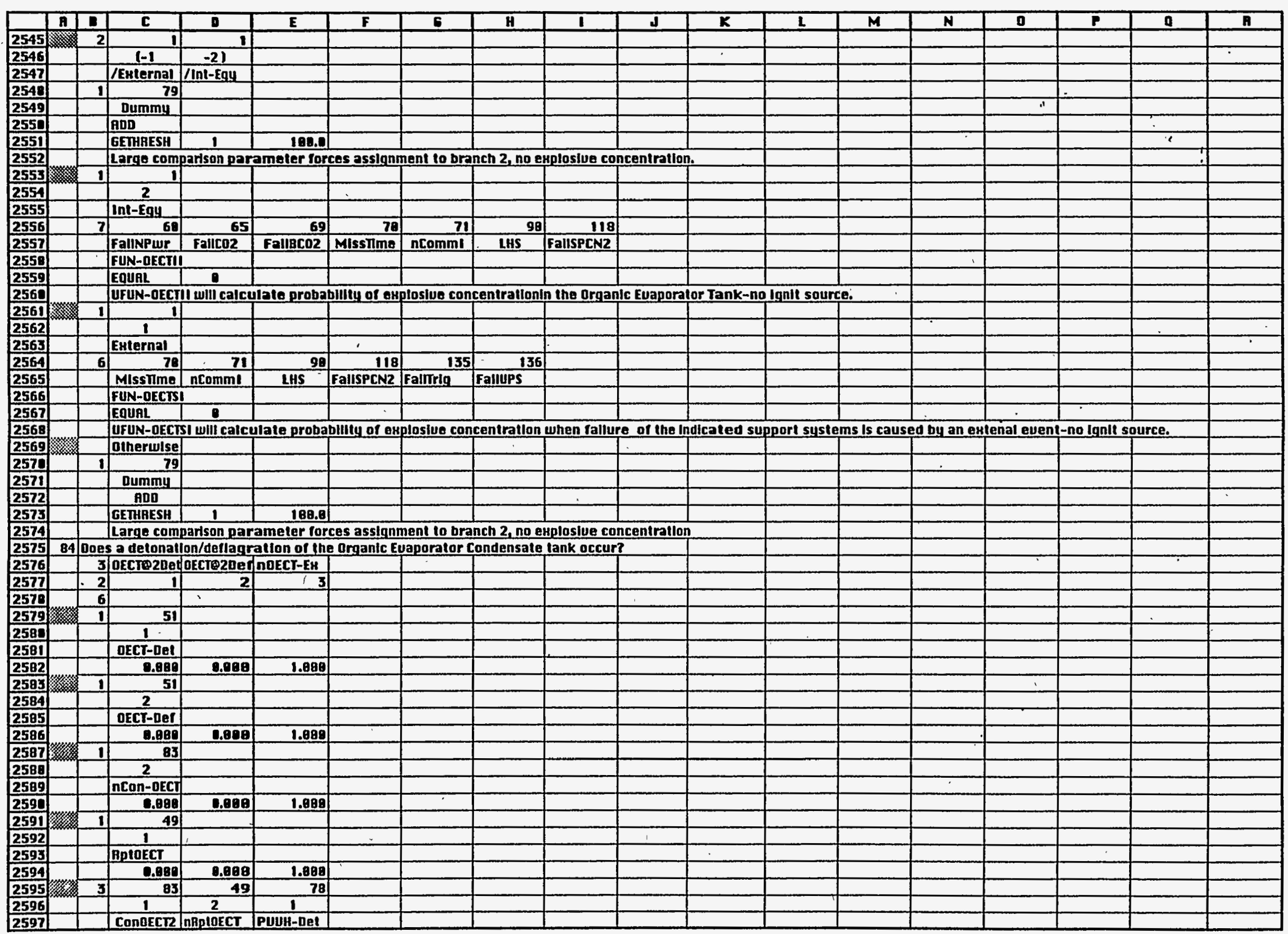


APPENDIK $A$

\begin{tabular}{|c|c|c|c|c|c|c|c|c|c|c|c|c|c|c|c|c|c|c|}
\hline & a & \pm & c & D & $E$ & $F$ & E & H & 1 & d & $\bar{x}$ & I & $M$ & $\bar{N}$ & 0 & $\bar{P}$ & a & $\bar{n}$ \\
\hline 2590 & & & 0.081 & 0.999 & 0.080 & & & & & & & & & & & & & \\
\hline 2599 & & & Dtherulse & & & & & & & & & & & & & & & \\
\hline 268 & & & $0.0 B 0$ & 8.000 & 1.080 & & & & & & & $\cdot$ & ${ }^{\circ}$ & & & & & \\
\hline 2681 & 85 & noes & s an aHplosi & ive concent & rollon accu & mulate in $P$ & reclpiltale & Aeaclor free & d Tank? & & & & & & & & & \\
\hline 2682 & & 2 & ConPAFr2 & nConPRFT & & & & & & & & & & & & & & \\
\hline 2683 & & 6 & 1 & 2 & & & & & & & & & & & & & $\cdot$ & \\
\hline 2684 & & 4 & E & & & & & & & & & & & & & $\div$ & & 1 \\
\hline 2685 & 3 & 2 & 1 & I) & & & & & & & & & & & & & & . \\
\hline 2696 & & & $(-1$ & -21 & & & & & & & & & & & & & & \\
\hline 2697 & & $=$ & /Enternal & /Int-Eq! & & & & & & & & & & & & & & \\
\hline 2688 & & 1 & 79 & & & & & &. & & & & & & & 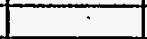 & & \\
\hline 2689 & & & Dummy & & & & & & & & & & & & & & 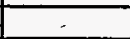 & \\
\hline 2610 & & & ADD & & & & & & & & & & & & & & & \\
\hline 2618 & & & GETHAESH & 1 & 100.0 & & & & & & & & & & & & & \\
\hline 2612 & & & Large com & partson pare & ampler forc & ces assignm & nent to bro & $\operatorname{nnch} 2, \mathrm{no}$ er & uplosive cor & ncentrallon & & & & & & & & \\
\hline 2613 & & 1 & 1 & & & & & & & & & & $\therefore$ & & & & & \\
\hline 2614 & & & 2 & & & & & & & & & & & & & & & \\
\hline 2615 & & & Int-Egu & & & & & & & $\therefore$ & & & & & & & & \\
\hline 2616 & & 7 & 60 & 65 & 69 & 70 & 71 & 90 & 118 & & & & & & & & & \\
\hline 2617 & & & FallNPwr & Fallco2 & Fallec02 & MIssIIme & ncomm! & LHS & FallSPLN2 & & & & & & & & & \\
\hline 2618 & & & FUN-PAFTII & & & & & & & & & & & & & & & \\
\hline 2619 & & & EqUAR & - & & & & & & & & 1. & & & & & & \\
\hline 2620 & & & UFUN-PAFI & II will calcu & late probat & bllity of oup & ploslue can & centraltonin & i the Precis & pllate Reac & tor Feed Ta & ank-no lgnit & t source. & & & & & \\
\hline 2621 & & 1 & 1 & & & & $\therefore$ & & & & & & & & & & & \\
\hline 2622 & & & 1 & & & & & 1 & & & & & & & & & & \\
\hline 2623 & & & Esternal & & & & & & & & & & & & & & & \\
\hline 2624 & & 6 & 79 & 71 & 99 & 118 & 135 & 136 & & & & & & & & & & \\
\hline 2625 & & & Missime & ncomml & LHS & FallsPCN2 & Falinrin & Fallups & & & & & & & & & & \\
\hline 2626 & & & FUN-PAFTS & & & & & & & & & $\because$ & & & & 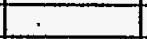 & & \\
\hline 2627 & & & EQUAL & 8 & & & & & & & & & & K & & & & $\div$ \\
\hline 2628 & & & UFUN-PRFIS & I wlll calcu & late probat & billty of oHp & ploslue con & centration & when fallur & fe of the in & dilcaled su & upport suste & ems is cause & ed by an er & utenal event & -no lgnit so & ource. & \\
\hline 2629 & & & Dingrwise & & & & & & & & 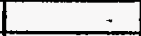 & & & & & & & \\
\hline 2630 & & 1 & 79 & & $\cdot$ & & & & & & & & & & & & & \\
\hline 2631 & & & Dummu & & & & & & & & & & & & & & & \\
\hline 2632 & & & 900 & & & & & & & & 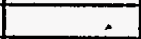 & & & & & & & \\
\hline 2633 & & & GETHRESH & 1 & 180.0 & & & & & & & & & & & & & \\
\hline 2634 & & & Large comp & arison para & meter forc & ces assignm & nent to bra & $\mathrm{nCh} 2, \mathrm{nO} \mathrm{eH}$ & iplosive con & ncentratlon & & & & & & & & \\
\hline 2635 & 86 & Does & a detonall & on/dellagre & atlon occur & In the Prec & Ipllate Ree & ctor Feed Ta & ank? & & & & & & & & & \\
\hline 2636 & & 3 & PAFTE2DEC & PAITO2Derf & nPAFT-EнP & & & & & $\cdot$ & & & & & & & & \\
\hline 2637 & & 2 & 1 & 2 & 3 & & & & & & & $\therefore$ & & & & & & \\
\hline 2638 & & 6 & & & & & & - & & & & & & & & & & \\
\hline 2639 & 璒 & 1 & 55 & & & & & & & & & & & & & & & \\
\hline 2640 & & & 1 & & & & & & & & & & & & & & & \\
\hline 2641 & & & \begin{tabular}{|l|} 
PRFT-Det \\
\end{tabular} & & & & & & & & & & & & & & & \\
\hline 2642 & & & 0.000 & 0.080 & 1.080 & & & & & & & & & & & & & \\
\hline 2643 & & 1 & 55 & & & & & & & & & & & & & & & \\
\hline 2644 & & & 2 & & & & & & & & & & & & & & & \\
\hline 2645 & & & PAFT-Der & & & & & & & & & & & & & & & \\
\hline 2646 & & & 0.080 & D.880 & 1.080 & & & & & & - & & & & & & & \\
\hline 2647 & 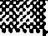 & 1 & 85 & & & & & & & & & & & & & & & \\
\hline 2648 & & & 2 & & & & & & & & & & $\therefore$ & & & & & \\
\hline 2649 & & & nConPRFrT & & & & & & & & & & & & & & & \\
\hline 2650 & & & 8.068 & 8.080 & 1.000 & $=$ & & & & & & & & & & & & \\
\hline
\end{tabular}


APPENDIK A

\begin{tabular}{|c|c|c|c|c|c|c|c|c|c|c|c|c|c|c|c|c|c|c|}
\hline & a & - & C & D & $E$ & $\mathbf{F}$ & $\boldsymbol{s}$ & H & $\mathbf{I}$ & \rfloor & $x$ & L & $M$ & $N$ & D & $\mathbf{p}$ & a & h \\
\hline 2651 & & 1 & 53 & & & & & & & & & & & & & & & \\
\hline 2652 & & & 1 & & & & & & & & & & & & & & & 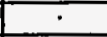 \\
\hline 2653 & & & BptPAFT & & & & & & & & & & & & & & & \\
\hline 2654 & & & 0.088 & 0.800 & $1.08 \mathrm{~B}$ & & & & & & & & & & & & & \\
\hline 2655 & & 3 & 85 & 53 & 78 & & & & & & & & & & . & & & \\
\hline 2656 & 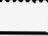 & & 1 & 2 & 1 & & & & & & & & & & & & $?$ & \\
\hline 2657 & & & ConPAFT & nBptPAFT & \begin{tabular}{|l|} 
PUUH-Dat \\
\end{tabular} & & & & & & & & & & & & 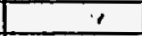 & \\
\hline 2650 & & & 0.001 & 0.999 & 0.080 & & & & & & & & & & & & & 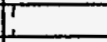 \\
\hline 2659 & & & otherwise & & & & & & & & & & & & & & & \\
\hline 2660 & & & 0.188 & 0.080 & 1.088 & & & & & & & & & & & & & \\
\hline 2661 & 87 & Does & s an enplosi & wa concent & rallon accu & Imulata $\ln 1$ & he Preciplt: & to Reactor & Bolloms $\mathrm{T}$ & ank IPABTI? & & & & & & & & \\
\hline 2662 & & 2 & ConPABT2 & nCOAPRBT & & & & & & & & & & & & & & \\
\hline 2663 & & 6 & If & 2 & & & & & & $\therefore$ & & & & & & & & \\
\hline 2669 & & 5 & & & & & & & & & & & & & & & & \\
\hline 2665 & & 2 & If & 1 & & & & & & & & & & & & & & \\
\hline 2666 & & & $(-1$ & -21 & & & & & & & & & & & & & & \\
\hline 2667 & & & /Esternai & /Int-Egu & & & & & & & & & & & & & & \\
\hline 2668 & & 1 & 79 & & & & & & & & & & & & & & & \\
\hline 2669 & & & Dummy & & & & & & & & & & & & & & & \\
\hline 2670 & & & fon & & & & & & & & & & $?$ & 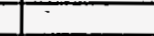 & & & & \\
\hline 2671 & & & GETHAESH & 1 & 180.0 & & & & & & & & & & & & & \\
\hline 2672 & & & Large comp & parison par & ameler for & ces assignn & nent & $n c h 2, n o$ e & Hploslue ca & ncentrallon & & & & & & & & \\
\hline 2673 & 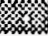 & 2 & II & 3 & & & & & & & & & & & & & & \\
\hline 2674 & & & 2 & 7 & & & & & & & & & & & & & & \\
\hline 2675 & & & $\ln 1-$ Egy & N2TkInli & & & & & & & & & & & & & & \\
\hline 2676 & & 1 & 79 & & & & & & & & & & & & & & & \\
\hline 2677 & & & Dummy & & & & & & & & & & & & & & & \\
\hline 2678 & & & $\mathrm{ADD}$ & & & & & & & & & & & & & & & \\
\hline 2679 & & & GEIHAESH & 1 & 180,8 & & & & & & & & & & & & & \\
\hline 2680 & 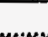 & & Large comp & parison par & ameter for & ces assignn & nent to bra & $\mathrm{nch} 2, \mathrm{no}$ e & yplosive co & ncentration & & & & & & & & \\
\hline 2681 & 40 & T & 1 & & & & & & & & & & & & & & & \\
\hline 2682 & & & 2 & & & & & & & & & & & & & & & \\
\hline 2683 & & & Int-Eg4 & & & & & & & & & & & 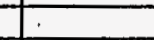 & & & & \\
\hline 2689 & & 8 & 68 & 66 & 67 & 95 & 78 & 71 & 98 & 108 & & & & & & & & \\
\hline 2685 & & & FallNPur & Falliar & FallN2IK & FalicPCN2 & Missnme & ncomm! & LHS & FallcPCIK & & & & & & & & \\
\hline 2686 & & & FUN-PABTII & & & & & & & & & & & & & & & \\
\hline 2687 & & & EQUALL & a & & & & & & & & & & & & & & \\
\hline 2688 & & & UFUN-PABI & 11 will calcu & Llate probe & blilly of en & ploslue con & centration & In the Prec & Ipltate Reac & Clar Boll & oms Tank-no & 0 Iqnil sourc & & & & & \\
\hline 2689 & 然 & 1 & II & & & & & & & & & & & & & & & \\
\hline 2690 & & & $I$ & & & & & & & & & & & & & & & \\
\hline 2691 & & & Esternal & & & & & & & & & & & & & & & \\
\hline 2692 & & 8 & 60 & 66 & 67 & 95 & 70 & 71 & 98 & 188 & & & & & & & & \\
\hline 2693 & & & FallNPur & Fallair & Fallin2Tk & FaIICPCN2 & MIssTlme & nComml & LLSS & FallcPCIK & & & & 7 & & & & \\
\hline 2694 & & & FUN-PABTSI & & & & & & & & & & & & & & & \\
\hline 2695 & & & EQUAL & a & & & & & & & & & & & & & & \\
\hline 2696 & & & UFUN-PRBT & sI will calct & ulate proba & bility of er & ploslue con & centrallon & when fallu & re of the in & Idicated & support syst & tems is caus & sed by an ef & eutenal event & -no Ignit se & ource. & \\
\hline 2697 & 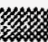 & & Otherwlse & & & & & & & & & & & & & & & \\
\hline 2698 & & 1 & 79 & & & & & & & & & & 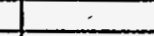 & $\therefore$ & & & & \\
\hline 2699 & & & Bummy & & & & & & & & & & & & & & & \\
\hline 2780 & & & ADD & & & & & & & & & & & & & & & \\
\hline 2701 & & & GETHBESH & 1 & 180,0 & & & & & & & & & & & & & \\
\hline 2782 & & & Large comp & parfson par & eter for & ces asslgnn & bral & $n \operatorname{ch} 2, n \in O$ & loslue co & ncentratlon & & & & & & & & \\
\hline 2783 & 88 & Does & sa detonall & lon/deflagr & allon accur & In the Prec & Iplifate Rea & ctar Balton & ms Iank IPH & 1877? & & & & & & & & \\
\hline
\end{tabular}




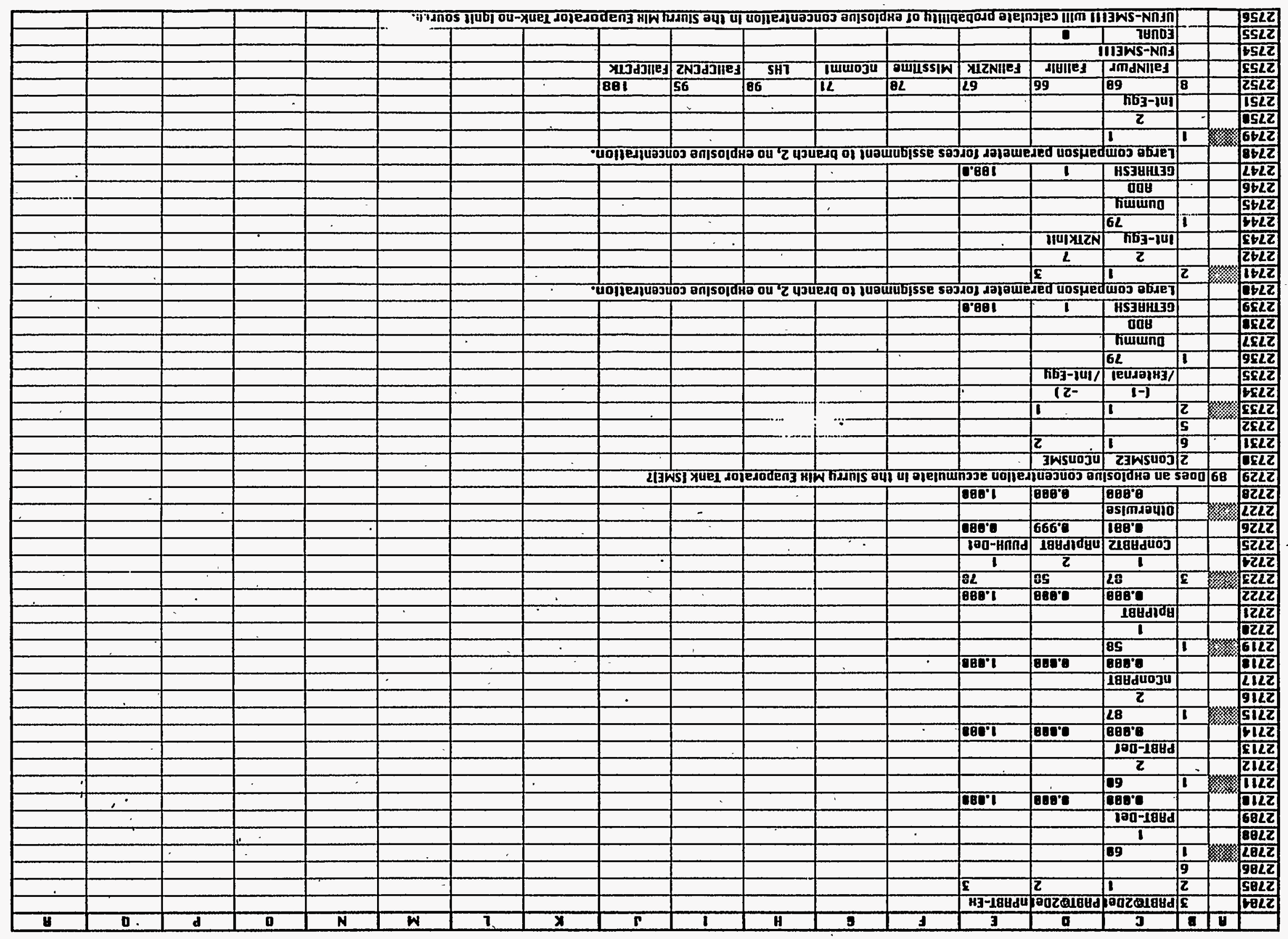

$2 L Z$ o6ed

c110-56-ü-Jusm

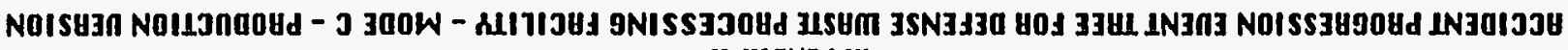

H BIONBddH 
APPENDIH ด

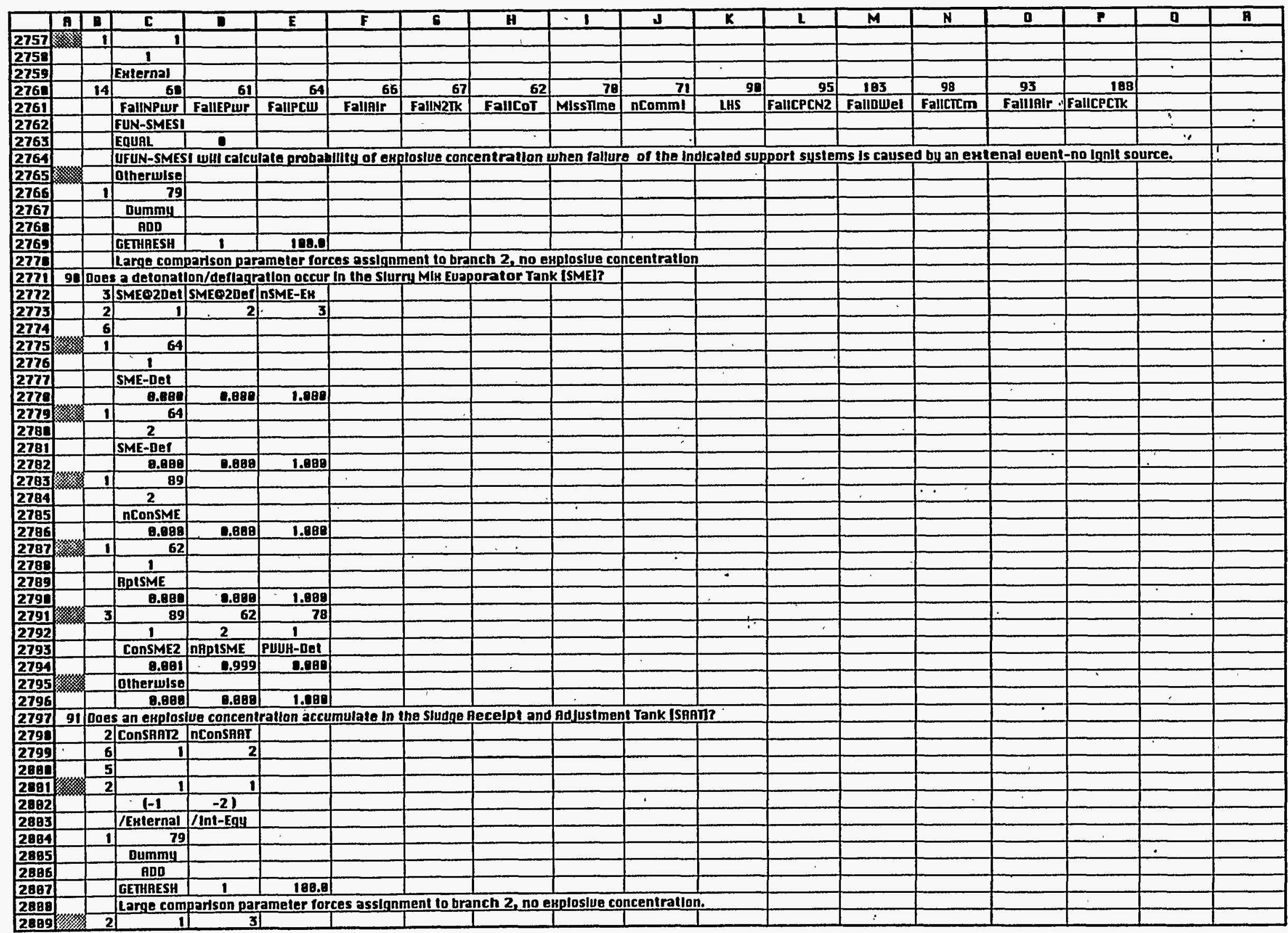




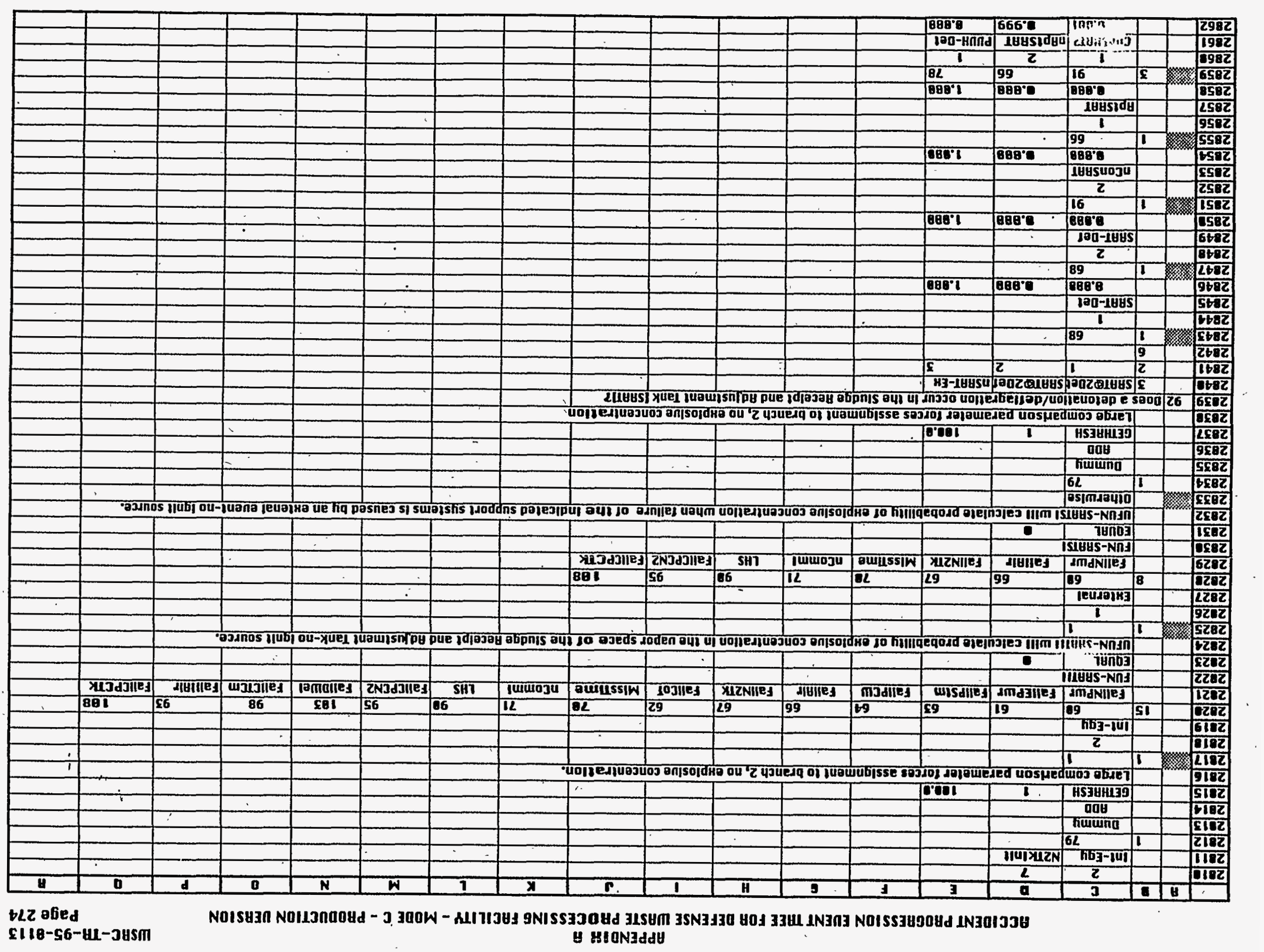


APPENDIH G

ACCIDENT PADGRESSION EUENT THEE FOR DEFENSE WASTE PROCESSING FACILITY - MODE C - PRODUCTION UEASIDN

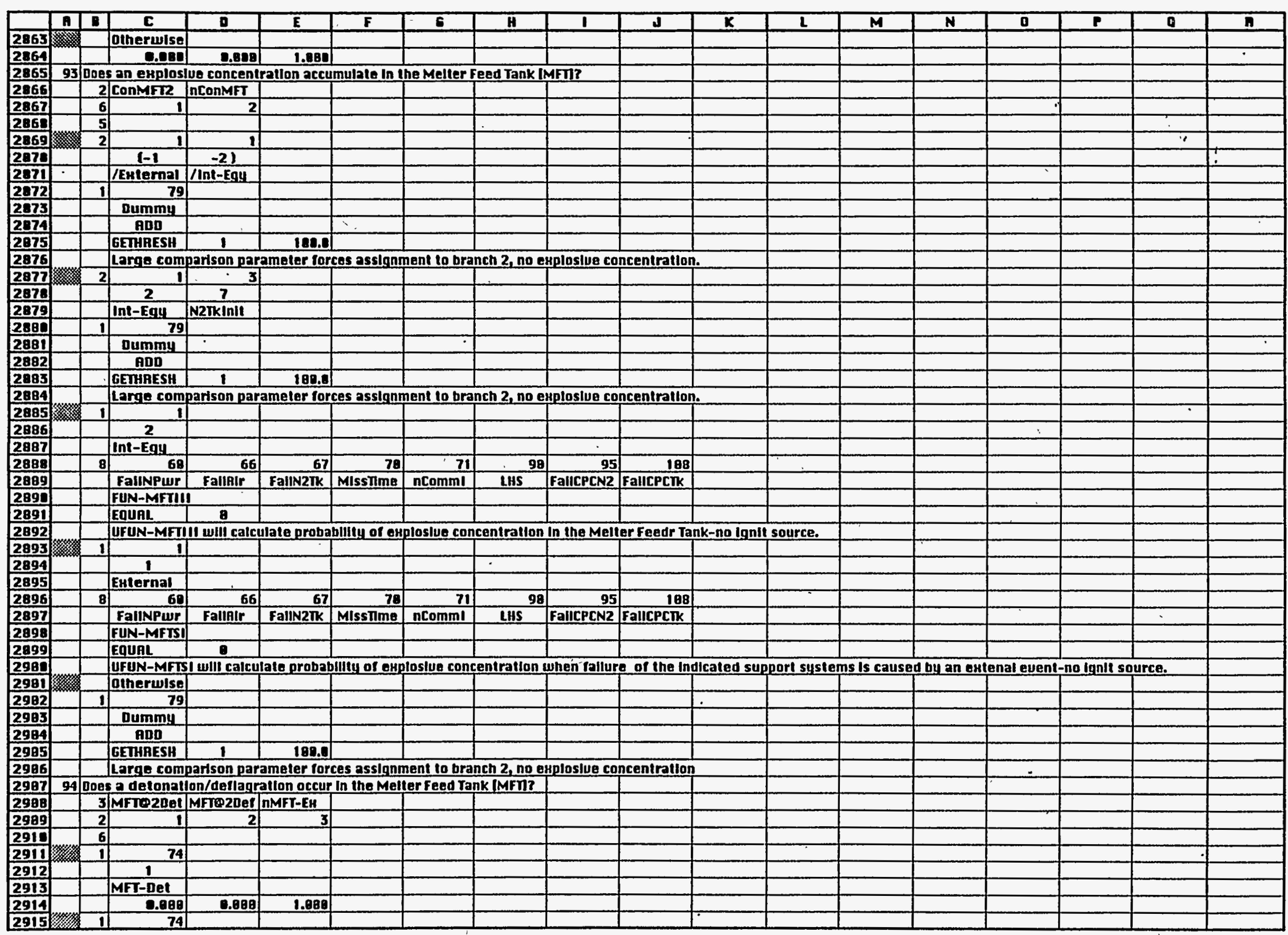




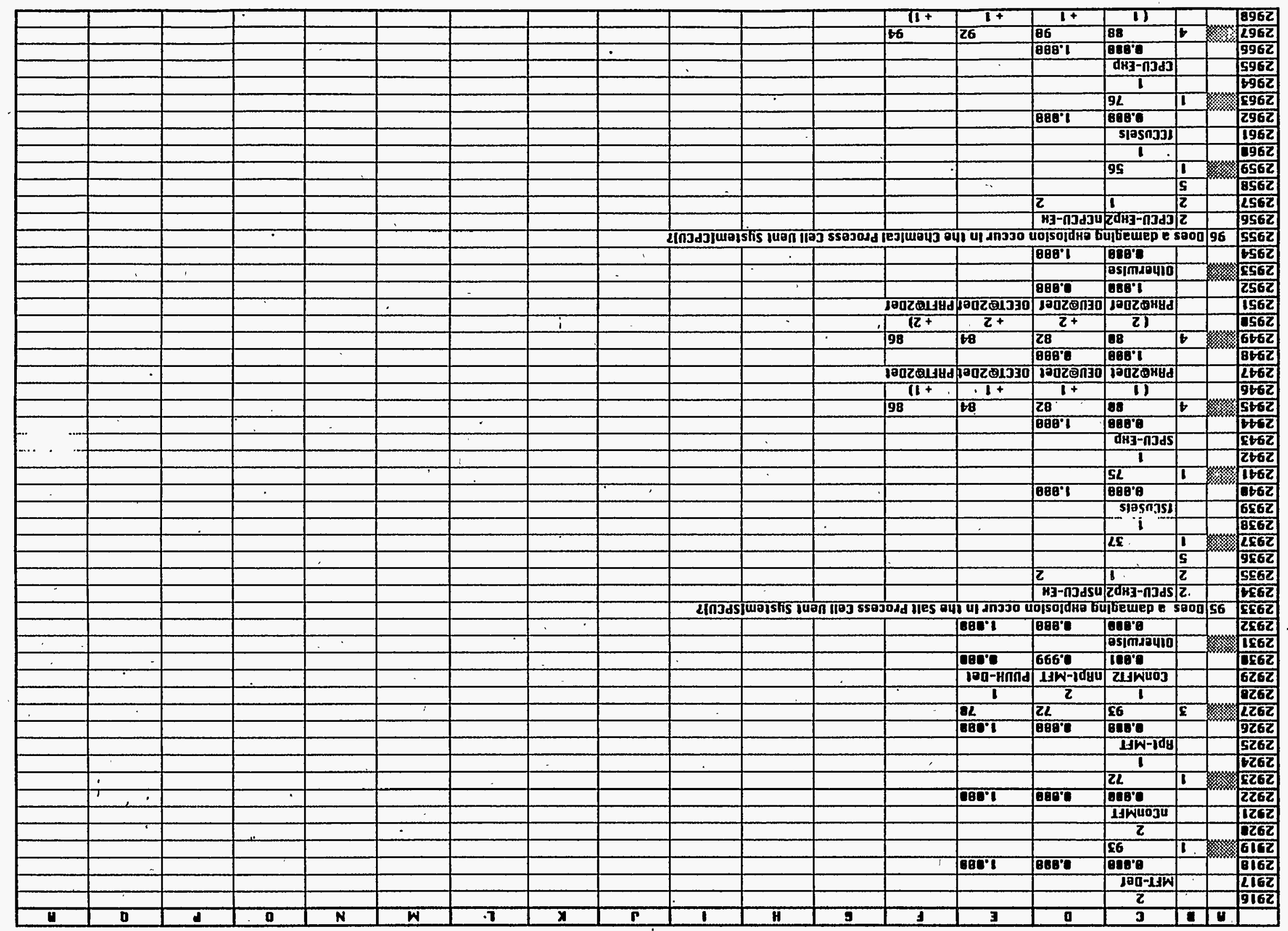

gLz abed

c110-56-4I-34Sm

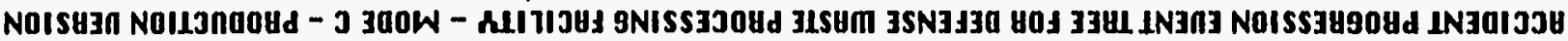


APPENDIH A

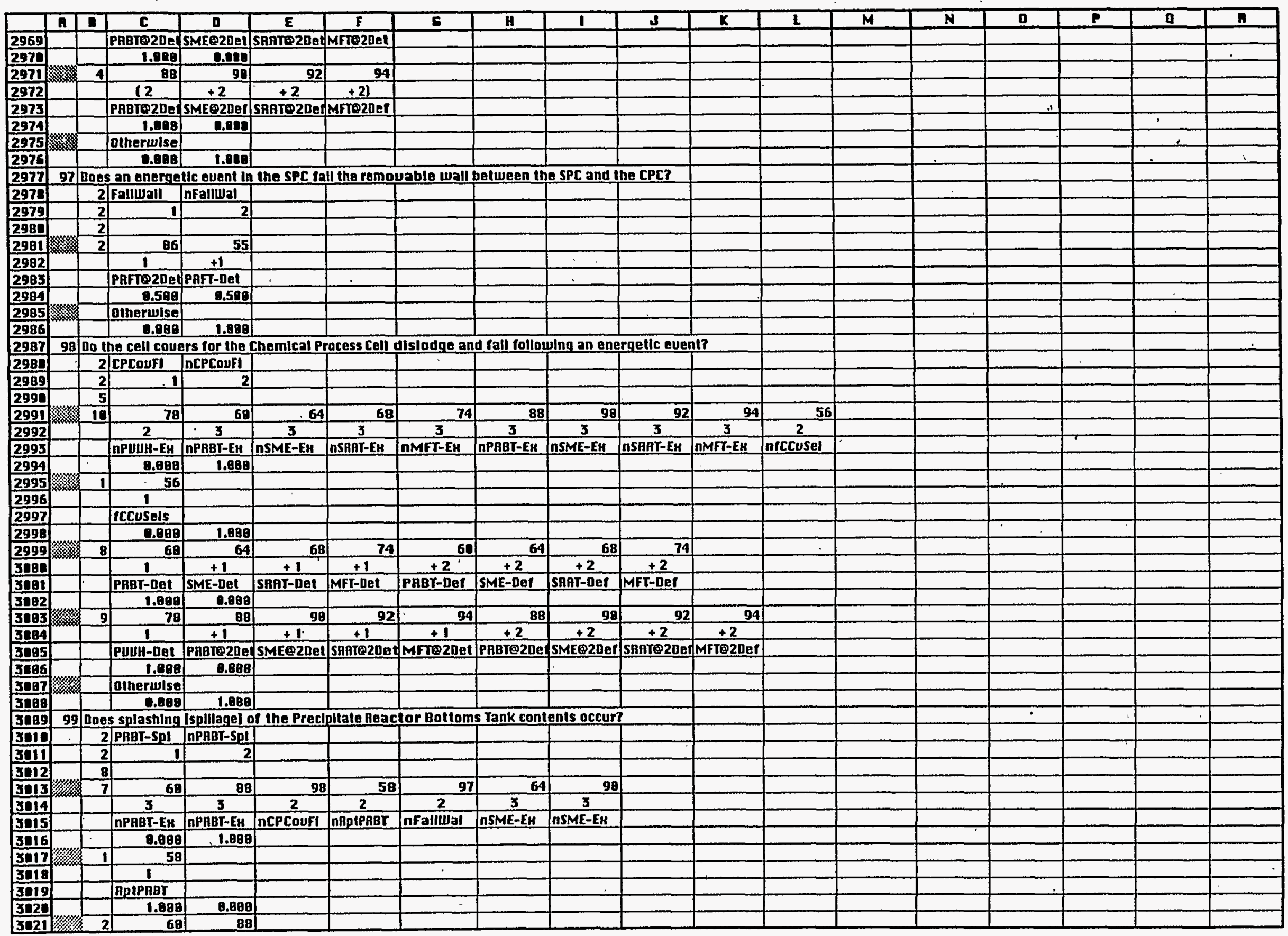




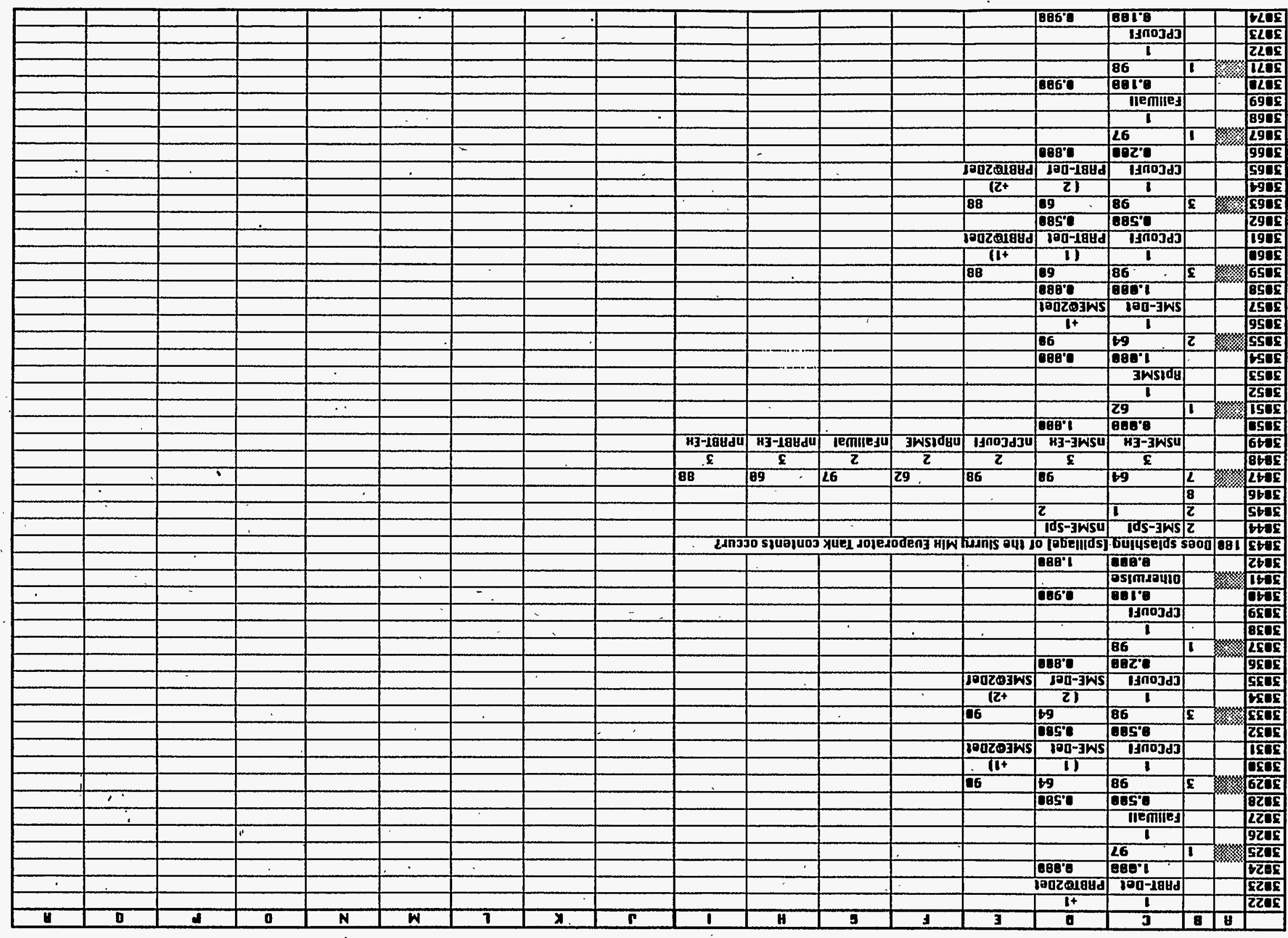

$8 \angle 2$ obed

EL18-56-HL-J4Sm

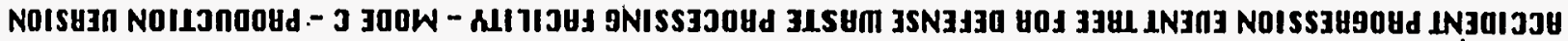

Y HIONJddH 
APPENDIY 月

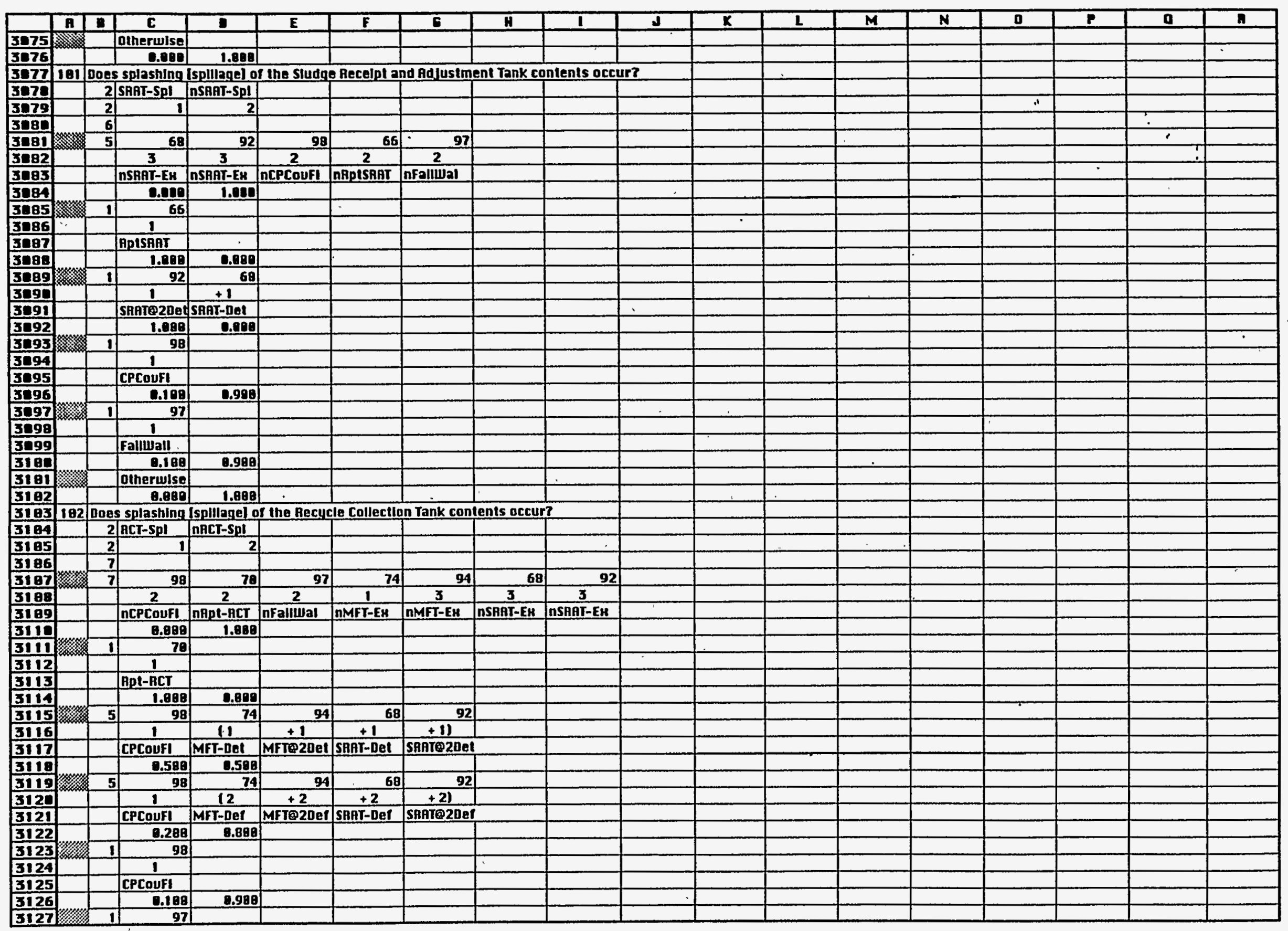




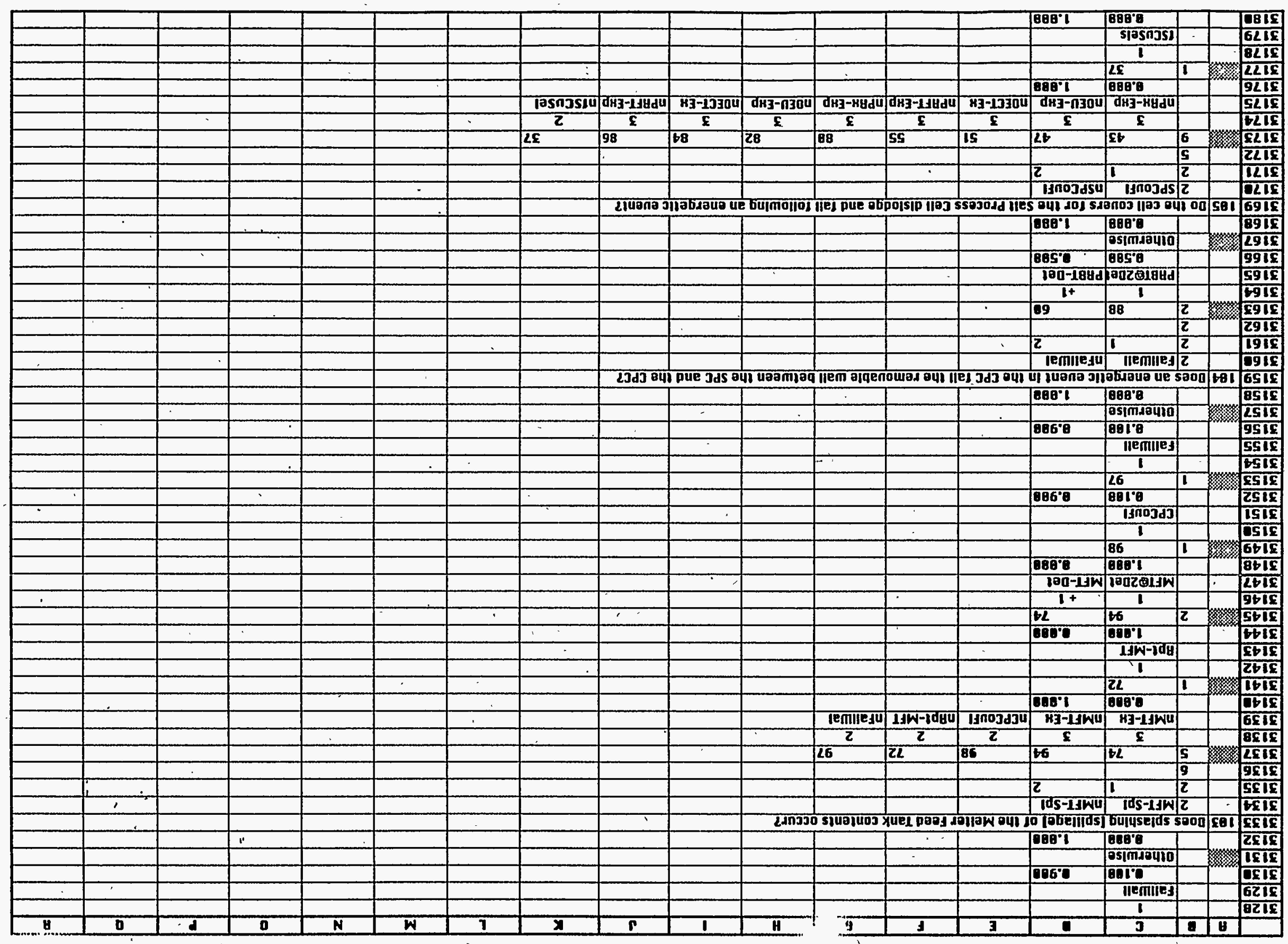

o8z o6ed

c116-S6-4L-J45T

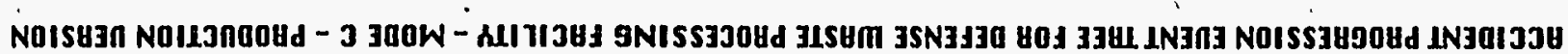

Y HIONZAdU 
APPENDIH A

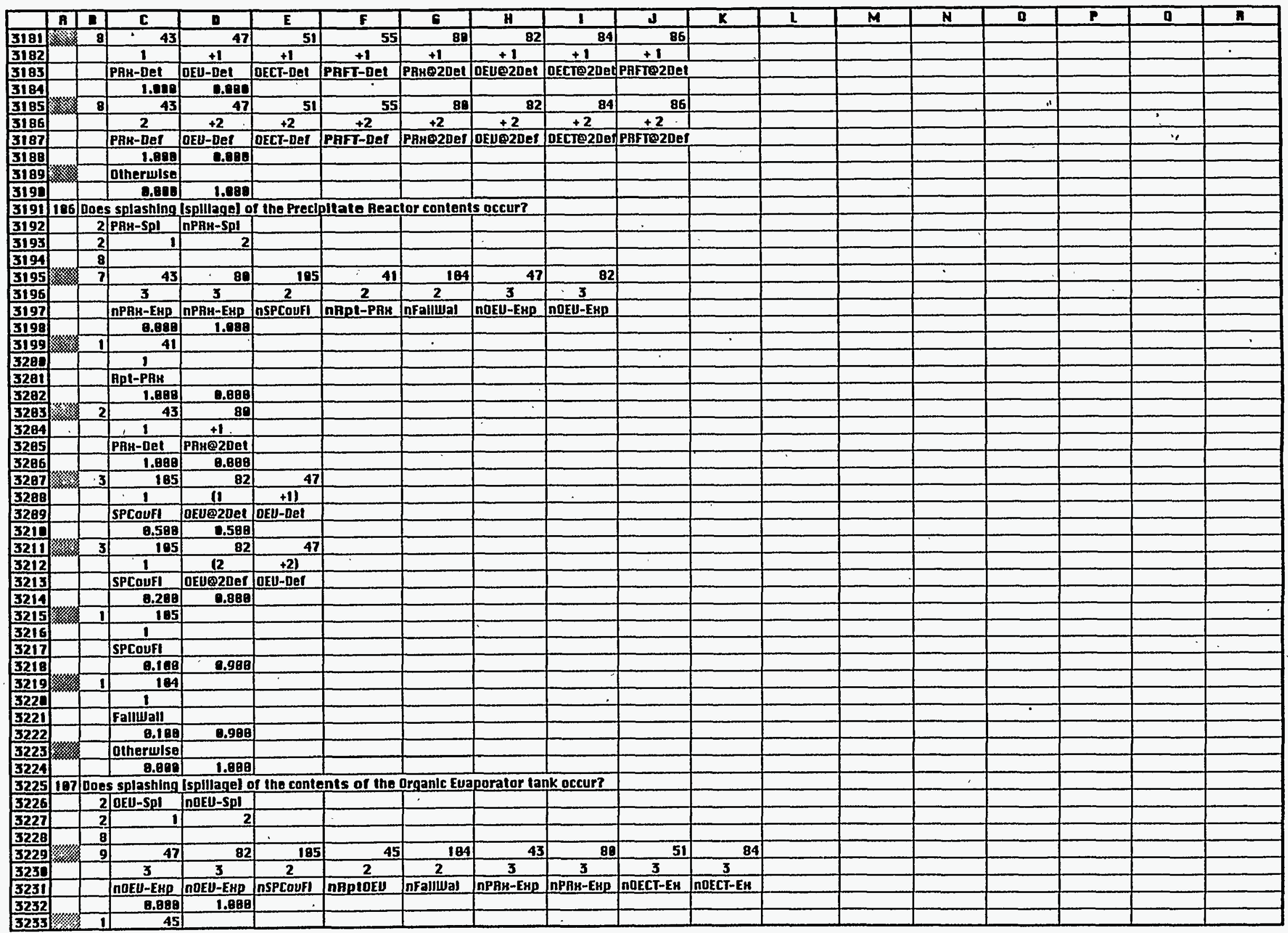


APPENBAH $\mathrm{A}$

ACCIDENT PROGRESSION EUENT TREE FOR DEFENSE WASTE PAOCESSING FACILITY - MDDE C - PRODUCTIDN UERSION

\begin{tabular}{|c|c|c|c|c|c|c|c|c|c|c|c|c|c|c|c|c|c|c|}
\hline & A & 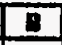 & $\mathbf{C}$ & E & $\mathbf{E}$ & $F$ & I & H & 1 & $d$ & $\bar{K}$ & L & $M$ & $N$ & D & $P$ & a & n \\
\hline 3234 & & & 1 & & & & & & & & & & & & & & & \\
\hline 3235 & & & BptoEU & & & & & & & & & & & & & & & \\
\hline 3236 & & & 1.000 & 0.000 & & & & & & & 7 & & & & & & & \\
\hline 3237 & & 2 & 82 & 47 & & & & & & & & & & & & & & \\
\hline 3230 & & & 1 & +1 & & & & $\cdot$ & & & & & & & & & & \\
\hline 3239 & & & OEUP2DEl & DEU-De! & & & & & & & & & & & & & - & \\
\hline 3241 & & & 1.808 & 0.080 & & & & & & & & & & & & & & 1 \\
\hline 3241 & & 5 & 185 & 43 & 80 & 51 & 84 & & & & & & & & & & & 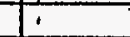 \\
\hline 3242 & & & 1 & 11 & +1 & +1 & +11 & & & & & & & & & & & \\
\hline 3243 & & & SPCoUfI & PAK-Det & PннQ2001 & DECT-Det & OECTD2DEl & & & & & & & & & & & \\
\hline 3244 & & & $0.58 \mathrm{~B}$ & 0.590 & $=$ & & & & & & & & & & & & & \\
\hline 3245 & & 5 & 105 & 43 & 80 & 51 & 84 & & & & & & & & & & & \\
\hline 3246 & & & 1 & 12 & +2 & +2 & +21 & & & & & & & & & & & \\
\hline 3247 & & & SPCOUFI & PAH-日ef & РRHQ2DAt & OECT-Def & DECTD2DEf & & & & & & & & & & & \\
\hline 3248 & & & 0.280 & 0.800 & & 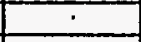 & & & & & & & & & & & & \\
\hline 3249 & & 1 & 105 & & & & & & & & $\cdot$ & & & & & & & \\
\hline 3250 & & & 1 & & & & & & & & & & & & & & $\bar{c}$ & \\
\hline 3251 & & $\therefore$ & SPCOUFI & & & & 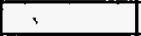 & & & & & & & & & & & \\
\hline 3252 & & & 0.180 & 0.900 & & & & & & & & & & & & & & \\
\hline 3253 & 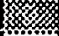 & 1 & 184 & & & & & & & & & & & - & & & & \\
\hline 3254 & & & 1 & & & & & & & & & & & & & & & \\
\hline 3255 & & & Falluall & & & & & & & & & & & & & & & \\
\hline 3256 & & & 8.108 & 0.908 & & & & & & & & & & & & & & \\
\hline 3257 & $x_{1}$ & & atherwise & & & & & , & & & 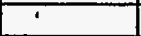 & & & & 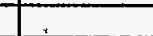 & & & \\
\hline 3258 & & & 8.880 & 1.880 & & & & & & & & & & & & & & \\
\hline 3259 & 108 & Does & s splashing & Isplllagel of & I the conte & n's of the & Organlc Eua & parator Co & ndensale ta & ank occur? & & & & & & & & \\
\hline 3269 & & 2 & OEECT-Spl & nDECT-Spl & & & & & & & & & & & & & & \\
\hline 3261 & & 2 & 1 & 2 & & & & & & & & & & & & & & \\
\hline 3262 & & 8 & & & & & & & & $E$ & & & & & & & & \\
\hline 3263 & & 9 & 51 & B4 & 105 & 49 & 184 & 47 & 82 & 55 & 86 & & & & & & & \\
\hline 3264 & & & 3 & 3 & 2 & 2 & 2 & 3 & 3 & 3 & 3 & & ? & 1 & & & & \\
\hline 3265 & & & nOECr-EH & ПOECT-EH & nSPCOUFI & nAPLIOECT & nfaillual & ПDEU-Енр & ПDEU-EHP & nPAFT-ERP & MPAFT-EHP & & & & & & & \\
\hline 3266 & & & 0.080 & 1.808 & & & & & & & & & & & & & & \\
\hline 3267 & & 1 & 49 & & & & & & & & & & & & & & & \\
\hline 3269 & & & 1 & & & & & & & & & & & & & & & \\
\hline 3269 & & & BPEOECT & & & & & & & & & & & & & & & \\
\hline 3270 & & & 1.080 & 0.800 & & & & & & & & & & & & & & \\
\hline 3271 & & 2 & 84 & 51 & & & & & & 5 & & & & & & & & \\
\hline 3272 & & & 1 & +1 & & & & & & & & & & & & & & \\
\hline 3273 & & & LecrQ20et & DECT-Det & & & & & & & & & & & & & & \\
\hline 3274 & & & 1.089 & 0.889 & & & & . & & & & & & & & & & \\
\hline 3275 & 聯 & 5 & 185 & 47 & 82 & 55 & 86 & & & & & & 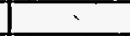 & & & & & \\
\hline 3276 & & & 1 & II & +1 & +1 & +11 & & & & . & & . & & & & & \\
\hline 3277 & & & SPCoufi & DEU-Del & DEUQ20et & PAFT-Det & Pafrozoet & & & & & & & & & & & \\
\hline 3278 & & & 0.580 & 0.588 & & & & & & & & & & & & & & \\
\hline 3279 & 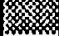 & 5 & 185 & 47 & 82 & 55 & 86 & & & & & & & & 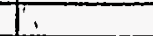 & & & \\
\hline 328 & & & 1 & 12 & +2 & +2 & +21 & & & & & & & & & & & \\
\hline 3281 & & & SPCoufI & DEU-Def & DEUQ20e! & PAFT-Det & PRFT@2DeI & & - & & & & & & & & & \\
\hline 3282 & & & 0.269 & 8.880 & & & & & & & & & & & $=$ & 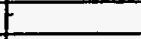 & & \\
\hline 3283 & 兹穪 & 1 & 104 & & & & & & & & & & & & & & & \\
\hline 3284 & & & 1 & & & & & & & & & & & & & & & \\
\hline 3285 & & & Faillall & & & & & & & & . & & & & & & & \\
\hline 3286 & L & & 0.188 & 0.988 & & & & & & & & & & - & & & & \\
\hline
\end{tabular}


APPENDIH A

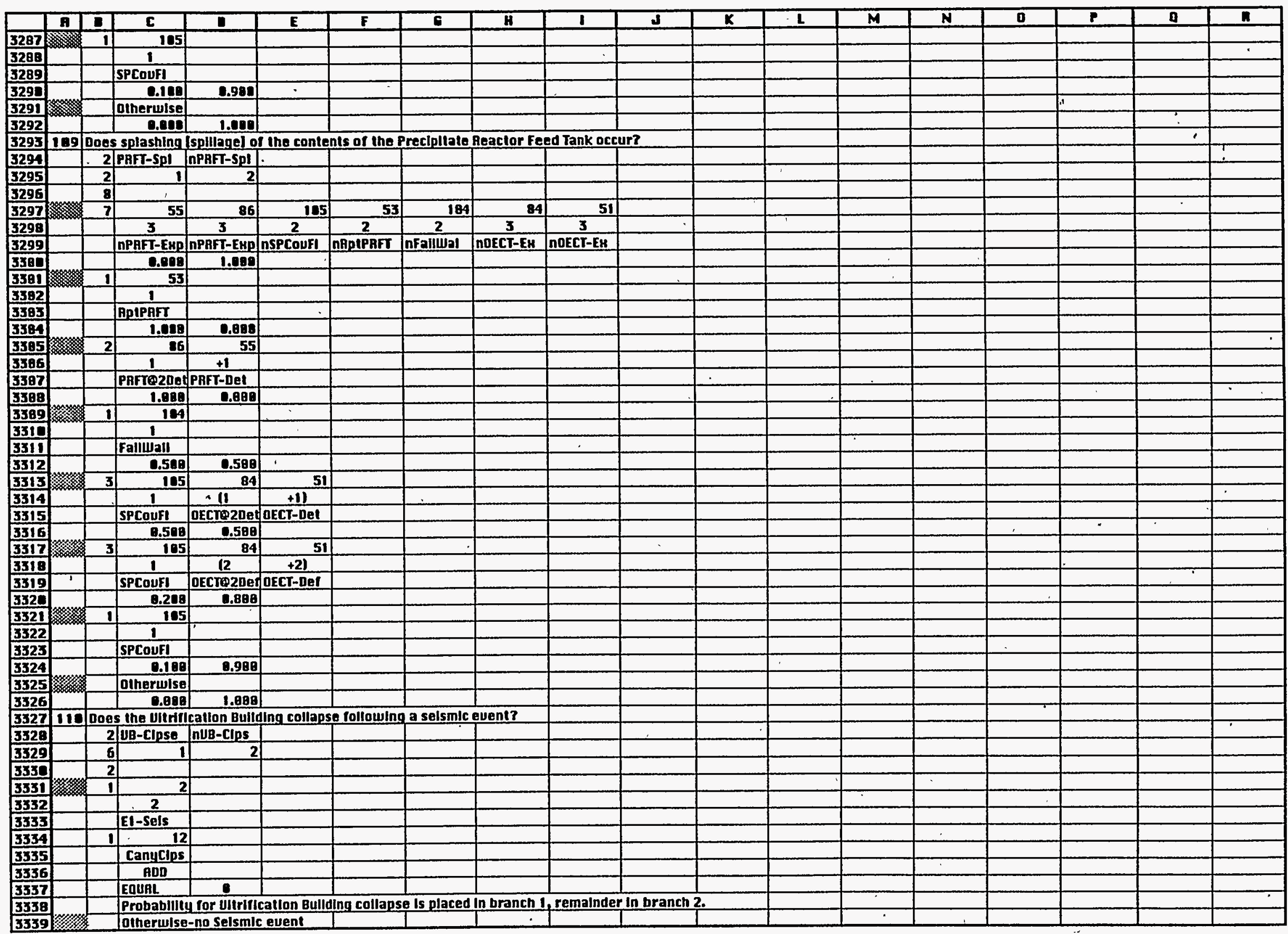


APPENDIK A

ACCIDENT PROGRESSION EUENT IREE FOA DEFENSE IUASTE PROCESSING FACILITY - MODE C - PADDUCTION UERSION

\begin{tabular}{|c|c|c|c|c|c|c|c|c|c|c|c|c|c|c|c|c|c|c|}
\hline & A & B & C & 8 & $E$ & $F$ & 5 & H & 1 & 3 & $\boldsymbol{K}$ & 1 & $M$ & $N$ & 0 & $P$ & 0 & R \\
\hline 3349 & & 1 & 79 & & & & & & & & & & & & & & & \\
\hline 3341 & & & Dummy & & & & & & & & & & & & & & $\therefore$ & 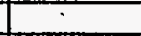 \\
\hline 3342 & & & ADO & & & & & & & & & & & & & & & \\
\hline 3343 & & & GETHRESH & 1 & 180,0 & & & & & & & & & ? & & & & \\
\hline 3344 & & & Lame com & parison par & ameter for & ces assignn & ment lo br & $\operatorname{anch} 2, \mathrm{nos}$ & Selsmic eue & nt, no colla & ase of ultru & Icatlon bul & Iding. & & & & & \\
\hline 3345 & 111 & Does & s the sand & Fulter functl & Ion followin & ig a selsmic & cuent? & 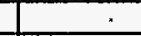 & & & & & & & & & & \\
\hline 3346 & & 2 & ISndFit & Inisndfit & & & & & & & & & & & & & & \\
\hline 3347 & & 6 & 1 & 2 & & & & & & & & & & & & & & $\cdot$ \\
\hline 3340 & & 2 & & & & & & & & & & & & & & & - & \\
\hline 3349 & & 1 & 2 & & & & & & & & $\cdot$ & & & & $\therefore$ & & & \\
\hline 335 & & & 2 & & & & 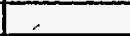 & & & & & & & & & & & \\
\hline 3351 & & & EI-Sels & & & & & & & & & & & & & - & & \\
\hline 3352 & & 1 & 14 & & & & & & & & & ? & & & & & & \\
\hline$\overline{\mathbf{3 3 5 3}}$ & & & Filtfr & & & & $:$ & & - & & & & & $\cdot$ & & & & \\
\hline 3354 & & & ADD & & & $\because$ & & & & & & & & 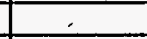 & & & & .. \\
\hline 3355 & & & EQUAL & 0 & & & & & & & & & & & & & & \\
\hline 3356 & & & Probabllit! & yol sand fil & Iter fallure & Is placed in & branch 1 , & remalnder & In branch 2 & & & & & & & & & \\
\hline 3357 & & & otherwlse & & & & & & & & & & & & & & & \\
\hline 3358 & & 1 & 79 & & & & & & & & & & & & & & & \\
\hline 3359 & & & Dummu & & & & & & & & & & & & & & & \\
\hline 3361 & & & ADD & & & & & & & $\dot{\square}$ & & & & & & & & \\
\hline 3361 & & & GETHAESH & 1 & 188.0 & & & & & & & & & & & & & \\
\hline 3362 & & & Larqe com & parison par & ameter tor & ces assignn & ment to br & $\operatorname{anch} 2$, nos & selsmic eue & nt, no tailur & e of the sa & nd filter. & & & & & & \\
\hline 3363 & 112 & Do II & he Bullding & Eshaust Du & cis irom Zo & ne 1 to the & Sand Fille & er funclion & followling a & seismlc eut & ent? & & & & & & & \\
\hline 3364 & & 2 & taltDuct & nfalloct & & & & & 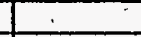 & & & & & & & & & \\
\hline 3365 & & 6 & 1 & 2 & & & & & & & & & & & & & & \\
\hline 3366 & & $\mathbf{3}$ & & & & & & & & & & & & & & & & \\
\hline 3367 & 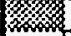 & 2 & 2 & 110 & & & & & & & & & & & & & & \\
\hline 3368 & & & 2 & 1 & & & & & & & & & & & - & I. & & \\
\hline 3369 & & & EI-Sels & UB-CIpse & & & & & & & &. & & & & & & \\
\hline 3570 & & 1 & 79 & & & & & & & & & & & & & & & \\
\hline 3371 & & & Dummy & & & & & & & & & & & & & & & \\
\hline 3572 & & & ADD & & & & & & & & & & & & & & & \\
\hline 3373 & & & THAESH & 1 & 8.88 & & & & & & & & & & & & & \\
\hline 3374 & & & compariso & on paramete & f forces as & slgnment if & o branch 1 & , if bullding & Collapses' & Inen rallure & ol erhaus & ducts. & & & & & & \\
\hline 3375 & & 2 & 2 & 118 & & & & & & & & & & & . & & & \\
\hline 3376 & & & 2 & 2 & & & & & & & & & & & & & & \\
\hline 3577 & & & E1-Sels & nUB-CIps & & & & & & & & & & & & & & \\
\hline 3370 & & 1 & 15 & & & & & & & & & & & & & & & \\
\hline 3379 & & & Ductfr & & & & & & & & & & & & & & & \\
\hline 3380 & & & $\mathrm{ADD}$ & & & 3 & & & & & & & & & & & $\cdot$ & \\
\hline 3381 & & & EQUAL & $a$ & & & & & & & & & & & & & & \\
\hline 3382 & & & Probablute & yof Eshaust & t Duct collap & pse Is place & ed In branc & ch 1 , remaln & duer In bran & $\cos 2$. & & & & & & & & \\
\hline 3393 & $\$$ & & Otherwise & & & & & & & & & & & & & & & \\
\hline 3384 & & 1 & 79 & & & . & & & & & . & & & & 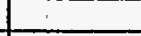 & & & \\
\hline 3385 & & & Dummy & & & & & & & & & & & & & & & \\
\hline 3386 & & & $\mathrm{ADD}$ & & & & & & & & & & & & & & & \\
\hline 3387 & & & GETHAESH & 1 & 188.0 & & & & & & 1. & & & & & & & \\
\hline 3388 & & & Large com & parison par & ameter fore & ces assignn & nent lo br & in $2, n 0 s$ & Selsmic eve & $\mathrm{nt}$, no fallur & ef the & Iust d & & & & & & \\
\hline 3389 & 113 & Wha & Is the con & ndition of in & 18 Zone 1 EH & Hhaust fan & System? & & & & & & & & & & & \\
\hline 3392 & & 2 & Fan-fail & Infanfall & & & & & & & & & & & & & & \\
\hline 3391 & & 8 & 1 & 2 & & & & & & & & & & & & & & \\
\hline 3392 & & 12 & & & & & 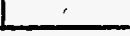 & & & & & & $\therefore$ & & & & & \\
\hline
\end{tabular}


APPENDIH 月

ACCIDENT PRQgRESSION EUENT tREE FOR DEFENSE WASTE PROCESSING FRCILITY - MODE C - PRODUCTION UERSION
WSAC-Th-95-R113

Page 285

\begin{tabular}{|c|c|c|c|c|c|c|c|c|c|c|c|c|c|c|c|c|c|c|}
\hline & h & $\mathbf{B}$ & c & D & $\mathbf{E}$ & $F$ & $\mathbf{E}$ & H & 1 & J & $\bar{K}$ & L & $M$ & $\mathbf{N}$ & D & $P$ & a & $\mathbf{R}$ \\
\hline 3393 & & 4 & 1 & 3 & 8 & 9 & & & & & & & & & & & & \\
\hline 3394 & & & 2 & 112 & +11 & 11 & & & & & & & & & & & & \\
\hline 3395 & & & Int-Egy & NPow/nit & NPOWEn & EPOWEn & & & & & & & & & & & & \\
\hline 3396 & & 1 & 79 & & & & & & & & & & & & & & & \\
\hline 3397 & & & Dummy & & & & & & & & & & & & & i. & & \\
\hline 3399 & & & AnD & & & & & & & & & & & & & & & \\
\hline 3399 & & & THAESH & 1 & 0.0 & & & & & & & & & & & & $\because$ & \\
\hline 3480 & & & Comparisor & in paramete & r wlil force & assignmen & it to branch & A1, Ians & all becau & or loss a & power. & & & & & & & 1 \\
\hline 3481 & & 1 & & & & & & & & & & & & & & & & \\
\hline 3492 & & 128 & 1.000 & 0.000 & & & sparamete & \% 4128,25 & sign value & $=1.8$ & & sparam. & $\$ 128$ & Fallifan & & & & \\
\hline 3493 & & 5 & 1 & 1 & 1 & 8 & 9 & & & & & & & & & & & \\
\hline 3489 & & & 13 & +5 & 4) & 1 & 1 & & & & & & & & & & & \\
\hline 3485 & & & Ltdleak & LtdCD & LIdMIsc & NPOWEn & EPOWEn & & & & & & & & & & & \\
\hline 3485 & & I) & 79 & & & & & & & & & & & & & & & \\
\hline 3487 & & & Dummy & & & & & & & & & & & & & & & \\
\hline 3480 & & & AnD & & & & & & & & & & $\dot{\square}$ & & & & & \\
\hline 3489 & & & THAESH & 1 & 0.0 & & & & & & & & & & & & & \\
\hline 3410 & & & Comparisor & In paramele & r will force & asslgnmen & Af to branch & \#1, Ians 1 & all becau & of loss o & pawer. & & & & & & & \\
\hline 3411 & & 1 & & I & & & & & & & & & & & & & & \\
\hline 3412 & & 128 & 1.089 & 2.089 & & & sparamete & $\# 128,25$ & slgn value & 1.8 & & sparàm. & $\# 128$ & Fallfan & & & & \\
\hline 3913 & 森 & 6 & 1 & 3 & 8 & 9 & 3 & 18 & & & & & & & & & & \\
\hline 3414 & & & 2 & 12 & +11 & 12 & -4 & 21 & & & & & & & & & & \\
\hline 3415 & & & $\ln 1$-Egu & NPowinis & NPOUEn & nEPOWEn & /IAIrInit & nlAirEn & & & & & & & & & & \\
\hline 3416 & & 2 & 44 & 56 & & & & & & & & & & & & & & \\
\hline 3417 & & & Fantmdal & FanTaut & & & & & & & & $\therefore$ & & & & & & \\
\hline 3418 & & & Uяบค1L & & & & & & & & & & & & & & & \\
\hline 3419 & & & EQUAL & 2 & & & & & & & & & & & & & & \\
\hline 3420 & & & Unauailabll & Illty of zone & 1 eHhaust & tans & & & L & 2 & & & & & & & & \\
\hline 3421 & & 1 & & & & & & & & & & & & & & & & \\
\hline 3422 & & 128 & 1.088 & . 8.008 & & & SParamete & $\# 128$, ass & sign value & 1.8 & & sParam. & $\# 128$ & Fallfan & & & & \\
\hline 3423 & x & 6 & 1 & 1 & 1 & 8 & 9 & 18 & & & & & & & & & & \\
\hline 3424 & & & 19 & +3 & +51 & 1 & 12 & 2) & & & & & & & & & & \\
\hline 3425 & & & LIOMISC & LIdLeak & LtdCo & NPOWEn & nEPaWEn & nifiren & & & & & & & & & & \\
\hline 3426 & & 2 & 44 & 56 & & & & & & & & & & & & & & \\
\hline 3427 & & & FanLmdal & Faniaul & & & & & & & & & & & & & & \\
\hline 3428 & & & UAUAIL & & & & & & & & & & & & & & & \\
\hline 3429 & & & EQUAL & D & & & & & & & & & & & & & & \\
\hline 343 & & & Unauallabit & Ility of Zone & 1 enhaust & fans & & & & & & & & & & & & \\
\hline 3431 & & 1 & & & & & & & & & & & & & & & & \\
\hline 3432 & & 128 & 1.008 & Q.8Bg & & & sparamete & \#128,ass & slgn value & & & sparam. & $\$ 128$ & Falifan & & & & \\
\hline 3433 & 纱 & 6 & 1 & 3 & 8 & 9 & 3 & 18 & & & & & & & & & & \\
\hline 3434 & & & 2 & 12 & +11 & 12 & 14 & +111 & & & & & & & & & & \\
\hline 3435 & & & Int-Eg4 & NPowinll & NPowEn & nEPOWEn & IAtrinit & IAIREN & & & & & & & & & & \\
\hline 3436 & & 2 & 124 & .125 & & & & & & & & & & & & & & \\
\hline 3437 & & & Fanlmda2 & FanTau2 & & & & & & & & & & & & & & \\
\hline 3438 & & & UAUAIL & & & & & & & & & & & & & & & \\
\hline 3439 & & & EqUAL & D & & & & & & & & & & & & & & \\
\hline 3440 & & & Unauallabll & lility of zone & 1 eshaust & fans & & & & & & & & & & & & \\
\hline 3441 & & 1 & & & & & & & & & & & & & & & 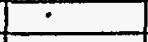 & \\
\hline 3442 & & 128 & 1.080 & 0.898 & & & Sparamete & \#128, & Ign value & 1.0 & & sparam. & \#128 & Fallfan & & & & \\
\hline 3443 & 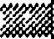 & 5 & 1 & 1 & 1 & 8 & 18 & & & & & & & & & & & \\
\hline 3444 & 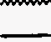 & & 14 & +3 & +51 & 1 & 1 & & & & & & & & & & & \\
\hline 3445 & $L$ & I & LtdMise & Ltdleak & Ltden & INPOWEn & Laliren & & & & & & & & & & & \\
\hline
\end{tabular}


APPENBAH A

ACCIDENT PAOGRESSION EUENT TREE FOR DEFENSE UASTE PROCESSING FACILITY - MODE C - PRODUCTION UERSION

\begin{tabular}{|c|c|c|c|c|c|c|c|c|c|c|c|c|c|c|c|c|c|c|}
\hline - & A & D & C & D & $\bar{E}$ & $F$ & 5 & H & 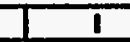 & $d$ & $\mathbf{K}$ & L & $\bar{M}$ & $N$ & 0 & $\mathbf{p}$ & a & in \\
\hline 3446 & & 2 & 124 & 125 & 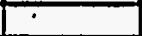 & & & & & & $\overline{7}$ & & & & & & . & \\
\hline 3447 & & & FanLmde2 & FanTau2 & & & & & & & & & & & & & & \\
\hline 3448 & & & UAUALL & & & & & & & & & & & & & & & \\
\hline 3449 & & & EQUAL & 0 & & & & & & & & & & & & & & \\
\hline 3450 & & & Unauallabl & IItey of Zons & 1 ehthaust & Fons & & & & & & & & & & & & \\
\hline 3451] & & i) & & & & & & & & & & & & & & & $\therefore$ & \\
\hline 3452 & & 128 & $1.0 \mathrm{BB}$ & 0.000 & & & sparamele & $\# 128$ & sslgn valu & $0=1.0$ & & sparam. & $\$ 128$ & Fallfan & & & & \\
\hline 3453 & 聯 & 4 & 1 & 1 & 1 & 1 & I & & $\cdot$ & & & & & & & & 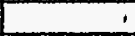 & \\
\hline 3454 & & & 2 & +3 & +4 & +5 & & & & & & & & & & & & \\
\hline 3455 & & & Int-Egy & LIdLeak & LLdMIsc & Ledco & & & & & & & & & & & & \\
\hline 3456 & & 2 & 126 & 127 & & & & & & & & & & & & & & \\
\hline 3457 & & & FanLmans & FanTau3 & & & & & & & & & & & & & & \\
\hline 3450 & & & UAUAIL & & & & & & & & & & & & & & & \\
\hline 3459 & & & EQUAL & \pm & & L & & & & & & - & & & & & & \\
\hline 3460 & & & Unauallabl & ility of Zone & 1 ehhaust & fans & & & & & & & & & & & & \\
\hline 3451 & & I & & & & & & & & & & & & & & & & \\
\hline 3462 & & 128 & 1.800 & 0.008 & & & sparamete & $\# 128$ & Issign ualu & $a=1.8$ & & sparam. & $\# 128$ & Fallfan & & & & \\
\hline 3463 & 㹡 & 3 & 1 & 8 & 9 & & & & & & & & & & & & & \\
\hline 3464 & & & 1 & 1 & 1 & & & & & & & & & & & & & \\
\hline 3465 & & & Eylernal & NPOUEn & EPOWEn & & & & & & & & & & & & & \\
\hline 3466 & & 1 & 79 & & & & & & & & & & & & & & & \\
\hline 3467 & & & Dummy & & - & & & & & & & & & & & & & \\
\hline 3468 & & & ADD & & & & & & & & & & & & & & & \\
\hline 3469 & & & THAESH & 1 & 8.8 & & & & & & & & & & & & & \\
\hline 347 & & & Compariso & in paramete & er will force & assignmen & nt to branch & \# $1, \mathrm{far}$ & 5 fall becal & Ise of loss & of power. & & & & & & & \\
\hline 3471 & & 1 & & & & & & & & & & & & & & & & \\
\hline 3472 & & 128 & 1.080 & 0.080 & & & sparamete & $\$ 12 B$ & sslgn valu & $e=1.8$ & & sParam. & $\# 128$ & Fallfan & & & & \\
\hline 3473 & 俻 & 4 & 1 & 8 & 9 & 18 & & & & & & & & & & & & \\
\hline 3474 & & & 1 & 1 & 2 & 2 & & & & & & & & & & & & \\
\hline 3475 & & & Emternal & NPOLEn & DEPOWEn & nifiren. & & & & & 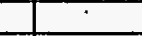 & & & & & & & \\
\hline 3476 & & 5 & 44 & 56 & 16 & 96 & 1 & & & & & & & & & & & \\
\hline 3477 & & & FanLmda & FanTaul & Fanfr & FanAnI & MsTImEH & & & & & & & & & & & \\
\hline 3478 & & & UAUAIL & & & & & & & & & & & & & & & \\
\hline 3479 & & & EQUAL & e & & & & & & $E$ & & & & & & & & \\
\hline 3480 & & & Unauallaal & Lity of Zone & 1 eHhaust & Ians, Irom & enternat es & yent, or & randomly 0 & luring perio & Id MSImEH & & & & & & & \\
\hline 3481 & & I & & & & & & & & & & & & & & & & \\
\hline 3482 & & 128 & 1.890 & 0.880 & & & sparamete & $\# 128$ & ssign valu & $\theta=1.0$ & & sParam. & $\# 128$ & Fallfan & & & & \\
\hline 3483 & & 4 & $T$ & 8 & 9 & 18 & & & & & & & & & & & & \\
\hline 3484 & & & 1 & 1 & 2 & 1 & & & & & & & & & & & & \\
\hline 3485 & & & Esternal & NPowEn & nEPoWEn & IRIIrEn & & & & & & & & & & & & \\
\hline 3486 & & 5 & 124 & 125 & 16 & 122 & 1 & & & & & & & & & & & \\
\hline 3487 & & & Fanlmde2 & FanTau2 & Fanfr & FanAn2 & MSImEH & & & & & & & & & & & \\
\hline 3480 & & & UAUAIL. & & & & & & & & & & & & & & & \\
\hline 3489 & & & \begin{tabular}{|l} 
EOURL \\
\end{tabular} & 0 & & & & & & & & & & & & & & \\
\hline 3490 & & & Unaupllab! & IIIty of Zane & 1 ehhaust & fans, from & entemal ey & yent, or & randomily 0 & luring perio & Id MsTmEH & & & & & & & \\
\hline 3491 & & 1 & & & & & & & & & & & i & & $\cdot$ & & & \\
\hline 3492 & & 178 & 1.808 & 8.880 & & & sparamete & $\# 128$ & Isslgn valu & $e=1.0$ & & sparam. & $\$ 128$ & Failfan & & & & \\
\hline 3493 & 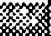 & 1 & 1 & & & & & & & & & & & & & & & \\
\hline 3494 & & & 1 & & & & & & & & & & & & & & & \\
\hline 3495 & & & Eyternal & & & & & 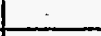 & & & & & & & & & & \\
\hline 3496 & & 5 & 126 & 127 & 16 & 123 & 1 & & & & & & & & & & & \\
\hline 3497 & & & FanLmda3 & FanTaus & Fanfr & Fanfin3 & MSTMER & & & & & & & & & & & \\
\hline 3490 & & 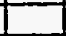 & URUAIL & & & & & & & & & & & & & & & \\
\hline
\end{tabular}


APPENDIH $A$

\begin{tabular}{|c|c|c|c|c|c|c|c|c|c|c|c|c|c|c|c|c|c|c|}
\hline & A & D & $\bar{c}$ & 0 & $\mathbf{E}$ & $F$ & $E$ & $\mathrm{H}$ & 1 & $J$ & $K$ & 1 & $\bar{M}$ & $N$ & $\mathbf{0}$ & $P$ & a & A \\
\hline 3499 & & & EQUAL & $\bar{E}$ & & & & & & & & & & & & & & \\
\hline 3580 & & & Unauallabll & lity of Zone & 1 eshausf & fans, from & ertemal et & uent, or ra & indomly dur & ing perlod & MSTmEH & & & & & & & $\cdot$ \\
\hline 3581 & & 1 & & & & & & & & & & & & & & & & \\
\hline 3582 & & 128 & 1.098 & 0.010 & & & sparamele & $* 128$, & slgn value = & $=1.0$ & & sparam. & 4128 & Fallfan & & & & \\
\hline 3593 & 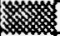 & & alherwise & & & & & & & & & & & & $\because$ & & & \\
\hline 359 & & $T$ & 79 & & & & & & & & & & & & & & & \\
\hline 3585 & & & Dummy & & & & & & & & & & & & & & $\because$ & \\
\hline 3586 & & & AnB & & & & & & & & & & & & & & & 1 \\
\hline 3587 & & & GETHAESH & $I$ & 108.0 & & & & & & & & & & & & & \\
\hline 3588 & & & Compariso & n paramete & forces as & signment I & 0 branch 2 & no $\operatorname{ran}$ fal & llure. & & & & & & & & & \\
\hline 3589 & & 1 & & & & & & & & & & & & & & & & \\
\hline 3510 & & 128 & 0.020 & 0.000 & & $\cdot$ & SParamele & 4128 ,as & sign value = & 1.0 & & SParam. & $\# 128$ & Fallfan & & & & \\
\hline 3511 & 114 & Does & sa deflagra & tabie conce & entrallon oc & ccur in the & SPC due 10 & Uessel Oue & pressurtza & tion? & & & & & & & & \\
\hline 3512 & & 2 & SPCOAfOP & ASPCDEROP & & & & & & & & & & & & & & \\
\hline 3513 & & 6 & 1 & 2 & & & & & 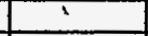 & & & & & & & & & \\
\hline 3514 & & 4 & & & & & & & & & & & & & & & & \\
\hline 3515 & 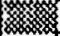 & 2 & 1 & 1 & & & & & & & - & & & & & & & \\
\hline 3516 & & & $(-1)$ & -21 & & & & & & & & & & & & & & \\
\hline 3517 & & & /Eнternal & $/ \operatorname{lnt-Egy}$ & & & & & & & & & & & & & & - \\
\hline 3518 & & 1 & 79 & & & & & & & & & & & & & & & \\
\hline 3519 & & & Dummy & & & & & & & & & & & & & & & \\
\hline 3520 & & & ADo & & & & & & & & & & & & & & & \\
\hline 3521 & & & GETHAESH & 1 & 108.8 & & & & & & & & & & & & & \\
\hline 3522 & & & Large com & parison par & amater for & ces assign & ment to bra & inch 2, nod & Jeflagratab! & le cancentr & atton. & & & & & & & \\
\hline 3523 & 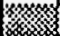 & 1 & 1 & & & & 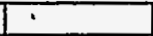 & & & & & &. & & & & & \\
\hline 3524 & 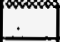 & & 2 & & & & & & & & & & & & & & & \\
\hline 3525 & & & Int-Egy & & & & & & & & & & & & & & & \\
\hline 3526 & & 5 & 69 & 78 & 71 & $9 \mathrm{~g}$ & 128 & & & & & & & & & & & \\
\hline 3527 & & & FalINPwr & Missime & \begin{tabular}{|c|} 
ncommi \\
\end{tabular} & LHS & Fallfan & & & & & & & & & & & \\
\hline 3528 & & & FUN-SPC & & & & & & & & & & & & & & & \\
\hline 3529 & & & EQUAL & 0 & & & & & & & $\therefore$ & & & & & & & \\
\hline 3553 & & & UFUN-SPC & wlil calculat & Io probabili & ty of defla & gratable co & ncentratio & in in the SPC & & & & & & & & & \\
\hline 3531 & 皪漯 & 1 & 1 & & & & & & & & & & & & & & & \\
\hline 3532 & & & 1 & & & & & & & & & & & & & & & \\
\hline 3535 & & & Euternal & & & & & & & & & & & & & & & \\
\hline 3534 & & 4 & 70 & 71 & 98 & 128 & & & & & & & & & & & & \\
\hline 3535 & & & MIssIIme & nComm! & LHS & Fallfan & & & & & & & & & & & & \\
\hline 3536 & & & FUN-SPCS & & & & & & & & & & & & & & & \\
\hline 3537 & & & EQUAL & 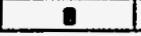 & & & & & & & & & & & & & - & \\
\hline 3538 & & & UFUN-SPCS & wlll calcula & le probabll & lity of detl & agratable c & ancentrallic & on due to an & $n$ estenal $\mathrm{e}$ & yent. & & & & & & & \\
\hline 3539 & 䇣 & & otherwlse & & & & & & & & & & & & & & & \\
\hline 3544 & & 1 & 79 & & & & & & & & & & 1 & & & & & \\
\hline 3541 & & & Dummy & & & & & & & & & & & & $\therefore$ & & & \\
\hline 3542 & & & ADB & & & & & & & & & & & & & & & \\
\hline 3543 & & & GETHAESH & 1 & 188.0 & & & & & & & & & & & & & \\
\hline 3544 & & & Large com & parison par & ameter Ior & ces assigni & ment to hra & $\operatorname{nch} 2, \mathrm{nod}$ & leflagratabl & e concentr & allon & & & & & & & \\
\hline 3545 & 115 & Doe: & 3 a vessel a & verpressuff & zzatlon in 11 & he Salt Pro & cess Cell re & sulf in a de & eflagratlon! & In the Salt & Process Ce & & & & & & & \\
\hline 3546 & & 2 & SPC-DefI & nSPCDef1 & & & & & & & & & & & & & & \\
\hline 3547 & & 2 & 1 & 2 & & & & & & & & & & & & & & \\
\hline 3548 & & $\underline{3}$ & & & & & & & & & & & & & & & & \\
\hline 3549 & z & 4 & 186 & 109 & 107 & 168 & & & & & & & & & & & & \\
\hline 3550 & & & $1+$ & $1+1$ & $1+$ & 1 & & & & & & & & & & & & \\
\hline 3551 & & & $P A H-5 P I$ & PBFT-SP! & QEU-Sp! & | DECT-Spl & & & & & & & & & & & & \\
\hline
\end{tabular}


APPENDIH A

ACCIDENT PRDGRESSION EUENT TAEE FOA DEFENSE UWSTE PROCESSING FACILITY - MODE C - PAODUCTION UERSION

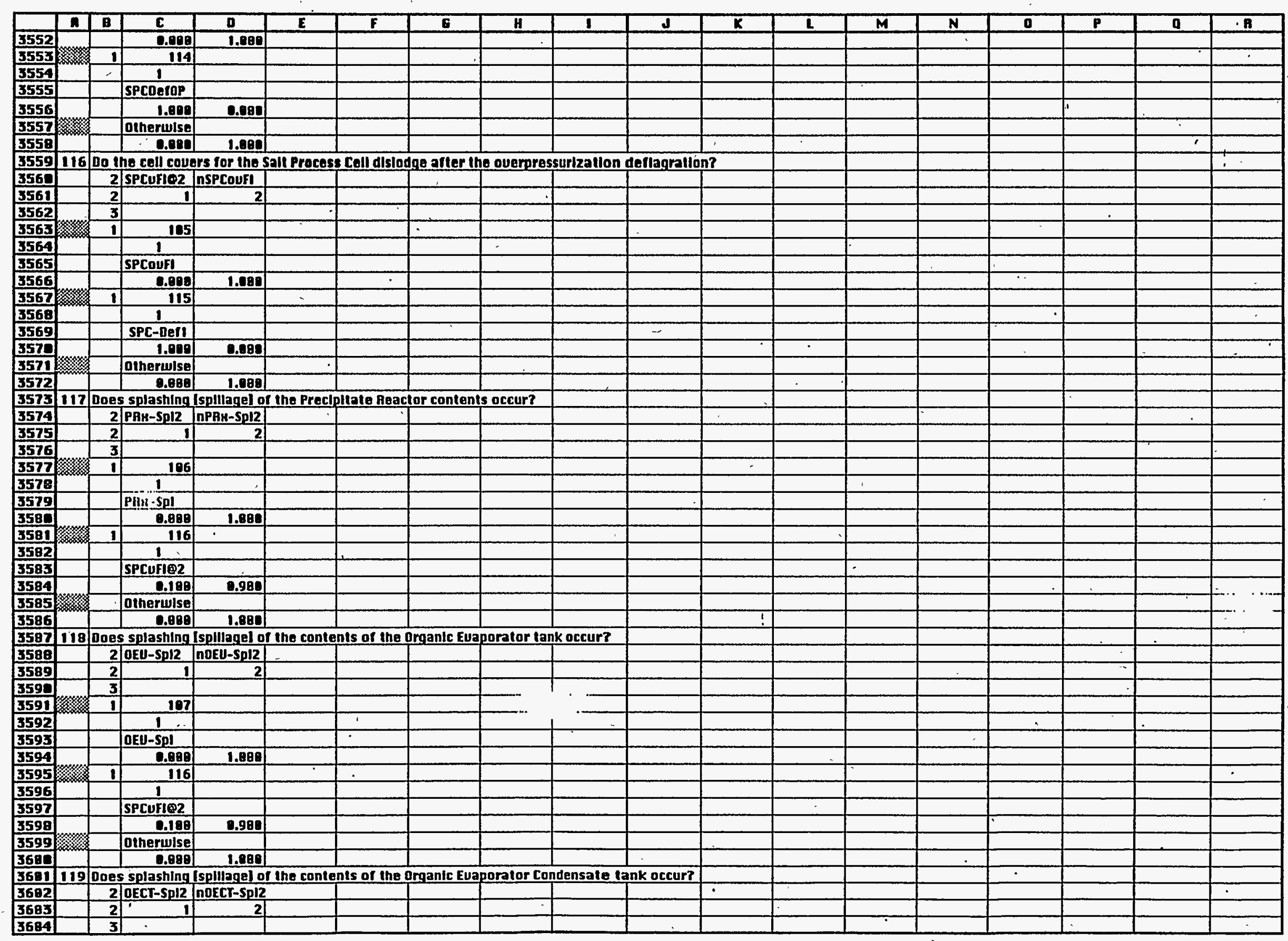


APPENDIK $\mathrm{A}$

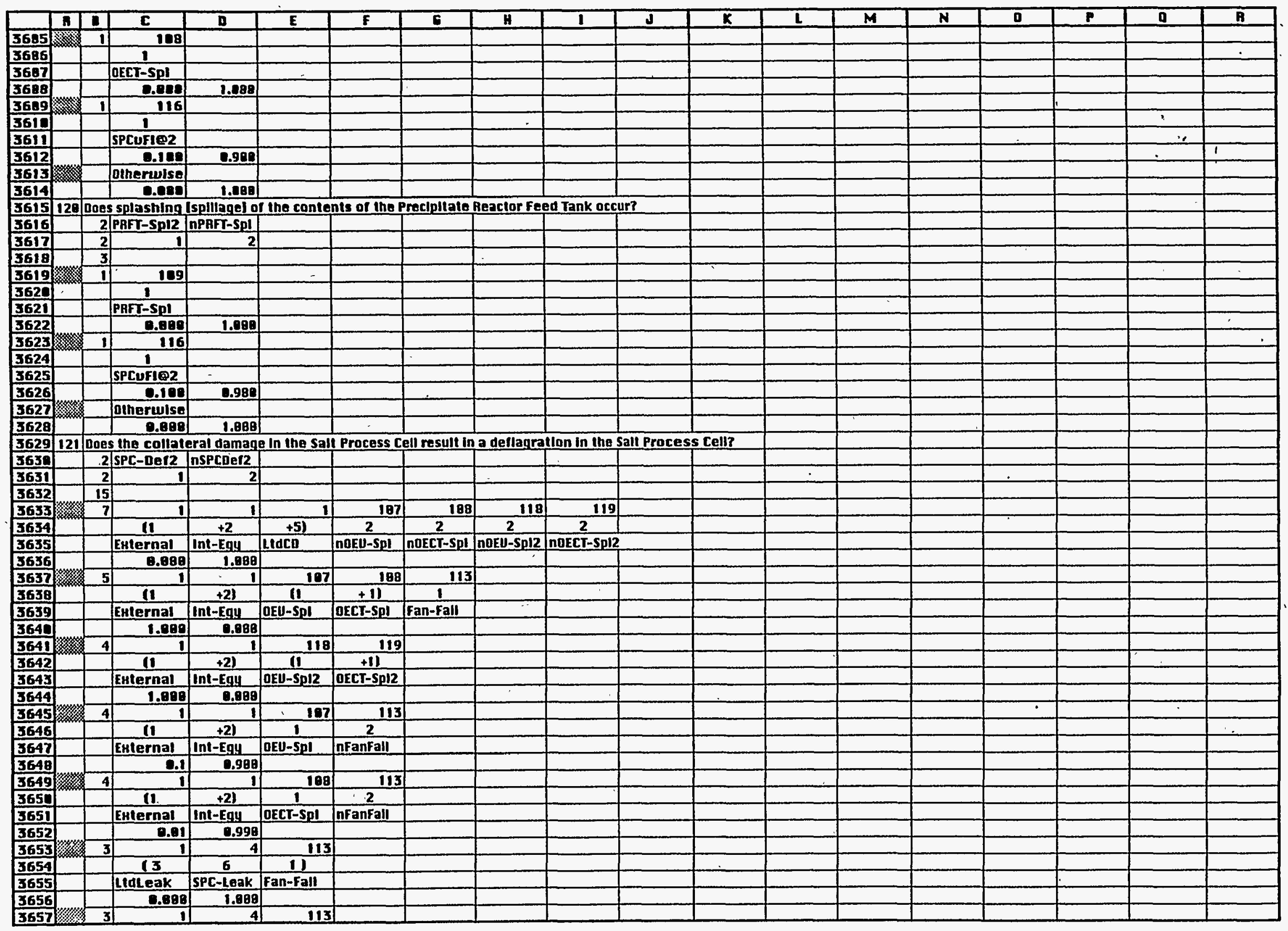


APPENDII 9

ACCIDENT PAOGRESSION EUENT TAEE FOR DEFENSE WUSTE PROCESSING FACILITY - MODE C - PAODUCTION DERSION

\begin{tabular}{|c|c|c|c|c|c|c|c|c|c|c|c|c|c|c|c|c|c|c|}
\hline & n & 8 & $\overline{\mathbf{C}}$ & D & $\bar{E}$ & $F$ & C & $H$ & 1 & 4 & $\bar{K}$ & $L$ & $M$ & $N$ & 0 & P & a & B \\
\hline 3658 & & & 13 & 6 & 2) & & & & & & & & & & & & & \\
\hline 3659 & & & LidLleak & SPC-Leak & nFanfail & & & & & & & & & & & & & \\
\hline 3660 & & & 0.080 & 1.000 & & & & & & & & & & & & & & \\
\hline 3669 & - & .3 & 1 & 4 & 113 & & & & $\dot{-}$ & & & & & & & & & \\
\hline 3662 & 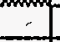 & & 13 & 11 & 1) & & & & & & & & & & & it & & \\
\hline 3663 & & & Ltaleak & SPC-0Ilow & Fan-Fall & & & & & & & & & & & & - & \\
\hline 3664 & & & 0.080 & 1.809 & & & & & & & & & & 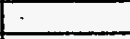 & & & 1 & \\
\hline 3665 & 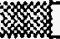 & 3 & 1 & 4 & 113 & & & & & & & & & & & & & 1 \\
\hline 3666 & & & 13 & 11 & 2) & & & & & & & & & & & & & \\
\hline 3667 & & & LtdLeak & SPC-0110W & nfonfall & & & & & & & & & & & & & \\
\hline 3668 & & & 8.080 & 1.088 & & & & & & & & & 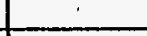 & & & & & \\
\hline 3669 & 聯 & 3 & (1) & 4 & 113 & & & - & & & & & & & - & & & \\
\hline 367 & & & 13 & 9 & 11 & & & & & & & & & & & & & \\
\hline 3671 & & & LidLeak & Bz-'onow & Fan-Fall & $=$ & & & & & & & & & & & & \\
\hline 3672 & & & 0.010 & 0.999 & & & & & & & & & & & & & & \\
\hline 3673 & 係聯 & 3 & 1 & 4 & 113 & & & & & & & & & & & & & \\
\hline 3674 & & & 13 & 9 & 21 & & & & & & & & & & & & & \\
\hline 3675 & & & Lidleak & Bz-0llow & nFanfall & & & & & & & & & & & & & \\
\hline 3676 & 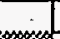 & & 8.099 & 1.080 & & & & & & & & & & & & & & \\
\hline 3672 & 嬨䊽 & 4 & 1 & 187 & 188 & 113 & & & & & & & & & & & & \\
\hline 3678 & & & 5 & (1) & +11 & 1 & & & & & & & & & & & & \\
\hline 3679 & & & Lidco & DEEU-SpI & DECT-Spl & Fan-Fall & & & & & & & & & & & & \\
\hline 3680 & & & 0.010 & 8.998 & & & & & & & & & & & & & & \\
\hline 3681 & 漯 & $\underline{\underline{3}}$ & 1 & 4 & 113 & & & & & & & & & & & & & \\
\hline 3682 & & & 13 & 10 & 1) & & & & & & & & & & & & & \\
\hline \begin{tabular}{|l|}
6683 \\
\end{tabular} & & & Lidleak & Bz-Leak & Fan-Fall & & & & & - & & & & & & & & \\
\hline 3684 & & & B.081 & 0.999 & & & & & & & & & & & & & & \\
\hline 3685 & s & 3 & 1 & 4 & 113 & & & & & & & 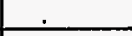 & & & & & & \\
\hline 3686 & & & 13 & 18 & 2) & & & & & 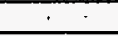 & & & & & & & & \\
\hline 3687 & & & LldLeaK & BZ-Leak & nfanfail & & & & & & $\ldots$ & & & & & & & \\
\hline 3689 & & & 8.800 & 1.880 & & i. & & & & & & & & & & & & \\
\hline 3689 & & & Diherwise & & & & & & & & & $\therefore$ & & & & $\therefore$ & & \\
\hline 3690 & & & 0.080 & 1.880 & & & & & & & & & & & & & & \\
\hline 3691 & 122 & Does & s the collat & eral damag & In the Sall & 1 Process Ce & ell result in & a tire in in & e Sall Proce & ess Celli? & & & & & & & & \\
\hline 3692 & & 2 & SPC-Fire & nsPCFIre & & & & & & & & & & & & & & \\
\hline 3693 & & 2 & 1 & 2 & & & & & & & & & & & & & & \\
\hline 3699 & & 18 & & & & & & & & & & & & & & & & \\
\hline 3695 & 留㡭 & 4 & 107 & 188 & 118 & 119 & & & & & & & & & & & $\cdot$ & \\
\hline 3696 & & & 2 & 2 & 2 & 2 & & & & & & & & & & & & \\
\hline 3697 & & & InOEU-Spt & nOECT-Spl & nOEU-Spl2 & nOECT-Sp12 & & & & & & & & & & & & \\
\hline 3698 & & & 8.080 & 1.880 & & & & & & & & & & & 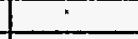 & & & \\
\hline 3699 & & 7 & 1 & 1 & 107 & 118 & 188 & 119 & 121 & & & & & & & & & \\
\hline 3780 & & & $(1$ & $+2)$ & 11 & +1 & +1 & +11 & 1 & & & $\therefore$ & & & & & & \\
\hline 3701 & & & Esternal & Int-Egy & DEE-Spl & OEU-Spl2 & OECr-Spl & OECT-Spl2 & SPC-Der2 & & & & & & & & & \\
\hline 3782 & & & 0.988 & 0.180 & & & & & & & & & & & & & & \\
\hline 3793 & & 7 & 1 & 1 & 187 & 118 & 108 & 119 & 121 & & & & & & & & & \\
\hline 3784 & & & 11 & $+2)$ & 11 & +1 & +1 & +11 & 2 & & & & - & & & & & \\
\hline 3785 & & & External & Int-Egu & OEU-Spl & OEU-Sp12 & OECI-SDI & OECT-Spl2 & DSPCDeI2 & & & & & & & & & \\
\hline 3786 & & & 0.188 & 0.988 & & & & & & & & & & & & & & \\
\hline 3707 & 聯 & 3 & 1 & 4 & 121 & & & & & & & & & & & & & \\
\hline 3768 & & & 3 & 9 & 1 & & & & & & & & & & & & & \\
\hline 3789 & & & Ltoleak & 8Z-0110w & SPC-Def2 & & & & & & & & & & & & & \\
\hline 3710 & & & 8.988 & 8.188 & & & & & & & & & & & & & & \\
\hline
\end{tabular}


APPENDIH R

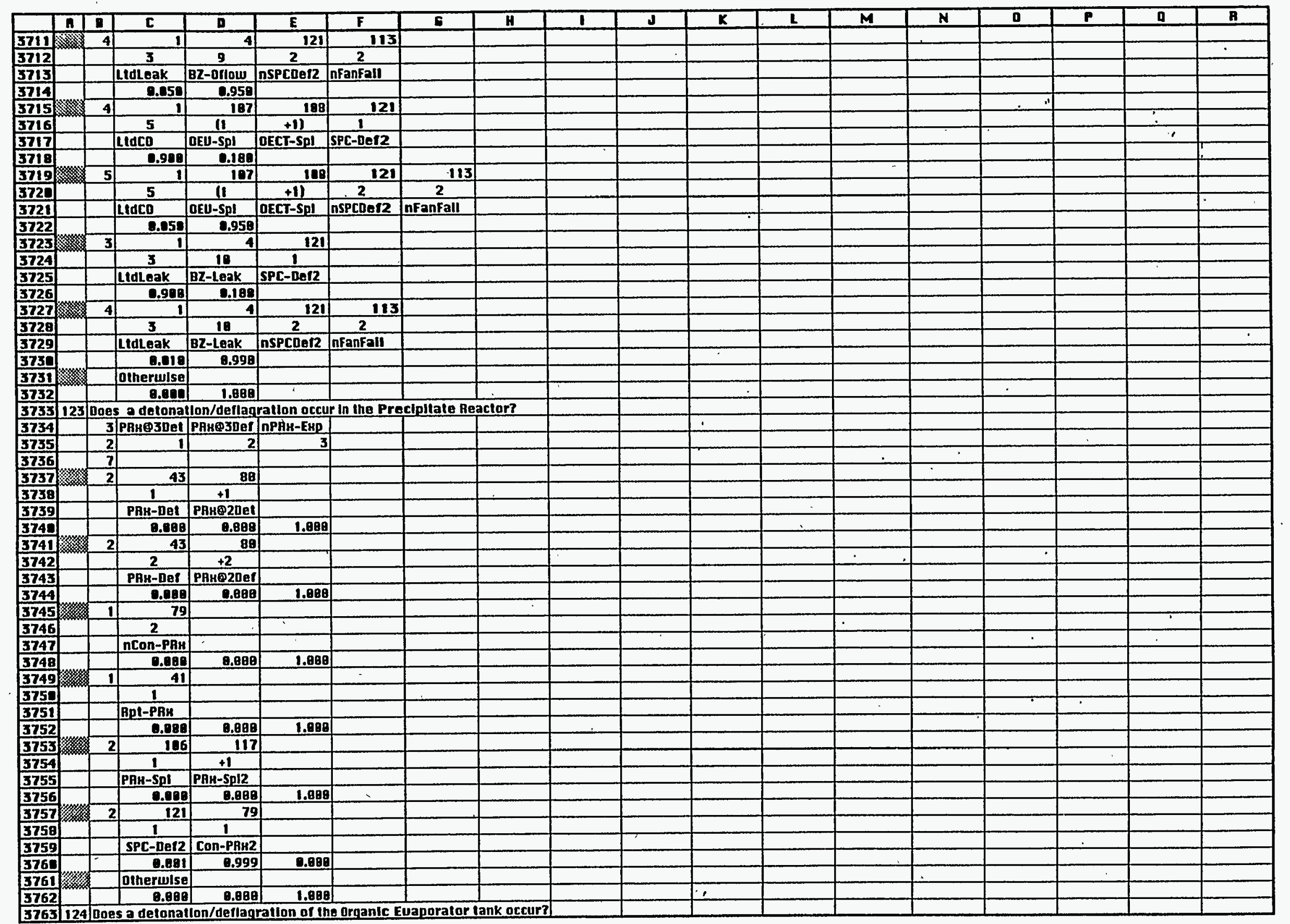




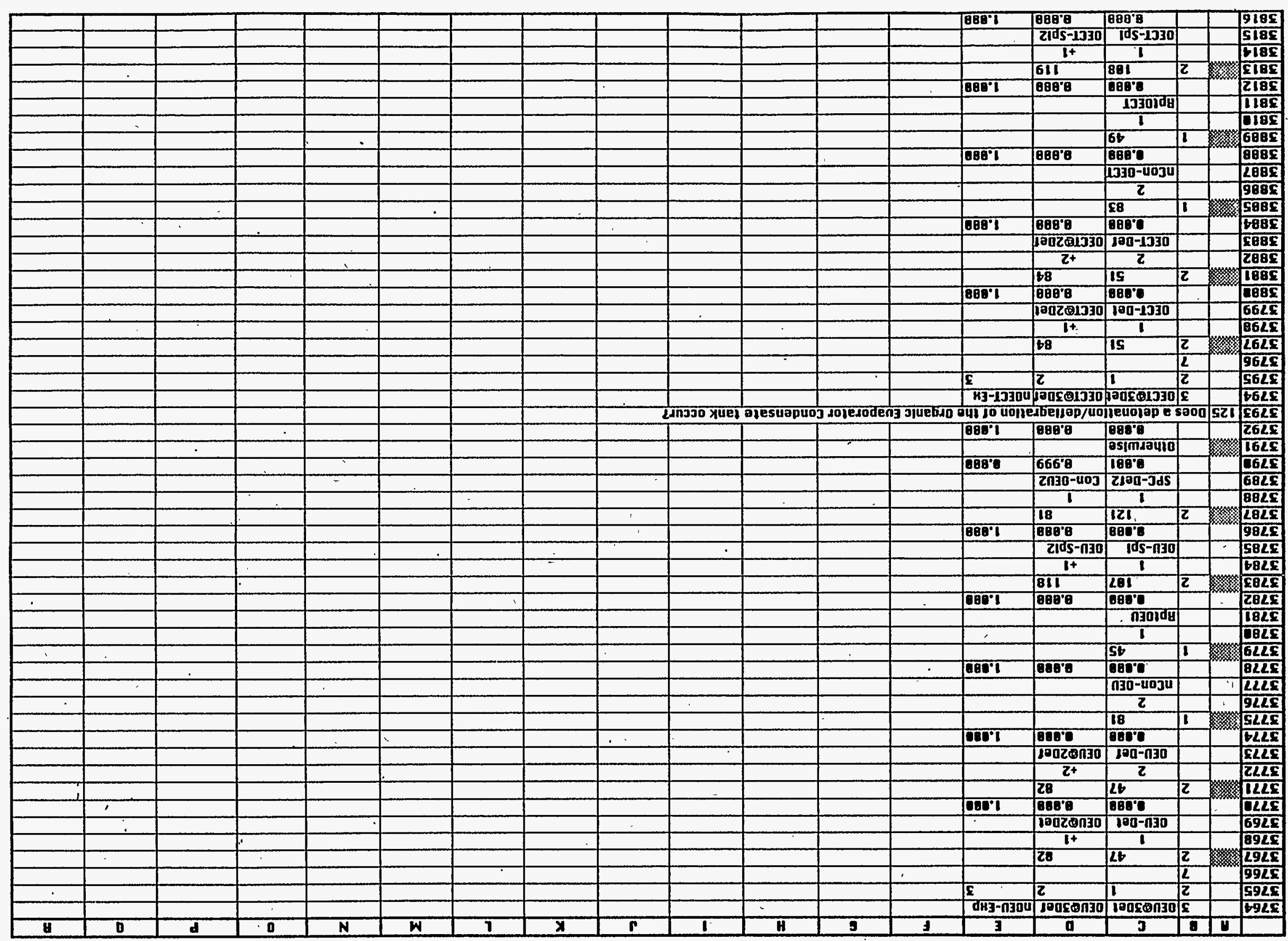


APPENDIH A

GCCIDENT PROGRESSION EUENT TREE FOR DEFENSE WASTE PROCESSING FACILITY - MODE C - PRODUCTION UERSION

USAC-TR-95-8113

Page 293

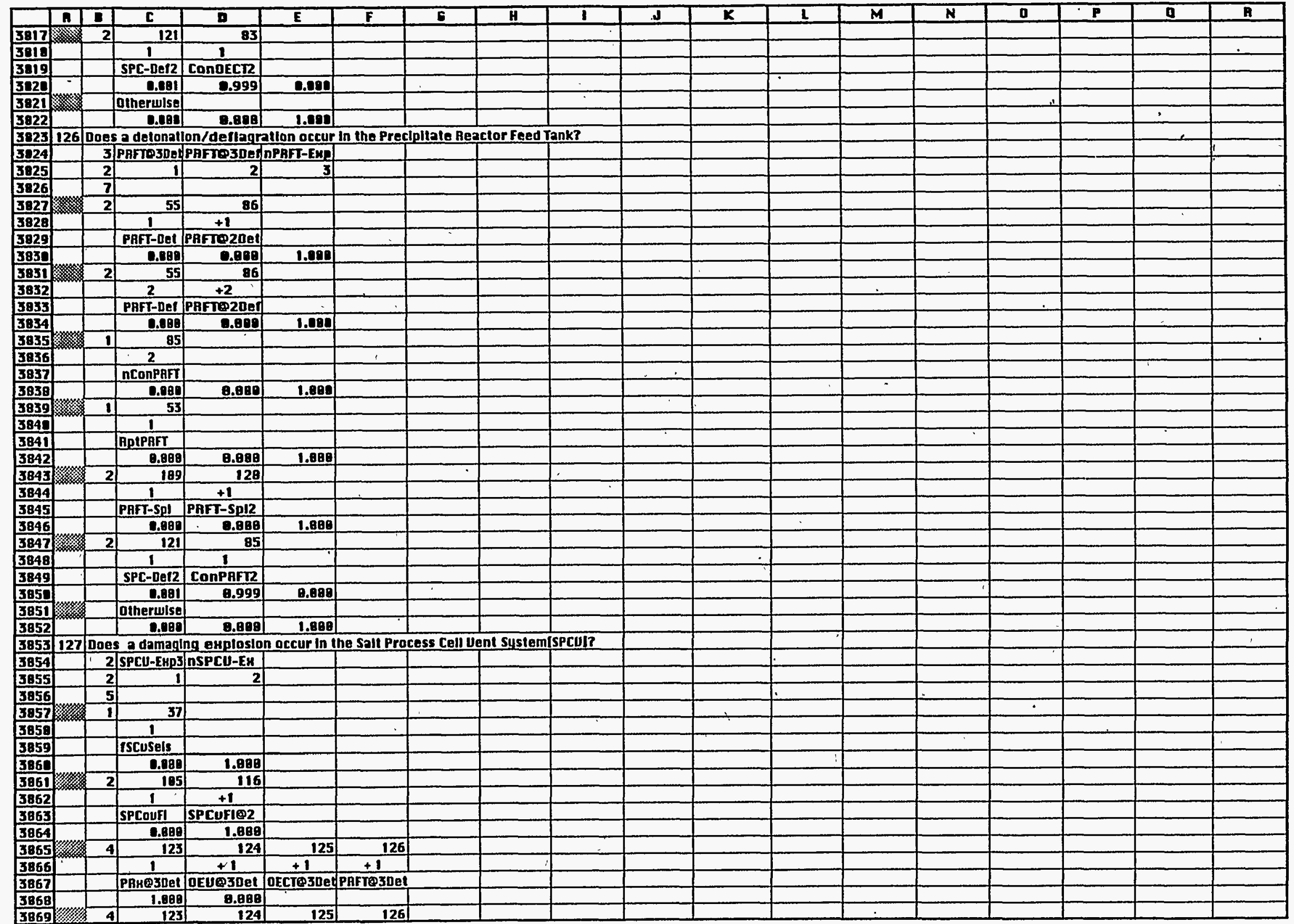




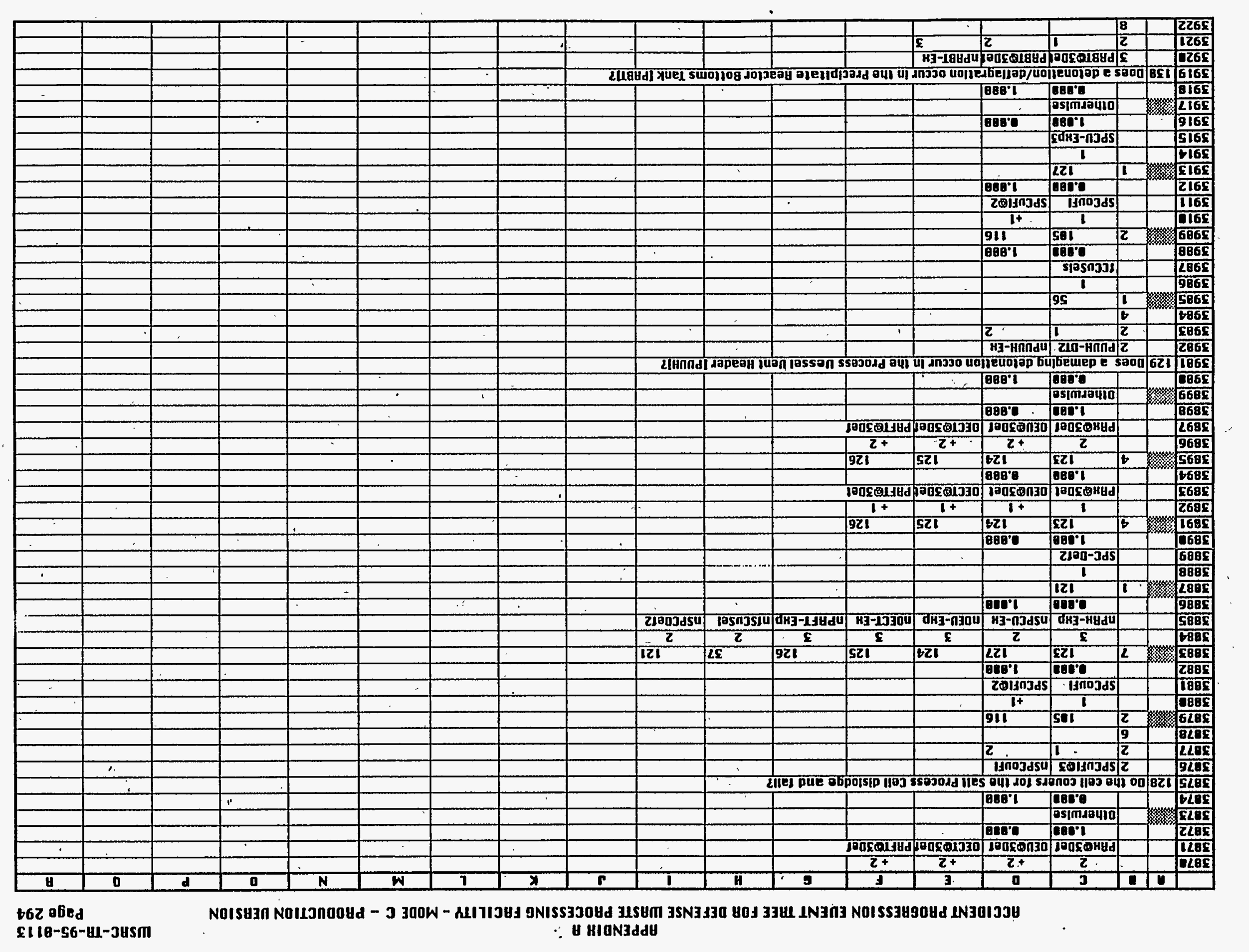




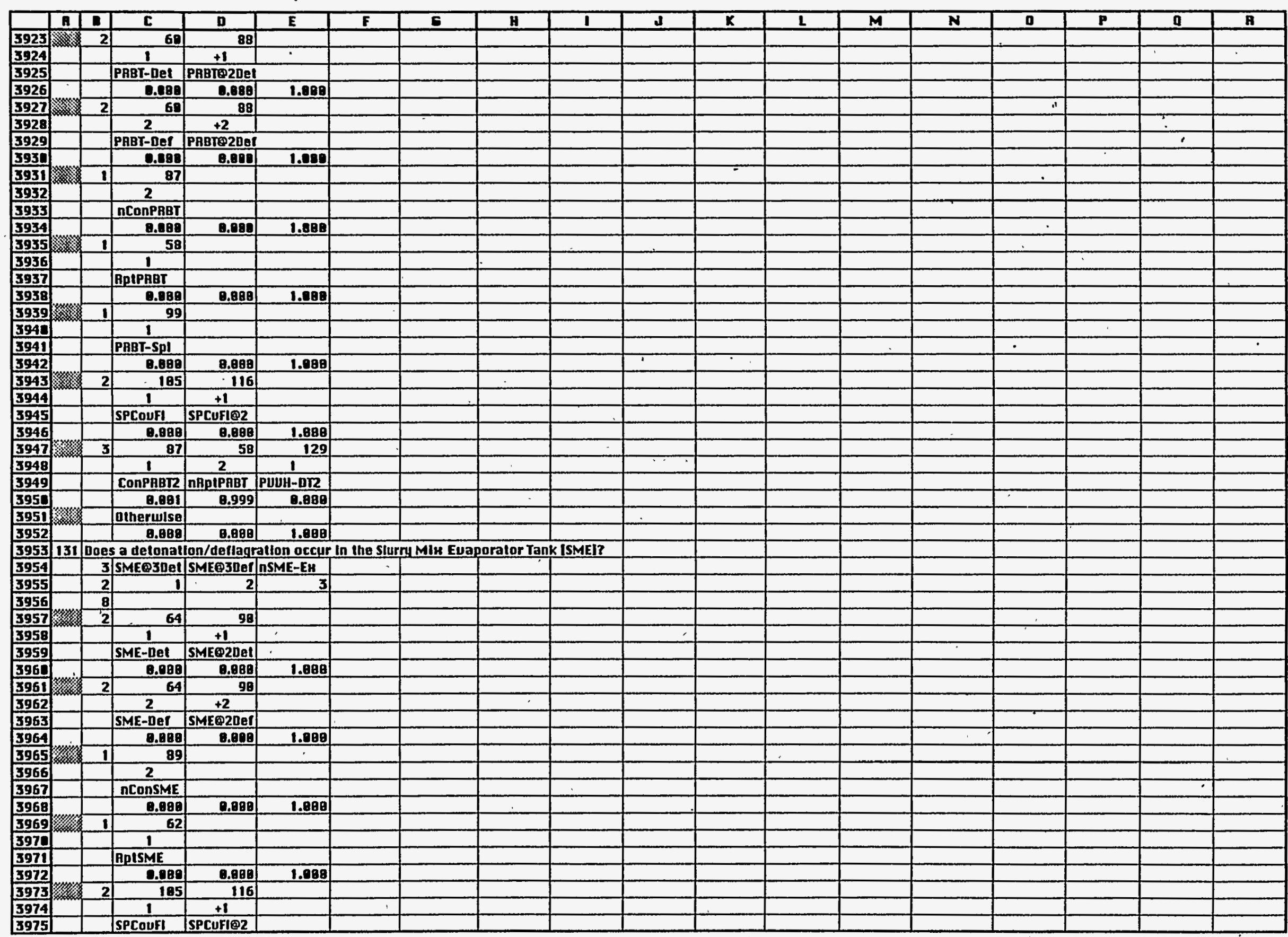




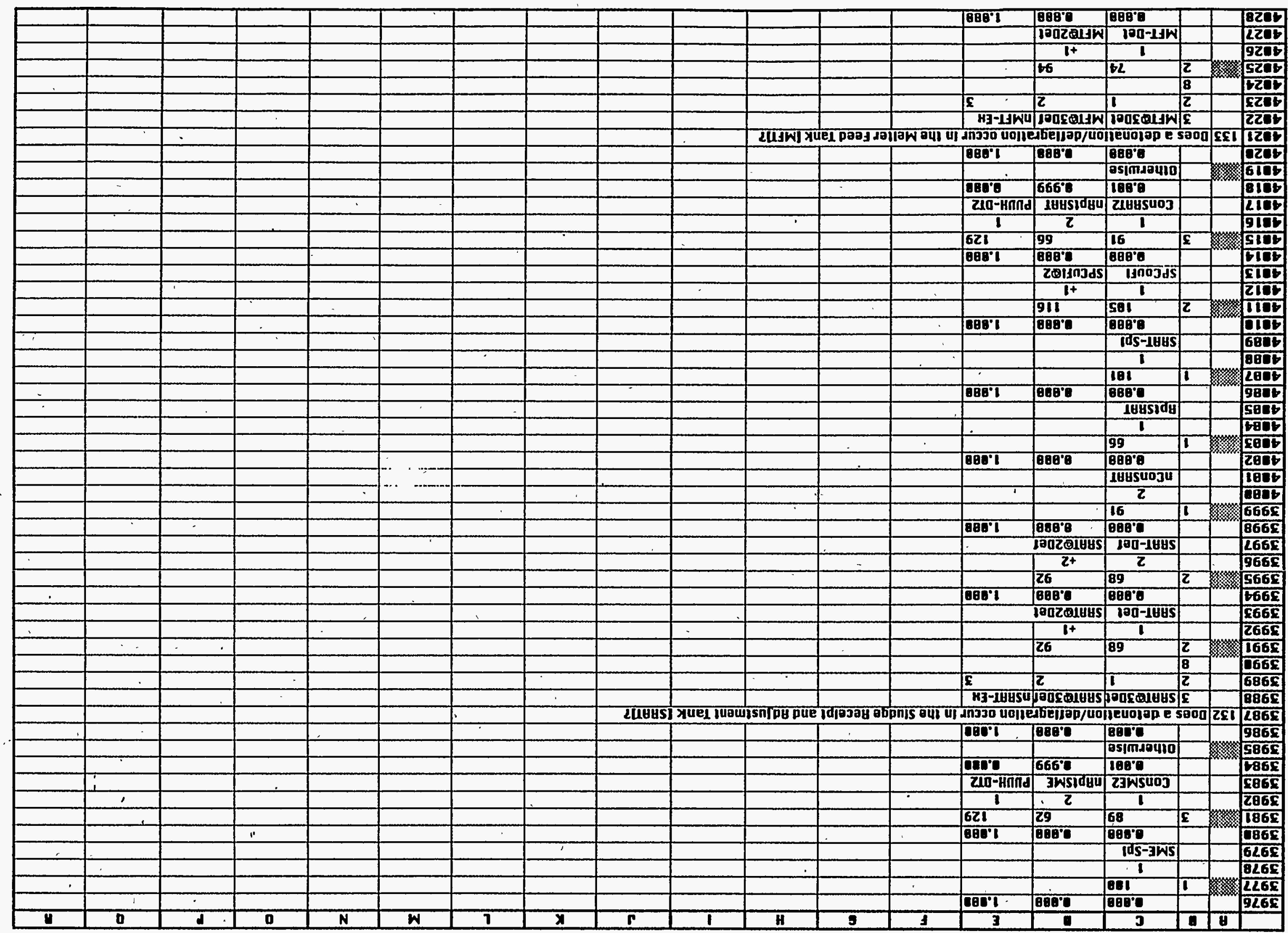

962 abed

2116-56-41-345ก!

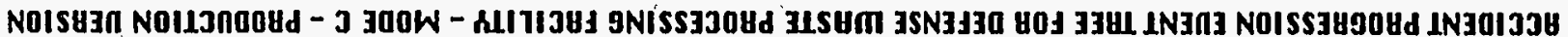




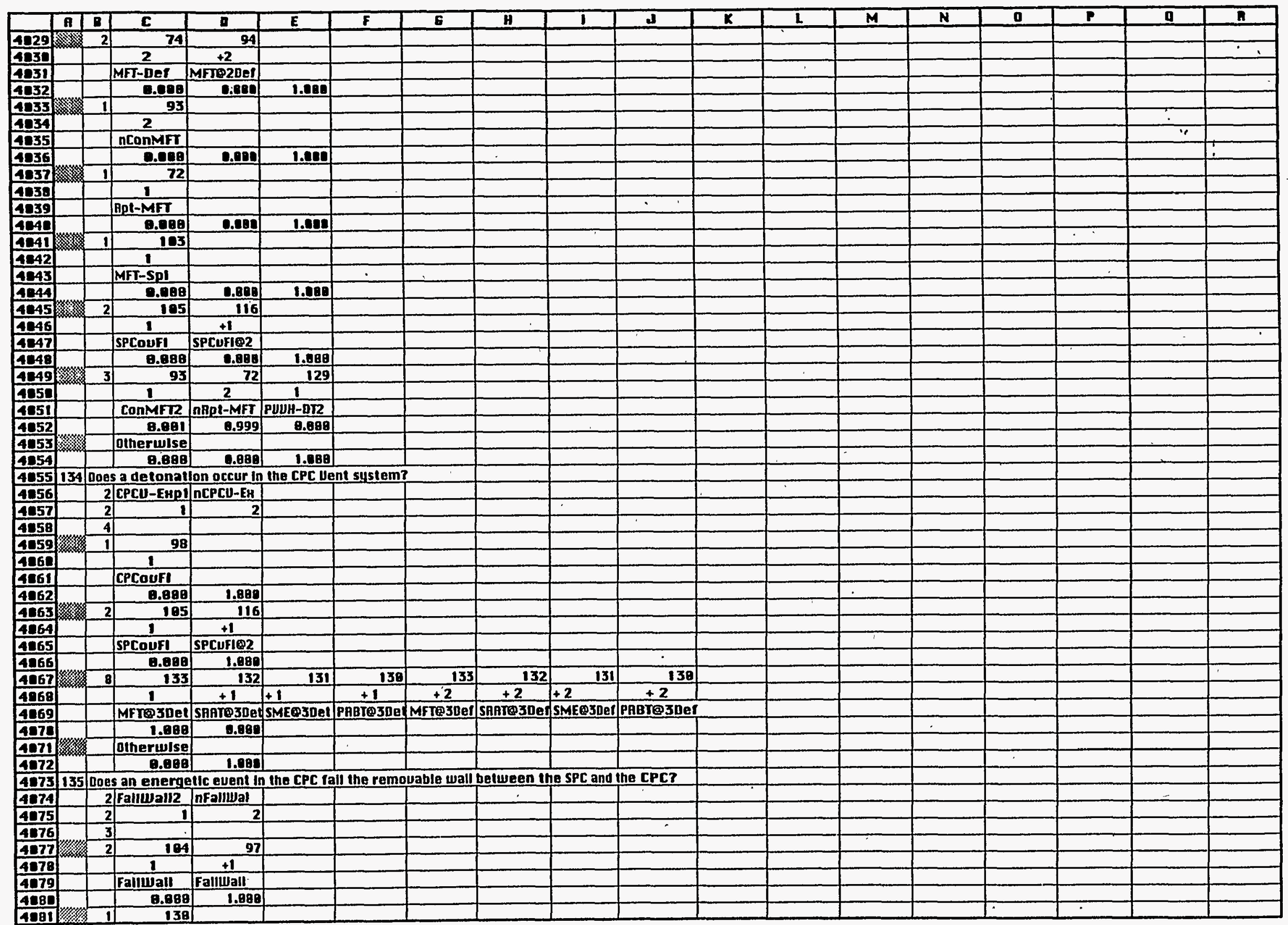


APPENDIH A

ACCIDENT PAOGRESSION EUENT TREE FOR DEFENSE WRSTE PROCESSING FACILITY - MODE C - PAODUCTION UERSION

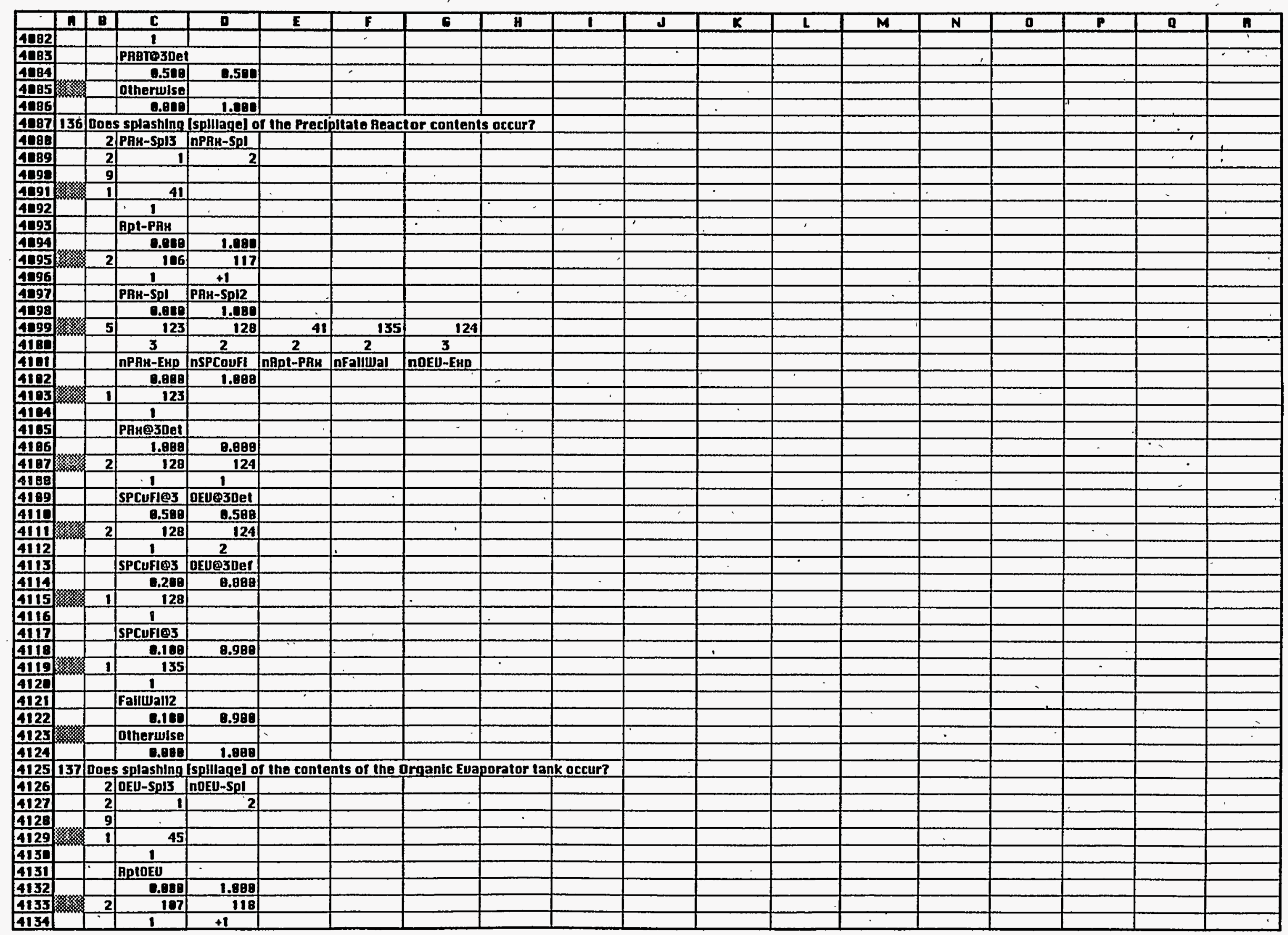


APPENDIK A

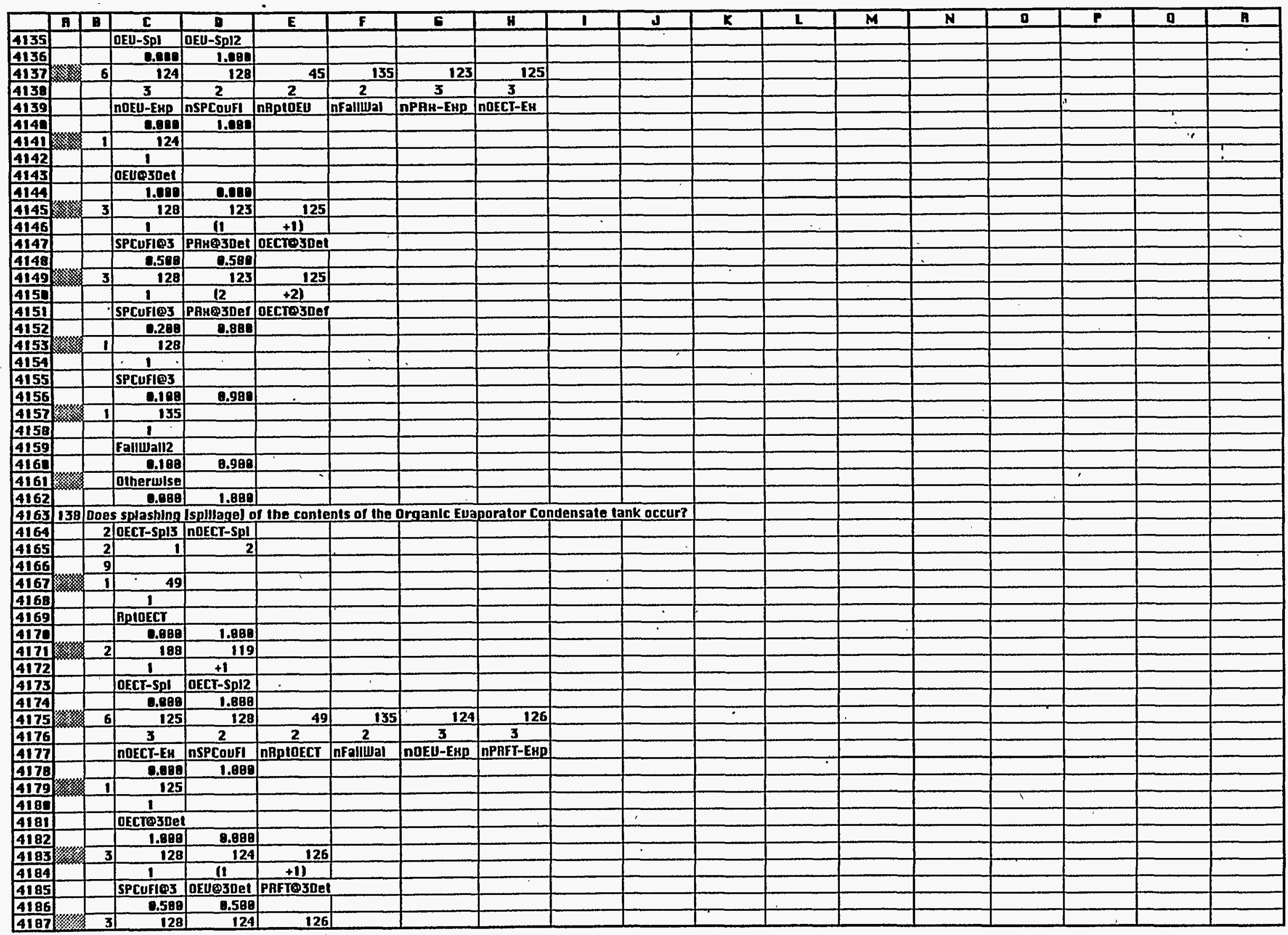




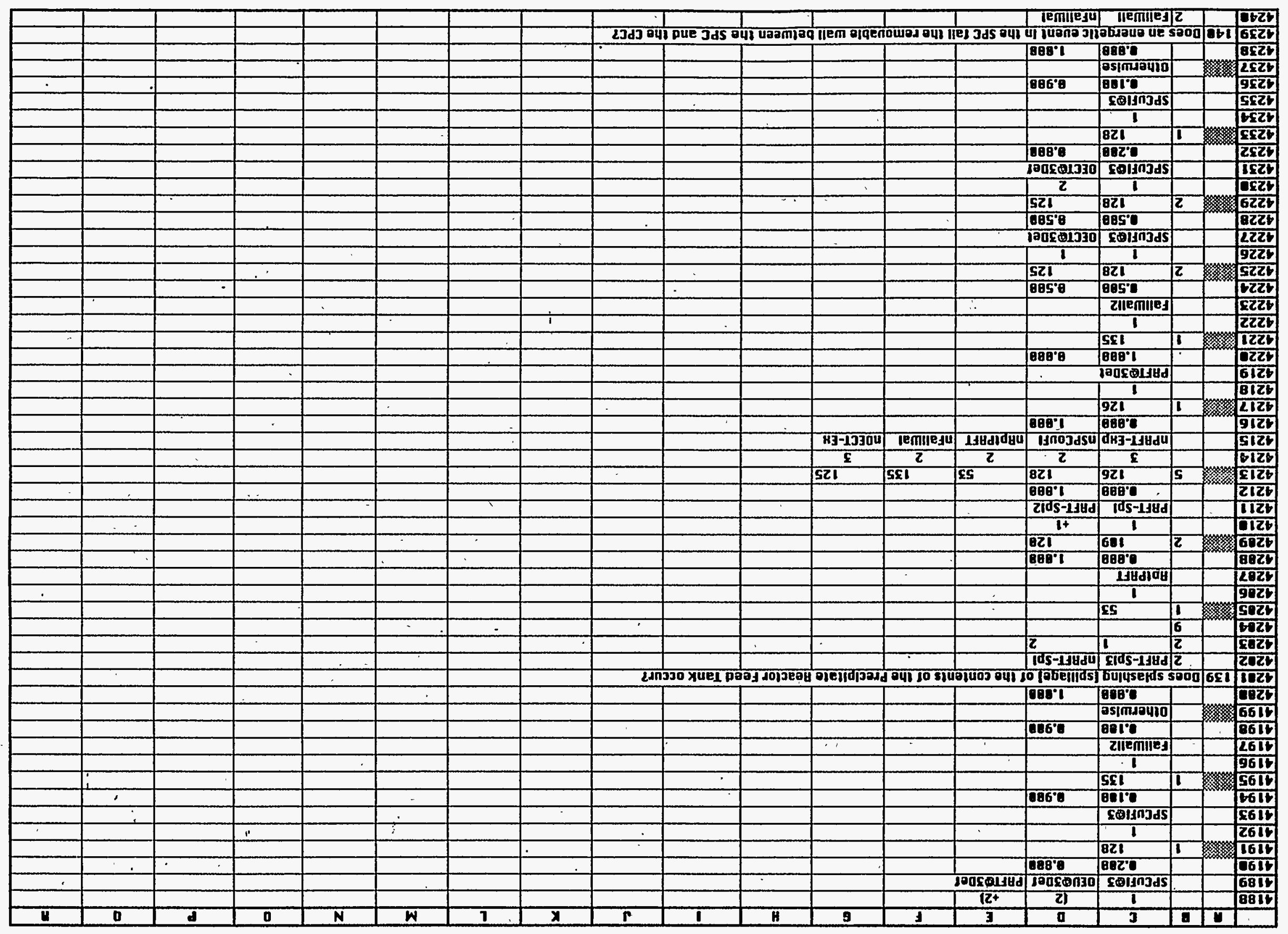

$88 \varepsilon$ o6ed

c118-56-4I-Jusm

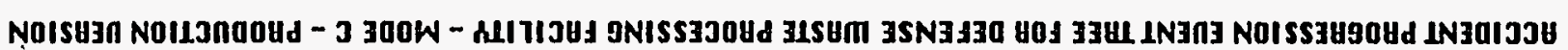

$\forall$ HIONZIdAH 
APPENDIH $\mathrm{A}$

\begin{tabular}{|c|c|c|c|c|c|c|c|c|c|c|c|c|c|c|c|c|c|c|}
\hline & n & B & C & D & $E$ & $F$ & 6 & $\mathbf{H}$ & 1 & 3 & $\bar{K}$ & $t$ & $M$ & $N$ & D & $P$ & a & A \\
\hline 1241 & & 2 & $\mathrm{I}$ & 2 & & & & & & & & & & & & & & \\
\hline 1242 & & 3 & & & & & & & & & & & & & & & & $\therefore$ \\
\hline 4243 & & 2 & 97 & 184 & & & & & & & & & & - & & & & \\
\hline 4244 & & & 1 & +1 & & & & & & & & & & & & & & \\
\hline 4245 & & & Fallwall & Fallwall & & & & & & & & & & & & & & \\
\hline 4246 & & & 0.889 & 1.180 & & & & & & & & & & & & & & \\
\hline 4247 & & 1 & 126 & & & & & & & & & & & & & & $\because$ & \\
\hline 4248 & & & 1 & & & $\approx$ & & & & & & & & & & & $\dot{2}$ & $T$ \\
\hline 4249 & & & PAFTQSDEI & & & & & & & & & & & & & & & \\
\hline 4250 & & & 0.500 & 0.588 & & & & & & & & & & & & & & \\
\hline 4251 & 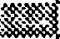 & & oinerulse & & & & & & & & & & & & & & & \\
\hline 4252 & & & 0.980 & 1.088 & & & & & & & & & & & & & $\therefore$ & \\
\hline 4253 & 141 & Do II & he cell caue & ars for the & Chemical Pr & rocess Cell o & dislodge an & & wing an ene & rgetic euen & & & & & & & & \\
\hline 4254 & & 2 & CPCoufIOZ & nCPCoufl & & & & & & & & & & & & & & \\
\hline 4255 & & 2 & 1 & 2 & & & & & & & & & & & & & & \\
\hline 1256 & & 4 & & & & & & & & & & & & & & & & \\
\hline 4257 & 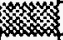 & 1 & 98 & & & & & & & & & & & & & & & 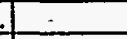 \\
\hline 4258 & & & 1 & & & & & & & & & & & & & & & \\
\hline 4259 & & & CPCOUFI & & & & & & & & & & & & & & & $\therefore$ \\
\hline 4260 & & & 8.608 & 1.800 & & & & & & & & & & & & & & \\
\hline 4261 & & 7 & 129 & 138 & 131 & 132 & 133 & 56 & 134 & & & & & & & & & \\
\hline 4262 & & & 2 & 3 & 3 & 3 & 3 & 2 & 2 & & & & & & & & & \\
\hline 4263 & & & DPUUH-EH & ПPABT-EM & ASME-EH & ISAAT-EH & nMFT-EH & niccusel & ПСРСU-EH & & & & & & & & & \\
\hline 4264 & 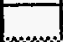 & & 8.088 & 1.080 & & & & & & & & & & & & & & \\
\hline 4265 & 濼 & 18 & 129 & 130 & 131 & 132 & 133 & 138 & 131 & 132 & 133 & 134 & & & & & & \\
\hline 4266 & & & 1 & +1 & +1 & +1 & +1 & +2 & +2 & +2 & +2 & +1 & & & & & & \\
\hline 4267 & & & PUUH-DT2 & PnBT@3De & SMEQ3Det & SARTQ 3BE & MFTQ30et & PABTQ3De & SMEQ30ef & SAATQ3DE & MFT@3Der & СРCU-ЕHPI & & 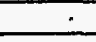 & & & & \\
\hline 4268 & & & $1.08 \mathrm{~g}$ & 0.808 & & & & & & & & & & & & & & \\
\hline 4269 & 给 & & otherwise & & & & & & & & & & & & & & & $\therefore$ \\
\hline 4270 & & & enge & 1.888 & & & & & & & & & & & & & & \\
\hline 4271 & 142 & Does & s splashing & Ispillagel o & f the Precip & pltate Beac & tor Bottoms & s Tank cont & ents occur? & & & & & & & & & \\
\hline 4272 & & 2 & PABT-SDI2 & InPABI-SpI & & & & & & & & & & & & & & \\
\hline 4273 & & 2 & 1 & 2 & & & & & & & & & & & & & & \\
\hline 4274 & & 9 & & & & & & & & & & & & & & & & \\
\hline 4275 & 慈 & 1 & 58 & & & & & & & & & & & & & & & \\
\hline 4276 & 2000 & & 1 & & & & & & & & & & & & & & & \\
\hline 4277 & 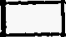 & & BPAPABT & & & & & & & & & & & & & & & \\
\hline 4278 & & $\dot{5}$ & Q.808 & 1.880 & & & & & & & & & & & & & & \\
\hline 4279 & 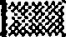 & 1 & 99 & & & & & & & & & & & & & & & \\
\hline 4280 & & & 1 & & & & & & & & & & & & & & & \\
\hline 4281 & & & PABT-SpI & & & & & & & & & & & & & & & \\
\hline 1282 & & & 0.088 & 1.080 & & & & & & & & & & & & & F & \\
\hline 4283 & 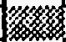 & 5 & 130 & 141 & 58 & 140 & 131 & & & & & & & & & & & \\
\hline 4284 & & & 3 & 2 & 2 & 2 & 3 & & & & & & & & & & & \\
\hline 4285 & & & IPABT-EH & nCPCOUFI & nRptPABT & nfallwal & ПSME-ЕЯ & & & & & & & & & & & \\
\hline 4286 & & & 0.008 & 1.068 & & & & & & & & & & & & & & \\
\hline 4287 & 慈 & 1 & 138 & & & & & & & & & & & & & & & \\
\hline $428 \mathrm{~B}$ & wh & & 1 & & & & & & & & & & & & & & & \\
\hline 4289 & & & PABTP 3DEl & & & & & & & & & & & & & & & \\
\hline 1290 & & & 1.080 & 8.888 & & & & & & & & & & & & & & \\
\hline 4291 & 1 & 1 & 148 & & & & & & & & & & & & & & & \\
\hline 1292 & 4 & & 1 & & & & & & & & & & & & & & & \\
\hline 4293 & it & & Falluall & & & & & & & & & & & & & & & \\
\hline
\end{tabular}


APPENDIH A

ACCIDENT PAOGAESSION EUENT IAEE FOA DEFENSE WASTE PAOCESSING FACILITY - MODE C - PRODUCTION UERSION

\begin{tabular}{|c|c|c|c|c|c|c|c|c|c|c|c|c|c|c|c|c|c|c|}
\hline & n & $D$ & $\mathbf{C}$ & D & $\boldsymbol{E}$ & $F$ & 6 & H & $I$ & $d$ & $\bar{K}$ & $L$ & $M$ & $\mathbf{N}$ & $\mathbf{0}$ & $\mathbf{P}$ & D & $\overline{8}$ \\
\hline 4294 & & & 0.589 & 0.580 & & & & & $\cdot$ & & & & & & & & & \\
\hline 4295 & 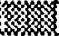 & 2 & .141 & 131 & & & & & & & & & & & & & & \\
\hline 4296 & & & 11 & 1) & & & & & & & & & & & & & & \\
\hline 4297 & & & CPCOUft@Z & SMEQ3DEl & & & & & & & & & & & & & & \\
\hline 1298 & & & 0.500 & 0.580 & - & & & & & & & $i$ & $\therefore$ & & 4 & & & \\
\hline 4299 & 燹 & $\overline{2}$ & 141 & 131 & & & & & & & & & & & 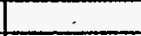 & & 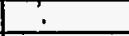 & \\
\hline 4380 & & & 11 & 2) & & & & & & & & & & & & & 1 & \\
\hline 4381 & & & CPCOUFI02 & SMEO3DEI & 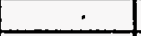 & & & & & & & & & & & & & \\
\hline 1392 & & & 0.200 & 0.890 & & & & . & & & & & & & & & & \\
\hline 4303 & & 1 & 141 & -1 & & & & & & & & & & & & & & \\
\hline 4389 & & & 1 & & & & & & & $\div$ & & & & & & & & \\
\hline 4385 & & & CPCOUFIФ2 & & & & & $\cdot$ & & & & & & & & & & \\
\hline 4386 & & & 0.180 & 0.980 & & & & & & & & & & & & & & \\
\hline 4307 & & & Otharwise & & & & & & & & & & & & & & & \\
\hline 4398 & & & 0.890 & 1.090 & & & & & & & & & & & & $\therefore$ & & \\
\hline 4399 & 143 & Does & s splashing & Isplllagel of & I the sturry & Miн Euapo & rator Tank & ontents o & ccur? & & & & & & & & & \\
\hline 4310 & & 2 & SME-Sp12 & nSME-Spl & & & & & & & & & $\dot{-}$ & & & & & \\
\hline 4311 & & 2 & 1 & 2 & & & & & & & & & & & & & & 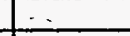 \\
\hline 4312 & & 9 & & & & & & & & & & & & & & & & \\
\hline 4313 & 纞 & 1 & 62 & & & & & 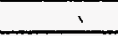 & & & & & & & & & & \\
\hline 4314 & & & 1 & & & & & & & & & & & & & & & \\
\hline 4315 & & & BpISME & & & & & & & & & & & & & & & \\
\hline 4316 & & & 0.680 & 1.080 & & & & 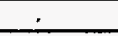 & & & & & & & & & & \\
\hline 4317 & 8 & 1 & 180 & & & $\therefore$ & & & & & & & & & & & & \\
\hline 4318 & & & 1 & & & & & & & & & & & & & & & . \\
\hline 4319 & & & SME-Spl & & & & - & & & & & & $\therefore$ & & & & & \\
\hline 4320 & & & Q.png & 1.880 & & & & & & & & & & & & & & \\
\hline 4321 & & 5 & 131 & 141 & 62 & 140 & 130 & & & & & & & & & & & \\
\hline 4322 & & & 3 & 2 & 2 & 2 & 3 & & & & & & & & & & & \\
\hline 4323 & & & ПSME-EH & nCPCOUFI & ARPISME & nFaillwal & nPBBT-EH & & & & & & & & & & & \\
\hline 4324 & & & 0.800 & 1.000 & & & & & & & & & & & & & & \\
\hline 1325 & 嬨 & 1 & 131 & & & & & & & & & & & & & & & \\
\hline 4326 & & & 1 & & & & & & & & & & & & & & & \\
\hline 4327 & & & SMEO3DEt & & & & & & & & & & & & - & & & \\
\hline 4328 & & & 1.088 & 0.800 & & & & & & & & & & 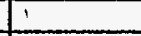 & & & & \\
\hline 4329 & 纱濨 & 2 & 141 & 130 & $;$ & & & & & & & & & & & & & \\
\hline 4333 & & 1 & 1 & 1 & & & & - & & & & & & & & & & \\
\hline 4331 & & & CPCOUFID2 & PABTE30El & & & & & & & & & & & & & & \\
\hline 4332 & & & 0.580 & 0.580 & & & & & & & & & & & & & & \\
\hline 4333 & & 2 & 141 & 130 & & & & & & & & & & & & & & \\
\hline 4334 & & & 1 & 2 & & & . & & & & & & & & & & & \\
\hline 4335 & & & CPCouflø2 & PABTQ3DEF & & $=$ & & & & & & & & & & & & \\
\hline 4336 & & & 8.280 & e.880 & & & & & & & & & & & & & & \\
\hline 4337 & 爍 & 1 & 141 & & & & & & & & & & & & & & & \\
\hline 4330 & & & 1 & & & & & & & & & & & & & & & \\
\hline 4339 & & & CPCOUFID2 & & & & & & & & & & & & & & & \\
\hline 4340 & & & 0.180 & 8.980 & & & & & & & & & & & & & & \\
\hline 4341 & & 1 & 148 & & & & & & & & & & & & & & & \\
\hline 4342 & & & 1 & & & & & & & & & & & & & & & \\
\hline 4343 & & & Fallwall & & & & & & & & & & & & & & & \\
\hline 4344 & & & 0.100 & 0.980 & & - & - & & & & & & & & & & & \\
\hline 4345 & 絲 & & Otherwlse & & & & & & & & & & & & & & & \\
\hline 4346 & 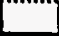 & 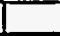 & 0.088 & 1.888 & & & & & & & & & & & & & & \\
\hline
\end{tabular}




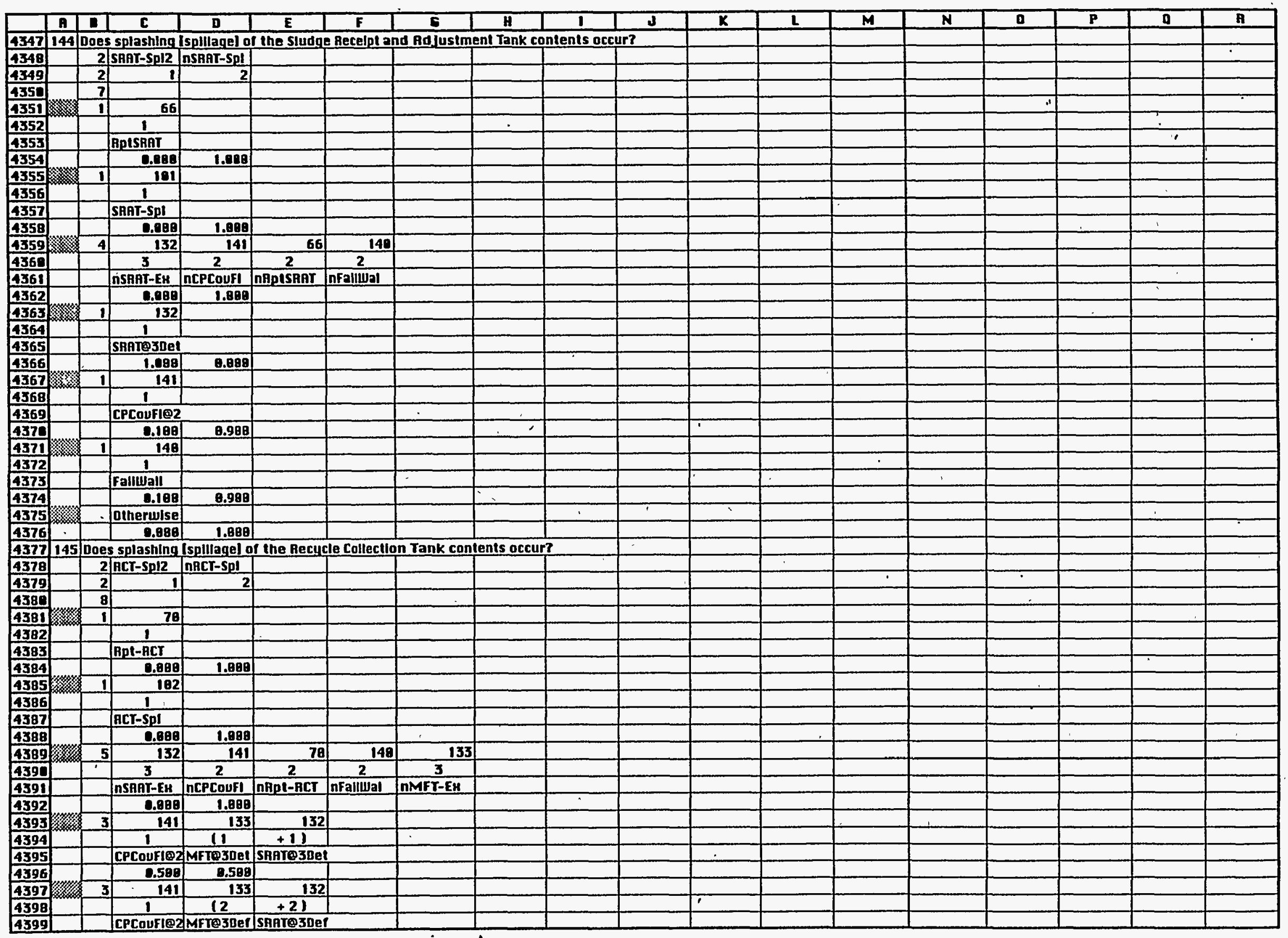




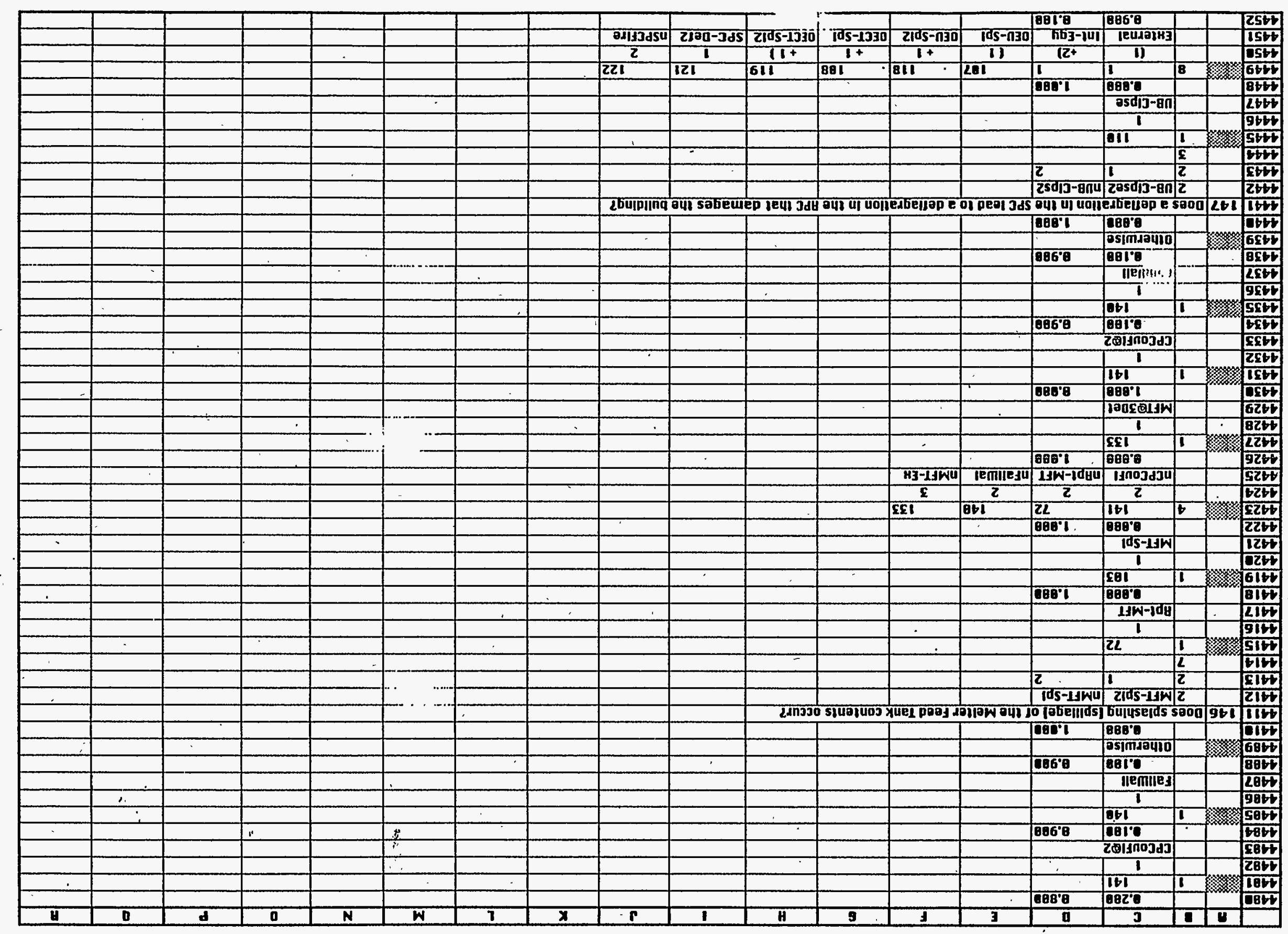

bec obed

รL16-56-HL-J45T

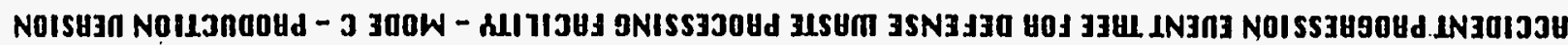

H HIONZMdU 


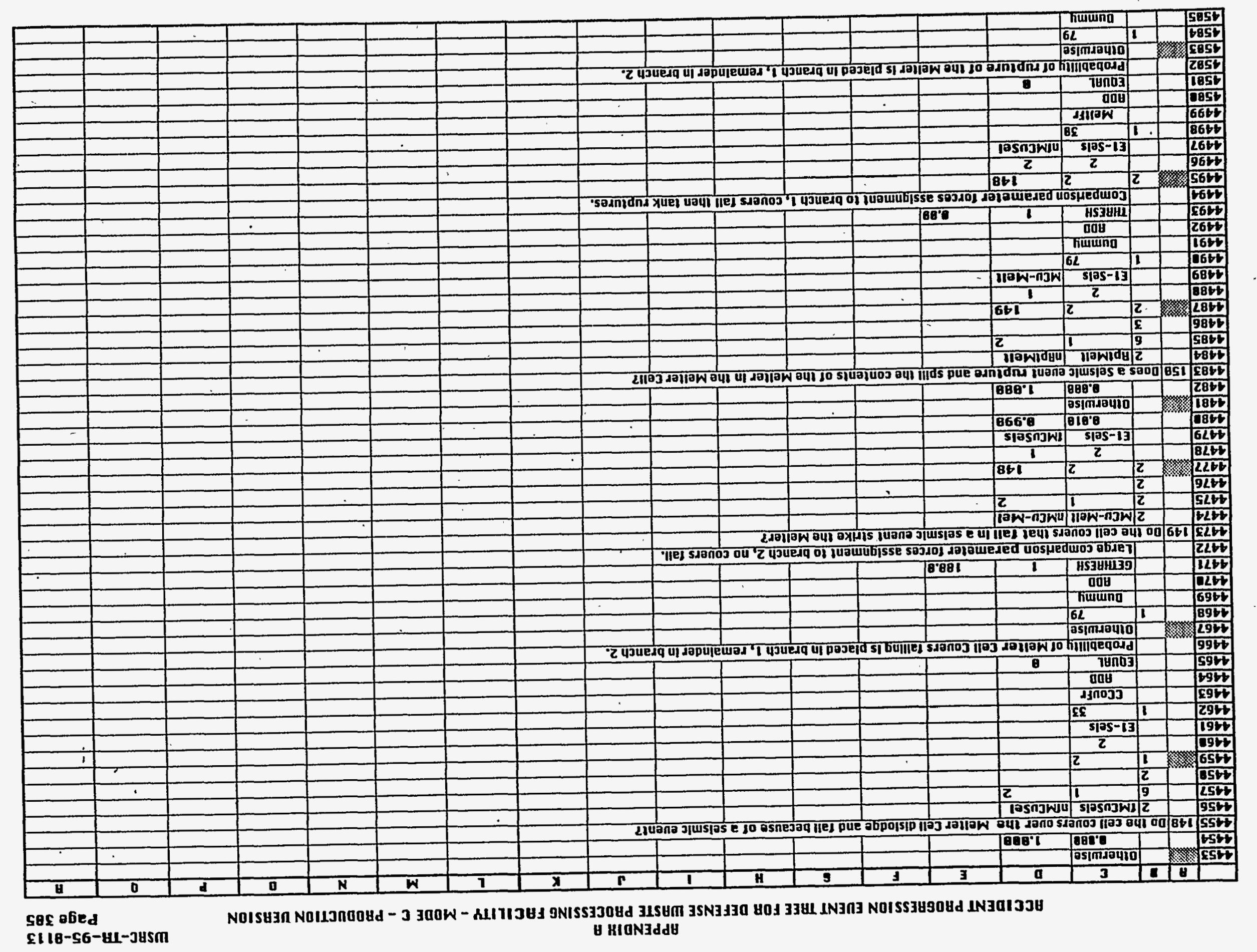




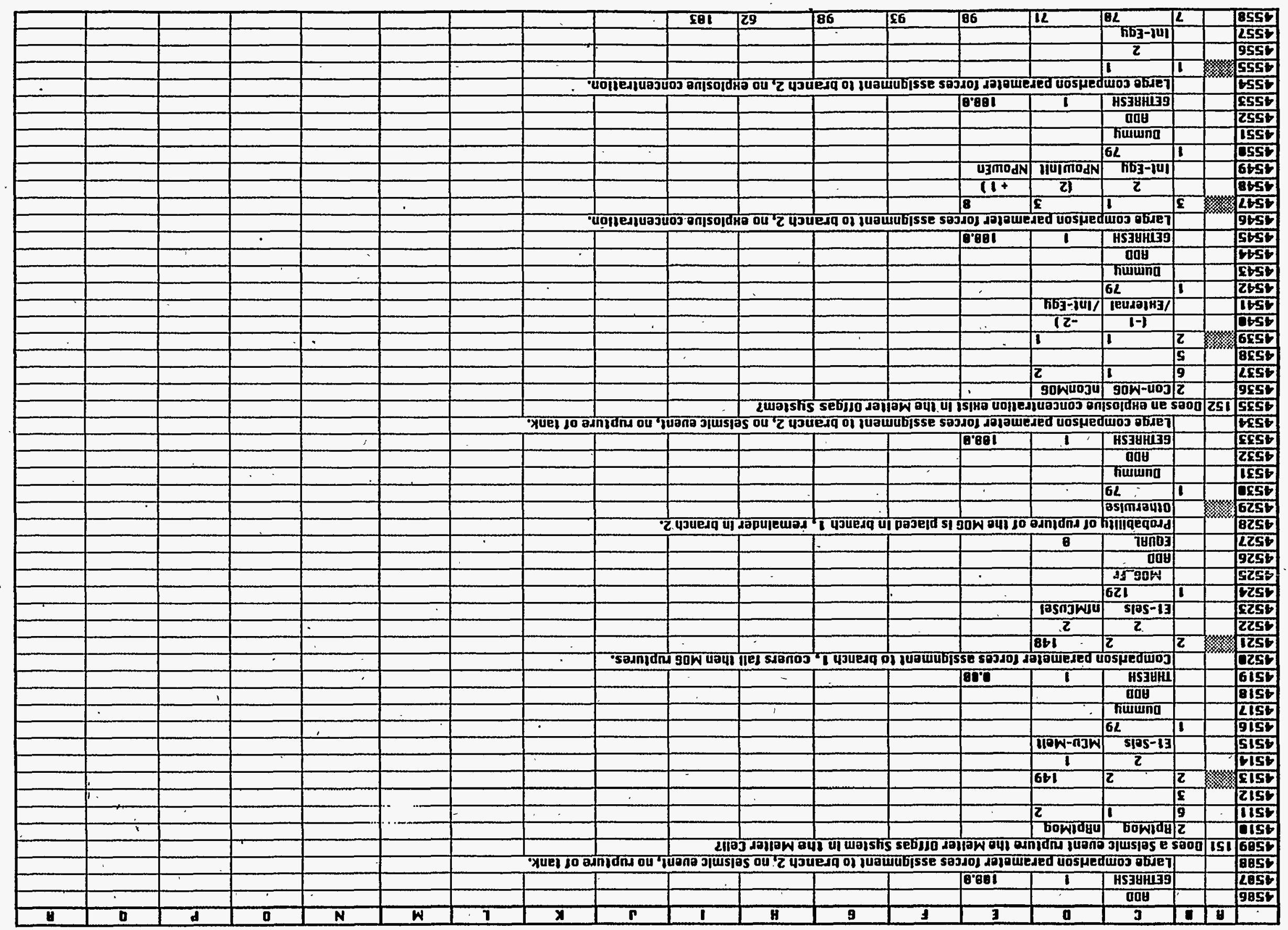

98E abed 
APPENDIH A

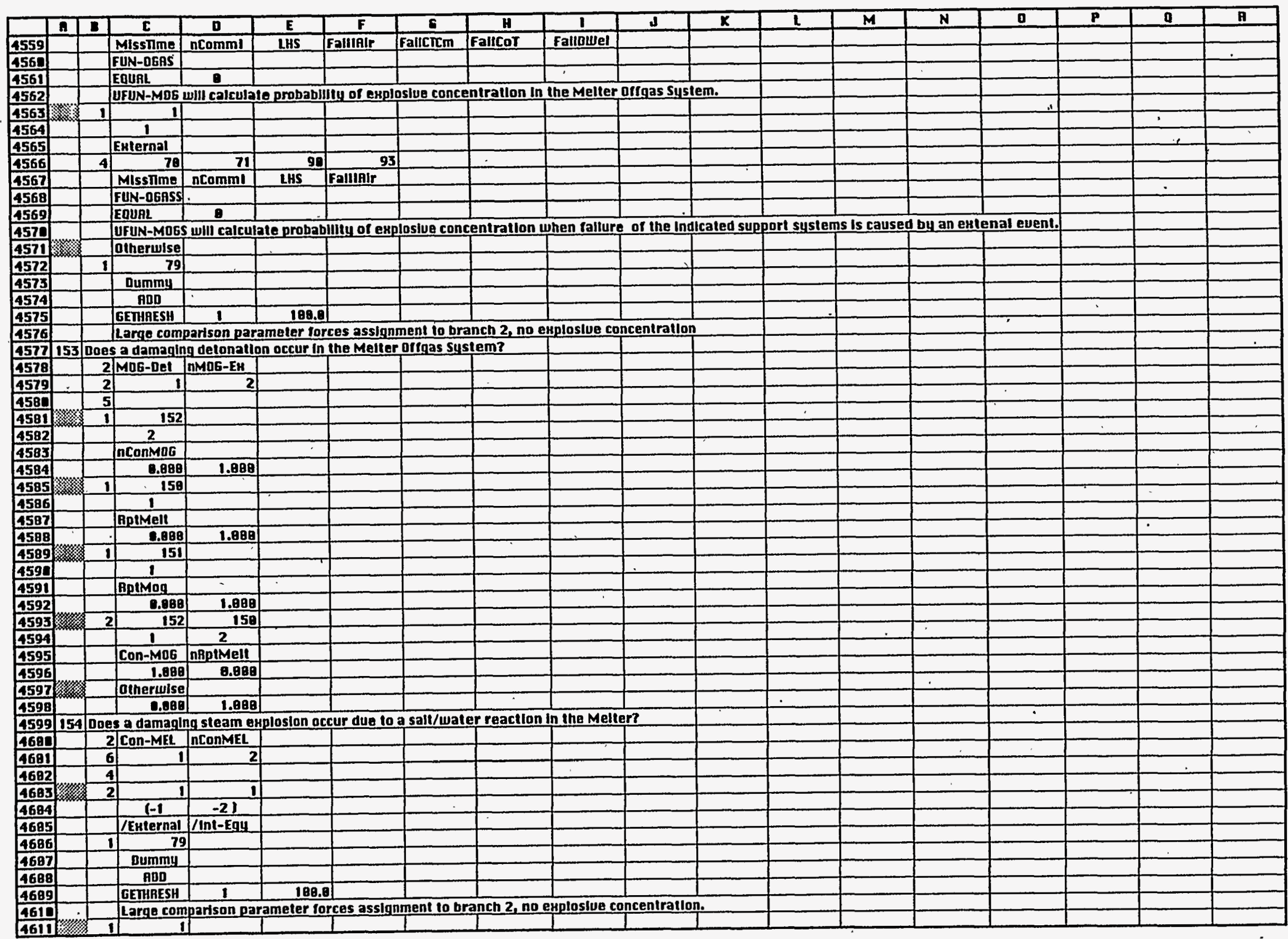




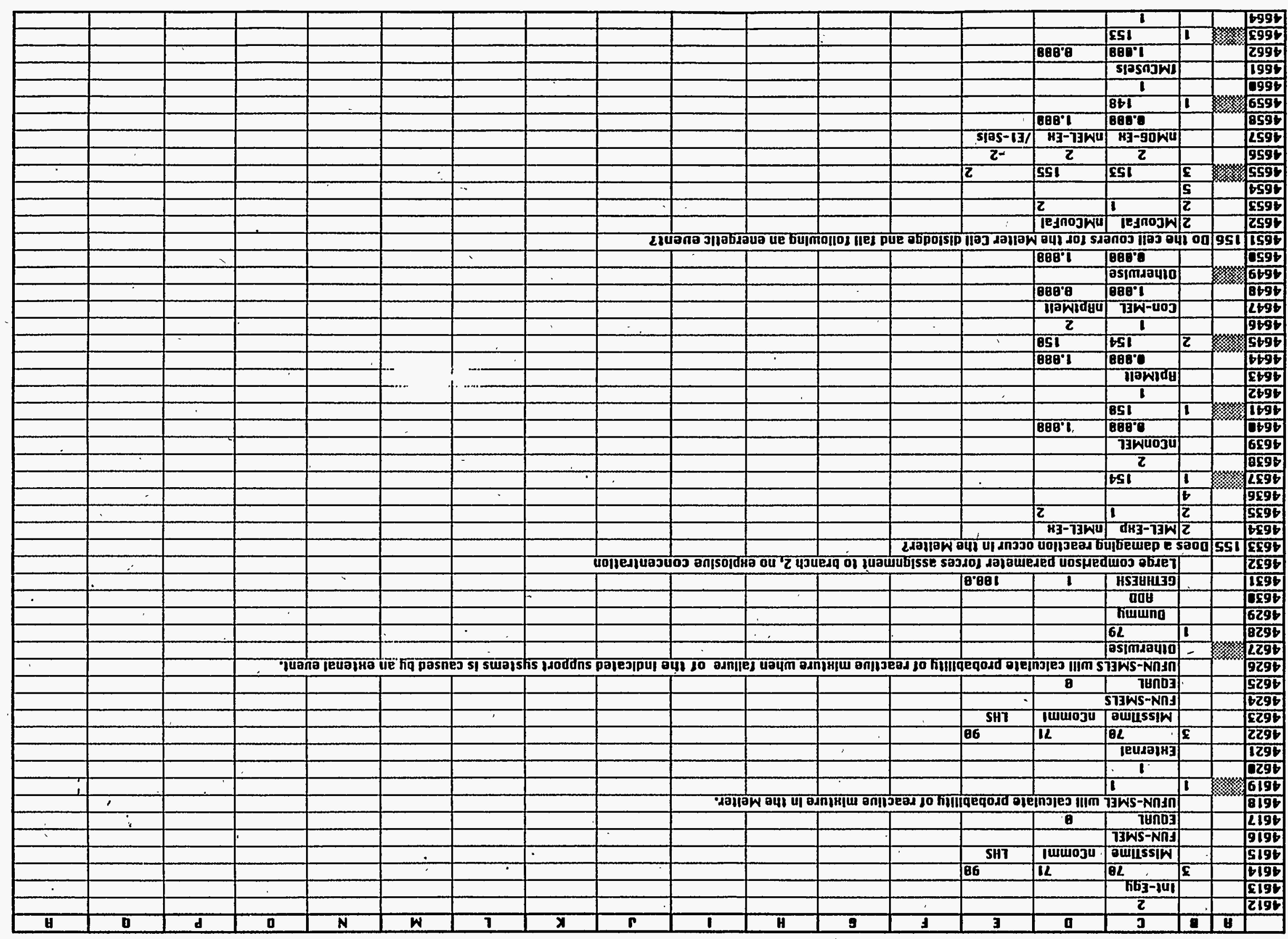

Boc abed

ร116-56-41-345m

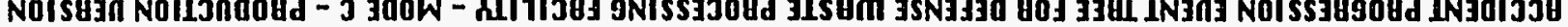

H HIONBdAt 
APPENDIH Q

GCCIDENT PAOGRESSION EUENT TREE FOR DEFENSE WASTE PROCESSING FACILITY - MODE C - PRODUCTION UERSION

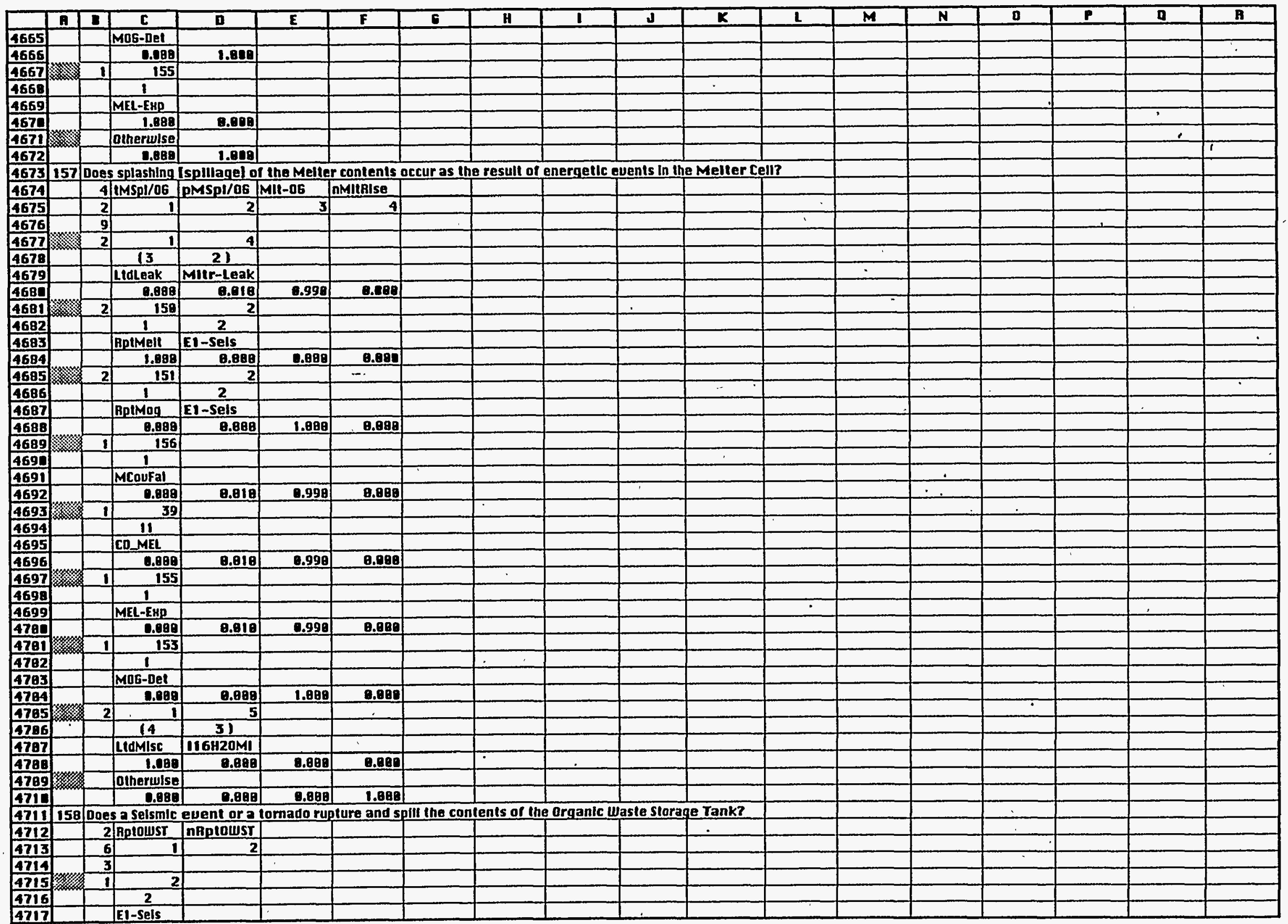




\begin{tabular}{|c|c|c|c|c|c|c|c|c|c|c|c|c|c|c|c|c|c|c|}
\hline & A & 旦 & $\boldsymbol{c}$ & D & $E$ & $F$ & $\overline{\mathbf{c}}$ & $H$ & 1 & I & $\bar{x}$ & $\underline{L}$ & $\bar{M}$ & N & 0 & $P$ & a & B \\
\hline 4710 & & 1 & 31 & & & & & & & & & & & & & & & \\
\hline 4719 & & & OWSIFr & & & & & & & & & & & & & & & 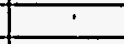 \\
\hline 1721 & & & ABD & & & & & & & & & & & & & & & \\
\hline 1721 & & & EQUARL & e & & & & & & & & & & & & & & \\
\hline 4722 & & & Probablilt & y of rupture & of the Org & tanlc Waste & Storage ta & nk Is placec & d In branch & 1, remain & der In branc & $\operatorname{ch~} 2$. & & & & & & \\
\hline 1723 & 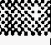 & 1 & 2 & & & & & & & & & & & & & & & \\
\hline 4724 & & & 3 & & & & & & & & & & & & & & 1 & \\
\hline 4725 & & & E2-Wind & & & & & & & & & & & & & & & \\
\hline 4726 & & 1 & & & & & & & & & & & & & & & & \\
\hline 1727 & & & aWSIr & & & 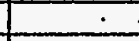 & & & & & & & & & & & & \\
\hline 4728 & 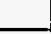 & & ADD & & & & & & & & & & & & & $\dot{\square}$ & & \\
\hline 4729 & & & EQUAL & E & & & & & & & & & & & & & & \\
\hline 4730 & & & Fragilley o & of the Organ & Ic waste St & torage Tank & Is placed in & branch I, & remalnder & in branch & & & & & & & & \\
\hline 1731 & 絸 & & otherwise & - & 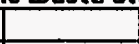 & (c) & 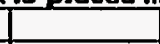 & s. & & & & & & & & & & \\
\hline 1732 & 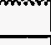 & 1 & & & & & & & & & & & & & & & & $I$ \\
\hline 4733 & $\cdot$ & & Dummy & & & & & & & & & & & & & & & \\
\hline 4734 & & & ADD & & & & & & & & & & & & & & & \\
\hline 1735 & & & GETHRESH & 1 & 198.0 & & & & & & & & & & & & & \\
\hline 4736 & & & Large com & apartson par & ameler ior & ces assign & meni lo bra & $\operatorname{nen} 2$, no S & elsmic ave & nt, no rupt & lure of tank & & & & & & & \\
\hline 1737 & 159 & Does & 8 an esplos & slue concent & fallon accu & umulato in & the oraanic & Wasle Stor & rage Tank? & & & & & & & & & \\
\hline 4738 & & 2 & Conowst & InConowst & & & & & & & & & & & & & & \\
\hline 4739 & & 6 & 1 & & & & & & & & & & & - & & & & \\
\hline 4741 & & 4 & & & & & & & & & & & & & & & & \\
\hline 1741 & x & 2 & & 1 & & & & & & & & & & & & & & \\
\hline 4742 & & & $(-1$ & -21 & & & & & & & & & & & & & & \\
\hline 4743 & & & fExternal & /nin-Eq4 & & & & & & & & & & & & & & \\
\hline 1724 & & 1 & 79 & & & & & & & & & & & & & & & \\
\hline 4745 & & & Dummy & & & & & & & & & & & & & & & \\
\hline 1746 & & & ADO & & & & & & & & & & & & & & & \\
\hline 4747 & & & GETHRESH & 1 & 180.0 & & & & & & & & & & & & & \\
\hline 4741 & & & Large com & aparison par & ameter for & reses assign & ment to bra & $\operatorname{lnch} 2$, no e & aploslue co & oncentratic & & & & & & & & \\
\hline 1749 & 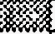 & 1 & & & & & & & & & & & & & & & & \\
\hline 1750 & & & 2 & & & & & & & & & & & & & & & \\
\hline 1751 & & & Int-Eg|l & & & & & & & & & & & & & & & \\
\hline 4752 & & 7 & & 61 & 67 & 94 & 70 & 71 & 98 & & & & & & & & & \\
\hline 4753 & & & FaliNPWur & FallePur & Falin2IK & FallbikN2 & Missnme & ncomml & LHS & & & & & & & & & \\
\hline 4754 & & & FUN-DUSST & & & & & & & & & & & & & & & \\
\hline 4755 & & & EQUAL & e & & & & & & & & & & & & & & \\
\hline 9756 & > & & UFUN-DWS & T wlll calcul & ale probab & flilly of ekp & loslue conc & entration in & n the oqgar & nic waste S & Storage Tant & & & & & & & \\
\hline 1757 & & 1 & & & & & & & & & & & & & & & & \\
\hline 9758 & & & 1 & & & & & & & & & & & & & & & \\
\hline 4759 & & & Esternal & & & & & & & & & & & & & & & \\
\hline 9760 & & 5 & 67 & .99 & 70 & 71 & 90 & & & & & & & & & & & \\
\hline 4761 & & & Falin2/K & FallBIkN2 & Mlssimme & ncomm! & LHS & & & & & & & & & & & \\
\hline 1762 & & & FUN-OWST & & & & & & & & & & & & & & & \\
\hline 4763 & & & EQURL & 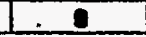 & & & & & & & & & & & & & & \\
\hline 9764 & sine & & UFUN-OWS & iss will calce & fate probal & bllily of eH & ploslue con & centrallon & when rallu & of the It & Indicaled su & ort & sis & by & nal eue & & & \\
\hline 4765 & 䜌 & & Otherwise & & & & & & & & & & & & & & & \\
\hline 1766 & & 1 & 79 & & & & & & & & & & & & & & & \\
\hline 4767 & & & \begin{tabular}{|l|} 
Dummy \\
\end{tabular} & & & & & & & & & & & $\div$ & & & & \\
\hline 4769 & & & $A 20$ & & & & & & & & & & & & & & & \\
\hline 4769 & & & GETHAESH & 1 & 188.0 & & & & & & & & & & & & & \\
\hline 4770 & & & Large cam & apartson par & ameler for & rees assign & ment lo bra & nch 2, noe & Hploslue co & ancent & & & & & & & & \\
\hline
\end{tabular}




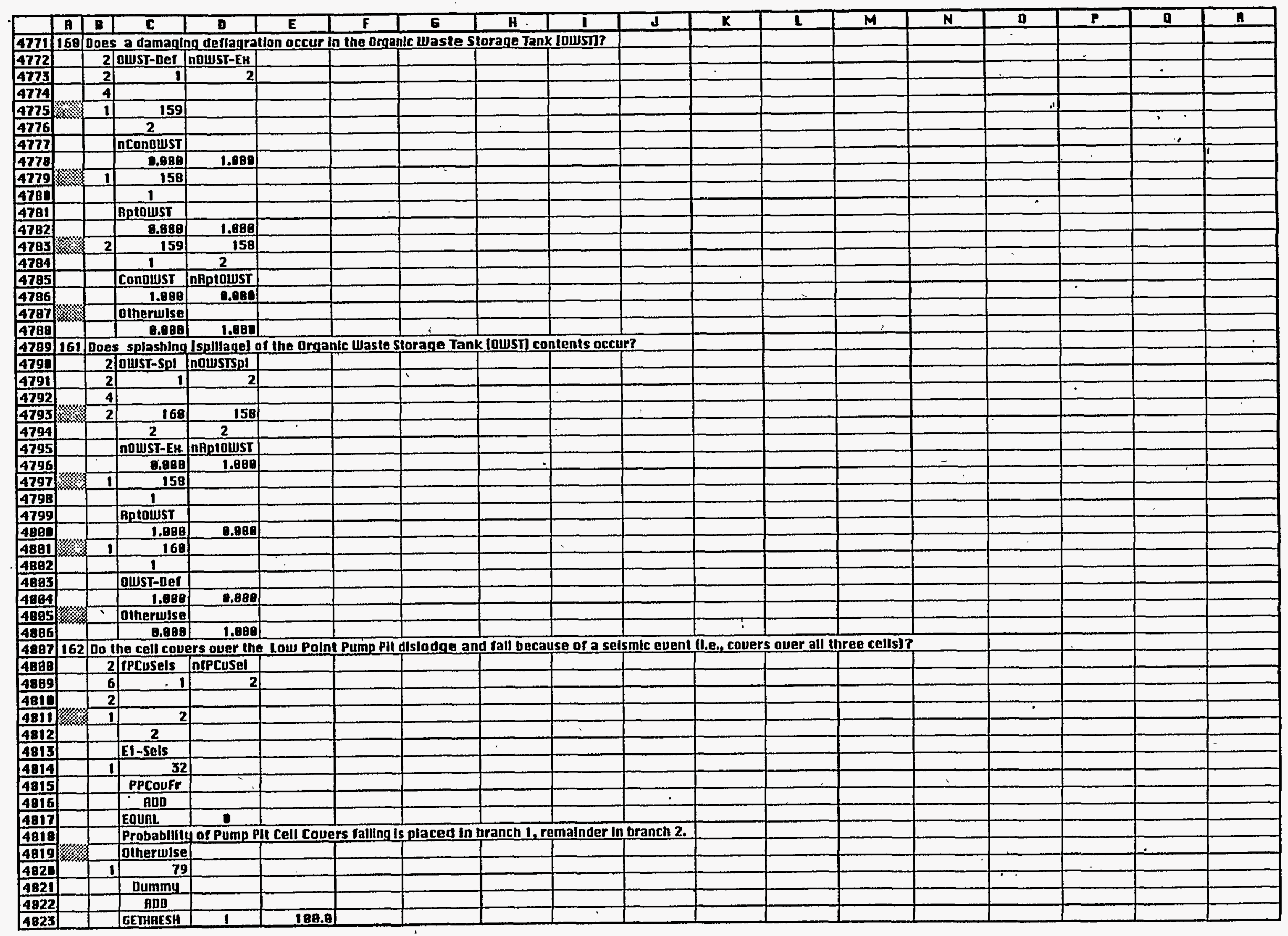




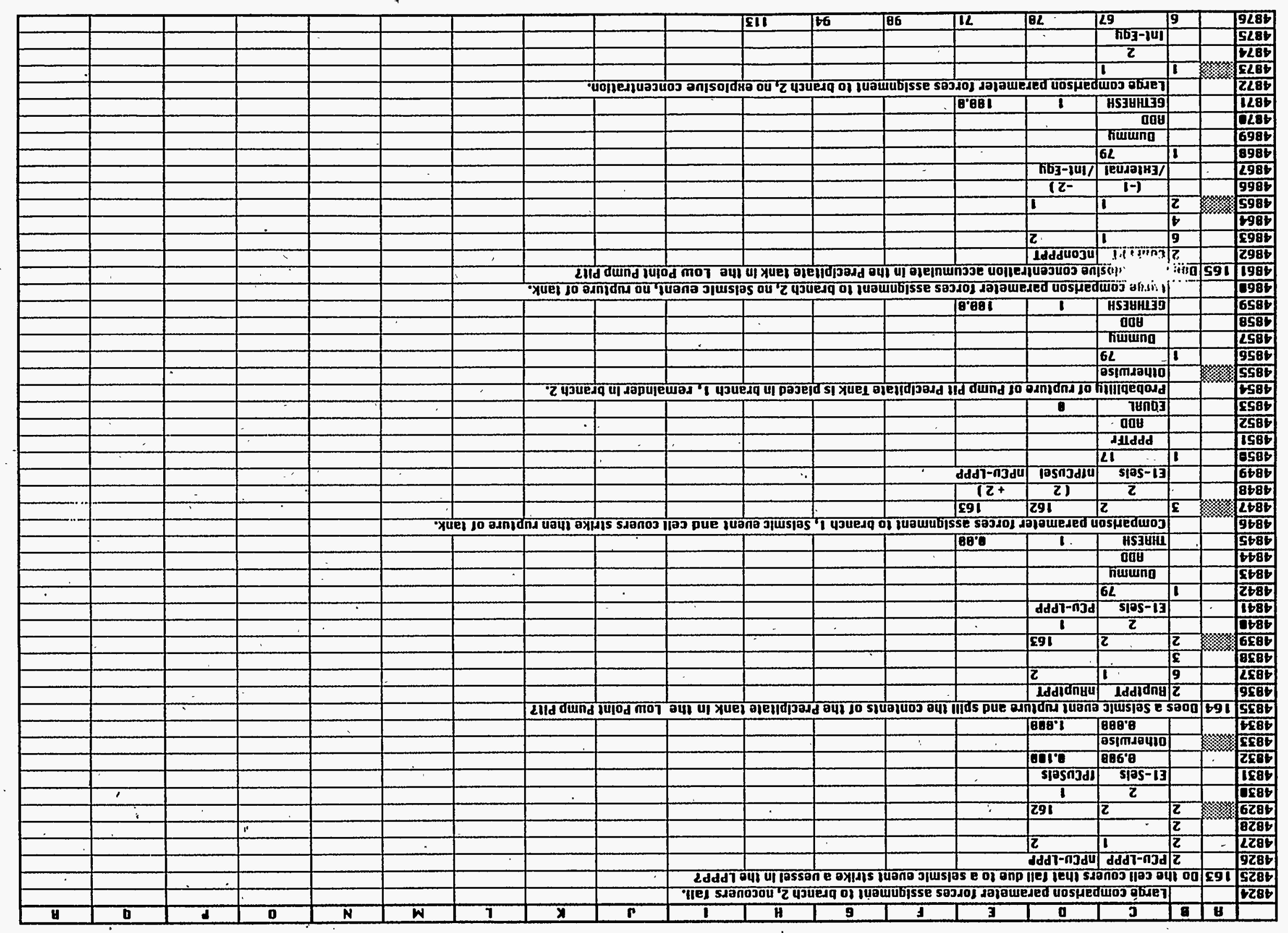

ZIE abed

ع118-56-412-J45m

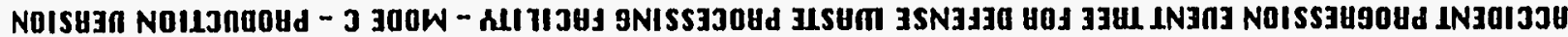

6 HIONZMdG 
APPENDIH A

\begin{tabular}{|c|c|c|c|c|c|c|c|c|c|c|c|c|c|c|c|c|c|c|}
\hline & ค & 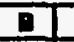 & $\bar{c}$ & D & $E$ & $F$ & 6 & H & $I$ & J & $\bar{x}$ & L & $M$ & $N$ & 0 & $\mathbf{P}$ & a & n \\
\hline 4877 & & & Falin2Ik & MIssTime & ncomml & LHS & FallbikN2 & FallPPN2 & & & & & & & & & & \\
\hline 4878 & & & FUN-PPPT & & & & & & & & & & & & & & & \\
\hline 4879 & & & Equal & E & & & & & & & & & & & & & & \\
\hline 488 & & & UFUN-PPPT & will calculat & le probabil & Ily of expl & Inslue conce & entratlon in & the Low $\mathrm{f}$ & OInt Pump & Pit Preclpit & ate Tank. & & & & & & \\
\hline$\frac{4889}{9897}$ & 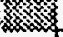 & 1 & 1 & & & & & & & & & & & & & & & \\
\hline \begin{tabular}{|l|l|}
4882 \\
19027
\end{tabular} & & & 1 & & & & & & & & . & & & & & & 7 & \\
\hline \begin{tabular}{|l|}
4883 \\
4884 \\
\end{tabular} & & 6 & $\begin{array}{r}\text { External } \\
67\end{array}$ & 70 & 71 & 98 & 94 & 113 & & & & & & & & & & \\
\hline 4885 & & & FallN2IK & MIssTime & ncomml & LHS & FallBikN2 & FallPPN2 & & & & & & & & & & \\
\hline 4886 & & & FUN-PPPIS & & & & & & & & & & & & & & & \\
\hline 4887 & & IE & EQUAR & e & & & & & & & & & & & & & & \\
\hline 4888 & & & UFUN-PPPT & 5 will calcule & late probab & IIIty of oHs & plosive conc & centrallon & When railu & re of the in & ndicated su & pport syste & ins is cause & d by a sels & imic euent. & & & \\
\hline 4889 & & & Ditherwise & & & & & & & & & & & & & & & \\
\hline 489 & & 1 & & & & & & & & & & & & & & & & \\
\hline 44891] & & & Dummy & & & & & & & & & & & & & & & \\
\hline 4892 & & If & ADD & & & & & & & & & & & & & & & \\
\hline 4893 & & & GEMHRESH & 1 & 180.0 & & & & & & & & & & & & & \\
\hline 4894 & & & Large comp & partson para & ameler for & ces assign & ment to bra & $\operatorname{anch} 2$, noe & Hplosive c & oncentrallo & & & & & & & & \\
\hline 4895 & 166 & Does & 5 a damagli & ing detonallo & on/deflagr & tion orcul & In the Prec & clpitate tan & $2 k$ In the $L$ & W Polnt Pu & Imp PII? & & & & & & & \\
\hline 4896 & & $3 / 1$ & PPPMEl & PPPDEI & пPPPTEнP & & & & & & & & & & & & & \\
\hline 4897 & 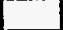 & 2 & & 2 & & & & & & & & & & & & & & \\
\hline 4898 & 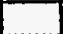 & 4 & & & & & & & & & & & & & & & & \\
\hline 4899 & & 1 & 165 & & & & & & & & & & & & & & & \\
\hline 498 & & I & 2 & & & & & & & & $L^{\circ}$ & & & & & & & \\
\hline [4981] & & It & nConpPPY & & & & & & & & & & & & & & & \\
\hline 4982 & & & 8.88日 & 8.880 & $1.88 \mathrm{~B}$ & & & & & & & & & & & & & \\
\hline 4983 & & 1 & 164 & & & &. & & & & & & & & & & & \\
\hline 4984 & & & 1 & & & & & & & & & & & & & & & \\
\hline 4985 & & I & AuptPPI & & & & & & & & & & & & & & & \\
\hline 49886 & & & 8.989 & Q.809 & 1.898 & & & & & & & & & & & & & \\
\hline 4987 & 13 & 2 & 165 & 164 & & & $\therefore$ & & & & & & & & & & & \\
\hline 4980 & & & 1 & 2 & & & & & & & & & & & & & & \\
\hline 4989 & & & Conpppi & nAuptPPY & & & & & & & & $\cdot$ & & & & & & \\
\hline 4910 & & & 8.081 & 0.999 & 0.808 & & & & & & & & & & & & & \\
\hline 4911 & 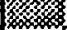 & & Ditherwise & & & & & & & & & & & & & & & \\
\hline 4912 & & & 8.68B & 8.080 & $1.88 \mathrm{~B}$ & & & & & & & & & & & & & \\
\hline 4913 & 167 & Does & s splasting & Isplllagel of & t the Pump & Plt Precipl & IIate Tankc & Contents oce & cur? & & & & & & & & & \\
\hline 4914 & & 2 & ppprspl & nPPPISp! & & & & & & & & & & & & & & \\
\hline 4915 & & 2 & 1 & $\mathbf{2}$ & & & & & & & & & & & & & & \\
\hline 4916 & & 4 & & & & & & & & & & & & & & & & \\
\hline 4917 & 㸚 & 2 & 166 & 164 & & & & & & & & & & & & & & \\
\hline 4918 & & & 3 & 2 & & & & & & & & & & & & & & \\
\hline 4919 & & 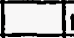 & InPPPIERP & nRuptPPT & & & & & & & & & & & & & & \\
\hline 4920 & & & 8.880 & $1.88 \mathrm{~g}$ & & & & & & & & & & & & & & \\
\hline 4921 & 濨 & 7 & 164 & & & & & & & & & & & & & & & \\
\hline 4922 & & & 1 & & & & & & & & & & & & & & & \\
\hline 4923 & & & ARupIPPT & & & & & & & & & & & & & & & \\
\hline 4924 & & & $1.88 \mathrm{~g}$ & C.89e8 & & & & & & & & & & & & & & \\
\hline 4925 & & 1 & 166 & & & & & & & & & & & & & & & \\
\hline 4926 & & & 1 & & & & & & & & & & & & & & & \\
\hline 4927 & & & PPPTER & & & & & & & & & & & & & & & \\
\hline 4928 & & & 1.880 & B.800 & & & & . & & & & & & & & & & \\
\hline 4929] & & & Dotherwise & & & & & & & & & & & & & & & \\
\hline
\end{tabular}




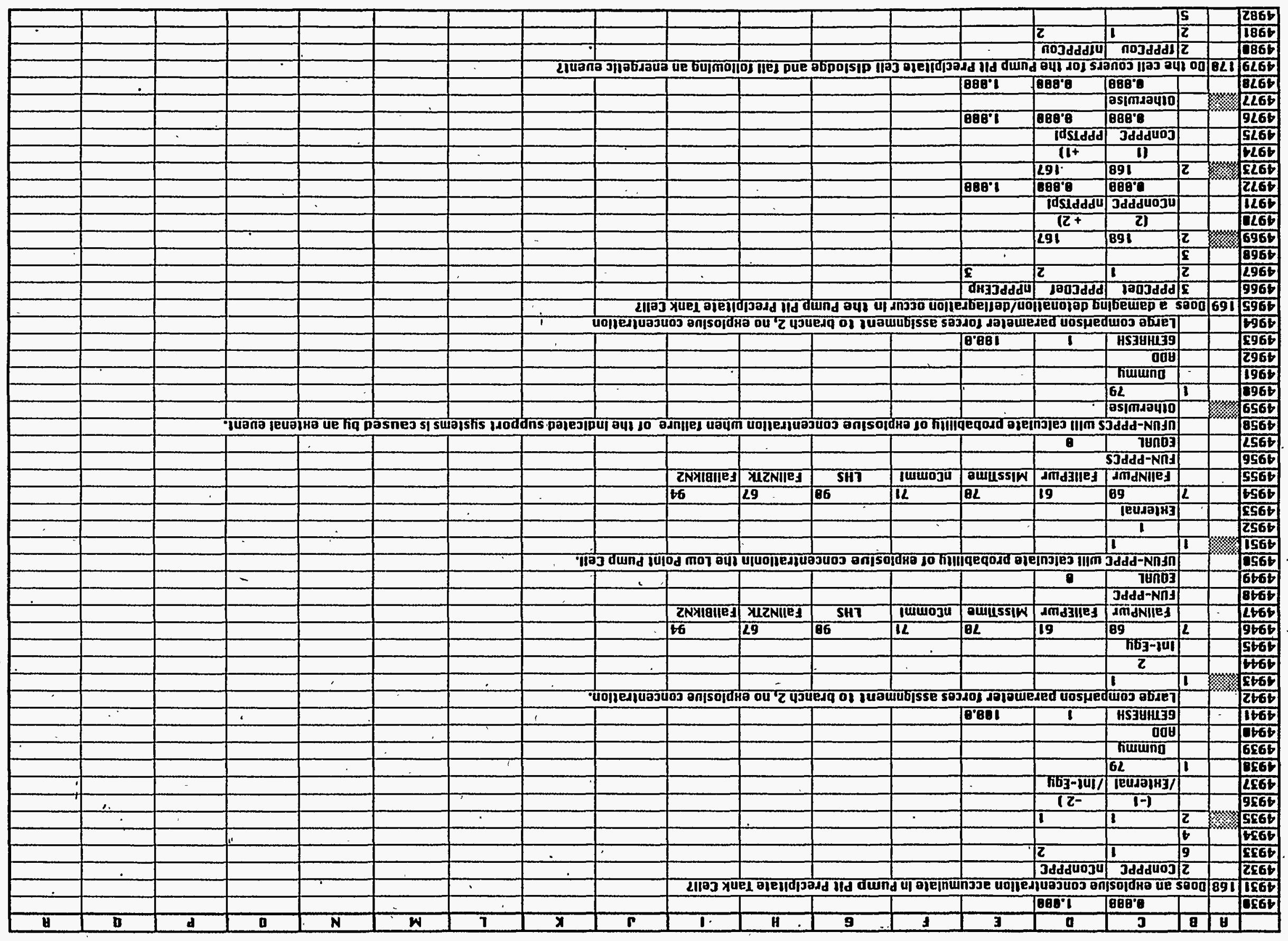

bI5 abed

S!18-56-HL-J4Sm

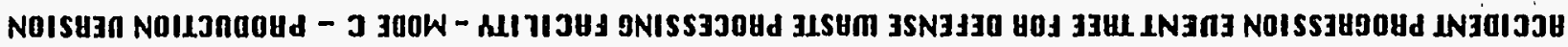

H HIONJddU 


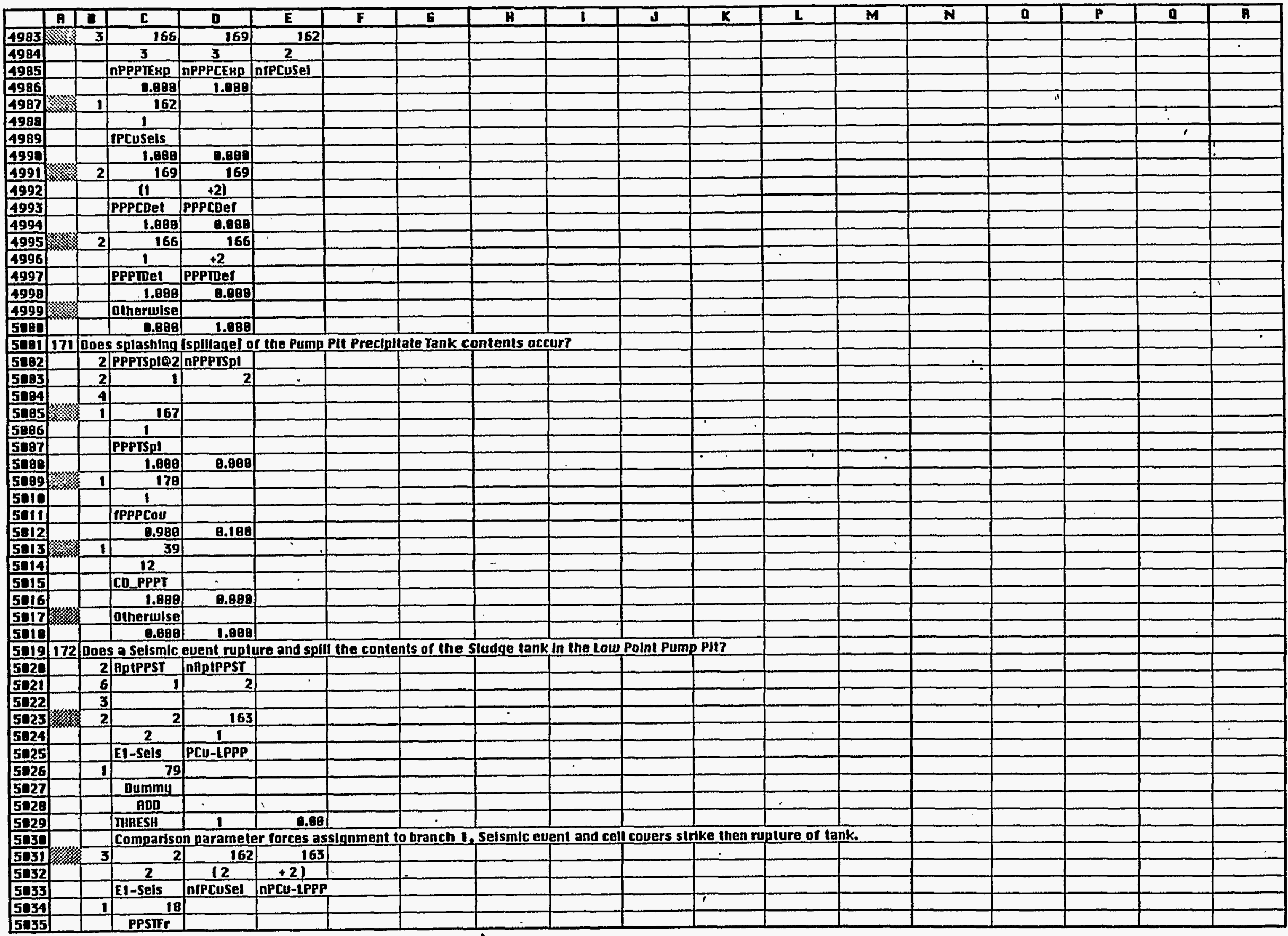




\begin{tabular}{|c|c|c|c|c|c|c|c|c|c|c|c|c|c|c|c|c|c|c|}
\hline & A & B & $\bar{C}$ & $\overline{0}$ & E & $F$ & 6 & $\bar{H}$ & I & $J$ & $\bar{K}$ & L & $M$ & $N$ & 0 & $p$ & a & A \\
\hline 5036 & & & $\mathrm{ADO}$ & & & & & & & & & & & & & & & \\
\hline 5037 & & & EQUAL & B & & & & & & & & & & & & & & \\
\hline 5038 & & & Probabillity & of ruplure & of Pump PI & If Sludge Ta & IK Is placed & d in branch & 1, remaind & er In branch & 2. & & & & & & & \\
\hline 5039 & & & Otherwise & & & & $\frac{T}{1}$ & & & & & & & & & . & & \\
\hline 5040 & & 1 & 79 & & & & & & & & & & & & it & & & \\
\hline 5241 & & & Durmmy & & & & & & & & & & & & & & \pm & \\
\hline 5042 & & & $\mathrm{ADD}$ & & & & $\therefore$ & & & 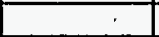 & & & 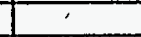 & & & & 1 & \\
\hline 5043 & & & GETHAESH & 1 & 100.0 & & & & & & & & & & & & - & 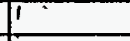 \\
\hline 5044 & & & Large comp & parison par & amoler tor & ces assignim & ent to bran & $\operatorname{nch} 2$, no Se & Ismlc even & $t$, no ruptur & re of tank. & & & & & & & \\
\hline 5045 & 173 & Does & an erplosil & ve concentr & rallon accu & Imulate in P & ump pit Sluc & Idge Tank? & & & & & & & & & & \\
\hline 5046 & & 2 & ConPPST & nConPPST & & & & & & & & & & & & & & \\
\hline 5847 & & $\overrightarrow{6}$ & 1 & 2 & & & & & & & & & & & & & & \\
\hline 5048 & & 4 & & & & & & & & & - & & & & & & i & \\
\hline 5049 & & 2 & 1 & 1) & & & & & & & & & , & & & & & \\
\hline 5050 & & & $(-1)$ & -21 & & & & & & & & & & & & & & \\
\hline 5051 & & & /Esternal & $\begin{array}{l}\operatorname{Int}-\mathrm{Eg} y \\
\end{array}$ & & & & & & & & & & & & & & \\
\hline 5052 & & 1 & 79 & & & & & & & & & & & & & & & \\
\hline 5053 & & & Dummy & & & & & & & & & & & & & & & \\
\hline 5059 & & & $\mathrm{ADO}$ & & & & & & & & & & & & & & & - \\
\hline 5055 & & & GETHAESH & 1 & 188.8 & & & & & & & & & & & & & \\
\hline 5056 & & & Large comp & parison par & ameler for & ces assignm & ent lo bran & nch 2, no es & splosive ca & ncentrallon & & 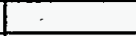 & & $=$ & $=$ & & & \\
\hline 5057 & & 1 & 11 & & & & & & & & & & & & & & & \\
\hline 5858 & & & 2 & & & & & & & & & & & & & & & \\
\hline 5059 & & & Int-Egy & & & & & & & & & & & & & & & \\
\hline 5960 & & 8 & 68 & 61 & 78 & 71 & 98 & 113 & 67 & 94 & & & & & & & & \\
\hline 5061 & & & FalinPwr & FallePwr & Missime & nComm! & LHS & FaltPPN2 & FaliN2IK & FallBIkN2 & & & & & & & & \\
\hline 5862 & & & FUN-PPST & & & & & & & & & & & & 5 & $\therefore$ & & \\
\hline 5263 & & & EQUAL & $\bar{B}$ & & & & & & & & & & & & & & \\
\hline 5064 & & & UFUN-PPST & will calcula & te probabll & IIty of esplo & sive conce & ntratlonin & The Pump P & It sluage ra & ank. & & $\therefore$ & & & &. & \\
\hline 5065 & 梦 & 1 & 1 & & & & & & & & & & & & & & & \\
\hline 5966 & & & 1 & & & & & & & & & & & & & & & \\
\hline 5067 & & & Euternal & & & & & & & & & & & & & & & \\
\hline 5068 & & 6 & 78 & 71 & 98 & 113 & 67 & 94 & & & & & & & & & & \\
\hline 5969 & & & MIssTIme & nCommi & LHS & FallPPN2 & Falin2IK & fallBIKN2 & & & & & & & & & & \\
\hline 5070 & & & FUN-PPSTS & & & & & & & & & & & & & & & \\
\hline 5071 & & & EQUAL & 8 & 5 & & & & & & & & & & & & & \\
\hline 5072 & & & UFUN-PPSTS & S will calcul & late probab & Iilty of esp & Iosiue conce & entrallon 4 & When fallur & of the ind & dicated sup & port syste & ms is & by an & flenal event. & & & \\
\hline 5073 & 8 & & otherwise & & & & & & & & & & & & 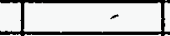 & & & \\
\hline 5474 & & 1 & 79 & & & & & & & & & & & & & & & \\
\hline 5075 & & & Dummy & & & & & & & & & & & & & & & \\
\hline 5976 & 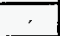 & & ADD & & & & & & & & & & & & & & & \\
\hline 5077 & & & GETHAESH & 1 & 188.0 & & & & & & & & & 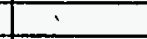 & & & & \\
\hline 5078 & & & Large comp & parison par & ameter for & ces assignn & ent to bran & $\operatorname{nch} 2, \mathrm{no}$ & uplosive co & ncentratlon & & & & & & & & \\
\hline 5079 & 174 & Doe: & s a damagt & ng delonall & Ion/deflagra & aflon occur & In the Pump & op PII Sludg & Tank? & & & & & & & & & \\
\hline 548 & & 3 & PPSIDel & PPSTREI & nPPSIEHP & & & & & & & & & & & & & \\
\hline 5081 & & 2 & 1 & 2 & 3 & & & & & & & & & & & & & \\
\hline 5082 & & 4 & & & & & & & & & & & & & & & & \\
\hline 5083 & & 1 & 173 & & & & & & & & & & & & & $\therefore$ & & \\
\hline 5984 & & & 2 & & & & & & & & & & & & & & & \\
\hline 5085 & & & nConPPSI & & & & & & & & 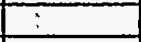 & & & & & & & \\
\hline 5186 & & & 0.088 & -8.080 & 1.080 & & & & & & & & & & & & & \\
\hline 5187 & 8 & 1 & 172 & & & & & & & & & & & & & & & \\
\hline 5088 & & 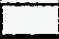 & $I$ & & & & & & & & & & & & & & & \\
\hline
\end{tabular}


APPENDIH A

ACCIDENT PROGRESSION EUENT TREE FOR DEFENSE WRSTE PROCESSING FACILITY - MODE C - PRODUCTION UERSION

WSAC-TR-95-811
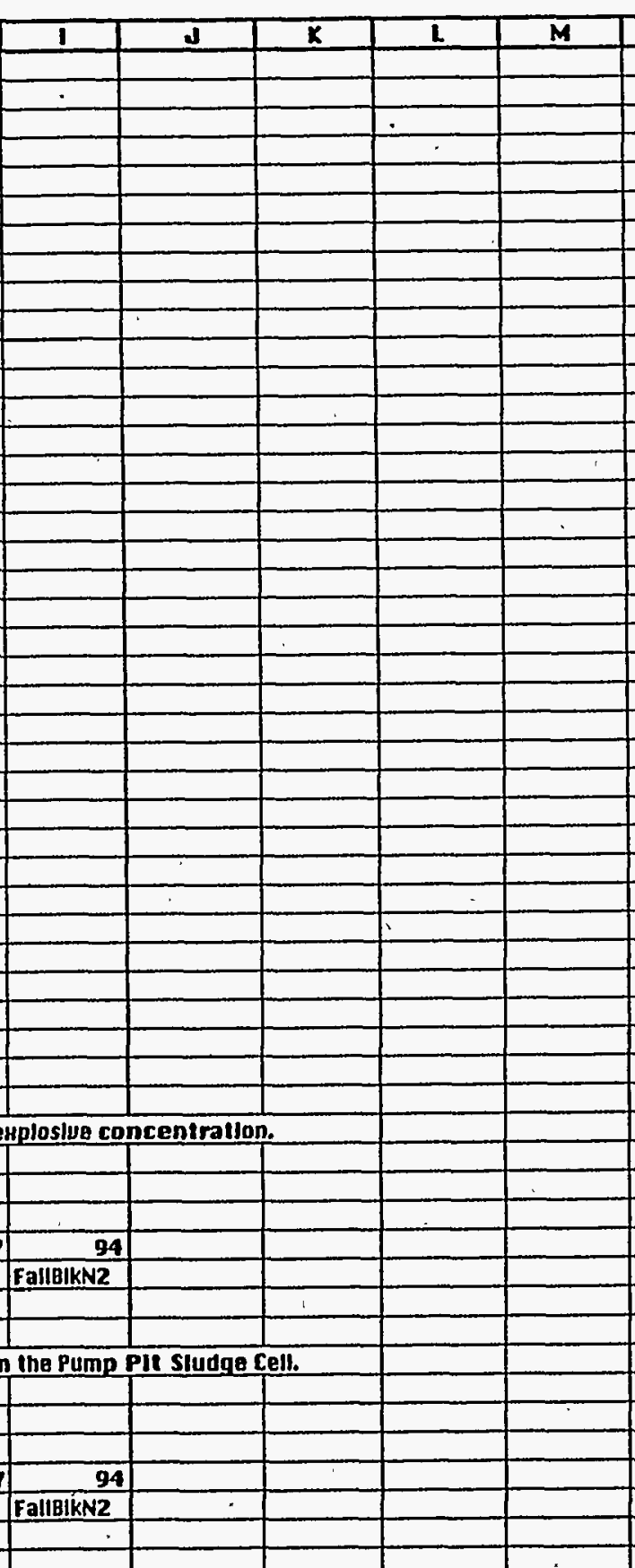

\begin{tabular}{|c|c|c|c|c|c|c|c|c|c|c|c|c|c|c|c|c|c|c|}
\hline & A & 2 & $\bar{c}$ & $D$ & $\underline{E}$ & $F$ & E & $\mathrm{H}$ & 1 & J & $x$ & L & $M$ & $N$ & D & $P$ & n & A \\
\hline 5289 & & & BpIPPST & & & & & & & & & & & & & & & \\
\hline 5090 & & & 0.080 & 0.000 & 1.008 & & & & $\therefore$ & & & & & & & & & $\dot{-}$ \\
\hline 5091 & 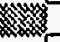 & 2 & 173 & 172 & & & & & & & & $\therefore$ & & & & & & \\
\hline 5092 & & & 1 & 2 & & & & & & & & $\therefore$ & & & & & & \\
\hline 5493 & & & ConPPST & nAptPPST & & & & & & & & & & & $\because$ & & & \\
\hline 5994 & & & e.e01 & 0.999 & 0.000 & & & & & & & & & & & & & \\
\hline 5095 & 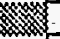 & & Oinerwise & & & & & & & & & & & & & & $\because$ & \\
\hline 5096 & & & 0.008 & 0.880 & 1.018 & & & & & & & & & & & & & \\
\hline $5=97$ & 175 & Does & splashing & Ispillagel of & the Pump & PII sludge 7 & Tank conter & its occur? & & & & & & & & & & \\
\hline 5098 & & 2 & PPSTSPI & nPPSTSpl & & & & & & & & & & - & & & & \\
\hline 5eg9 & & 2 & 1 & 2 & & & & & & & & & & & & & & \\
\hline 5180 & & 4 & - & & & & & & & & & & & & & & & \\
\hline 5101 & 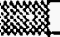 & 2 & 174 & 172 & & & & & & & & & & & & & & \\
\hline 5182 & & & 3 & 2 & & & & & & & & & & & & & & \\
\hline 5103 & & & nPPSIEYP & ARPIPPST & & & & & & & & & & & & & & \\
\hline 5184 & & & $\begin{array}{r}0.088 \\
\end{array}$ & 1.089 & & & & & & & & & & & & & & \\
\hline 5185 & 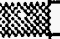 & 1 & 172 & & & & & & & & & & & & & & & \\
\hline 518 & & & 1 & & & & & & & & & & & & & & & \\
\hline 5107 & & & HPIPPSI & & & & & & & & & & & & & & & \\
\hline 5180 & & & 1.880 & 0.880 & & & & & & & & & & & & & & \\
\hline 5189 & 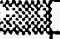 & 1 & 174 & & & & & & & & & & & & & & & \\
\hline 5110 & & & 1 & & & & & & & & & & & & & & & \\
\hline 511 & & & PPSTDEI & & & & & & & & & & & & & & & \\
\hline 5112 & & & 1.808 & 8.898 & & & & & & & & & & & & & & \\
\hline $5 ! 13$ & 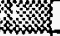 & & Otherwlse & & & & & & & & & & & & & & & \\
\hline 5114 & & & 2.88日 & $1.8 \mathrm{BB}$ & & & & & & & & & & & & & & \\
\hline 5115 & 176 & Does & 5 an erploslu & Ue concent & rallon accur & mulate in P & ump PII SIU & Idge Cell? & & & & & & & & & & \\
\hline 5116 & & 2 & ConpPSC & nconPPSC & & & & & & & & & & & & & & \\
\hline 5112 & & 6 & 1 & 2 & & & & & & & & & & & & & & \\
\hline 5118 & & 4 & & & & & & & & & & & & & & & & \\
\hline 5119 & 慈 & 2 & 1 & 1 & & & & & & & & $E$ & & & & & & \\
\hline 5120 & & & $1-1$ & -21 & & & & & & & & & & & & & & \\
\hline 5121 & & & |Enternal & /Int-Eg4 & & & & & & & & & & & & & & \\
\hline 5122 & & 1 & 79 & & & & & & & & & & & & & & & \\
\hline 5123 & & & Dummy & & & & & & & & & & & & & & & \\
\hline 5124 & & & ADDD & & & & & & & & & & & & & & & \\
\hline 5125 & & & GETHAESH & 1 & 180.0 & & & & & & & & & & & & & \\
\hline 5126 & & & Large comp & parison par & ameter forc & ces assignn & nent lo bra & nch 2, no e & Hploslue Co & ncentrallo & & & & & & & & \\
\hline 5127 & 嬨 & 1 & 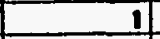 & & & & & & & & & & & & & & & \\
\hline 5128 & & & 2 & & & & & & & & & & & & & & & \\
\hline 5129 & & & Int-Egy & & & & & & & & & & & & & & & \\
\hline 5130 & & 7 & 69 & 61 & 78 & 71 & 98 & 67 & 94 & & & & & & & & & \\
\hline 5131 & & & FallNPwr & Fallepwr & Miss IIme & \begin{tabular}{|l|} 
ncommI \\
\end{tabular} & LHS & FaliN2TK & fallolkN2 & & & & & & & & & \\
\hline 5132 & & & FUN-PPSC & & & & & & & & 1 & & & & & & & \\
\hline 5133 & & & EOUAL & 0 & & & & & & & & & & & & & & \\
\hline 5134 & & & UFUN-PPSC & will calcula & ale probabl & IIty of eHpl & oslue conce & miratlon in & the Pump & PIt Sludge & Cell. & & & & & & & \\
\hline 5135 & 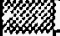 & 1 & 1 & & & & & & & & & & & & & & & \\
\hline 5136 & & & 1 & & & & & & & & & & & & & & & \\
\hline 5137 & & & Esternal & & & & & & & & & & & & & & & \\
\hline 5138 & & 7 & 68 & 61 & 78 & 71 & 90 & 67 & 94 & & & & & & & & & \\
\hline 5139 & & & FallNPwr & FallEPur & Missnme & \begin{tabular}{|c|} 
ncommi \\
\end{tabular} & LHS & FalIN2TK & FallolikN2 & $\therefore$ & & & & & & & & \\
\hline 5140 & & & FUN-PPSCS & & & & & & & & & & & & & & & \\
\hline 5141 & & & EQUAL & 8 & & & & & & & & & & & & & & \\
\hline
\end{tabular}


APPENBAH $\mathrm{f}$

ACCIDENT PROGRESSION EUENT MAEE FOR DEFENSE UASTE PROCESSING FACILITY - MODE C - PAODUCTION UERSION

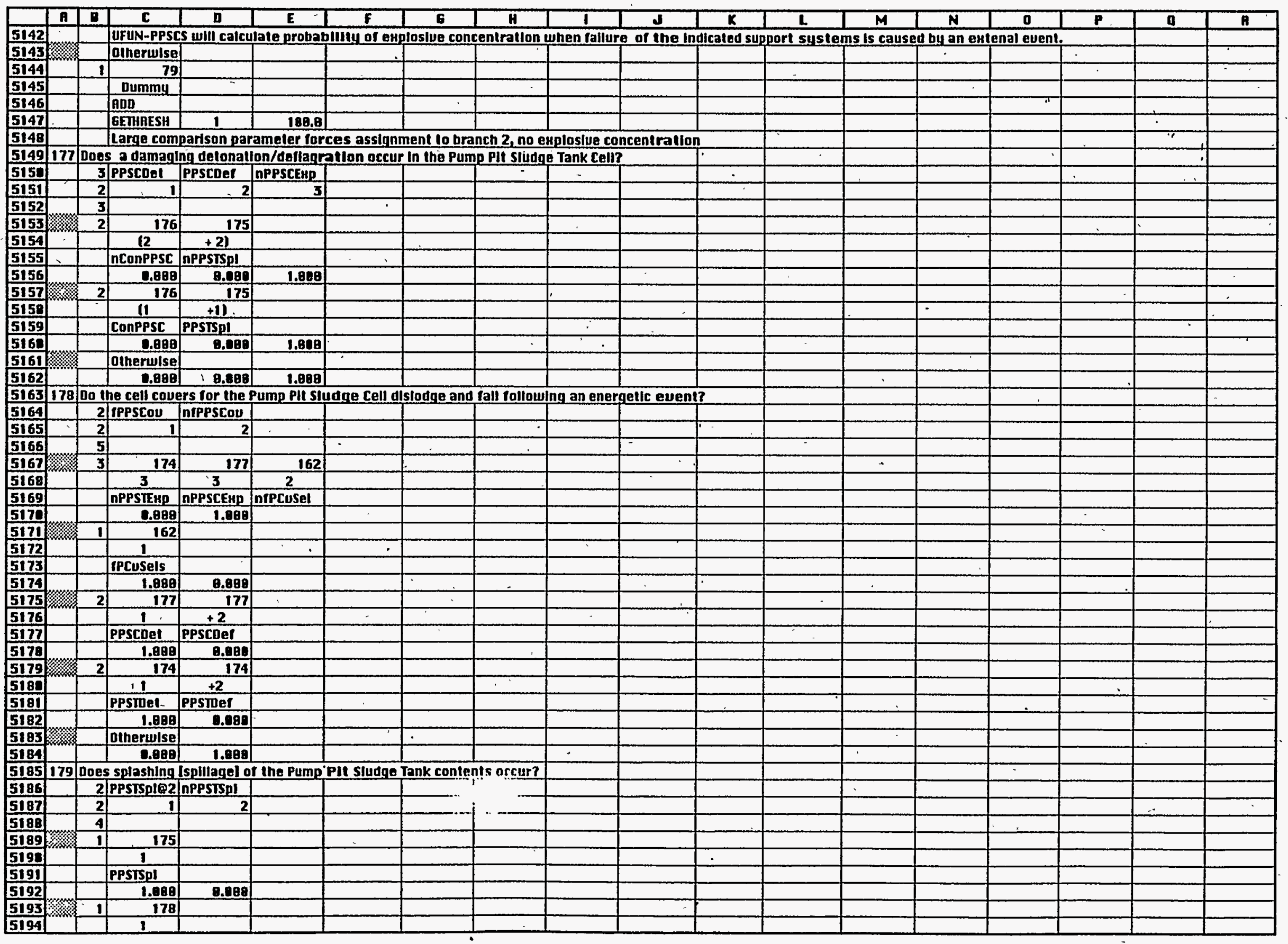


APPENDIH A

ACCIDENT PROgRESSION EUENT TREE FOR DEFENSE WASTE PROCESSING FACILITY - MODE C - PRODUCTION UERSION

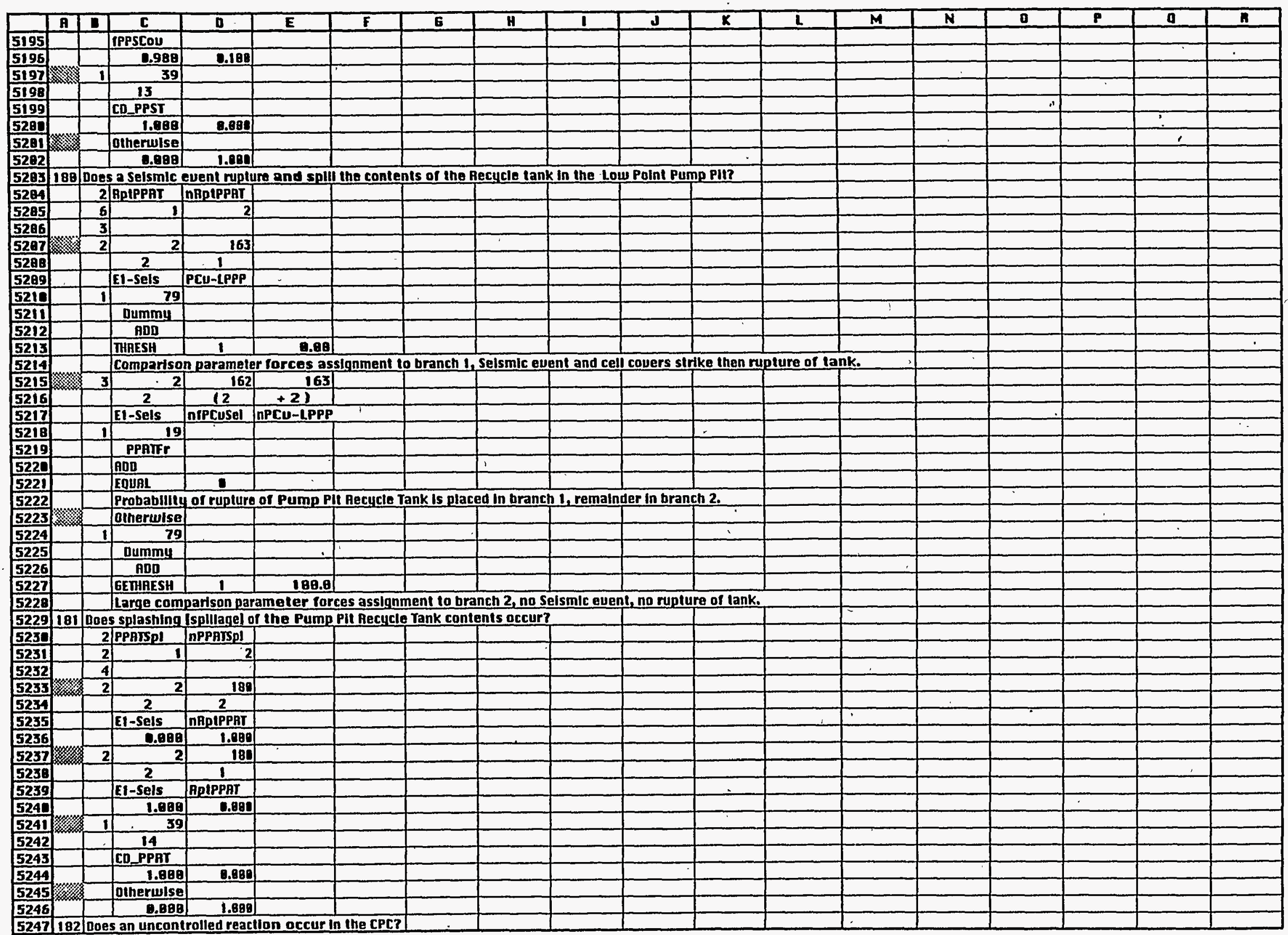




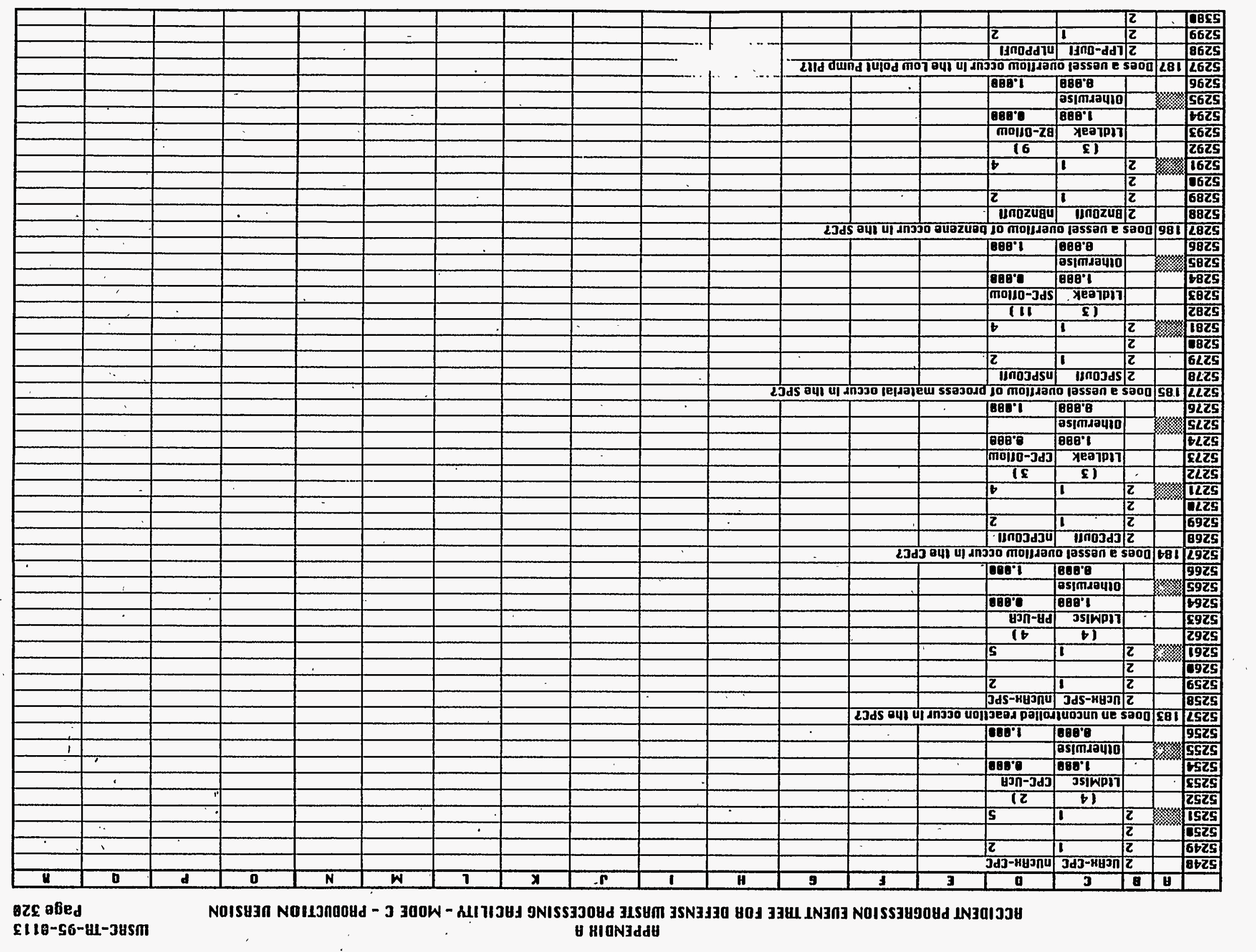


APPENDIH A

ACCIDENT PROGRESSION EUENT TREE FOR DEFENSE WASTE PROCESSING FACILITY - MDDE C - PRODUCTION UERSION

USAC-TH-95-8113

Page 321

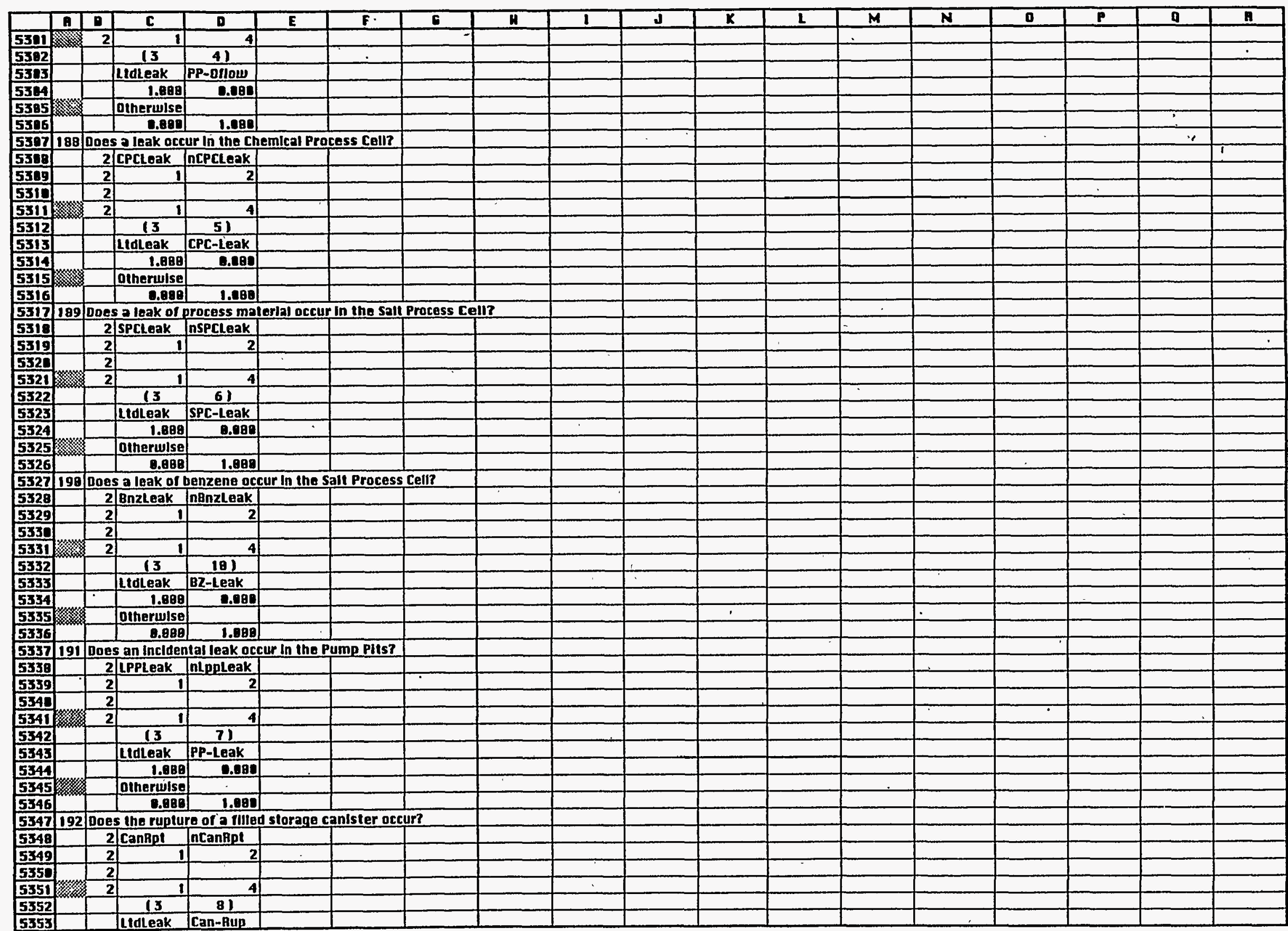




\begin{tabular}{|c|c|c|c|c|c|c|c|c|c|c|c|c|c|c|c|c|c|c|}
\hline & $\overline{\text { A }}$ & $\bar{B}$ & C & D & $\bar{E}$ & $\mathbf{F}$ & G & $\bar{H}$ & 1 & $J$ & $\bar{K}$ & $\mathbf{L}$ & $\bar{M}$ & $\bar{N}$ & D & $p$ & D & $\mathbf{R}$ \\
\hline 5354 & & & 1.098 & 8.800 & & & & 8 & & & & , & & & & & & \\
\hline 5355 & & & Otherwise & & & & & & & & & & & & & & & $\bar{T}$ \\
\hline 5356 & & & 8.080 & 1.088 & & & & & & & & & & & & & & \\
\hline 5357 & 193 & Wha & I Is the sta & tes of the & sentliation & for the Lou & Polnt Pum & p PII Bulld| & $\mathrm{ng?}$ & & & & & & & & & \\
\hline 5358 & & 4 & PPnFn/日r & PPnF/nBr & PPFan/Br & LPPUent & & & & & & & & & 1 & & & \\
\hline 5359 & & 2 & 1 & 2 & 3 & 4 & & - & & & & & & & & & & \\
\hline 5360 & & 7 & & & & & & & & & - & & & & & t. & $\because$ & $\because$ \\
\hline 5361 & 㱛 & 2 & $\overline{2}$ & $\overline{2}$ & & & & & & & . & $=$ & & & & & & \\
\hline 5362 & & & 12 & $+3 \mid$ & 1 & & & & & 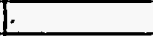 & & & & & & & " & \\
\hline 5363 & & & E1-Sels & E2-Wind & & & & & & & & & & & & & & \\
\hline 5364 & & & 1.080 & 8,080 & 0.808 & 0.898 & & & & & & & & & & . & & \\
\hline 5365 & 㮡 & 8 & 166 & 166 & 169 & 169 & 170 & 3 & B & 9 & & & & & & & & \\
\hline 5366 & & & 111 & +2 & +1 & +21 & 11 & 112 & +11 & 113 & & & & $\therefore$ & & & & \\
\hline 5367 & & & PPPIDel & PPPIDeI & PPPCDel & PPPCDEI & IPPPCOU & NPowInlt & NPOWEn & EPOWEn & & & 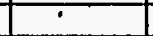 & & & & & \\
\hline 5368 & & & $1.08 \mathrm{~B}$ & 0.080 & 0.088 & 0.080 & & & & & & & & & & & & \\
\hline 5369 & & 8 & 174 & 174 & 177 & 177 & 178 & 3 & $\overline{8}$ & 9 & & & & & & 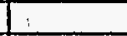 & & \\
\hline 5370 & & & If 1 & +2 & +1 & +21 & 11 & 112 & +11 & 113 & & & & & & & . & \\
\hline 5371 & & & PPSTIDet & PPSIDET & PPSCDet & PPSCDel & IPPSCOU & NPowin!t & NPOWEN & EPOWEN & & & & & & 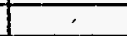 & & \\
\hline 5372 & & & 1.888 & 0.080 & 0.888 & 0.080 & & & & & & & & & & & & \\
\hline 5373 & & $\mathbf{3}$ & 3 & 8 & 9 & 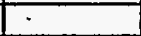 & & & & & & & & & & & & \\
\hline 5374 & & & 112 & +11 & (1) & 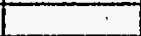 & & & & & & & & & & & & \\
\hline 5375 & & & NPow/nit & NPOWEn & EPOWEn & & & & & & & & & & & & & \\
\hline 5376 & & & Q.988 & $1.8 \mathrm{Bg}$ & $.88 \mathrm{~B}$ & 8.080 & & & & & & & & & & & & \\
\hline 5377 & & 5 & 166 & 166 & 169 & 169 & 178 & & & & & & & & & & & \\
\hline 5378 & & & 111 & +2 & +1 & +21 & (1) & & & & & & & & & & & \\
\hline 5379 & & & PPPYDet & PPPTDef & PPPCDEI & PPPCDEI & IPPPCOU & & & & $\therefore$ & & & & & & & \\
\hline 5380 & & & 0.088 & 0.080 & 1.800 & 0.808 & & & & & & & & & & & & \\
\hline 5381 & 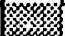 & 5 & 174 & 174 & $: 177$ & 177 & 178 & & & & & & & & & & & \\
\hline 5382 & & & 111 & +2 & +1 & +21 & 11 & & & & & & & & & & & \\
\hline 5383 & & & ppsmet & pPsIDef & Ppscoel & PPSCDEI & IPPSCOU & 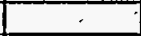 & & & & & & & & & & \\
\hline 5384 & & & 0.888 & 8.808 & 1.080 & $\theta .8 B \theta$ & & & & & & & & & & & & \\
\hline 5385 & 䊽 & & Ditherwise & & & & & & & & & & & & & & & \\
\hline 5386 & & & 0.098 & $\theta .880$ & 8.888 & $1.68 \mathrm{~g}$ & & & & & & & & & & & & \\
\hline 5387 & 193 & & & & & & & & & & , & & & & & & & \\
\hline 5388 & & & & & & & & & $\therefore$ & & & & & & & & & \\
\hline 5389 & & & & & & & & & & & & & & & & & & \\
\hline 5390 & & & & & & & & & & & & & & & & & & \\
\hline 5391 & & & & & & & & & & & & & & & . & & & \\
\hline 5392 & & & & & & & & & & & & & & & & & & \\
\hline 5393 & & & & & & & & & & & & & & & & & & \\
\hline 5394 & & & & & & & & & . & & & & & & & . & & \\
\hline 5 & & & & & & $=$ & & & & & & & & & & & & $\overline{5}$ \\
\hline 5396 & & & & & & & & & & & & & & & & & & \\
\hline 5397 & & & 7 & & & & & & & & & & & & & & & \\
\hline 5398 & Inill & al Ou & Ientificallor & D - Source 1 & Serm Binning & G Flle for DI & UPF-C7, $8 / \mathrm{S}$ & 9/94 & & & & & & & & & - & \\
\hline 5399 & 19 & & OUSTank & PPSTank & PPPTank & PpaTank & PaIank & & & & & & & & & & & \\
\hline 5400 & & & PAFTank & PABTank & SMETank & SAATank & MfTank & & & & & & & & & & & \\
\hline 5491 & & & BCrank & Melter & SPC-DEI & SPC-FIre & CPCCQU & & & & & & & & & & & \\
\hline 5402 & & & SPCCOU & Mitcou & Canyuent & LPPPUent & & & & & & & & & & & & \\
\hline 5483 & & & & & & & & & & & & & & & & & & \\
\hline 5484 & 3 & 3 & DUSTDEf & OWS-T/Sp & nOWSTREl & & & & & & & Sorganic L & Vaste Tank & & 1 & & & \\
\hline 5485 & 1 & 1 & 168 & & & & & & & & & & & & & & & \\
\hline 5486 & & & 1 & & & & & & & & & & & & & & & \\
\hline
\end{tabular}




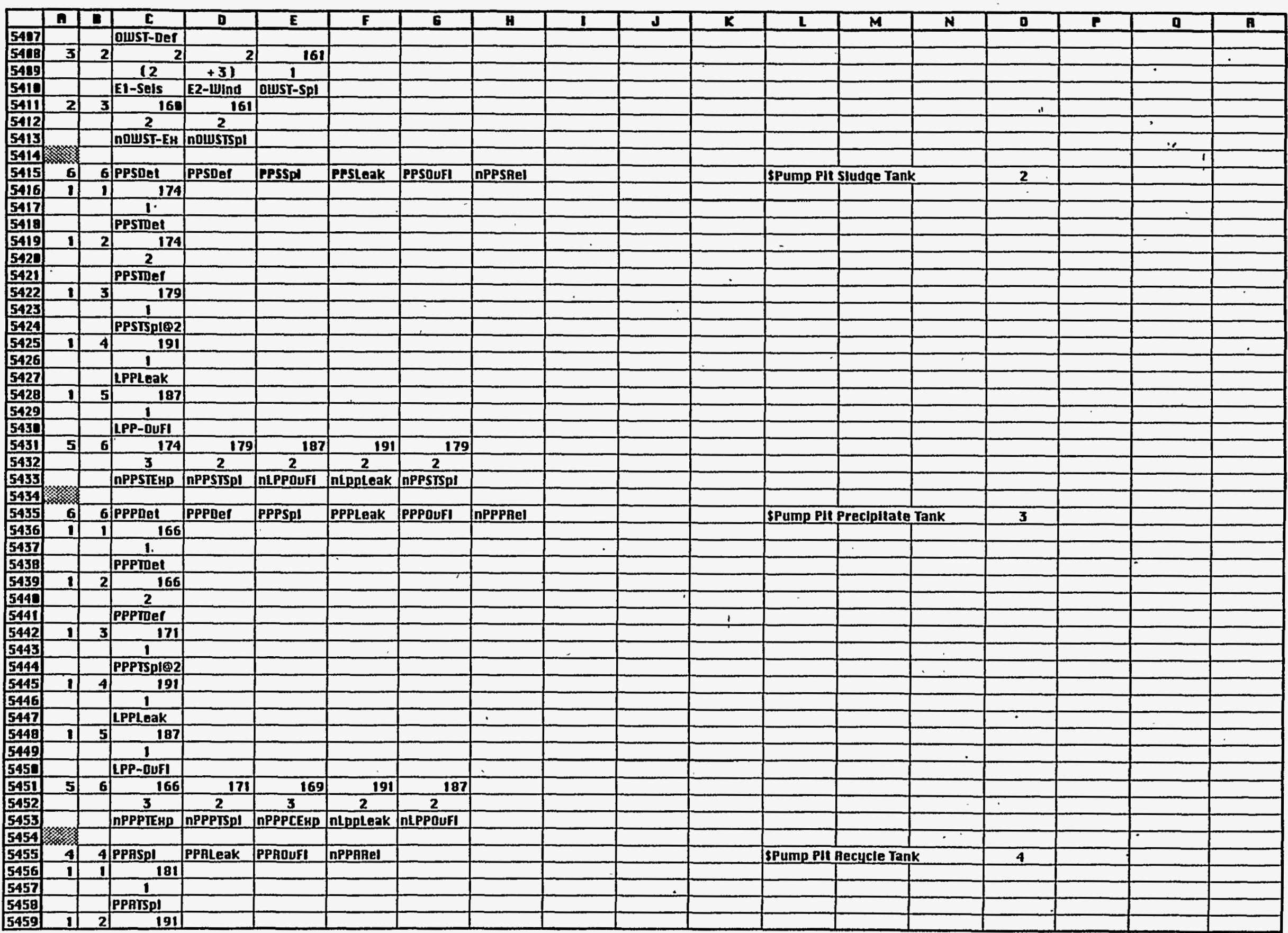


APPENDIH

ACCIDENT PROGAESSION EUENT TREE FOR DEFENSE WASTE PAOCESSING FACILITY - MODE C - PAODUCTION UEASION

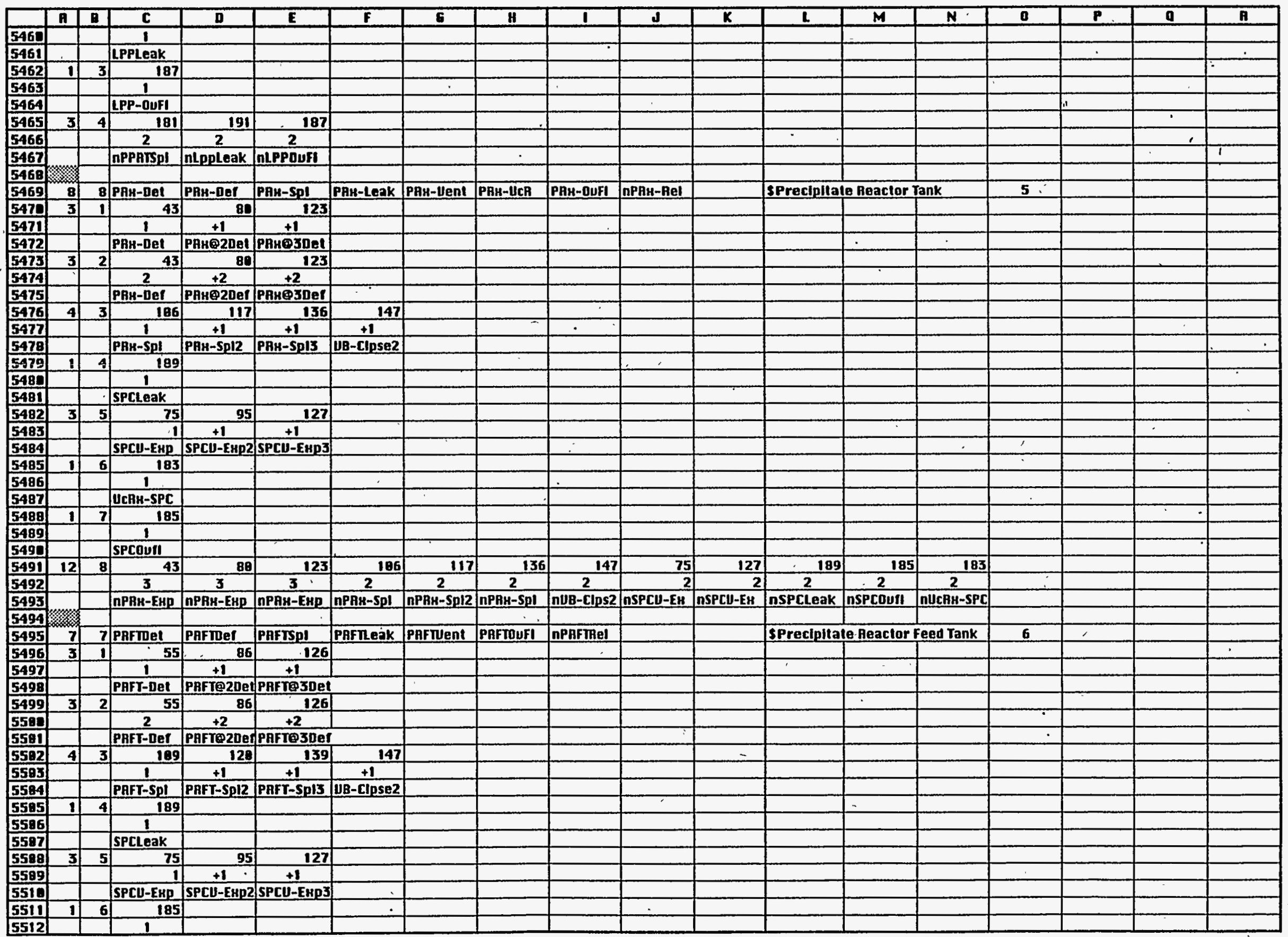




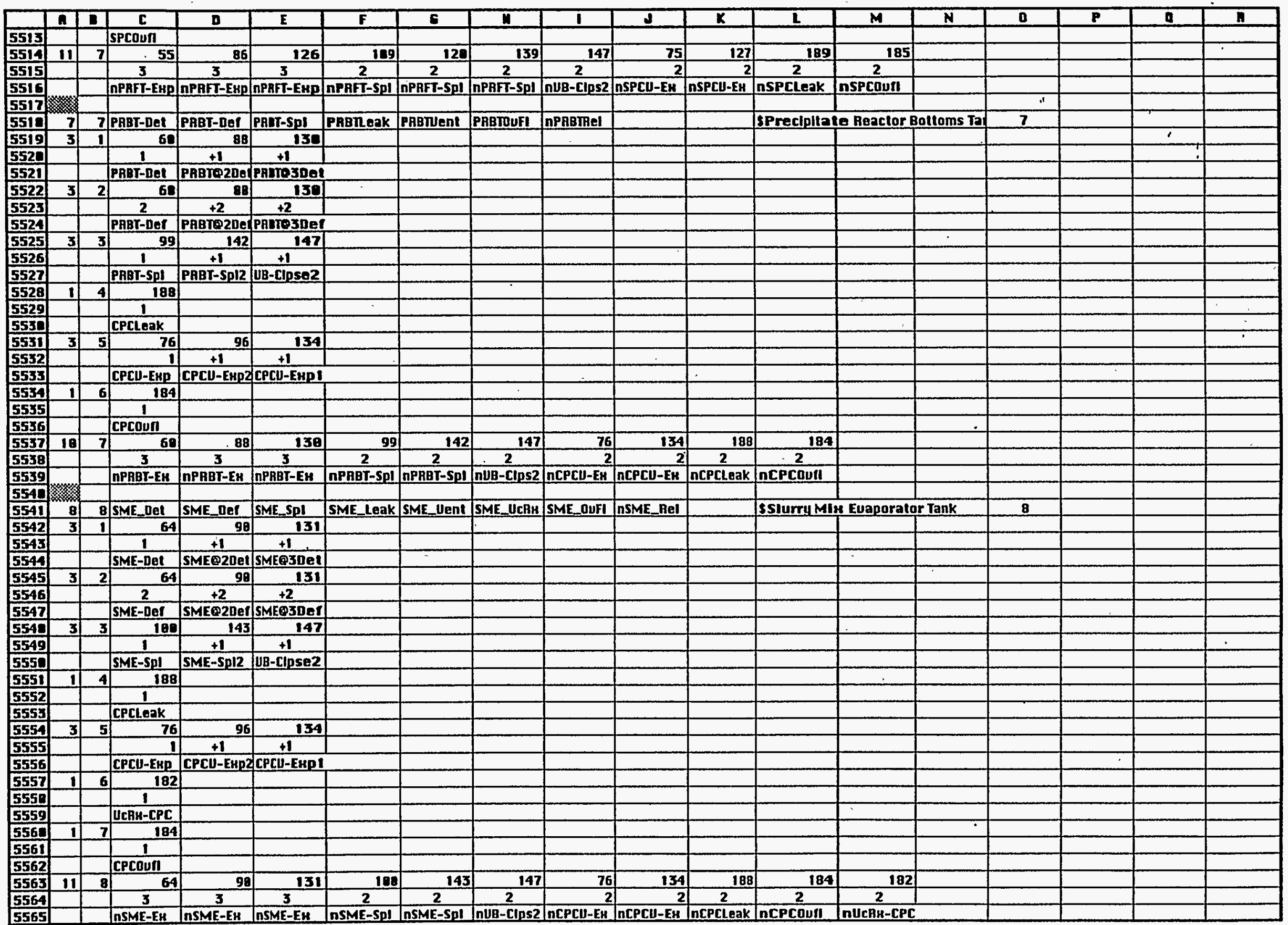


GPPENDIH A

ACCIDENT PROGRESSION EUENT TREE FOR DEFENSE WASTE PROCESSING FACILITY - MOOE C - PAODUCTION UERSION

UUSAC-Th-95-8113

Page 326

\begin{tabular}{|c|c|c|c|c|c|c|c|c|c|c|c|c|c|c|c|c|c|c|}
\hline & A & E & $\boldsymbol{c}$ & $\mathbf{D}$ & $\bar{E}$ & $F$ & 6 & H & 1 & $d$ & $\underline{K}$ & L & $M$ & $N$ & 0 & $P$ & a & H \\
\hline 5566 & & & & & & & & & & & & & & & 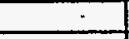 & & & \\
\hline 5567 & 8 & 8 & Shamel & SAATEA & SAATSPI & SBAn.eak & SARTEnt & SRATUCAH & SRAJOUFI & nSARIRel & & \multicolumn{3}{|c|}{ ssludge Recelpt and fdjustment } & 9 & & & $\div$ \\
\hline 5568 & 3 & 1 & 68 & 92 & 132 & & & & & & & & & & & & . & \\
\hline 5569 & & & 1 & +1 & +1 & & & & & & & & & & & & & - \\
\hline 5570 & & & SAAT-Det & SAATE2DEE & SBAMOZDet & & & & & & & & & $\div$ & $\therefore$ & & & \\
\hline 5571 & 3 & 2 & 68 & 92 & \begin{tabular}{r|}
132 \\
\end{tabular} & & & & & & & & & & & & & \\
\hline 5572 & & & 2 & +2 & +2 & & & & & & & & & & $\sigma$ & & I & \\
\hline 5573 & & & SABT-DEI & SARTE2DEI & SARTO5Del & & & & & & & & & & $\therefore$ & & & \\
\hline 5574 & 3] & 3 & 181 & 144 & 142 & & & & & & & & & & & & & \\
\hline 5575 & & & 1 & +1 & +1 & & & & & & & & & & & & & \\
\hline 5576 & & & SART-SDI & SARI-Spl2 & UB-CIpse2 & & & & & & & & & & & & & \\
\hline 5572 & 1 & -4 & 188 & & & & & & & & & & & & & & & \\
\hline 5578 & & & $I$ & & & & & & & & & & & & & & & \\
\hline 5579 & & & CPCLEak & & & & & & & & & & & & & & & \\
\hline 5580 & 3 & 5. & 76 & 96 & 134 & & & & & & & & & & & & & \\
\hline 5580 & & & I & +1 & +1 & & & & & & & & & & & & & $\div$ \\
\hline 5582 & & & CPCU-EYP & CPCU-Еяр2 & CPCU-EHPI & & & & & & & & & & & & & \\
\hline 5583 & 1 & 6 & 182 & & .7 & & & & & & & & & & & & & \\
\hline 5584 & & & 1 & & & & & & & & & & & & & & & \\
\hline 5585 & & & UСАН-СРC & & & & & & & & & & & & & & & \\
\hline 5586 & 1 & 7 & 184 & & & & & & & & & & & & & & & \\
\hline 5587 & & & 1 & & & & & & & & & & & & & & & \\
\hline $558 \mathrm{~B}$ & & & CPCOUI & $\therefore$ & & & & & & & & - & & & & & & \\
\hline 5589 & 11 & 8 & 68 & 92 & 132 & 181 & 144 & 147 & 76 & 134 & 188 & 184 & 182 & & & & & \\
\hline 5590 & & & 3 & 3 & 3 & 2 & 2 & 2 & 2 & 2 & 2 & 2 & 2 & & & & & \\
\hline 5591 & & & ПSARAT-EH & ПSAAT-EH & ПSRAT-EH & nSRAT-Spl & nSART-Spl & \begin{tabular}{|l|} 
nUB-CIps2 \\
\end{tabular} & nCPCU-EH & nCPCU-EK & ncPCleak & nCPCOUf & nUCAR-CPC & & & & & \\
\hline 5592 & & & & & & & & & & & & & & & & & & , \\
\hline 5593 & 7 & 7 & MFT-Del & MFT-DeI & MFT-Spl & MFT-Leak & MFT-Uent & MFT_OUFI & nMFTRel & & & sMelter Fe & ed Tank & & 18 & & & \\
\hline 5594 & 3 & 1 & 74 & 94 & 133 & & & & & & & & & & & $\therefore$ & & \\
\hline 5595 & & & 1 & +1 & +1 & & & & & & & & & & & & & \\
\hline 5596 & & & MFT-Det & MFTQ2Det & MFT@30et & & & & & & & & & & & & & \\
\hline 5597 & 3 & 2 & 74 & 94 & 133 & & & & & & & & & & & & & \\
\hline 5598 & & & 2 & +2 & +2 & & & & & & & & & & & & & \\
\hline 5599 & & & MFT-DEI & MFTQ2DE1 & MFT@3Der & & & & ? & & & & & $i$ & & & & \\
\hline 5680 & 3 & 3 & 183 & 146 & 147 & & & & & & & & & & & & & \\
\hline 5681 & & & 1 & +1 & +1 & & & & & & & & & & & & & \\
\hline 5602 & & & MFT-Spl & MFT-Spl2 & UB-CIpse2 & & & & & & & & & & & & & \\
\hline 56893 & 1 & 4 & 188 & & & & & & & & & & & & & & & \\
\hline 5684 & & & 1 & & & & & & & & & & & $\dot{-}$ & & & & \\
\hline 5685 & & & CPCLeak & & & & & &. & & & & & & & & & \\
\hline 5686 & 3. & 5 & 76 & 96 & 134 & & & & $\therefore$ & & & & & & & & & \\
\hline 5687 & & & 1 & +1 & +1 & & & & & & & & & & & & & \\
\hline 5680 & & & СРCU-ЕНP & СРСИ-ЕнР2 & CPCU-EHPI & & & & & & & & & & & & & \\
\hline 5689 & 1 & 6 & 184 & & & & & & & & & & & & & & & \\
\hline 5610 & & & 1 & & & & & & & & & & & & & & & \\
\hline 5611 & & & CPCOUn & & & & & & & & & & & & & & & \\
\hline 5612 & 18 & 7 & 74 & 94 & 135 & 183 & 146 & 147 & 76 & 134 & 188 & 184 & & & & & & \\
\hline 5613 & & & 3 & 3 & 3 & 2 & 2 & 2 & 2 & 2 & 2 & 2 & & & & & & \\
\hline 5614 & & & nMFT-EH & IMFT-EH & nMFT-ER & nMFT-Spl & nMFT-Spl & nUB-CIps2 & ПСРСU-ЕH & nCPCU-EH & nCPCLeak & nCPCOUN & & & & & $\because$ & \\
\hline 5615 & & & & & & & & & & & & & & & & & & \\
\hline 5616 & 6 & 6 & |ACI_SpI & ACT_Leak & BCT_Uent & BCT_UCAH & ACT_OUFI & nACTHEI & & & & SRecycle Co & olleclion $\mathrm{Ta}$ & & 11 & & & \\
\hline 5617 & 3 & 1 & 102 & 145 & 147 & & & & & & & & & & & & & \\
\hline 5618 & & & 1 & +1 & \pm 1 & & & & & & & & & & & & & \\
\hline
\end{tabular}


APPENDIH A

ACCIDENT PRDGRESSION EUENT TREE FOR DEFENSE UASTE PROCESSING FACILITY - MODE C - PRODUCTION UERSION

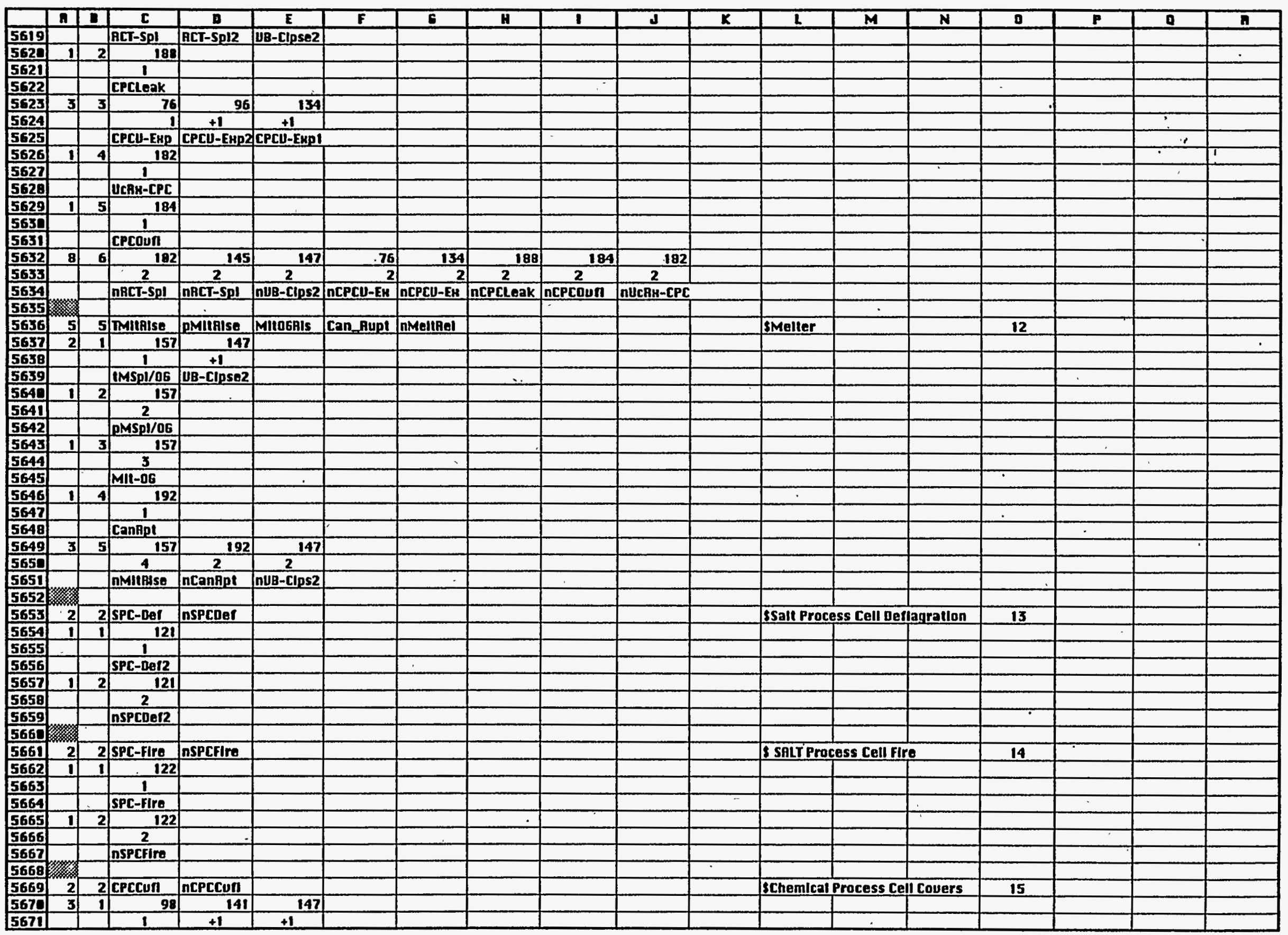


APPENDIS $\mathrm{A}$

ACCIDENT PROgRESSION EUENT TREE FOR DEFENSE WASTE PROCESSING FACILITY - MODE C - PRODUCTION UERSION

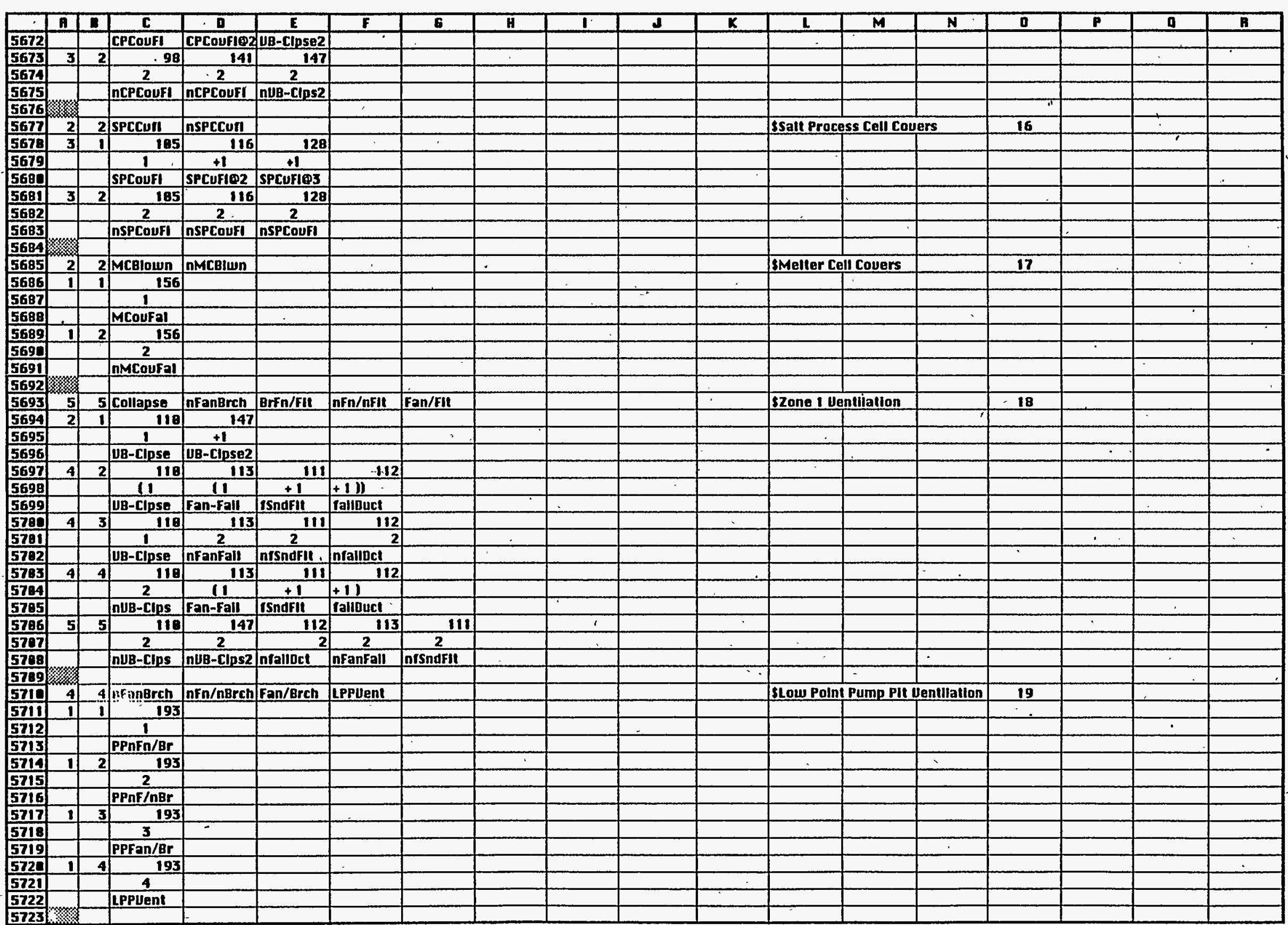

